\title{
SAUERSTOFFISOTOPE ZUR KLÄRUNG DER HERKUNFT NICHTMETALLISCHER AUSSCHEIDUNGEN (CLOGGING) BEIM STRANGGIESSEN VON STAHL
}

\author{
Dissertation \\ zur \\ Erlangung des Doktorgrades \\ Doctor rerum naturalis (Dr. rer. nat.) \\ der \\ Mathematisch-Naturwissenschaftlichen Fakultäten \\ der \\ Georg-August-Universität zu Göttingen
}

vorgelegt von

Christoph Toulouse

aus Aachen

Göttingen 2007 
D 7

Referent: Prof. Dr. Andreas Pack

Korreferent:Prof. Dr. Matthias Göbbels

Tag der mündlichen Prüfung: 20.12.2007 
Sauerstoffisotope zur Klärung der Herkunft nichtmetallischer Ausscheidungen (Clogging) beim Stranggießen von Stahl

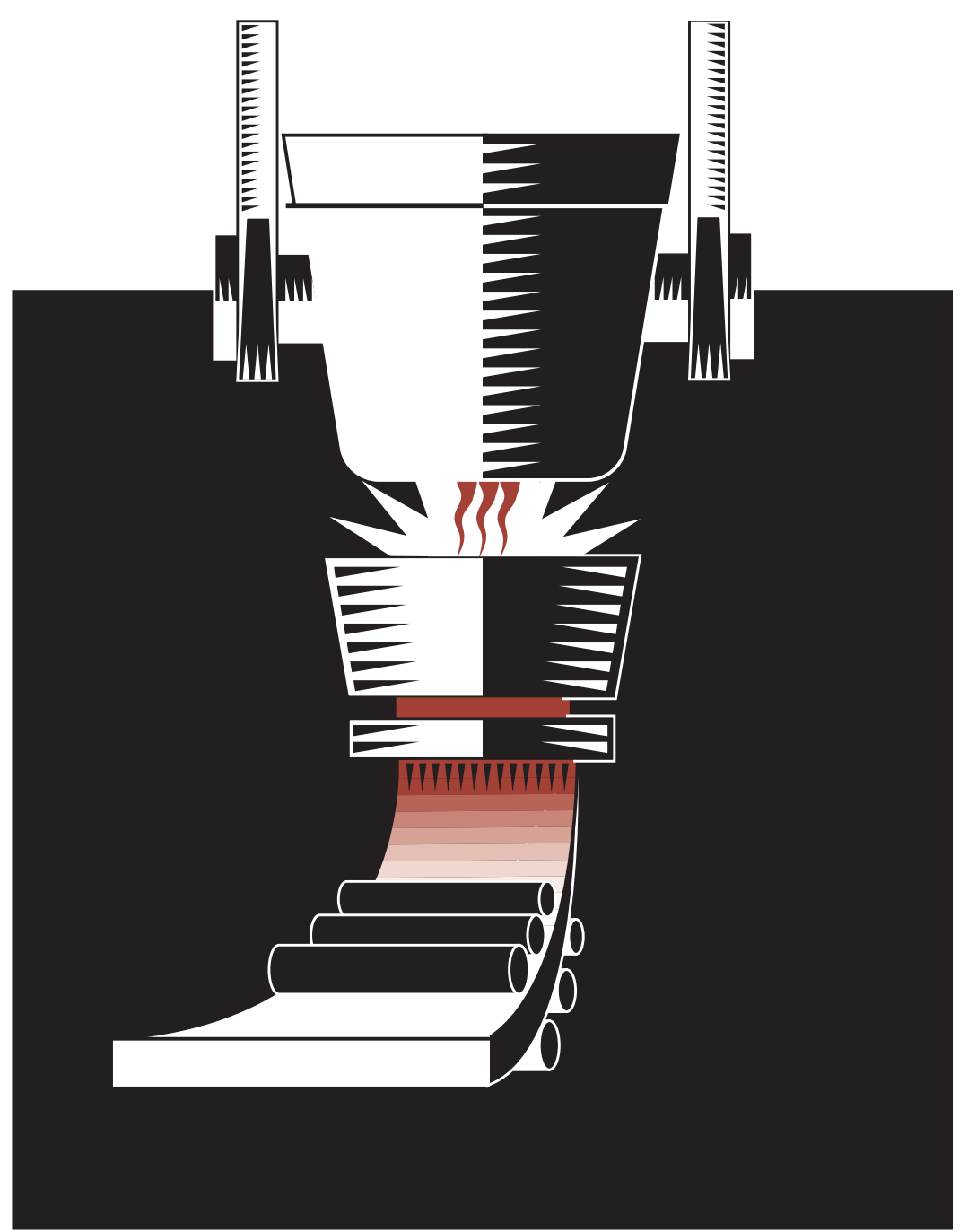

US DEPARTMENT OF ENERGY (2007) 
Diese Arbeit ist meinen Eltern

Christine Toulouse-Lingnau und Denis Toulouse gewidmet. 


\section{Vorwort}

Die vorliegende Arbeit entstand im Rahmen des von der ThyssenKrupp Steel AG geförderten Forschungsprojektes Isotopensignatur. Ziel war die Klärung der Herkunft nichtmetallischer Ausscheidungen bei der Stahlherstellung. Das Projekt erfolgte in enger Zusammenarbeit mit der ThyssenKrupp Steel AG, die sämtliche untersuchte Proben zur Verfügung stellte. Die Kooperation mit mehreren Universitäten und einem Forschungsinstitut ermöglichte den raschen Fortschritt des Projektes. Isotopenmessungen wurden an der Georg-August-Universität Göttingen, an der Ruhr-Universität Bochum und am Consiglio Nazionale delle Ricerche (CNR) in Pisa (Italien) durchgeführt. Hochtemperaturexperimente erfolgten an der FriedrichAlexander-Universität Erlangen-Nürnberg und kristallographische Untersuchungen an der Leibniz Universität Hannover.

Allen Mitarbeitern der Abteilung Qualitätswesen und Verfahrenstechnik der ThyssenKrupp Steel AG sei an dieser Stelle ausdrücklich für Ihre Mithilfe gedankt, ohne die diese Arbeit nicht möglich gewesen wäre, insbesondere Herrn Dr. Stefan Petry, Herrn Dr. Alfred Ender, Herrn Dipl.-Ing. René Krieg, Herrn Günter Merschjohann und Herrn Kemal Kahraman.

Herrn PD Dr. Thorsten Gesing aus Hannover sei für die Hilfe bei der Rietveldanalyse gedankt. Sowohl Frau Dr. Ulrike Schulte aus Bochum als auch Herrn Dr. Luigi Dallai aus Pisa danke ich für die Ermöglichung von Isotopenmessungen.

Ganz besonders möchte ich mich bei Herrn Prof. Dr. Matthias Göbbels für die Übernahme des Korreferates danken, ferner für die Bereitstellung seiner Laborräume zur Durchführung der Hochtemperaturexperimente und für die sehr angenehme Unterbringung während meiner Zeit in Erlangen. Bei den Experimenten stand er mir zu jeder Zeit - auch zu später Stunde - mit Rat und Tat zur Seite.

Der größte Dank gilt meinem Doktorvater Herrn Prof. Dr. Andreas Pack für sein Vertrauen, die zahlreichen Hilfestellungen, die ständige Diskussionsbereitschaft sowie die stetige Unterstützung und Förderung. Das geradezu freundschaftliche Verhältnis ermöglichte durch eine außergewöhnlich gute und direkte Kommunikation die sehr erfolgreiche Zusammenarbeit. Weiter möchte ich meiner Familie und meinen Freunden danken, die mich stets unterstützt haben, insbesondere meiner Freundin Laila und meinem besten Freund Christian. Euch allen vielen, vielen Dank und weiterhin Glück auf! 


\section{INHALT}

Vorwort

$1 \quad$ Einleitung ................................................................................................................................. 1

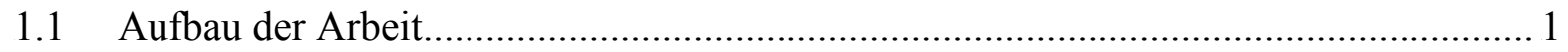

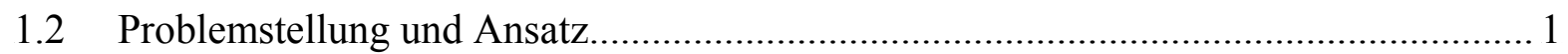

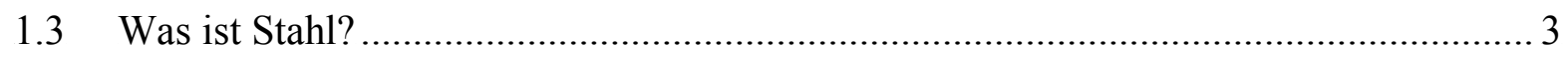

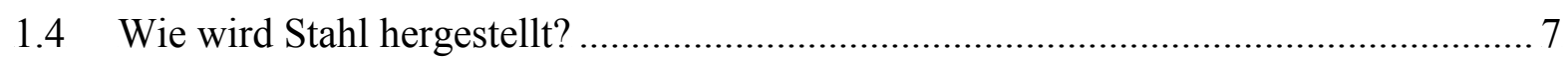

1.5 Nichtmetallische Einschlüsse im Stahl und Clogging............................................. 18

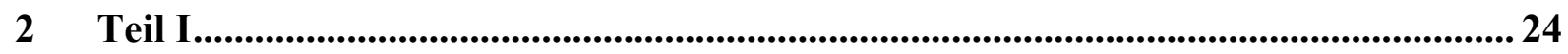

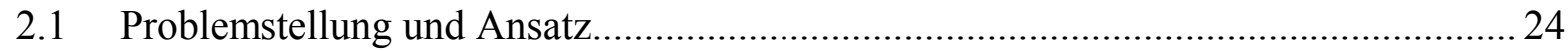

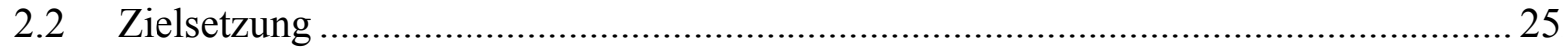

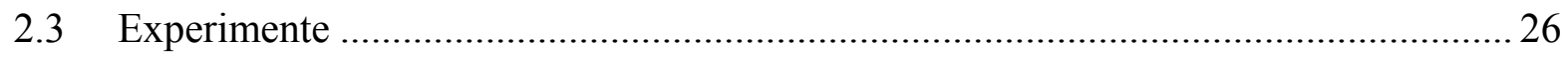

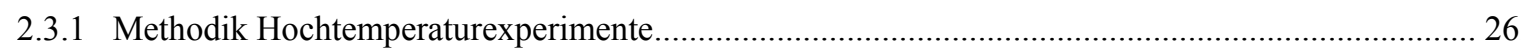

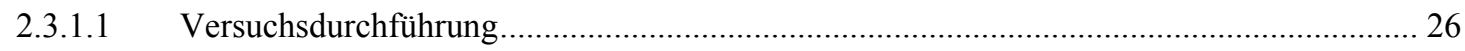

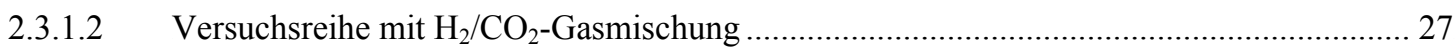

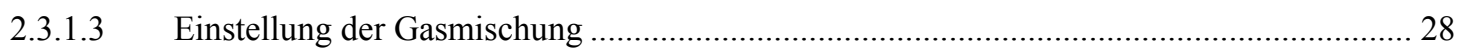

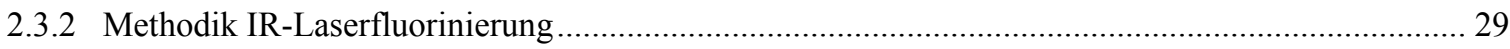

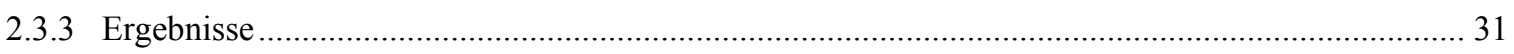

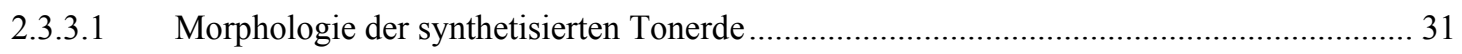

2.3.3.2 Chemische Zusammensetzung der Legierungen.................................................................. 31

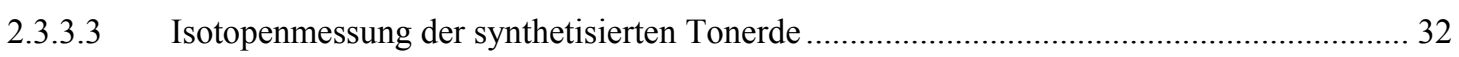

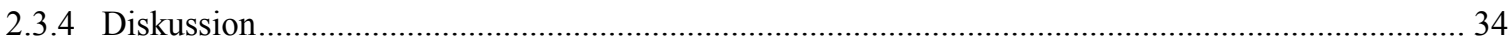

2.4 Analyse von Clogging und Prozessgasen............................................................... 40

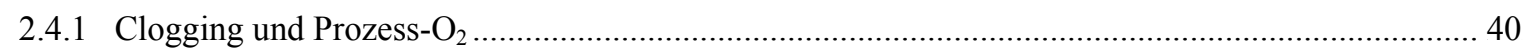

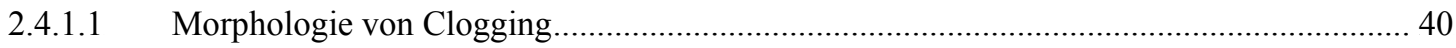

2.4.1.2 Chemische Zusammensetzung von Clogging ................................................................ 41

2.4.1.3 Mineralogische Zusammensetzung von Clogging ............................................................ 42

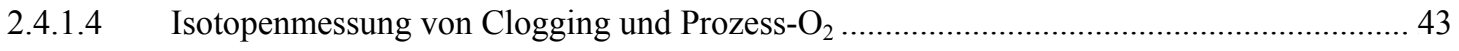

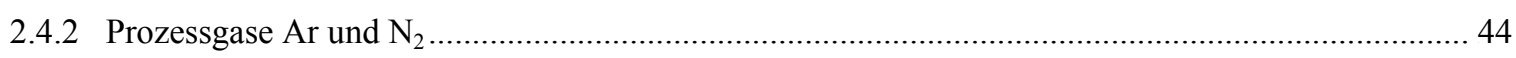

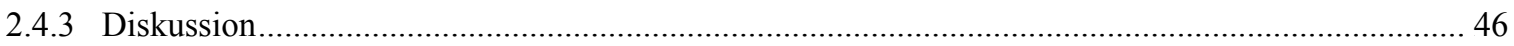

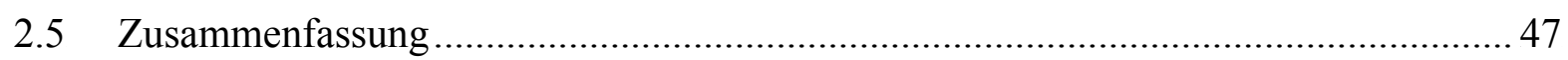

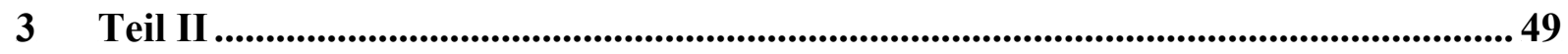

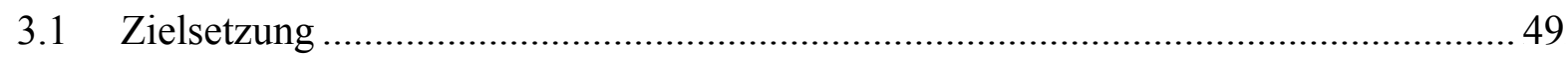

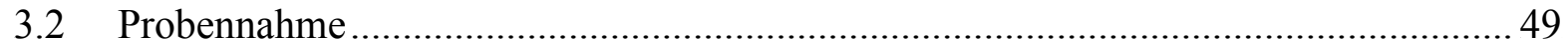

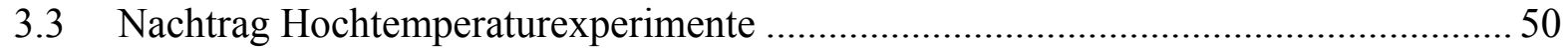

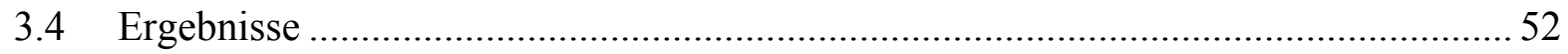

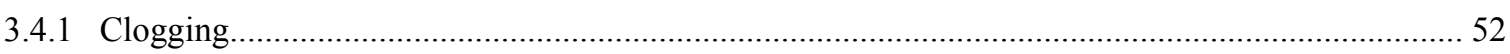

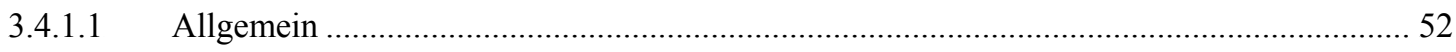

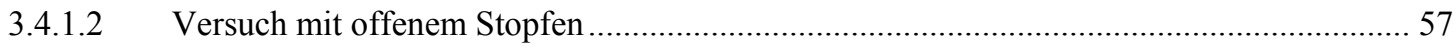




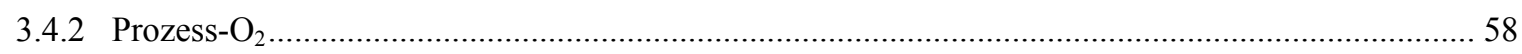

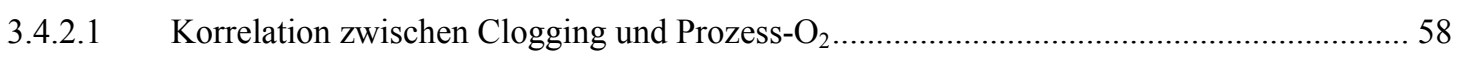

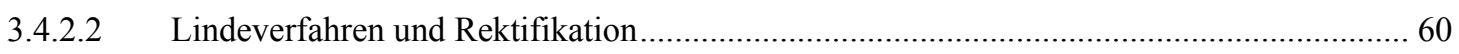

3.4.2.3 Experiment zur Bestimmung der Fraktionierung zwischen GOX und LOX ......................62

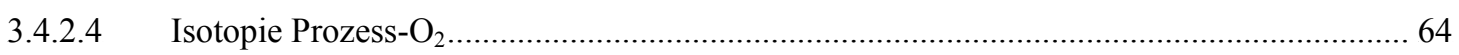

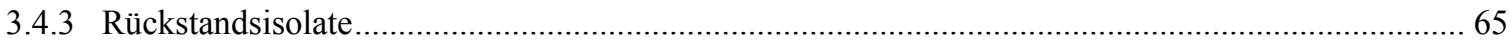

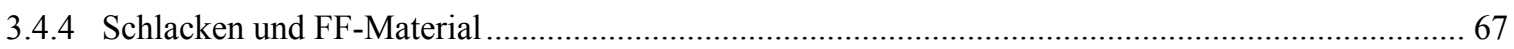

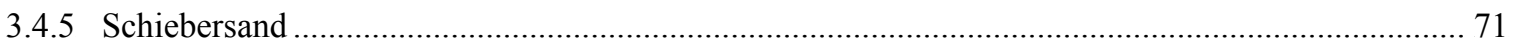

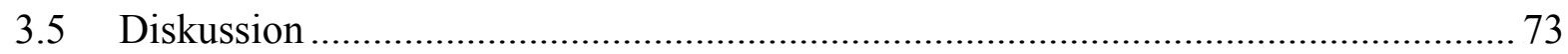

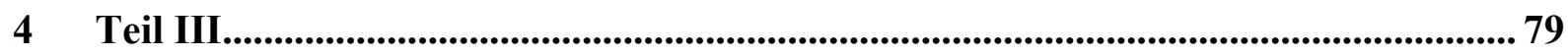

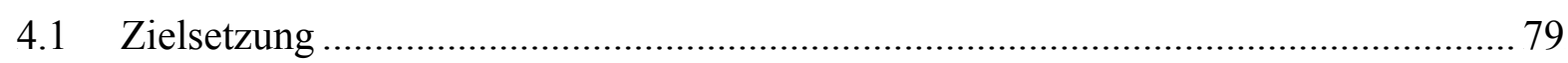

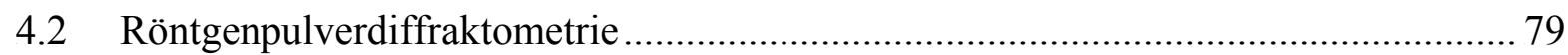

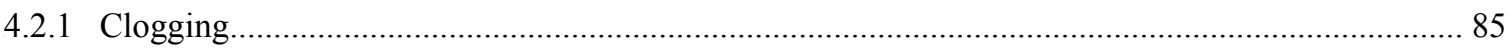

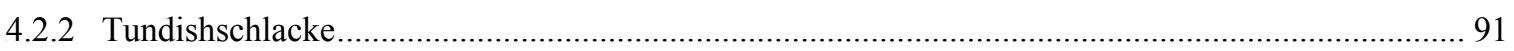

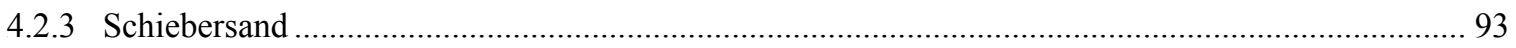

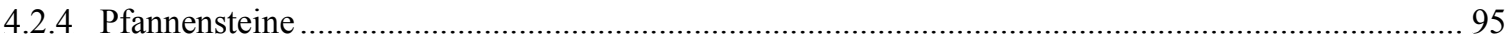

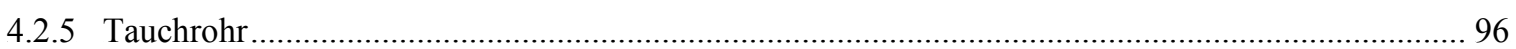

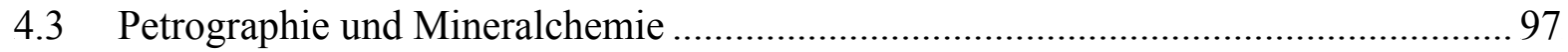

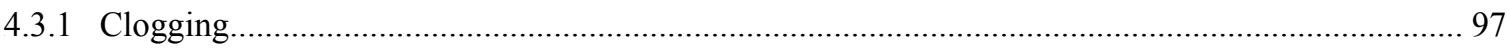

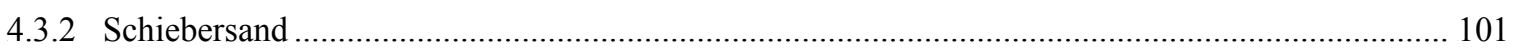

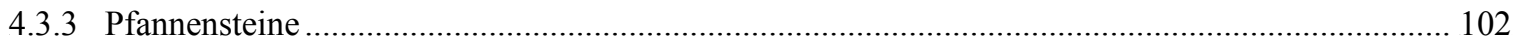

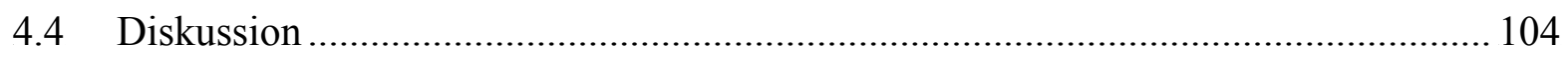

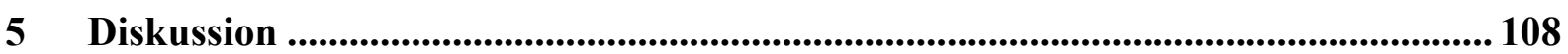

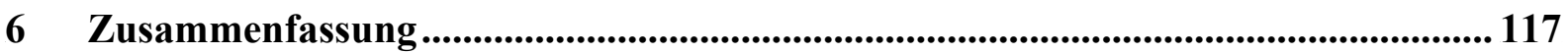

7 Ausblick...................................................................................................................... 118

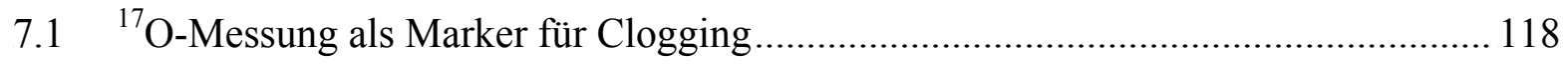

7.2 Weitere Möglichkeiten der Untersuchung von Clogging ……................................. 125

$8 \quad$ Literatur .................................................................................................................... 127

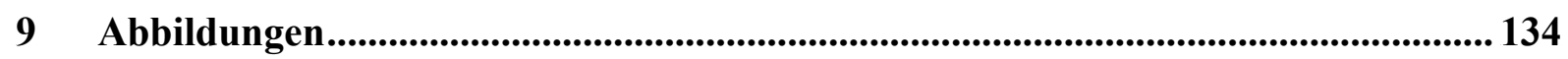

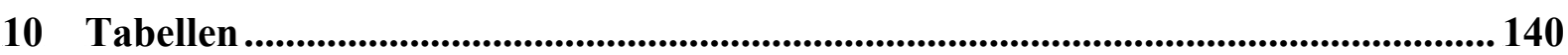

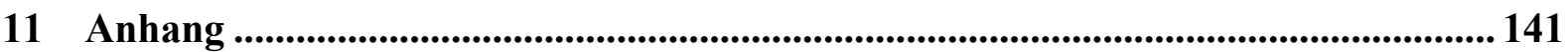

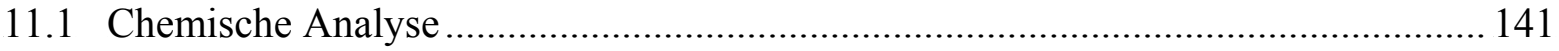

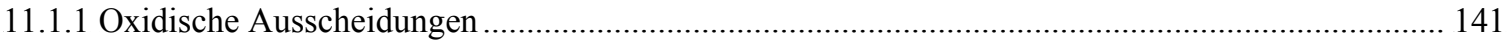

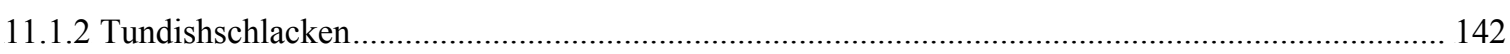

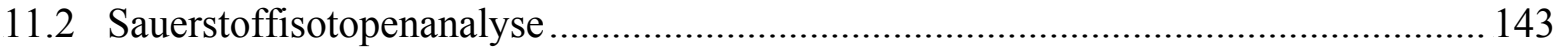

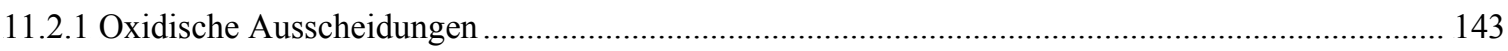

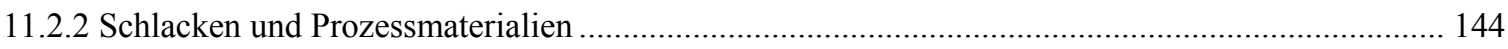

11.2.3 ${ }^{17} \mathrm{O}-$ Messung von Prozess- $\mathrm{O}_{2}$, Chromit und Clogging ............................................................. 145 


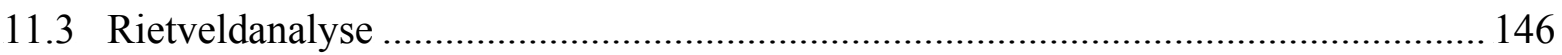

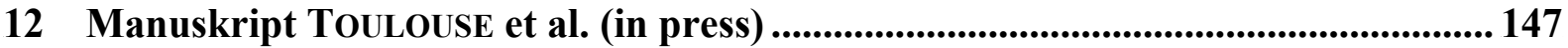

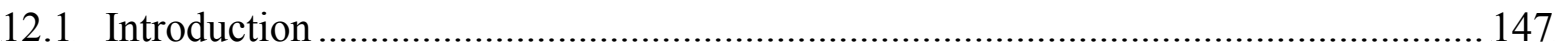

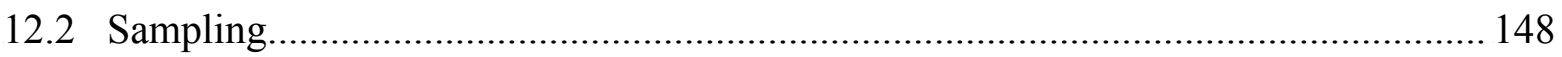

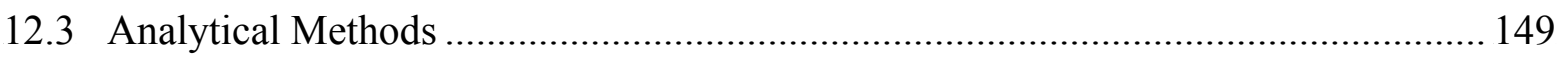

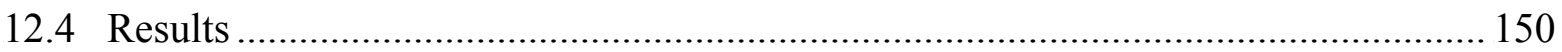

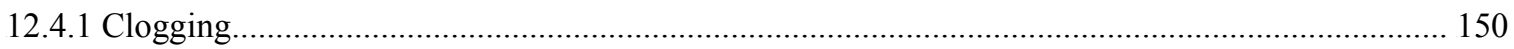

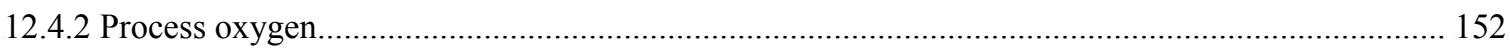

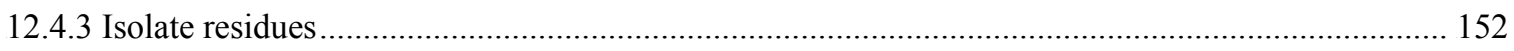

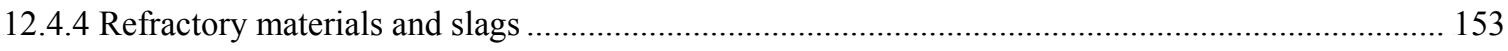

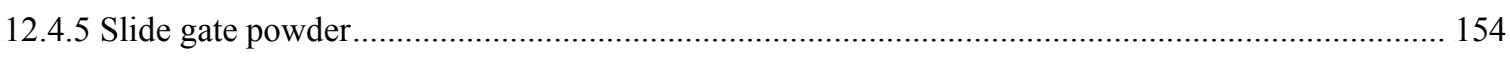

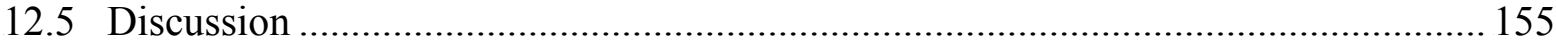

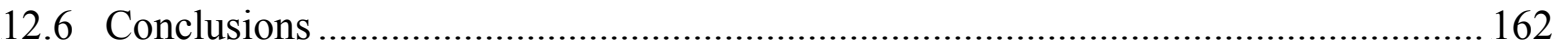

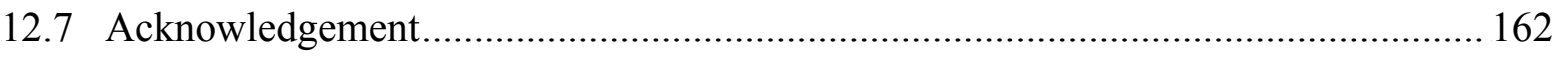

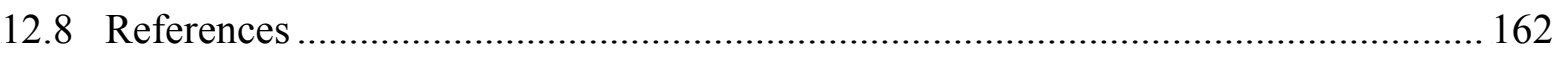

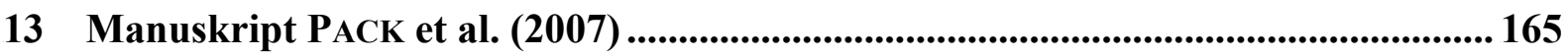

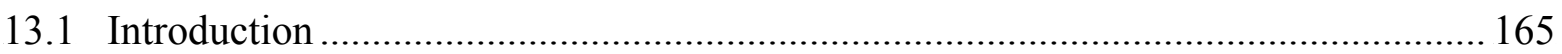

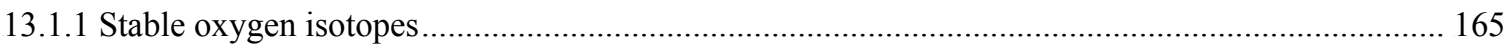

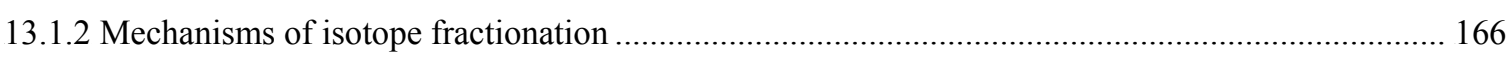

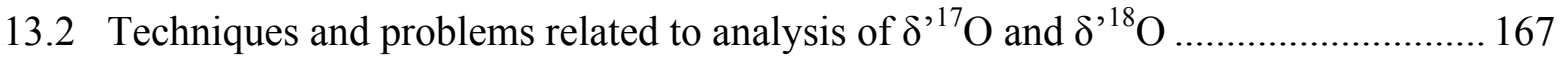

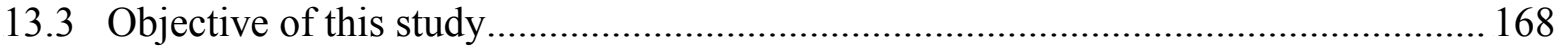

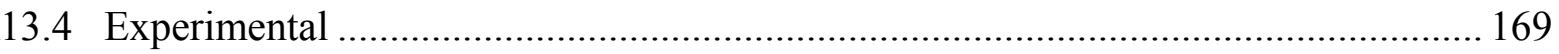

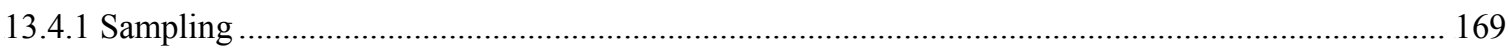

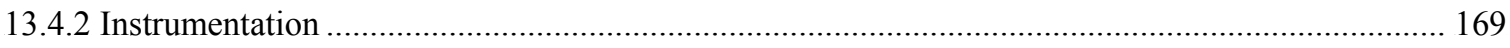

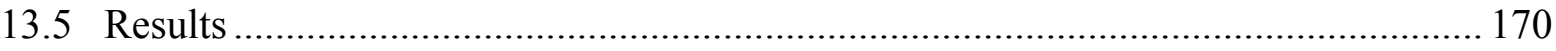

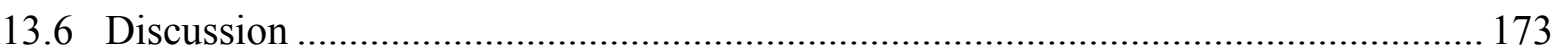

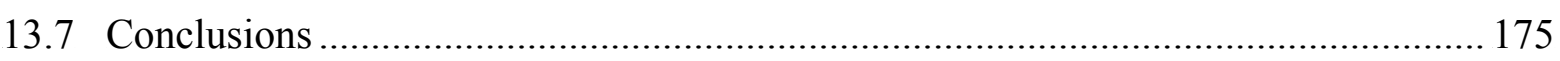

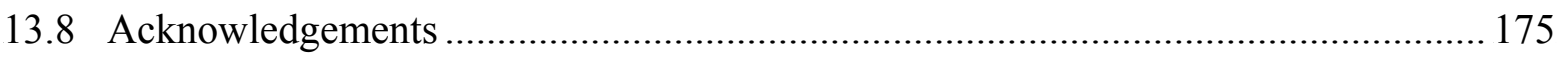

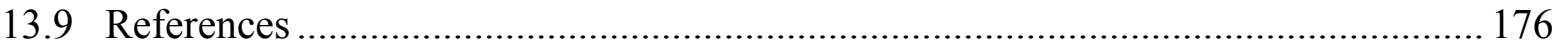

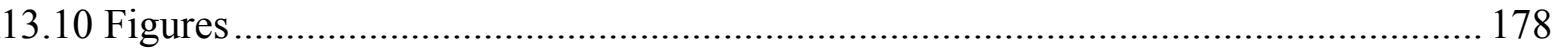

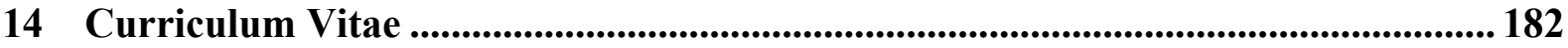




\section{Einleitung}

\subsection{Aufbau der Arbeit}

Die Ergebnisse dieser Studie wurden in regelmäßigen Zeitabschnitten zu in sich abgeschlossenen Teilen zusammengefasst. Dieser Aufbau stellte die kontinuierliche Auswertung, Diskussion und Zusammenfassung der Ergebnisse sicher. Darüber hinaus wurde ein Manuskript als Erstautor verfasst, das bei der Zeitschrift Steel Research International eingereicht wurde und sich zurzeit im Druck befindet (siehe Kapitel 12). Dieses Manuskript soll als Gesamtdiskussion dienen. Bei einem weiteren Manuskript, das bei der Zeitschrift Rapid Communications in Mass Spectrometry eingereicht wurde, bin ich Zweitautor (siehe Kapitel 13). Mein Anteil an letzterer Arbeit ist die Messung von ${ }^{17} \mathrm{O}$ an Silikaten und Oxiden. Des Weiteren habe ich einen Anteil an der Klarheit der Argumentationskette.

\subsection{Problemstellung und Ansatz}

Beim Stranggießen von Stahl werden nichtmetallische Einschlüsse ausgeschieden, die negative Einflüsse auf das Produkt und den Prozess der Stahlherstellung haben (siehe Kapitel 1.5). Ziel dieser Studie ist die Klärung der Herkunft nichtmetallischer Ausscheidungen in Tauchrohren (Clogging) (siehe Abbildung 29 und in Kapitel 12.2 Abbildung 1). Hierfür wurden Sauerstoffisotope als Marker benutzt, um mögliche O-Quellen zu identifizieren, die für die Bildung dieser meist oxidischen Ausscheidung verantwortlich gemacht werden. Mit Hilfe von Massenbilanzierungen wurde ein quantitatives Modell zur Herkunft von oxidischen Ablagerungen in Tauchrohren entwickelt (vgl. TOULOUSE et al. in press).

Die Grundlage zur Verwendung von stabilen Isotopen als Marker liegt in der unterschiedlichen isotopischen Signatur potentieller Clogging-Verursacher. Eine isotopische Charakterisierung ist vergleichbar mit einer chemischen Charakterisierung (PACK 2000). Bei der Stahlherstellung eignen sich O-Isotope zur Ursachenklärung für Clogging, da es eine Reihe möglicher O-Quellen für nichtmetallische Einschlüsse/Ablagerungen gibt.

Es gibt drei stabile O-Isotope, das häufigste ist das ${ }^{16} \mathrm{O}$ mit $\sim 99,8 \%$, gefolgt vom ${ }^{18} \mathrm{O}$ mit $\sim 0,2 \%$ und dem ${ }^{17} \mathrm{O}$ mit $\sim 0,04 \%$ (PACK et al. 2007). Die Variationen der Verhältnisse ${ }^{18} \mathrm{O} /{ }^{16} \mathrm{O}$ und ${ }^{17} \mathrm{O} /{ }^{16} \mathrm{O}$ sind sehr gering und werden daher relativ zu einem Standard in Promilleabweichungen angegeben. Daher führten MCKINNEY et al. (1950) die $\delta$-Notation ein: 


$$
\delta^{x} \mathrm{O}_{\text {vSMOW }}=\left(\frac{\left(\frac{{ }^{x} \mathrm{O}}{{ }^{16} \mathrm{O}}\right)_{\text {Probe }}}{\left(\frac{{ }^{x} \mathrm{O}}{{ }^{16} \mathrm{O}}\right)_{\text {vSMOW }}}-1\right) \times 1000[\%] \text { mit } x=17,18
$$

1968 wurde von der International Atomic Energy Agency (IAEA) Vienna Standard Mean Ocean Water (VSMOW) als internationaler Standard etabliert, der sich von seiner Zusammensetzung am Ozeanwasser orientiert. Vor fast 40 Jahren wurden 70 Liter dieses Standards hergestellt. Die sich erschöpfenden Vorräte wurden letztes Jahr von der IAEA durch einen 300 Liter umfassenden Standard ersetzt, der lediglich um $<0,01 \%$ im $\delta^{18} \mathrm{O}$ vom ursprünglichen VSMOW abweicht (InTERnATIONAL ATOMIC ENERgy AGENCy 2007). Definitionsgemäß ist $\delta^{18} \mathrm{O}=\delta^{17} \mathrm{O}=0 \%$ (für VSMOW). Ein positiver $\delta^{x} \mathrm{O}$-Wert bedeutet ein erhöhtes Verhältnis von ${ }^{x} \mathrm{O} /{ }^{16} \mathrm{O}$ in der Probe gegenüber dem im Standard. Ein negativer $\delta^{x} \mathrm{O}$-Wert zeigt ein niedrigeres ${ }^{x} \mathrm{O} /{ }^{16} \mathrm{O}$-Verhältnis in der Probe gegenüber dem im Standard an. Das ${ }^{18} \mathrm{O} /{ }^{16} \mathrm{O}$ Verhältnis schwankt in Gesteinen, natürlichen Fluiden und atmosphärischen Gasen zwischen $-80 \%$ (FUJITA and ABE 2006) und +100\%o (SAVARINO et al. 2007) relativ zum Meerwasser (COPLEN et al. 2002).

Das chemische Verhalten von Elementen ist durch deren Elektronenkonfiguration bestimmt. Isotope eines Elements verhalten sich aufgrund gleicher Elektronenkonfiguration chemisch weitgehend gleich (PACK 2006). Unterschiedliche Neutronenzahlen im Atomkern und damit verbundene kleine Massenunterschiede bedingen jedoch leicht unterschiedliches physikalisches Verhalten der Isotope. Isotopeneffekte jeglicher Art beruhen damit allein auf unterschiedlichen Massen isotopisch substituierter Phasen. Nach PACK (2006) werden vier verschiedene Prozesse unterschieden, die zu Isotopenvariationen führen:

- Kinetische massenabhängige Fraktionierung während einer Reaktion wie z. B. der Diffusion

- Massenabhängige Fraktionierung im Gleichgewicht zwischen zwei oder mehr koexistierenden Phasen

- Mischung von Reservoirs unterschiedlicher Isotopenverhältnisse

- Massenunabhängige Fraktionierung

O-Isotopenfraktionierungen, die bei Prozessen entsprechend den ersten drei Punkten entstehen, werden in den Kapiteln 2.3.4, 3.5 und 5 diskutiert. Die massenunabhängige Fraktionie- 
rung wird in Kapitel 7.1 im Zusammenhang mit der Anwendung von ${ }^{17} \mathrm{O}$ als weiterem Marker zur Herkunftsklärung nichtmetallischer Ausscheidungen bei der Stahlherstellung erläutert.

\subsection{Was ist Stahl?}

In WedDige (2001) findet sich folgende Definition: ,, Stahl ist wissenschaftich als Eisenwerkstoff, der weder Reineisen noch Gusseisen ist, zu definieren “. Nach DIN (2000) ist Stahl „[...] ein Werkstoff, dessen Massenanteil an Eisen größer ist als der jedes anderen Elementes, dessen Kohlenstoffgehalt im Allgemeinen kleiner als 2\% ist und der andere Elemente enthält. Eine begrenzte Anzahl von Chromstählen kann mehr als $2 \%$ C enthalten, aber $2 \%$ ist die übliche Grenze zwischen Stahl und Gusseisen“. Darüber hinaus ist die Warmformbarkeit ein typisches Stahlmerkmal (BERNS 1999, Verein Deutscher Eisenhüttenleute 1989). Entsprechend Tabelle 1 werden Stähle weiter nach ihrer chemischen Zusammensetzung und nach Hauptgüteklassen unterteilt.

Tabelle 1: Einteilung der Stähle nach chemischer Zusammensetzung und Hauptgüteklassen (DIN 2000).

\begin{tabular}{|c|c|}
\hline \hline $\begin{array}{c}\text { Einteilung nach der chemischen } \\
\text { Zusammensetzung }\end{array}$ & Einteilung nach Hauptgüteklassen \\
\hline \hline unlegierte Stähle & $\begin{array}{c}\text { unlegierte Qualitätsstähle } \\
\text { unlegierte Edelstähle }\end{array}$ \\
\hline nichtrostende Stähle & nichtrostende Stähle \\
\hline andere, legierte Stähle & $\begin{array}{c}\text { legierte Qualitätsstähle } \\
\text { andere legierte Edelstähle }\end{array}$ \\
\hline
\end{tabular}

Bei unlegierten Stählen erreicht kein Legierungselement den vorgegebenen Grenzwert. Legierte Stähle erreichen bei mindestens einem Legierungselement den vorgegebenen Grenzwert bzw. überschreiten diesen (siehe Tabelle 2). 


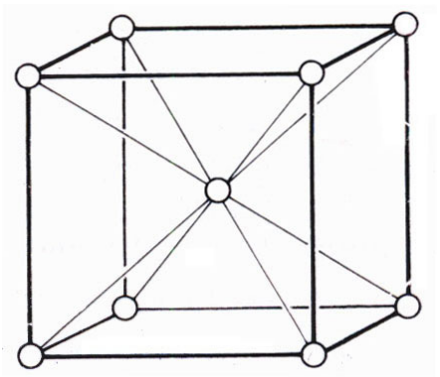

Abbildung 1: Kubisch-innenzentriertes Gitter des Ferrits (KLEBER et al. 1990)

Die maximalen C-Gehalte für IF-Güten in den beprobten Stahlwerken liegen zwischen 0,002 und 0,02 Gew.-\%. IF-Stähle besitzen daher eine hohe Streckgrenze (DIN 2005), da das Wandern von Versetzungen nicht durch Kohlenstoffatome gehemmt wird. Bei der Abkühlung nach Warmumformung wird Kohlenstoff in den starken Carbid-Bildnern Ti und $\mathrm{Nb}$ gebunden (RICHARD 1969), die sich an Korngrenzen ausscheiden und zu einer Abbindung der restlichen interstitiell gelösten Kohlenstoffatome führen. Dadurch wird die benötigte Festigkeit der Tiefziehteile erreicht. Eine typische Zusammensetzung eines ULC-IF-Stahls ist in Abbildung 2 dargestellt.

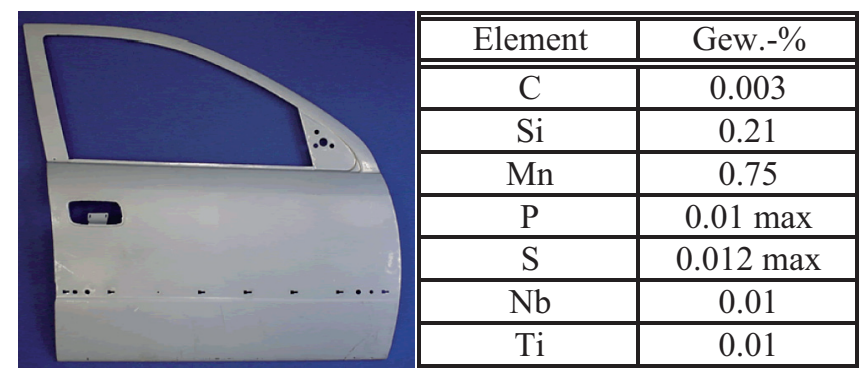

Abbildung 2: Photo eines Karosseriebauteils für PKWs und dessen typische chemische Zusammensetzung (ULC-IF-Stahl) (INTERNATIONAL IRON AND STEEL INSTITUTE 2007). 


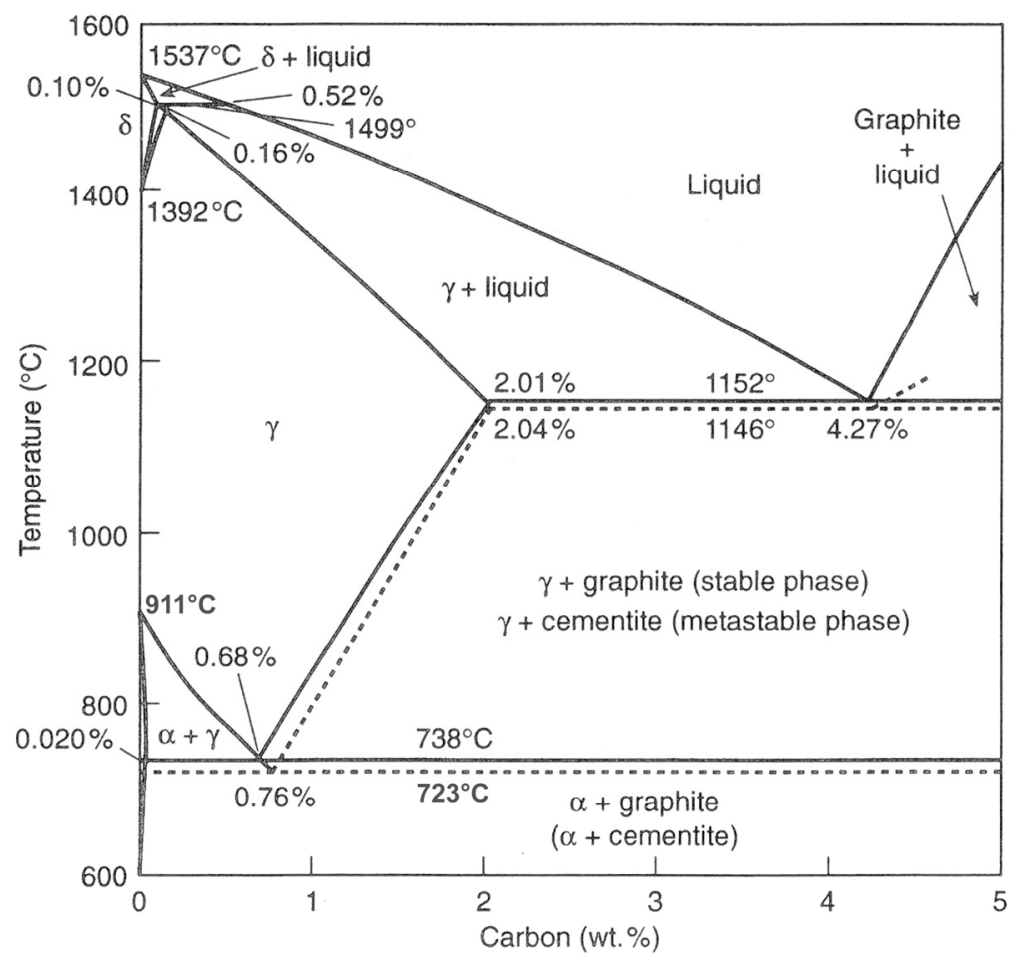

Abbildung 3: Der für die Stahlherstellung relevante Teil des Fe-C-Diagramms. Die gestrichelten Linien begrenzen Stabilitätsfelder des metastabilen Systems $\left(\mathrm{Fe}_{-}-\mathrm{Fe}_{3} \mathrm{C}\right)$, die durchgezogenen Linien die des stabilen Systems (Fe-C). Letzteres ist in der Praxis der Stahlherstellung weniger von Bedeutung (verändert nach TURKDOGAN 1996).

Bei Erwärmung auf eine Temperatur von $911^{\circ} \mathrm{C}$ unter Atmosphärendruck klappt das Gitter reinen Eisens in die kubisch-flächenzentrierte Zelle des $\gamma$-Eisens oder Austenits um (siehe Abbildung 3 und Abbildung 4). Die $\alpha / \gamma$-Umwandlung ist nur mit einer geringen Lageveränderung der Fe-Atome verbunden (BERNS 1999). Dieser Gittertyp begünstigt die Einlagerung von $\mathrm{C}$ in die Kristallstruktur und erhöht dadurch die Zugfestigkeit des Stahls. Interstitiell werden darüber hinaus H, B, N und O im Austenit gelöst (TURKDOGAN 1996).

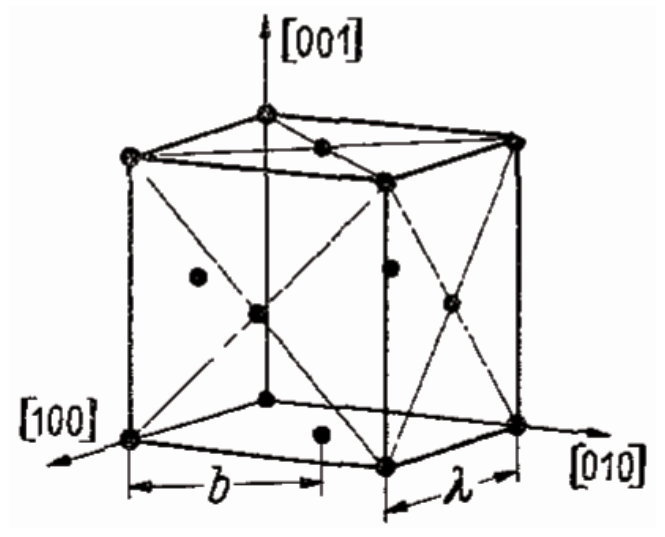

Abbildung 4: Kubisch-flächenzentrierte Elementarzelle mit $h k l$-Achsenindizierung. Beim Austenit ist Kohlenstoff im Zentrum der Kristallstruktur gelöst (LEIBFRIED and DIETRICH 1951). 
Bei $1392^{\circ} \mathrm{C}$ erfolgt die Umwandlung in $\delta$-Eisen, welches wiederum durch eine kubischraumzentrierte Elementarzelle aufgebaut ist (siehe Abbildung 1 und Abbildung 3). Bei $1537^{\circ} \mathrm{C}$ ist der Schmelzpunkt von Eisen erreicht. In Abbildung 5 ist die Variation des Gitterparameters a in Abhängigkeit von der Temperatur der zwei möglichen kristallinen Modifikationen reinen Eisens dargestellt. Das Volumen der Elementarzelle des $\gamma$-Eisens ist etwa um den Faktor 2 größer als das des $\alpha$ - bzw. $\delta$-Eisens (siehe Abbildung 5).

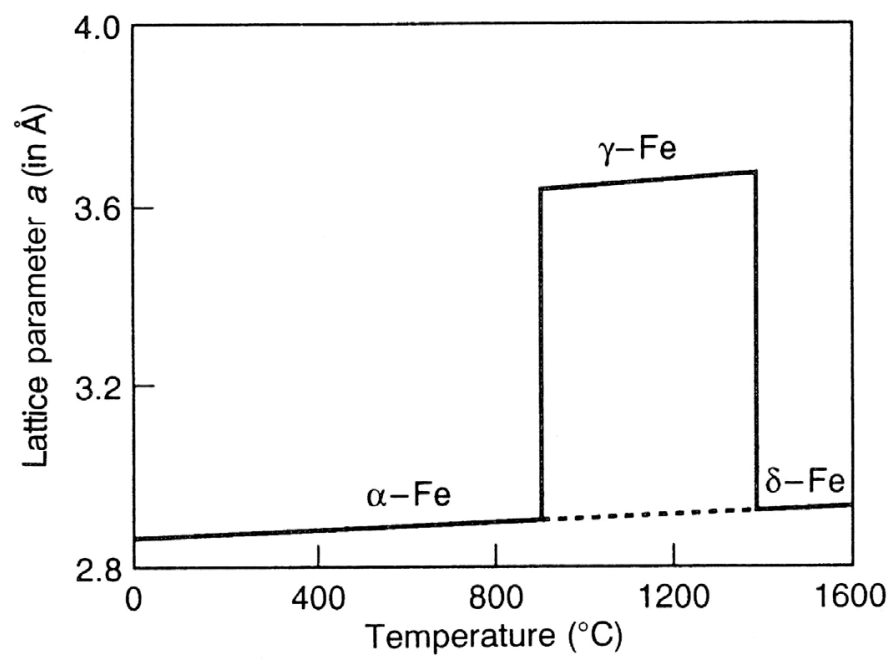

Abbildung 5: Variation des Gitterparameters a von reinem Eisen als Funktion der Temperatur (modifiziert nach TURKDOGAN 1996).

Als Faustformel gilt, dass die Löslichkeit von Legierungselementen in der Kristallstruktur des Eisens abhängig von deren Gittertyp ist. Im Stabilitätsbereich des Austenits werden bevorzugt Elemente eingebaut, die ein kubisch-flächenzentriertes Gitter besitzen (bei $25^{\circ} \mathrm{C}$ und 1 bar) wie Ni und Co. Diese Elemente erhöhen dadurch den Temperaturstabilitätsbereich des Austenits. Substitutionsmischkristalle mit den kubisch-innenzentriert kristallisierenden Elementen $\mathrm{Si}$, Cr und V (bei $25^{\circ} \mathrm{C}$ und 1 bar) hingegen erhöhen die Stabilität des Ferrits (TURKDOGAN 1996).

\subsection{Wie wird Stahl hergestellt?}

Die bedeutendste Verfahrensroute zur Herstellung von Stahl führt über die Erzeugung von flüssigem Roheisen im Hochofen, dessen Umwandlung zu Stahl im Konverter, einer Pfannenbehandlung zur Einstellung der gewünschten chemischen Zusammensetzung und über das Vergießen des Stahls bis hin zum Walzen. Darüber hinaus ist die Verfahrenslinie Elektrolichtbogenofen, Pfannenbehandlung, Gießbetrieb und Walzwerk in den Industrieländern von wesentlicher Bedeutung (siehe Abbildung 3) (PleschiUtsCHNIGG 1989). 


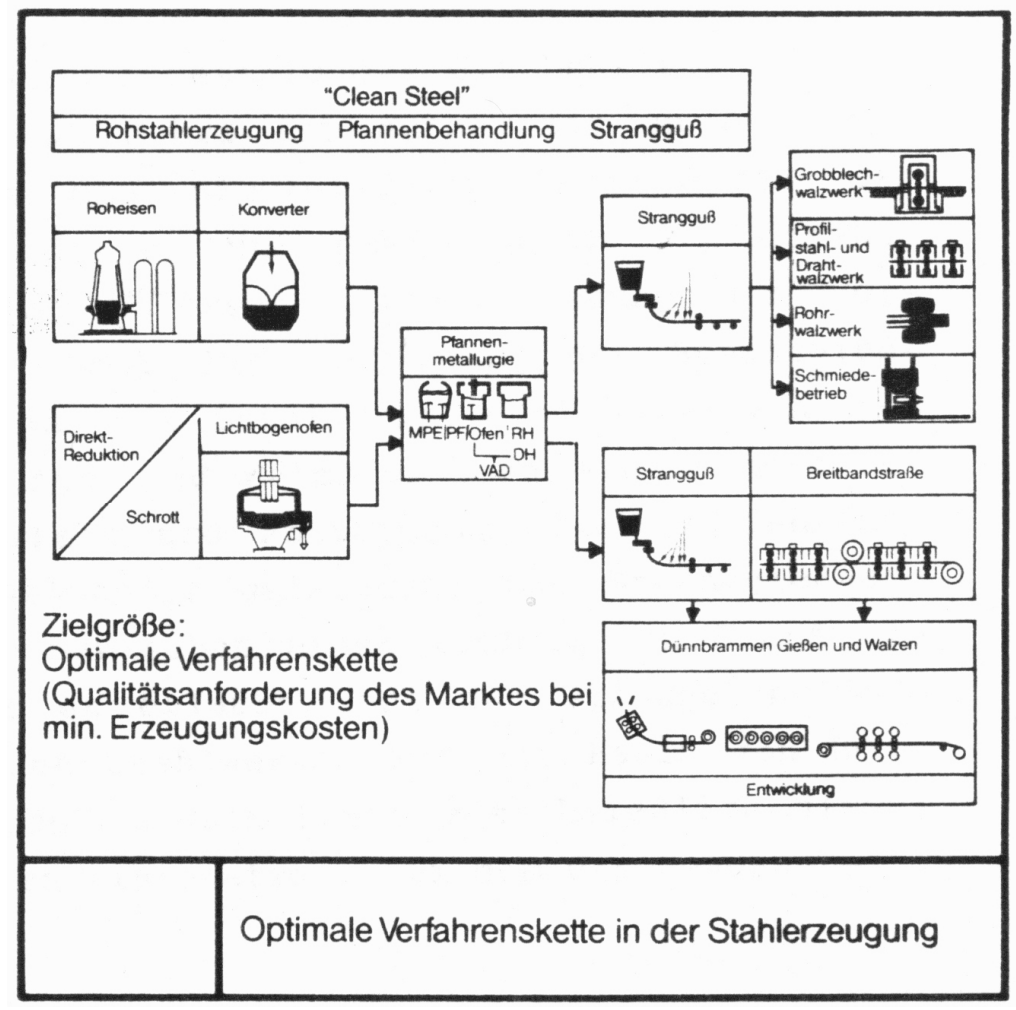

Abbildung 6: Dargestellt sind die beiden wichtigsten Verfahrensrouten: Hochofen - Konverter - Pfannenbehandlung - Gießen - Walzen und Lichtbogenofen - Konverter - Pfannenbehandlung - Gießen Walzen (PLESCHIUTSCHNIGG 1989).

Der Großteil der Erze wird im Hochofen verhüttet (GUDENAU 1989b). Daher wird hier nur auf diese Verfahrensroute eingegangen, die auch in den untersuchten Stahlwerken der ThyssenKrupp Steel AG Anwendung findet. Einsatzstoffe für den Hochofenprozess sind neben den Eisenerzen Zuschläge wie Kalkstein $\left(\mathrm{CaCO}_{3}\right)$, Dolomit $\left(\mathrm{MgCa}\left[\mathrm{CO}_{3}\right]_{2}\right)$ und $\mathrm{Kies}\left(\mathrm{SiO}_{2}\right)$, die als Möller bezeichnet werden. Koks dient als Brennstoff und Reduktionsmittel im Verhüttungsprozess und bildet mit dem Möller die Beschickung des Hochofens. Der Hochofen arbeitet als Schachtofen auf der Grundlage des Gegenstromprinzips, bei dem die Beschickung von oben nach unten sinkt. Gleichzeitig steigen die bei der Verbrennung des Koks entstehenden Reduktionsgase von unten nach oben. Hauptprodukte des Hochofens sind Roheisen, Gichtgas und Schlacke (siehe Abbildung 7) (GUDENAU 1989a, GUDENAU 1989b). 


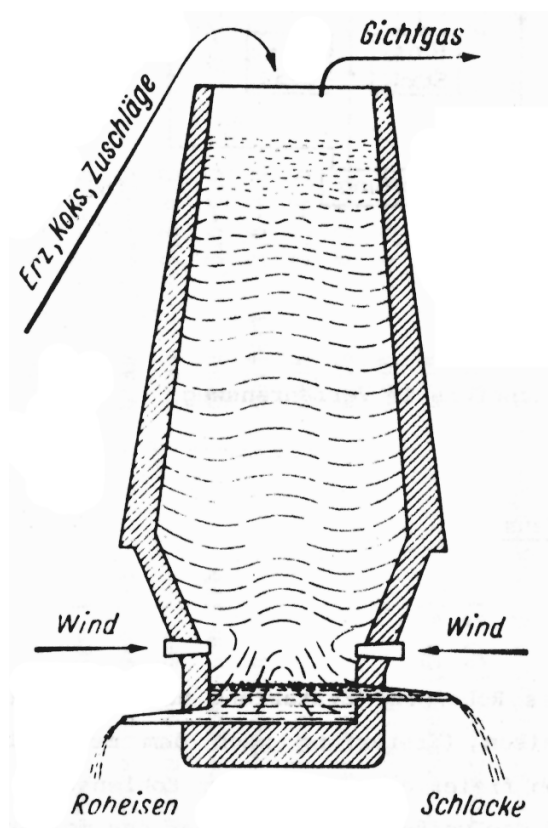

Abbildung 7: Einsatzstoffe und Produkte des Hochofens (GUDENAU 1989a).

Die direkte Reduktion von festen Eisenoxiden durch Koks ist für die Roheisenerzeugung von untergeordneter Bedeutung und verläuft nach GUDENAU (1989a) als gekoppelte Reaktion über:

$$
\begin{aligned}
& \mathrm{FeO}+\mathrm{CO}=\mathrm{Fe}+\mathrm{CO}_{2} \\
& \frac{\mathrm{CO}_{2}+\mathrm{C}=2 \mathrm{CO}}{\mathrm{FeO}+\mathrm{C}=\mathrm{Fe}+\mathrm{CO}}
\end{aligned}
$$

Sie ist mit einem erheblichen Wärmeverbrauch verbunden und verschiebt das BoudouardGleichgewicht $\mathrm{C}+\mathrm{CO}_{2}=2 \mathrm{CO}$ auf die Eduktseite. Bei Temperaturen unter $400^{\circ} \mathrm{C}$ liegt das Gleichgewicht nahezu vollständig auf der $\mathrm{CO}_{2}$-Seite (siehe Abbildung 8). Mit zunehmendem Druck wird das Zustandsfeld des $\mathrm{CO}_{2}$ immer größer, um die Gasmenge des Systems zu erniedrigen (Prinzip des kleinsten Zwangs von Le Chatelier). Je höher der $\mathrm{CO}_{2}$-Anteil des Gasgemisches ist, desto höher ist das Sauerstoffpotential und desto niedriger ist das Reduktionsvermögen (GUDENAU 2002). Deswegen ist die direkte Reduktion gering zu halten. 


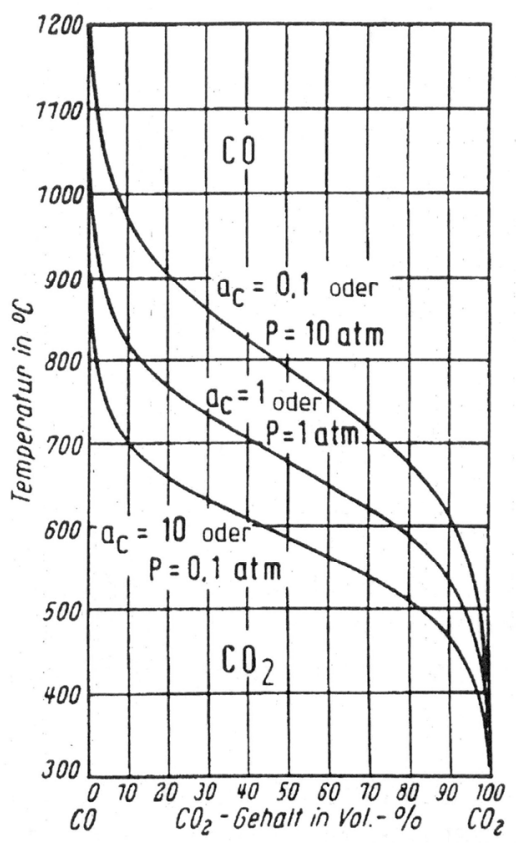

Abbildung 8: Temperatur- und Druckabhängigkeit der Aktivität von Kohlenstoff in $\mathrm{CO} / \mathrm{CO}_{2}-$ Gasgemischen (GUDENAU 2002).

Von wesentlicher Bedeutung ist die indirekte Reduktion der Eisenoxide über die Gasphase. Die Reduktion des Eisenoxids mit dem höchsten Sauerstoffanteil, des Hämatits $\left(\mathrm{Fe}_{2} \mathrm{O}_{3}\right)$ mit 30,06 Gew.-\% Sauerstoff, verläuft mit dem Reduktionsmittel CO nach GUDENAU (1989a) über folgende Reaktionen ab:

$$
\begin{array}{ll}
3 \mathrm{Fe}_{2} \mathrm{O}_{3}+\mathrm{CO}=2 \mathrm{Fe}_{3} \mathrm{O}_{4}+\mathrm{CO}_{2} & 400-550^{\circ} \mathrm{C} \\
\mathrm{Fe}_{3} \mathrm{O}_{4}+\mathrm{CO}=3 \mathrm{FeO}+\mathrm{CO}_{2} & 570-800^{\circ} \mathrm{C} \\
\frac{\mathrm{FeO}+\mathrm{CO}=\mathrm{Fe}+\mathrm{CO}_{2}}{\mathrm{Fe}_{2} \mathrm{O}_{3}+3 \mathrm{CO}=2 \mathrm{Fe}+3 \mathrm{CO}_{2}} & \text { ab } 650^{\circ} \mathrm{C}
\end{array}
$$

Das erzeugte Roheisen hat typischerweise eine Zusammensetzung wie in Tabelle 3 aufgelistet.

Tabelle 3: Typische Zusammensetzung von in Deutschland erzeugtem Roheisen in Gew.-\% (GUDENAU 2002).

\begin{tabular}{|c|c|c|c|c|}
\hline \hline $\mathbf{C}$ & $\mathbf{S i}$ & $\mathbf{M n}$ & $\mathbf{P}$ & $\mathbf{S}$ \\
\hline \hline $4,2-4,5$ & $0,25-0,8$ & $0,2-0,6$ & $0,07-0,13$ & $0,01-0,035$ \\
\hline
\end{tabular}

Bei der Reduktion der Erze wird Schwefel in das Roheisen eingebracht, der zu 80\% vom Koks und zu 20\% vom Möller stammt. Schwefel hat negative Auswirkungen z. B. auf Formbarkeit, Zähigkeit, Korrosionsanfälligkeit und die Schweißbarkeit des Stahls. Der Hochofen ist ein guter metallurgischer Reaktor zur Entschwefelung über die basische Schlackenphase, 
da hierfür reduzierende Bedingungen benötigt werden (TURKDOGAN 1996). Die Hochofenschlacke besteht aus den nichtreduzierten Anteilen der Erze (Gangart), den Zuschlägen und der Koksasche. In Tabelle 4 ist eine typische Hochofenschlackenzusammensetzung dargestellt.

Tabelle 4: Hochofenschlackenzusammensetzung in Gew.-\% nach GUDENAU (1989a).

\begin{tabular}{|c|c|c|c|}
\hline \hline $\mathbf{S i O}_{2}$ & $\mathbf{C a O}$ & $\mathbf{M g O}$ & $\mathbf{A l}_{\mathbf{2}} \mathbf{O}_{\mathbf{3}}$ \\
\hline \hline $33-35$ & $40-43$ & $5-10$ & $10-13$ \\
\hline
\end{tabular}

Als Reduktionsmittel zur Entschwefelung kommen Metalle in Frage, deren Sulfide ein Schwefelpotential haben, das niedriger ist als das der Eisensulfide. Dies sind $\mathrm{Ca}$ und $\mathrm{Mg}$, die als Oxide zugesetzt werden. Im Stahlwerk wird Branntkalk $(\mathrm{CaO})$ eingesetzt, der mit Eisensulfid reagiert:

$$
(\mathrm{CaO})+[\mathrm{FeS}]=(\mathrm{CaS})+(\mathrm{FeO})
$$

Wie in der hüttenmännischen Praxis üblich, stehen runde Klammern für flüssige Reaktanden, geschweifte für gasförmige, eckige für gelöste und spitze für feste. Da die Aufnahmefähigkeit der Schlacke für $\mathrm{CaS}$ und $\mathrm{FeO}$ begrenzt ist, wird fortlaufend ein Schlackenwechsel vorgenommen. Darüber hinaus wird Eisenoxid nach folgender Gleichung weiter reduziert:

$$
(\mathrm{FeO})+[\mathrm{C}]=[\mathrm{Fe}]+\{\mathrm{CO}\}
$$

Daraus lässt sich folgende Nettoreaktion ableiten:

$$
[\mathrm{FeS}]+(\mathrm{CaO})+[\mathrm{C}]=(\mathrm{CaS})+[\mathrm{Fe}]+\{\mathrm{CO}\}
$$

Aus dem Hochofen wird massenmäßig mehr als doppelt so viel Gichtgas ausgebracht wie Roheisen und Schlacke. Im Durchschnitt enthält das Gichtgas 20-22\% $\mathrm{CO}_{2}, 20-22 \% \mathrm{CO}, 1$ $4 \% \mathrm{H}_{2}$ und 54-56\% $\mathrm{N}_{2}$ (GUDENAU 1989a).

Das Roheisen enthält 4,2-4,5 Gew.-\% C und andere unerwünschte Begleitelemente, die seinen begrenzten Nutzen als Werkstoff bedingen (siehe Tabelle 3). Im Konverter wird durch das Herausbrennen von $\mathrm{C}$ aus Roheisen Stahl erzeugt. Der Prozess wird Frischen genannt und dient neben der Entkohlung auch der Entfernung von Si, Mn, P und S. Bei dem Prozess werden Elemente, die eine höhere Affinität zu Sauerstoff besitzen als Eisen, aus der Schmelze entfernt und von der Schlacke absorbiert oder sie entweichen als Gase. Die Einsatztemperatur liegt im Konverter bei 1295 bis $1350^{\circ} \mathrm{C}$ (GUDENAU 2002). Die Oxidationsreaktionen sind 
stark exotherm und erfordern keine Energiezugabe, vielmehr wird mit Hilfe von Schrott das Stahlbad gekühlt. Nach GUDENAU (2002) laufen folgende Frischreaktionen ab:

$$
\begin{aligned}
{[\mathrm{C}]+\frac{1}{2}\left\{\mathrm{O}_{2}\right\} } & =\{\mathrm{CO}\} & -92,01 \\
{[\mathrm{Mn}]+\frac{1}{2}\left\{\mathrm{O}_{2}\right\} } & =(\mathrm{MnO}) & -378,45 \\
{[\mathrm{Si}]+\left\{\mathrm{O}_{2}\right\} } & =\left(\mathrm{SiO}_{2}\right) & -895,75 \\
2[\mathrm{P}]+\frac{5}{2}\left\{\mathrm{O}_{2}\right\} & =\left(\mathrm{P}_{2} \mathrm{O}_{5}\right) & -1236,37 \\
{[\mathrm{~S}]+\frac{1}{2}\left\{\mathrm{O}_{2}\right\} } & =\left\{\mathrm{SO}_{2}\right\} & -271,57 \\
{[\mathrm{Fe}]+\frac{1}{2}\left\{\mathrm{O}_{2}\right\} } & =(\mathrm{FeO}) & -271,50
\end{aligned}
$$

Die angegebenen $\Delta \mathrm{H}$-Werte charakterisieren die frei werdende Reaktionswärme. Es gibt verschiedene metallurgische Reaktionsgefäße für den Frischprozess, an dieser Stelle soll beispielhaft der LD-Konverter (Linz-Donawitz) beschrieben werden. In Abbildung 9 sind die Geometrie des LD-Konverters und die verschiedenen Reaktionsbereiche während des Frischens gekennzeichnet.

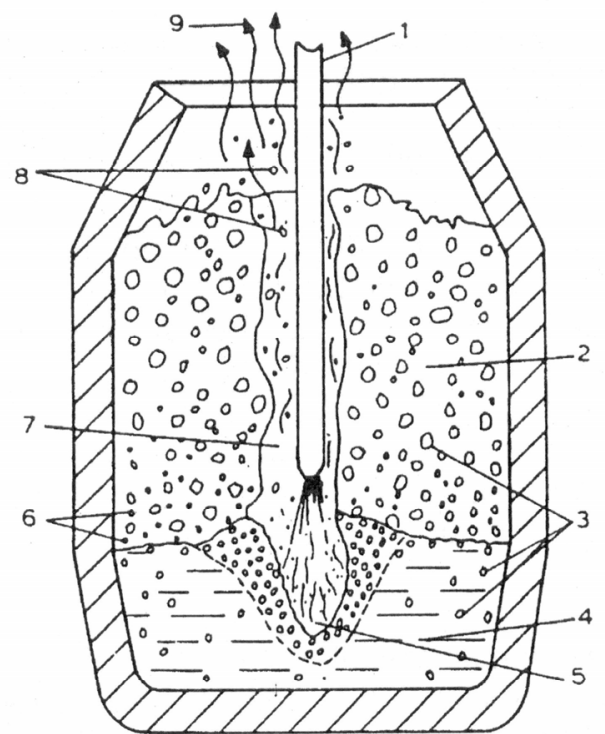

1. Blaslanze; 2. Schaumschlacke; 3. CO-Blasen; 4. Schmelze; 5. Brennfleck;

6. Eisentropfen; 7. Lanzenkanal; 8. versprühte Eisentropfen; 9. Brauner Rauch

Abbildung 9: Skizze des LD-Konverters während der Hauptphase der Entkohlung (GUDENAU 2002).

Das wichtigste Merkmal des LD-Konverters ist das Einblasen von Prozess- $\mathrm{O}_{2}$ über eine gekühlte Toplanze von oben. Bei der ThyssenKrupp Steel AG in Duisburg werden im Stahlwerk Bruckhausen zwei LD-Konverter mit einem Chargengewicht von 380 t eingesetzt. In den Stahlwerken in Duisburg wird ein kombiniertes Blasen zur besseren Durchmischung der Schmelze über Bodendüsen mit den Inertgasen $\mathrm{N}_{2}$ und Ar durchgeführt. Über die Lanze wird als Schlackenbildner staubförmiges $\mathrm{CaO}$ eingeblasen, das mit $\mathrm{FeO}$ niedrigschmelzende $\mathrm{Eu}-$ 
tektika bildet. $\mathrm{CaO}$ dient der Erhöhung der Basizität der Schlacke und vermindert dadurch den Verschleiß der feuerfesten Auskleidung des Konverters.

$\mathrm{Zu}$ Beginn des Kohlenstoffabbrandes wird eine flüssige FeO-Schlacke gebildet, die später wieder reduziert wird und die die hohe Entkohlungsgeschwindigkeit ermöglicht. Etwa $2 / 3$ der Entkohlung findet in der Schlacke statt, ein weiterer großer Teil direkt im Brennfleck (siehe Abbildung 9). Aufgrund der starken Verwirbelung liegt im Brennfleck eine Vier-

Komponenten-Dispersion aus Eisentropfen, Schlacke ( $\left.\mathrm{CaO}-\mathrm{SiO}_{2}-\mathrm{FeO}-\mathrm{MnO}\right)$, Gasblasen (CO) und festen Partikeln $\left(\mathrm{SiO}_{2}\right.$ und $\mathrm{CaO}$ ) vor (GudENAU 2002). Das sich bildende CO kann durch einen Kanal um die Lanze entweichen. Aus dem Brennfleck herausgeschlagene Fe-Tropfen werden in der Schlacke oxidiert oder gelangen zurück in die Schmelze.

Der Abbrandverlauf der Elemente beim Frischen ist in Abbildung 10 dargestellt. Zu Beginn des Frischens wird vor allen Dingen Si verschlackt. Nach dem Richardson-Jeffes-Diagramm (TURKDOGAN 1996) besitzt Si ein niedriges Sauerstoffpotential und wird deshalb am schnellsten abgebaut. Dabei bildet es durch seine hohe O-Affinität die stabilsten Oxide. So wird FeO aus der Schlacke in Anwesenheit von hohen Si-Konzentrationen direkt reduziert. Nach 25\% der Gesamtblaszeit ist der gewünschte Si-Gehalt der Schmelze eingestellt. Die MnVerschlackung verläuft ähnlich der von Si. In Abbildung 10 ist zu erkennen, dass der MnGehalt der Schmelze beim Frischen nach 3/4 der Zeit ein Maximum erreicht. Dies liegt an der Reduktion von $\mathrm{MnO}$ aus der Schlacke nach Beendigung des Si-Abbrandes. Zum Ende der Blaszeit sinkt der Mn-Gehalt der Schmelze erneut.

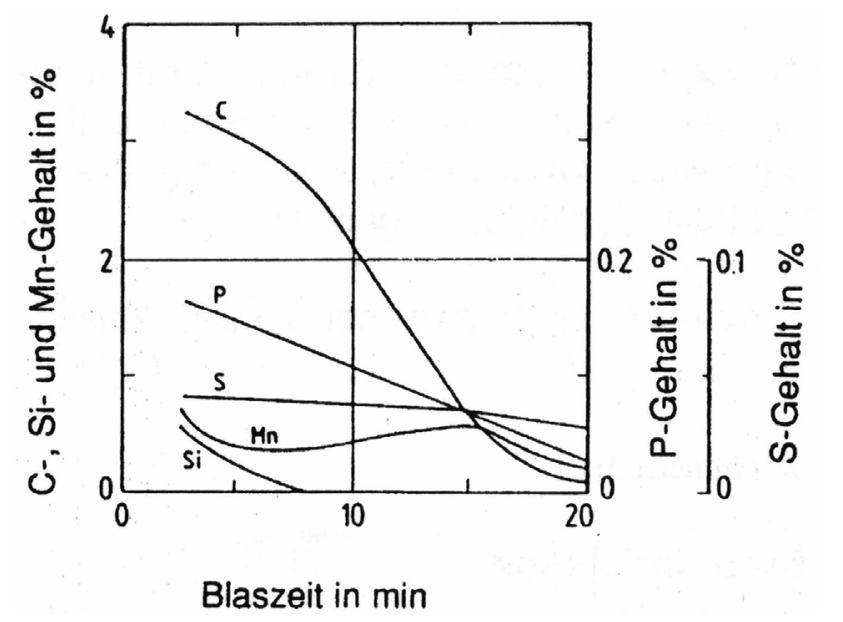

Abbildung 10: Abbrandverlauf der Elemente beim Konverterprozess (GUDENAU 2002).

Der Konverter ist durch die oxidierenden Bedingungen kein besonders gutes Aggregat zur Entschwefelung (siehe Abbildung 10), für die reduzierende Bedingungen und eine hohe 
Kalkaktivität benötigt werden. Eine geringe Entschwefelung verläuft jedoch über die Schlackenphase unter Bildung von Calciumsulfiden ab:

$$
(\mathrm{CaO})+[\mathrm{S}]=(\mathrm{CaS})+[\mathrm{O}]
$$

Die Tiefentschwefelung findet bei der Nachbehandlung in der Pfanne statt. Am Ende des Frischens werden Sauerstoffgehalte im Stahl von 500 bis 700 ppm erreicht, die Abstichstemperatur beträgt ca. $1650^{\circ} \mathrm{C}$ (DEKKERS 2002). Nach ZHANG and THOMAS (2002) liegt der O-Gehalt beim Abstich zwischen 450 bis 800 ppm. Nach INTERNATIONAL IRON AND STEEL INSTITUTE (2007) kann der O-Gehalt bis 1000 ppm betragen. Dabei ist der Sauerstoff in atomarer Form im Stahl gelöst (TURKDOGAN 1996). Die Löslichkeit von Sauerstoff im Stahl ist temperaturabhängig und nimmt mit sinkender Temperatur stark ab. Die maximale Löslichkeit von $\mathrm{O}$ in reinem Eisen beträgt 0,16 Gew.-\% (TURKDOGAN 1996). Beim Abkühlen von Stahlschmelzen sinkt die Löslichkeit von O bis zum vollständig erstarrten Stahl von 5 ppm auf annähernd null (DEKKERS 2002).

Früher war nach dem Konverterprozess die Stahlherstellung „,vollendet“ (VEREIN DEUTSCHER EISENHÜTTENLEUTE 1989). Der Stahl wurde dann im Blockgussverfahren als Block in unten geschlossene Kokillen vergossen. Heute schließen sich an das Frischen Nachbehandlungsverfahren an, die bis zum Erstarren des Stahls in der Kokille als Sekundärmetallurgie bezeichnet werden (GUDENAU 2002). Diese dient der Erhöhung der Qualität des Werkstoffs durch Ablauf folgender metallurgischer Arbeiten (VEREIN DEUTSCHER EISENHÜTTENLEUTE 1989):

- Legierungseinstellung

- Homogenisierung von Temperatur und Zusammensetzung

- Entkohlung

- Entschwefelung

- Entphosphorung

- Entfernung von Spurenelementen

- Entgasung

- Desoxidation

- Einschlusseinformung

- Verbesserung des Reinheitsgrades

- Beeinflussung des Erstarrungsgefüges

Der hohe Sauerstoffgehalt, den der Rohstahl nach dem Frischen hat, muss vor dem Stranggießen abgesättigt werden, da der Stahl sonst beim Erkalten anfangen würde zu „kochen“. Der gelöste Sauerstoff würde mit C zu CO reagieren und den Stahl aufschäumen lassen. Um dies 
zu verhindern, wird der Restsauerstoff bei der Desoxidation durch geeignete sauerstoffaffine Elemente, die meist während des Abstichs zugegeben werden, abgebunden (VEREIN DEUTSCHER EISENHÜTTENLEUTE 1989). Der Hüttenkundler spricht vom Beruhigen des Stahls. Al ist als Desoxidationsmittel weitverbreitet (DEKKERS et al. 2001, OvTCHINNIKOV 2001). Das Al reagiert mit dem Sauerstoff $\mathrm{zu} \mathrm{Al}_{2} \mathrm{O}_{3}$, das sich in der Pfannenschlacke abscheidet. Unterstützt wird das Aufsteigen der Desoxidationsprodukte durch Inertgasspülen. Grund für den Einsatz von $\mathrm{Al}$ als Desoxidationsmittel ist zum einen seine hohe Effizienz bei vergleichsweise geringen Kosten (ROBINSON et al. 1979), zum anderen schwimmt das beim Desoxidieren gebildete $\mathrm{Al}_{2} \mathrm{O}_{3}$ schnell in die Schlacke auf (PLÖCKINGER 1963). Darüber hinaus gibt Al dem Stahl eine feinkörnige Struktur (ROBINSON et al. 1979). Bereits bei einem Gehalt von 0,01 Gew.-\% Al liegt der O-Gehalt im Stahl bei $1600^{\circ} \mathrm{C}$ bei ca. 0,00045 Gew.-\% (siehe Abbildung 11). Bemerkenswert ist, dass bei einem Al-Gehalt von ca. 0,5 Gew.\% der O-Gehalt wieder steigt. In der Praxis wird der Al-Gehalt auf 0,03 bis 0,05 Gew.-\% eingestellt, was eine fast vollständige Umsetzung des Sauerstoffs gewährleistet (GUDENAU 2002).

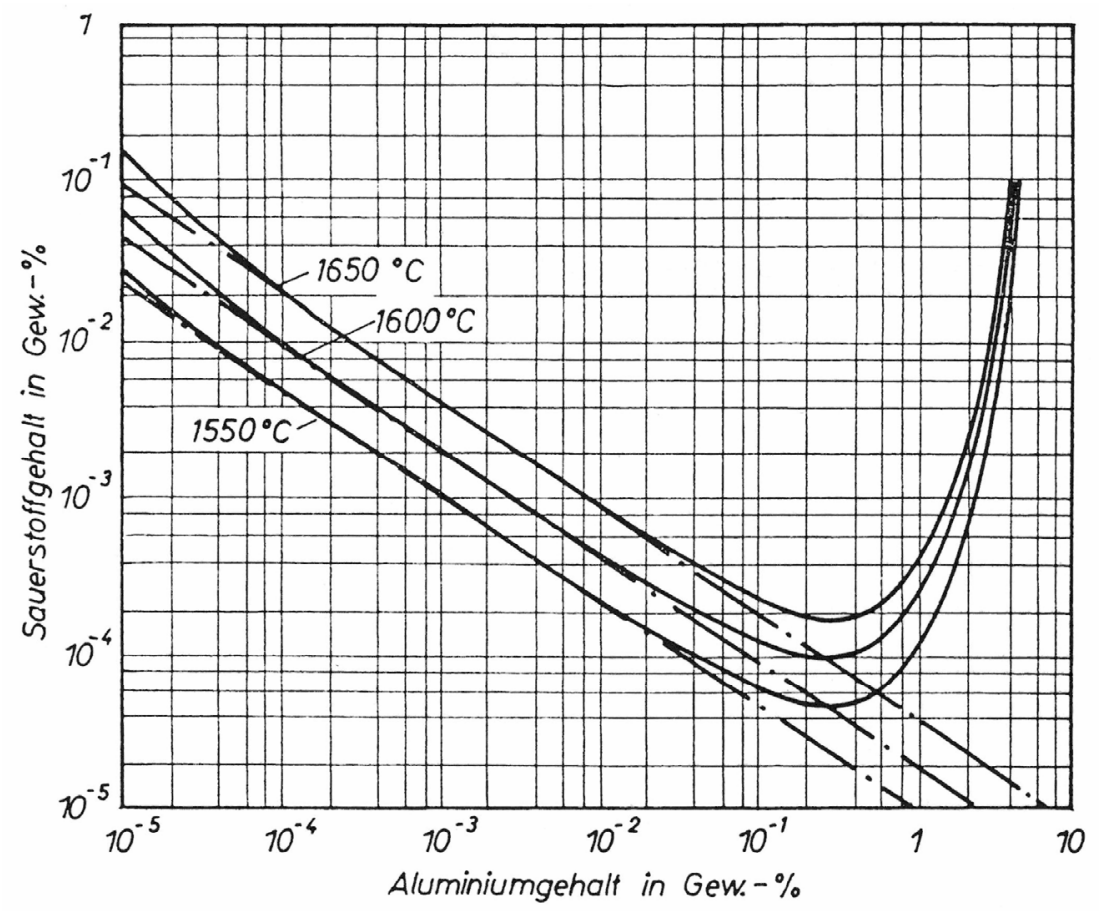

Abbildung 11: O-Gehalt in Fe-Schmelzen in Abhängigkeit vom Al-Gehalt (KNÜPPEL 1970).

Bei der Vakuumbehandlung wird unter vermindertem Druck von 1 bis 3 Torr (Grobvakuum) die gütespezifische Legierungseinstellung vorgenommen. Beim Vakuumfrischen wird der Kohlenstoffgehalt weiter gesenkt. Beim RH-Vakuumverfahren (Rheinstahl-Heraeus) wird ein Vakuumgefäß, welches zwei feuerfeste Eintauchstutzen besitzt, in die Pfanne eingetaucht (siehe Abbildung 12). Der atmosphärische Druck drückt die Schmelze in das evakuierte Vakuumgefäß, Fördergas (Argon) lässt die Schmelze umlaufen. Über eine Schleuse können Le- 
gierungsmittel zugegeben werden. In der Vakuum-Umlaufanlage erfolgen die Tiefenentkohlung und eine Entfernung von Wasserstoff und Stickstoff.

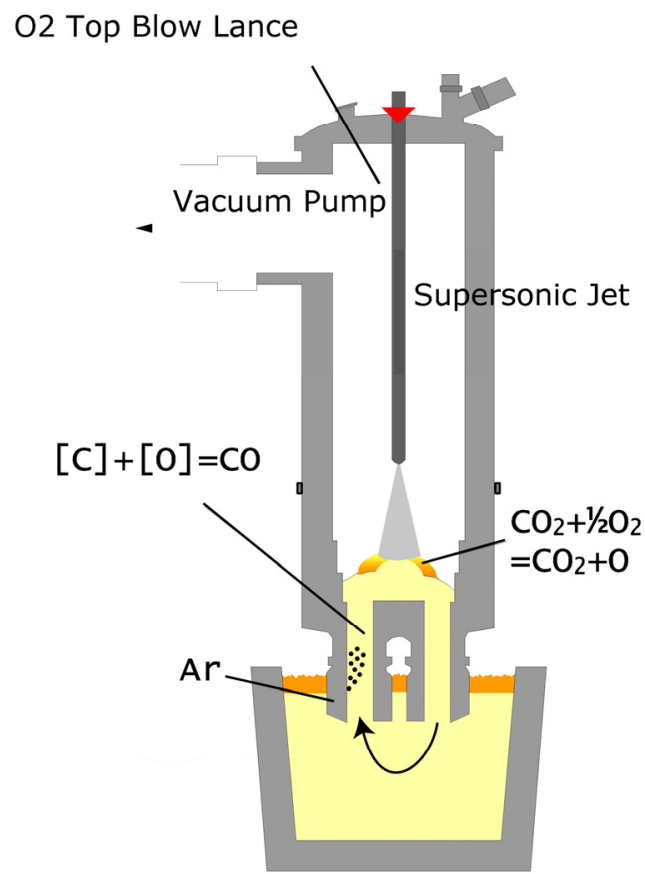

Abbildung 12: RH-Vakuumanlage mit feuerfesten Einlaufstutzen zur Überführung der Schmelze aus der Pfanne in den Unterteil des Vakuumbehandlungsgefäßes (verändert nach INTERNATIONAL IRON AND STEEL INSTITUTE 2007).

Nach der Vakuumbehandlung ist das Legieren des Stahls abgeschlossen und die Pfanne wird zur Stranggusshalle transportiert. Im Gegensatz zum Blockguss wird hier der Stahl in eine unten offene Durchlaufform gegossen, die die Stahlherstellung revolutionierte. 1887 wurde von R. M. Daelen die wassergekühlte Durchlaufkokille erstmals vorgeschlagen (ELFSBERG 2003). Erst mit der oszillierenden Kokille, die von Siegfried Junghans 1933 eingeführt wurde, konnte das Problem von Metallanfrierungen an der Kokille und Seigerungen beseitigt werden. Zunächst wurde erst Nichteisenmetall im Strang vergossen. Ernsthafte Versuche, Stahl im Stranggussbetrieb zu verarbeiten, gab es Mitte der 1940er Jahre in den USA und in Deutschland (GUDENAU 2002).

Beim Stranggießen wird der Stahl aus einer Gießpfanne über ein Schattenrohr in den Verteiler (engl. Tundish) vergossen (siehe Abbildung 13). Unterhalb der Pfanne befindet sich ein System von Schieberplatten, mit dessen Hilfe die Pfanne geöffnet werden kann. Um die Platten bei befüllter Pfanne bewegen zu können, werden sie zuvor mit Schiebersand bedeckt. Dieser verflüssigt sich nicht bei Kontakt mit der Schmelze in der Pfanne, sondern wird hierbei lediglich oberflächlich gesintert. Das Schattenrohr schützt den Gießstrahl vor Reoxidation durch Luft- $\mathrm{O}_{2}$. Das Feuerfest-Material (FF-Material) der Pfanne besteht aus kohlenstoffgebundenem 
Schmelzmagnesia (siehe Kapitel 4.2.4 und 4.3.3), das Schattenrohr überwiegend aus $\mathrm{Al}_{2} \mathrm{O}_{3}$ und Kohlenstoff.

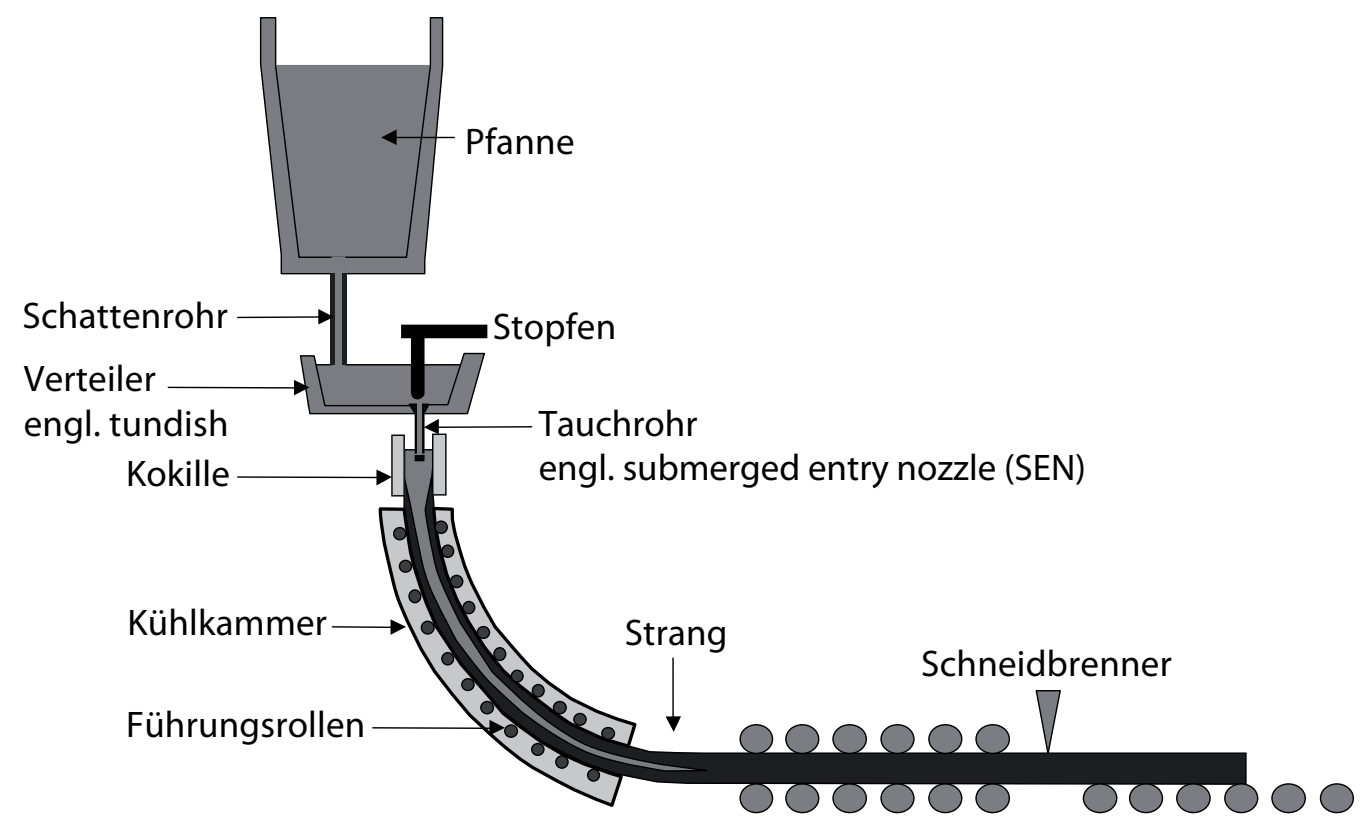

Abbildung 13: Skizze eines Querschnitts einer Stranggussanlage (verändert nach BINNS et al. 2001).

Der Tundish ist ein Zwischengefäß, in dem die Schmelze beim Brammenstranggießen auf zwei Stränge verteilt wird. Beim Pfannenwechsel dient der Tundish als Pufferaggregat zwischen Pfanne und Kokille und ermöglicht damit ein kontinuierliches Gießen von mehreren Schmelzen (Sequenzguss). Darüber hinaus dient er der Abscheidung nichtmetallischer Einschlüsse in die Schlacke, die durch Wehre begünstigt wird. Die Tundishschlacke besteht aus einer heterogenen, teilweise flüssigen $\mathrm{SiO}_{2}-\mathrm{Al}_{2} \mathrm{O}_{3}-\mathrm{CaO}$-Masse. Diese wird aus zwei Schichten gebildet, die nacheinander auf die Schmelze gegeben werden und sich bei ihrer Verflüssigung mischen. Die untere Schicht besteht aus flüssigem Abdeckpulver (Calciumaluminat), das die Schmelze vor Reoxidation durch Luft- ${ }_{2}$ schützt. Darüber befindet sich eine Schicht aus teilweise flüssiger Reisschalenasche ( $>95 \mathrm{Gew} .-\% \mathrm{SiO}_{2}$ ), die den Stahl vornehmlich thermisch isoliert. Reisschalenasche entsteht bei Verbrennung von Reisschalen und besteht fast ausschließlich aus biogenem $\mathrm{SiO}_{2}$. Das Verschleißfutter (Spritzmasse oder Verteilerrinnenmasse, kurz VTR-Masse) des Tundishs besteht nahezu vollständig aus Periklas (MgO) und muss nach einer Gießsequenz (meist vier bis sieben Schmelzen) neu zugestellt werden. Über zwei Tauchrohre gelangt der Stahl vom Tundish in die wassergekühlten Kupferdurchlaufkokillen. Über Stopfen kann die Durchflussmenge durch das Tauchrohr reguliert werden. Der Stopfen besitzt entlang seiner Längsachse einen durchgehenden Hohlraum. Über diesen Kanal wird Argon eingeleitet, das am Stopfenende in den Stahl gelangt. Das aufsteigende Inertgas fördert die Abscheidung von Oxideinschlüssen im Tundish. Das FF-Material des Tauchrohres besteht 
überwiegend aus $\mathrm{Al}_{2} \mathrm{O}_{3}, \mathrm{SiC}$ und Kohlenstoff (siehe Kapitel 4.2.5). In der Kokille erstarrt der Stahl am Kontakt zum Kupfer direkt. Es bildet sich eine feste Strangschale, die dem im Inneren befindlichen, flüssigen Stahl (Sumpf) Halt verschafft. Die Kokille wird mit Gießpulver abgedeckt, das überwiegend aus $\mathrm{Al}_{2} \mathrm{O}_{3}$ und $\mathrm{SiO}_{2}$ zusammengesetzt ist und die Kokillenschlacke bildet. Beim Abziehen des Strangs wird dieser kontinuierlich gekühlt, Schneidbrenner zerschneiden das Halbzeug zu Brammen. Eine detailliertere, graphische Darstellung einer Brammenstranggussanlage findet sich in Kapitel 12.5, Abbildung 7.

\subsection{Nichtmetallische Einschlüsse im Stahl und Clogging}

In den letzten Jahrzehnten sind die Anforderungen an die Stahlhersteller bezüglich der Quantität wie auch insbesondere der Qualität der erzeugten Stähle stetig gestiegen

(PleschiUtschnigg 1989). Die Verbesserung des Reinheitsgrades von Stählen erfordert sehr niedrige Gehalte von S, O, P, N, H, C und nichtmetallischen Einschlüssen (OvTCHINNIKOV 2001, Zhang and Thomas 2002, Zhang and Thomas 2003b, LANGe 1988, Morrison 1989, AHLBORG et al. 2003). Letztere beeinflussen durch Anzahl, Morphologie und Art den Reinheitsgrad (DEKKERS et al. 2004b, LUO 2001). Zur Bewertung des Reinheitsgrades wird in der Industrie der Gesamtsauerstoffgehalt des Stahls herangezogen. Da der Gehalt an gelöstem O im Stahl konstant ist, gibt der Gesamtsauerstoffgehalt Aufschluss über nichtmetallische Einschlüsse im Stahl (DEKKERS et al. 2004b). In Abhängigkeit von ihrer Herkunft werden sie als exogen oder endogen klassifiziert (VAN DER EIJK et al. 2000, OVTCHINNIKOV 2001, JUVONEN 2004, ZHANG and THOMAS 2003b, PACK et al. 2005). Erstere stammen von erodiertem FFMaterial (z. B. Verteilerauskleidung, Pfannensteine) und von Mitlaufschlacke aus der Pfanne und/oder dem Verteiler (OvTCHINNIKOV et al. 2003). Letztere entstehen bei der Oxidation des legierten Aluminiums in der Schmelze (PACK et al. 2005). In der modernen metallurgischen Praxis treten exogene Einschlüsse im Stahl weit gegenüber endogenen zurück. Nach OVTCHINNIKOV (2001) werden Oxideinschlüsse entsprechend ihrer chemischen Zusammensetzung unterteilt in:

- Typ FeO mit diadochem Einbau von Mangan (MnO oder (Fe,Mn)O)

- Tonerdetyp $\left(\mathrm{Al}_{2} \mathrm{O}_{3}\right)$

- Spinelltyp, meist Spinell $\left(\mathrm{MgAl}_{2} \mathrm{O}_{4}\right)$, bei oxidierenderen Bedingungen Hercynit $\left(\mathrm{FeAl}_{2} \mathrm{O}_{4}\right)$, Galaxit $\left(\mathrm{MnAl}_{2} \mathrm{O}_{4}\right)$

- Silikattyp: zu diesem Einschlusstyp gehören Mullit $\left(\mathrm{Al}_{6} \mathrm{Si}_{2} \mathrm{O}_{13}\right)$ und Rhodonit (Ca,Mn) $\mathrm{SiO}_{3}$

- $2 \mathrm{MnO} * 2 \mathrm{Al}_{2} \mathrm{O}_{3} * 5 \mathrm{SiO}_{2}, \mathrm{CaO} \mathrm{SiO}_{2}$, Fayalit $\left(\mathrm{Fe}_{2} \mathrm{SiO}_{4}\right)$ und (Fe,Mn)-Silikate von unterschiedlicher Zusammensetzung 
Nach dem Zeitpunkt der Entstehung bezeichnen OvTCHINNIKOV et al. (2003) und OvTCHINNIKOV (2001) primäre Desoxidationsprodukte als Einschlüsse, die direkt nach Zugabe des Desoxidationsmittels entstehen. Sekundäre Desoxidationsprodukte kristallisieren während der Abkühlung der Stahlschmelze bis zur Solidustemperatur. Diese scheiden sich aufgrund ihrer geringen Größe und steigender Viskosität der Schmelze mit abnehmender Temperatur schlechter ab. Die Löslichkeit des Desoxidationsmittels und insbesondere die von O sind stark temperaturabhängig und nehmen mit sinkender Temperatur ab. Dies führt im Stabilitätsfeld zwischen Liquidus und Solidus (siehe Abbildung 3) sowohl zu einer Anreicherung von O als auch zu einer Anreicherung des Desoxidationsmittels in der Restschmelze. Die dabei gebildeten tertiären Desoxidationsprodukte werden kaum abgeschieden und beeinflussen somit stark die Materialeigenschaften des Stahls. Einschlüsse, die erst im durcherstarrten Zustand wachsen, werden als Quartäreinschlüsse bezeichnet (OVTCHINNIKOV 2001). In dieser Studie sind mit Desoxidationsprodukten stets Einschlüsse gemeint, die beim Desoxidieren in der Pfanne gebildet werden.

In aluminiumberuhigten Stählen bilden Tonerdeeinschlüsse (trigonal-rhomboedrische $\alpha$ Modifikation des Korunds) den vorherrschenden Typ nichtmetallischer Einschlüsse (TOUlOuse et al. in press, BANNENBERG 1995, PlesCHIUTSCHNIGG 1989, ZimMERMANN 1974). Das Aluminium reagiert mit Sauerstoff zu festen Tonerdeeinschlüssen, die durch Absorption in Pfannen-, Verteiler- und Kokillenschlacke zum überwiegenden Teil aus der Schmelze entfernt werden (SHANNON and SRIDHAR 2004).

Nichtmetallische Einschlüsse haben zwei schädigende Einflüsse. Zum einen wirken sie sich produkt-, zum anderen prozessschädigend aus. Die Art des Einschlusses hat großen Einfluss auf mechanische Eigenschaften des Stahls (KIESSLING 1997, NiCHOLSON and GLADMAN 1986). In Bezug auf den vergossenen Stahl sind das nach ZHANG and THOMAS (2002) folgende Auswirkungen durch oxidische und sulfidische Einschlüsse:

- Formbarkeit (Anisotropie)

- Kerbschlagzähigkeit

- Tiefziehfähigkeit

- Zähigkeit

- Dauerfestigkeit

Nach DEKKERS (2003) verhalten sich Einschlüsse im Stahl beim Walzen auf drei verschiedene Weisen (siehe Abbildung 14). 
(a)

(b)
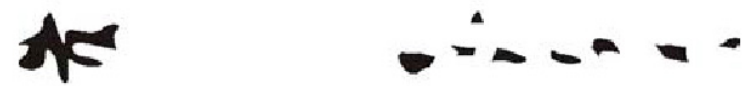

(c)
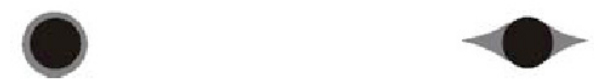

Abbildung 14: Form von Einschlüssen im Stahl vor (links) und nach dem Walzen (rechts). (a) duktile Einschlüsse, (b) spröde Einschlüsse und (c) nicht verformbare, sphärische Einschlüsse mit einem duktilen Rand (DEKKERS 2003).

Plastisch lassen sich MnS-Einschlüsse verformen (a), $\mathrm{Al}_{2} \mathrm{O}_{3}$-Einschlüsse werden beim Walzen spröde verformt und zerbrechen zu irregulären Partikeln (b). Die runden Calciumaluminateinschlüsse werden nicht verformt (c) (siehe Abbildung 14). WIJK (1995) unterteilt vier verschiedene Arten von deformierten Einschlüssen, HILTY and KAY (1985) fünf. Dabei handelt es sich um weitere Unterteilungen der drei Haupttypen.

Die Größe nichtmetallischer Einschlüsse variiert zwischen $<50 \mu \mathrm{m}$ bis zu einigen hundert Mikrometern (Zimmermann 1974, Pleschiutschnigg 1989, PACK 2000). Der hohe Reinheitsgrad von IF-Stählen bedingt maximale Einschlussgrößen von $100 \mu \mathrm{m}$ (ZHANG and THOMAS 2002).

Nichtmetallische Einschlüsse können aber nicht nur das Produkt schädigen, sondern wirken sich auch negativ auf den Stranggießprozess selbst aus (DEKKERS et al. 1999). Beim Gießen lagern sich Einschlüsse innerhalb des Tauchrohres ab - es entsteht Clogging (engl. das Verstopfen). Oxidische Ablagerungen finden sich auch an der Tauchrohrunterseite (siehe Abbildung 38 und in Kapitel 12.2 Abbildung 1). Das keramische Tauchrohr wächst durch die Präzipitate von außen nach innen zu. Dies kann zu einer Verringerung der Durchflussmenge bis hin zum Gießabbruch führen (ToulousE et al. in press). Das lässt die Produktionskosten steigen (RACKERS and THOMAS 1995), da weniger Schmelzen vergossen werden können (JÖNSSON et al. 2005) und Tauchrohre häufiger gewechselt werden müssen (THOMAS and BAI 2001). Clogging kann einen asymmetrischen Gießstrahl verursachen, wodurch es zu unerwünschten Strömungsverhältnissen in der Kokille (THOMAS and BAI 2001) und zu asymmetrischer Einschlussverteilung im Strang kommt (siehe Abbildung 15) (ZHANG and THOMAS 2003a). 


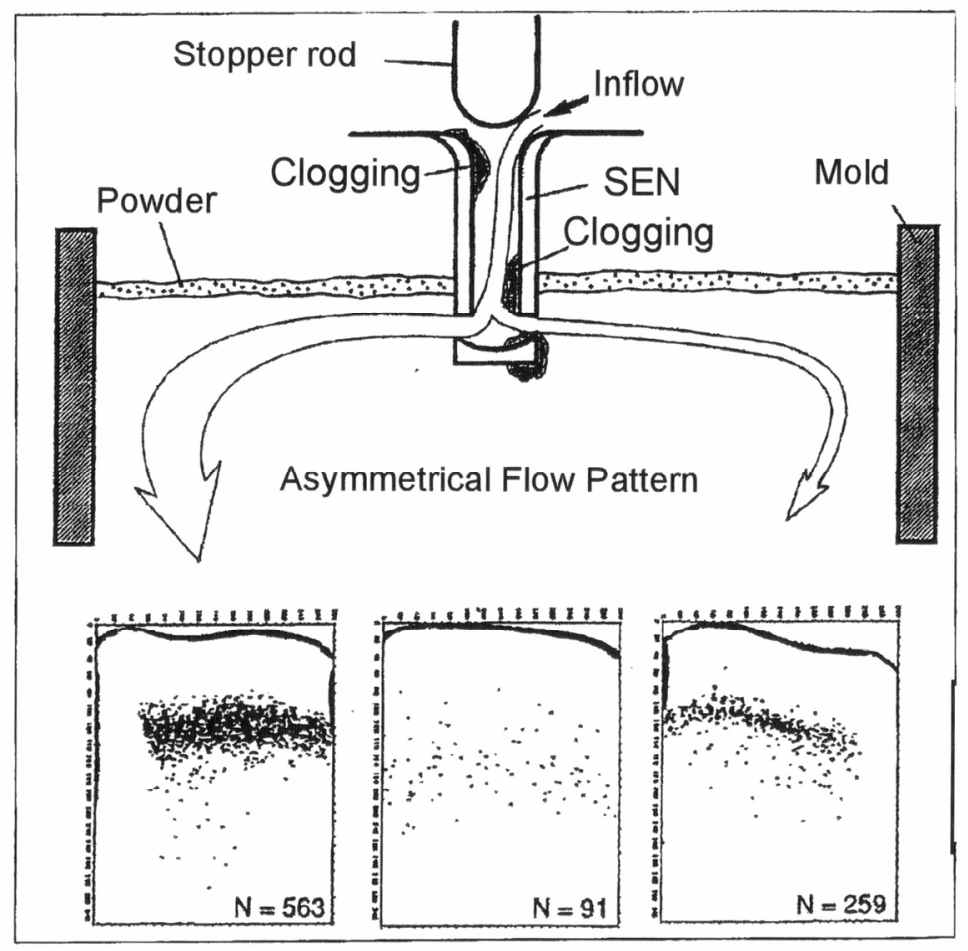

Abbildung 15: Asymmetrische Einschlusskontamination eines Strangs, verursacht durch asymmetrischen Gießstrahl aufgrund von Clogging im Tauchrohr (ZHANG and THOMAS 2003b).

Erodiertes Clogging gelangt in die Schmelze und kann zu Oberflächendefekten des Stahls führen (DEKKERS et al. 2002, DEKKERS 2003). Große, mitgerissene Cloggingfragmente führen zu einer deutlichen Herabsetzung des Reinheitsgrades.

THOMAS and BAI 2001 (2001) und KEMEY (1998) unterscheiden abhängig von dem Entstehungsmechanismus folgende Typen von Clogging:

- Ablagerung von im Stahl vorliegenden Oxiden an der Tauchrohrwandung

○ Desoxidationsprodukte

- Reoxidationsprodukte durch Kontakt der Schmelze mit Luft

○ Mitlaufschlacke

○ exogene Einschlüsse mit anderen Quellen

- Einschlussmodifikation durch chemische Reaktionen

- Bildung von Oxiden in der Nähe der oder direkt an der Tauchrohrwandung

- Aspiration von Luft in das Tauchrohr

- Chemische Reaktion zwischen FF-Material des Tauchrohres und Stahl

- Stahlanfrierungen 
○ geringe Vorheizung des Tauchrohres oder hohe Wärmeverlustrate

○ lokale Erhöhung der Liquidustemperatur durch Entmischung

In der Praxis besteht Clogging häufig aus einer Kombination von mehreren Typen, wobei es schwierig ist, die genaue Ursache festzustellen (THOMAS and BAI 2001). In der weitergehenden Arbeit ist mit Clogging stets die nichtmetallische Fraktion gemeint. Die Arbeiten von DEKKERs et al. (2003), DEKKERS et al. (2002), DEKKERs (2002), und DEKKERS et al. (2004c) zu Einschlüssen im Stahl und Clogging konzentrieren sich auf morphologische Untersuchungen. Danach ist Reoxidation der Grund für die Entstehung von Clogging. BASU et al. (2004) schreiben, dass Reoxidation von Stahl zu Clogging führen kann. Daher sollten z. B. die Anteile an reduzierbaren Komponenten $\left(\mathrm{SiO}_{2}+\mathrm{FeO}_{\mathrm{x}}+\mathrm{MnO}\right)$ im Schiebersand weniger als 10 Gew.-\% ausmachen. DAWSON (1990) führt Strömungsexperimente durch und kommt zu dem Schluss, dass das Zusetzen von Tauchrohren durch eine Separation des Gießstrahls verursacht wird. Die Aufteilung des Gießstrahls geht danach einher mit der Bildung von Wirbeln, wodurch Einschlüsse direkt an der Tauchrohrwandung abgelagert werden. FUHR and CICUTTI (2003) schließen aus morphologischen, chemischen und mineralogischen Untersuchungen, dass Clogging in den meisten Fällen mit der Zusammensetzung von im Stahl vorliegenden nichtmetallischen Einschlüssen zusammenhängt und dass Clogging entsteht, wenn die Menge an festen Einschlüssen einen kritischen Wert überschreitet. MUKAI and ZEZE (2003) schließen aus Experimenten in wässrigen Lösungen, dass die Bewegung feiner Teilchen eine Folge von Grenzflächenspannungsradienten ist und dies in enger Beziehung zur Cloggingproblematik steht. SASAI and MizUKAMI (2003) untersuchen Clogging von calciumbehandelten Stahlschmelzen und entwickeln ein Tonerde-Adhäsions-Modell. Sie führen Clogging auf Partikel zurück, die bereits in der Schmelze vorlagen, bevor diese abgelagert wurden. SASAI and MIZUKAMI (2002) untersuchen Clogging während auftretender Reoxidation von Stahl im Tundish. Danach entsteht im Tundish flüssiges FeO, was nicht reduziert, sondern in das Tauchrohr und die Kokille weitertransportiert wird. Sie leiten ab, dass flüssiges $\mathrm{FeO}$ in diesem Fall als Binder für die Anhaftung von $\mathrm{Al}_{2} \mathrm{O}_{3}$ an die Tauchrohrwandung fungiert. VERMEULEN et al. (2000) schlagen vor, dass die Reduktion von $\mathrm{SiO}_{2}$ aus dem FF-Material des Tauchrohres ODonator für die Bildung von Clogging ist. Dabei reagiert $\mathrm{SiO}_{2}$ mit $\mathrm{C}$ zu gasförmigem $\mathrm{SiO}$ und $\mathrm{CO}$, die das legierte $\mathrm{Al}$ im Stahl zu Tonerde oxidieren. Die In-Situ-Bildung von $\mathrm{Al}_{2} \mathrm{O}_{3}$ im Tauchrohr durch SiO ist bei dieser Studie nicht möglich, da das Tauchrohr kein $\mathrm{SiO}_{2}$ enthält (siehe Kapitel 4.2.5). HÖLlER (1999) schreibt darüber hinaus, dass die Reduktion von $\mathrm{Na}_{2} \mathrm{O}$ und $\mathrm{K}_{2} \mathrm{O}$ aus dem FF-Material die Ansatzbildung in Tauchrohren verstärkt. Die Alkalien stammen danach aus der Graphitgangart. Als Hauptursache für Clogging sieht er die Oxidati- 
on von $\mathrm{Al}$ durch $\mathrm{CO}$ an der Tauchrohrwandung. Ein vollständiges Verschwinden von Quarz im FF-Material des Tauchrohres würde häufig beobachtet werden und durch den starken Unterdruck im Tauchrohr zur Einsaugung der Gasphasen führen. Dabei würde es zu Oxidationsreaktionen am Kontakt Stahl/FF-Material kommen. OGIBAYASHI (1994) führt morphologische Untersuchungen von Clogging durch. Danach besteht Clogging aus einem Netzwerk von Tonerde. Als Bildungsmechanismus wird der gleiche wie bei VERMEULEN et al. (2000) angenommen (siehe oben). Auch THOMAS and BAI (2001) berichten, dass C aus dem FF-Material von Tauchrohren mit Bindern und Verunreinigungen zu CO reagiert, welches dann zur Reoxidation des Stahls führt. Nach POIRIER and THILlou (1994) geht Clogging auf Gastransfer durch das Tauchrohr zurück, wodurch der Luftdurchlässigkeit des FF-Materials eine essentielle Bedeutung zukommt. Eine etwaige Permeabilität des Tauchrohres ist aus sauerstoffisotopischer Sicht weder bei dieser Studie noch bei den Studien von PACK (2000) und PACK et al. (2005) festgestellt worden. KEMEY (1998) schlägt die Einrichtung eines Clogging-Indikators durch Messung bestimmter Prozessgrößen vor. Bei Erreichen bestimmter Indikationswerte wie z. B. einer erniedrigten Gießgeschwindigkeit könnten geeignete Gegenmaßnahmen wie z. B. Calciumzugabe erfolgen. JÖNSSON et al. (2005) führen In-Situ-Experimente im Labor zur Cloggingbildung durch. Mit morphologischen Untersuchungen und thermodynamischen Berechnungen wird versucht, die Mechanismen, die hinter Clogging stehen, besser zu verstehen. KEMEY (1998) schreibt, dass es aufgrund von unzureichender quantitativer Analyse häufig schwierig ist, die Änderung von Prozessparametern in Bezug zu setzen zu der Änderung des Ausmaßes von Clogging. Ein quantitatives Modell zur Herkunft von Clogging wird in Kapitel 3.5 und bei TOULOUSE et al. (in press) in Kapitel 12.5 beschrieben.

Die Literaturauswertung zeigt, dass sich die bisherigen Untersuchungen von Clogging auf phänomenologische Betrachtungen konzentrierten und Lösungsvorschläge daher lediglich Symptome bekämpfen konnten. Dabei blieb die Ursache und der Entstehungsmechanismus von Clogging ungeklärt (HIRAGA et al. 1995). Die Herangehensweise mit O-Isotopen ist somit völlig neu und zielt auf die eigentliche Ursache von Clogging als Lösungsansatz ab. 


\section{Teil I}

\subsection{Problemstellung und Ansatz}

Mit Hilfe von Sauerstoffisotopen als Marker soll die Herkunft von Clogging beim Stranggießen geklärt werden. Als Hauptursache für Clogging ist die Ablagerung von Tonerde anzusehen, die u. a. bei der Desoxidation entsteht. Später liegt Aluminium von der Desoxidation legiert in der Stahlschmelze vor, typischerweise am Ende der Pfannenbehandlung in einer Konzentration von 0,03 bis 0,05 Gew.-\% Al (OETERS 1989). Das Aluminium reagiert mit Sauerstoff zu Tonerde nach:

$$
2[\mathrm{Al}]^{\text {Stahl }}+3 \mathrm{O}^{\text {Atmosphare } \text { Stahl/FF-Material }} \rightleftharpoons\left\langle\mathrm{Al}_{2} \mathrm{O}_{3}\right\rangle^{\text {Einschluss }}
$$

Beim Vergießen des Stahls werden die Oxide abgelagert, es entsteht Clogging (DEKKERS 2003). Neben der Bildung von Clogging durch Ablagerung von oxidiertem Aluminium (=Tonerde) ist auch die Erosion und spätere Ablagerung von FF-Material und Schlacke zu nennen, die ebenfalls zu Clogging beitragen kann. Auch kann die Reduktion von FF-Material zur Entstehung von nichtmetallischen Einschlüssen führen, die dann, wenn sie abgelagert werden, ebenfalls zum Clogging beitragen (PACK et al. 2003). Um die O-Quelle für Clogging herauszufinden, wird der isotopische „Fingerabdruck“ der O-Quelle genutzt.

Es gibt drei stabile Sauerstoffisotope, von denen in diesem Kapitel lediglich das ${ }^{16} \mathrm{O}(99,8 \%)$ und das ${ }^{18} \mathrm{O}(0,2 \%)$ von Bedeutung sind. Bei Sauerstoffisotopenzusammensetzungen geht es hier um das Verhältnis von ${ }^{18} \mathrm{O}$ zu ${ }^{16} \mathrm{O}$. Die Variationen in den Sauerstoffisotopenverhältnissen sind i. A. gering und werden in Promilleabweichungen als $\delta^{18} \mathrm{O}-$ Wert relativ zu einem Standard $^{1}$ angegeben.

$$
\delta^{18} \mathrm{O}=\left(\frac{\left(\frac{{ }^{18} \mathrm{O}}{{ }^{16} \mathrm{O}}\right)_{\text {Probe }}}{\left(\left(\frac{{ }^{18} \mathrm{O}}{{ }^{16} \mathrm{O}}\right)_{\text {Standard }}\right.}-1\right) \times 1000[\%]
$$

Der Standard ist Meerwasser, dem ein $\delta^{18} \mathrm{O}$ von $0 \%$ zugeschrieben wurde. Variationen sind in der Größenordung von einigen Zehnern Promille.

\footnotetext{
${ }^{1}$ Alle Isotopendaten sind hier relativ zum O-Isotopenstandard VSMOW (Vienna Standard Mean Ocean Water) angegeben.
} 
Je höher der $\delta^{18} 0$-Wert, desto größer das Verhältnis von ${ }^{18} 0 /{ }^{16} 0$ in der Probe. Je niedriger der $\delta^{18} 0$-Wert, desto kleiner das Verhältnis von ${ }^{18} 0 /{ }^{16} 0$ in der Probe.

Für die Oxidation des legierten Aluminiums kommen verschiedene O-Quellen in Frage:

1. Frischsauerstoff

2. Luftsauerstoff

3. Prozessgase $\mathrm{Ar}$ und $\mathrm{N}_{2}$

4. FF-Material (Pfanne, Verteiler)

5. Schlacken

Die meisten dieser O-Quellen unterscheiden sich in ihrer Sauerstoffisotopenzusammensetzung (PACK et al. 2005).

\subsection{Zielsetzung}

Eine Grundlage für die Verwendbarkeit von 0-Isotopen zur Klärung der Herkunft von Clogging ist die Annahme, dass bei der Oxidation von Al im flüssigen Stahl keine oder nur eine geringe Verschiebung der Isotopenverhältnisse auftritt, $d$. $h$. dass die Tonerde die Isotopie ihrer Sauerstoffquelle übernimmt.

Die Herangehensweise an obige Problemstellung bestand in Teil I in der experimentellen Bestimmung der O-Isotopenfraktionierung ${ }^{2}$ bei der Oxidation von Al-legiertem Stahl bei $1600^{\circ} \mathrm{C}$. Neben diesem vorrangigen Ziel wurden zusätzlich bereits erste Cloggingproben, Probenmaterialien und Gasproben aus den Stahlwerken A und B analysiert.

Sowohl von den Cloggingproben als auch von den Probenmaterialien sollte $\delta^{18} \mathrm{O}$ bestimmt werden. Von der ThyssenKrupp Steel AG wurden sowohl zwei Tauchrohre samt Clogging sowie eine Cloggingprobe aus einem weiteren Tauchrohr als auch Schiebersand der Hersteller B und A02 bereitgestellt.

Des Weiteren wurden Proben der Prozessgase $\mathrm{O}_{2}$ (Stahlwerk B, 1 Flasche am 20.12.2005, 2 Flaschen am 24.01.2006), Ar und $\mathrm{N}_{2}$ (Stahlwerk B, 1 Flasche Ar am 05.12.2005, 1 Flasche $\mathrm{N}_{2}$ am 20.12.2005) genommen. Bei den Inertgasen Ar und $\mathrm{N}_{2}$ war das Ziel, zu untersuchen, ob $\mathrm{O}_{2}$ in den Gasen vorhanden ist und ob dieses für die Bildung von Clogging verantwortlich gemacht werden kann. Dafür sollte das $\mathrm{O}_{2}$ aus den Inertgasen extrahiert, der $\delta^{18} \mathrm{O}-\mathrm{Wert}$ be-

\footnotetext{
${ }^{2}$ O-Isotopenfraktionierung: Hier die Änderung/Verschiebung des Verhältnisses von ${ }^{18} \mathrm{O}$ zu ${ }^{16} \mathrm{O}$ zwischen koexistierenden Phasen im Laufe einer chemischen Reaktion.
} 
stimmt und mit dem $\delta^{18} \mathrm{O}$-Wert des Cloggings verglichen werden. Der $\delta^{18} \mathrm{O}$ des Frischsauerstoffs sollte direkt gemessen werden.

\subsection{Experimente}

\subsubsection{Methodik Hochtemperaturexperimente}

Ziel der hier dargestellten Untersuchungen ist, zu klären, ob es unter Bedingungen, wie sie bei der Stahlherstellung herrschen, bei der Oxidation von Al aus Stahlschmelzen Isotopenfraktionierung zwischen dem Oxidationsmittel und der gebildeten Tonerde gibt.

In Vorversuchen wurde gezeigt, dass bei experimenteller Oxidation von $\mathrm{Al}$ aus einer $\mathrm{Fe}, \mathrm{Al}-$ Legierung tatsächlich nur ein geringer Unterschied zwischen der Isotopie der 0-Quelle und der der gebildeten Oxide, hier Tonerde $\left(\mathrm{Al}_{2} \mathrm{O}_{3}\right)$, Hercynit $\left(\mathrm{FeAl} \mathrm{O}_{4}\right)$ und Wüstit $(\mathrm{FeO})$, zu beobachten ist.

Bei den aktuellen Experimenten wurden die Oxidationsversuche unter stärker reduzierenden Bedingungen $\left(\log f \mathrm{O}_{2}=-14,8\right)$ als in den Vorversuchen $\left(\log f \mathrm{O}_{2}=-4,0\right)$ durchgeführt, um Bedingungen wie bei der Stahlherstellung einzustellen. Thermodynamischen Berechnungen zufolge liegt die Sauerstofffugazität ${ }^{3}$ in der Pfanne bei einem Al-Gehalt von 0,03 Gew.\% bei $1600^{\circ} \mathrm{C}$ bei $\log f \mathrm{O}_{2}=-14,1$.

\subsubsection{Versuchsdurchführung}

Neun Legierungen mit Aluminiumgehalten von 0,28, 0,68 und 0,87 Gew.\% und zwei Proben aus reinem $\mathrm{Al}$ wurden bei $1600^{\circ} \mathrm{C}$ aufgeschmolzen und in zwei Versuchsreihen jeweils eine Stunde kontrolliert oxidiert. In einer Versuchsreihe wurden die Proben mit Argon 4.5, in einer anderen in einem $\mathrm{H}_{2} / \mathrm{CO}_{2}$-Gasstrom oxidiert (siehe Tabelle 5).

\footnotetext{
${ }^{3}$ Fugazität: Effektiver Partialdruck eines Gases. Die Fugazität verhält sich zu Drücken wie Aktivitäten zu Konzentrationen.
} 
Tabelle 5: Hochtemperaturexperimente $\left(T=1600^{\circ} \mathrm{C}\right)$.

\begin{tabular}{|c|c|c|c|c|c|c|}
\hline Versuchsdatum & Exp\# & $\mathrm{Al}_{\text {vor }}$ [Gew.\%] & $\mathrm{Al}_{\text {nach }}$ [Gew.\%] & Atmosphäre & Synthesezeit [min] & $\delta^{18} \mathrm{O} \mathrm{Al}_{2} \mathrm{O}_{3}[\%$ ] \\
\hline 06.02 .2006 & 1 & 0.87 & 0.17 & $\mathrm{Ar}$ & 60 & - \\
\hline 06.02 .2006 & 2 & 0.87 & 0.80 & $\mathrm{Ar}$ & 60 & - \\
\hline 06.02 .2006 & 3 & 0.68 & 0.57 & $\mathrm{Ar}$ & 60 & - \\
\hline 06.02 .2006 & 4 & 0.28 & 0.19 & $\mathrm{Ar}$ & 60 & - \\
\hline 07.02 .2006 & 5 & 100 & 100 & $\mathrm{Ar}$ & 60 & +0.5 \\
\hline 07.02 .2006 & 6 & 0.87 & 0.62 & $\mathrm{H}_{2} / \mathrm{CO}_{2}$ & 60 & - \\
\hline 07.02 .2006 & 7 & 0.68 & 0.00 & $\mathrm{H}_{2} / \mathrm{CO}_{2}$ & 60 & - \\
\hline 07.02 .2006 & 8 & 0.68 & 0.08 & $\mathrm{H}_{2} / \mathrm{CO}_{2}$ & 60 & +11.8 \\
\hline 07.02 .2006 & 9 & 0.28 & 0.00 & $\mathrm{H}_{2} / \mathrm{CO}_{2}$ & 60 & +13.0 \\
\hline 08.02 .2006 & 10 & 0.87 & 0.57 & $\mathrm{H}_{2} / \mathrm{CO}_{2}$ & 60 & +11.3 \\
\hline 08.02 .2006 & 11 & 100 & 100 & $\mathrm{H}_{2} / \mathrm{CO}_{2}$ & 60 & +12.7 \\
\hline
\end{tabular}

Die Sauerstofffugazität wurde mit der $\mathrm{H}_{2} / \mathrm{CO}_{2}$-Mischungen auf $\log f \mathrm{O}_{2}=-14,8$ eingestellt. Nach thermodynamischen Berechnungen sollte sich nur Tonerde $\left(\mathrm{Al}_{2} \mathrm{O}_{3}\right)$, aber weder Hercynit $\left(\mathrm{FeAl}_{2} \mathrm{O}_{4}\right)$ noch Wüstit $(\mathrm{FeO})$ bilden.

Als Probenhalter dienten Korundtiegel, die an einem Draht aufgehängt in die $1600^{\circ} \mathrm{C}$ heiße Heißzone des vertikalen Gasmischungsofens abgesenkt wurden (siehe Abbildung 16).
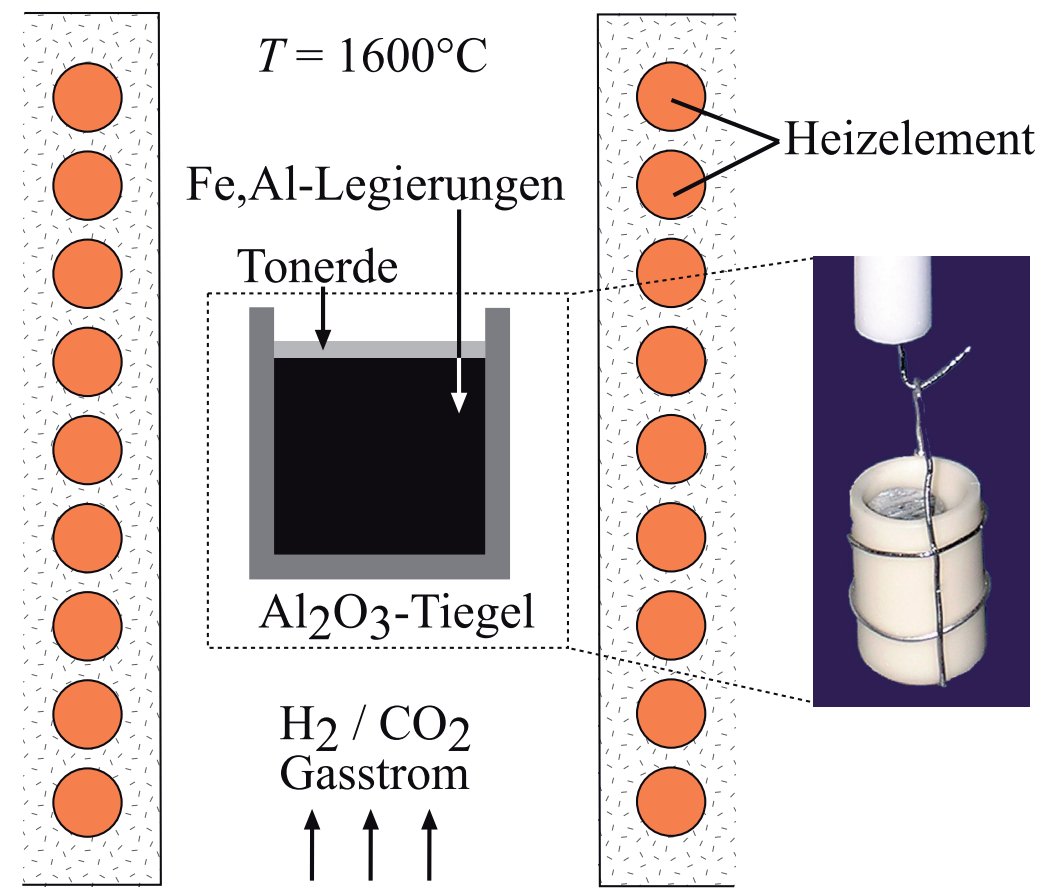

Abbildung 16: Versuchsaufbau zur Oxidation der Fe,Al-Legierungen in einem vertikalen Gasmischungsofen. Die Proben wurden in einem Korundtiegel unter reduzierender Atmosphäre partiell oxidiert (rechts Photo des Probenhalters im Rohrofen).

\subsubsection{Versuchsreihe mit $\mathrm{H}_{2} / \mathrm{CO}_{2}$-Gasmischung}

Zunächst wurde der Ofen zur Vermeidung einer Knallgasreaktion durch im Ofen verbliebenen Sauerstoff 20 Minuten mit Argon gespült. Danach wurden die $\mathrm{H}_{2} / \mathrm{CO}_{2}$-Gasmischung eingelassen, die Proben in die Heißzone abgesenkt, der Ar-Gasfluss gestoppt und die Proben jeweils eine Stunde oxidiert. Nach Beendigung des Versuchs wurden die Proben durch Ziehen am 
oberen Ende des Aufhängedrahtes in einem mit Sand gefüllten Kieselglasbehälter abgeschreckt (quenchen) (siehe Abbildung 17), der $\mathrm{H}_{2} / \mathrm{CO}_{2}$-Gasfluss abgestellt und der Spülvorgang mit Argon erneut gestartet.

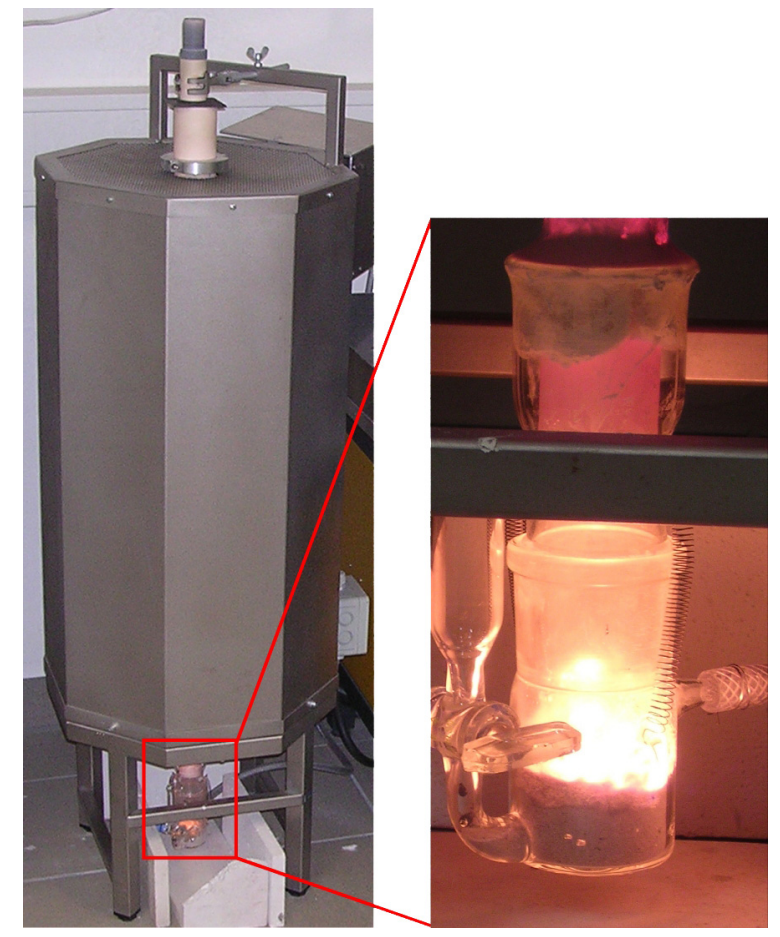

Abbildung 17: Vertikaler Gasmischungsofen (links), Kieselglasbehälter zum Quenchen des Tonerdetiegels nach Beendigung des Experimentes (rechts).

Nach weiteren 20 Minuten war der Ofen hinreichend mit Argon gespült, so dass die Proben gefahrlos entnommen werden konnten. Die Versuchsdurchführung unter Argon 4.5-Atmosphäre war bis auf das entfallende Nachspülen identisch mit obiger Beschreibung.

\subsubsection{Einstellung der Gasmischung}

Um im Gasgemisch $\mathrm{H}_{2} / \mathrm{CO}_{2}$ bei $1600^{\circ} \mathrm{C}$ eine Sauerstofffugazität von $\log f \mathrm{O}_{2}=-14,8$ zu erreichen, muss die Zusammensetzung des Gasgemisches nach DEINES et al. (1976) 0,04 Vol.\% $\mathrm{CO}_{2}$ enthalten. In einem ersten Schritt wurde ein $\mathrm{H}_{2} / \mathrm{CO}_{2}$-Gasgemisch im Volumenverhältnis 98:2 mittels einer computergestützten Gasmischanlage mit elektronischen Massenflussreglern hergestellt. In einem zweiten Schritt wurde die anfängliche Gasmischung in einer analogen Gasmischanlage wieder mit $\mathrm{H}_{2}$ im gleichen Verhältnis 98:2 gemischt. Produkt dieser Kaskadenmischung war eine $\mathrm{H}_{2} / \mathrm{CO}_{2}$-Gasmischung mit $0,04 \mathrm{Vol} \% \mathrm{CO}_{2}$, was ein $\log f \mathrm{O}_{2}$ von $-14,8$ puffert. 


\subsubsection{Methodik IR-Laserfluorinierung}

Um Sauerstoffisotopenverhältnisse der synthetisierten Tonerde messen zu können, muss das $\mathrm{Al}_{2} \mathrm{O}_{3}$ aufgeschlossen und der Sauerstoff extrahiert werden. Um das refraktäre Oxid aufzuschließen, wird es mit einem Laser aufgeheizt und einem stärkeren Oxidationsmittel als Sauerstoff ausgesetzt. Als Aufschlussreagenz dient $\mathrm{F}_{2}$, da Fluor das einzige Element ist, dessen Elektronegativität $\left(E N F_{2}=4,0 ; E N \mathrm{O}_{2}=3,5\right)$ größer ist als die des $\mathrm{O}_{2}$ (HAGEN 2002). Das Fluorgas muss für die Analyse sehr rein sein. Durch ein Verfahren nach ASPREY (1976) wird in gleichnamigen Öfen, in denen das Gas mehrere Reinigungsstufen durchläuft, hochreines $\mathrm{F}_{2}$ erzeugt (siehe Abbildung 18).

Das im Überschuss zugeführte Fluorgas ist dafür verantwortlich, dass der freigesetzte Sauerstoff in der Gasphase bleibt und die freigesetzten Kationen abgesättigt werden. Hierbei wird die Tonerde zu Aluminiumtrifluorid umgesetzt nach:

$$
2 \mathrm{Al}_{2} \mathrm{O}_{3}+6 \mathrm{~F}_{2} \rightarrow 4 \mathrm{AlF}_{3}+3 \mathrm{O}_{2}
$$

Mit dieser Methode können reaktionsträge Materialien wie $\mathrm{Al}_{2} \mathrm{O}_{3}$ aufgeschlossen werden. Die benötigten Probenmengen sind dabei sehr gering. Für eine Analyse ist ca. $1 \mathrm{mg}$ Tonerde ausreichend. Für detaillierte Beschreibungen der IR-Laserfluorinierung wird auf HAGEN (2002), PACK (2000), PACK et al. (2005) und SHARP (1990) verwiesen.

Bei der IR-Laserfluorinierung heizt ein $\mathrm{CO}_{2}$-Laser die Probe auf über $2000^{\circ} \mathrm{C}$ auf. Beim Einkoppeln der Probe in den Laserstrahl wird sie aufgeschmolzen, und das Fluorgas reagiert direkt mit der Schmelze.

Der freigesetzte Sauerstoff gelangt über eine Edelstahlhochvakuumanlage online in ein Gasmassenspektrometer, wo die Sauerstoffisotopie gemessen wird. Auf dem Weg zum Massenspektrometer wird der Sauerstoff über eine Tauscherkolonne und mehrere Kühlfallen geleitet, wo er vom Fluorgas gereinigt wird (siehe Abbildung 18 und Abbildung 19). Um $F_{2}$ von $\mathrm{O}_{2}$ zu trennen, wird am CNR in Pisa eine KCl-Tauscherkolonne verwendet, in der $\mathrm{F}_{2} \mathrm{zu}$ Kaliumfluorid umgesetzt wird; als Nebenprodukt entsteht Chlorgas:

$$
2 \mathrm{KCl}+\mathrm{F}_{2} \rightleftharpoons 2 \mathrm{KF}+\mathrm{Cl}_{2}
$$

Das bei der Reaktion freigesetzte Chlorgas wird in Kühlfallen bei $-196^{\circ} \mathrm{C}\left(\mathrm{LN}_{2}\right)$ überfroren und kann so später entsorgt werden. Das $\mathrm{O}_{2}$ wird auf einem Molekularsieb des Typs $13 \mathrm{X}$ gegettert, das in flüssigen $\mathrm{N}_{2}$ getaucht ist, ehe der Sauerstoff in das Gasmassenspektrometer gelangt (siehe Abbildung 18). 


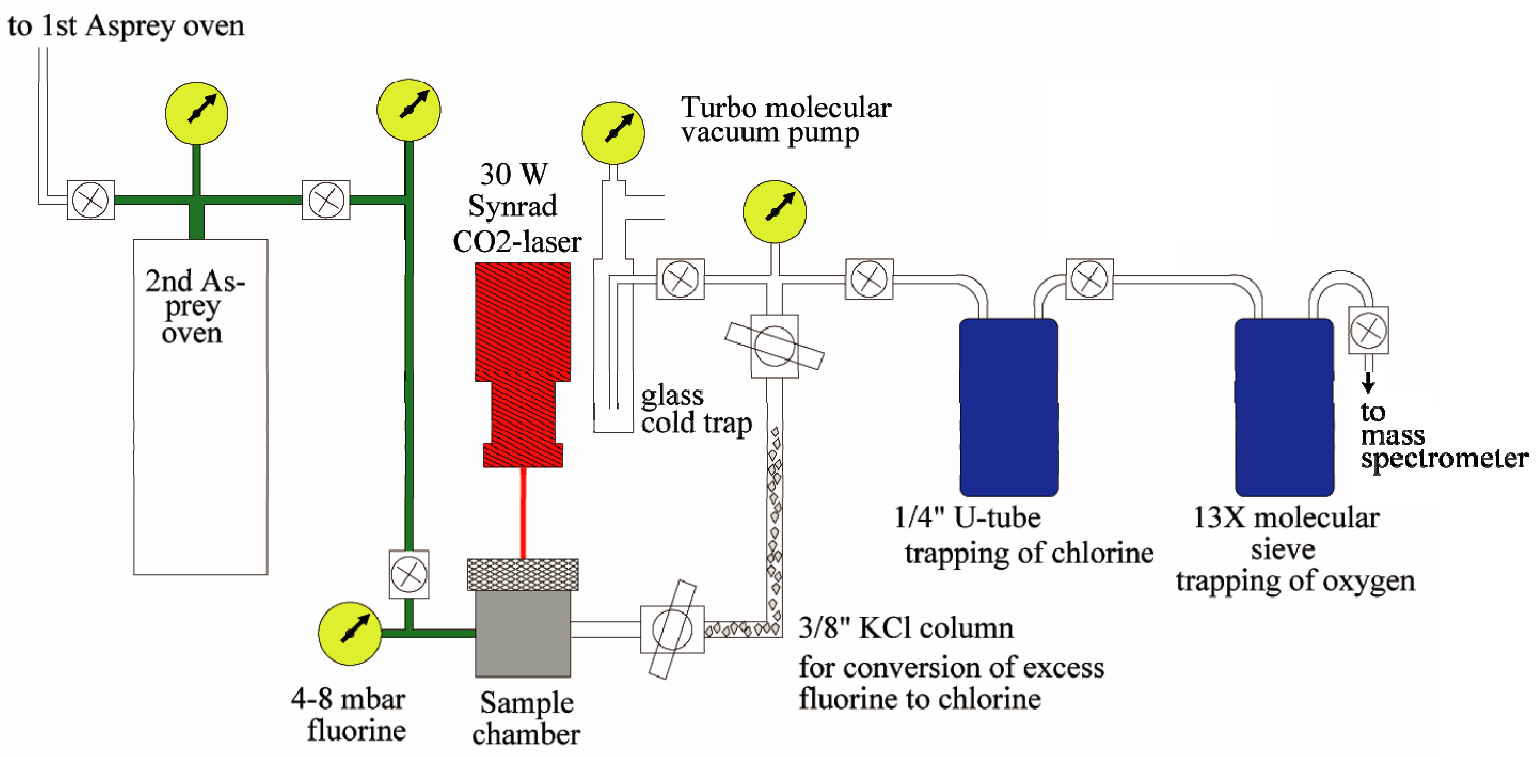

Abbildung 18: IR-Laserfluorinierungsanlage am Mineralogisch-Petrologischen Institut der Rheinischen Friedrich-Wilhelms-Universität Bonn (verändert nach PACK 2000).

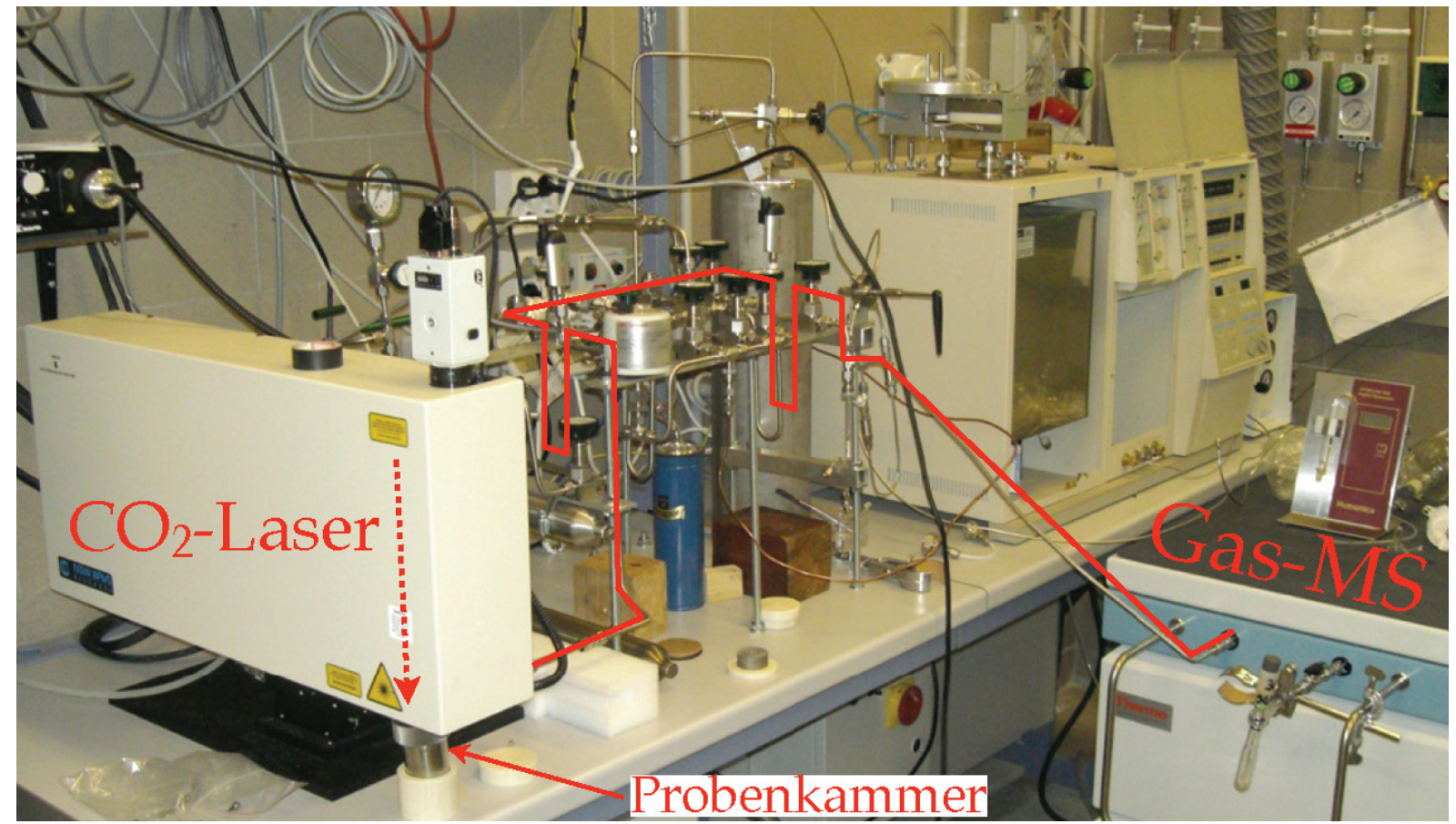

Abbildung 19: IR-Laserfluorinierungsanlage mit $\mathrm{CO}_{2}$-Laser, Hochvakuumline (Weg des Gases durch rote Linie vereinfacht angedeutet) und Gasmassenspektrometer am CNR in Pisa (Italien).

Mit der IR-Laserfluorinierung können extrem refraktäre Phasen wie Tonerde aufgeschlossen und deren Sauerstoffisotopie online gemessen werden. Für die Analyse werden ca. $1 \mathrm{mg}$ Probenmaterial benötigt, eine Analyse dauert etwa $15 \mathrm{~min}$. Bei pulverigen Proben wie Clogging wird ein Fehler von $\pm 0,4 \% 0(1 \sigma)$ angegeben. Für gesinterte Proben wird lediglich ein Fehler von $\pm 0,2 \% 0(1 \sigma)$ erreicht. 


\subsubsection{Ergebnisse}

Die Stahlschmelze konnte in allen Experimenten kontrolliert oxidiert werden. Durch die reduzierende Atmosphäre des $\mathrm{H}_{2} / \mathrm{CO}_{2}$-Gasstromes wurde eine vollständige Oxidation der Legierung effektiv verhindert. Durch das niedrige $f \mathrm{O}_{2}$ wurde nur Tonerde, aber nicht wie in den Vorversuchen Hercynit oder Wüstit gebildet. Anhand der Präparate in den Vorversuchen konnte gezeigt werden, dass die Oxidation schrittweise abläuft - mit Tonerde als erstem Oxidationsprodukt, gefolgt von Hercynit und Wüstit. Die Reihenfolge der Oxidation spiegelt die hohe Affinität von $\mathrm{Al}$ zu O wider.

\subsubsection{Morphologie der synthetisierten Tonerde}

Von der in den Experimenten gebildeten Tonerde wurden mit einem Rasterelektronenmikroskop (REM) Sekundärelektronenbilder angefertigt. Mittels energiedispersivem System (EDS) wurde die chemische Zusammensetzung gemessen.

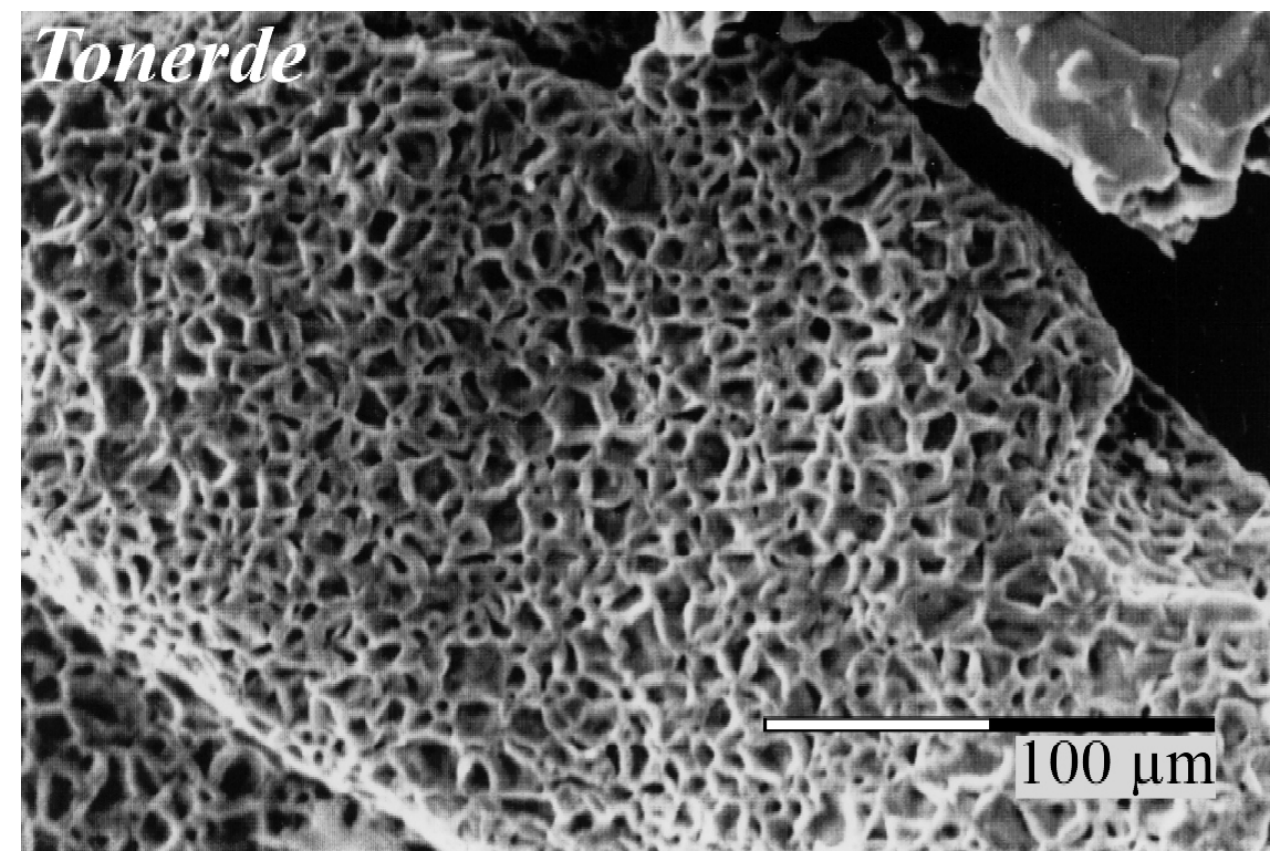

Abbildung 20: Rasteraufnahme der synthetisierten Tonerde (REM, EDS).

Die Tonerde ist in einer korallenartigen Struktur kristallisiert, die ein dreidimensionales Netzwerk aufspannt.

\subsubsection{Chemische Zusammensetzung der Legierungen}

Die Legierungen wurden nach den Experimenten mit einer Elektronenstrahlmikrosonde (EMS) mittels wellenlängendispersivem System mikrochemisch analysiert. In allen Fe,AlLegierungen, die mit der $\mathrm{H}_{2} / \mathrm{CO}_{2}$-Gasmischung oxidiert wurden, war der Al-Gehalt nach dem Experiment deutlich niedriger als in der Ausgangslegierung (siehe Abbildung 21). Bei den 
Experimenten unter $\mathrm{H}_{2} / \mathrm{CO}_{2}$-Gasmischung wurde bei den 0,87 -Legierungen etwa ein Viertel des Aluminiums oxidiert. Bei den 0,68- und 0,28-Legierungen wurde fast das gesamte Al oxidiert. Die Experimente mit Ar zeigen (mit einer Ausnahme) eine Abnahme des Aluminiumgehaltes von $8 \%$ für die 0,87-Legierung, über $16 \%$ für die 0,68 -Legierung und bis zu $32 \%$ für die 0,28-Legierung. Allein die 0,87-Legierung von Experiment 1 zeigt eine Abnahme im Al-Gehalt von $80 \%$.

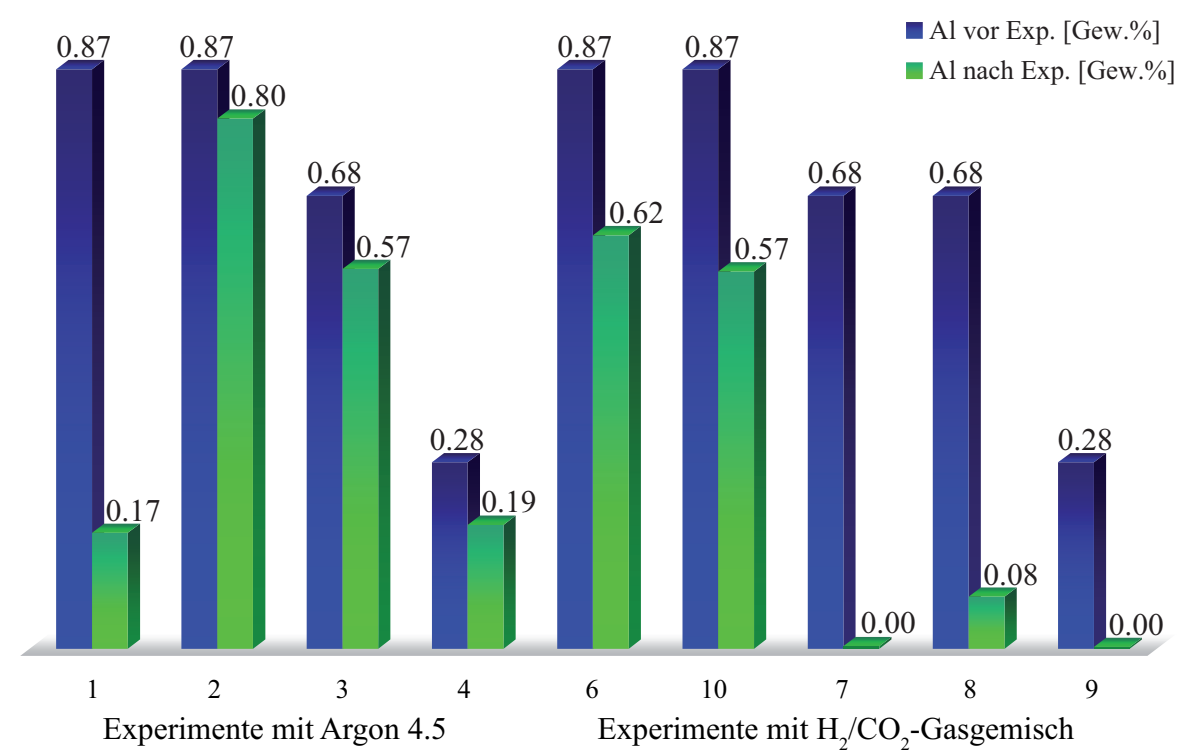

Abbildung 21: Fe,Al-Legierungen mit 0,28, 0,68 und 0,87 Al [Gew.\%] Ausgangszusammensetzung (blaue Säulen) und Al-Gehalte nach den Experimenten (grüne Säulen).

\subsubsection{Isotopenmessung der synthetisierten Tonerde}

Die Syntheseprodukte (=Tonerde) der Experimente wurden isotopenchemisch untersucht. In Abbildung 22 sind die Ergebnisse der O-Isotopenmessungen sowohl der in den Experimenten gebildeten Tonerde (unter $\mathrm{H}_{2} / \mathrm{CO}_{2}$-Gasstrom) als auch der Ofenatmosphäre dargestellt. Insgesamt konnten fünf Proben analysiert werden. Vier Proben, die in dem eingestellten $\mathrm{H}_{2} / \mathrm{CO}_{2}$ Gasstrom oxidiert wurden, und eine, die unter Argon 4.5 oxidiert wurde. Der $\delta^{18} \mathrm{O}$-Wert von letzterer Probe liegt bei $+0,5 \pm 0,4 \%$, der der oxidierenden Spezies im Argon konnte allerdings nicht gemessen werden. Daher ist eine mögliche Fraktionierung zwischen der Ofenatmosphäre mit Argon 4.5 und der gebildeten Tonerde nicht bestimmbar. 


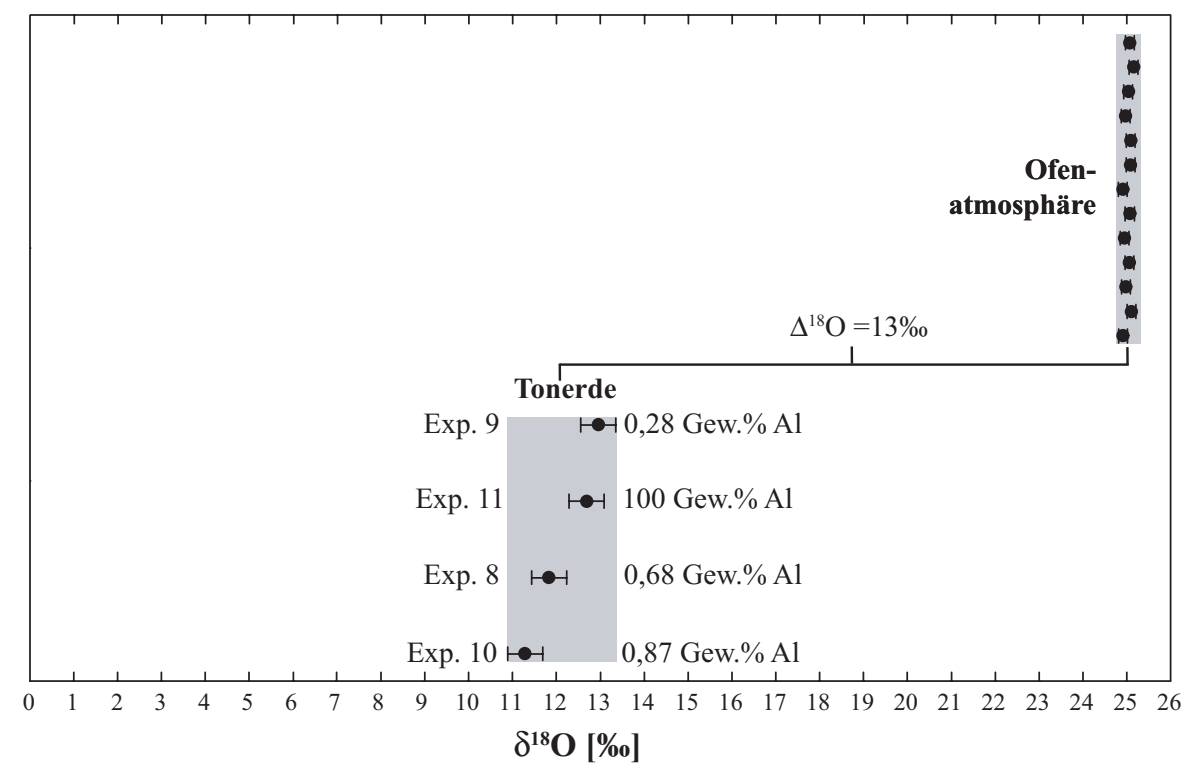

Abbildung 22: O-Isotopenanalyse der synthetisierten Tonerde unter $\mathrm{H}_{2} / \mathrm{CO}_{2}$-Gasstrom und die der Ofenatmosphäre $\left(\mathrm{H}_{2} / \mathrm{CO}_{2}\right.$-Gasstrom).

Der $\delta^{18} 0$-Wert der Ofenatmosphäre ist $+25,1 \pm 0,1 \%$ (Ergebnis von 13 Messungen). Die Tonerde liegt im $\delta^{18} 0$ zwischen $+11,3 \pm 0,4 \%$ für Experiment $10(0,87 \mathrm{Gew} . \% \mathrm{Al})$ und $+13,0 \pm 0,4 \%$ für Experiment 9 (0,28 Gew.\% Al). Der Mittelwert der vier Experimente liegt bei $+12,2 \%$. Bei den aktuellen Experimenten ist damit eine Fraktionierung zwischen Ofenatmosphäre $\left(\log \mathrm{fO}_{2}=-14,8\right)$ und gebildeter Tonerde von $\Delta^{18} \mathrm{O}=+13 \%$ zu beobachten. In den Vorversuchen war $\Delta^{18} \mathrm{O}=+3 \%$; die Sauerstofffugazität lag bei $\log \mathrm{fO}_{2}=$ $-4,0$.

Um zu untersuchen, ob der $\delta^{18} \mathrm{O}$-Wert mit dem Grad der Oxidation zusammenhängt, ist der $\delta^{18} \mathrm{O}$-Wert gegen den Grad der Oxidation aufgetragen (siehe Abbildung 23).

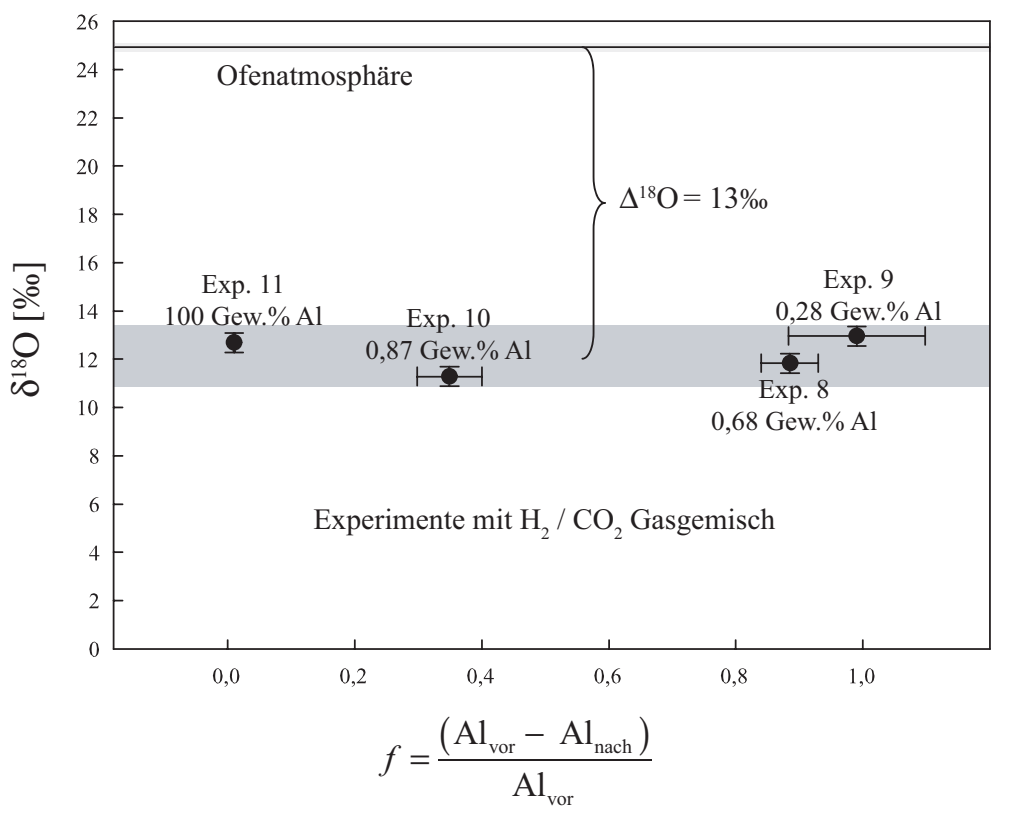

Abbildung 23: Abgetragen ist $\delta^{18} \mathrm{O}$ von Ofenatmosphäre und Tonerde gegen die anteilig gebildete Tonerde $f$. 
In Abbildung 23 ist zu sehen, dass mit abnehmendem Al-Gehalt der Startlegierungen der Anteil gebildeter Tonerde zunimmt. Ebenso abgetragen ist der $\delta^{18} \mathrm{O}-$ Wert der Ofenatmosphäre. Man sieht, dass zwischen beiden Größen kein systematischer Zusammenhang besteht.

Die Isotopie der experimentell gebildeten Tonerde ist weder abhängig von dem AlAusgangsgehalt der Legierungen noch von ihrem Oxidationsgrad (siehe Abbildung 23).

\subsubsection{Diskussion}

Bei den aktuellen Experimenten wurde eine Differenz zwischen Ofenatmosphäre und den Oxiden von $\Delta^{18} \mathrm{O}_{\text {Ofenatmopshäre-Tonerde }}=+13 \%$ festgestellt. Um diesen deutlichen Unterschied zu erklären, werden nachfolgend drei mögliche Fraktionierungsmechanismen diskutiert.

1. Gleichgewichtsfraktionierung ${ }^{4}$

2. Rayleighfraktionierung ${ }^{5}$

3. kinetische Fraktionierung ${ }^{6}$

Zu 1.: Wenn die gebildete Tonerde im chemischen Gleichgewicht mit der Ofenatmosphäre stünde, würde die Gleichgewichtsfraktionierung den entscheidenden Effekt auf deren Isotopie ausüben. Die O-Isotopenfraktionierung im Gleichgewicht geht auf die unterschiedlichen Nullpunkt- und Bindungsenergien von ${ }^{18} \mathrm{O}$ - und ${ }^{16} \mathrm{O}$-haltigen Molekülen zurück. Diese Isotopeneffekte beruhen auf unterschiedlichen quantenmechanischen Eigenschaften isotopisch substituierter Phasen, die durch das Vorhandensein von unterschiedlichen diskontinuierlichen Energiestufen zustande kommen (WILLI 1983).

Die Gleichgewichtsfraktionierung ist temperaturabhängig, und zwar zwischen koexistierenden Phasen etwa proportional zu $1 / T^{2}$, wobei $T$ die Temperatur in Kelvin ist (dies gilt für $t>$ $500^{\circ} \mathrm{C}$ ) (PACK et al. 2005). Die Gleichgewichtsfraktionierung wird bei sehr hohen Temperaturen, wie sie bei der Stahlherstellung herrschen, immer unbedeutender, da sie mit steigender Temperatur gegen null geht (PACK 2000). Nach ZHENG (1991) lässt sich die Fraktionierung zwischen Tonerde und $\mathrm{H}_{2} \mathrm{O}$ für einen Temperaturbereich von 0 bis $1200^{\circ} \mathrm{C}$ berechnen. Extrapoliert man diese Daten auf Temperaturen von $1600^{\circ} \mathrm{C}$, ergibt sich eine Fraktionierung von $\Delta^{18} \mathrm{O}_{\text {Tonerde-H2O }} \approx-2,4 \%$. Durch Extrapolation der theoretisch berechneten Sauerstoffisotopen-

\footnotetext{
${ }^{4}$ Gleichgewichtsfraktionierung: Fraktionierung im chemischen Gleichgewicht $(\mathrm{A} \leftrightarrow \mathrm{B})$, wenn sich die thermodynamischen Eigenschaften isotopisch substituierter Verbindungen unterscheiden.

${ }^{5}$ Fraktionierung im Laufe einer Reaktion, bei der Produkte von Edukten getrennt werden.

${ }^{6}$ kinetische Fraktionierung: Fraktionierung im Zusammenhang mit unidirektionalen Prozessen wie z. B. Diffusion $(\mathrm{A} \rightarrow \mathrm{B})$ aufgrund unterschiedlicher Geschwindigkeiten von Molekülen/Atomen.
} 
fraktionierung zwischen $\mathrm{H}_{2} \mathrm{O}$ und $\mathrm{O}_{2}$ nach RICHET et al. (1977) auf eine Temperatur von $1600^{\circ} \mathrm{C}$ ergibt sich ein $\Delta^{18} \mathrm{O}_{\mathrm{H} 2 \mathrm{O}-\mathrm{O} 2} \approx+2,0 \%$. Führt man diese Daten zusammen, errechnet sich ein $\Delta^{18} \mathrm{O}_{\mathrm{O} 2 \text {-Tonerde }}=+0,4 \%$ (PACK et al. 2005). Da die Experimente in einem $\mathrm{H}_{2} / \mathrm{CO}_{2}$ Gasstrom durchgeführt wurden, ist die Gleichgewichtsfraktionierung zwischen der oxidierenden Spezies und Tonerde von Bedeutung. Bei Temperaturen von $1600^{\circ} \mathrm{C}$ liegen in einem $\mathrm{H}_{2} / \mathrm{CO}_{2}$-Gasstrom neben den Einsatzgasen vorwiegend $\mathrm{CO}$ und $\mathrm{H}_{2} \mathrm{O}$ als oxidierende Spezies vor nach:

$$
\mathrm{H}_{2}+\mathrm{CO}_{2} \rightleftharpoons \mathrm{H}_{2} \mathrm{O}+\mathrm{CO}
$$

Nach RICHET et al. (1977) beträgt die Gleichgewichtsfraktionierung bei $1300^{\circ} \mathrm{C}$ zwischen $\mathrm{O}_{2}$ und $\mathrm{CO}_{2} 3,1 \%$, die zwischen $\mathrm{O}_{2}$ und $\mathrm{CO} 3,7 \%$ ond die zwischen $\mathrm{H}_{2} \mathrm{O}$ und $\mathrm{O}_{2}$ 2,4\%o. Bei $1600^{\circ} \mathrm{C}$ ist die Gleichgewichtsfraktionierung zwischen den Spezies noch kleiner als bei $1300^{\circ} \mathrm{C}$. Daraus wird abgeleitet, dass die oxidierenden Spezies bei den Experimenten einen annähernd gleichen $\delta^{18} \mathrm{O}$ haben.

Die Berechnungen zeigen, dass es nur eine geringe Gleichgewichtsfraktionierung zwischen Ofenatmosphäre und Tonerde gibt. Damit kann die große Differenz von +13\%o nicht erklärt werden.

Zu 2.: Ein anderer Prozess, der zu Isotopenpartitionierung führen kann, wird als Rayleighfraktionierung bezeichnet. Dabei handelt es sich um eine Fraktionierung im Laufe einer Reaktion, wenn Produkte von Edukten getrennt werden. Die Kondensation von Wasser aus einer Wolke ist ein Beispiel für Rayleighfraktionierung. Dabei geht das schwerere ${ }^{18} \mathrm{O}$ bevorzugt in die flüssige Phase über, was zu seiner Abreicherung in der verbleibenden Dampfphase führt. Im Falle der Oxidation von $\mathrm{Al}$ aus einer flüssigen Stahlschmelze würde sich durch eine stetige Entfernung der gebildeten Tonerde sowohl die Isotopie des verbleibenden Sauerstoffs in der Schmelze (bei endlicher Sauerstoffmenge) als auch die der sich im weiteren Verlauf bildenden Tonerde ändern. Die sich im Laufe eines Rayleighprozesses ändernden $\delta^{18} \mathrm{O}$-Werte des Sauerstoffs und der Tonerde sind gegeben durch:

$$
\begin{aligned}
& \delta^{18} \mathrm{O}_{\mathrm{O}_{2}}=\left[\delta^{18} \mathrm{O}_{i}+1000\right] \times f^{\left(1-\alpha_{\mathrm{O}_{2} \text {-Tonerde }}\right)}-1000 \\
& \delta^{18} \mathrm{O}_{\mathrm{Al}_{2} \mathrm{O}_{3}}=\delta^{18} \mathrm{O}_{\mathrm{O}_{2}}-1000 \times \ln \alpha_{\mathrm{O}_{2} \text {-Tonerde }}
\end{aligned}
$$

$\delta^{18} \mathrm{O}_{i}$ ist das anfängliche Isotopenverhältnis des Sauerstoffs in der Schmelze, $f$ der Anteil des in der Schmelze verbleibenden Restsauerstoffs und $\alpha$ der Fraktionierungsfaktor zwischen $\mathrm{O}_{2}$ und $\mathrm{Al}_{2} \mathrm{O}_{3}$ (PACK et al. 2005). Mit Hilfe des Fraktionierungsfaktors $\alpha$ lässt sich die Fraktionierung zwischen zwei Substanzen A und B berechnen nach: 


$$
\alpha_{\mathrm{A}-\mathrm{B}}=\frac{\mathrm{R}_{\mathrm{A}}}{\mathrm{R}_{\mathrm{B}}} \text { mit } \mathrm{R}=\frac{{ }^{18} \mathrm{O}}{{ }^{16} \mathrm{O}}\left[\text { es gilt: } 1000 \times \ln \alpha_{\mathrm{O}_{2} \text {-Tonerde }} \approx \Delta^{18} \mathrm{O}_{\mathrm{O}_{2}-\text { Tonerde }}=\delta^{18} \mathrm{O}_{\mathrm{O}_{2}}-\delta^{18} \mathrm{O}_{\text {Tonerde }}\right]
$$

Hierbei sind $\mathrm{R}_{\mathrm{A}, \mathrm{B}}$ die Verhältnisse des schweren zum leichten Isotop. Die Isotopie der Tonerde ist von $f$ und $\alpha$ abhängig nach:

$$
\delta^{18} \mathrm{O}_{\mathrm{Al}_{2} \mathrm{O}_{3}}=\left(\left[\delta^{18} \mathrm{O}_{i}+1000\right] \times f^{\left(1-\alpha_{\mathrm{O}_{2} \text {-Tonerde }}\right)}-1000\right)-1000 \times \ln \alpha_{\mathrm{O}_{2} \text {-Tonerde }}
$$

Wie oben gezeigt, ist die Gleichgewichtsfraktionierung $\Delta^{18} \mathrm{O}_{\mathrm{O}_{2} \text {-Tonerde }}=+0,4 \%$ o $\left(\alpha_{\mathrm{O}_{2} \text {-Tonerde }}=1,0004\right)$. Für $\alpha_{\mathrm{O}_{2} \text {-Tonerde }}>1$ und $f \rightarrow 0$ wird der $\delta^{18} \mathrm{O}$-Wert der Tonerde immer größer. Für $\alpha_{\mathrm{O}_{2} \text {-Tonerde }}<1$ und $f \rightarrow 0$ wird der $\delta^{18} \mathrm{O}$-Wert der Tonerde immer kleiner (siehe Abbildung 24).

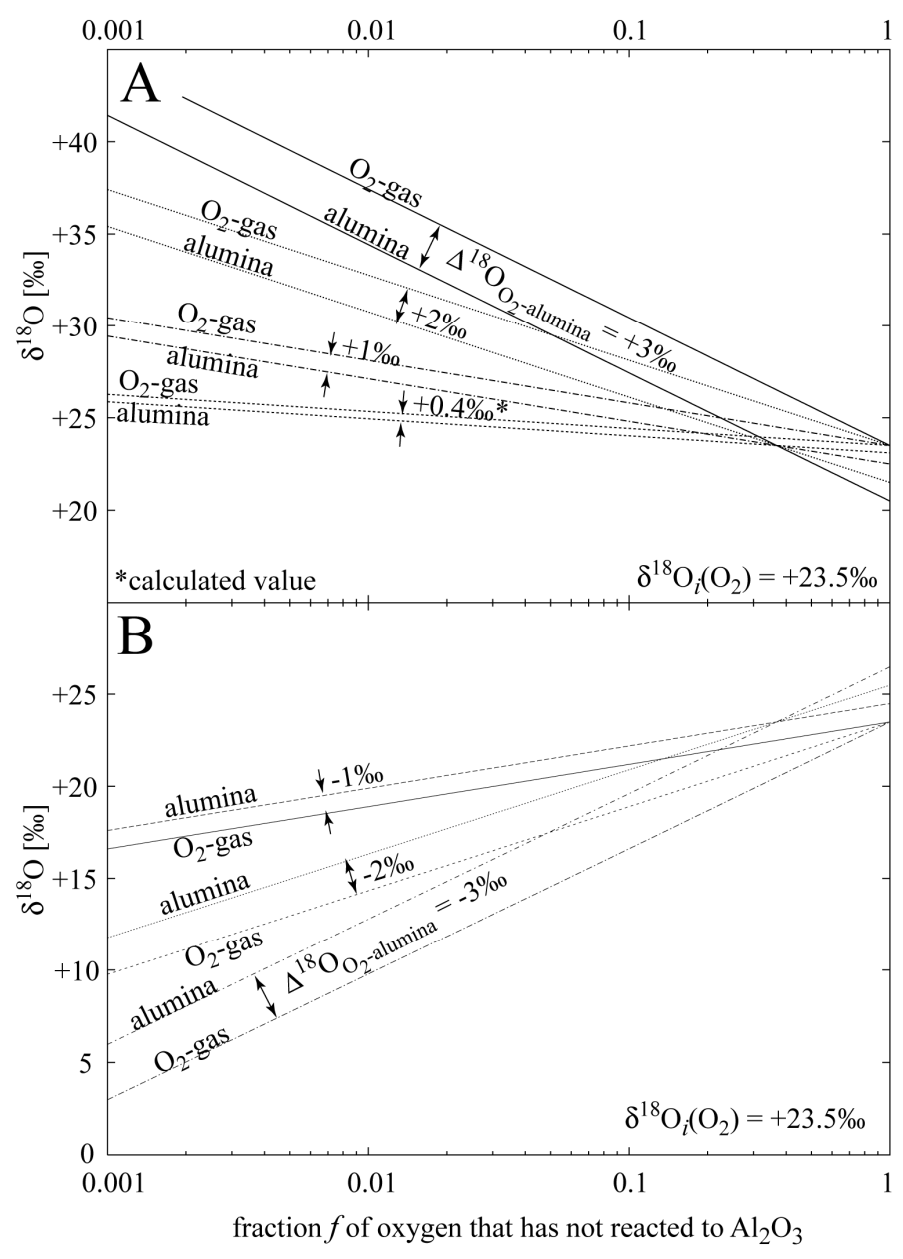

Abbildung 24: $\delta^{18} \mathrm{O}$ von Sauerstoff und Tonerde als Funktion des noch nicht reagierten Sauerstoffanteils $(f)$ und des Fraktionierungsfaktors $(\alpha)$ zwischen Sauerstoff und Tonerde. (A) $\delta^{18} \mathrm{O}$ von $\mathrm{O}_{2}$ und Tonerde für unterschiedliche Fraktionierungsfaktoren $\left(\alpha_{\mathrm{O}_{2} \text {-Tonerde }}>1\right)$. Die aktuell berechnete Fraktionierung zwischen $\mathrm{O}_{2}$ und Tonerde ist $0,4 \%$. (B) $\delta^{18} \mathrm{O}$ von $\mathrm{O}_{2}$ und Tonerde für unterschiedliche Fraktionierungsfaktoren $\left(\alpha_{\mathrm{O}_{2} \text {-Tonerde }}<1\right)$ (aus PACK et al. 2005). 
Da die Experimente jedoch in einem kontinuierlichen Gasstrom durchgeführt wurden, dessen $\delta^{18} \mathrm{O}$ stets konstant war, kann ein Rayleighprozess die Isotopologe bei den Experimenten nicht fraktioniert haben.

Bei den experimentellen Untersuchungen kann es nicht zu Rayleighfraktionierung kommen, da im Gasstrom immer das gleiche Sauerstoffangebot gegeben ist.

Angewandt auf die Stahlherstellung ergeben sich nachfolgend beschriebene Zusammenhänge. Durch das Frischen im Konverter liegt eine fixe Menge an Sauerstoff in der Schmelze vor, die im Laufe der Pfannenbehandlung mit Al abgesättigt wird. Das sich während der Beruhigung der Schmelze bildende $\mathrm{Al}_{2} \mathrm{O}_{3}$ wird aus der Schmelze entfernt. Damit sind Bedingungen für einen Rayleighprozess gegeben. $\mathrm{Da} \alpha_{\mathrm{O}_{2} \text {-Tonerde }}>1$ ist, müsste die sich durch Rayleighfraktionierung bildende Tonerde isotopisch schwerer sein als die Sauerstoffquelle. Da der Sauerstoffabbau beim Desoxidieren allerdings annähernd quantitativ erfolgt, spielt Rayleighfraktionierung wahrscheinlich keine bedeutende Rolle. So sind O-Gehalte nach dem Frischen zwischen 500 und 700 ppm typisch (DEKKERS 2002), in den untersuchten Stahlwerken werden bis zu 1000 ppm erreicht. Nach DEKKERS et al. (2004a) und DEKKERS et al. (2004b) liegt der Gesamtsauerstoffgehalt des Stahls im Tundish für ULC-Güten im Mittel bei ca. 27 ppm. Bei DEKKERS (2003) und VERMEULEN et al. (2000) findet sich ein O-Gehalt nach dem Desoxidieren von ca. 37 ppm. Damit werden zwischen 93 und 97\% des gelösten Sauerstoffs umgesetzt.

$\mathrm{Zu}$ 3.: Bei den Experimenten wird die Stahlschmelze von einem kontinuierlichen Gasstrom umspült (siehe Kapitel 2.3.1.1). Aus der kinetischen Gastheorie geht hervor, dass sich leichte Moleküle/Atome schneller bewegen als schwere. Infolgedessen ist auch die Stoßfrequenz von ersteren höher, was dazu führt, dass die schnelleren Moleküle/Atome häufiger Reaktionen mit der Legierung auslösen als langsamere (=schwere Moleküle/Atome). Daher werden bei der Bildung der Tonerde während der Experimente mehr ${ }^{16} \mathrm{O}$-haltige Moleküle bzw. ${ }^{16} \mathrm{O}$-Atome eingebaut. Dies wird in Abbildung 25 schematisch durch die Situationsdarstellung während der Experimente aus kinetischer Sicht gezeigt. 


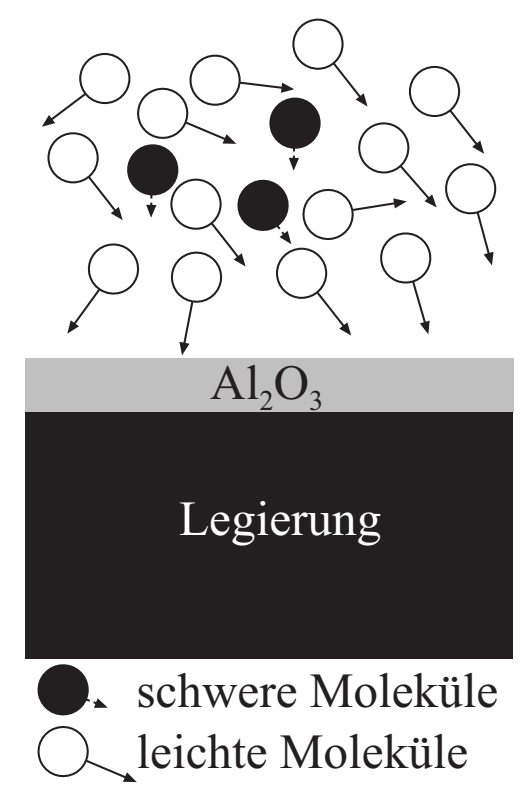

Abbildung 25: Modell der experimentellen Versuchsbedingungen mit leichten Molekülen mit hohen Geschwindigkeiten (lange Pfeile) und schweren Molekülen mit niedrigen Geschwindigkeiten (kurze Pfeile).

Bei gegebener Temperatur ist die kinetische Energie $E_{k i n}$ in idealen Gasen pro Molekül gleich nach:

$$
E_{k i n}=\frac{1}{2} m_{1} v_{1}^{2}=\frac{1}{2} m_{2} v_{2}^{2}[\text { mit } m \text { für die Masse und } v \text { für die Geschwindigkeit }]
$$

Damit gilt für ${ }^{16} \mathrm{O}$ und ${ }^{18} \mathrm{O}$ :

$$
\frac{v_{l}\left({ }^{16} \mathrm{O}\right)}{v_{2}\left({ }^{18} \mathrm{O}\right)}=\sqrt{\frac{m_{2} 18}{m_{1} 16}}=1,061
$$

Folglich ist die Geschwindigkeit von ${ }^{16} \mathrm{O}$ um $6,1 \%$ höher als die von ${ }^{18} \mathrm{O}$. Da der Geschwindigkeitsunterschied zwischen zu betrachtenden Spezies gleich $\alpha$ ist, lässt sich durch Einsetzen in die $\delta$-Notation leicht die Fraktionierung zwischen Ofenatmosphäre und Tonerde berechnen. Auf diese Weise kann man für verschiedene Spezies mögliche Fraktionierungen ausrechnen. Wenn ${ }^{12} \mathrm{C}^{16} \mathrm{O}$ mit dem $\mathrm{Al}$ reagiert, wäre die zuerst gebildete Tonerde maximal 34,5\%o leichter $^{7}$ als das CO (siehe Tabelle 6).

\footnotetext{
${ }^{7}$ leichte Probe $=$ niedrige $\delta^{18} \mathrm{O}-\mathrm{Werte}$, schwere Probe $=$ hohe $\delta^{18} \mathrm{O}-\mathrm{Werte}$
} 
Tabelle 6: Geschwindigkeitsquotient $\left(v_{1} / v_{2}\right)$ und Fraktionierung $(\Delta)$ zwischen Gasspezies und der zuerst gebildeten Tonerde.

\begin{tabular}{|c|c|c|c|}
\hline Gasspezies & Fraktionierung zwischen & $v_{1} / v_{2}$ & $\Delta[\%]$ \\
\hline \hline $\mathrm{O}$ & ${ }^{16} \mathrm{O} \mathrm{\&}{ }^{18} \mathrm{O}$ & 1.061 & 58.9 \\
\hline $\mathrm{H}_{2} \mathrm{O}$ & ${ }^{1} \mathrm{H}_{2}{ }^{16} \mathrm{O} \&{ }^{1} \mathrm{H}_{2}{ }^{18} \mathrm{O}$ & 1.054 & 52.7 \\
\hline $\mathrm{CO}$ & ${ }^{12} \mathrm{C}^{16} \mathrm{O} \&{ }^{12} \mathrm{C}^{18} \mathrm{O}$ & 1.035 & 34.5 \\
\hline $\mathrm{O}_{2}$ & ${ }^{16} \mathrm{O}^{16} \mathrm{O} \&{ }^{16} \mathrm{O}^{18} \mathrm{O}$ & 1.031 & 30.3 \\
\hline $\mathrm{CO}_{2}$ & ${ }^{12} \mathrm{C}^{16} \mathrm{O}^{16} \mathrm{O} \&{ }^{12} \mathrm{C}^{16} \mathrm{O}^{18} \mathrm{O}$ & 1.022 & 22.2 \\
\hline
\end{tabular}

Wie oben gezeigt, führt die kinetische Fraktionierung dazu, dass die gebildete Tonerde isotopisch leichter ist als die Ofenatmosphäre. Um zu verstehen, warum sich die Fraktionierung in den Vorversuchen von der bei den aktuellen Experimenten unterscheidet, muss man die Bildung von $\mathrm{Al}_{2} \mathrm{O}_{3}$ differenziert in Hin- und Rückreaktion betrachten. In Abbildung 26 ist die Fraktionierung als Funktion der Zeit für unterschiedliche $\mathrm{O}_{2}$-Fugazitäten zu sehen. Die zuerst gebildete Tonerde (Hinreaktion) hat den niedrigsten $\delta^{18} \mathrm{O}$-Wert. Mit zunehmender Reaktionszeit nimmt der $\delta^{18} \mathrm{O}-$ Wert zu, da die Rückreaktion einsetzt und die Einstellung des isotopischen Gleichgewichts zwischen Tonerde und Ofenatmosphäre angestrebt wird.

Die Daten zeigen eine Abhängigkeit der Fraktionierung von der Sauerstofffugazität. Bei konstanter Zeit ist die Fraktionierung bei den Experimenten allein abhängig von der Sauerstofffugazität. Je niedriger die Sauerstofffugazität bei der Oxidation von Al-legiertem Stahl ist, desto höher ist die Fraktionierung zwischen Ofenatmosphäre und gebildeter Tonerde.

Aus dem Modell geht hervor, dass der Anteil kinetischer Fraktionierung bei den aktuellen Experimenten wesentlich größer ist als bei den Vorversuchen (siehe Abbildung 26). Bei letzteren war der Anteil von Gleichgewichtsfraktionierung deutlich höher (Datenpunkt liegt nahe am $\delta^{18} \mathrm{O}$-Wert der Ofenatmosphäre und damit nahe dem isotopischen Gleichgewicht). Bei angenommener Gleichgewichtsfraktionierung zwischen oxidierender Spezies und Tonerde von annähernd null ergibt sich ein Anteil an kinetischer Fraktionierung bei $\Delta^{18} \mathrm{O}_{\text {Ofenatmopshäre- }}$ Tonerde $=+13 \%$ für CO als oxidierende Spezies von $\mathrm{rd}$. $37 \%$. 


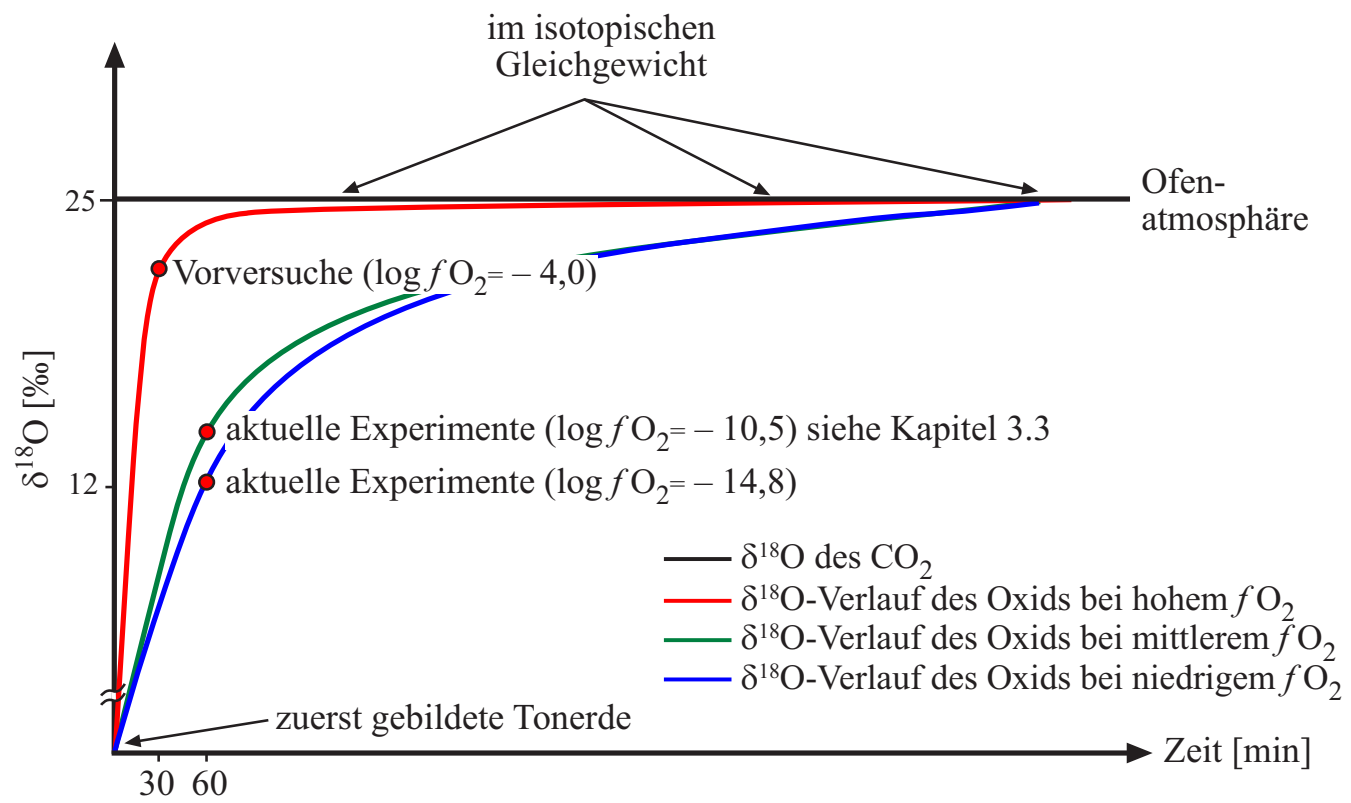

Abbildung 26: Fraktionierung zwischen Ofenatmosphäre und gebildeter Tonerde für unterschiedliches $f \mathrm{O}_{2}$ (semiquantitative Darstellung).

Die Experimente haben gezeigt, dass kinetische Fraktionierung bei hohen Temperaturen von $1600^{\circ} \mathrm{C}$ bei der Oxidation von Al-legiertem Stahl von Bedeutung sein kann.

In Anwendung auf die Stahlherstellung kann folglich angenommen werden, dass kinetische Fraktionierung eine bedeutende Rolle einnimmt, wenn die Bildung von $\mathrm{Al}_{2} \mathrm{O}_{3}$ und damit die von Clogging z. B. auf Diffusion von Luftsauerstoff durch Tauch- und Schattenrohre zurückgeht. Möglicherweise könnte hierbei metallisches Si des FF-Materials z. B. von Tauchrohren zu $\mathrm{SiO}_{2}$ oxidiert werden. Die $\delta^{18} \mathrm{O}$-Werte der Oxide könnten in diesem Fall möglicherweise von außen nach innen abnehmen. Dann würde die kinetische Fraktionierung den entscheidenden Effekt auf die O-Isotopie des Materials ausüben.

\subsection{Analyse von Clogging und Prozessgasen}

\subsubsection{Clogging und Prozess- $\mathrm{O}_{2}$}

\subsubsection{Morphologie von Clogging}

Von den Cloggingproben wurden Rasteraufnahmen angefertigt. In Abbildung 27 ist die gut kristallisierte, poröse Tonerde zu sehen, deren chemische Zusammensetzung mittels EDS analysiert wurde. Dabei stellte sich heraus, dass es sich um reines $\mathrm{Al}_{2} \mathrm{O}_{3}$ handelt. 


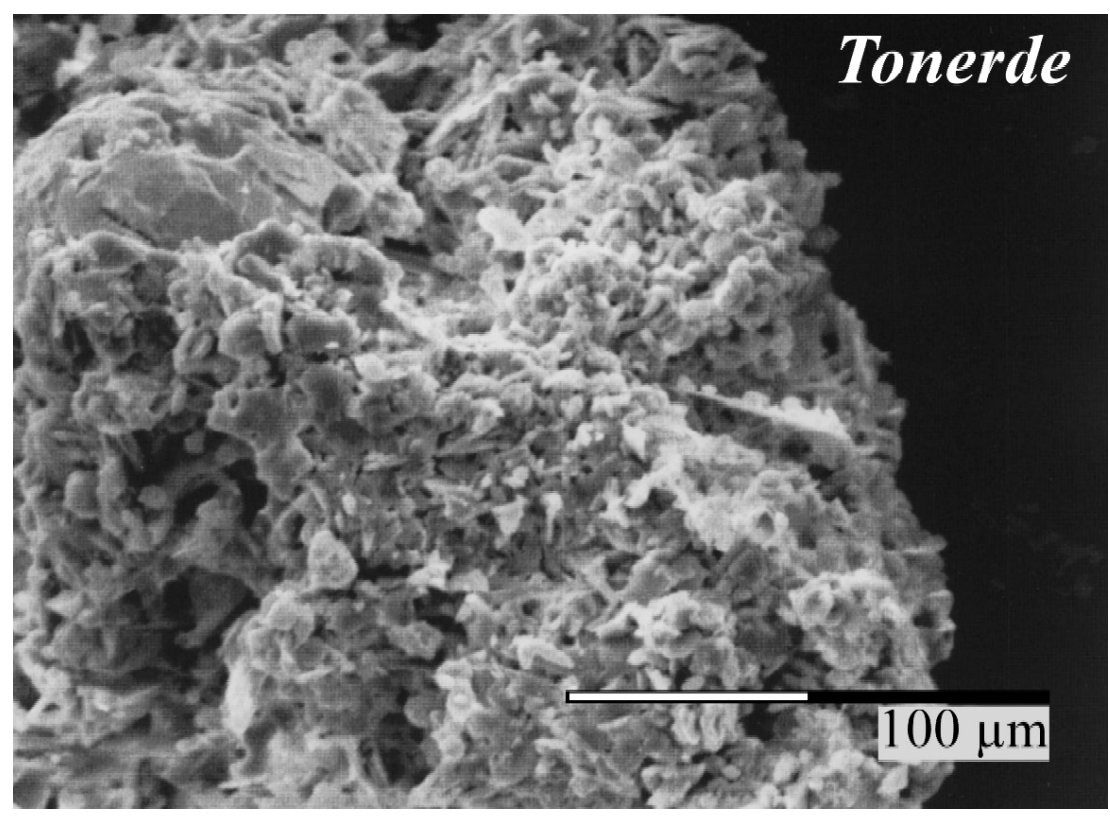

Abbildung 27: Sekundärelektronenbild der Cloggingprobe vom 05.09.05, Strang 2, Stahlwerk A.

\subsubsection{Chemische Zusammensetzung von Clogging}

Von den Cloggingproben wurden Röntgenfluoreszenzanalysen (RFA) gemacht, um die chemische Zusammensetzung zu ermitteln. In Tabelle 7 sind die Ergebnisse in oxidischer Form dargestellt. Alle Proben wurden auf Fe-freie Basis normalisiert. Der Al-Gehalt dominiert alle Proben mit $>80$ Gew.\%. $\mathrm{MnO}$ und $\mathrm{TiO}_{2}$ liegen für beide Stahlwerke unter 0,5 Gew.\%. $\mathrm{SiO}_{2}$ nimmt 0,2 bis 3,2 Gew.\% im Stahlwerk A ein und liegt im Stahlwerk B bei 0,5 bis 0,7 Gew.\%. Der MgO-Anteil liegt im Stahlwerk A zwischen 5,9 und 10,3 Gew.\%. Im Stahlwerk B liegt er bei 0,5 Gew.\%. Damit sind die MgO-Gehalte im Stahlwerk A bis zu 21-mal so hoch wie im Stahlwerk B. Auch der CaO-Anteil des Cloggings im Stahlwerk A übersteigt weit den des Stahlwerks B. Im Stahlwerk A macht $\mathrm{CaO}$ zwischen 1,3 und 2,6 Gew.\% aus. Die beiden Proben aus dem Stahlwerk B weisen 0,2 und 0,3 Gew.\% CaO auf. Der CaO-Gehalt liegt damit im Stahlwerk A bis zu 13-mal höher als im Stahlwerk B.

Tabelle 7: RFA von Tauchrohrclogging aus Stahlwerk A und B (Auszug der RFA).

\begin{tabular}{|c|c|c|c|c|c|c|c|}
\hline & \multirow{2}{*}{$\begin{array}{c}\text { MnO } \\
\text { Gew.-\% } \\
\end{array}$} & \multirow{2}{*}{$\begin{array}{c}\mathrm{SiO}_{2} \\
\text { Gew.-\% } \\
\end{array}$} & \multirow{2}{*}{$\begin{array}{c}\mathrm{TiO}_{2} \\
\text { Gew.-\% } \\
\end{array}$} & \multirow{2}{*}{$\begin{array}{c}\mathrm{Al}_{2} \mathrm{O}_{3} \\
\text { Gew.-\% } \\
\end{array}$} & \multirow[b]{2}{*}{$\begin{array}{c}\mathrm{CaO} \\
\text { Gew.-\% }\end{array}$} & \multirow[b]{2}{*}{$\begin{array}{c}\text { Mg0 } \\
\text { Gew.- } \%\end{array}$} \\
\hline & & & & & & & \\
\hline \multirow{7}{*}{ Stahlwerk A } & $1 \mathrm{~A}$ innen & 0.2 & 1.7 & 0.1 & 85.3 & 2.2 & 9.2 \\
\hline & $1 \mathrm{~B}$ innen & 0.2 & 0.2 & 0.2 & 80.9 & 2.6 & 10.3 \\
\hline & 1 E innen & 0.4 & 3.2 & 0.1 & 86.4 & 1.5 & 7.8 \\
\hline & $1 \mathrm{~F}$ innen & 0.2 & 1.1 & 0.2 & 81.7 & 2.0 & 7.3 \\
\hline & $2 \mathrm{C}$ innen & 0.2 & 0.4 & 0.0 & 89.5 & 1.3 & 7.9 \\
\hline & $2 \mathrm{D}$ innen Zone 1 & 0.4 & 2.2 & 0.1 & 89.1 & 1.5 & 5.9 \\
\hline & $2 \mathrm{D}$ innen Zone 2 & 0.4 & 0.2 & 0.0 & 86.2 & 2.0 & 9.3 \\
\hline \multirow{2}{*}{ Stahlwerk B } & Probe 7 innen & 0.0 & 0.5 & 0.0 & 98.3 & 0.2 & 0.5 \\
\hline & Probe 7 außen & 0.0 & 0.7 & 0.0 & 98.0 & 0.3 & 0.5 \\
\hline
\end{tabular}


Auffällig sind die hohen $\mathrm{CaO}-$ und $\mathrm{MgO}-$ Gehalte im Stahlwerk A gegenüber denen im Stahlwerk B.

\subsubsection{Mineralogische Zusammensetzung von Clogging}

Von den Cloggingproben wurden röntgenpulverdiffraktometrische Aufnahmen gemacht. Dabei wurden die Proben sowohl einer qualitativen als auch einer quantitativen Phasenanalyse unterzogen (siehe Abbildung 28 und Abbildung 29). Letztere erfolgte mit Hilfe der Rietveldmethode, welche auf einer mathematischen Anpassung der gemessenen Pulverdiagramme beruht (RIETVELD 1967). Zur näheren Beschreibung der Röntgenpulverdiffraktometrie und der Rietveldmethode siehe Kapitel 4.2.

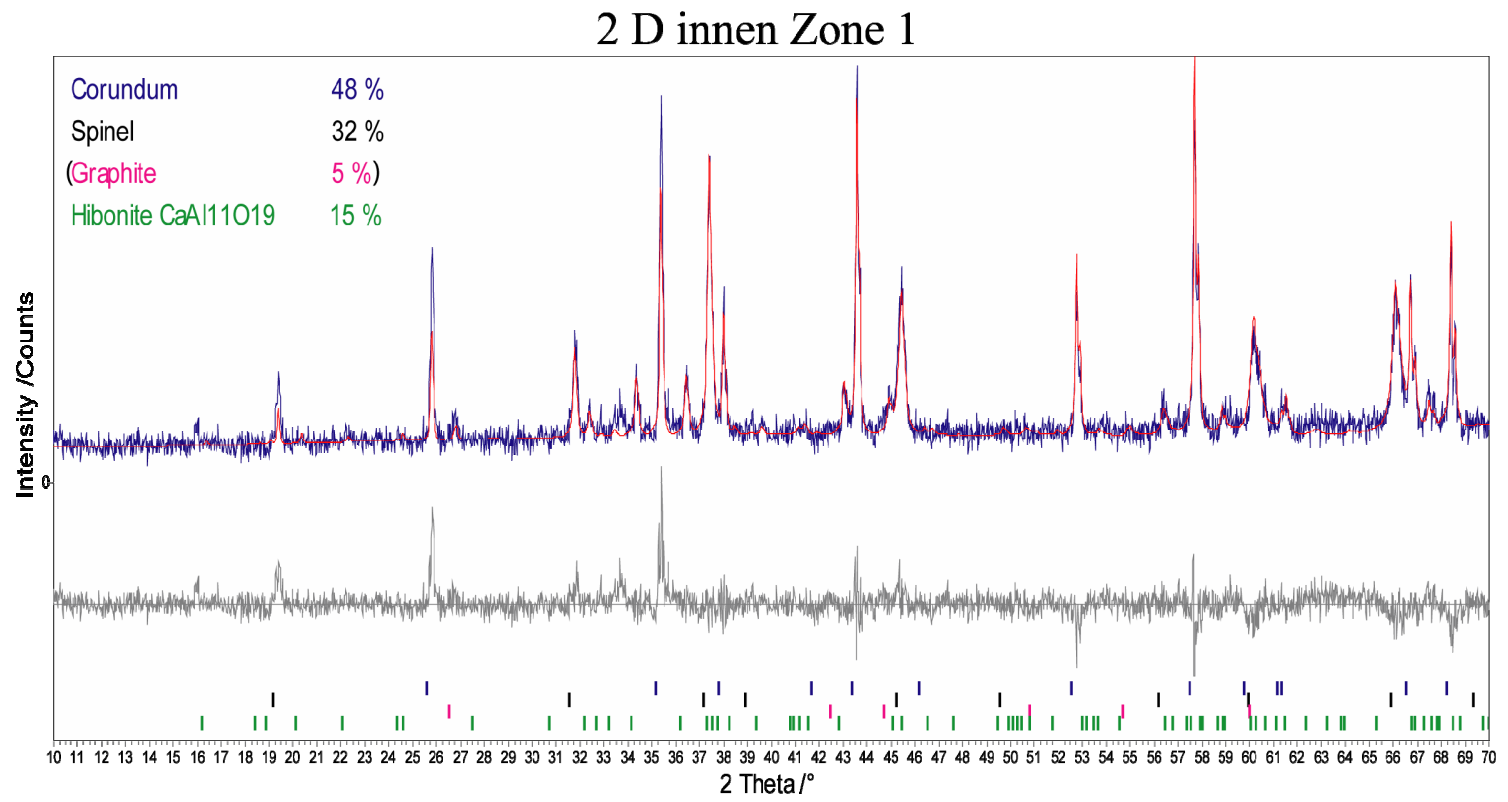

Abbildung 28: Diffraktogramm und Rietveldanalyse von Tauchrohrclogging; blaue Linie = gemessenes Diffraktogramm, rote Linie $=$ angepasstes Diffraktogramm (Rietveldanalyse), graue Line $=$ Differenzkurve, senkrechte Striche $=$ Peaklagen der Phasen.

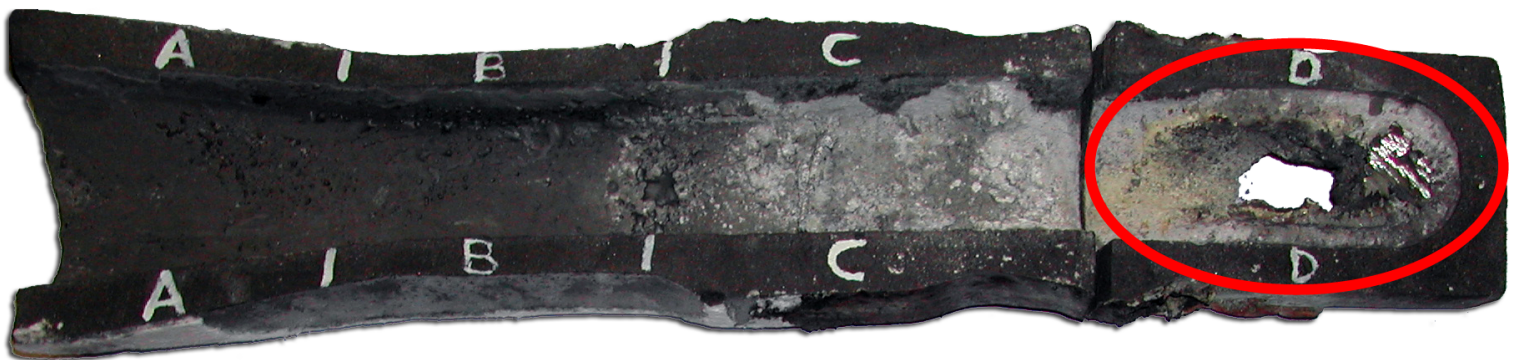

Abbildung 29: Photo von Tauchrohr 2 mit Clogging. Der rote Kreis zeigt den Bereich der Probennahme (05.09.05, Strang 2, Stahlwerk A). Das Tauchrohr hat eine Länge von rd. einem Meter und einen Innendurchmesser von rd. $9 \mathrm{~cm}$ im Bereich D. 
Auffällig sind die hohen Gehalte an Spinell $\left(\mathrm{MgAl}_{2} \mathrm{O}_{4}\right)$ und Hibonit $\left(\mathrm{CaAl}_{12} \mathrm{O}_{19}\right)$ im Clogging aus Stahlwerk A. Damit schlagen sich die hohen Mg- und Ca-Gehalte (siehe auch Kapitel 4.2.1) auch im Phasenbestand nieder.

\subsubsection{Isotopenmessung von Clogging und Prozess- $\mathrm{O}_{2}$}

Die Cloggingproben wurden mittels IR-Laserfluorinierung aufgeschlossen und der freigesetzte Sauerstoff online in einem SIRMS Finnigan Delta Plus Gasmassenspektrometer gemessen (siehe Kapitel 2.3.2.) In Abbildung 30 sind die Ergebnisse der aktuellen Untersuchung zusammen mit den Ergebnissen der Voruntersuchung sowie Daten aus PACK (2000) (Stahlwerk Dortmund-Hörde) als Häufigkeitsverteilung dargestellt.

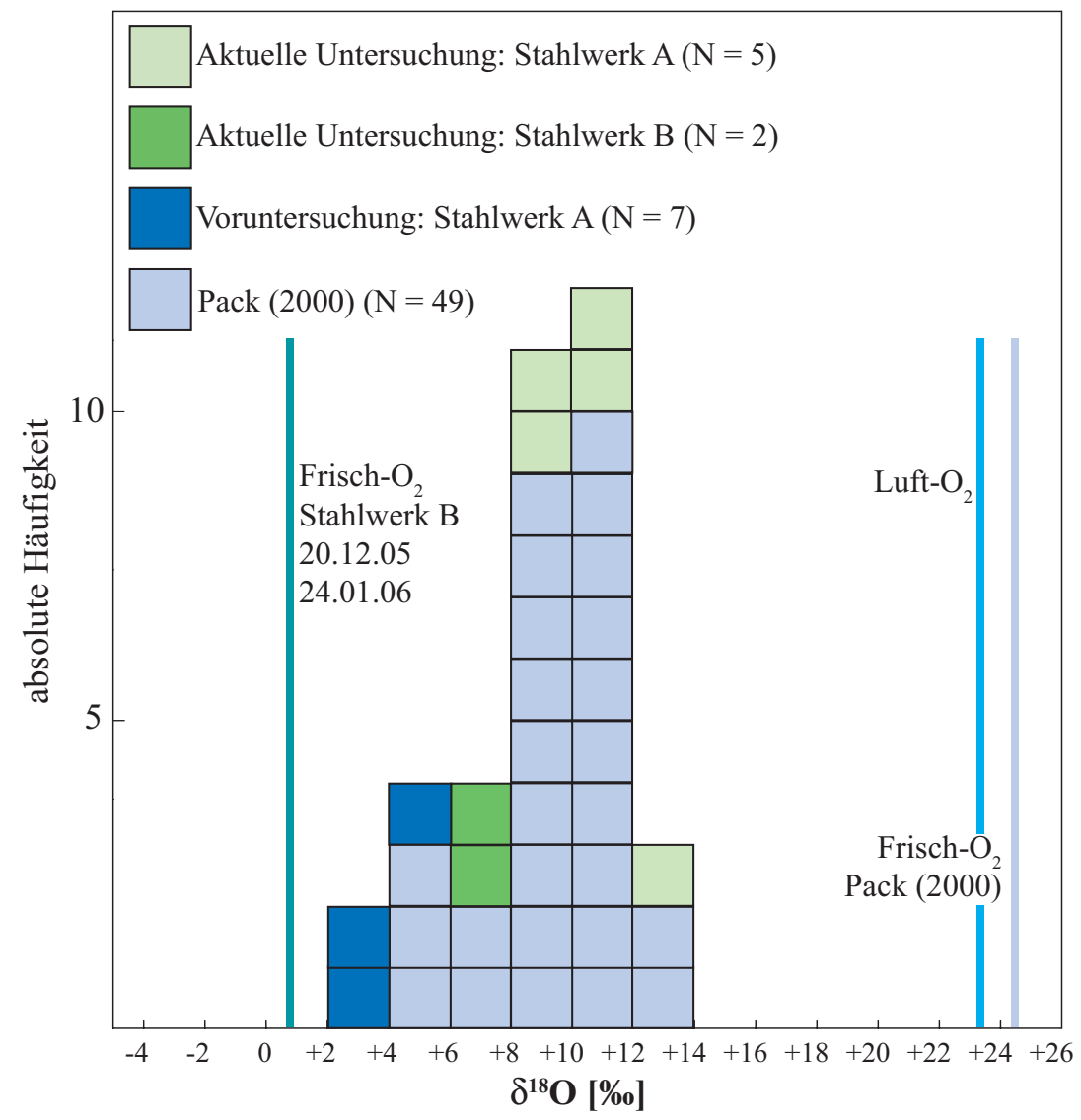

Abbildung 30: Zusammenfassendes Histogramm der O-Isotopie von Tauchrohrclogging und einige mögliche O-Quellen. Dargestellt sind alle bisher gemessenen Tauchrohrcloggingproben.

Tauchrohrclogging aus den Stahlwerken in Duisburg variiert im $\delta^{18} \mathrm{O}$ zwischen +2 und +13\%. Das von PACK (2000) untersuchte Tauchrohrclogging aus Dortmund liegt zwischen +5 und $+14 \%$. Die Proben der Voruntersuchung aus dem Stahlwerk A streuen zwischen +2 und $+6 \%$. Die der aktuellen Untersuchung liegen für das Stahlwerk A bei +8 bis $+13 \%$ ond für das Stahlwerk B zwischen +6 und $+7 \%$. 


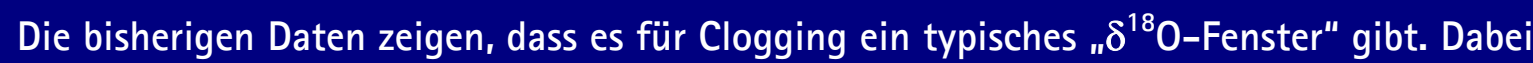
liegen etwa 2/3 des Cloggings in einem engen Bereich zwischen +8 bis $+12 \%$. In diesem Bereich liegt auch der Hauptteil der Proben der aktuellen Untersuchung.

Einige mögliche Sauerstoffquellen für die Bildung von Clogging sind in Abbildung 30 dargestellt. Luftsauerstoff kann als weltweit konstant angenommen werden und liegt bei einem $\delta^{18} \mathrm{O}$ von $+23,5 \%$ (Dole et al. 1954). Der beprobte Prozesssauerstoff aus dem Stahlwerk B hat im Mittel einen $\delta^{18} \mathrm{O}$-Wert von $+0,9 \%$. Die Probe vom 20.12.05 weist einen $\delta^{18} \mathrm{O}-\mathrm{Wert}$ von $+1,0 \pm 0,1 \%$ auf, jene vom 24.01.06 einen von $+0,7 \pm 0,1 \%$. Der Frischsauerstoff aus dem Stahlwerk Dormund-Hörde liegt bei $+24,3 \%$ und ist damit wesentlich schwerer als das Clogging.

Der in einem Abstand von einem Monat beprobte Frischsauerstoff aus dem Stahlwerk B liegt im Mittel bei $\delta^{18} 0=+0.9 \%$ und ist damit leichter als das Clogging.

Vergleicht man den Frischsauerstoff der Stahlwerke, ist der große Unterschied zwischen Dortmund und Duisburg bemerkenswert.

\subsubsection{Prozessgase Ar und $\mathrm{N}_{2}$}

Für die $\mathrm{O}_{2}$-Extraktion wurde eine Apparatur gebaut, bei der die Inertgase bei $920^{\circ} \mathrm{C}$ über Spektralkohle geleitet wurden und das $\mathrm{O}_{2} \mathrm{zu} \mathrm{CO}_{2}$ konvertiert werden sollte.

Mit Hilfe von Spektralkohle sollte in einem Ofen bei $920^{\circ} \mathrm{C}$ eine $\mathrm{O}_{2}-\mathrm{Konvertierung} \mathrm{zu} \mathrm{CO}_{2}$ nach

$$
\mathrm{O}_{2}+\mathrm{C} \rightleftharpoons \mathrm{CO}_{2}
$$

erfolgen. Das gebildete $\mathrm{CO}_{2}$ sollte dann in einem Kühlfinger bei Temperaturen von $-196^{\circ} \mathrm{C}$ quantitativ ausgefroren werden. Der Kühlfinger konnte nach dem Überfrieren abgeschweißt und schließlich der $\delta^{18} \mathrm{O}$-Wert des Kohlendioxids gemessen werden.

In Abbildung 31 und Abbildung 32 ist der Aufbau der $\mathrm{CO}_{2}$-Linie zu sehen. Nach der Evakuierung der Apparatur wurde zunächst jeweils ein Teil des Gases in ein Edelstahl-U-Rohr expandiert. Auf diese Weise konnte der Druck gemindert und mehrere Aliquote nacheinander gewonnen werden.

Nach umfassenden Testreihen mit Luft- und Flaschensauerstoff, um die Anlage zu kalibrieren, wurde mit der $\mathrm{O}_{2}$-Extraktion aus den Spülgasen $\mathrm{Ar}$ und $\mathrm{N}_{2}$ begonnen. 
Es stellte sich heraus, dass mit diesem Versuchsaufbau keine $\mathrm{O}_{2}$-Extraktion aus den Inertgasen möglich war. Dies lässt den Schluss zu, dass der $\mathrm{O}_{2}$-Gehalt in den Gasen $\operatorname{Ar}$ und $\mathrm{N}_{2}$ weit unter $1 \%$ sein muss. Das entspricht der von Linde angegebenen Reinheit für Argon

4.5 von $>99,995 \%$.

Es konnte mit dem unten gezeigten Versuchsaufbau kein $\mathrm{O}_{2}$ nachgewiesen werden. Konstruktionsbedingt können mit dieser Apparatur nur Beimengungen von $\mathrm{O}_{2}>1$ Vol.\% nachgewiesen werden.

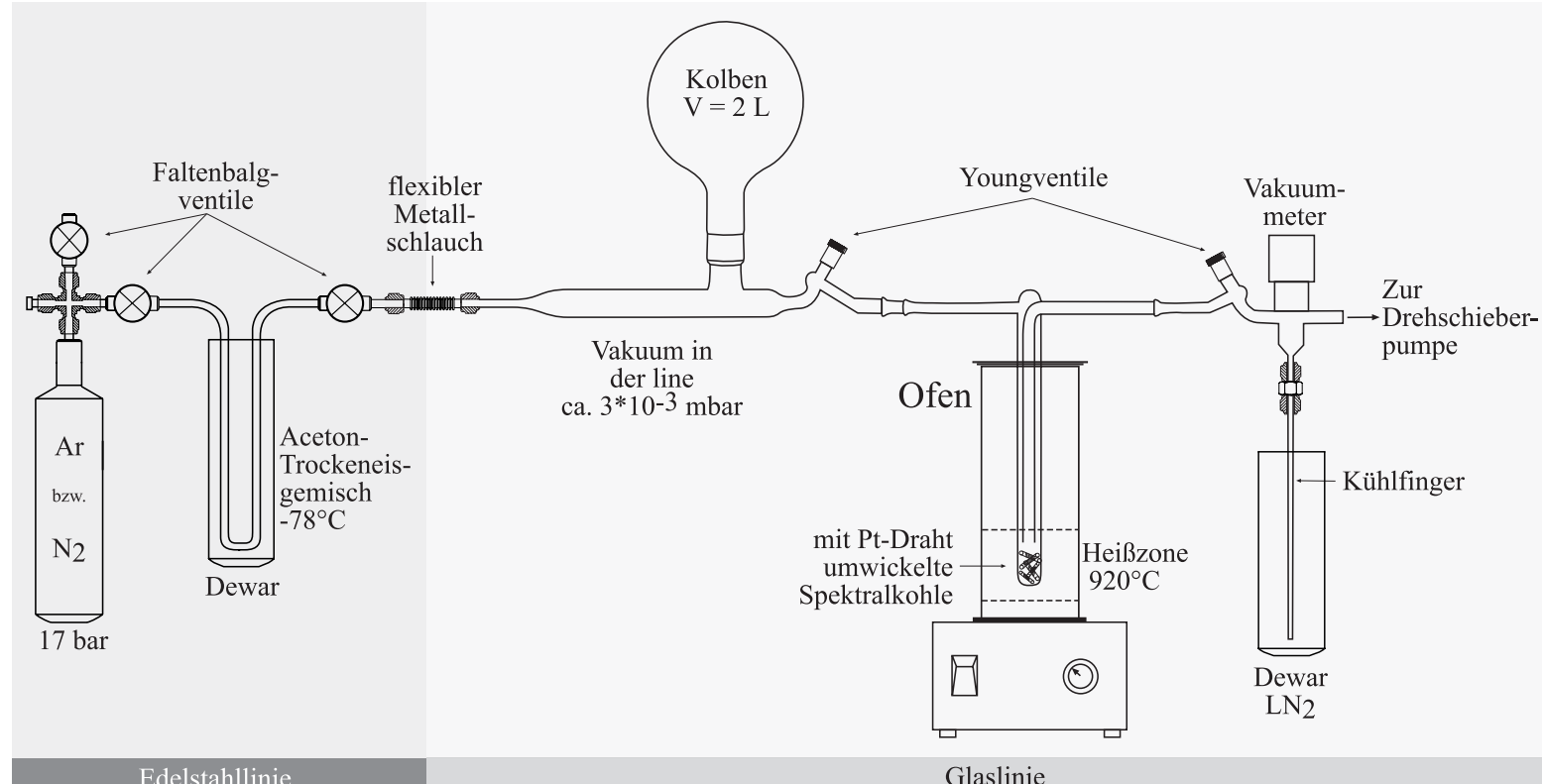

Abbildung 31: Zeichnung des Versuchsaufbaus zur Extraktion von $\mathrm{O}_{2}$ aus den Inertgasen $\mathrm{Ar}$ und $\mathrm{N}_{2}$.

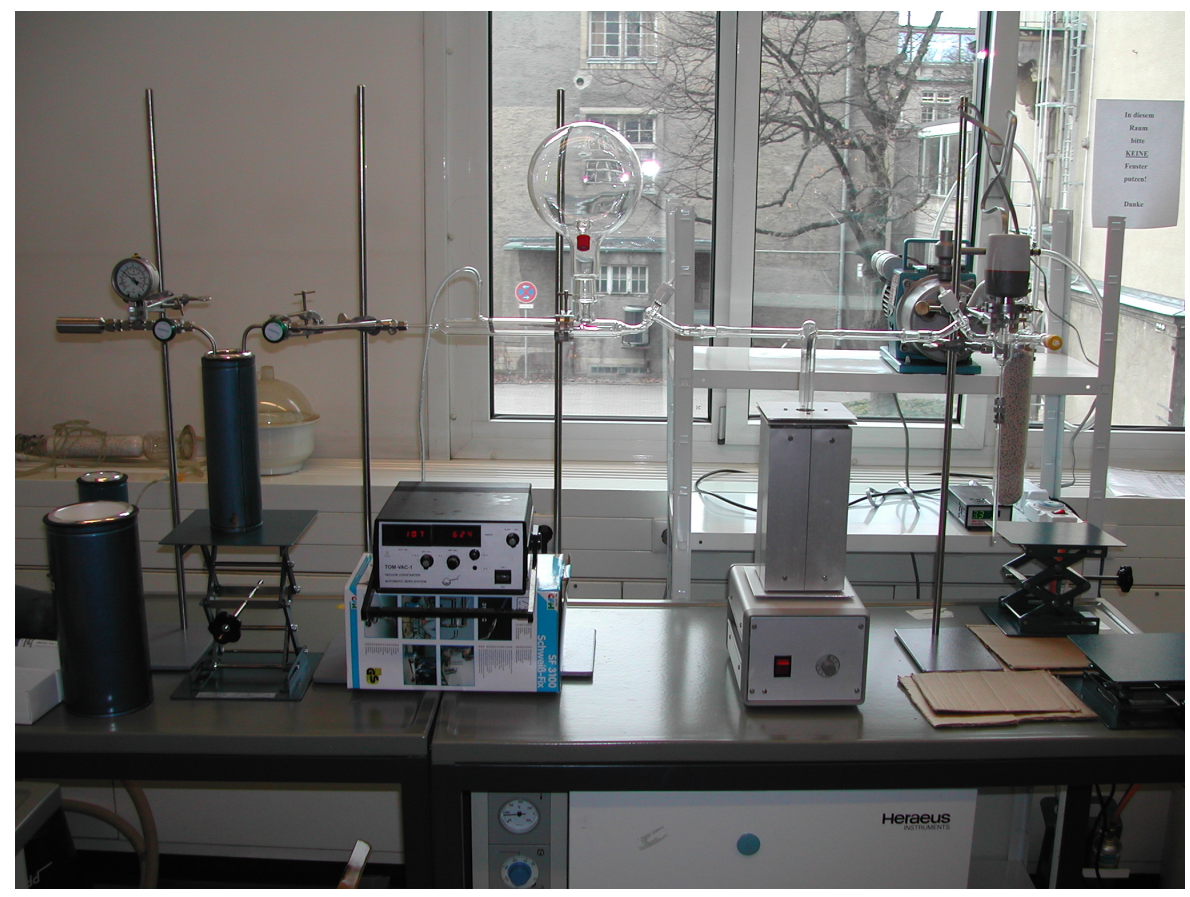

Abbildung 32: Photo der $\mathrm{CO}_{2}$-Linie mit Probengasflasche (links), Ofen zur $\mathrm{O}_{2}$-Konvertierung (Mitte rechts) und Drehschieberpumpe (hinten rechts) zur Evakuierung der Anlage. 


\subsubsection{Diskussion}

\section{Chemie und Mineralogie}

Die Ergebnisse der chemischen und mineralogischen Charakterisierung zeigen einen deutlichen Unterschied zwischen Stahlwerk A und Stahlwerk B. Erhöhte Mg- und Ca-Gehalte von Proben aus dem Stahlwerk A korrelieren mit hohen Spinell- und Hibonitgehalten im Stahlwerk A. Möglicherweise sind die hohen Gehalte Produkt der Ablagerung von Mitlaufschlacke aus Pfanne und Verteiler. Typischerweise würde erwartet werden, dass die Schlacke an $\mathrm{CaO}$ gegenüber $\mathrm{MgO}$ angereichert ist, da das Abdeckpulver der Tundishschlacke zu rd. 43 Gew.-\% aus $\mathrm{CaO}$ und lediglich zu rd. $1 \mathrm{Gew} .-\%$ aus $\mathrm{MgO}$ besteht. Auch die Pfannenschlacke ist mit rd. 35 Gew.-\% CaO und rd. 10 Gew.-\% MgO reicher an Calcium. Die Clogginganalysen weisen jedoch höhere $\mathrm{MgO}$ - als $\mathrm{CaO}-\mathrm{Geh} a l t e$ auf (siehe Kapitel 2.4.1.2). Da die Schlacke CaOreicher ist, deuten hohe MgO-Gehalte im Clogging eher auf Erosion von FF-Material aus Pfanne und Verteiler hin als auf die Ablagerung von Mitlaufschlacke, da dieses hohe MgOGehalte von $>95$ Gew.-\% aufweist (siehe auch Kapitel 4.2.4 und 4.3.3). Eine andere Erklärung, die für Mitlaufschlacke spräche, wäre, dass der CaO-Anteil der Schlacke nicht im Tauchrohr abgelagert, sondern in die Kokille weitertransportiert wird. So bildet $\mathrm{CaO}$ mit $\mathrm{Al}_{2} \mathrm{O}_{3}$ in einem Mischungsverhältnis von ungefähr 1:1 ein Eutektikum. Aus dieser flüssigen Mitlaufschlacke könnte sich bei niedrigeren Temperaturen im Tauchrohr Spinell ausscheiden und damit eine Separation von $\mathrm{CaO}$ und $\mathrm{MgO}$ auftreten, welche die höheren $\mathrm{MgO}-\mathrm{Gehalte}$

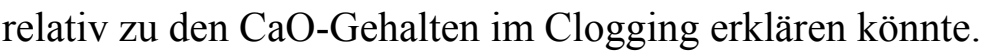

Im Stahlwerk B zeigt die RFA wesentlich geringere Gehalte an $\mathrm{Mg}$ und $\mathrm{Ca}$, die mit niedrigen Spinell- und Hibonitgehalten verbunden sind.

Das Clogging aus Stahlwerk $\mathrm{A}$ ist gegenüber dem aus Stahlwerk B reicher an MgO und $\mathrm{CaO}$. Das deutet auf einen reineren Gießprozess im Stahlwerk B als im Stahlwerk A hin.

\section{Isotopie}

Tauchrohrclogging liegt zwischen +2 und $+13 \%$. In Abbildung 30 sind neben dem Clogging mit Luft- und Frischsauerstoff zwei mögliche Sauerstoffquellen für die Bildung von Clogging dargestellt. Der Frischsauerstoff aus Stahlwerk B $\left(\delta^{18} \mathrm{O}=+0,9 \%\right)$ ist isotopisch leichter als das Clogging.

Auch wenn Frischsauerstoff die Quelle für Clogging ist, könnte, wie in Kapitel 2.3.4 gezeigt, ein Rayleighprozess nicht zu schwererem Clogging relativ zum Frischsauerstoff führen, da der Frischsauerstoff beim Desoxidieren annähernd quantitativ umgesetzt wird und damit Reservoireffekte auszuschließen sind. 
Luftsauerstoff liegt weltweit konstant bei $+23,5 \%$ und ist damit deutlich schwerer als das Clogging. Wenn Clogging auf die Diffusion von Luftsauerstoff durch FF-Material (z. B. Tauchrohre) zurückginge, könnte man niedrigere $\delta^{18} \mathrm{O}$-Werte für das Clogging relativ zum Luftsauerstoff erwarten. Wie die experimentellen Untersuchungen zeigen, führt ein kinetisch dominierter Prozess, wie es die Diffusion von Luftsauerstoff durch Tauchrohre wäre, zu leichterem Clogging relativ zur Sauerstoffquelle. Weiter wurde in Kapitel 2.3.4 gezeigt, dass der Grad der Rückreaktion bei konstanter Zeit abhängig von der Sauerstofffugazität ist.

Falls die Diffusion von Luft- $\mathrm{O}_{2}$ durch Tauchrohre entscheidend für die Bildung von Clogging ist, würden hohe $\delta^{18} 0$-Werte des Cloggings auf eine schlechte Luftabschirmung beim Gießen hinweisen und niedrige $\delta^{18} 0$-Werte auf eine gute Luftabschirmung.

Die experimentellen Ergebnisse zeigen, dass bei diesem unidirektionalen Prozess die Fraktionierung zwischen Sauerstoffquelle und der gebildeten Tonerde mit sinkender Sauerstofffugazität zunimmt (siehe Kapitel 2.3.4).

\subsection{Zusammenfassung}

\section{Experimente}

Bei den aktuellen Experimenten ist bei $\log f \mathrm{O}_{2}=-14,8$ eine Fraktionierung zwischen Ofenatmosphäre und gebildeter Tonerde von $\Delta^{18} \mathrm{O}_{\text {Ofenatmosphäre-Tonerde }}=+13 \%$ zu beobachten. Die der Voruntersuchung weisen lediglich einen $\Delta^{18} \mathrm{O}_{\text {Ofenatmosphäre-Tonerde }}$ von $+3 \%$ auf. Die Ursache für die größere Fraktionierung bei der aktuellen Untersuchung liegt an der niedrigeren Sauerstofffugazität.

Je niedriger die Sauerstofffugazität bei der Oxidation von Al-legiertem Stahl ist, desto höher die resultierende Fraktionierung zwischen Ofenatmosphäre und gebildeter Tonerde.

\section{Isotopenanalyse von Clogging}

Das Clogging aus dem Stahlwerk A der aktuellen Untersuchung liegt im $\delta^{18} \mathrm{O}$-Wert zwischen +8 und $+13 \%$, das der Voruntersuchung zwischen +2 und $+6 \%$. Im Stahlwerk B liegt es bei +6 bis $+8 \%$ (aktuelle Untersuchung). Cloggingproben aus dem Stahlwerk in Dortmund-

Hörde haben einen $\delta^{18} \mathrm{O}$ von +5 bis $+14 \%$ (PACK 2000).

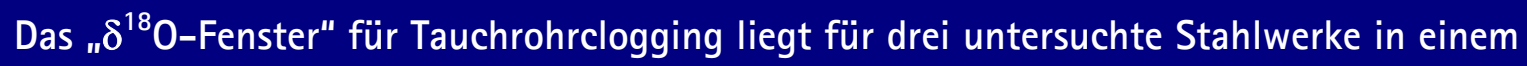
Bereich von +2 bis $+14 \%$. 


\section{Analyse der Prozessgase $\mathrm{O}_{2}$, Ar und $\mathrm{N}_{2}$}

Der beprobte Prozesssauerstoff aus dem Stahlwerk B hat im Mittel einen $\delta^{18} \mathrm{O}$ von $+0,9 \%$.

Der Frischsauerstoff aus dem Stahlwerk in Dortmund-Hörde liegt bei $\delta^{18} \mathrm{O}=+24,3 \%$.

\section{Der Prozess- $\mathrm{O}_{2}$ zeigt zwischen diesen beiden Stahlwerken einen großen Unterschied im} $\delta^{18} 0$.

Aus den Inertgasen Ar und $\mathrm{N}_{2}$ war keine $\mathrm{O}_{2}$-Extraktion möglich, somit muss deren $\mathrm{O}_{2}$-Gehalt weit unter $1 \%$ liegen.

Wie oben gesehen, ist das Hauptziel der ersten Projektphase erreicht und ein deutlicher Fortschritt im Hinblick auf das Prozessverständnis der O-Isotopenfraktionierung bei der Stahlherstellung zu verzeichnen. Ausgehend von diesen neuen und bisher unbekannten Zusammenhängen, kann die Interpretation von O-Isotopenmessungen von Cloggingproben bzw. nichtmetallischen Einschlüssen noch konsistenter erfolgen. 


\section{Teil II}

\subsection{Zielsetzung}

Im Mittelpunkt von Teil II stand eine breit durchgeführte O-Isotopenmesskampagne mit dem Ziel der Beantwortung drei zentraler Fragen:

1. Geht Clogging auf die Desoxidation zurück?

2. Entsteht Clogging durch die Reaktion/Erosion von FF-Material und Schlacke?

3. Übernimmt Clogging die Isotopie von Luft- ${ }_{2}$, wenn Luft an den Stahl kommt?

$\mathrm{Zu}$ 1.: Es wurden parallel Proben von Prozesssauerstoff und Clogging im Stahlwerk genommen, um eine mögliche Korrelation in der Isotopie aufzudecken.

Zu 2.: Die Fragestellung wurde insbesondere um die Frage nach der Bedeutung der Reaktion der Stahlschmelze mit Schiebersand ergänzt.

Zu 3.: Um zu untersuchen, ob Clogging die Isotopie des Luftsauerstoffs übernimmt, wurde ein Versuch mit offenem Stopfen an einem Strang durchgeführt. Dafür wurde die Ar-Spülung beim Gießen abgestellt, so dass Luftsauerstoff durch den Stopfen an den Stahl gelangen sollte.

\subsection{Probennahme}

Über einen Zeitraum von insgesamt neun Tagen wurden im August, September und Oktober 2006 Proben von Clogging und Schlacken im Stahlwerk B genommen (siehe Tabelle 8 und Tabelle 9).

Tabelle 8: Übersicht über die genommenen Proben nichtmetallischer Ablagerungen in Tauchrohren und an deren Unterseiten im Stahlwerk B (US = Ablagerungen an der SEN-Unterseite).

\begin{tabular}{|c|c|c|c|c|}
\hline Datum & Strang 3 & Strang 3 US & Strang 4 & Strang 4 US \\
\hline \hline 28.08 .2006 & $\mathrm{X}$ & & $\mathrm{X}$ & \\
29.08 .2006 & $\mathrm{X}$ & & & \\
31.08 .2006 & $\mathrm{X}$ & & $\mathrm{X}$ & \\
01.09 .2006 & & & & \\
04.09 .2006 & $\mathrm{X}$ & $\mathrm{X}$ & $\mathrm{X}$ & $\mathrm{X}$ \\
05.09 .2006 & $\mathrm{X}$ & $\mathrm{X}$ & $\mathrm{X}$ & $\mathrm{X}$ \\
23.10 .2006 & & & $\mathrm{X}$ & $\mathrm{X}$ \\
24.10 .2006 & $\mathrm{X}$ & $\mathrm{X}$ & $\mathrm{X}$ & $\mathrm{X}$ \\
26.10 .2006 & & & $\mathrm{X}$ & $\mathrm{X}$ \\
\hline
\end{tabular}


Tabelle 9: Übersicht über die genommen Schlackenproben im Stahlwerk B.

\begin{tabular}{|c|c|c|c|c|}
\hline \multirow{2}{*}{ Datum } & \multicolumn{2}{|c|}{ Tundishschlacke } & \multirow{2}{*}{ Pfannenschlacke } & \multirow{2}{*}{ Konverterschlacke } \\
\hline & Str. 3 & Str. 4 & & \\
\hline 28.08 .2006 & $\mathrm{X}$ & $\mathrm{X}$ & & \\
\hline 29.08 .2006 & $X$ & $X$ & & \\
\hline 31.08 .2006 & $\mathrm{X}$ & $\mathrm{X}$ & & \\
\hline 01.09 .2006 & $\mathrm{X}$ & $\mathrm{X}$ & & \\
\hline 04.09 .2006 & & & & \\
\hline 05.09 .2006 & $\mathrm{X}$ & $\mathrm{X}$ & & \\
\hline 23.10 .2006 & $\mathrm{X}$ & $\mathrm{X}$ & $\mathrm{X}$ & $\mathrm{X}$ \\
\hline 24.10 .2006 & $\mathrm{X}$ & $\mathrm{X}$ & $\mathrm{X}$ & $\mathrm{X}$ \\
\hline 26.10 .2006 & $\mathrm{X}$ & $\mathrm{X}$ & $\mathrm{X}$ & $\mathrm{X}$ \\
\hline
\end{tabular}

Des Weiteren wurden Schiebersande der Hersteller A, B und C beprobt (siehe auch Kapitel 4.2.3 und Kapitel 4.3.1). Es wurden Proben von Pfannensteinen, Reisschalenasche, Abdeckpulver, VTR-Masse und Gießpulvern genommen (siehe auch Kapitel 4.2.4 und Kapitel 4.3.3). Von drei Lollyproben $(23.10 .06,24.10 .06,26.10 .06)$ aus dem Konverter wurden Rückstandsisolate angefertigt und isotopisch analysiert. Von Prozess- $\mathrm{O}_{2}$ wurde neben der parallelen Probennahme mit Clogging auf dem Werksgelände von Linde in Duisburg-Ruhrort eine Probe von LOX (flüssiger Sauerstoff) und GOX (gasförmiger Sauerstoff) genommen.

\subsection{Nachtrag Hochtemperaturexperimente}

In Teil I wurde bei Hochtemperaturexperimenten eine Fraktionierung bei der Oxidation flüssiger Fe,Al-Schmelzen zwischen O-Quelle und gebildeter Tonerde festgestellt (siehe Kapitel 2.3.3.3). Daraufhin wurden im Mai 2006 zwei weitere Oxidationsexperimente durchgeführt, um die Datenbasis zu erweitern und den festgestellten Zusammenhang zwischen $\mathrm{fO}_{2}$ (Sauerstofffugazität) und $\Delta^{18} \mathrm{O}$ (Fraktionierung zwischen O-Quelle und Tonerde) zu überprüfen (siehe Tabelle 10).

Tabelle 10: Übersicht über die im Mai 2006 durchgeführten Hochtemperaturexperimente.

\begin{tabular}{|c|c|c|c|c|c|c|c|}
\hline Datum & Versuch & $\begin{array}{c}\mathbf{A l}_{\text {vor }} \\
{[\mathbf{G e w . - \% ]}}\end{array}$ & $\begin{array}{c}\mathbf{A I}_{\text {nach }} \\
{[\mathbf{G e w . - \%}]}\end{array}$ & Atmosphäre & $\mathbf{T}\left[{ }^{\circ} \mathbf{C}\right]$ & $\log \boldsymbol{f} \mathbf{O}_{\mathbf{2}}$ & $\delta^{\mathbf{1 8}} \mathbf{O ~ A l}_{\mathbf{2}} \mathbf{O}_{\mathbf{3}}[\mathbf{\%} \mathbf{0}]$ \\
\hline \hline 29.05 .2006 & 1 & 0.68 & 0.29 & $\mathrm{H}_{2} / \mathrm{CO}_{2}$ & 1600 & $-11,90$ & keine Messung \\
29.05 .2006 & 2 & 0.68 & 0.68 & $\mathrm{H}_{2} / \mathrm{CO}_{2}$ & 1600 & $-10,50$ & +14.5 \\
\hline
\end{tabular}

Ziel der Hochtemperaturexperimente war es, herauszufinden, ob es Fraktionierung bei der Bildung von Tonerde aus Stahlschmelzen zwischen der 0-Quelle und den gebildeten Oxiden gibt, und inwiefern diese mit dem Angebot von Sauerstoff $\left(\mathrm{fO}_{2}\right)$ zusammenhängt. 
Zwei Legierungen mit Gehalten von jeweils 0,68 Gew.- $\%$ Al wurden bei $1600^{\circ} \mathrm{C}$ geschmolzen und eine Stunde lang in einer $\mathrm{H}_{2} / \mathrm{CO}_{2}$-Gasmischung kontrolliert oxidiert. Zur näheren Beschreibung von Versuchsaufbau und -durchführung siehe Kapitel 2.3.1. Bei beiden Versuchen im Mai 2006 wurde eine höhere Sauerstofffugazität als bei den vorangegangenen Experimenten vom Februar 2006 eingestellt. Beim ersten Versuch wurde ein Kohlendioxidgehalt in der Gasmischung von 1 Vol.-\% eingestellt, was nach DEINES et al. (1976) ein $\log f \mathrm{O}_{2}=$ $-11,90$ puffert. Beim zweiten Versuch wurde mit 5 Vol.- $\% \mathrm{CO}_{2}$ ein $\log f \mathrm{O}_{2}=-10,50$ gewählt. Die Experimente vom Februar 2006 wurden alle mit $\log f \mathrm{O}_{2}=-14,80$ unter stärker reduzierenden Bedingungen durchgeführt. Bei den Experimenten der Voruntersuchung waren die Bedingungen mit $\log f \mathrm{O}_{2}=-4,0$ am oxidierensten (siehe Abbildung 33).

Beim ersten Versuch wurde nicht ausreichend Tonerde gebildet, um eine Isotopenanalyse durchführen zu können, wobei beide Legierungen nur teiloxidiert wurden. Aus Mikrosondenuntersuchungen ergab sich beim ersten Versuch, dass 35 Gew.-\% des Al oxidiert wurden, beim zweiten waren es 57 Gew.-\% (siehe Abbildung 34). Der $\delta^{18} \mathrm{O}-$ Wert des zweiten Versuchs lag bei $+14,5 \%$, der der Ofenatmosphäre bei $+25,1 \%$ (siehe Abbildung 33). Der $\delta^{18} \mathrm{O}-$ Wert und die Sauerstofffugazität des Versuches 2 vom Mai 2006 liegen zwischen denen vom Februar 2006 (stärker reduzierend) und denen der Voruntersuchung (stärker oxidierend). Die Daten vom Mai 2006 bestätigen die bereits beobachtete Abhängigkeit der Fraktionierung von $f \mathrm{O}_{2}$.

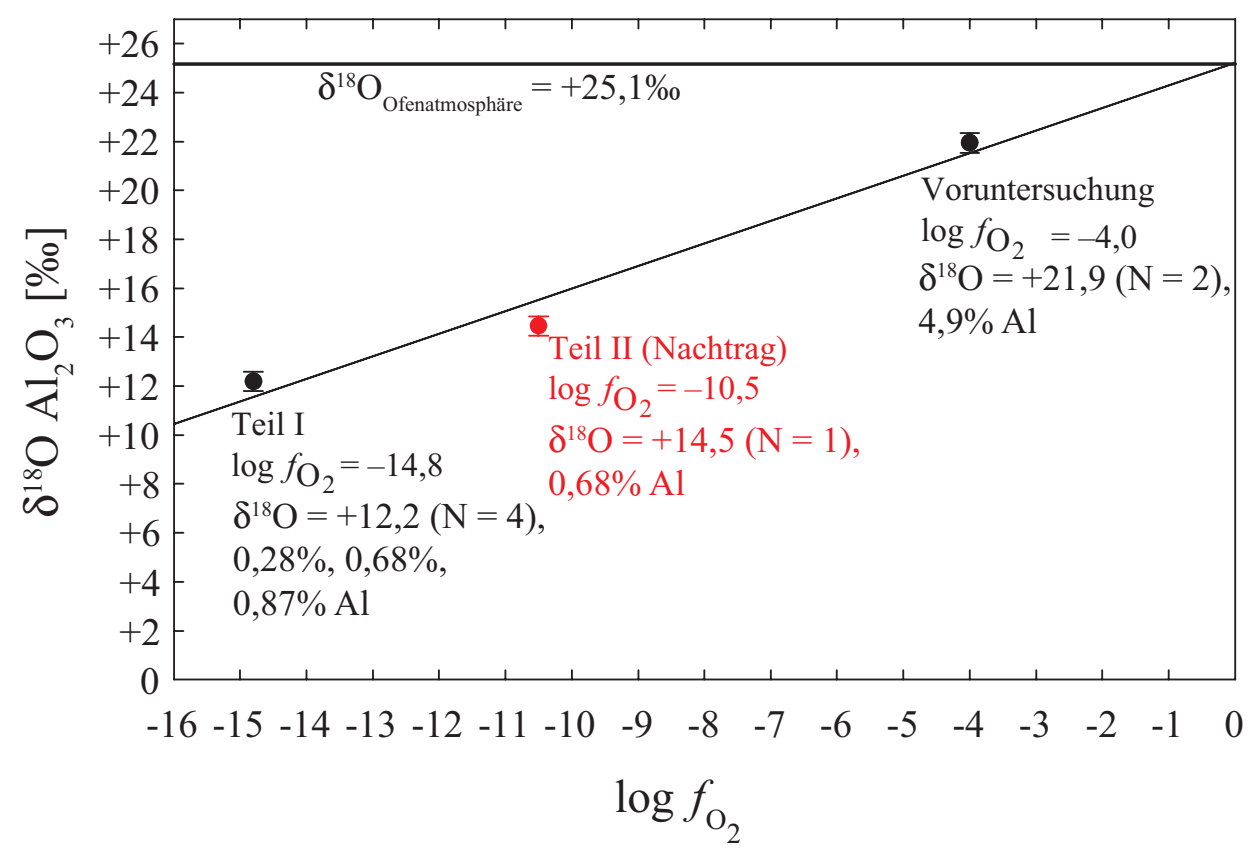

Abbildung 33: Aufgetragen ist der $\delta^{18} \mathrm{O}-$ Wert der gebildeten Tonerde gegen $\log f \mathrm{O}_{2}$. Der $\delta^{18} \mathrm{O}$-Wert der Ofenatmosphäre ist als horizontale Linie dargestellt. 


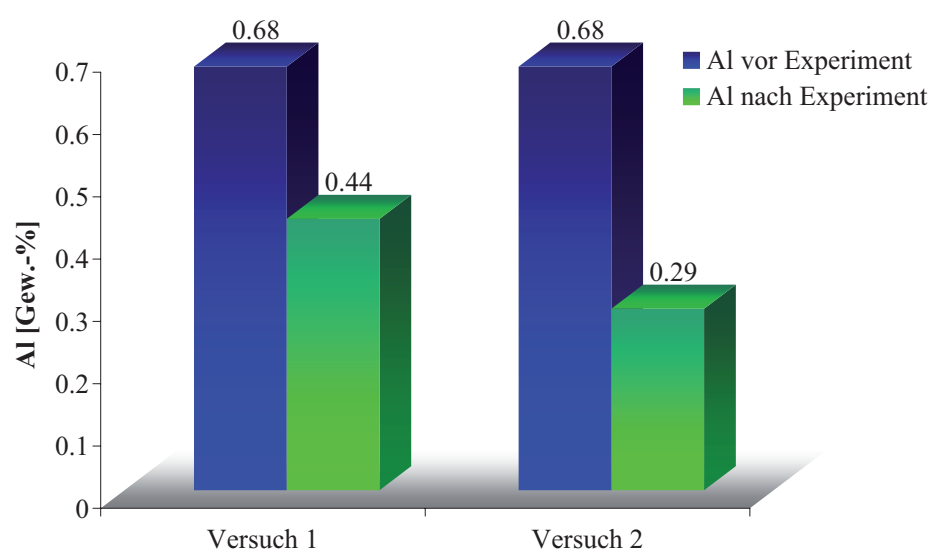

Abbildung 34: Zusammensetzung der Fe,Al-Legierungen vor (blaue Säulen) und nach (grüne Säulen) den Experimenten (die Legierungen wurden nach den Experimenten mikrochemisch analysiert).

Je höher die Sauerstofffugazität, desto niedriger die Fraktionierung zwischen 0-Quelle und gebildeter Tonerde bei der Oxidation flüssiger Stahlschmelzen.

\subsection{Ergebnisse}

\subsubsection{Clogging}

\subsubsection{Allgemein}

Von 23 Proben wurden chemische Analysen gemacht. Mit einem Handmagneten wurden die makroskopisch erkennbaren Stahlpartikel aus den Proben entfernt. Aufgrund der stark reduzierenden Bedingungen ist $\mathrm{Fe} \gg \mathrm{Fe}^{2+}>\mathrm{Fe}^{3+}$. Da der $\mathrm{Fe}_{2} \mathrm{O}_{3}$-Gehalt aus der chemischen Analyse zwischen 1 und 23 Gew.-\%. variiert, wurde er bei allen Proben subtrahiert und die Analysen auf 100 Gew.-\% normalisiert, um die Daten vergleichbarer zu machen. Die Hauptkomponenten im Clogging sind $\mathrm{Al}_{2} \mathrm{O}_{3}, \mathrm{CaO}, \mathrm{MgO}$ und $\mathrm{TiO}_{2}$. Bei den Elementoxiden unter 2 Gew.$\%$ sind $\mathrm{Na}_{2} \mathrm{O}, \mathrm{SiO}_{2}$ und $\mathrm{ZrO}_{2}$ die massenmäßig bedeutendsten (siehe Tabelle 18). Die vorherrschende Komponente des Cloggings ist $\mathrm{Al}_{2} \mathrm{O}_{3}$ mit Gehalten zwischen 89 und 98 Gew.-\%. Die Gehalte für $\mathrm{CaO}$ variieren zwischen 0,1 und 7,7 Gew.-\%, die für $\mathrm{MgO}$ zwischen 0,1 und 6,8 Gew.-\%. Erstere stehen in Zusammenhang mit der Bildung von Hibonit, letztere mit der Bildung von Spinell im Clogging. Die Gehalte für $\mathrm{TiO}_{2}$ erreichen bis zu 2,5 Gew.-\%, alle anderen Hauptelemente liegen unterhalb dieser Grenze. Die Ablagerungen an der SEN-Unterseite sind etwa dreimal so reich an $\mathrm{Na}_{2} \mathrm{O}$ und $\mathrm{K}_{2} \mathrm{O}$ wie das eigentliche Tauchrohrclogging (siehe Tabelle 18). Dies schlägt sich in entsprechend hohen Gehalten an Na- $\beta$-Aluminaten bzw. Diaoyudaoit nieder (siehe auch Kapitel 4.2.1). Ihr $\mathrm{Al}_{2} \mathrm{O}_{3}$-Gehalt liegt zwischen 89 und 93 Gew.\%. $\mathrm{CaO}$ variiert zwischen 1,2 und 6 Gew.-\%. Der MgO-Gehalt liegt bei 0,3 bis 1,6 Gew.-\%, $\mathrm{TiO}_{2}$ bei 1 bis 5 Gew.- $\%$ und $\mathrm{SiO}_{2}$ bei bis zu 2 Gew.- $\%$. 
An 33 Proben nichtmetallischer Ablagerungen in Tauchrohren (das eigentliche Clogging) aus Duisburg und an deren Unterseiten wurden 70 Isotopenmessungen durchgeführt. In Abbildung 35 sind zusammenfassend die Ergebnisse von Teil I und II, der Voruntersuchung und die Daten von PACK (2000) aus Dortmund dargestellt, und zwar die rechten Intervallgrenzen geschlossener, äquidistanter Klassen. Die Klassenbreite beträgt 1\%o.

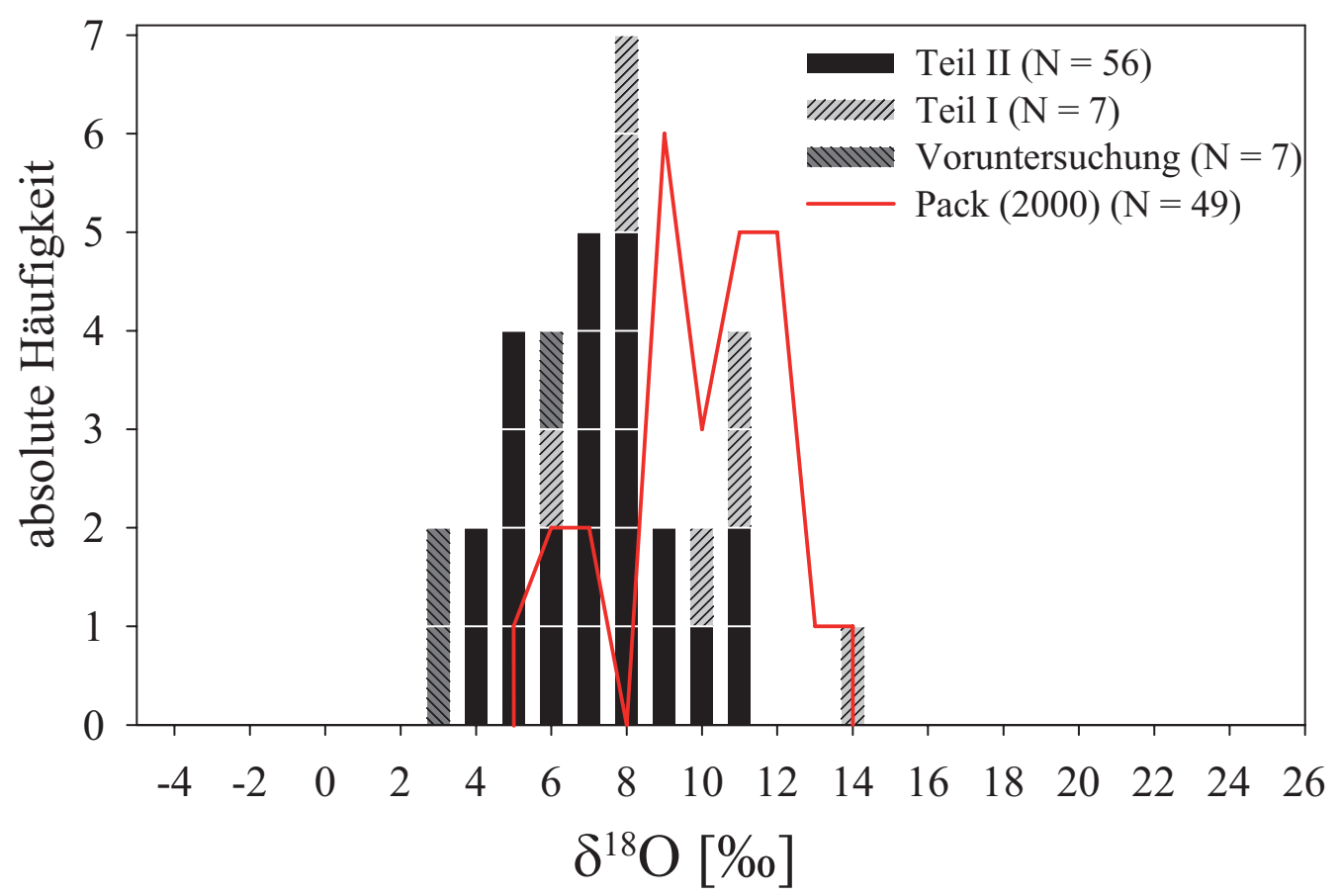

Abbildung 35: Zusammenfassendes Histogramm aller bisher gemessenen $\delta^{18} \mathrm{O}-$ Werte von nichtmetallischen Ablagerungen im Tauchrohr und an dessen Unterseite.

Die $\delta^{18} 0$-Werte nichtmetallischer Ablagerungen aus Teil II liegen zwischen $+3 \%$ und $+11 \%$. Der Mittelwert für nichtmetallische Ablagerungen aus Duisburg liegt bei $+6,75 \%$, der für Ablagerungen aus Dortmund bei $+9,4 \%$.

Im Vergleich zu Teil II sind die $\delta^{18} \mathrm{O}-$ Werte aus Teil I etwas höher und die der Voruntersuchung etwas niedriger. Insgesamt zeigen sowohl die Daten aus Duisburg (Voruntersuchung, Teil I und II) als auch die Daten aus Dortmund-Hörde annähernd eine Normalverteilung, wobei das Maximum in Duisburg bei niedrigeren $\delta^{18} \mathrm{O}-$ Werten liegt als in Dortmund. Nichtmetallische Ablagerungen aus Dortmund sind im Mittel rd. 3\%o schwerer als jene aus Duisburg. In Abbildung 36 sind die $\delta^{18} \mathrm{O}$-Werte von Clogging und SEN-Unterseiten-Ablagerungen aus dem Teil II von Strang 3 und 4 dargestellt. Das Diagramm zeigt, dass es nicht an jedem Tag Clogging in beiden Tauchrohren gab, das Gleiche gilt für SEN-Unterseiten-Ablagerungen. Mit einem Mittelwert von $\delta^{18} \mathrm{O}=+5,9 \%$ sind diese Ablagerungen etwas leichter als das eigentliche Tauchrohrclogging mit einem Mittelwert von $\delta^{18} \mathrm{O}=+7,2 \%$. 


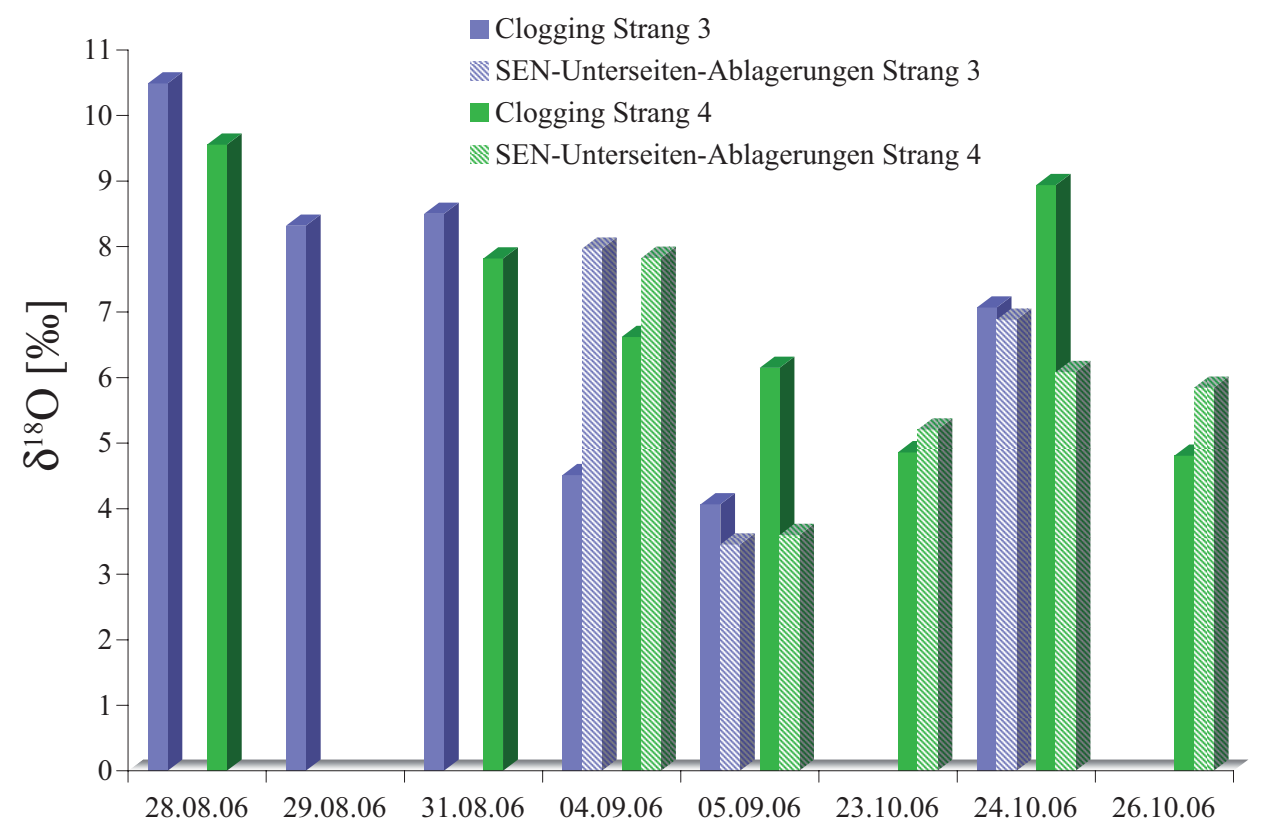

Abbildung 36: $\delta^{18} \mathrm{O}-$ Werte von Clogging und SEN-Unterseiten-Ablagerungen aus Teil II an acht Tagen.

Bei den SEN-Unterseiten-Ablagerungen wurde eine isotopische Zonierung festgestellt, wie sie auch von PACK et al. (2005) beobachtet wurde (siehe Abbildung 37).

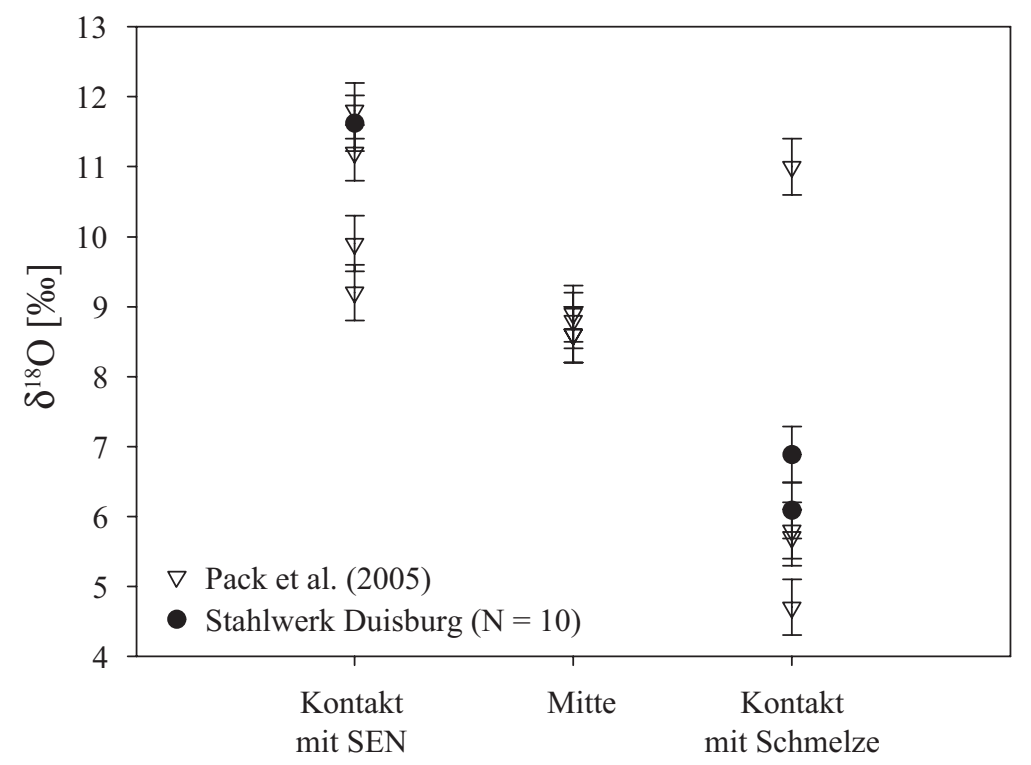

Abbildung 37: Aufgetragen ist der $\delta^{18} \mathrm{O}-$ Wert von SEN-Unterseiten-Ablagerungen gegen den Abstand vom FF-Material der SEN-Unterseite (Teil II) und Daten aus PACK et al. (2005).

Die Ablagerungen, die Kontakt zum FF-Material der SEN-Unterseite haben, zeigen höhere $\delta^{18} 0$-Werte als Ablagerungen am Kontakt zur Stahlschmelze. Das heißt, dass "frühe" Ablagerungen isotopisch leichter sind als "späte".

Abbildung 38 zeigt ein massives SEN-Unterseiten-Präzipitat, das eine Mächtigkeit von bis zu $1,5 \mathrm{~cm}$ aufweist. 


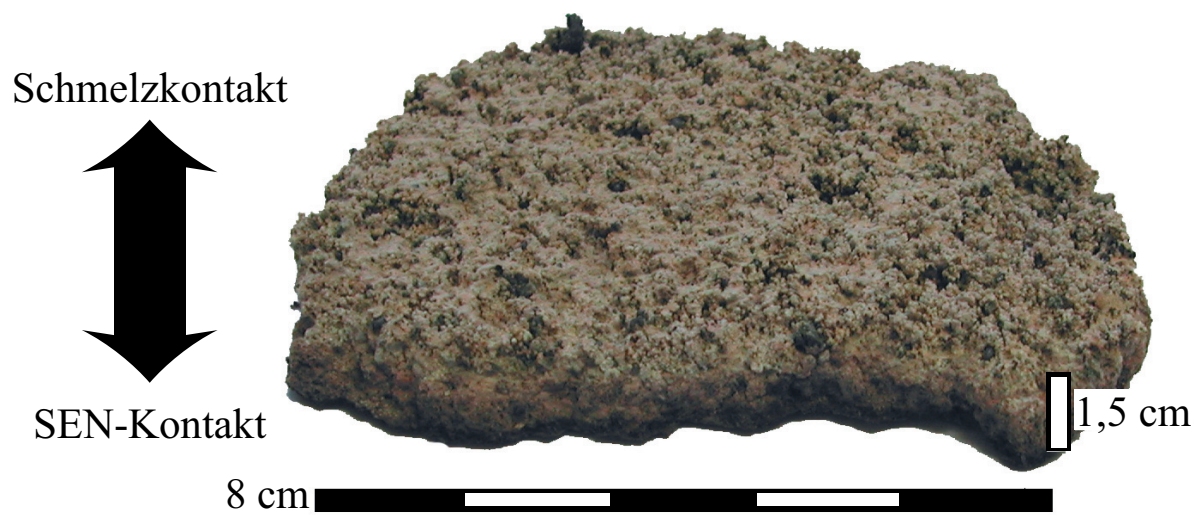

Abbildung 38: Dargestellt ist die SEN-Unterseiten-Ablagerung vom 24.10.06, Strang 4, Stahlwerk B.

Ein Gradient im $\delta^{18} \mathrm{O}$ wurde auch bei den Cloggingproben (Ablagerungen innerhalb des Tauchrohres) vom 24.10.2006 beobachtet. Hier ist die Isotopie von Clogging, das Kontakt zur Schmelze hatte (= später abgelagerte Partikel), mit einem $\delta^{18} \mathrm{O}$ von $+6,6 \%$ (Strang 3 ) bzw. $+7,5 \%$ (Strang 4) ebenfalls niedriger als die des Cloggings mit einem $\delta^{18} \mathrm{O}$ von $+7,5 \%$ (Strang 3) bzw. +10,4\%o (Strang 4), das Kontakt zum FF-Material hatte (= früher abgelagerte Partikel).

Clogging von Strang 3 und 4 ist im $\delta^{18} \mathrm{O}$ positiv korreliert. Dasselbe gilt für die SENUnterseiten-Ablagerungen, die entlang einer Geraden liegen. Das bedeutet, dass die Ablagerungen in beiden Strängen durch denselben Prozess entstanden und/oder aus demselben Reservoir stammen (siehe Abbildung 39).

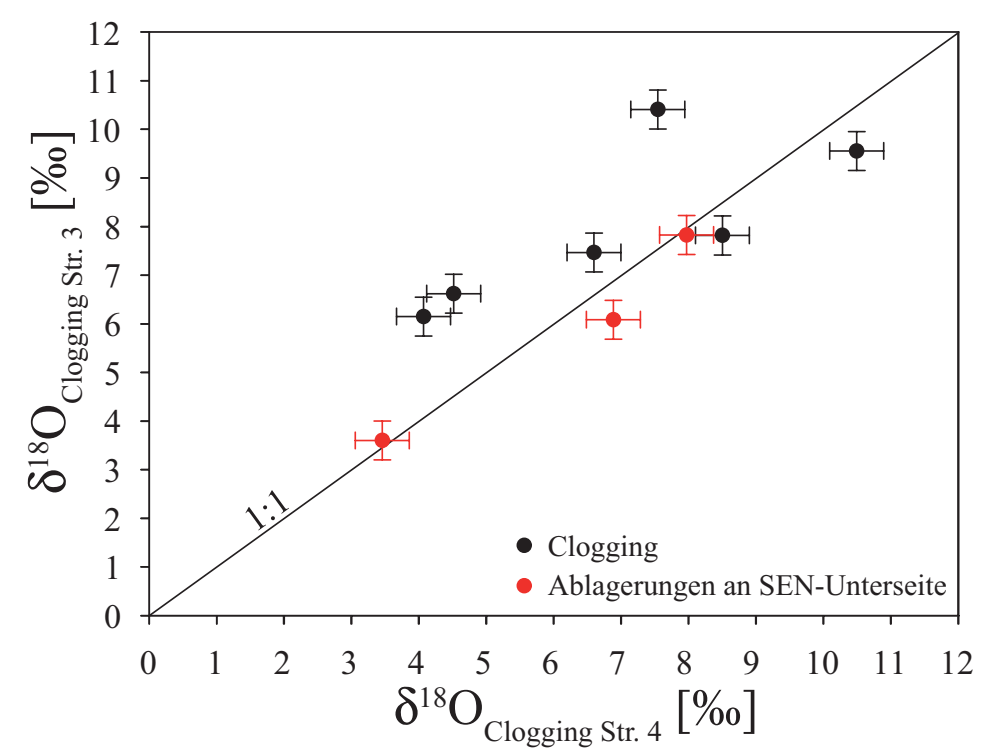

Abbildung 39: Zusammenhang zwischen der Isotopie von oxidischen Ablagerungen beim Stranggießen von Strang 3 und 4.

Trägt man die Gehalte der Elementoxide der analysierten oxidischen Ablagerungen von Strang 3 gegen die von Strang 4 auf, zeigt sich die gleiche Korrelation (siehe Abbildung 40). 


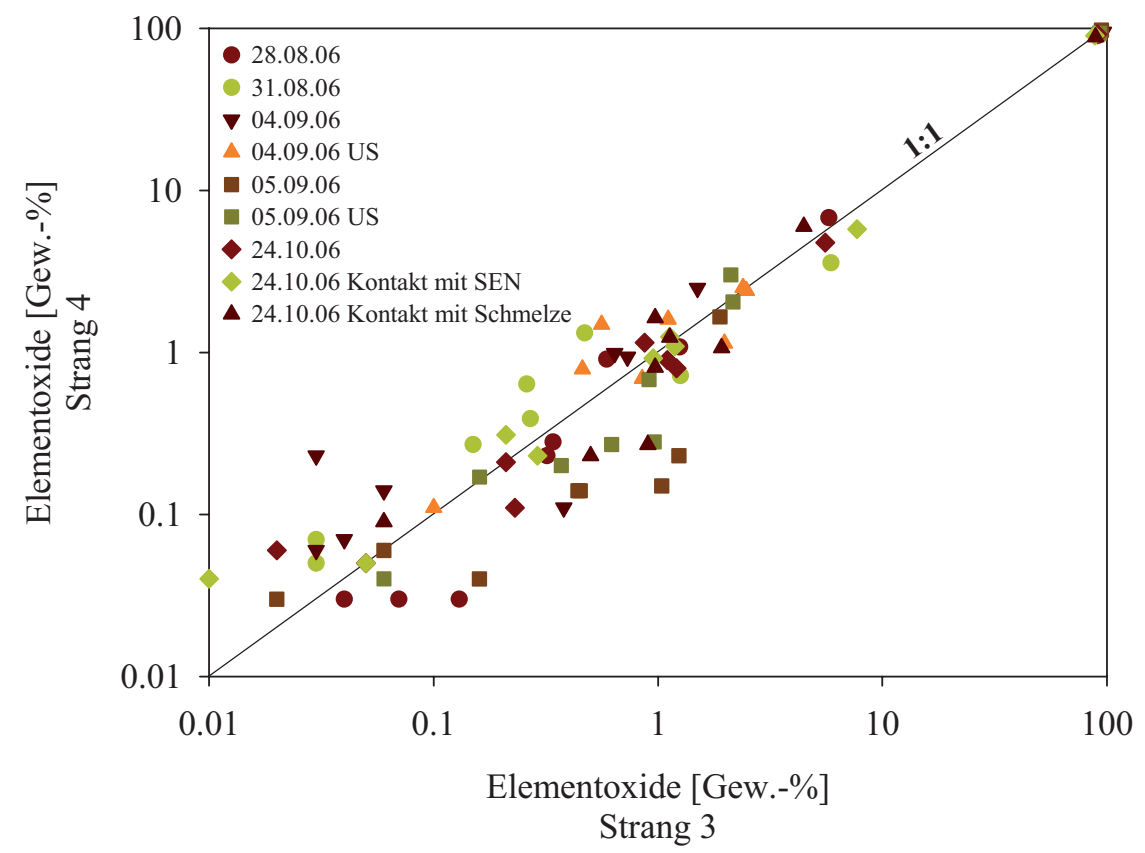

Abbildung 40: Zusammenhang zwischen der Chemie oxidischer Ablagerungen von Strang 3 und 4.

Oxidische Ablagerungen aus Tauchrohren eines Verteilers haben die gleiche Isotopie und Chemie und sind daher vermutlich durch den gleichen Prozess, aus dem gleichen Reservoir entstanden.

Trägt man den $\delta^{18} \mathrm{O}$ von Clogging gegen dessen $\mathrm{Al}_{2} \mathrm{O}_{3}$-Gehalt auf, zeigt sich eine negative Korrelation (siehe Abbildung 41).

Clogging mit den größten Anteilen an $\mathrm{Al}_{2} \mathrm{O}_{3}$ (>94 Gew.- $\%$ ) hat mit $\delta^{18} 0 \approx+5 \%$ die niedrigsten $\delta^{18} 0$-Werte.

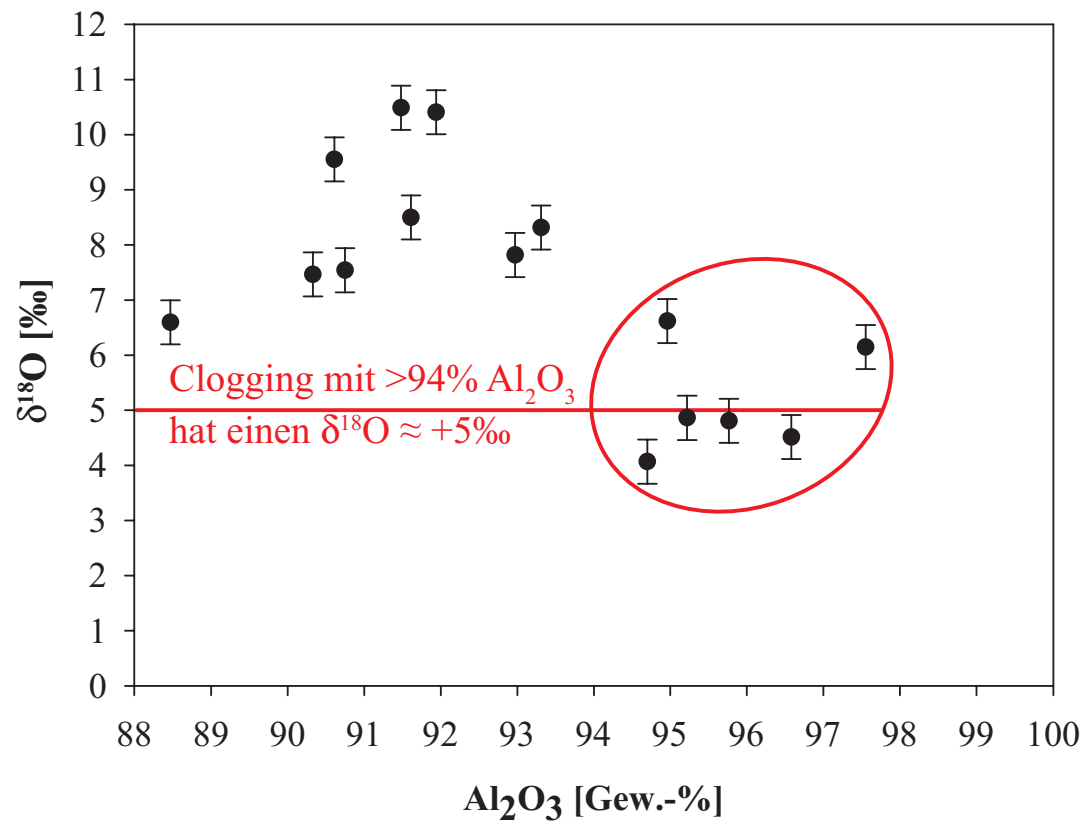

Abbildung 41: Korrelation zwischen $\delta^{18} \mathrm{O}$ und $\mathrm{Al}_{2} \mathrm{O}_{3}$-Gehalt von Clogging. 
Clogging mit $>94$ Gew.- $\% \mathrm{Al}_{2} \mathrm{O}_{3}$ hat im Mittel einen $\delta^{18} \mathrm{O}$ von ungefähr $+5 \%$ (siehe Abbildung 41).

\subsubsection{Versuch mit offenem Stopfen}

Am 26.10.06 wurde bei einer 2er-Sequenz (IF-Stahl mit 0,095 bis 0,124\% Ti) am Strang 4 die Argonspülung abgestellt und der Stopfen offen gelassen, so dass Luft eingesogen werden sollte. Ziel war es, zu überprüfen, ob das Clogging die Isotopie des Luftsauerstoffs übernimmt.
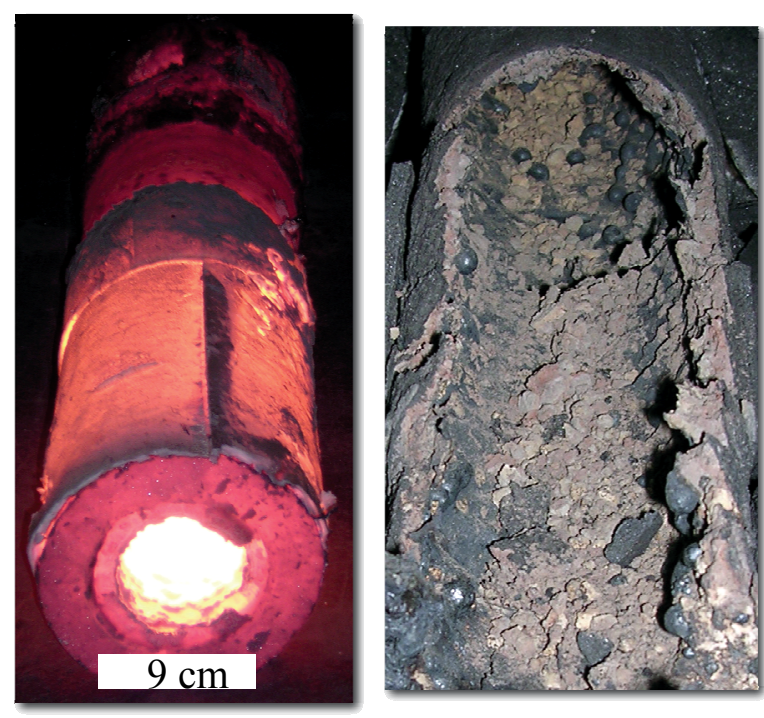

Abbildung 42: Photos des Tauchrohres nach dem Versuch mit offenem Stopfen. Links sind etwa $1 \mathrm{~cm}$ mächtige Anfrierungen von Stahl unmittelbar nach dem Gießen zu sehen. Rechts ist Clogging aus dem oberen SEN-Bereich (Richtung Stopfen) abgebildet. Die Anfrierung besteht aus Stahl mit einem Netzwerk aus Clogging.

Bei dem Versuch waren erhebliche Mengen an Stahl im Tauchrohr auffällig. Die Anfrierungen von Stahl waren mit einem Netzwerk von Clogging agglomeriert (siehe Abbildung 42). Es wurden nur an diesem Tag zwei Schmelzen vergossen, so dass keine direkten Vergleiche der Cloggingmenge gegenüber anderen Tagen gemacht werden können. Das Clogging erstreckte sich fast über die gesamte Tauchrohrlänge. Auffallend war, dass auch im oberen SEN-Bereich, das heißt in Richtung des offenen Stopfens, viele Präzipitate auftraten. Der Strang 3, bei dem die Argonspülung regulär betrieben wurde, hatte kein Clogging und keine Anfrierungen. Ebenso auffallend ist bei diesem Versuch der um 55\% höhere Gehalt von $\mathrm{TiO}_{2}$ gegenüber dem Mittelwert von Clogging (siehe Tabelle 18).

In Abbildung 43 sind die $\delta^{18} \mathrm{O}$-Werte von Clogging bei offenem Stopfen und die des Luftsauerstoffs dargestellt. Abbildung 44 zeigt die $\delta^{18} \mathrm{O}$-Werte von Clogging am 26.10.06 von Strang 4 in den verschiedenen Probennahmebereichen. Diese variieren zwischen $\delta^{18} \mathrm{O}=+3,6 \%$ und $\delta^{18} \mathrm{O}=+6,0 \%$. 
Die $\delta^{18} 0$-Werte von Clogging bei offenem Stopfen liegen mit +4 bis $+6 \%$ durchschnittlich $18 \%$ o tiefer als die des Luftsauerstoffs $\left(\delta^{18} 0=+23,5 \%\right)$. Damit wurde die Isotopie des Luftsauerstoffs nicht übernommen.

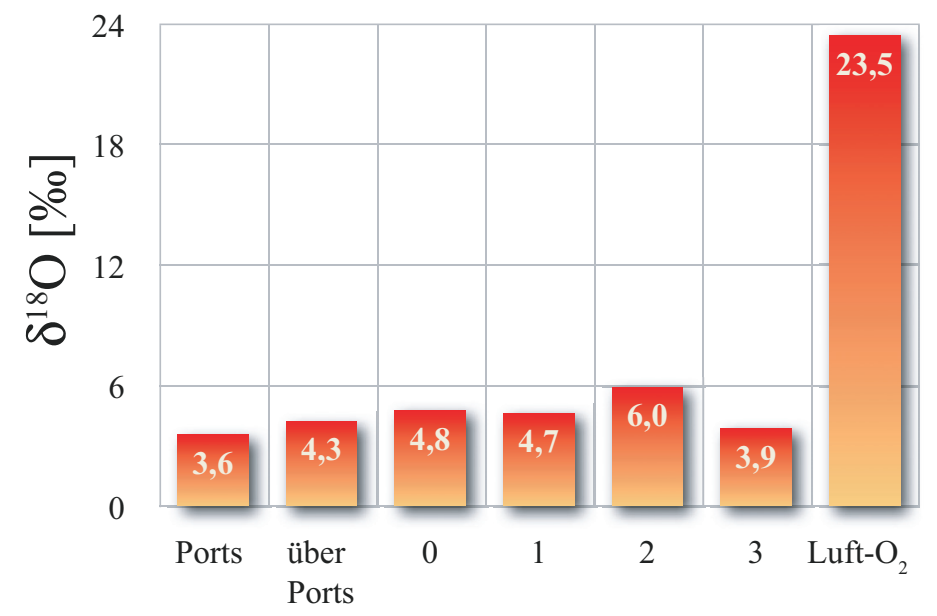

Abbildung 43: Isotopie von Clogging und von $\mathrm{Luft}^{-} \mathrm{O}_{2}$ beim Versuch mit offenem Stopfen.

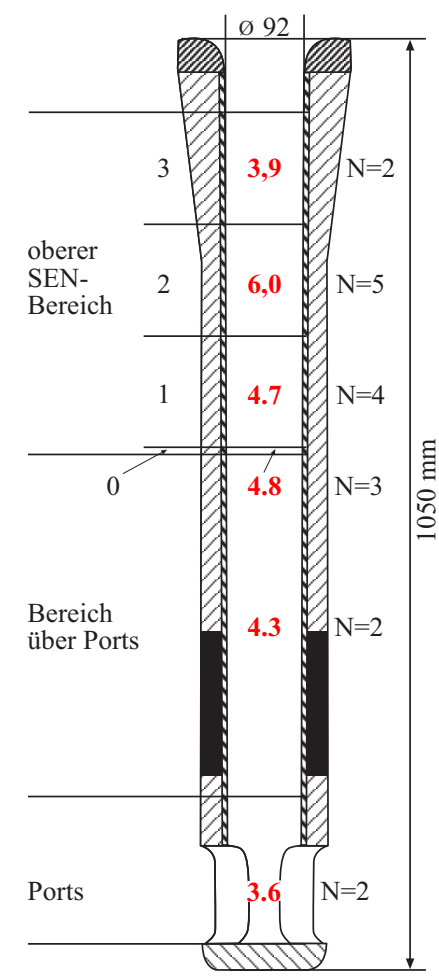

Abbildung 44: Zeichnung des Tauchrohres, das beim Versuch mit offenem Stopfen zum Einsatz kam. In rot sind die $\delta^{18} \mathrm{O}-$ Werte von Clogging in Promille angegeben.

\subsubsection{Prozess- $\mathrm{O}_{2}$}

\subsubsection{Korrelation zwischen Clogging und Prozess- $\mathrm{O}_{2}$}

Ein Hauptziel von Teil II war es, herauszufinden, ob es einen Zusammenhang zwischen der Isotopie von Clogging und von Frischsauerstoff gibt. Falls sich eine eindeutige Korrelation 
feststellen ließe, könnte man die O-Quelle für Clogging dem Blassauerstoff zuordnen. Wie in Abbildung 36 gezeigt, kann Clogging im $\delta^{18} \mathrm{O}$ um mehrere Promille von einem auf den anderen Tag variieren. Falls sich dies beim Prozess- $\mathrm{O}_{2}$ auch nachweisen ließe, könnte ein Zusammenhang überprüft werden.

In Teil I wurden im Dezember 2005 und Januar 2006 Proben von Frischsauerstoff im Stahlwerk B genommen (siehe Kapitel 2.4.1.4). Diese beiden Proben aus Duisburg waren mit einem $\delta^{18} \mathrm{O}-$ Wert von $+0,9 \%$ sehr leicht. Bei PACK (2000) findet sich ein $\delta^{18} \mathrm{O}$ von Blassauerstoff von $+24,3 \%$. Damit variierte der Prozess- $\mathrm{O}_{2}$ zwischen den Daten aus Dormund und denen aus Duisburg um mehr als zwei Zehner Promille. Nun war die Frage zu klären, ob die Isotopie des Prozess- $\mathrm{O}_{2}$ aus Duisburg immer solch niedrige Werte hat, oder ob es eine Variation im $\delta^{18} \mathrm{O}-$ Wert gibt.

Um den Prozess der Sauerstoffherstellung besser verstehen und damit mögliche Schwankungen in der Isotopie abschätzen zu können, wurde Kontakt zu Linde in Duisburg-Ruhrort hergestellt. Es fand ein Treffen bei Linde statt, bei dem das Lindeverfahren, die Luftzerlegung und die Anlagentechnik zur Sauerstoffherstellung von Linde-Mitarbeitern eingehend erläutert wurden. Bei einem weiteren Treffen wurden zwei Gasproben, eine von LOX (flüssiges $\mathrm{O}_{2}$ ) und eine von GOX (gasförmiges $\mathrm{O}_{2}$ ), auf dem Werksgelände von Linde aus den Versorgungsleitungen für die ThyssenKrupp Steel AG genommen (siehe Abbildung 45). Aufgrund beschränkter technischer Möglichkeiten konnte LOX nur gasförmig beprobt werden. Bei der Probennahme des GOX wurde sichergestellt, dass zu diesem Zeitpunkt kein LOX ins Netz eingespeist wurde.

Linde liefert etwa zwei Drittel des Prozesssauerstoffs, ein Drittel kommt von Air Liquide, wobei rd. $80 \%$ des $\mathrm{O}_{2}$ von Linde GOX sind und rd. 20\% erst flüssig in zwei Tanks gelagert werden und bei Bedarf über einen Verdampfer zusätzlich in die Leitungen eingespeist werden können (siehe Abbildung 45). 


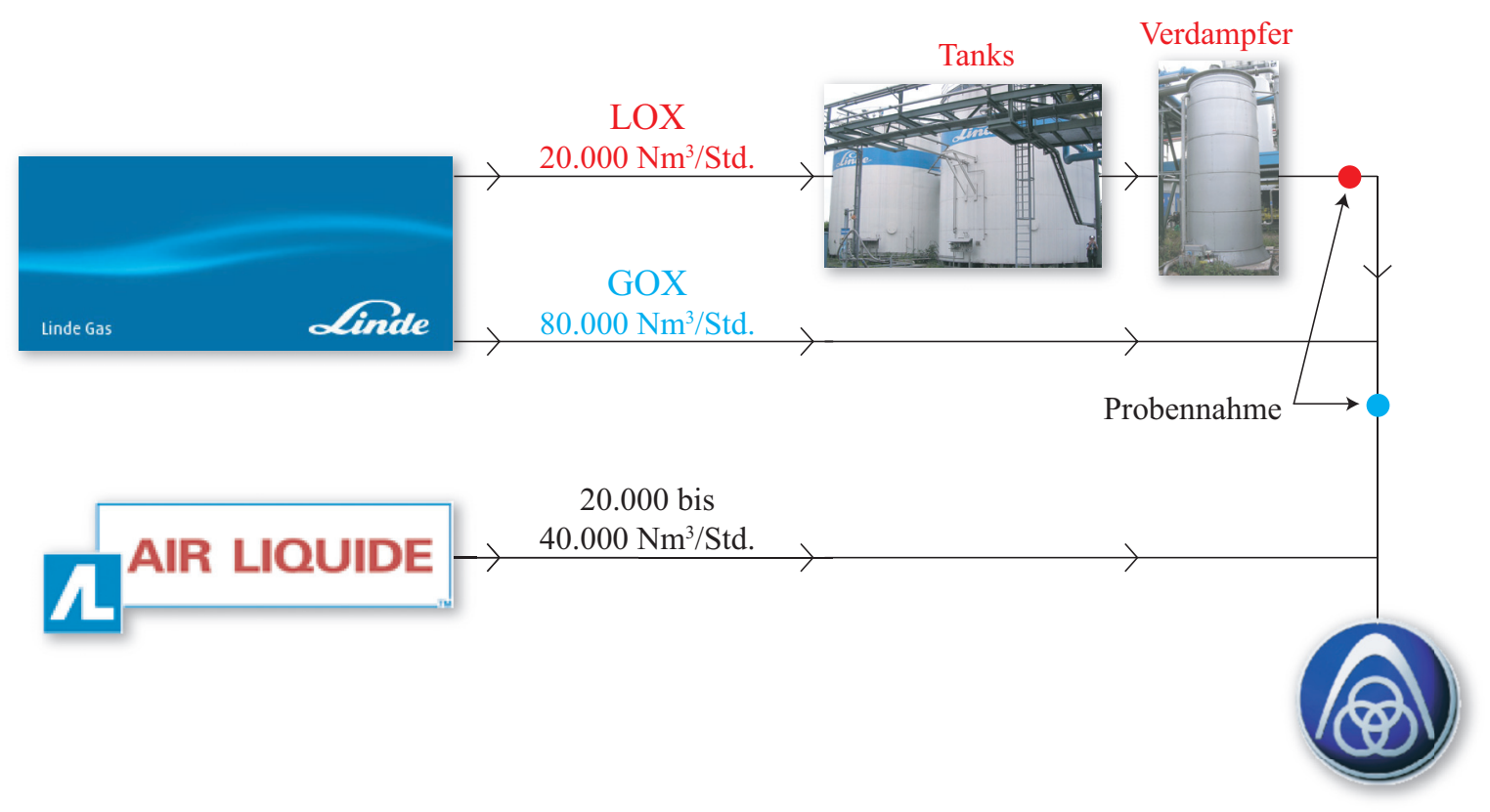

Abbildung 45: Organigramm über die Blassauerstofflieferung von Linde und Air Liquide an die ThyssenKrupp Steel AG. Zwei Drittel des $\mathrm{O}_{2}$ kommen von Linde, ein Drittel stammt von Air Liquide.

\subsubsection{Lindeverfahren und Rektifikation}

Beim Lindeverfahren wird Luft mittels Kühlung fast isotherm verdichtet. Unter Ausnutzung des Joule-Thomson-Effektes erfolgt eine Abkühlung und Verflüssigung. Der Effekt beruht auf der Tatsache, dass ein komprimiertes Gas bei Expansion abkühlt. Beim Entspannen wird Arbeit gegen die intermolekularen Anziehungskräfte geleistet. Die Energie hierfür stammt aus der kinetischen Energie der Gase, was zur Folge hat, dass die Gase abkühlen. Eine weitere Möglichkeit der Entspannung ist die durch eine Expansionsmaschine, bei der die Luft ebenfalls weiter abgekühlt wird. Mit diesen Möglichkeiten der Luftentspannung allein wird eine Luftverflüssigung allerdings nicht erreicht, da sie nicht ausreichen, das Gas unterhalb seines kritischen Punktes ${ }^{8}$ zu bringen, wo es flüssig wird. Mit Hilfe des von Carl von Linde entwickelten Gegenstrom-Wärmeüberträgers wird der Abkühlungseffekt auf tiefere Temperaturniveaus übertragen und es werden die für eine Verflüssigung notwendigen Temperaturen erreicht. Die verflüssigte Luft wird in einen Luftzerleger eingebracht, in dem Stickstoff von Sauerstoff getrennt wird (siehe Abbildung 46).

\footnotetext{
${ }^{8}$ Kritischer Punkt: Zustand eines Stoffes, der sich durch Angleichen der Dichten von flüssiger Phase und Gasphase kennzeichnet.
} 


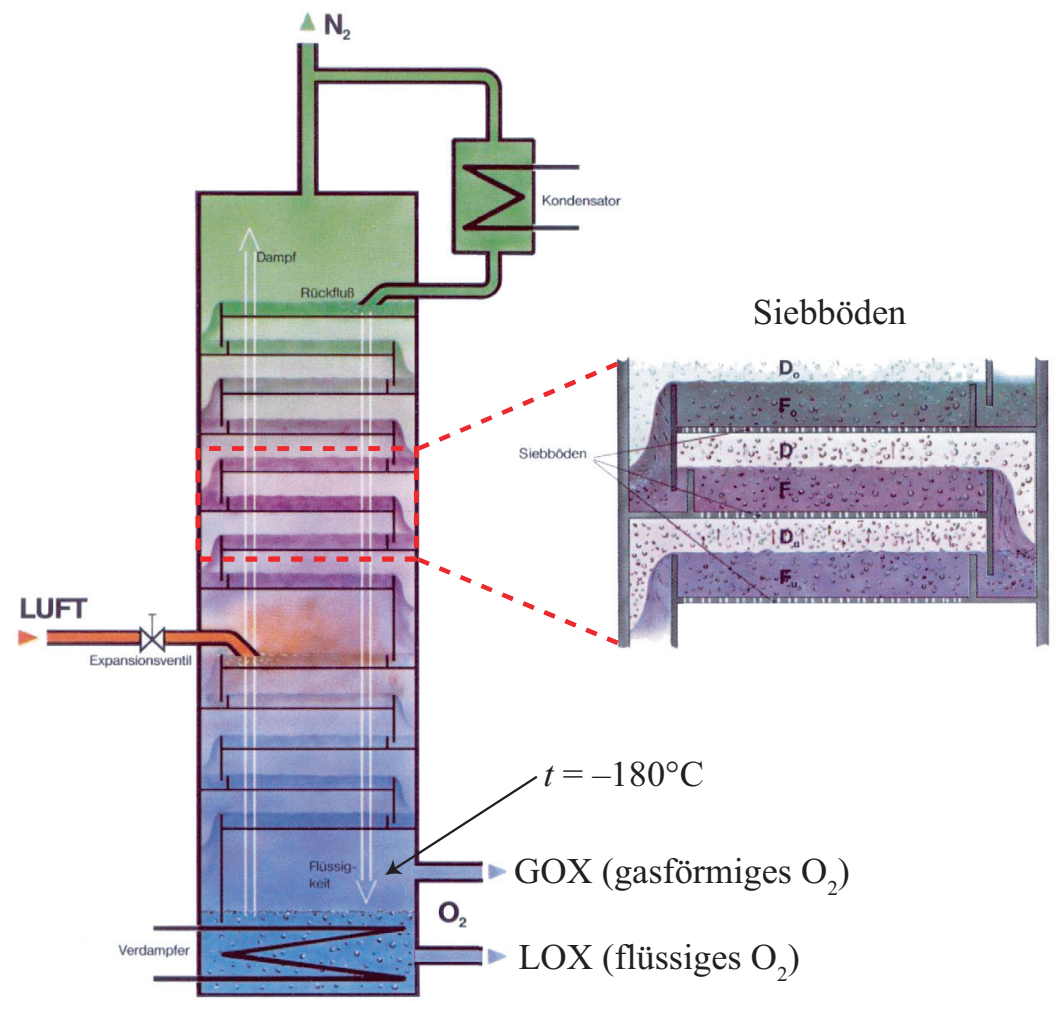

Abbildung 46: Luftzerleger (links) mit den Produkten GOX und LOX am Boden und $\mathrm{N}_{2}$ am Kopf der Säule und ein Ausschnitt (rechts), der den prinzipiellen Aufbau einer Rektifikationssäule zeigt (verändert nach LINDE).

\section{Das Prinzip der Rektifikation ${ }^{9}$ ist die Gegenstromführung von Flüssigkeit und Gas, wobei} beide in engen Kontakt miteinander kommen.

Bei der Rektifikation fließt die verflüssigte Luft über Siebböden in der Säule abwärts und wird dabei von Wehren aufgehalten (siehe Abbildung 46). Am Boden des Luftzerlegers sammelt sich reiner, flüssiger Sauerstoff, darüber befindet sich reines, gasförmiges $\mathrm{O}_{2}$. Im Kopfbereich der Säule ist reines $\mathrm{N}_{2}$. Betrachtet man einen beliebigen Siebboden aus der Rektifikationssäule, so ist die $\mathrm{O}_{2}$-Konzentration im $\mathrm{N}_{2} / \mathrm{O}_{2}$-Flüssigkeitsgemisch höher als die in der darüber befindlichen Gasphase, da Stickstoff einen höheren Dampfdruck als Sauerstoff hat. Die Gasphase steigt durch die Siebböden in die darüberliegende Flüssigphase auf und kondensiert. Dabei wird Kondensationswärme frei, die zu einer weiteren Verdampfung der Flüssigkeitsphase führt. Auf diese Weise entsteht eine Gegenstromdestillation, die zur Trennung von Stickstoff (oben) und Sauerstoff (unten) führt.

\footnotetext{
${ }^{9}$ Rektifikation: Zerlegung flüssiger und dampfförmiger Gemische.
} 


\subsubsection{Experiment zur Bestimmung der Fraktionierung zwischen GOX und LOX}

Wie in Abbildung 46 dargestellt, werden bei einer Temperatur von $-180^{\circ} \mathrm{C}$ die Produkte GOX und LOX voneinander getrennt. Die Frage war, ob es eine Fraktionierung zwischen den im Gleichgewicht stehenden Phasen GOX und LOX gibt. Wasser hat z. B. im Gleichgewicht mit Wasserdampf bei $+20^{\circ} \mathrm{C}$ eine Fraktionierung von $\Delta^{18} \mathrm{O}_{\text {flüssig-gasförmig }}=9,8 \%$. Bei $0^{\circ} \mathrm{C}$ hat Wasser im Gleichgewicht mit Wasserdampf bereits eine Fraktionierung von $\Delta^{18} \mathrm{O}_{\text {flüssig-gasförmig }}=11,6 \%$ ( (siehe Abbildung 47).

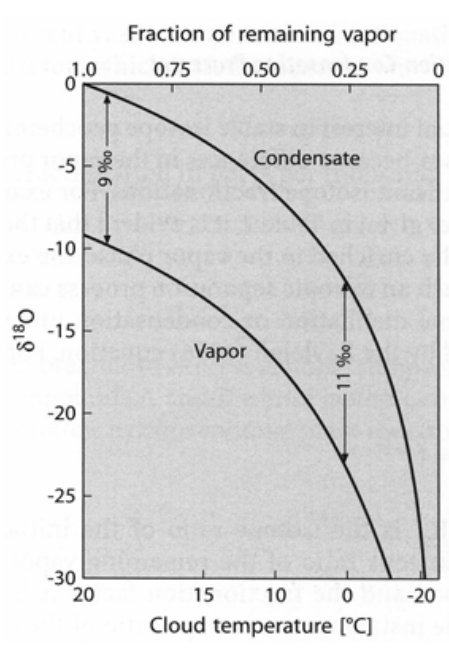

Abbildung 47: Fraktionierung zwischen Dampf und Wasser bei Kondensation aus einer Wolke nach dem Rayleighschen Destillationsgesetz (siehe auch Kapitel 2.3.4) (HOEFS 2004).

Die Gleichgewichtsfraktionierung ist also temperaturabhängig und nimmt in der Regel mit sinkender Temperatur zu. Daher könnte man erwarten, dass bei Temperaturen von $-180^{\circ} \mathrm{C}$ eine große Fraktionierung zwischen GOX und LOX auftritt und somit auch große Variationen im $\delta^{18} \mathrm{O}$ von Prozesssauerstoff erklärt werden können.

Um die Fraktionierung zwischen GOX und LOX bestimmen zu können, wurden Experimente durchgeführt, bei denen Sauerstoff bei der Temperatur von flüssigem Stickstoff in einem Edelstahlrohr verflüssigt wurde (=LOX). Der Dampfdruck des $\mathrm{O}_{2}$ beträgt bei dieser Temperatur 0,2 bar (=GOX). Mehrere Inkremente von GOX wurden in ein Teilstück der Edelstahllinie expandiert und dort unterschiedlich lange bei geöffnetem Ventil zum LOX stehengelassen. Nach den jeweiligen Equilibrierungszeiten wurde das Ventil zum LOX wieder geschlossen und die Portionen in das Massenspektrometer eingelassen (siehe Abbildung 48). 


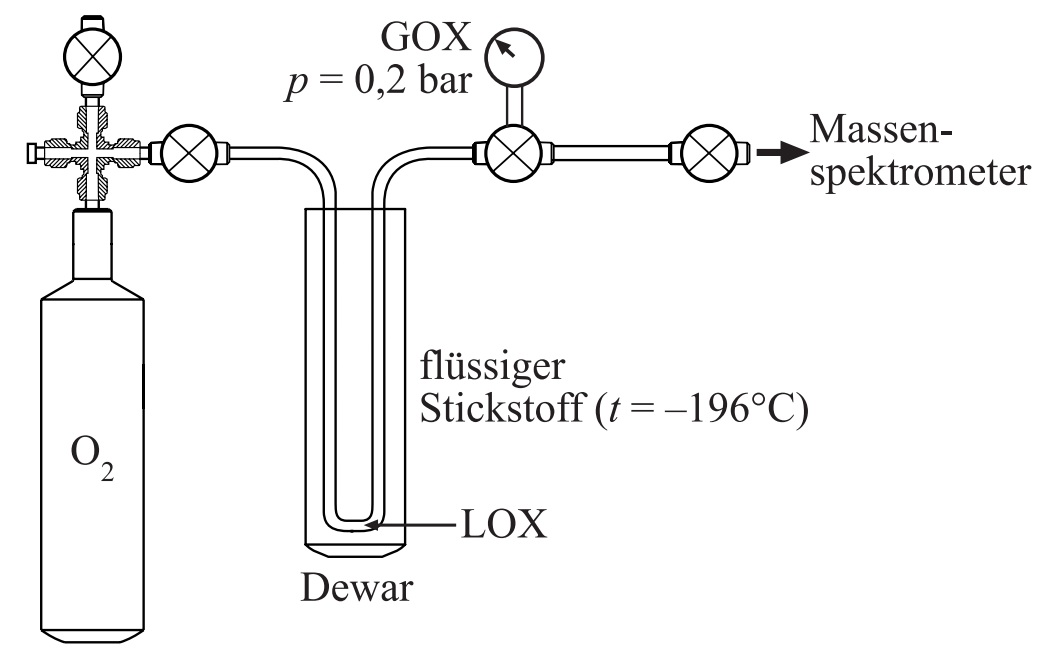

Abbildung 48: Versuchsaufbau zur Bestimmung der Fraktionierung zwischen GOX und LOX.

Die Ergebnisse der Messungen sind in Abbildung 49 dargestellt. Die gemessene Fraktionierung zwischen LOX und GOX liegt bei 5 -7\%, der Mittelwert beträgt $\Delta \Delta^{18} \mathrm{O}_{\text {LOX-GOX }}=+5,9 \pm 0,2 \%$. Die Daten für $\Delta^{18} \mathrm{O}_{\text {LOX-GOX }}$ zeigen bei diesen Experimenten keine Zeitabhängigkeit und streuen mit $\pm 0,7 \%$ um den Mittelwert. Nach CHIALVOA and HORITA (2006) liegt die Gleichgewichtsfraktionierung bei $-196^{\circ} \mathrm{C}$ bei $9,5 \%$ (siehe Abbildung 50 ).

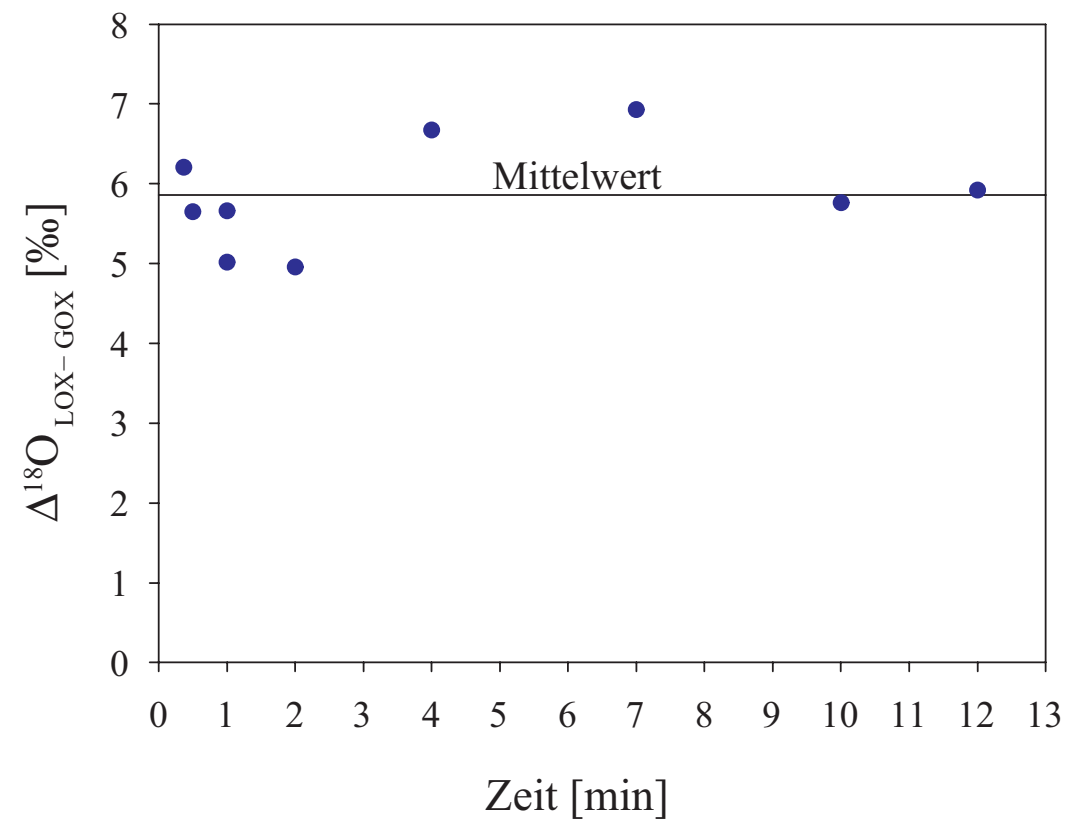

Abbildung 49: Aufgetragen ist der $\Delta^{18} \mathrm{O}_{\text {LOX-GOX }}$ gegen unterschiedliche Equilibrierungszeiten $\left(\Delta^{18} \mathrm{O}_{\text {LOX-GOX }}=\delta^{18} \mathrm{O}_{\text {LOX }}-\delta^{18} \mathrm{O}_{\text {GOX }}\right)$. 


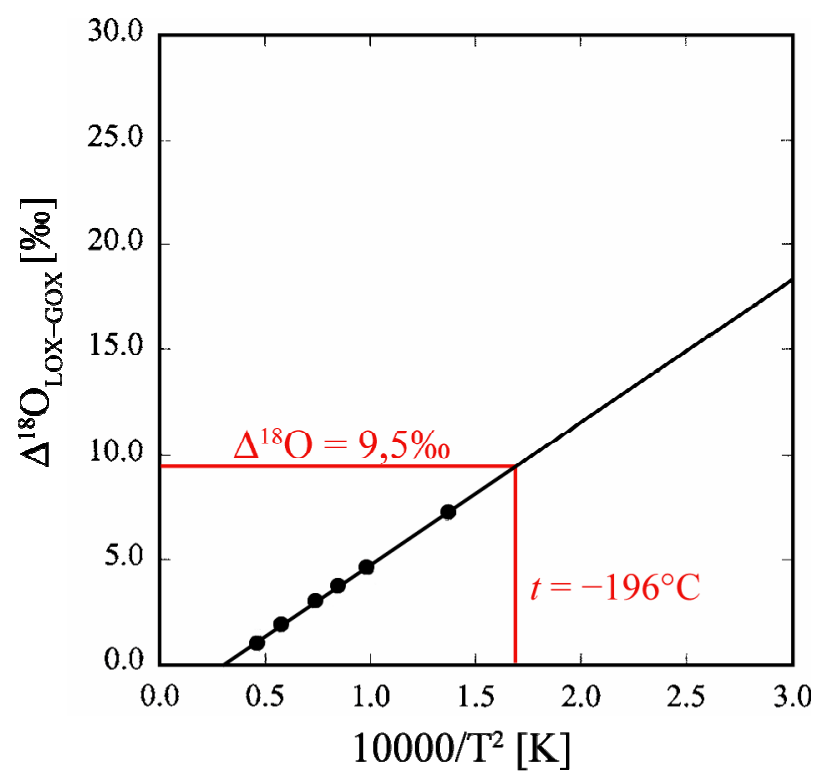

Abbildung 50: Aufgetragen ist der $\Delta^{18} \mathrm{O}_{\text {LOX-GOX }}$ gegen die Temperatur (verändert nach CHIALVOA and HORITA 2006).

Bei einer Temperatur von $-180^{\circ} \mathrm{C}$ liegt die Gleichgewichtsfraktionierung zwischen GOX und LOX nach CHIALVOA and HORITA (2006) bei 5,5\%.

\subsubsection{Isotopie Prozess- $\mathrm{O}_{2}$}

In Abbildung 51 sind die Ergebnisse aller Isotopenmessungen der aktuellen Untersuchung dargestellt. Über einen Zeitraum von drei Monaten (August, September und Oktober 2006) ist keine Variation im $\delta^{18} \mathrm{O}$ des Blassauerstoffs festzustellen. Diese Proben haben mit einem Mittelwert von $+24,3 \%$ die gleiche Isotopie wie der Prozess- $\mathrm{O}_{2}$ von 1998 aus dem Stahlwerk in Dortmund-Hörde (PACK 2000). Der beprobte GOX hatte einen $\delta^{18} \mathrm{O}$ von $+24,0 \pm 0,1 \%$, der LOX einen $\delta^{18} \mathrm{O}$ von $+24,7 \pm 0,1 \%$. Damit gab es zwischen dem beprobten Prozess- $\mathrm{O}_{2}$ im Stahlwerk B und den genommenen Gasproben auf dem Werksgelände bei Linde keine signifikanten Unterschiede in der Isotopie.

In Teil II wurde keine isotopische Variation des Prozess- $\mathrm{O}_{2}$ festgestellt. Damit war für diesen Zeitraum keine Korrelation zwischen Clogging und Prozess $-\mathrm{O}_{2}$ möglich. Prozess $-\mathrm{O}_{2}$ hat ein $\delta^{18} 0$ von $+24,3 \%$ und ist somit ähnlich wie Luft $(+23,5 \% 0)$. 


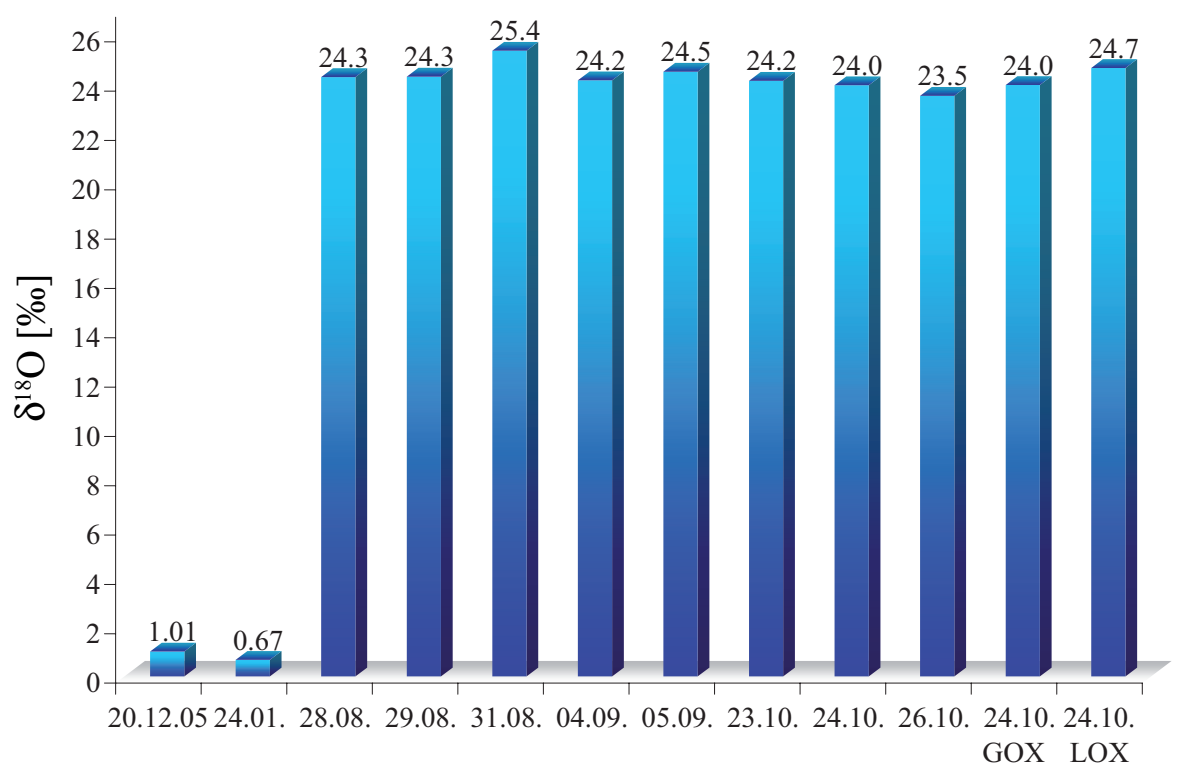

Abbildung 51: $\delta^{18} \mathrm{O}$ des Prozess- $\mathrm{O}_{2}$ von Teil I und II inkl. der Proben von GOX und LOX (alle Proben außer GOX und LOX wurden im Stahlwerk B genommen).

\subsubsection{Rückstandsisolate}

Von drei Lollyproben $(23.10 .06,24.10 .06,26.10 .06)$, die jeweils am Ende des Frischens vor Zugabe des Desoxidationsmittels aus dem Konverter gezogen wurden, wurden Rückstandsisolate angefertigt. Dabei wird ein lollyähnliches Probengefäß, das im Überschuss Aluminium enthält, in die Schmelze getaucht. Der in das Gefäß eindringende Stahl wird dabei durch das Aluminium beruhigt. Mit Hilfe potentiostatischer Isolierung wird das gebildete $\mathrm{Al}_{2} \mathrm{O}_{3}$ aus dem Stahl extrahiert. Ziel war es, die Isotopie des gelösten Sauerstoffs im Stahl zu bestimmen und damit auf die Isotopie von Desoxidationsprodukten in der Pfanne schließen zu können. Dieser Schluss ist deswegen zulässig, da in der Pfanne ein annähernd quantitativer Umsatz des O erfolgt und damit Reservoireffekte ausgeschlossen werden können. Nach DEKKERS (2002) liegt der Gesamtsauerstoffgehalt nach dem Desoxidieren bei 30 ppm. Bei angenommenem 1000 ppm gelöstem O im Stahl nach dem Frischen werden damit 97\% des Sauerstoffs beim Beruhigen umgesetzt.

Lollyproben können aus dem Konverter weder inertisiert noch unter Vakuum gezogen werden. Daher enthält das Probengefäß Luft-O 2 , der mit der flüssigen Stahlschmelze in Kontakt kommt. In Tabelle 11 ist der Anteil von Luft- ${ }_{2}$ bei einer Lollyprobe aus dem Konverter dargestellt. Die Isotopie der Rückstandsisolate ist zu ca. 6,7\% durch die Isotopie des Luft- $\mathrm{O}_{2}$ $\left(\delta^{18} \mathrm{O}=+23,5 \%\right)$ geprägt. Die gemessenen $\delta^{18} \mathrm{O}-$ Werte der Rückstandsisolate sind deshalb ca. 0,1\%o niedriger als die um den Luftanteil korrigierten Werte. Da dieser Anteil vernachlässigbar klein ist, ist die Isotopie der Rückstandsisolate die des im Stahl gelösten Sauerstoffs. 
Tabelle 11: Einfluss von Luft- $\mathrm{O}_{2}$ am Gesamt-O bei nicht inertisiert gezogenen Lollyproben aus dem Konverter.

\begin{tabular}{|cccccccc|}
\hline $\begin{array}{c}\text { Proben- } \\
\text { gefäßgröße } \\
{[\mathbf{l}]}\end{array}$ & $\begin{array}{c}\mathbf{n}_{\mathbf{O} 2} \text { in Luft } \\
{[\mathbf{m o l}]}\end{array}$ & $\begin{array}{c}\mathbf{m}_{\mathbf{O}} \text { im Stahl } \\
{[\mathbf{g}]}\end{array}$ & $\begin{array}{c}\mathbf{n}_{\mathbf{O}} \text { im Stahl } \\
{[\mathbf{m o l}]}\end{array}$ & $\begin{array}{c}\mathbf{n}_{\mathbf{O} 2} \text { aus Stahl } \\
{[\mathbf{m o l}]}\end{array}$ & $\begin{array}{c}\mathbf{n}_{\mathbf{O} 2} \text { gesamt } \\
{[\mathbf{m o l}]}\end{array}$ & $\begin{array}{c}\text { Anteil } \mathbf{O}_{2} \text { aus } \\
\text { Stahl [\%] }\end{array}$ & $\begin{array}{c}\text { Anteil } \mathbf{O}_{2} \text { aus } \\
\text { Luft [\%] }\end{array}$ \\
\hline \hline 0.10 & 0.00 & 0.42 & 0.03 & 0.01 & 0.01 & 93.35 & 6.65 \\
\hline
\end{tabular}

Die Isotopenanalyse von Rückstandsisolaten ist aufgrund der geringen Partikelgröße der Isolate extrem schwierig. Bei der Voruntersuchung war deswegen eine Analyse von Rückstandsisolaten nicht möglich. Daraufhin wurde eine Methode entwickelt, bei der die Proben vor der Fluorinierung im Vakuum angeschmolzen werden, wodurch eine Analyse erst ermöglicht wird. Dennoch lassen sich die Rückstandsisolate nur schwer aufschließen und erfordern überdies eine erfahrene Hand am Laser. Die Tonerde koppelt schlecht in die infrarote Strahlung des $\mathrm{CO}_{2}$-Lasers ein und reagiert bei Energiezugabe sehr spontan und unkontrolliert.

In Abbildung 52 sind die Ergebnisse der Isotopenmessung der Rückstandsisolate relativ zum Frisch- $\mathrm{O}_{2}$ dargestellt.

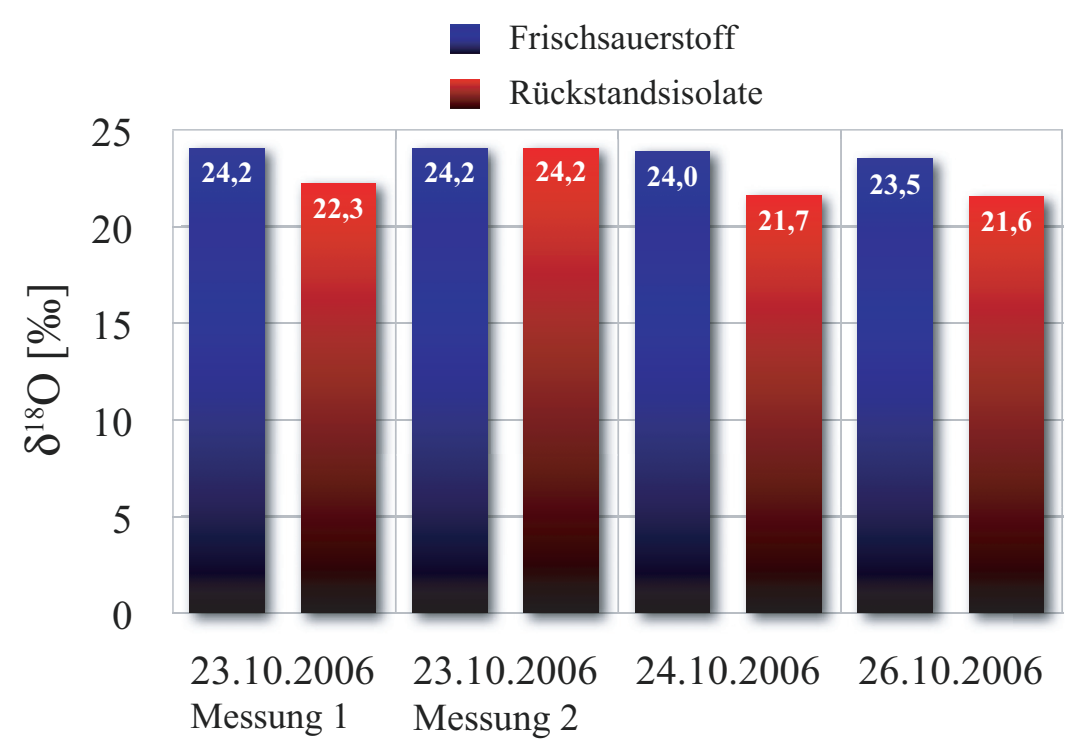

Abbildung 52: Isotopie von Frisch- $\mathrm{O}_{2}$ und Rückstandsisolaten aus dem Konverter an drei Tagen.

Die $\delta^{18} \mathrm{O}$-Werte der Rückstandsisolate liegen mit $+21,6 \%$ bis $+24,2 \%$ im Mittel um $2 \%$ niedriger als die des Prozess- $\mathrm{O}_{2}$. Nur die Messung 2 vom 23.10.2006 hat die gleiche Isotopie wie der Prozess- $\mathrm{O}_{2}$. Diese Probe war die Einzige, bei der ausreichend Probengas zur Verfügung stand, um den Fehler der Messung im Bereich von $\pm 1 \%$ zu halten. Bei den anderen Messungen war nicht ausreichend Probengas vorhanden, so dass ein größerer Fehler von $+3 \%$ o und $-1 \%$ anzusetzen ist.

Der große Fehler bei diesen Proben zu höheren $\delta^{18} \mathrm{O}$-Werten hin liegt an folgenden Zusammenhängen: Je geringer der Probenumsatz bei thermischen Reaktionen ist, das heißt hier, je 
weniger Probengas frei wird, desto größer ist der mögliche Anteil kinetischer Fraktionierung. Bei Aufschluss der Tonerde werden durch kinetische Fraktionierung die schweren Isotope/Moleküle tendenziell im Reaktionsprodukt $\left(\mathrm{O}_{2}\right)$ abgereichert. Dies liegt an der geringeren Mobilität schwerer Isotope/Moleküle, die niedrigere thermische Geschwindigkeiten und Diffusivitäten besitzen (siehe auch Kapitel 2.3.4). Die gegenüber dem Prozess- $\mathrm{O}_{2}$ um $2 \%$ niedrigeren $\delta^{18} \mathrm{O}$-Werte der Rückstandsisolate sind damit möglicherweise präparationsbedingt.

Der gelöste Sauerstoff im Stahl nach dem Frischen in der Pfanne hat die Isotopie des Blassauerstoffs. Die Rückstandsisolate geben Aufschluss über dessen Isotopie.

\subsubsection{Schlacken und FF-Material}

In Abbildung 53 ist die Lage der beprobten Tundishschlacken im Dreistoffsystem CaO$\mathrm{Al}_{2} \mathrm{O}_{3}-\mathrm{SiO}_{2}$ dargestellt. Die Schlacken liegen in den Primärausscheidungsfeldern von Gehlenit $\left(\mathrm{Ca}_{2} \mathrm{Al}_{2} \mathrm{SiO}_{7}\right)$ und Anorthit $\left(\mathrm{CaAl}_{2} \mathrm{Si}_{2} \mathrm{O}_{8}\right)$ (flüssig). Zwei Proben liegen im Stabilitätsfeld des Mullits und weisen die niedrigsten $\delta^{18} \mathrm{O}-$ Werte auf.

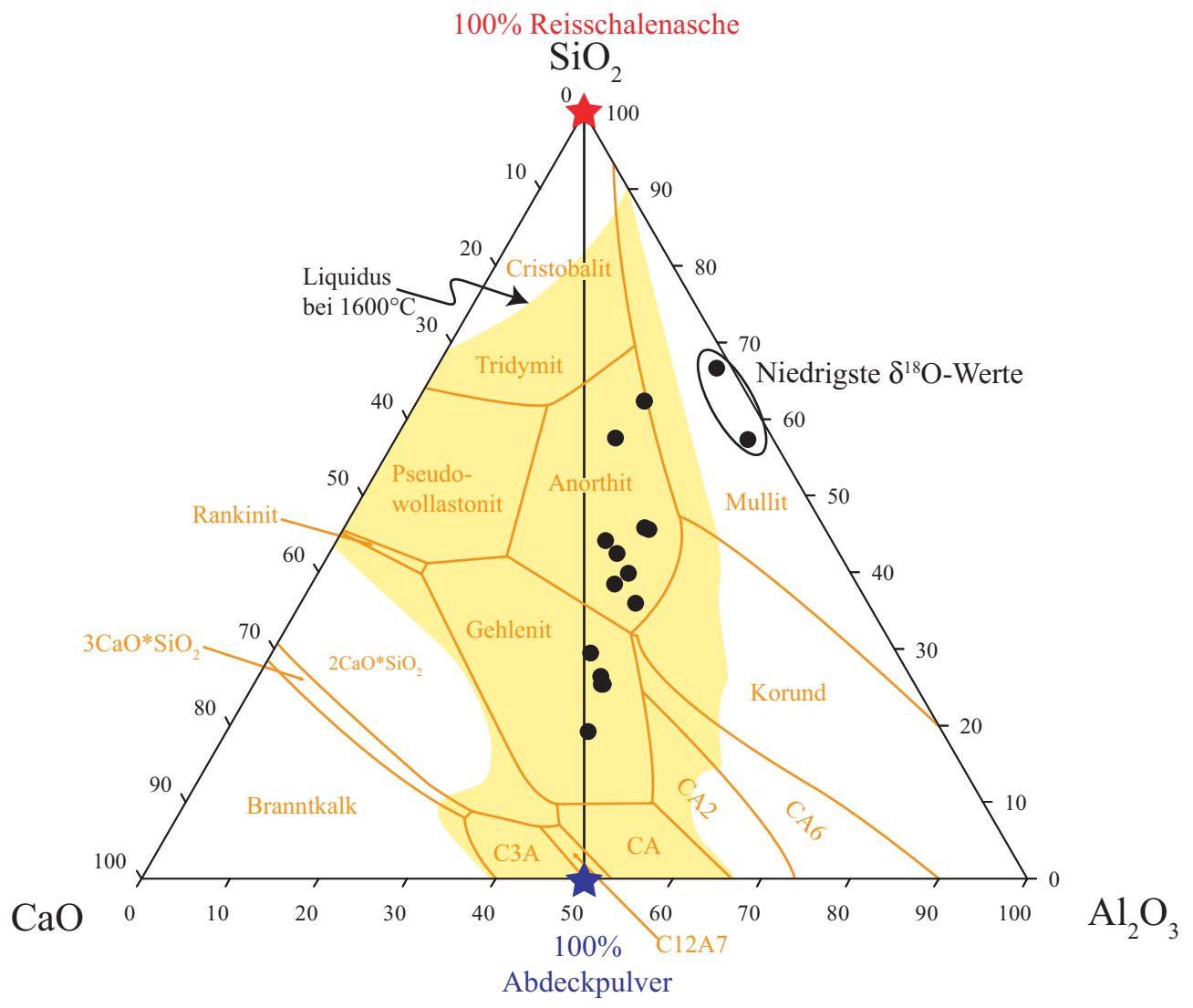

Abbildung 53: Dargestellt sind Tundishschlackenanalyen als Ergebnis der chemischen Analyse im System $\mathrm{CaO}-\mathrm{Al}_{2} \mathrm{O}_{3}-\mathrm{SiO}_{2}$. Bis auf zwei Proben liegen alle im Liquidusfeld $\left(1600^{\circ} \mathrm{C}\right)$.

In Abbildung 54 wurde die Tundishschlacke als Dreikomponentenmodell bestehend aus Tonerde, Reisschalenasche und Abdeckpulver angenommen. Unter Tonerde sind hier Tonerdeein- 
schlüsse zu verstehen, die aus dem Stahl in die Schlacke aufgestiegen sind und in der Schlacke gelöst wurden. Die Kontamination der Tundishschlacke durch Tonerde liegt im Mittel bei 10\% ihrer Masse. Allein die Proben vom 29.08.06 sind mit 30 und 37 Gew.-\% extrem reich an Tonerde, die aus dem Stahl in die Tundishschlacke aufgestiegen ist. Dies sind die Proben, die auch die niedrigsten $\delta^{18} \mathrm{O}-$ Werte aufweisen.

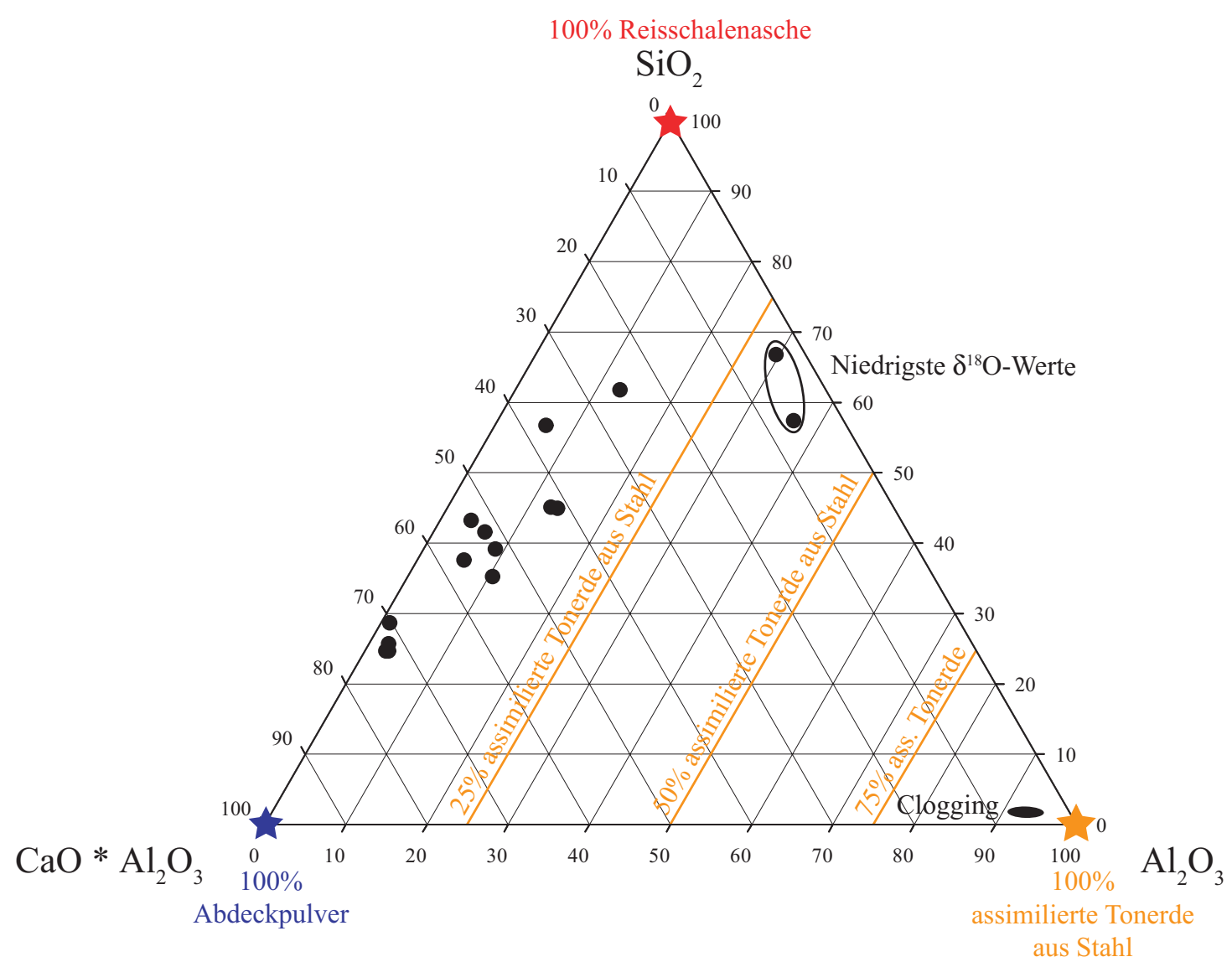

Abbildung 54: Dreikomponentenmodell von Tundishschlacke bestehend aus assimilierter Tonerde aus dem Stahl, Reisschalenasche und Abdeckpulver.

Durch Aufstellen eines Vierkomponentenmodells konnte zusätzlich der Anteil von VTRMasse aus dem Tundish an der Tundishschlacke bestimmt werden. Dazu wurde pro Schlackenanalyse ein lineares Gleichungssystem mit vier Gleichungen und vier Unbekannten mit Hilfe des Gaußverfahrens gelöst (siehe Abbildung 55). Der Lösungsvektor diente abgesehen von der jeweiligen Anteilsangabe der Komponenten zur Berechnung der Gesamtisotopie der Tundishschlacke. In Abbildung 56 sind die Ergebnisse der Berechnungen dargestellt. Die berechneten $\delta^{18} \mathrm{O}$-Werte beschreiben einen ähnlichen Verlauf wie die gemessenen. Damit lässt sich die Konsistenz des Modells überprüfen. Die Probe vom 29.08.06 fällt mit 57 Gew.-\% Reisschalenasche, 34 Gew.- $\%$ assimilierte Tonerde und den niedrigsten $\delta^{18} \mathrm{O}$-Werte auf. Die VTR-Masse des Tundishs liegt an allen Tagen unter 10 Gew.-\%. Die massenmäßig bedeu- 
tendsten Komponenten sind die Schlackenbildner Abdeckpulver und Reisschalenasche mit durchschnittlich 48 Gew.-\% bzw. 37 Gew.-\% (siehe Abbildung 56).

$$
\begin{aligned}
& \chi_{(\mathrm{RA})} * \% \mathrm{CaO}_{(\mathrm{RA})}+\quad \chi_{(\mathrm{AP})} * \% \mathrm{CaO}_{(\mathrm{AP})}+\chi_{\text {(Tо) }} * \% \mathrm{CaO}_{(\mathrm{To})}+\chi_{(\mathrm{VTR})} * \% \mathrm{CaO}_{(\mathrm{VTR})}=\% \mathrm{CaO}_{(\mathrm{TS})} \\
& \chi_{\text {(RA) }} * \% \mathrm{Al}_{2} \mathrm{O}_{3(\mathrm{RA})}+\chi_{\text {(AP) }} * \% \mathrm{Al}_{2} \mathrm{O}_{3(\mathrm{AP})}+\chi_{\text {(To) }} * \% \mathrm{Al}_{2} \mathrm{O}_{3(\mathrm{To})}+\chi_{\text {(VTR })} * \% \mathrm{Al}_{2} \mathrm{O}_{3(\mathrm{VTR})}=\% \mathrm{Al}_{2} \mathrm{O}_{3(\mathrm{TS})} \\
& \chi_{\text {(RA) }} * \% \mathrm{SiO}_{2(\mathrm{RA})}+\chi_{\text {(AP) }} * \% \mathrm{SiO}_{2(\mathrm{AP})}+\chi_{\text {(To) }} * \% \mathrm{SiO}_{2(\mathrm{TO})}+\chi_{\text {(VTR })} * \% \mathrm{SiO}_{2 \text { (VTR })}=\% \mathrm{SiO}_{2(\mathrm{TS})} \\
& \chi_{(\mathrm{RA})} * \% \mathrm{MgO}_{(\mathrm{RA})}+\chi_{(\mathrm{AP})} * \% \mathrm{MgO}_{(\mathrm{AP})}+\chi_{(\mathrm{To})} * \% \mathrm{MgO}_{(\mathrm{To})}+\chi_{(\mathrm{VTR})} * \% \mathrm{MgO}_{(\mathrm{VTR})}=\% \mathrm{MgO}_{(\mathrm{TS})} \\
& \text { RA }=\text { Reisschalenasche } \\
& \text { AP }=\text { Abdeckpulver } \\
& \text { To }=\text { Tonerde, die aus dem Stahl in die Schlacke aufgestiegen ist } \\
& \text { VTR }=\text { VTR-Masse } \\
& \text { TS }=\text { Tundishschlacke }
\end{aligned}
$$

Abbildung 55: Lineares Gleichungssystem mit vier Gleichungen und vier Unbekannten zur Berechnung der Anteile von RA, AP, To und VTR an der Tundishschlacke.

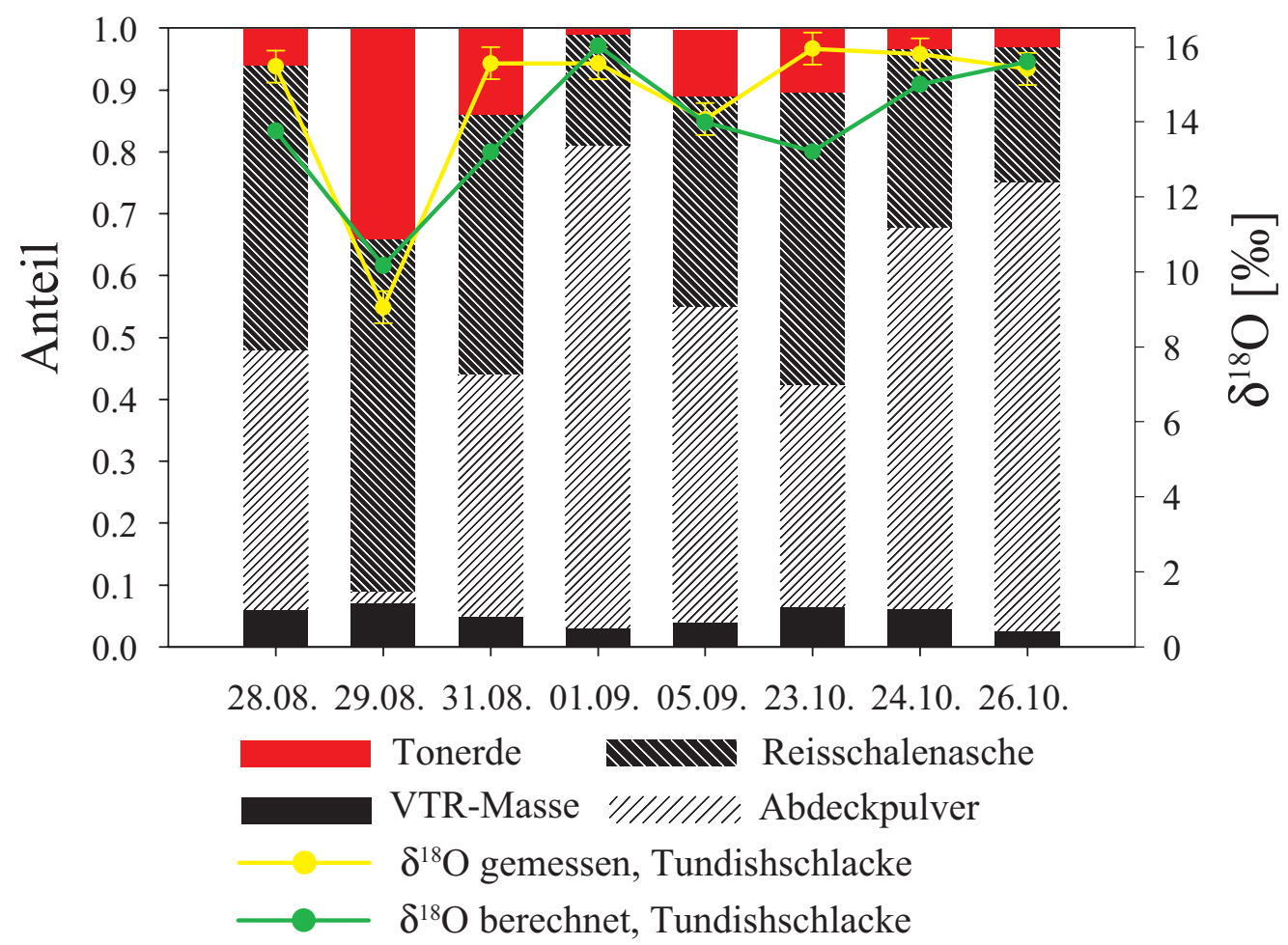

Abbildung 56: Berechnete Zusammensetzung der Tundishschlacke und ihre $\delta^{18} \mathrm{O}-$ Werte (gemessen und berechnet).

Vierkomponentenmodell: Je höher der Gehalt an assimilierter Tonerde aus dem Stahl in der Tundishschlacke ist, desto niedriger ist der $\delta^{18} 0$ der Tundishschlacke. Daraus folgt, dass Tonerdeeinschlüsse im Stahl im Tundish niedrige $\delta^{18} 0$-Werte aufweisen. Bei diesem Modell wird der Einfluss von Chromit auf den $\delta^{18} 0$ der Tundishschlacke nicht berücksichtigt (siehe hierzu Kapitel 4.4).

Mitlaufschlacken aus Pfanne und Verteiler haben je nach Menge einen mehr oder weniger großen Anteil an oxidischen Ablagerungen im Tauchrohr. In Abbildung 57 ist die Isotopenzu- 
sammensetzung der Schlacken relativ zum Clogging dargestellt. Die Tundishschlacke hat etwas höhere $\delta^{18} \mathrm{O}-$ Werte als das Clogging. Der Mittelwert der Tundishschlacke liegt bei $+13,7 \%$. Ohne die zwei sehr leichten Proben vom 29.08.06 liegt er bei +14.5\%o. Die Pfannenund Konverterschlacken sind mit einem Mittelwert von $+17,5 \%$ ozw. $+19,3 \%$ o schwerer als die Tundishschlacke.

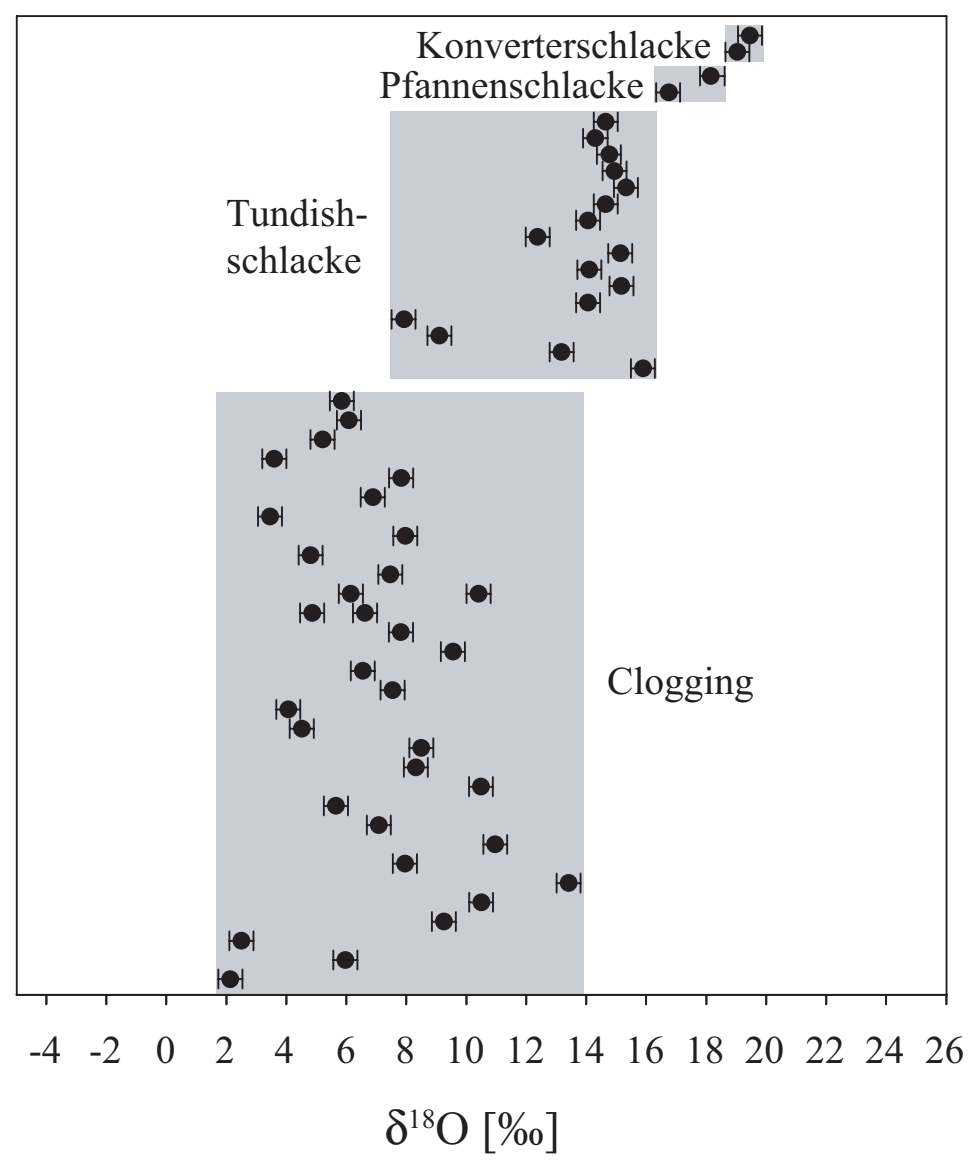

Abbildung 57: Isotopie der Schlacken relativ zum Clogging.

In Abbildung 58 ist die Isotopenzusammensetzung von FF-Materialien und von Prozess- $\mathrm{O}_{2}$ in einem $\delta^{18} \mathrm{O}$-Variationsdiagramm dargestellt. Die Einsatzstoffe der Tundishschlacke, Abdeckpulver $\left(\delta^{18} \mathrm{O}=+17 \%\right)$ und Reisschalenasche aus Dortmund-Hörde $\left(\delta^{18} \mathrm{O}=+20,3 \%\right.$, PACK 2000), sind etwas schwerer als die Tundishschlacke. Erst mit Kontamination der Tundishschlacke durch aufgestiegene Tonerdeeinschlüsse aus dem Stahl wird der $\delta^{18} \mathrm{O}$ der Tundishschlacke zu niedrigeren Werten hin verschoben (siehe Abbildung 56). Nach TOULOUSE et al. (in press) haben aufsteigende Chromite und Spinelle ebenfalls einen Einfluss auf die Isotopie der Tundishschlacke. Die Konverter- und Pfannenschlacke werden isotopisch durch aufgestiegene FeO- bzw. $\mathrm{Al}_{2} \mathrm{O}_{3}$-Partikel geprägt. Die $\delta^{18} \mathrm{O}$-Werte der Pfannensteine liegen zwischen $+7,0$ und $+9,4 \%$, die der VTR-Masse zwischen $+11,5$ und +13,3\%. Da MgO auch unter stark reduzierenden Bedingungen wie beim Gießen nur schwer reduziert werden kann, 
sind Pfannensteine und VTR-Masse wahrscheinlich keine bedeutenden O-Donatoren für Clogging. Zur näheren Beschreibung der Pfannensteine siehe auch Kapitel 4.2.4 und 4.3.3.

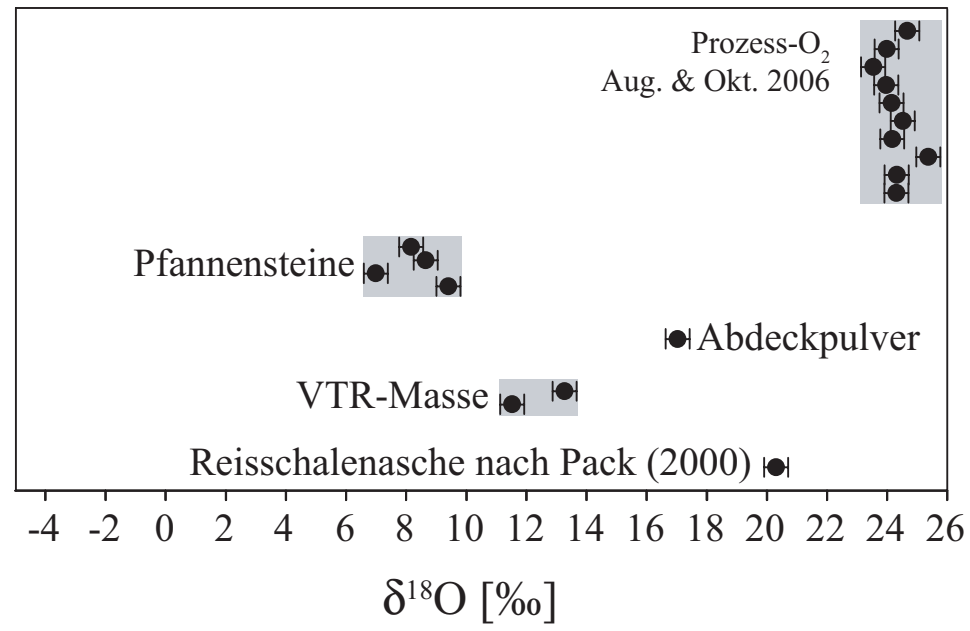

Abbildung 58: Isotopie von FF-Materialien und relativ dazu die Lage von Blassauerstoff aus Teil II.

\subsubsection{Schiebersand}

Der Schiebersand wird nach Öffnen der Schieberplatten mit der Schmelze in den Tundish eingebracht, wo er als dispergierte Phase in der Schmelze aufsteigt. Durch die feine Verteilung des Schiebersandes in der Schmelze im Tundish besitzt er eine große Oberfläche, wodurch er leichter reduziert wird als in der Pfanne und damit ein bedeutender O-Donator für die Bildung von Einschlüssen im Stahl sein kann.

Bei der isotopischen Charakterisierung der Prozessmaterialien fehlt bis zu diesem Zeitpunkt eine leichte O-Quelle, mit der auch Clogging von $\delta^{18} \mathrm{O}=+3,5 \%$ erklärt werden kann. Als eine bedeutende O-Quelle kann ein Teil des Schiebersandes angesehen werden, nämlich die reduzierbare Komponente des Schiebersandes, der Chromit.

Der Schiebersand aller untersuchten Hersteller besteht aus drei Phasen, wobei Chromit mit rd. 70 Gew.- $\%$ die dominierende Phase ist. Die untersuchten Chromite haben mit einem $\delta^{18} \mathrm{O}$ Wert von $-4,1$ bis $-3,0 \%$ im Vergleich zu anderen FF-Materialien eine extrem niedrige Isotopie (siehe Abbildung 59). Der Quarzanteil beträgt rd. 30 Gew.-\%, Enstatit kommt nur in sehr geringen Anteilen unter 3 Gew.-\% vor.

Schiebersand besteht zu 70 Gew.-\% aus Chromit und zu 30 Gew.-\% aus Quarz. Chromit ist isotopisch mit einem Mittelwert von $\delta^{18} 0=-3,5 \%$ das einzige extrem leichte Prozessmaterial. 


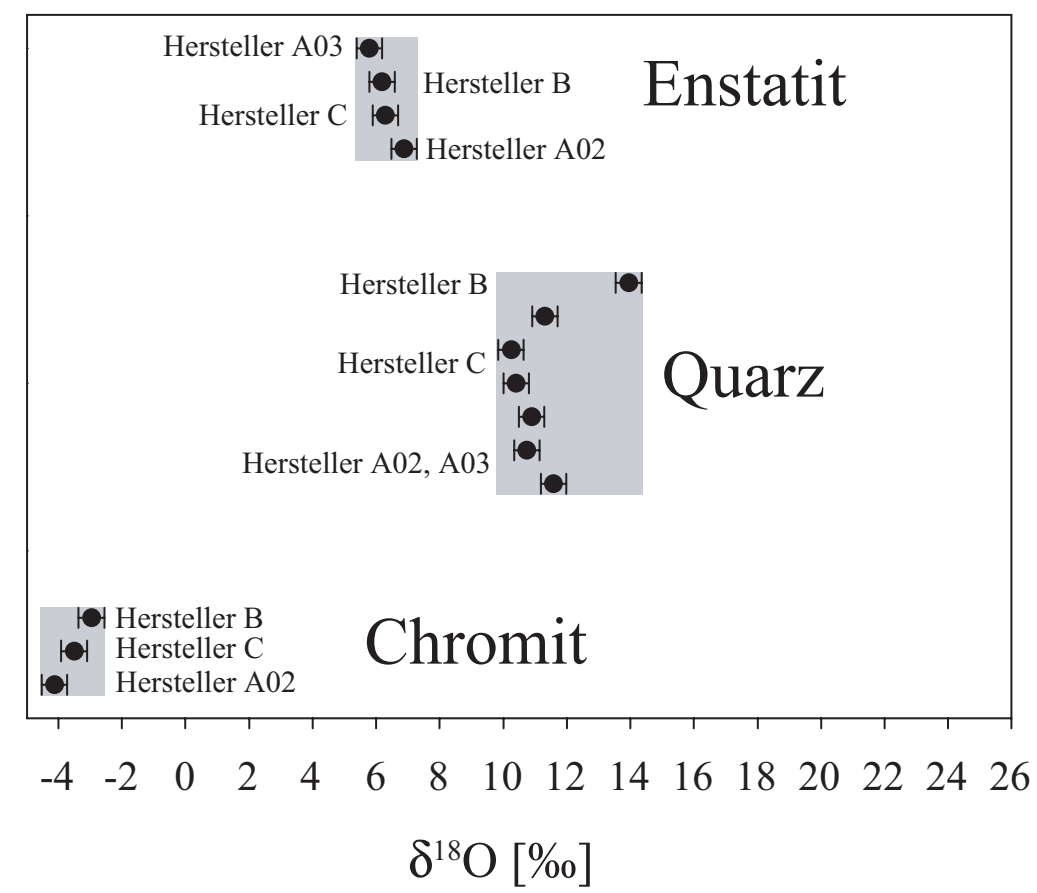

Abbildung 59: Isotopie der verschiedenen Phasen von Schiebersanden unterschiedlicher Hersteller.

In Abbildung 60 ist ein Enstatitkorn mit Verwachsungen von Chromit und Klinopyroxen des Schiebersandes A03 dargestellt. Der Enstatit der Schiebersande kommt als geringe Verunreinigung im Chromitrohstoff vor.

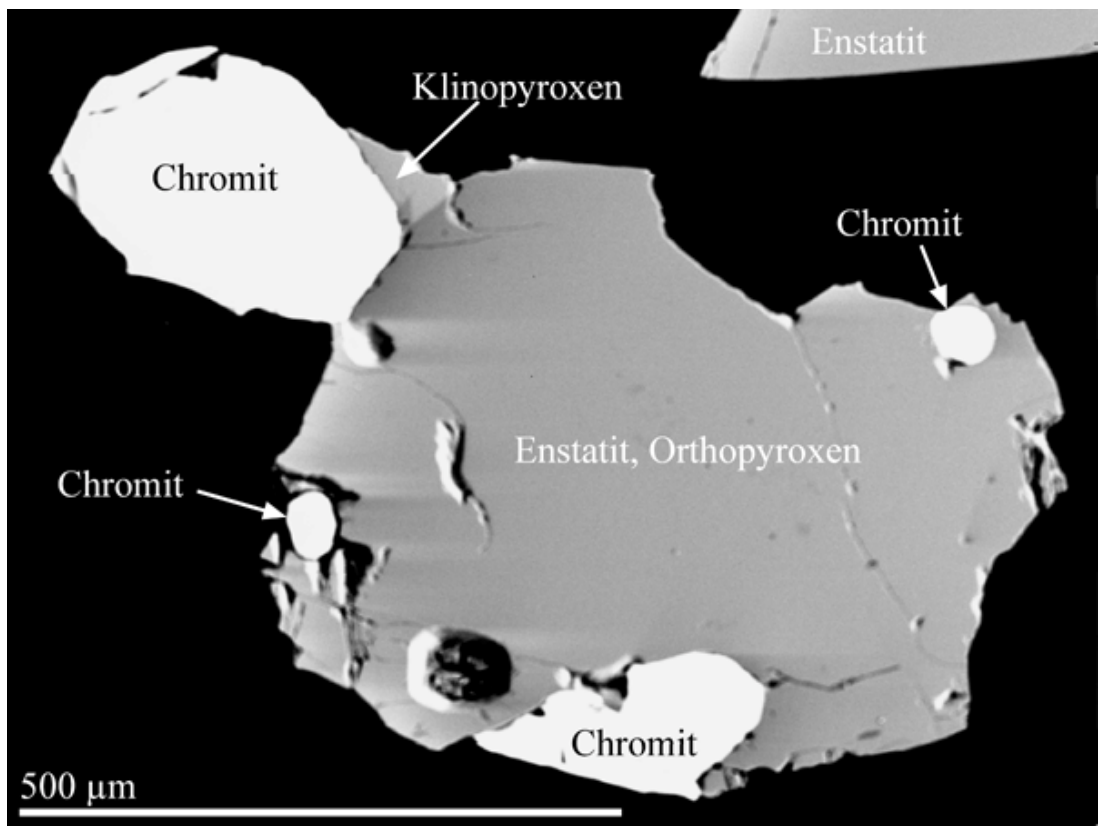

Abbildung 60: Rückstreuelektronenbild eines Enstatitkorns mit Verwachsungen von Chromit und Klinopyroxen (Schiebersand Hersteller A03).

Zur näheren Beschreibung des Schiebersandes siehe Kapitel 4.2.3 und 4.3.2. 


\subsection{Diskussion}

\section{Prozess- $\mathrm{O}_{2}$}

Die niedrige Isotopie von Prozess- $\mathrm{O}_{2}$ vom Dezember 2005/Januar $2006\left(\delta^{18} 0=+1 \%\right.$ ) kann nicht mit der Gleichgewichtsfraktionierung zwischen GOX und LOX erklärt werden, diese beträgt nur $\sim 5,5 \%$.

Für die niedrigen $\delta^{18} \mathrm{O}$-Werte des Blassauerstoffs vom Dezember 2005/Januar 2006 wurde keine hinreichende Erklärung gefunden. Der mittlere $\delta^{18} \mathrm{O}$ von Prozess- $\mathrm{O}_{2}$ zwischen August und Oktober 2006 hat mit $+24,3 \%$ die gleiche Isotopie wie der Prozess- $\mathrm{O}_{2}$ aus Dortmund von 1998. Bei einer Gleichgewichtsfraktionierung zwischen GOX und LOX von rd. 5,5\% bedeutet dies, dass LOX in den Tanks bei Linde einen $\delta^{18} \mathrm{O}$ von rd. $+29,5 \%$ hat, wenn GOX einen $\delta^{18} \mathrm{O}$ von $+24 \%$ aufweist.

Eine mögliche Erklärung ist kinetische Fraktionierung zwischen GOX und LOX in diesem Zeitraum. Im November 2005 wurde ein Luftzerleger bei Linde zwecks Wartung außer Betrieb genommen, Mitte Dezember wurde dieser dann wieder betrieben. Falls in diesem Zeitraum GOX und LOX nicht im Gleichgewicht miteinander standen, könnte kinetische Fraktionierung wie bei den Hochtemperaturexperimenten den Unterschied in der Isotopie erklären. Bei kinetischer Fraktionierung zwischen GOX und LOX würden im GOX vermehrt die leichten $\left({ }^{16} \mathrm{O}\right.$-haltigen) Moleküle angereichert und im LOX die schwereren ( $\left({ }^{18} \mathrm{O}\right.$-haltigen) Moleküle. In Tabelle 6 sind Geschwindigkeitsquotienten und Fraktionierungen zwischen Gasspezies dargestellt. Die Geschwindigkeit von ${ }^{16} \mathrm{O}^{16} \mathrm{O}$ ist danach um 3,1\% höher als die von ${ }^{16} \mathrm{O}^{18} \mathrm{O}$, damit ergibt sich eine Fraktionierung zwischen den Spezies von maximal 30,3\%o. Dies zeigt, dass durch kinetische Fraktionierung erheblich leichterer GOX gegenüber LOX zu erwarten ist.

\section{Rückstandsisolate}

Wie in Kapitel 3.4.3 gezeigt, gibt es beim Frischen keine Fraktionierung zwischen Blassauerstoff und im Stahl gelöstem Sauerstoff. Damit gilt: Desoxidationsprodukte haben die Isotopie des gelösten Sauerstoffs im Stahl nach dem Frischen, da beim Beruhigen der Sauerstoff annähernd quantitativ umgesetzt wird (siehe Kapitel 2.4.3.) Auch ist die Isotopie der Desoxidationsprodukte gleich der des Blassauerstoffs. Das bedeutet:

$\delta^{18} \mathrm{O}_{\text {Blassauerstoff }}=\delta^{18} \mathrm{O}_{\text {gelöster } 0 \text { nach Frischen }}=\delta^{18} \mathrm{O}_{\text {Desoxidationsprodukte }} \approx+24 \%$ 


\section{Schiebersand}

Die niedrige Isotopie des Chromits ist ungewöhnlich; für magmatische Oxide sind $\delta^{18} \mathrm{O}$ Werte zwischen +2 und $+4 \%$ o typisch. Bei TAYLOR (1968) findet sich ein $\delta^{18} \mathrm{O}-\mathrm{Wert}$ für Chromit aus Dunit von $+4,5 \%$. Nach dem Richardson-Jeffes-Diagramm (TURKDOGAN 1996) wird Chromit leichter reduziert als Quarz. Zudem weist Quarz $\left(\rho=2,65 \mathrm{~g} / \mathrm{cm}^{3}\right)$ gegenüber Chromit ( $\rho=4,8 \mathrm{~g} / \mathrm{cm}^{3}$ ) einen größeren Dichteunterschied zu flüssigen Stahlschmelzen auf, so dass auf Quarz eine größere Auftriebskraft wirkt und dieser schneller aus der Stahlschmelze im Tundish in die Schlacke aufsteigt. Daher ist der Quarz als O-Donator wahrscheinlich nur von untergeordneter Bedeutung.

Chromit aus dem Schiebersand ist mit einem Mittelwert von $\delta^{18} 0=-3,5 \%$ die einzige extrem leichte 0-Quelle beim Stranggussverfahren. Alle untersuchten Schiebersande verwenden den gleichen Chromitrohstoff.

\section{Clogging}

In Abbildung 37 ist dargestellt, dass Einschlüsse, die zuletzt aus dem Stahl ausgeschieden werden, die isotopisch leichtesten sind. Zudem ist die Isotopie des Cloggings eine Funktion des $\mathrm{Al}_{2} \mathrm{O}_{3}$-Gehalts (siehe Abbildung 41).

Je länger Stahl vergossen wird, desto niedriger sind die $\delta^{18} 0$-Werte und desto höher sind die $\mathrm{Al}_{2} \mathrm{O}_{3}-\mathrm{Gehalte}$ oxidischer Präzipitate. Bisher wird dies nicht für einzelne Schmelzen aufgelöst, sondern nur sequenzweise ${ }^{10}$ beobachtet.

Clogging, das zu $>94$ Gew.- $\%$ aus $\mathrm{Al}_{2} \mathrm{O}_{3}$ besteht, hat mit $\delta^{18} \mathrm{O} \approx+5 \%$ die niedrigste Isotopie (siehe Abbildung 41). Solche annähernd monomineralischen Ablagerungen sind zum überwiegenden Teil Produkt der Ausscheidung von reinen Tonerdeeinschlüssen aus dem Stahl. (siehe auch Kapitel 4.2.1).

\section{$\delta^{18} \mathrm{O}_{\text {sehr Al203-reiches Clogging }} \approx+5 \%$}

Wie in Kapitel 3.4.2.4 gezeigt, hat Prozess- $\mathrm{O}_{2}$ als eine mögliche O-Quelle für Clogging einen $\delta^{18} \mathrm{O}$ von ungefähr $+24 \%$. Damit hat Blassauerstoff einen ähnlichen $\delta^{18} \mathrm{O}-$ Wert wie Luft- $\mathrm{O}_{2}$ $\left(\delta^{18} \mathrm{O}=+23,5 \%\right)$. Wenn man annimmt, Desoxidationsprodukte seien die alleinige Ursache für Clogging und dass die O-Isotope nicht fraktioniert werden, müsste Clogging einen $\delta^{18} \mathrm{O}$ von $+24 \%$ aufweisen. Dies wurde weder in dieser Studie noch in denen von PACK (2000) und

\footnotetext{
${ }^{10}$ Eine Gießsequenz besteht aus mehreren Schmelzen. Durch die Tauchrohre, aus denen Clogging entnommen wurde, sind zwischen zwei und sieben Schmelzen vergossen worden.
} 
PACK et al. (2005) beobachtet. Unter dieser Annahme kann die Isotopie von Clogging allein durch Tonerdeeinschlüsse aus der Pfanne nicht erklärt werden. Es wird ein leichter O-Donator benötigt, um $\delta^{18} \mathrm{O}-$ Werte von $+5 \%$ zu erreichen. Daher wird Chromit als einziges isotopisch extrem leichtes Prozessmaterial neben Desoxidationsprodukten aus der Pfanne der folgenden Modellvorstellung zugrunde gelegt.

Bei der Stahlherstellung wird der Al-Gehalt nach der Desoxidation in der Regel auf 0,03 Gew.-\% eingestellt. Bei angenommenen 265 Tonnen Stahl pro Pfanne könnten damit maximal $150 \mathrm{~kg} \mathrm{Al}_{2} \mathrm{O}_{3}$ gebildet werden, wenn ausreichend $\mathrm{O}$ zur Verfügung stünde (siehe Tabelle 12).

Tabelle 12: Menge an Tonerde, die pro Pfanne maximal aus Al gebildet werden könnte, wenn ausreichend Sauerstoff zur Verfügung stünde.

\begin{tabular}{|c|c|c|c|c|c|}
\hline \hline $\mathrm{Al}_{\text {Stahl }}[\%]$ & Pfanne $[\mathrm{t}]$ & $\mathrm{Al}_{\text {Stahl }}[\mathrm{g}]$ & $\mathrm{Al}_{\text {Stahl }}[\mathrm{mol}]$ & $\mathrm{Al}_{2} \mathrm{O}_{3 \text { Stahl }}[\mathrm{mol}]$ & $\mathrm{Al}_{2} \mathrm{O}_{3 \text { Stahl }}[\mathrm{kg}]$ \\
\hline \hline 0.03 & 265 & 79500 & 2947 & 1473 & 150 \\
\hline
\end{tabular}

Nach dem Beruhigen der Schmelze liegt der Gesamtsauerstoffgehalt für ULC-Güten bei rd. 27 ppm (DeKKERS et al. 2004a, DEKKERS et al. 2004b). Damit können maximal $15 \mathrm{~kg} \mathrm{Al}_{2} \mathrm{O}_{3}$ pro Pfanne aus Frisch- $\mathrm{O}_{2}$ gebildet werden (siehe Tabelle 13).

Tabelle 13: Menge an Tonerde, die pro Pfanne bei gegebenem Sauerstoffgehalt maximal gebildet werden kann.

\begin{tabular}{|c|c|c|c|c|c|}
\hline \hline $\mathrm{O}_{\text {Stahl nach Desox. }}[\%]$ & Pfanne $[\mathrm{t}]$ & $\mathrm{O}_{\text {Stahl }}[\mathrm{g}]$ & $\mathrm{O}_{\text {Stahl }}[\mathrm{mol}]$ & $\mathrm{Al}_{2} \mathrm{O}_{3 \text { Stahl }}[\mathrm{mol}]$ & $\mathrm{Al}_{2} \mathrm{O}_{3 \text { Stahl }}[\mathrm{kg}]$ \\
\hline \hline 0.00270 & 265 & 7155 & 447 & 149 & 15 \\
\hline
\end{tabular}

Somit ist genügend $\mathrm{Al}$ in der Schmelze vorhanden und $\mathrm{O}$ ist der limitierende Faktor bei der Bildung von Tonerdeeinschlüssen im Stahl.

Pro Pfanne werden etwa $80 \mathrm{~kg}$ Schiebersand eingesetzt. Bei einem Anteil von 70 Gew.-\% Chromit im Schiebersand ergeben sich $56 \mathrm{~kg}$ Chromit pro Pfanne. Damit lassen sich pro Pfanne $36 \mathrm{~kg} \mathrm{Al}_{2} \mathrm{O}_{3}$ aus Chromit bilden (siehe Tabelle 14).

Tabelle 14: Maximale Menge an Tonerde, die pro Pfanne aus Chromit gebildet werden kann.

\begin{tabular}{|c|c|c|c|c|c|}
\hline \hline $\mathrm{O}_{\text {Chromit }}[\%]$ & Chromit $[\mathrm{kg}]$ & $\mathrm{O}_{\text {Chromit }}[\mathrm{g}]$ & $\mathrm{O}_{\text {Chromit }}[\mathrm{mol}]$ & $\mathrm{Al}_{2} \mathrm{O}_{3 \text { Stahl }}[\mathrm{mol}]$ & $\mathrm{Al}_{2} \mathrm{O}_{3 \text { Stahl }}[\mathrm{kg}]$ \\
\hline \hline 30 & 56 & 16800 & 1050 & 350 & 36 \\
\hline
\end{tabular}

Somit können durch Chromit und Prozess- $\mathrm{O}_{2} 51 \mathrm{~kg} \mathrm{Al}{ }_{2} \mathrm{O}_{3}$ pro Pfanne gebildet werden, von denen angenommen wird, dass sie zusammen einen $\delta^{18} \mathrm{O}$ von Clogging von $\mathrm{rd}$. $+5 \%$ verursachen. Es ergeben sich Anteile von rd. 70\% Chromit und 30\% Prozess- $\mathrm{O}_{2}$. 


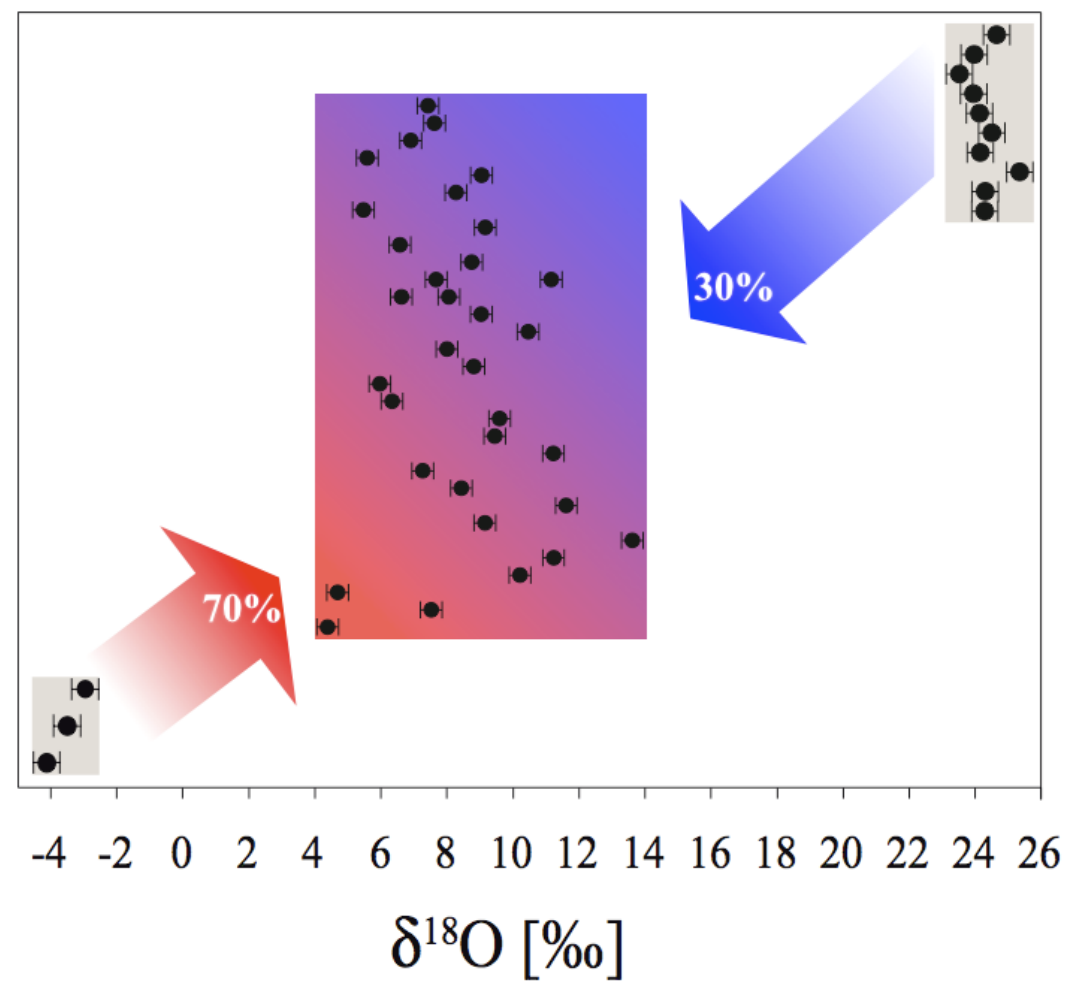

Abbildung 61: Illustration des Zweikomponentenmodells für die Herkunft von Clogging.

Bei dieser Modellvorstellung wird angenommen, dass weder bei der Reduktion des Chromits noch bei der Bildung von $\mathrm{Al}_{2} \mathrm{O}_{3}$ aus Frisch- $\mathrm{O}_{2}$ Fraktionierung auftritt. Falls die Tonerde, die aus dem frei werdenden Sauerstoff bei der Chromitreduktion hervorgegangen ist, kinetisch fraktioniert wäre, müsste der Anteil von Prozess- $\mathrm{O}_{2}$ am Clogging größer sein, um die beobachteten $\delta^{18} \mathrm{O}$-Werte der oxidischen Ablagerungen zu erreichen. $\delta^{18} \mathrm{O}$-Werte von Clogging bis $\mathrm{zu}+1 \%$ können zum einen durch einen Anteil von kinetischer Fraktionierung des Blassauerstoffs erklärt werden oder zum anderen durch höhere Anteile von Chromit am Clogging. Durch kinetische Fraktionierung zwischen ${ }^{18} \mathrm{O}$ und ${ }^{16} \mathrm{O}$ von Prozess- $\mathrm{O}_{2}$ könnten $\delta^{18} \mathrm{O}$-Werte von $+5 \%$ erreicht werden. Um Clogging von bis zu $+14 \%$ erklären zu können, muss der Anteil anderer Verursacher, wie z. B. der Spritzmasse aus dem Tundish mit einem $\delta^{18} \mathrm{O}=+11 \%$ bis $+13 \%$, im Clogging quantifiziert werden.

Wie in Kapitel 2.3.4 gezeigt, spielen Gleichgewichts- und Rayleighfraktionierung wahrscheinlich keine bedeutende Rolle beim Desoxidieren. Erstere liegt bei $\Delta^{18} \mathrm{O}_{\mathrm{O} 2 \text {-Tonerde }}=$ $+0,4 \%$. Nach PACK et al. (2005) ist der Fraktionierungsfaktor $\alpha_{\mathrm{O} 2 \text {-Tonerde }}>1$. Damit müsste die sich bei Rayleighfraktionierung bildende Tonerde $\delta^{18} \mathrm{O}$-Werte größer als $+24 \%$ aufwei- 
sen. Solch hohe $\delta^{18} \mathrm{O}-$ Werte wurden nie beobachtet. Für $\alpha_{\mathrm{O} 2 \text {-Tonerde }}<1$ könnte $\delta^{18} \mathrm{O}=+5 \%$ erreicht werden. Vor dem Hintergrund eines annähernd vollständigen Umsatzes des gelösten Sauerstoffs in der Pfanne erscheint Rayleighfraktionierung jedoch als vernachlässigbar (siehe Kapitel 2.3.4).

\section{Versuch mit offenem Stopfen}

Wie in Kapitel 3.4.1.2 gezeigt, waren die ausgeprägten Anfrierungen von Stahl nach lediglich zwei Schmelzen besonders auffällig bei diesem Versuch. BASU et al. (2004) berichten, dass Ti-legierte ULC-Stähle mit 0,050 Gew.-\% Ti gegenüber Ti-freien Stählen verstärkt zum Zusetzen der Tauchrohre mit Anfrierungen von Stahl neigen. Danach erfolgt ein Einbau von Stahl in $\mathrm{TiO}_{\mathrm{x}}-\mathrm{Al}_{2} \mathrm{O}_{3}$-Einschlusscluster aufgrund ihrer Benetzungscharakteristik. Bei pulverdiffraktometrischen Untersuchungen der Präzipitate vom 26.10.2006 wurden jedoch keine Tihaltigen Phasen identifiziert (siehe Kapitel 4.2.1). Allein bei zwei anderen Proben wurde bis zu 1 Gew.-\% Rutil $\left(\mathrm{TiO}_{2}\right)$ in den Ablagerungen gefunden. EMS-Untersuchungen der Cloggingprobe vom 04.09.06 (Strang 3) ergaben die Substitution von Al durch Ti und Mg in der Kristallstruktur von Korund mit der Mineralformel $\mathrm{Mg}_{0,027} \mathrm{Ti}_{0,028} \mathrm{Al}_{1,938} \mathrm{O}_{3}$. THOMAS and BAI (2001) und RACKERS and THOMAS (1995) beschreiben den Mechanismus von Stahlanfrierungen im Tauchrohr zu Beginn des Gießens bei nicht ausreichender Vorheizung der Tauchrohre oder zu geringer Gießgeschwindigkeit. Da bei dem Versuch lediglich zwei Schmelzen vergossen wurden und es sich dabei um eine Ti-legierte ULC-Güte handelte, erscheinen beide Prozesse auf den ersten Blick als Ursache für die mächtigen Stahlanfrierungen denkbar. Das Tauchrohr von Strang 3 wies an diesem Tag jedoch keine Stahlanfrierungen auf. Daher sind die beschriebenen Prozesse als Ursache für die Anfrierungen und das Clogging unwahrscheinlich bzw. auszuschließen.

Nachfolgend werden drei mögliche Prozesse diskutiert, welche die stark abweichende Isotopie des Cloggings vom Luft- $\mathrm{O}_{2}$ erklären sollen:

1. Bei diesem Versuch sollte Clogging durch den offenen Stopfen provoziert werden. Die Isotopie des Luftsauerstoffes wurde jedoch nicht übernommen. Falls die Versuchsbedingungen wie geplant funktionierten und $\mathrm{Luft}^{-} \mathrm{O}_{2}$ über den Stopfen an den Stahl geleitet würde, wäre kinetische Fraktionierung der Sauerstoffisotope wie bei den Hochtemperaturexperimenten (siehe Kapitel 3.3) eine mögliche Erklärung für die niedrigen $\delta^{18} \mathrm{O}$-Werte des Cloggings. 
2. Eine andere Möglichkeit wäre, dass auch bei offenem Stopfen der Einfluss von Luft-O nicht entscheidend für die Bildung von Clogging ist, weil die Tonerde, die hauptsächlich zum Clogging beiträgt, bereits im Tundish gebildet wird. Nach THOMAS and BAI (2001) und TOuLOusE et al. (in press) sind Einschlüsse, die bereits vor dem Erreichen des Tauchrohres im Stahl vorliegen, die bedeutendste Ursache für Clogging. Das würde bedeuten, dass Aluminium, das erst im Tauchrohr durch eingesogene Luft oxidiert wird, nicht oder nur untergeordnet im Tauchrohr abgelagert und zum überwiegenden Teil in die Kokille transportiert wird.

3. Eine weitere Möglichkeit wäre, dass der ferrostatische Druck beim Gießen so hoch war, dass Stahl im Stopfen stand und daher kein Luft- $\mathrm{O}_{2}$ an den Stahl kam. Bei dieser Überlegung wird ebenfalls davon ausgegangen, dass die Herkunft von Clogging auf die Bildung und spätere Ablagerung von Tonerde aus dem Tundish zurückgeht. Der Bildungsprozess wäre also der gleiche wie bei Punkt 2. Daher müssten die Cloggingmengen im Tauchrohr bei diesem Szenario denen von Punkt 2 entsprechen. Der einzige Unterschied wäre, dass hierbei keine Luft über den Stopfen an den Stahl kommen könnte und damit weniger Tonerde in die Kokille und die Bramme transportiert würde.

Da bei Abstellung der Argonspülung beim Gießen ein Unterdruck am Stopfenende festzustellen ist, erscheint die dritte Möglichkeit unwahrscheinlich. An dieser Stelle sei darauf hingewiesen, dass es bei dem Versuch keine Messung eines etwaigen Unterdrucks gab. Sowohl Punkt 1 als auch Punkt 2 erscheinen möglich, wobei die genaue Ursache für die niedrigen $\delta^{18} \mathrm{O}$-Werte des Cloggings anhand dieses Versuches nicht eindeutig geklärt werden kann. Hilfreich wäre die Messung des Stickstoffgehaltes im Stahl gewesen, diese würde bei hohen Gehalten auf Luftzufuhr deuten. Auch hätte der beim Versuch eingesetzte Stopfen Auskunft darüber geben können, ob Stahl im Stopfen stand. Zudem hätten am Stopfenende abgelagerte oxidische Ausscheidungen beprobt werden können. 


\section{Teil III}

\subsection{Zielsetzung}

Im zurückliegenden Teil II lag der Schwerpunkt auf $\delta^{18} \mathrm{O}-\mathrm{Messungen} \mathrm{mit} \mathrm{einer} \mathrm{umfassenden}$ isotopischen Charakterisierung von Clogging, Prozess- $\mathrm{O}_{2}$ und Prozessmaterialien. Auf dieser Basis und mit Hilfe chemischer Analysen erfolgte eine erste Modellierung des Prozesses zur Entstehung von Clogging.

In Teil III wurde ein Schwerpunkt auf die Mineralogie gelegt. Mit Hilfe quantitativer Phasenanalyse sollte z. B. der Anteil von Spinell am Clogging bestimmt werden. Eine Quelle für $\mathrm{MgO}$ im Spinell ist z. B. die Spritzmasse des Verteilers, die mit einem $\delta^{18} \mathrm{O}-\mathrm{Wert}$ von $+11 \%$ bis $+13 \%$ ein deutlich schwererer O-Donator als Chromit ist. Des Weiteren wurden anhand petrographischer Untersuchungen die verschiedenen Lithologien von Clogging beschrieben sowie eine Charakterisierung einiger Prozessmaterialien vorgenommen.

\subsection{Röntgenpulverdiffraktometrie}

Zur Phasenidentifizierung und quantitativen Phasenanalyse wurden von den untersuchten Cloggingproben und von Prozessmaterialien röntgenpulverdiffraktometrische Aufnahmen gemacht.

Die Grundlage der Röntgenpulverdiffraktometrie beruht auf der von Max von Laue Anfang des letzten Jahrhunderts entdeckten Reflexion von Röntgenlicht, das an Netzebenen gebeugt wird (ALLMANN 2003). Letztere werden aus Atomen des Kristallgitters aufgebaut, die eine beliebig orientierte Ebene im Kristall aufspannen. Die Periodizität der Kristallstruktur bedingt ihre Zuordnung zu einer Netzebenenschar, die mit Hilfe der Millerschen Indizes (hkl) indiziert wird (KLUG and AlEXANDER 1974, KRISCHNER 1990). Die Besetzungsdichten der Netzebenen und der Netzebenenabstand d beschreiben die Kristallgitter (FIX 2004). Für einen an einer Netzebene reflektierten Röntgenstrahl gilt Einfallswinkel $\theta_{0}=$ Ausfallswinkel $\theta$. Dabei kommt es zu Interferenzen des an verschiedenen Netzebenen reflektierten Röntgenlichtes. Diese können sich nur dann zu einem messbaren Effekt addieren, wenn die Gangunterschiede von Röntgenstrahlen an benachbarten Netzebenen gleich der Wellenlänge $\lambda$ der eingesetzten Strahlung ist oder ein ganzzahliges Vielfaches davon $(\lambda \times \mathrm{n})$. Nach der Braggschen Gleichung können nur in Phase schwingende Röntgenstrahlen mit dem Gangunterschied $2 \mathrm{~d} \times \sin \theta \mathrm{zu}$ einem Reflex führen für: 


$$
\mathrm{n} \times \lambda=2 \mathrm{~d} \times \sin \theta
$$

In Abbildung 62 ist der Beugungsvorgang an einer Netzebenenschar dargestellt. Der untere Strahl legt einen längeren Weg zurück, die Wegdifferenz entspricht der rechten Seite der Braggschen Gleichung.

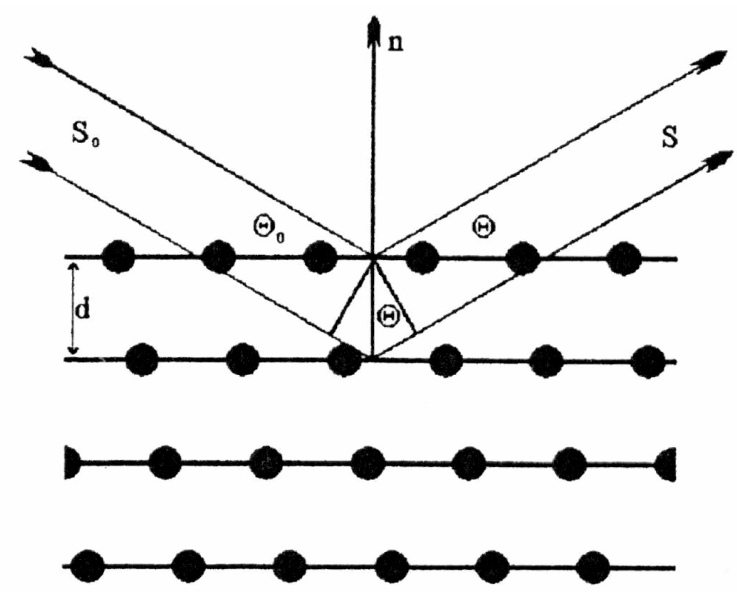

Abbildung 62: Prinzip der Braggschen Röntgenbeugung an einer Netzebenenschar. $d$ ist der Netzebenenabstand, $\theta$ ist der Glanz- oder Braggwinkel.

Die Diffraktogramme wurden mit CuK $\alpha$-Stahlung (1,54060 ̊̊) von 5 bis 85 Grad $2 \theta$ mit einer Schrittweite von 0,02 Grad $2 \theta$ aufgenommen. Pro Schritt wurde 1,6 Sekunden lang gemessen. Die Proben wurden in Achat-Reibschalen zu Pulvern gemörsert. Bei ausreichender Probenmenge wurden Presspräparate, bei geringen Probenmengen Schwemmpräparate angefertigt. Letztere erhöhen durch ihre unregelmäßige Oberfläche den Präparathöhenfehler der Messung. Die polykristallinen Materialien wurden in Reflexion mit einem PHILIPS PW 1800 Diffraktometer mit sekundärem Graphitmonochromator in Bragg-Brentano-Geometrie gemessen (siehe Abbildung 63). Bei einem Bragg-Brentano-Diffraktometer wird die monochromatische Röntgenstrahlung beim Durchtritt einer Sollerblende axial und durch eine variable Divergenzblende vertikal fokussiert. Dies ermöglicht es, die bestrahlte Fläche während der Messung konstant zu halten. Anschließend trifft das Röntgenlicht auf die Proben, wo es gebeugt wird. Der reflektierte Strahl passiert erneut ein System von Blenden, bevor er auf den Detektor trifft. Bei einigen Diffraktometern ist ein sekundärer Monochromator zwischengeschaltet, der wie im Falle des PHILIPS PW 1800 die Unterbindung von Fluoreszenzstrahlung z. B. durch eisenhaltige Cloggingproben ermöglicht. Allerdings geht bei der Absorption Intensität verloren, wodurch die Qualität der Messergebnisse erniedrigt werden kann. 


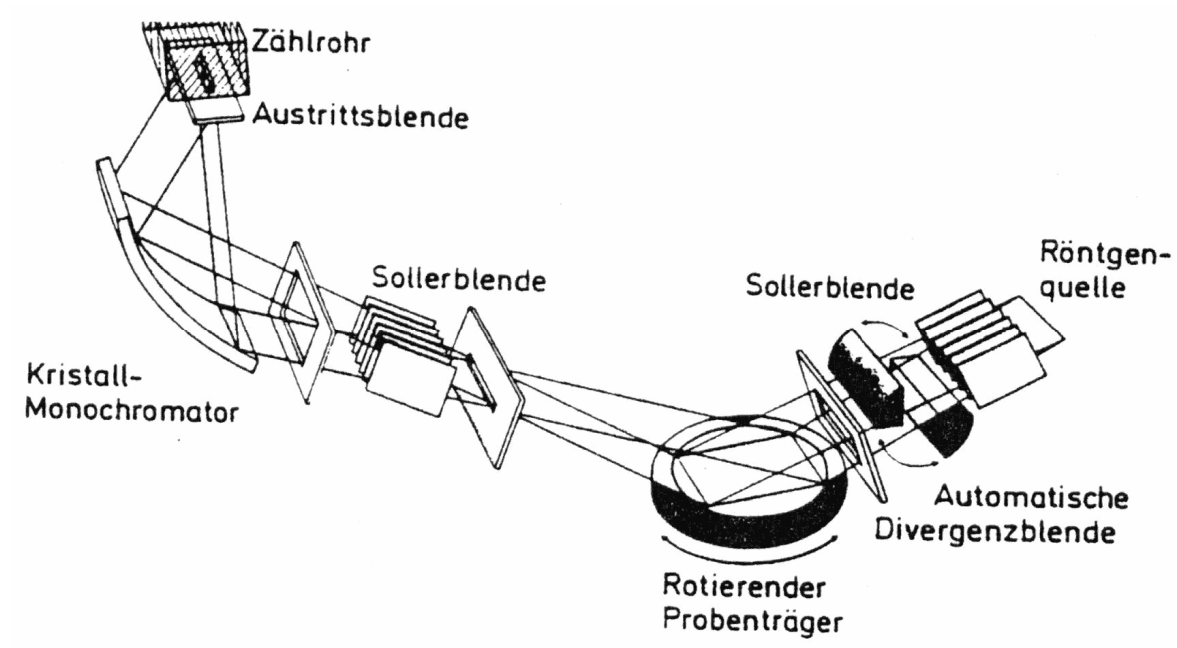

Abbildung 63: Strahlengang eines Pulverdiffraktometers mit Bragg-Brentano-Geometrie mit Blendensystem und sekundärem Monochromator (ALLMANN 2003).

Die qualitative und eine erste quantitative Untersuchung erfolgte mit dem Programm Win XPOW der Firma STOE \& Cie GmbH. Um den Fehler der quantitativen Analyse mittels Win XPOW abschätzen zu können, wurden fünf Pulver mit unterschiedlichen Anteilen von Korund und Spinell hergestellt (siehe Tabelle 15).

Tabelle 15: Einwaagen der fünf Korund-Spinell-Mischungen und die jeweiligen prozentualen Gewichtsanteile von Korund und Spinell.

\begin{tabular}{|cccccc|}
\hline \hline Mischung & $\begin{array}{c}\text { Spinell } \\
{[\mathrm{g}]}\end{array}$ & $\begin{array}{c}\text { Korund } \\
{[\mathrm{g}]}\end{array}$ & $\begin{array}{c}\text { Summe } \\
{[\mathrm{g}]}\end{array}$ & $\begin{array}{c}\text { Spinell } \\
{[\text { Gew.-\%] }}\end{array}$ & $\begin{array}{c}\text { Korund } \\
{[\text { Gew.-\%] }}\end{array}$ \\
\hline \hline 1 & 0.04 & 0.11 & 0.14 & 25.00 & 75.00 \\
2 & 0.05 & 0.09 & 0.14 & 32.97 & 67.03 \\
3 & 0.05 & 0.05 & 0.10 & 50.24 & 49.76 \\
4 & 0.05 & 0.13 & 0.18 & 28.76 & 71.24 \\
5 & 0.06 & 0.09 & 0.15 & 39.59 & 60.41 \\
\hline
\end{tabular}

In Tabelle 16 sind die Ergebnisse und die Fehler der Analysen dargestellt. Der mittlere Fehler der quantitativen Phasenanalyse liegt bei rd. $\pm 2 \%$. 
Tabelle 16: Ergebnisse der quantitativen Phasenbestimmung mit Win XPOW.

\begin{tabular}{|cccc|}
\hline \hline Mischung & $\begin{array}{c}\text { Spinell } \\
\text { [Gew.-\%] }\end{array}$ & $\begin{array}{c}\text { Korund } \\
\text { [Gew.-\%] }\end{array}$ & $\begin{array}{c}\text { Fehler } \\
{[\%]}\end{array}$ \\
\hline \hline 1 & 25 & 75 & 0.00 \\
2 & 29 & 71 & 5.92 \\
3 & 51 & 49 & 1.52 \\
4 & 30 & 70 & 1.74 \\
5 & 40 & 60 & 0.68 \\
\hline Mittelwert & & & 1.97 \\
\hline
\end{tabular}

In Abbildung 64 sind die Ergebnisse graphisch zu sehen. Für Korund-Spinell-Mischungen können mit Hilfe von Win XPOW gute Gehaltsangaben ermittelt werden. Der Fehler bei der quantitativen Analyse von Clogging und/oder Prozessmaterialien wird mit \pm 20 Gew.- $\%$ abgeschätzt und liegt an nachfolgend erläuterten Zusammenhängen.

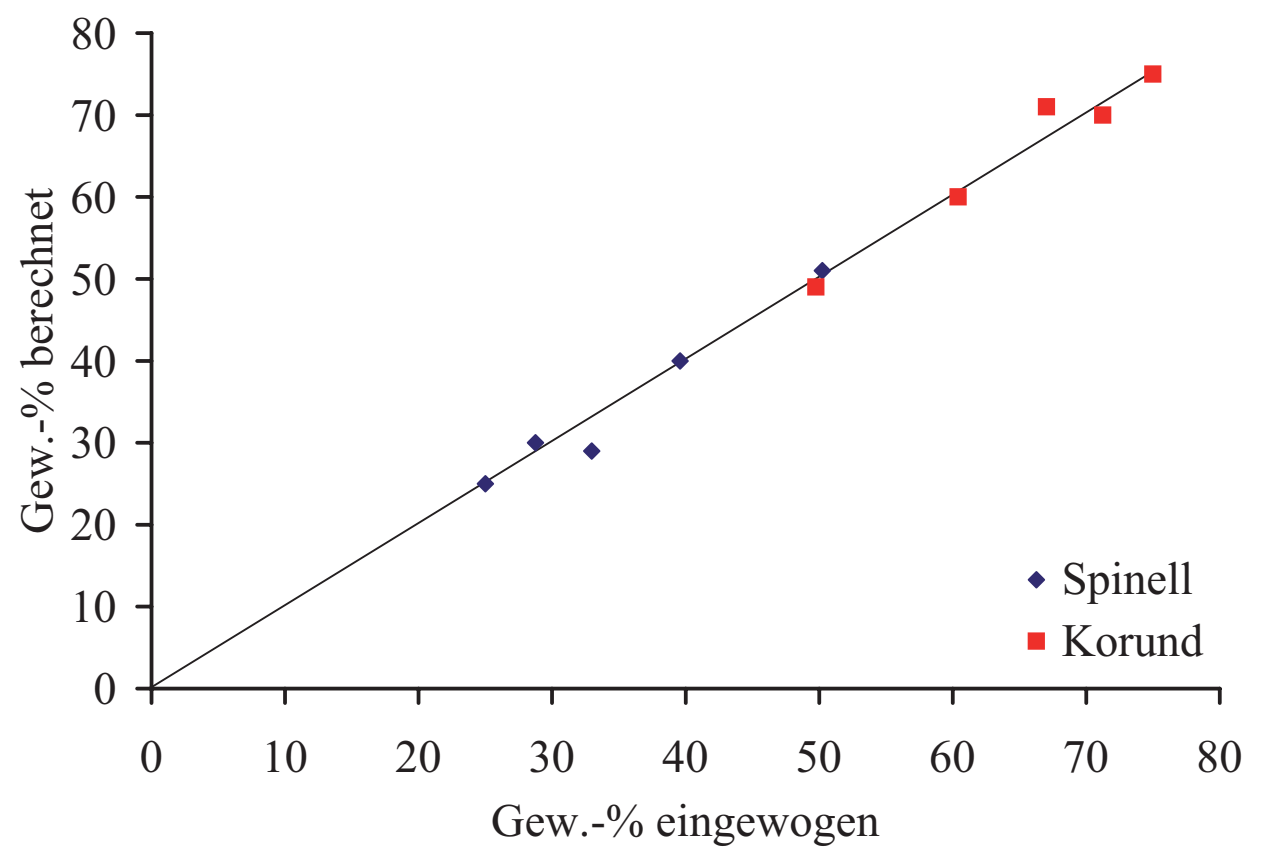

Abbildung 64: Dargestellt sind die Gehalte in Gew.-\% der eingewogenen Korund-Spinell-Mischungen gegen die berechneten Gehalte. Das Diagramm zeigt nur geringe Abweichungen (bis rd. 6\%) zwischen eingewogenen und berechneten Gehalten bei der quantitativen Analyse der untersuchten KorundSpinell-Mischungen mittels Win XPOW.

Die quantitative Analyse bei Win XPOW beruht auf der RIR-Methode (Reference Intensity Ratio Method). Hierbei werden alle Beugungsreflexe einer zu untersuchenden Phase auf einen Standard normiert. Als internationaler Standard dient Korund, auf den alle Phasen für die quantitative Analyse mit dem Verhältnis $\mathrm{I} / \mathrm{I}_{\text {cor }}$ referenziert sein sollten. In der Powder Diffrac- 
tion File (PDF) sind die I/I $\mathrm{I}_{\text {cor }}$-Werte referenzierter Phasen enthalten. Nach HALWAX (1998) errechnet sich das RIR-Verhältnis einer Phase j zu:

$$
\operatorname{RIR}_{j}=\frac{x_{\text {cor }}}{x_{j}} \times \frac{I_{j}(\max )}{I_{\text {cor }}(\max )}
$$

$\mathrm{I}_{\mathrm{j}}(\max )$ und $\mathrm{I}_{\text {cor }}(\max )$ sind die Netto-Intensitäten des höchsten Reflexes der Phase $\mathrm{j}$ und die von Korund in einer Pulvermischung mit Gewichtsanteilen von $\mathrm{x}_{\mathrm{j}}=\mathrm{x}_{\mathrm{cor}}=0.5$. Für Spinell ist das $\mathrm{I} / \mathrm{I}_{\text {cor }}$-Verhältnis im PDF enthalten. Deswegen ist der Fehler der quantitativen Phasenanalyse der Mischungen relativ klein (Abbildung 64). Für nicht referenzierte Phasen sind in den PDFs I/I $\mathrm{I}_{\text {cor }}$-Werte von 1 tabelliert. Daher sind quantitative Aussagen über den Phasenbestand für solche Gemische mit Win XPOW nicht möglich.

Mit Hilfe der Rietveldmethode lässt sich standardlos der quantitative Phasenbestand bestimmen. Die Rietveldanalyse wurde mit dem Programm TOPAS der Firma Bruker AXS durchgeführt. Die Methode geht auf die von RIETVELD (1967) eingeführte Strukturverfeinerung von Neutronenbeugungsdaten zurück. Das Prinzip der Methode beruht auf der Beschreibung aller Messpunkte eines Beugungsdiagramms mit Hilfe analytischer Funktionen (ALLMANN 2003). Die Funktionsparameter werden dabei mittels der Methode der kleinsten Fehlerquadrate an ein beobachtetes Beugungsdiagramm angepasst. In einem iterativen Prozess wird versucht, eine möglichst gute Anpassung des gefitteten Pulverdiagramms an das gemessene zu erreichen. Damit ist das Ziel der Rietveldmethode die Minimierung der Abweichung zwischen beobachteten und berechneten Daten (Young 1993). Nach ALlMANN (2003) ergeben sich die gemessenen Intensitäten y an einer Stützstelle i zu:

$$
\mathrm{y}_{\mathrm{i}}(\mathrm{obs})=\mathrm{y}_{\mathrm{S}_{\mathrm{i}}}(\mathrm{obs})+\mathrm{y}_{\mathrm{b}_{\mathrm{i}}}(\mathrm{obs})
$$

mit $\mathrm{y}_{\mathrm{i}}(\mathrm{obs})=$ Gesamtintensität an der Stelle $\mathrm{i}, \mathrm{y}_{\mathrm{S}_{\mathrm{i}}}(\mathrm{obs})=$ Strukturanteile an der Stelle i und $\mathrm{y}_{\mathrm{b}_{\mathrm{i}}}(\mathrm{obs})=$ Untergrundsanteil an der Stelle $\mathrm{i}$.

Bei jedem Schritt der Verfeinerung werden neue Struktur- und Profilparameter berechnet, die wiederum als Ausgangswerte für den nächsten Verfeinerungsschritt in die zu minimierende Funktion des Least-Square-Algorithmus eingesetzt werden. Bei letzterem ist die Summe S der gewichteten Abweichungsquadrate der berechneten von den beobachteten Intensitäten zu minimieren:

$$
\mathrm{S}=\Sigma \mathrm{w}_{\mathrm{i}}\left[\mathrm{y}_{\mathrm{i}}(\mathrm{obs})-\mathrm{y}_{\mathrm{i}}(\text { calc })\right]^{2} \rightarrow \min
$$


Der Gewichtungsfaktor $\mathrm{w}_{\mathrm{i}}$ an der Stelle $\mathrm{i}$ ist die reziproke Varianz der gemessenen Intensität am Punkt i:

$$
\mathrm{w}_{\mathrm{i}}=1 / \sigma_{\mathrm{i}}^{2}=1 / \mathrm{y}_{\mathrm{i}}(\mathrm{obs})=\mathrm{Z} \times \mathrm{t}
$$

mit $\mathrm{Z}=$ Zählrate [Impulse/Sekunde] und $\mathrm{t}=$ Messzeit am Punkt i in Sekunden (ALLMANN 2003). Die Güte einer Rietveldverfeinerung lässt sich zum einen graphisch mittels einer Differenzkurve von gemessenen minus berechneten Intensitäten beurteilen und zum anderen numerisch durch einige Kenngrößen. Bei letzteren handelt es sich um R-Werte oder Residuen, von denen grundsätzlich zwei verschiedene Typen zu unterscheiden sind. Profil-R-Werte beschreiben die Anpassung an den gemessenen Stützpunkt $y_{i}$, Struktur-R-Werte berücksichtigen die integrale Intensität der Reflexe. Erstere beschreiben die Qualität der Anpassung an das gesamte Profil, letztere geben Aufschluss über die Anpassungsgüte der einzelnen Phasen. Die wichtigsten R-Werte zur Beurteilung des Verfeinerungsprozesses sind die Größen, die die zu minimierende gewichtete Fehlerquadratsumme im Zähler enthalten. Das ist zum einen der gewichtete Profil- $\mathrm{R}_{\mathrm{wp}}-$ Wert:

$$
\mathrm{R}_{\mathrm{wp}}=\left[\sum_{\mathrm{i}}\left(\mathrm{w}_{\mathrm{i}} \times \Delta_{\mathrm{i}}^{2}\right) / \sum_{\mathrm{i}}\left(\mathrm{w}_{\mathrm{i}} \times \mathrm{y}_{\mathrm{oi}}\right)^{2}\right]^{1 / 2}
$$

mit $\Delta_{\mathrm{i}}=\mathrm{y}_{\mathrm{oi}}-\mathrm{y}_{\mathrm{ci}}$, o für beobachtet und c für berechnet, zum anderen der GOF-Wert (goodness of fit):

$$
\mathrm{GOF}=\left[\sum_{\mathrm{i}}\left(\mathrm{w}_{\mathrm{i}} \times \Delta_{\mathrm{i}}^{2}\right) /(\mathrm{N}-\mathrm{n})\right]^{1 / 2}
$$

mit $\mathrm{N}=$ Intensitätswert und $\mathrm{n}=$ Parameter der Modellfunktion. Am Ende einer Verfeinerung streuen im Idealfall die Abweichungen $\Delta_{\mathrm{i}}=\mathrm{y}_{\mathrm{oi}}-\mathrm{y}_{\mathrm{ci}}$ um null.

Grundlage einer jeden Rietveldanalyse ist das Vorhandensein eines Startmodells für die Strukturverfeinerung, das bereits sehr nahe an der wirklichen Struktur liegt. Neben Kenntnis der Phasen und ihrer Gitterparameter müssen die Atompositionen in der Elementarzelle bekannt sein. Für Phasen, von denen keine Strukturparameter bekannt sind, kann weder deren Kristallstruktur verfeinert noch können deren quantitative Anteile in Pulvergemischen bestimmt werden. Im Verfeinerungsprozess können globale wie lokale Parameter verändert (=verfeinert) werden. Erstere sind z. B. die Nullpunktskorrektur, die Präparathöhe und die Eindringtiefe. Lokal, das heißt für jede einzelne Phase, können z. B. die Vorzugsorientierung von Mineralen, die Gitterparameter, der Halbhöhenparameter, die Flankenform, die Asymmetrie, die relative Atomkoordination und der Besetzungsfaktor verfeinert werden (ALLMANN 2003). 
In Abbildung 65 sind übereinander zwei Rietveldverfeinerungen derselben Probe unterschiedlicher Güte und ihre Differenzkurven dargestellt. Bei Fit B hat ein verfeinerter Gitterparameter einer Phase zu große Werte angenommen, was in einer schlechten Anpassung resultiert. Der GOF von Fit A liegt bei 2,38, der $\mathrm{R}_{\mathrm{wp}}$ bei 19,72. Fit B weist einen GOF von 4,36 und einen $\mathrm{R}_{\mathrm{wp}}$ von 36,15 auf.

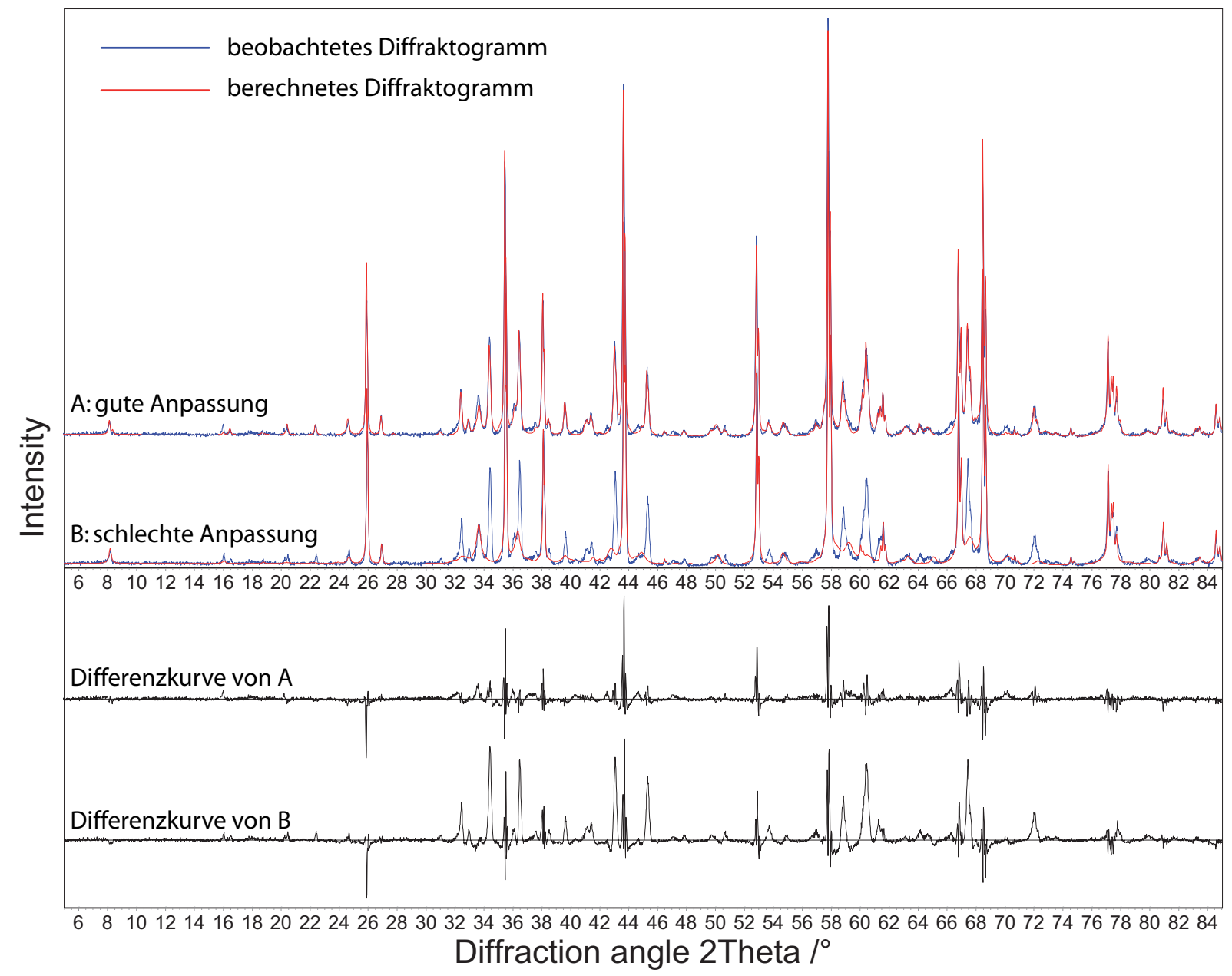

Abbildung 65: Vergleichendes Beispiel einer Rietveldverfeinerung der SEN-Unterseiten-Ablagerung vom 05.09.06, Strang 4. Der obere Fit zeigt eine deutlich bessere Anpassung an das beobachtete Diffraktogramm. Beim unteren Fit werden deutlich mehr Peaks nicht abgedeckt, die Restintensität schlägt sich in einer nach oben abweichenden Differenzkurve nieder.

\subsubsection{Clogging}

In 15 Cloggingproben und 8 SEN-Unterseiten-Präzipitaten, die zwischen August und Oktober 2006 im Stahlwerk B in Duisburg genommen wurden, wurden neun verschiedene Phasen identifiziert: Korund/Tonerde $\left(\mathrm{Al}_{2} \mathrm{O}_{3}\right)$, Hibonit $\left(\mathrm{CaAl}_{12} \mathrm{O}_{19}\right)$, Spinell $\left(\mathrm{MgAl}_{2} \mathrm{O}_{4}\right)$, Diaoyudaoit $\left(\mathrm{NaAl}_{11} \mathrm{O}_{17}\right)$, Na- $\beta$-Aluminat $\left(\mathrm{Na}_{2} \mathrm{Al}_{22} \mathrm{O}_{34}\right)$, Hämatit $\left(\mathrm{Fe}_{2} \mathrm{O}_{3}\right)$, Na-Mg-Aluminat $\left(\mathrm{NaMg}_{2} \mathrm{Al}_{15} \mathrm{O}_{25}\right)$, Graphit $(\mathrm{C})$ und Rutil $\left(\mathrm{TiO}_{2}\right)$. In Tabelle 23 sind die Ergebnisse der Rietveldanalyse von Clogging und von SEN-Unterseiten-Präzipitaten zusammenfassend dargestellt. Die Hauptphase in $\mathrm{Al}_{2} \mathrm{O}_{3}$-reichen oxidischen Ausscheidungen ist Tonerde, die in den 
untersuchten Proben Anteile zwischen 10 und 95 Gew.-\% ausmacht. Tonerde ist die einzige Phase, die sowohl im Clogging als auch in den SEN-Unterseiten-Ablagerungen in jeder Probe vorkommt. Hibonit liegt zwischen 5 und 90 Gew.-\%. Spinell hat einen Anteil von 2 bis 33 Gew.-\%. Diaoyudaoit besitzt Anteile von 9 bis 30 Gew.-\%. Die Gehalte für Na- $\beta$-Aluminat liegen zwischen 1 und 4 Gew.-\%. Hämatit erreicht Gehalte bis zu 6 Gew.-\%, Na-MgAluminat bis zu 3 Gew.-\%, Graphit und Rutil erreichen bis zu 1 Gew.-\%.

Im Clogging macht Tonerde einen Anteil von 10 bis 95 Gew.-\% aus. Hibonit liegt zwischen 5 und 90 Gew.-\%, Spinell bei 4 bis 33 Gew.-\%. Hämatit erreicht 3 bis 6 Gew.-\%, Na-MgAluminat bis zu 3 Gew.-\% und Na- $\beta$-Aluminat bis zu 2 Gew.-\%. Diaoyudaoit, Graphit und Rutil wurden nicht identifiziert.

Die Ablagerungen an der Tauchrohrunterseite haben Tonerdegehalte von 10 bis 81 Gew.-\%. Der Hibonitanteil liegt zwischen 12 und 78 Gew.-\%, der des Spinells zwischen 2 und 10 Gew.-\%. Diaoyudaoit trägt mit 9 bis 30 Gew.-\% zu der Mächtigkeit der Ablagerungen bei. Der Anteil von Na- $\beta$-Aluminat wurde auf 2 bis 4 Gew.-\% quantifiziert. Hämatit kommt in Gehalten zwischen 2 und 6 Gew.-\% vor. Graphit und Rutil erreichen bis zu 1 Gew.-\%. In Abbildung 66 ist das Diffraktogramm der Probe vom 04.09.06, Strang 3 zu sehen. Das hochkristalline Pulver ist annähernd monomineralisch und besteht $\mathrm{zu} \geq 95$ Gew.- $\%$ aus Tonerde. Mindestens eine Phase wurde nicht identifiziert (Peak bei 45 Grad 20). Das Beugungsdiagramm der SEN-Unterseiten-Ablagerung vom 24.10.06, Strang 3 hat mit Diaoyudaoit die typische Phase der Ablagerungen an der SEN-Unterseite (siehe Abbildung 67). Diese Präzipitate weisen mit bis zu 30 Gew.-\% im Vergleich zum eigentlichen Clogging im Tauchrohr mit maximal 2 Gew.-\% deutlich höhere Gehalte an Diaoyudaoit bzw. Na- $\beta$-Aluminat auf. 


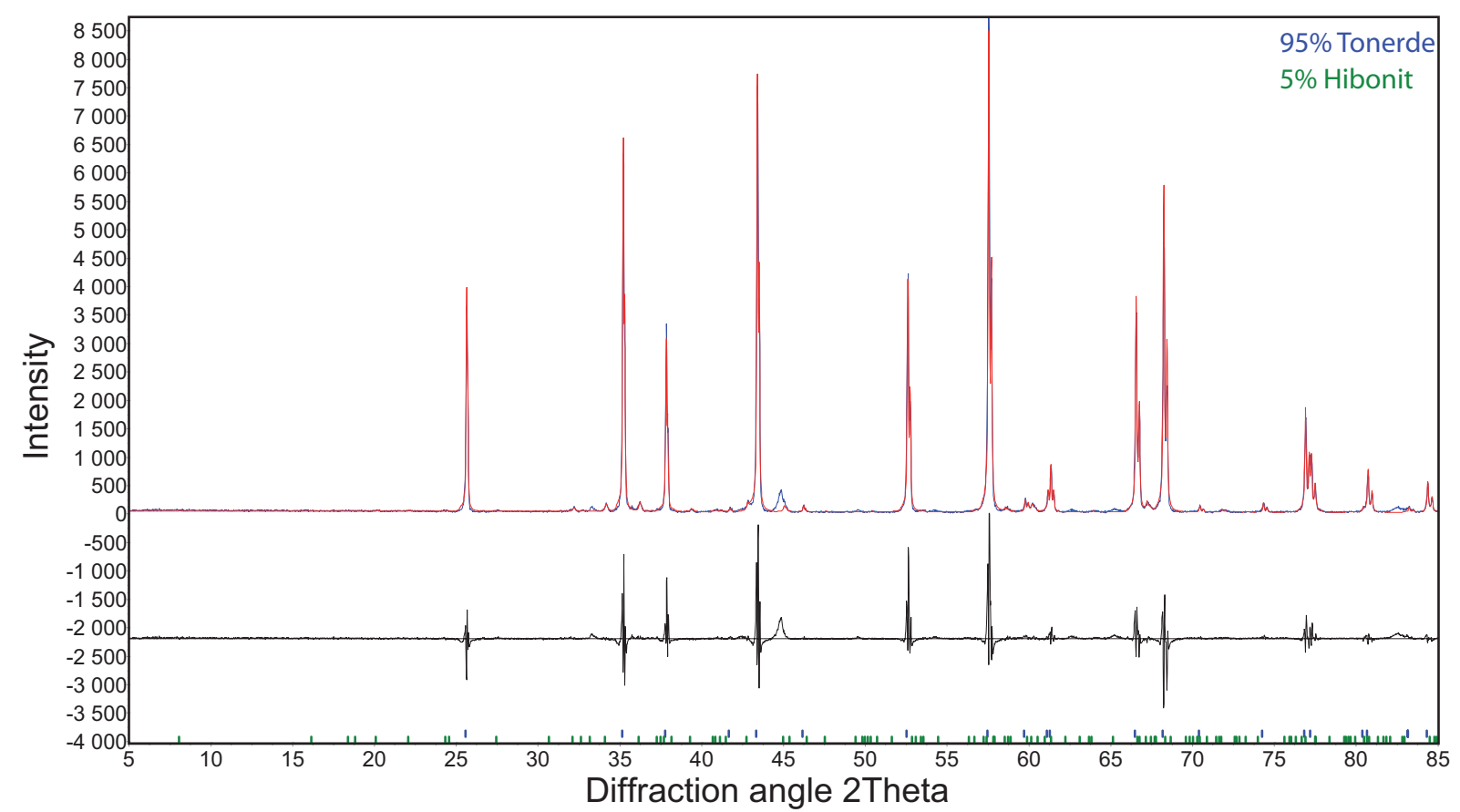

Abbildung 66: Beugungsdiagramm und Rietveldplot von Clogging vom 04.09.06, Strang 3. Die Probe hat einen Tonerdegehalt von $\geq 95$ Gew.- $\%$. Bei 45 Grad $2 \theta$ ist ein unidentifizierter Peak, der möglicherweise zu elementarem Eisen gehört.

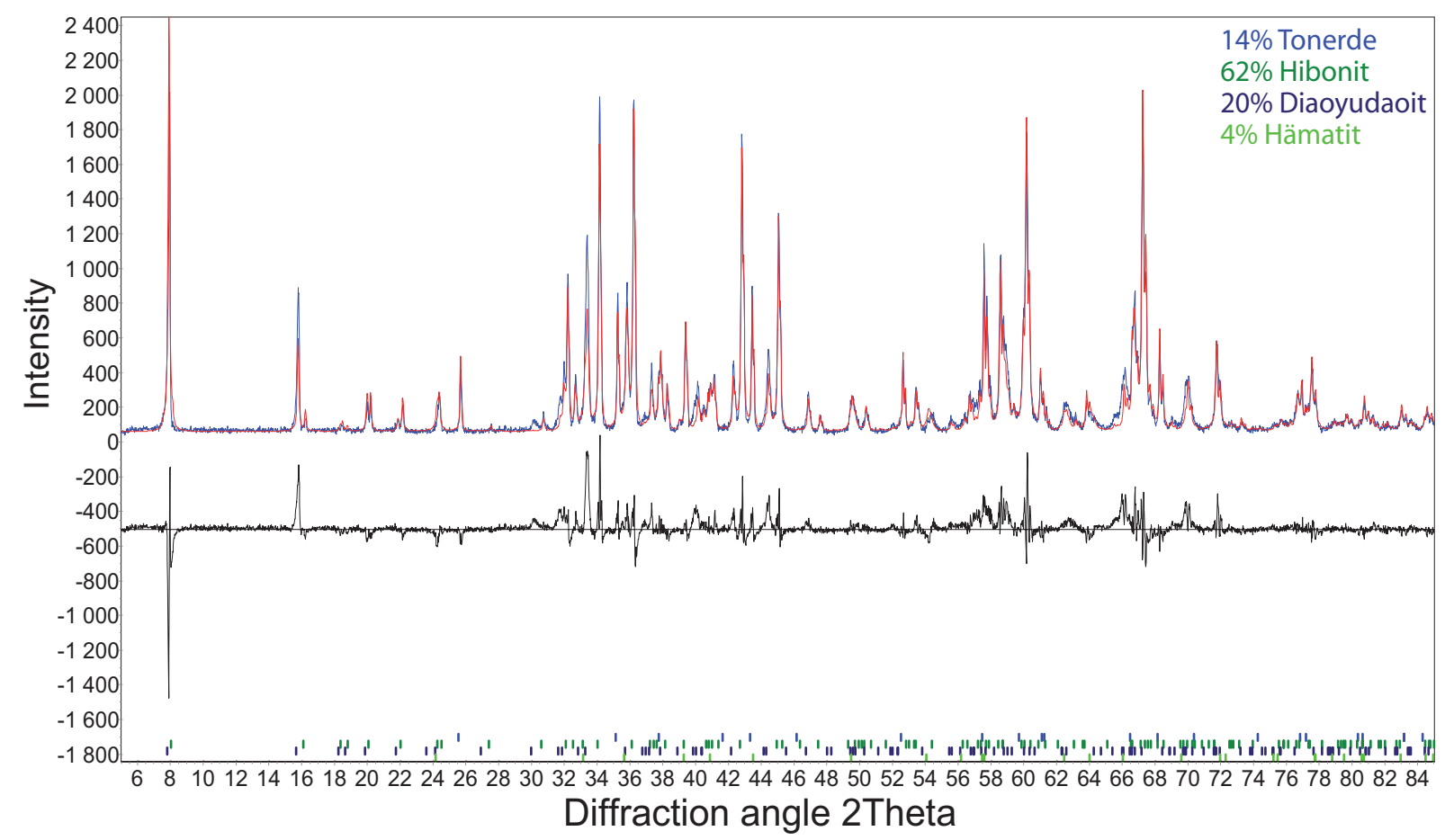

Abbildung 67: Beugungsdiagramm und Rietveldplot der SEN-Unterseiten-Ablagerung vom 24.10.06, Strang 3.

Die Maßzahl $r^{2}$ zeigt einen Zusammenhang zwischen dem Spinell- und dem MgO-Gehalt von Clogging von 0,96 an (siehe Abbildung 68). Die Spinellgehalte streuen mit $\pm 2,9$ Gew.- $\%$ um die Regressionsgerade. 


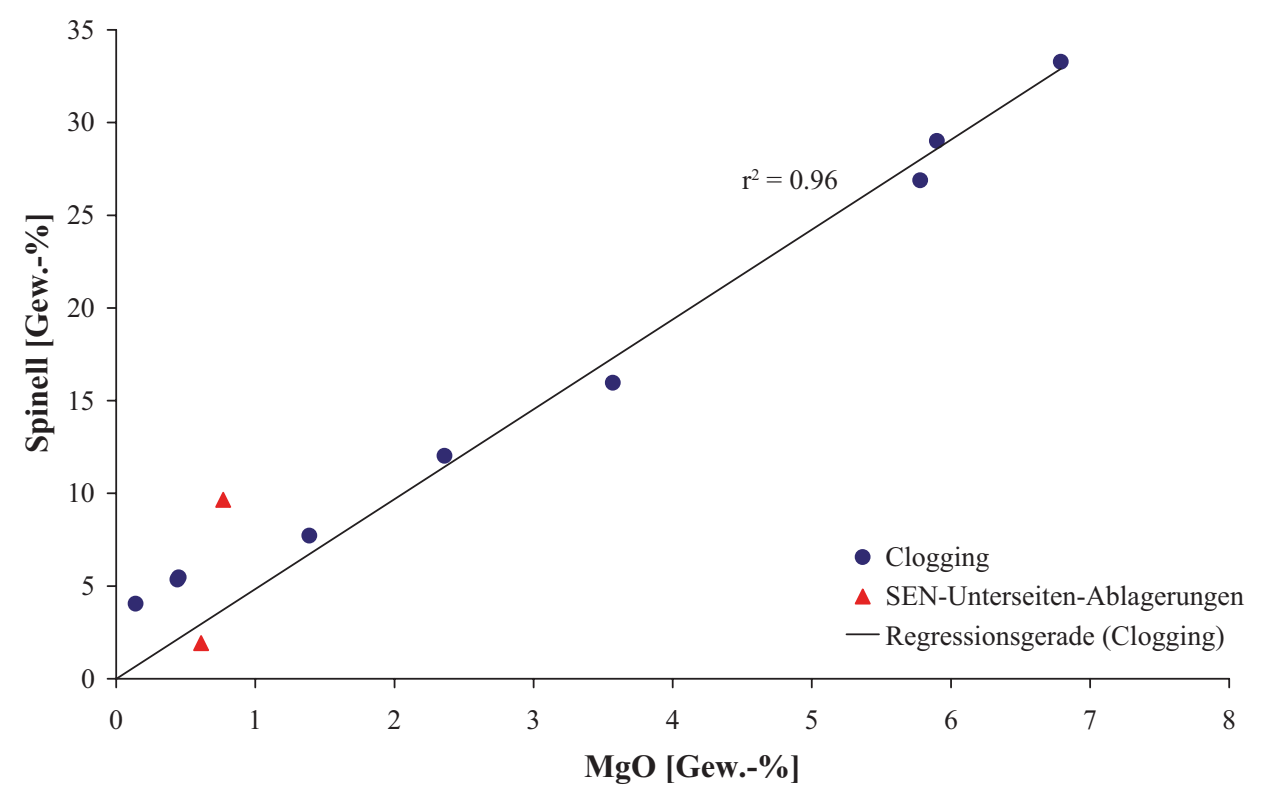

Abbildung 68: Spinell- gegen MgO-Gehalt von Clogging und SEN-Unterseiten-Ablagerungen.

In Abbildung 69 ist der Gitterparameter $\mathrm{a}_{0}$ von Spinellen der untersuchten oxidischen Ablagerungen (schwarze Punkte) und relativ dazu Daten aus der Literatur dargestellt. Der Gitterpa-

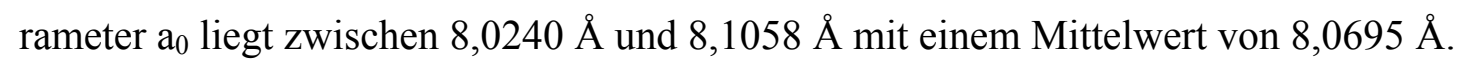

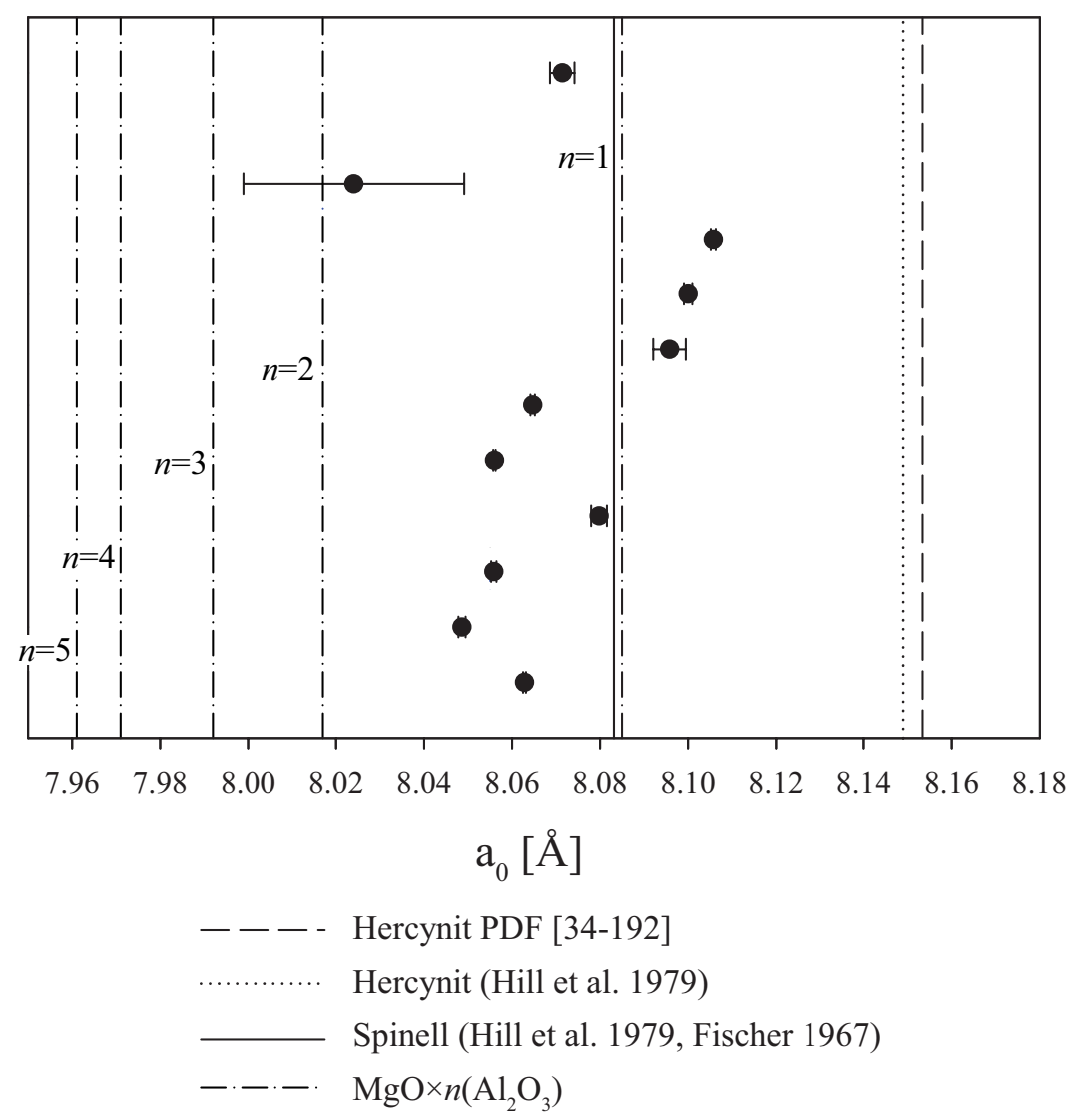

Abbildung 69: Gitterparameter $\mathrm{a}_{0}$ von Spinellen $\left(\mathrm{MgAl}_{2} \mathrm{O}_{4}\right)$ der oxidischen Ablagerungen im Tauchrohr und an dessen Unterseite (schwarze Punkte). Relativ dazu ist die Lage von Hercynit $\left(\mathrm{FeAl}_{2} \mathrm{O}_{4}\right)$, Spinell $\left(\mathrm{MgAl}_{2} \mathrm{O}_{4}\right)$ und nichtstöchiometrischen Spinellen $\left[\mathrm{MgO} \times n\left(\mathrm{Al}_{2} \mathrm{O}_{3}\right)\right]$ aus Literaturdaten dargestellt. Für $n>1$ sitzt $\mathrm{Al}^{3+}$ auf der $\mathrm{Mg}^{2+}$-Position im Spinell. 
Stellt man die massenmäßigen Gehalte von Hibonit gegen die von $\mathrm{CaO}$ in Cloggingproben dar, ergibt sich ein Zusammenhang von $\mathrm{r}^{2}=0.92$ (Abbildung 70). Die mittlere Abweichung zwischen gemessenen und berechneten Gehalten liegt bei \pm 7,4 Gew.- $\%$.

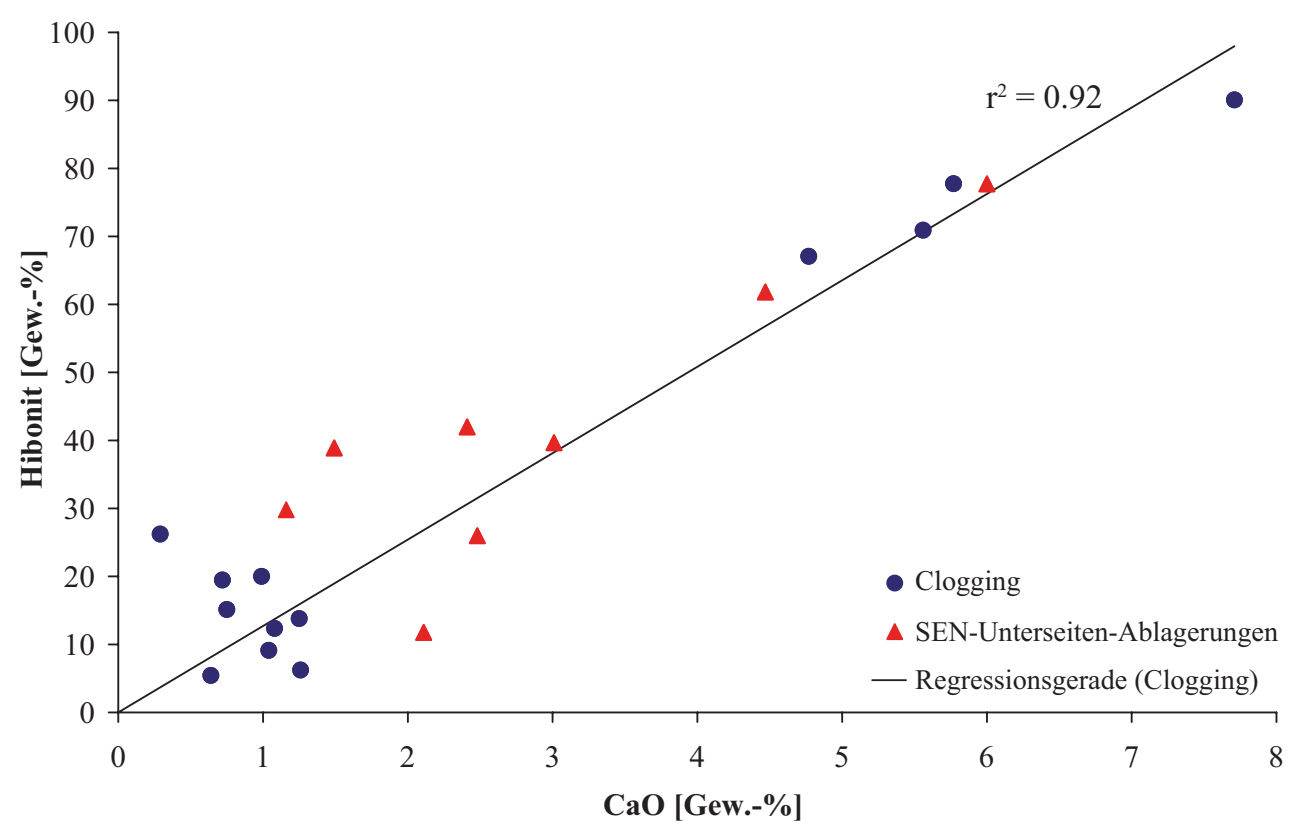

Abbildung 70: Hibonit- gegen CaO-Gehalt von Clogging und SEN-Unterseiten-Ablagerungen.

Trägt man Tonerdegehalt und Netto- $\mathrm{Al}_{2} \mathrm{O}_{3}$-Gehalt von Clogging gegeneinander auf, ergibt sich mit $\mathrm{r}^{2}=0,99$ eine sehr gute Korrelation (Abbildung 71). Der Netto- $\mathrm{Al}_{2} \mathrm{O}_{3}$-Gehalt berechnet sich aus der Differenz vom Gesamt- $\mathrm{Al}_{2} \mathrm{O}_{3}$-Gehalt der Proben abzüglich der $\mathrm{Al}_{2} \mathrm{O}_{3}$-Gehalte der aluminiumhaltigen Phasen. Die Regressionsgerade hat allerdings bei $\mathrm{y}=0$ einen $\mathrm{x}$-Wert von 13 (Abbildung 71). 


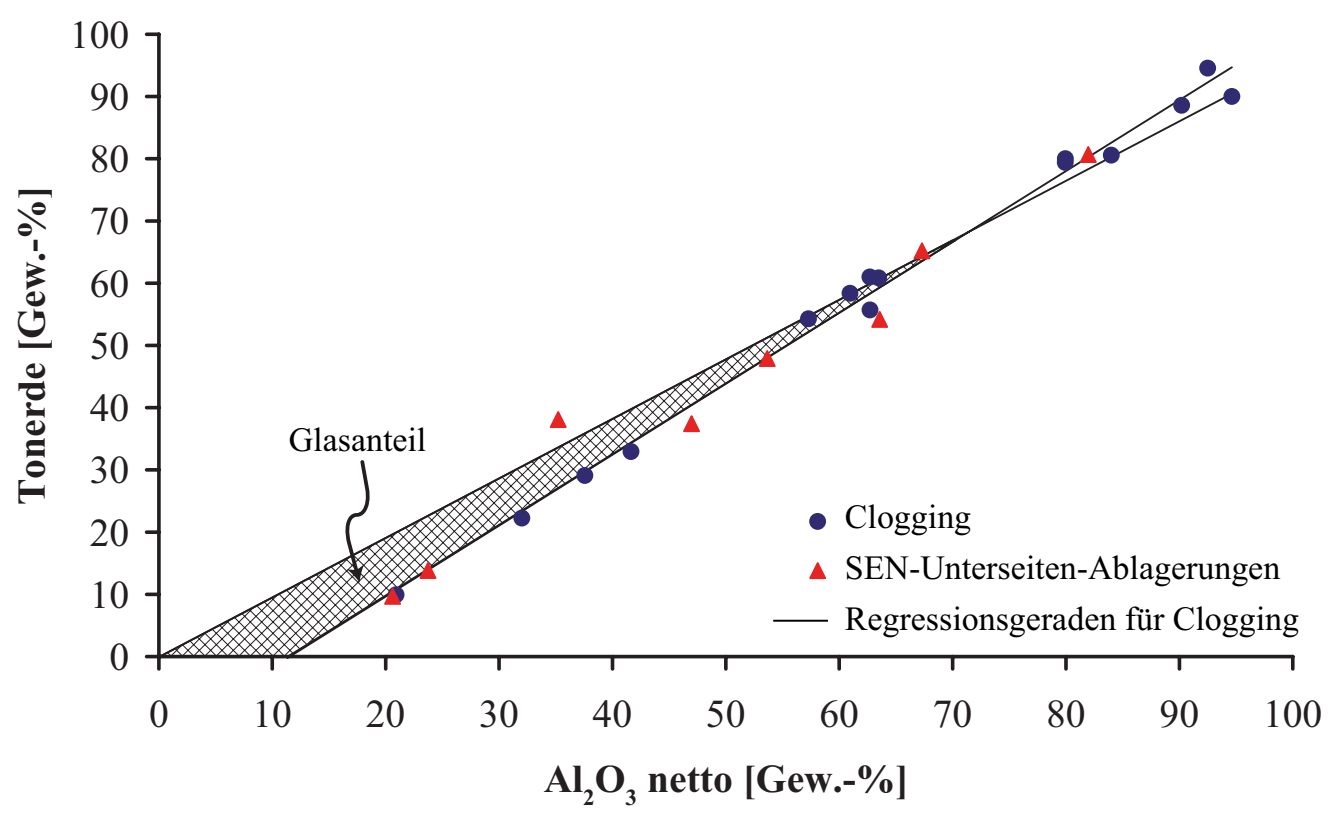

Abbildung 71: Tonerdegehalt gegen Netto- $\mathrm{Al}_{2} \mathrm{O}_{3}$-Gehalt von Clogging. Die Lage von SENUnterseiten-Präzipitaten ist ebenfalls dargestellt.

Trägt man $\delta^{18} \mathrm{O}$ gegen den Tonerdegehalt von Clogging auf, ergibt sich für Proben mit $>80$ Gew.- $\%$ Tonerde ein durchschnittlicher $\delta^{18} \mathrm{O}$ von $+5 \%$ (siehe Abbildung 72).

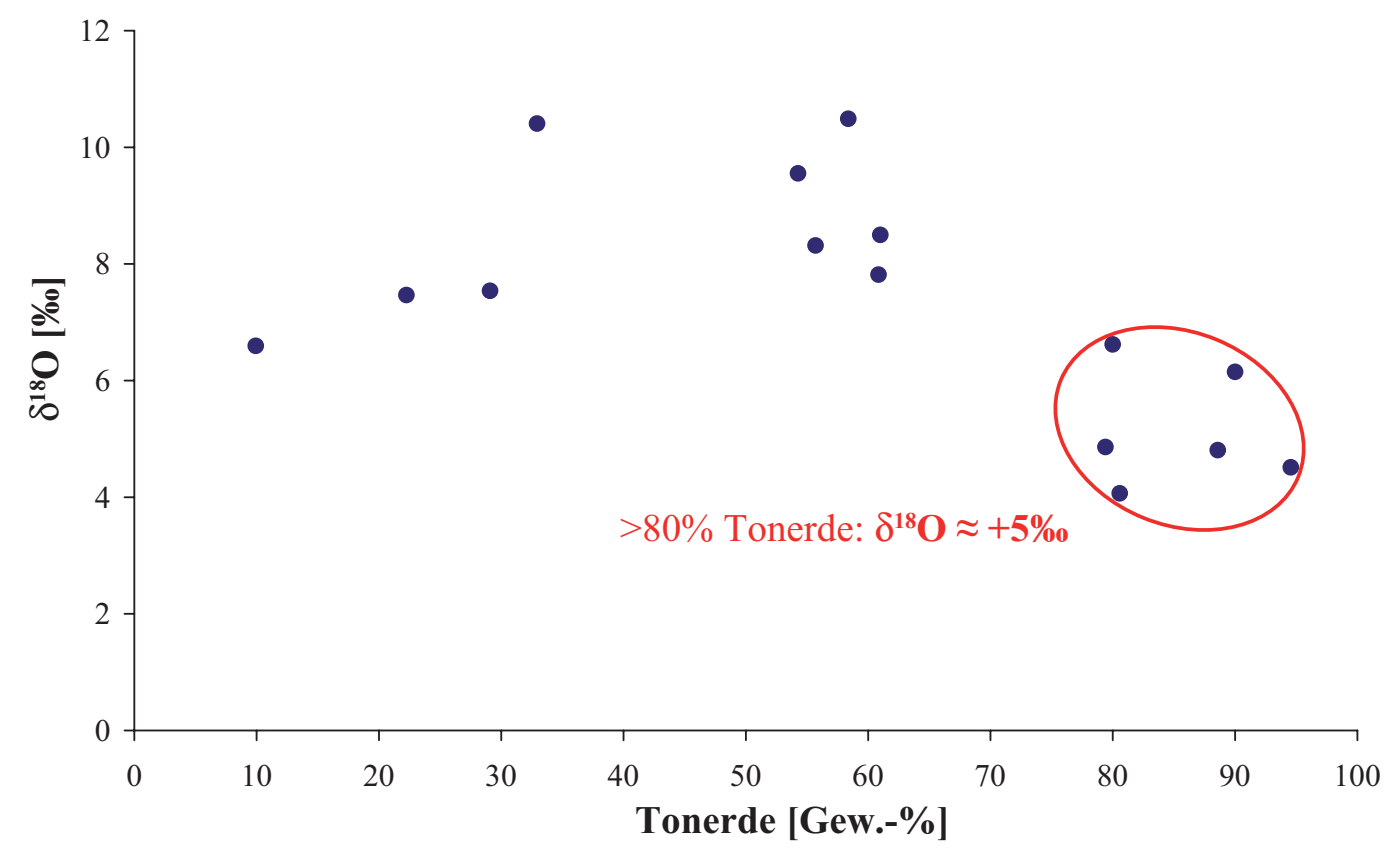

Abbildung 72: $\delta^{18} \mathrm{O}$ gegen Tonerdegehalte von Clogging.

Der $\delta^{18} \mathrm{O}$ von Clogging korreliert mit dem Spinellgehalt der Proben mit $r^{2}=0,74$ (Abbildung 73). 


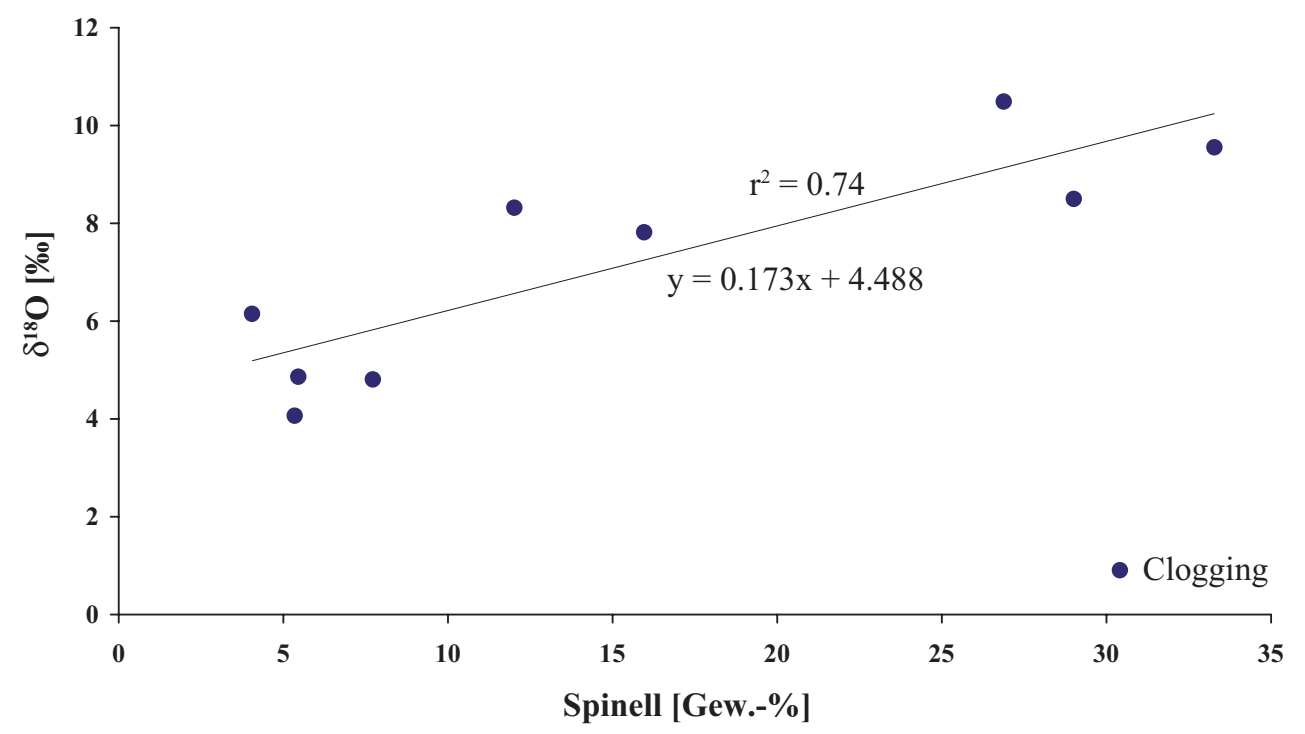

Abbildung 73: $\delta^{18} \mathrm{O}$ gegen Spinellgehalt von Clogging.

\subsubsection{Tundishschlacke}

16 Tundishschlackenproben wurden röntgenographisch untersucht. Tundishschlacken sind sehr heterogen und meist von glasiger Struktur (Abbildung 74).

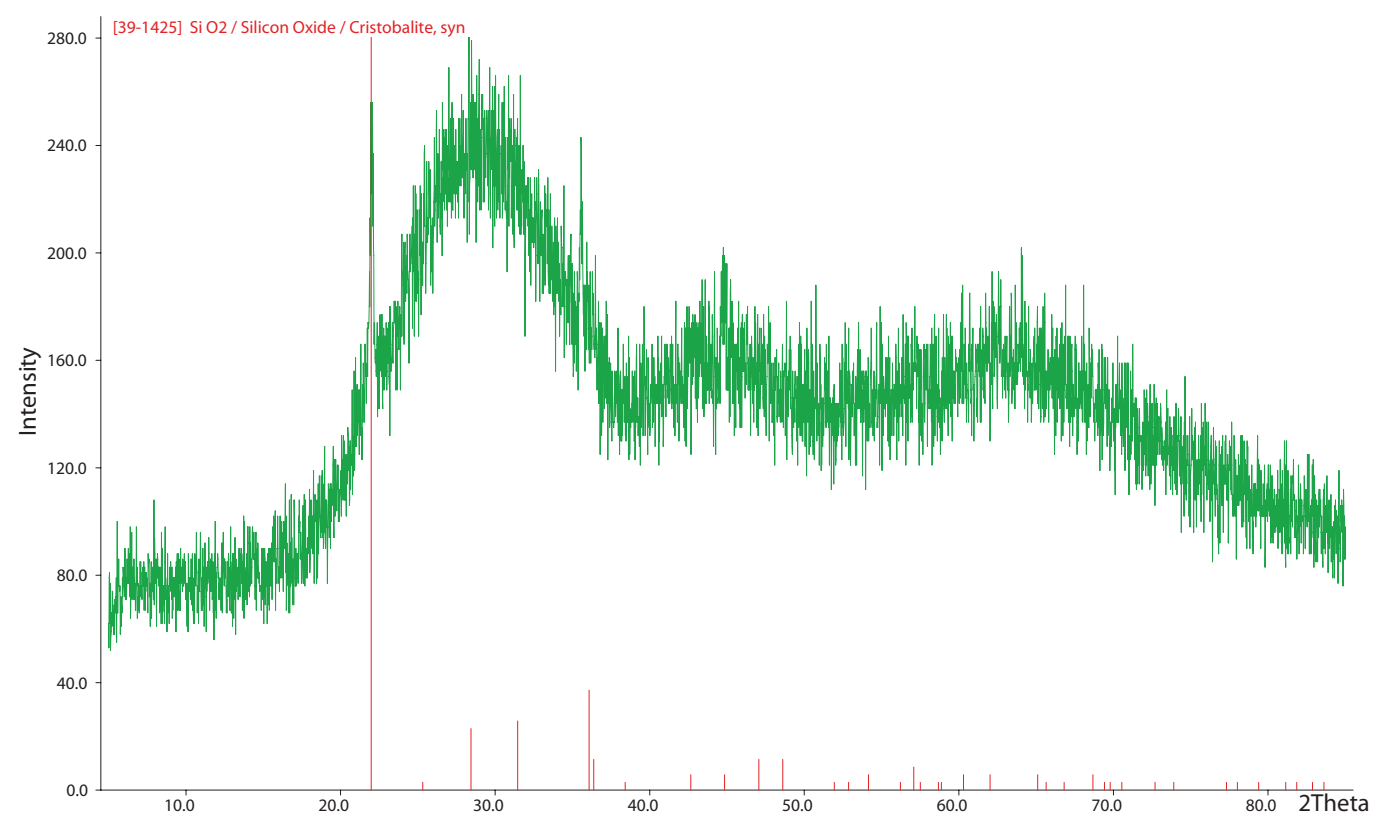

Abbildung 74: Tundishschlackenprobe vom 28.08.06, Strang 3. Das Diffraktogramm zeigt einen hohen Glasanteil im Pulver. Als einzige kristalline Phase wurde Cristobalit $\left(\mathrm{SiO}_{2}\right)$ identifiziert.

Zehn der untersuchten Proben weisen einen deutlichen Glasanteil entsprechend Abbildung 74 auf. Bei diesen Proben konnten daher keine Phasen identifiziert werden. Allein Cristobalit $\left(\mathrm{SiO}_{2}\right)$ bzw. Coesit $\left(\mathrm{SiO}_{2}\right)$ wurde in einigen Diffraktogrammen gefunden. Die Proben vom 24.10.06, Strang 3 und vom 01.09.06, Strang 3 und 4 weisen geringere Glasanteile auf und als dominierende Phase Gehlenit $\left(\mathrm{Ca}_{2} \mathrm{Al}_{2} \mathrm{SiO}_{7}\right)$ (siehe Abbildung 75). 


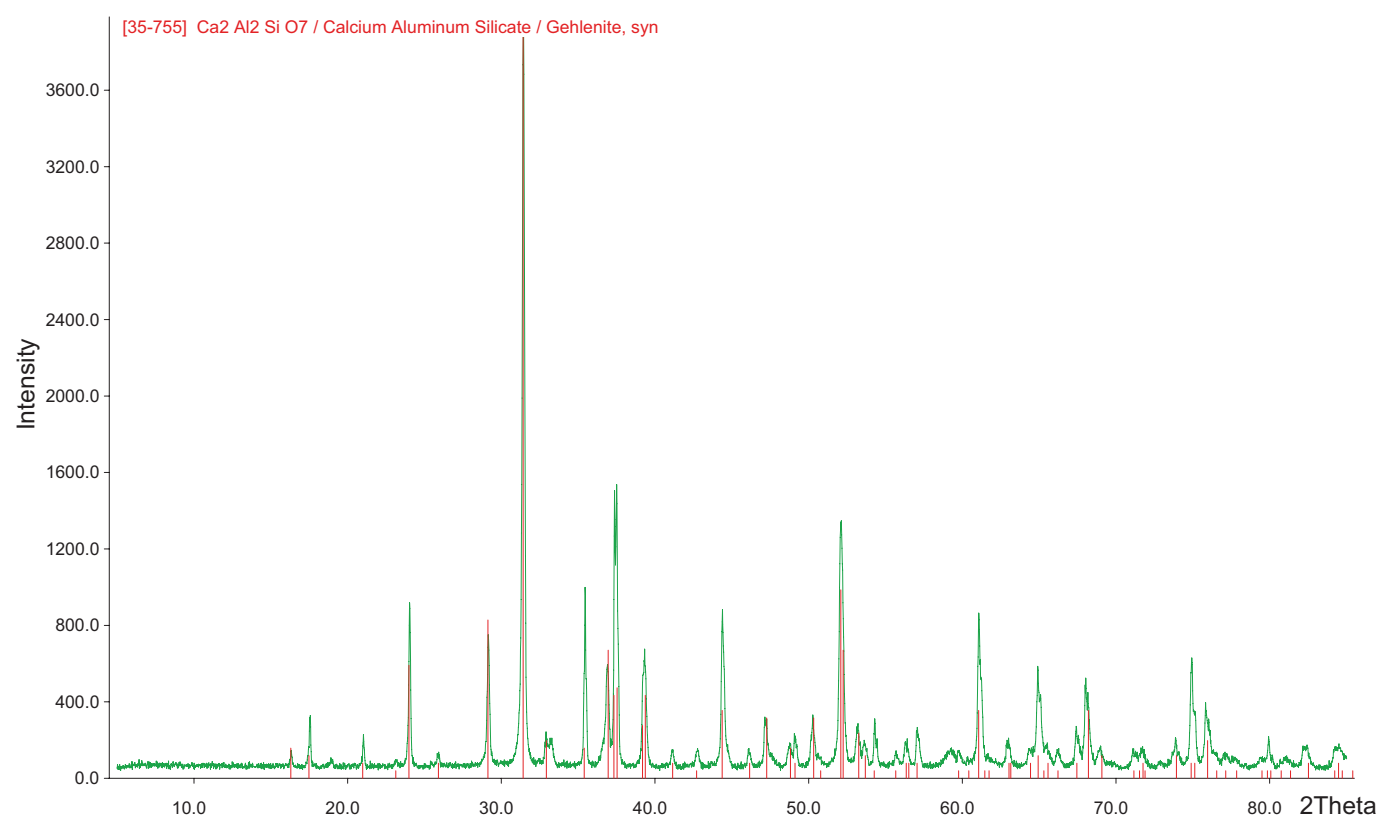

Abbildung 75: Tundishschlackenprobe vom 01.09.06, Strang 3. Als einzige Phase wurde Gehlenit $\left(\mathrm{Ca}_{2} \mathrm{Al}_{2} \mathrm{SiO}_{7}\right)$ identifiziert.

Am 29.08.06, Strang 3 wurden in der Schlackenprobe Chromit $\left((\mathrm{Mg}, \mathrm{Fe})(\mathrm{Cr}, \mathrm{Al})_{2} \mathrm{O}_{4}\right)$, Hercynit $\left(\mathrm{FeAl}_{2} \mathrm{O}_{4}\right)$, Cristobalit und Quarz gefunden.

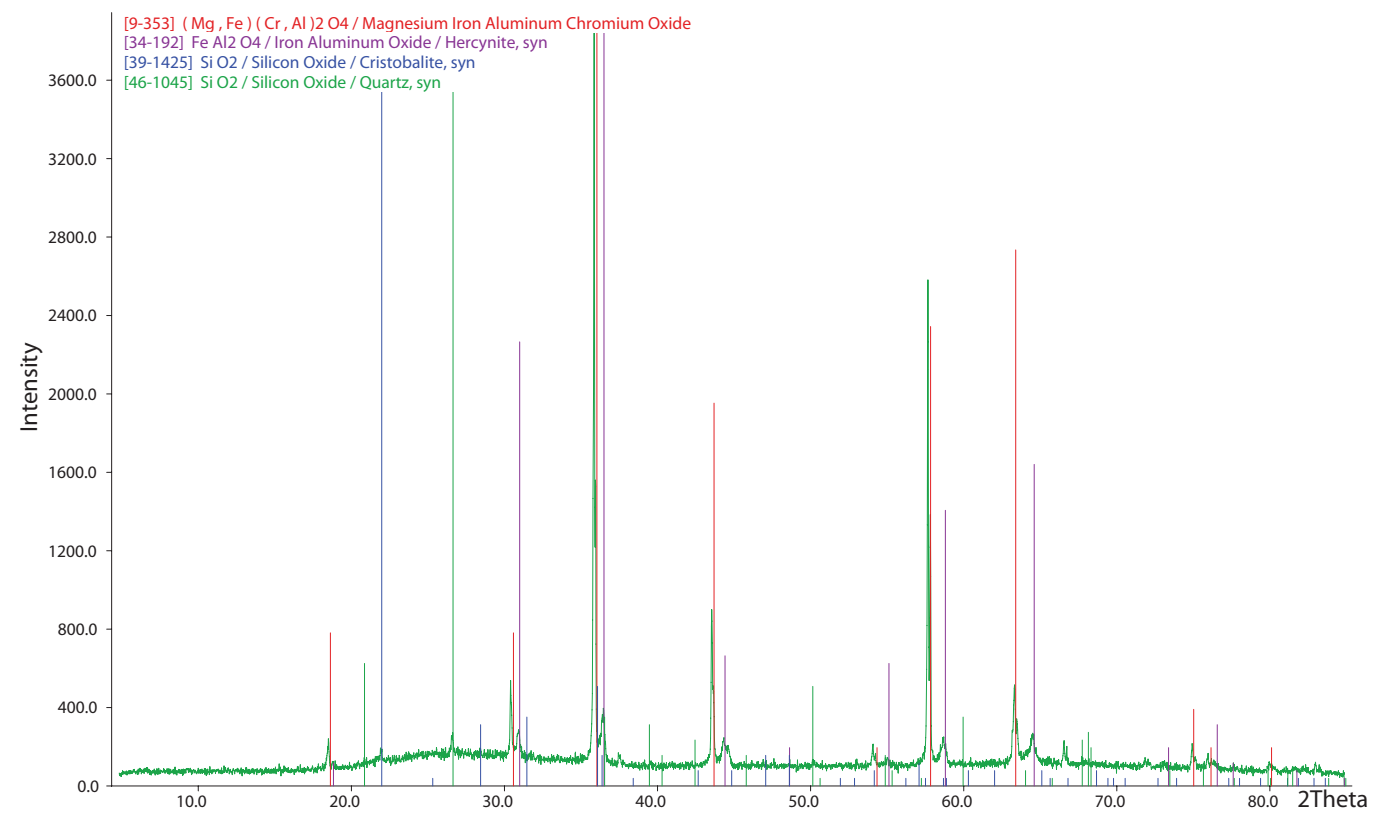

Abbildung 76: Tundishschlackenprobe vom 29.08.06, Strang 3. Auffallend ist der Chromit, der insgesamt nur in drei Proben beobachtet wurde.

Am Strang 4 wurde an diesem Tag die gleiche Zusammensetzung gefunden. Hier wurde allein Hercynit nicht identifiziert. Diese Probe weist gegenüber der von Strang 3 einen deutlich höheren Glasanteil auf (Abbildung 77). 


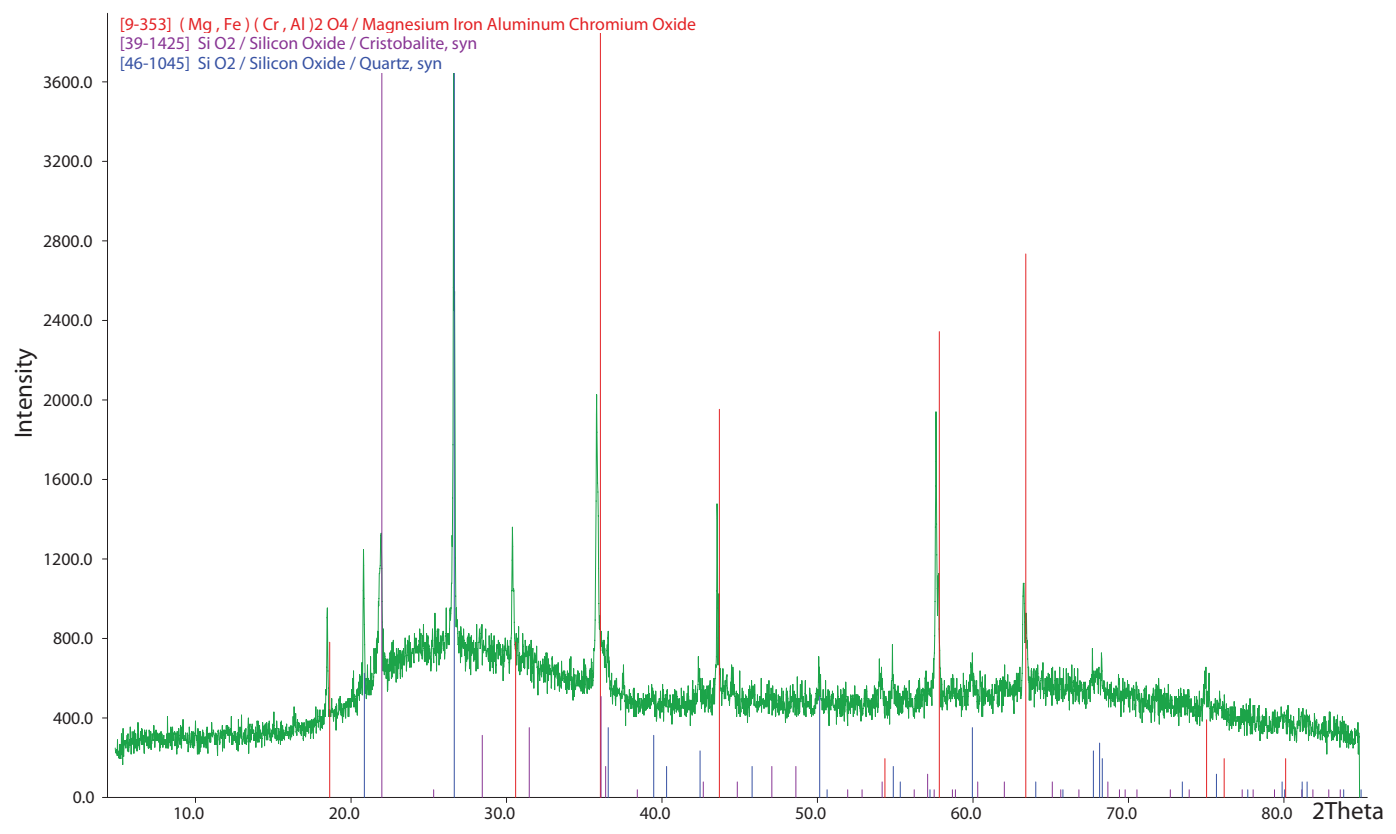

Abbildung 77: Tundishschlackenprobe vom 29.08.06, Strang 4.

Am 23.10.06 wurde am Strang 3 erneut Chromit gefunden. Die Probenzusammensetzung ähnelt der vom 29.08.06, allein elementares Eisen wurde hier als weitere Phase identifiziert (Abbildung 78).

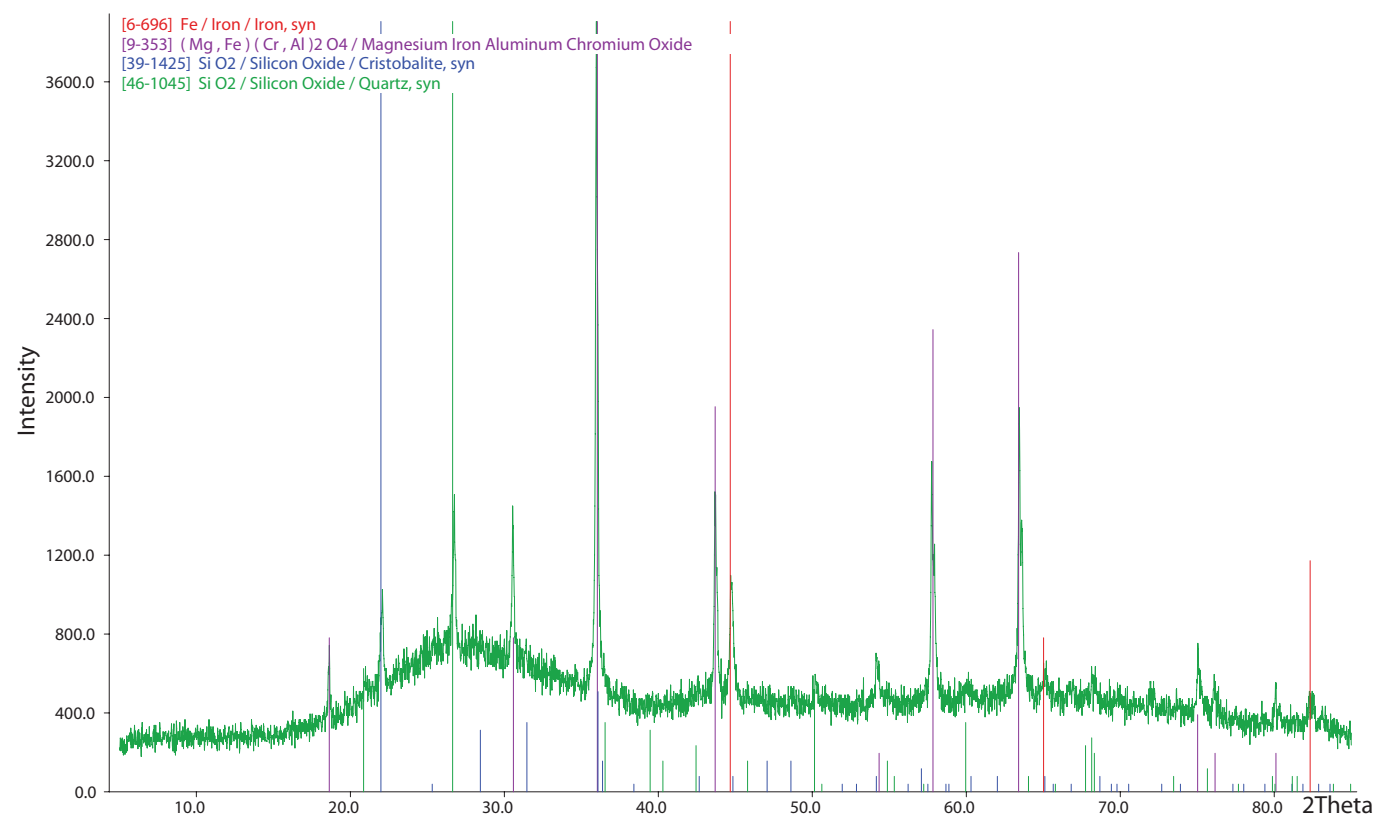

Abbildung 78: Tundishschlackenprobe vom 23.10.06, Strang 3.

\subsubsection{Schiebersand}

An den Schiebersanden wurden sowohl Gesamtanalysen durchgeführt als auch

Mineralseparate geröntgt. Erstere zeigten bei allen analysierten Schiebersanden Chromit und 
Quarz als dominierende Phasen. Die quantitative Analyse des Schiebersandes von Hersteller B mittels Win XPOW ergab 74 Gew.-\% Chromit und 26 Gew.-\% Quarz. Beim Schiebersand des Herstellers A02 waren es 85 Gew.-\% Chromit und 15 Gew.-\% Quarz.

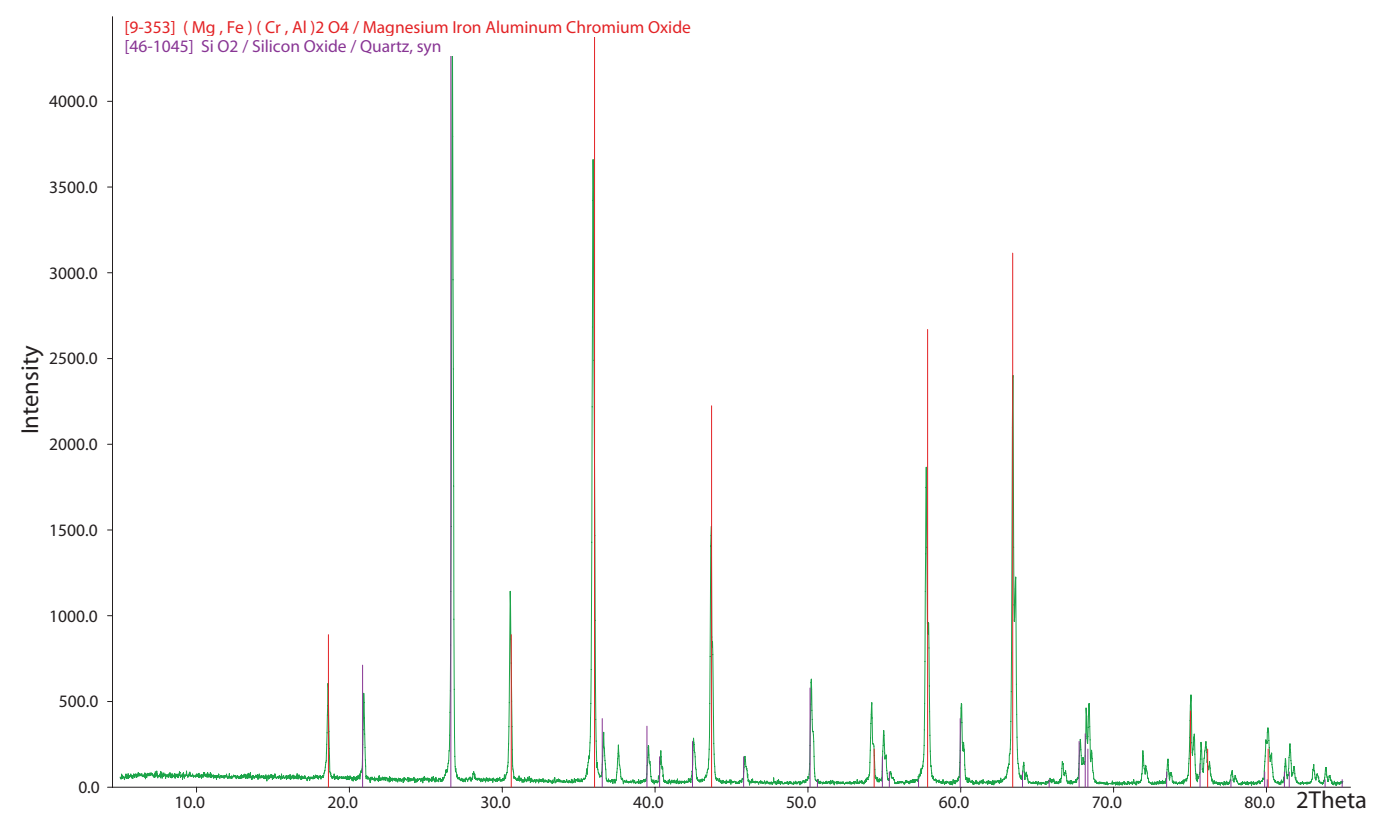

Abbildung 79: Das Beugungsdiagramm des Schiebersandes von Hersteller B (Gesamtanalyse) hat den stärksten Reflex bei 27 Grad $2 \theta$ (Chromit). Der zweitstärkste Reflex liegt bei 36 Grad $2 \theta$ und gehört zum Quarz.

Unter einem Binokular wurden die Schiebersande für die Röntgenpulverdiffraktometrie und für eine detaillierte isotopenchemische Charakterisierung separiert. Erstere ergab als dritte, weitere Phase das orthorhombisch kristallisierte Inosilikat Enstatit $\left(\mathrm{Mg}_{2} \mathrm{Si}_{2} \mathrm{O}_{6}\right)$. Es gehört zur Gruppe der Orthopyroxene und enthält rd. 40 Gew.-\% MgO. 


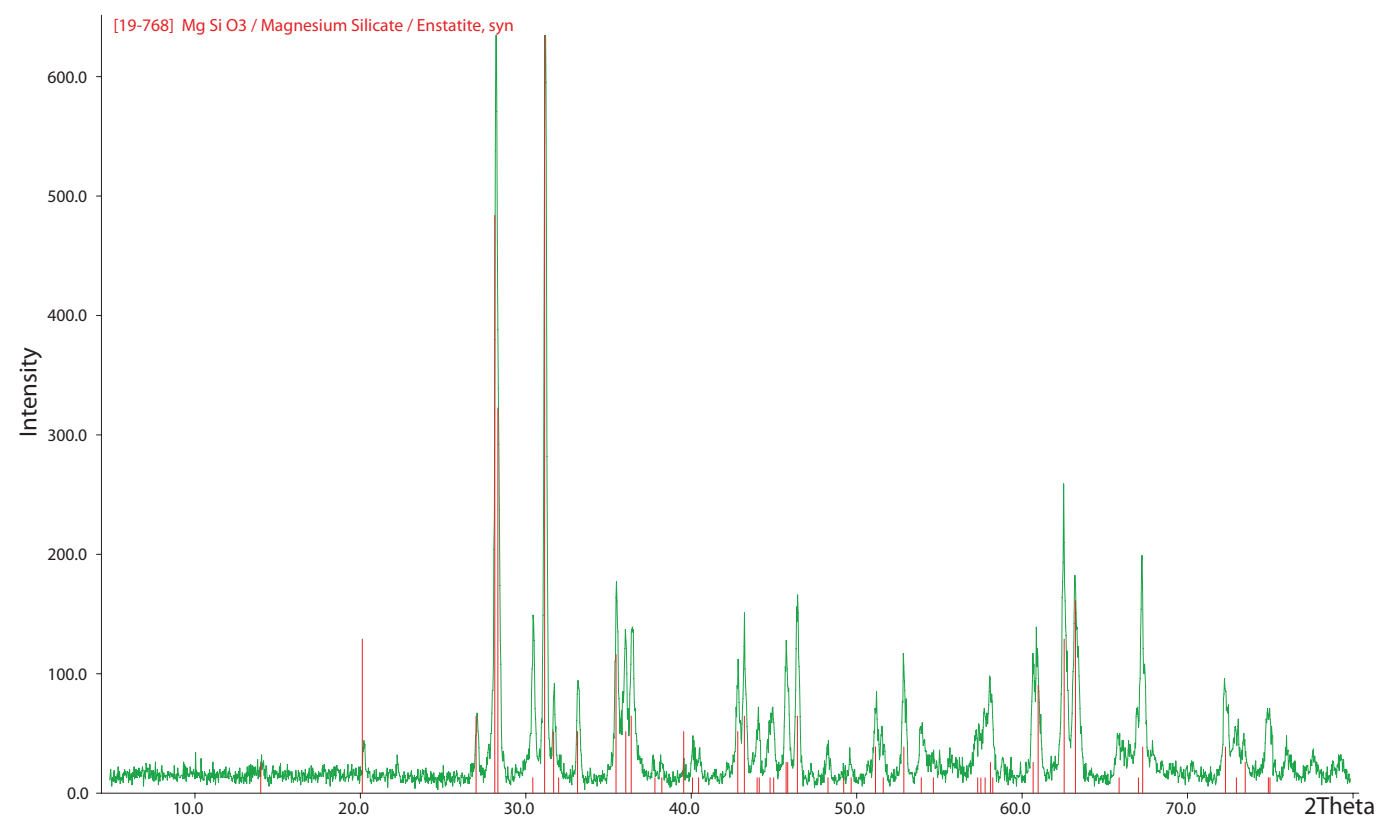

Abbildung 80: Dargestellt ist das Beugungsdiagramm des separierten Enstatit. Der Untergrund ist durch amorphe Anteile im Pulver erhöht. Das Pulver wurde aufgrund geringer Probenmenge als Schwemmpräparat gemessen.

\subsubsection{Pfannensteine}

Bei den untersuchten Feuerfestzustellungen der Pfannen handelt es sich um pechgebundene und getemperte Schmelzmagnesia. Die röntgenographischen Untersuchungen ergaben bei allen Steinen Periklas und Graphit als alleinige Phasen. In Abbildung 81 ist beispielhaft das Beugungsdiagramm des Pfannensteins $\mathrm{C}$ zu sehen.

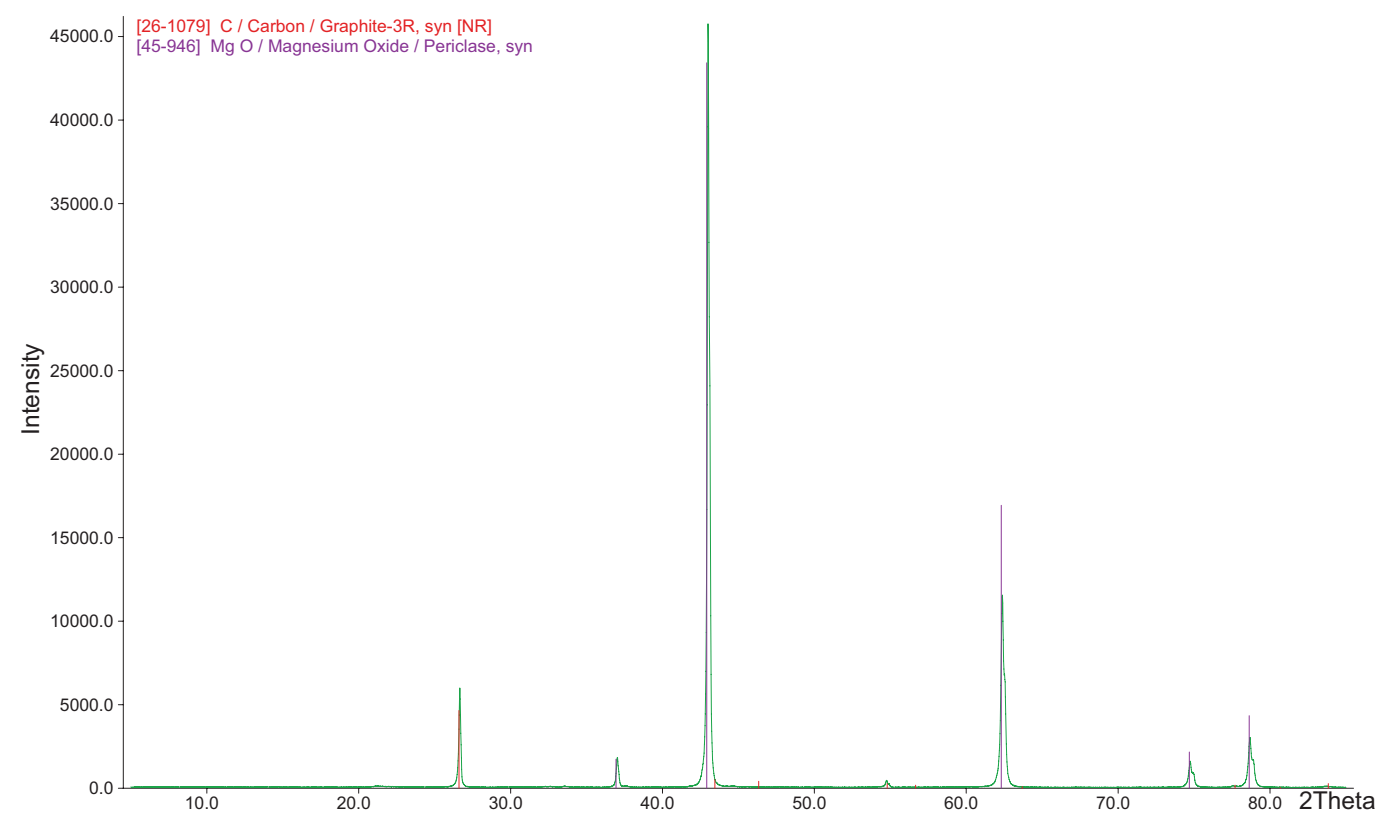

Abbildung 81: Das Beugungsdiagramm zeigt den hochsymmetrischen Periklas des Pfannensteins C, der nur vier Reflexe aufweist. Bei 26 Grad $2 \theta$ liegt der stärkste Peak des Graphits. Ein nennbarer Untergrund ist nicht zu erkennen, was auf einen sehr hohen kristallinen Anteil im Pulver schließen lässt. 
Die quantitative Analyse ergab Periklasanteile zwischen 83 und 96 Gew.-\%. Die Graphitanteile lagen entsprechend von 4 bis 17 Gew.-\% (siehe Tabelle 17). Eine Diffraktometeraufnahme der Spritzmasse des Tundishs ergab ebenfalls Periklas als Hauptphase.

Tabelle 17: Übersicht über die röntgenographisch untersuchten feuerfesten Erzeugnisse der Pfanne.

\begin{tabular}{|c|c|c|c|c|c|c|}
\hline Pfannensteine & $\begin{array}{c}\text { Periklas } \\
\text { Gew.-\% }\end{array}$ & $\begin{array}{c}\text { Graphit } \\
\text { Gew.-\% }\end{array}$ & Bindungsart & Klassifikation & Rohstoffbasis & Anwendung \\
\hline \hline A & 96 & 4 & $\begin{array}{c}\text { getempert, arom. } \\
\text { Kohlenwasserst. }\end{array}$ & $\begin{array}{c}\text { Magnesia- } \\
\text { kohlenstoffstein }\end{array}$ & Schmelzmagnesia & Boden + Wände \\
\hline B & 90 & 10 & $\begin{array}{c}\text { getempert, } \\
\text { pechgebunden }\end{array}$ & $\begin{array}{c}\text { Magnesia- } \\
\text { kohlenstoffstein }\end{array}$ & $\begin{array}{c}\text { Schmelzmagnesia, } \\
\text { Graphit }\end{array}$ & Schlackenzone \\
\hline C & 89 & 11 & - & $\begin{array}{c}\text { Magnesia- } \\
\text { Carbonstein }\end{array}$ & Schmelzmagnesia & Boden + Wände \\
\hline D & 83 & 17 & $\begin{array}{c}\text { getempert, } \\
\text { pechgebunden }\end{array}$ & $\begin{array}{c}\text { Magnesia- } \\
\text { kohlenstoffstein }\end{array}$ & $\begin{array}{c}\text { hochwertige } \\
\text { Schmelzmagnesia }\end{array}$ & Schlackenzone \\
\hline E & 95 & 5 & $\begin{array}{c}\text { getempert, } \\
\text { pechgebunden }\end{array}$ & $\begin{array}{c}\text { Magnesiastein } \\
\text { Schmelzmagnesia }\end{array}$ & Boden + Wände \\
\hline F & 83 & 17 & - & $\begin{array}{c}\text { Magnesia- } \\
\text { Carbonstein }\end{array}$ & Schmelzmagnesia & Schlackenzone \\
\hline
\end{tabular}

\subsubsection{Tauchrohr}

Das FF-Material eines gebrauchten Tauchrohres, das Kontakt zur Stahlschmelze hatte, wurde nach dem Gießen beprobt. Im Beugungsdiagramm in Abbildung 82 wurden Tonerde, Siliziumcarbid und Graphit identifiziert.

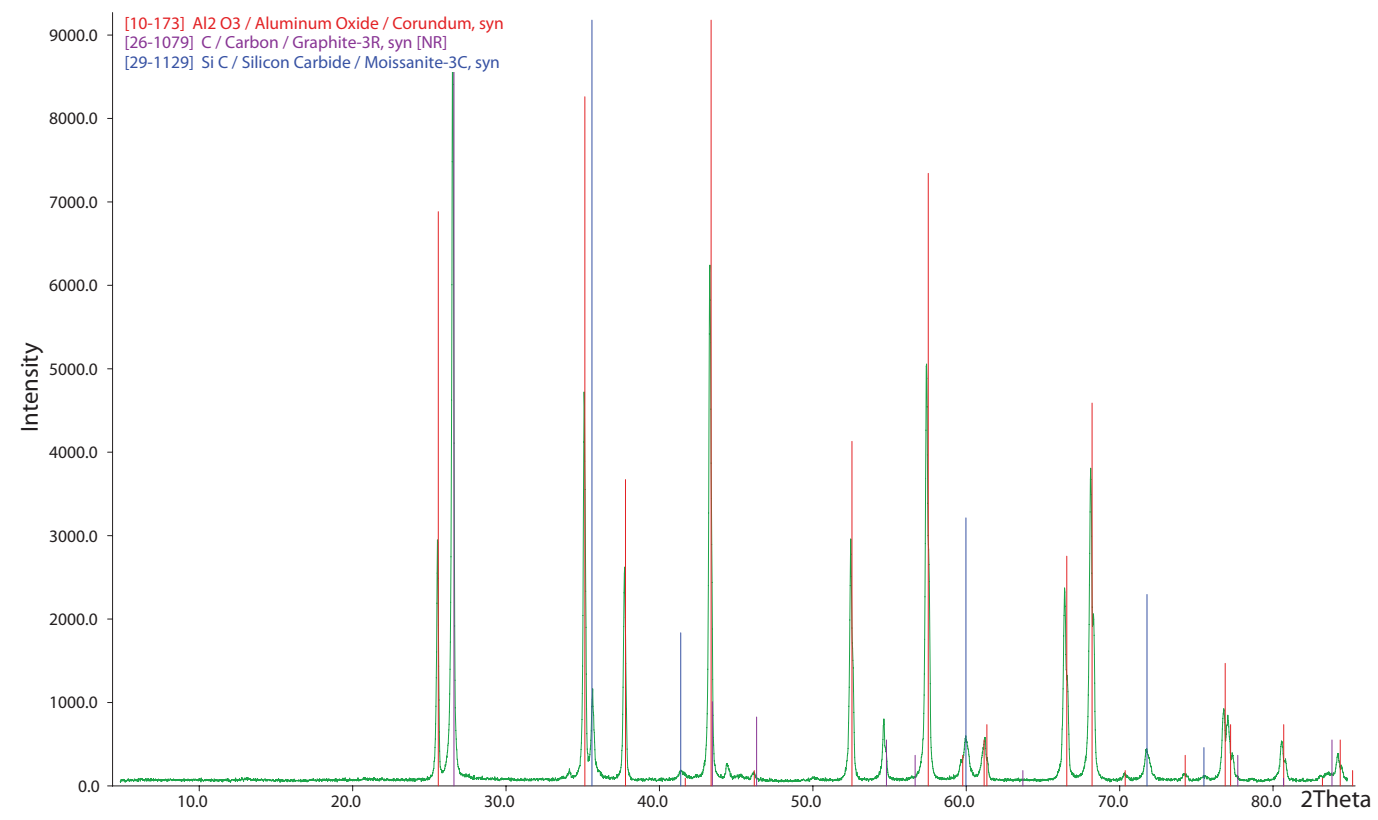

Abbildung 82: Diffraktogramm des FF-Materials eines Tauchrohres, das direkten Kontakt zur Schmelze hatte (nach dem Gießen). 


\subsection{Petrographie und Mineralchemie}

\subsubsection{Clogging}

Die Cloggingproben wurden im Auflicht (Leica-Polarisationsmikroskop) und mittels EMS (JEOL JXA 8900 RL) elektronenoptisch untersucht. Bei den untersuchten Proben lassen sich drei verschiedene Hauptlithologien unterscheiden.

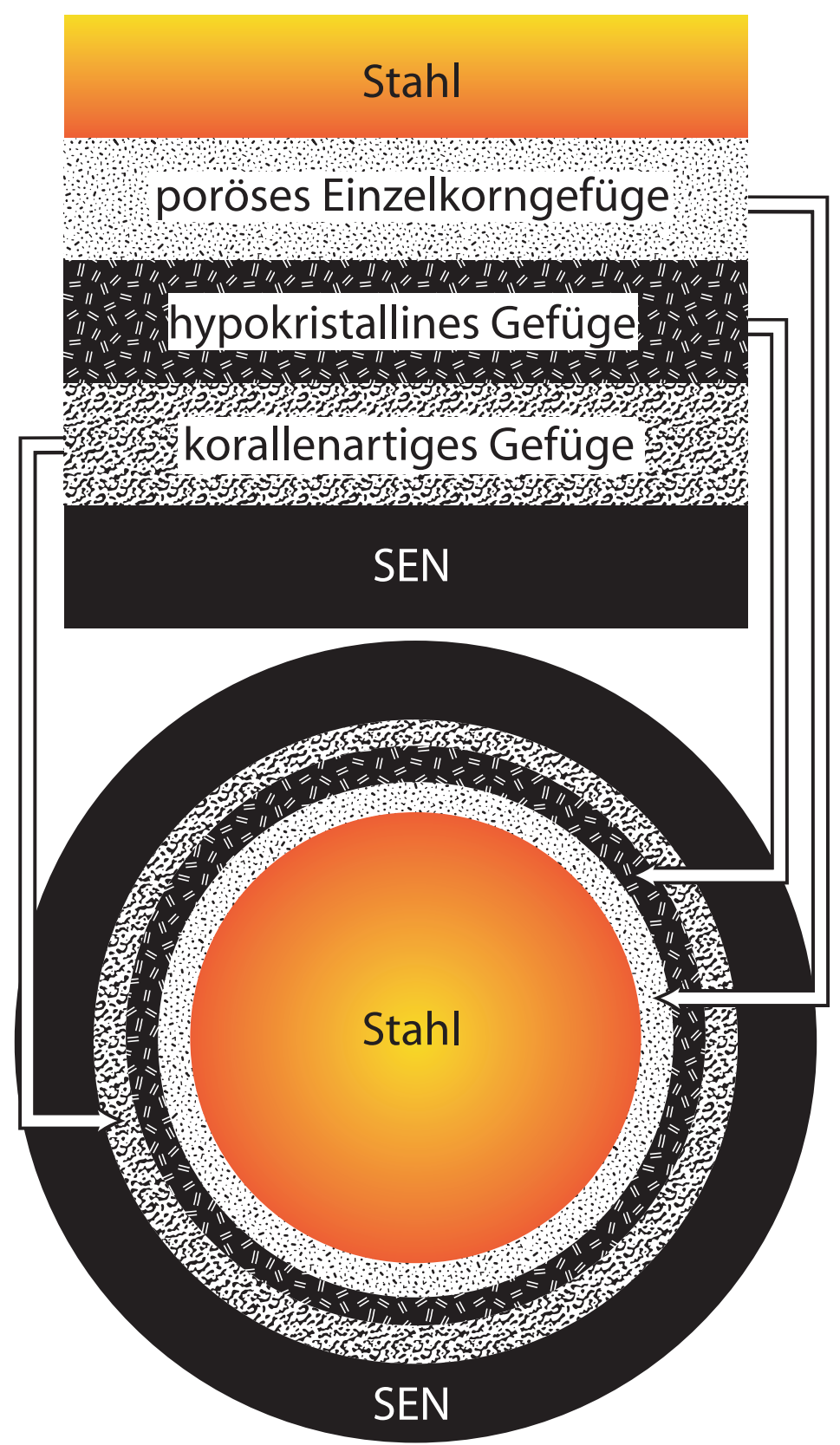

Abbildung 83: Unterteilung von Clogging in drei Hauptlithologien (Aufsichtschnitt senkrecht zur Längsachse des Tauchrohres). Am Kontakt zur Stahlschmelze treten die Körner meist in einem losen Verband auf. In einem Zwischenbereich sind meist isometrische bis leistenförmige Einsprenglinge in einer glasigen Matrix zu beobachten. Am Kontakt zum FF-Material des SEN bildet das Clogging typischerweise ein korallenartiges Netzwerk aus. 
Am Kontakt zur Schmelze hat Clogging eine poröse Struktur. Die mittlere Korngröße liegt bei einigen Mikrometer, die Körner sind von hyp- bis idiomorpher Ausprägung. Der Habitus reicht von rundlichen bis zu nadelig kristallisierten Formen, letztere sind meist kryptokörnig (Korndurchmesser $<2 \mu \mathrm{m}$ ). Die Körner liegen vorwiegend einzeln oder z. T. als Verwachsungsgefüge heterogranular vor. Das Clogging ist in diesen Bereichen korundreich (Abbildung 84). In den Tonerdeeinschlüssen sind bis zu rd. 2 Gew.-\% $\mathrm{TiO}_{2}$ gelöst.

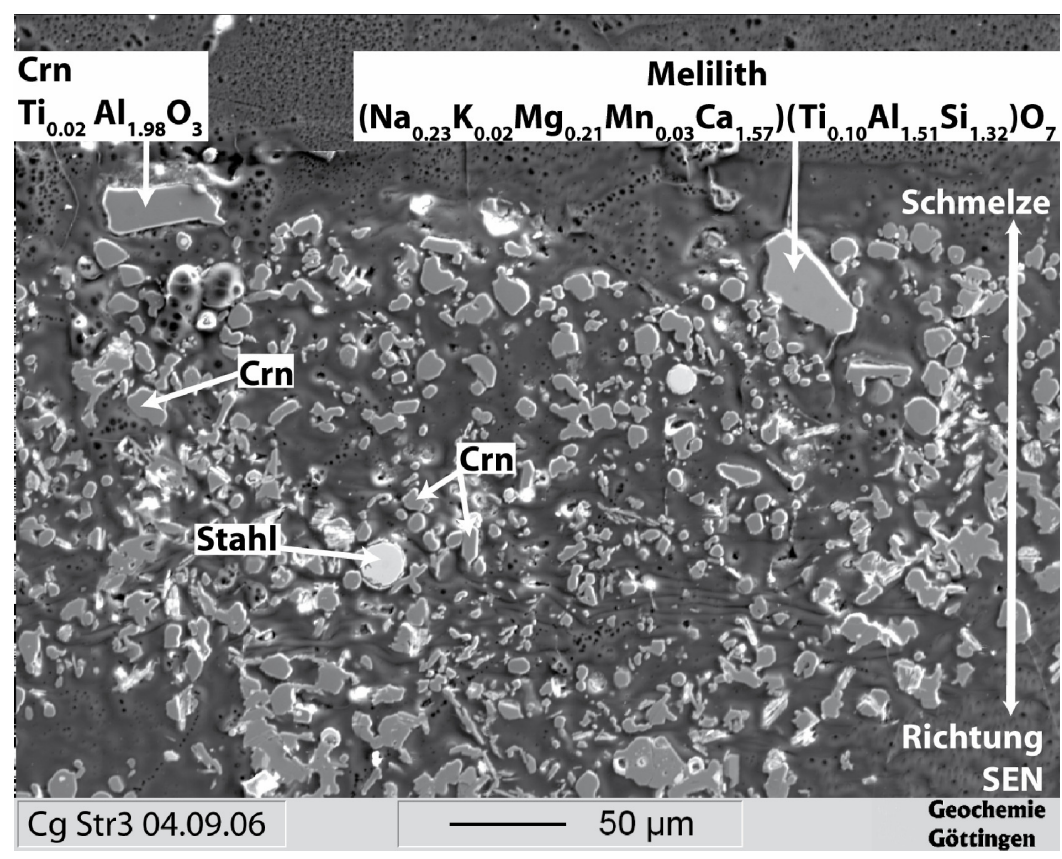

Abbildung 84: Sekundärelektronenbild von porösem, korundreichem Clogging am Kontakt zur Stahlschmelze (Probe vom 04.09.06, Strang 3). Auffallend ist hier ein relativ großes Melilithkorn.

In einem weiteren Hauptbereich, der eine „Sandwichposition“ zwischen dem Clogging mit Schmelzkontakt und dem mit SEN-Kontakt einnimmt, treten Kristalle und Glas nebeneinander auf (hypokristalline Gefügeelemente). Beim Glas handelt es sich durchweg um eine Al-, Si-, Ca-reiche Matrix ( $\sim 30$ Gew.- $\% \mathrm{Al}_{2} \mathrm{O}_{3}, \sim 30$ Gew.-\% $\mathrm{SiO}_{2}, \sim 30$ Gew.-\% CaO). Abbildung 85 zeigt einen typischen Ausschnitt aus diesem Lithologiebereich. Das Gefüge reicht von porphyrisch bei einer bimodalen Korngrößenverteilung zwischen feinkörniger Grundmasse und größeren Einsprenglingen über glomerophyrisch bei mehreren kleinen Agglomeraten aus halbidiomorphen Einsprenglingen und intersertal bei leistenförmigen Hibonitkristallen (siehe Abbildung 86) bis zum panidiomorphen Gefüge bei überwiegend idiomorphen Körnern mit wenig Zwickelmasse (Abbildung 87). Letztere werden vorwiegend durch Spinell- und Wüstitphasen gebildet. In diesem Hauptbereich und weiter Richtung Tauchrohrwandung wurden mehrfach Na- $\beta$-Aluminat-Körner beobachtet mit der durchschnittlichen Mineralformel: $\left(\mathrm{Na}_{0,79} \mathrm{~K}_{0,73} \mathrm{Ca}_{0,02} \mathrm{Mg}_{0,33} \mathrm{Mn}_{0,05} \mathrm{Fe}_{0,12}\right)\left(\mathrm{Al}_{21,37} \mathrm{Ti}_{0,29} \mathrm{Si}_{0,30}\right) \mathrm{O}_{34}$. 


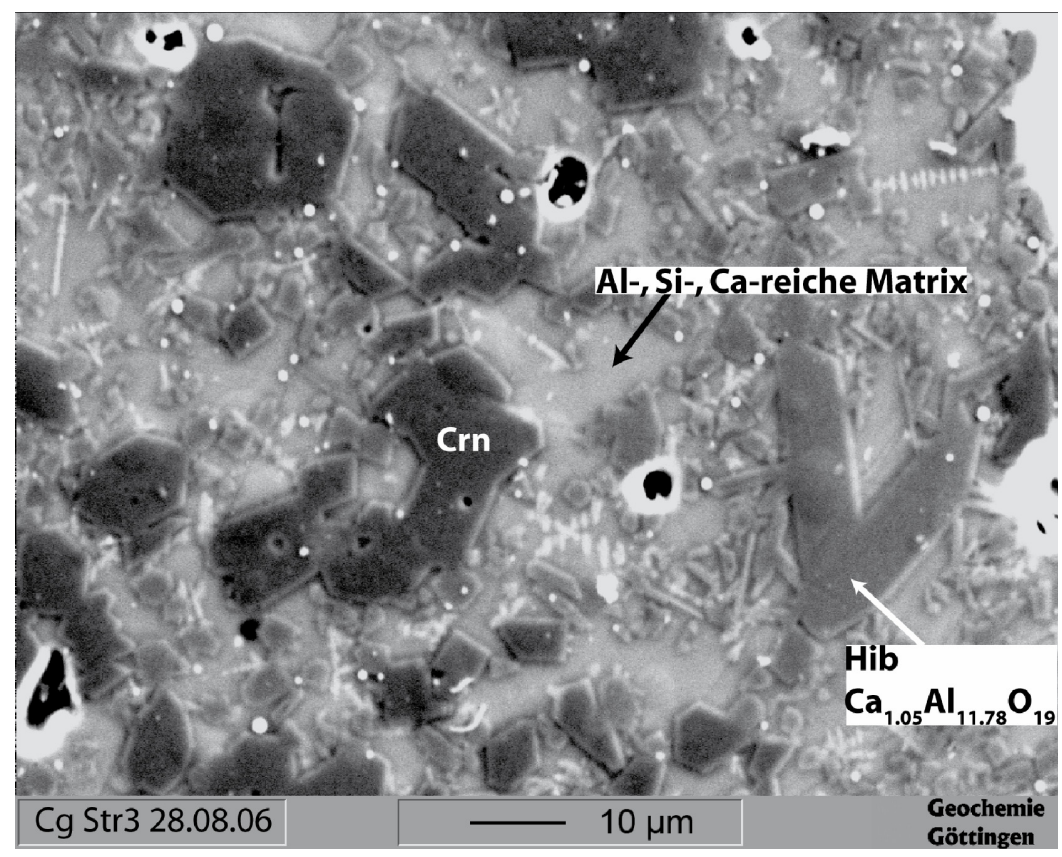

Abbildung 85: Sekundärelektronenbild von Clogging (28.08.06, Strang 3) mit idiomorphen Korundund Hibonitkristallen in einer glasigen Al-, Si-, Ca-Matrix. Aufgrund seiner größeren mittleren Ordnungszahl erscheint der Hibonit relativ zum Korund heller im elektronenoptischen Bild.

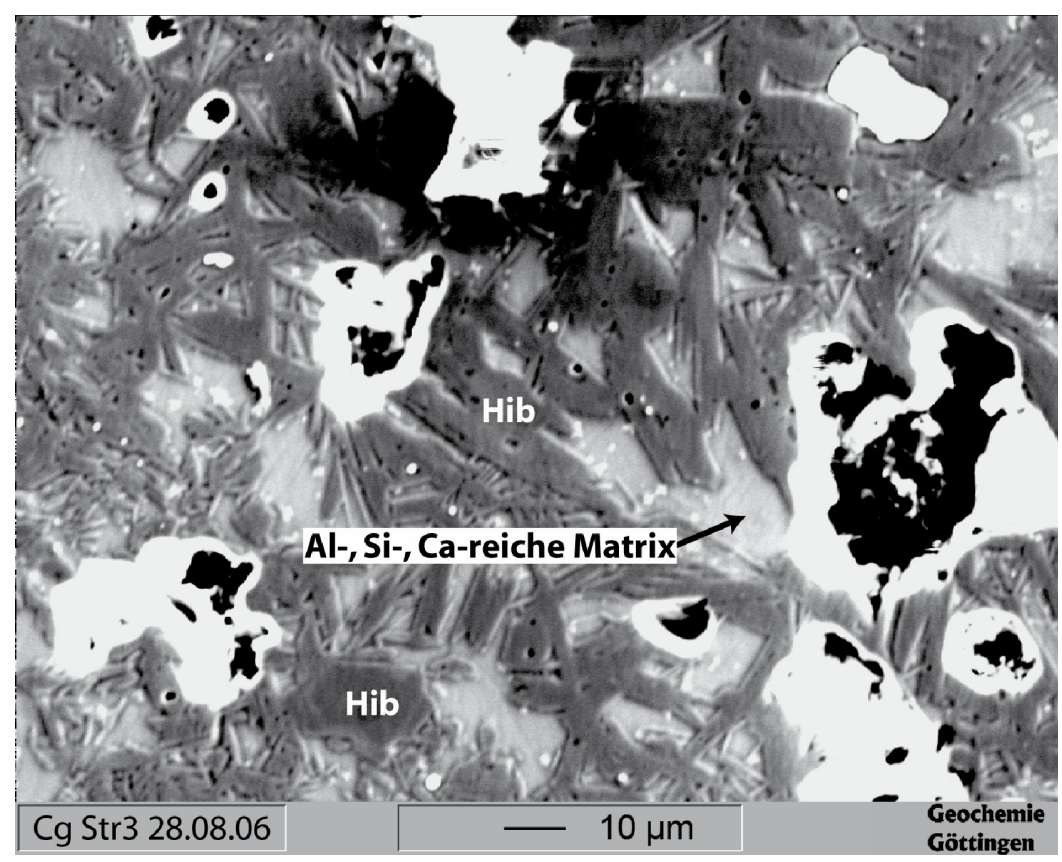

Abbildung 86: Sekundärelektronenbild von Clogging (28.08.06, Strang 3). Die leistenartigen Hiboniteinsprenglinge bilden ein sperriges intersertales Gefüge in glasiger Grundmasse. 


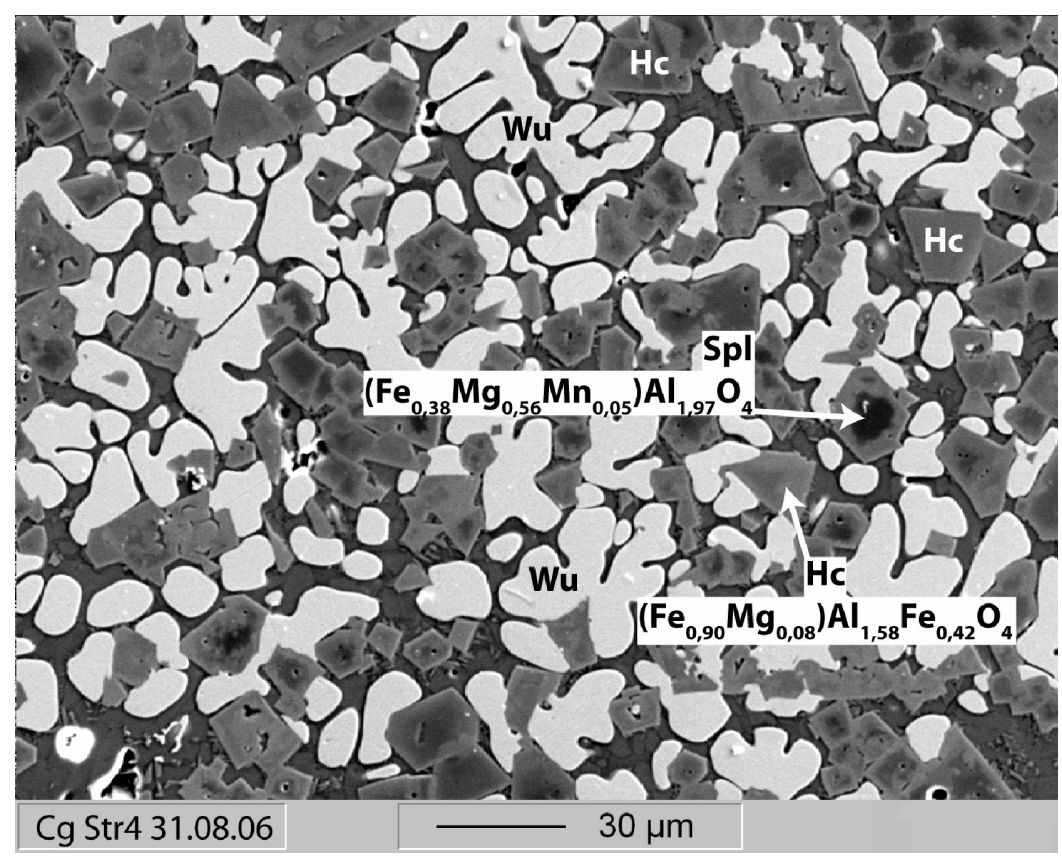

Abbildung 87: Sekundärelektronenbild von Clogging (31.08.06, Strang 4). Hypidiomorphe Hercynitkörner in einer dichten Anordnung mit rundlichen Wüstitkristallen.

Der dritte Hauptlithologiebereich hat direkten Kontakt zum FF-Material des Tauchrohres. Das Clogging bildet hier ein korallenartiges Netzwerk aus (Abbildung 88).

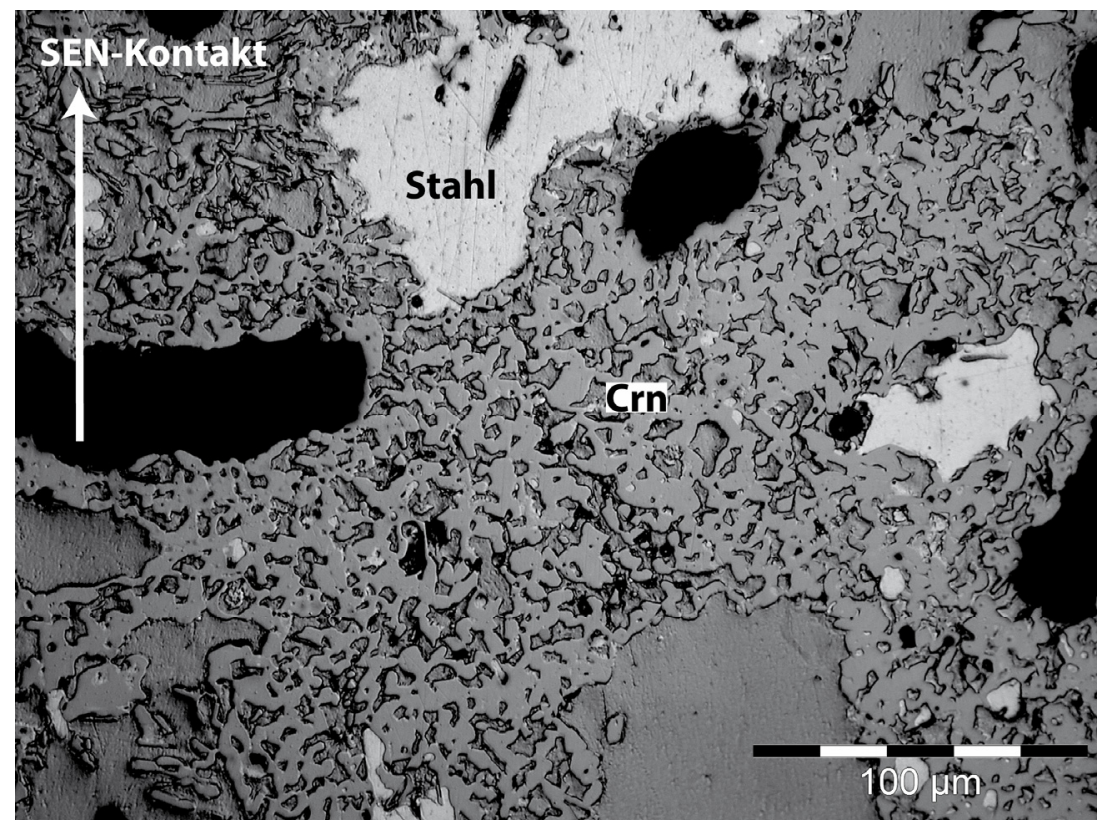

Abbildung 88: Auflichtmikrophotographie von Clogging am Kontakt zum Tauchrohr (Probe vom 29.08.06, Strang 3).

In Abbildung 89 ist ein Überblick über den Grenzbereich von Clogging zum FF-Material des Tauchrohres dargestellt. 


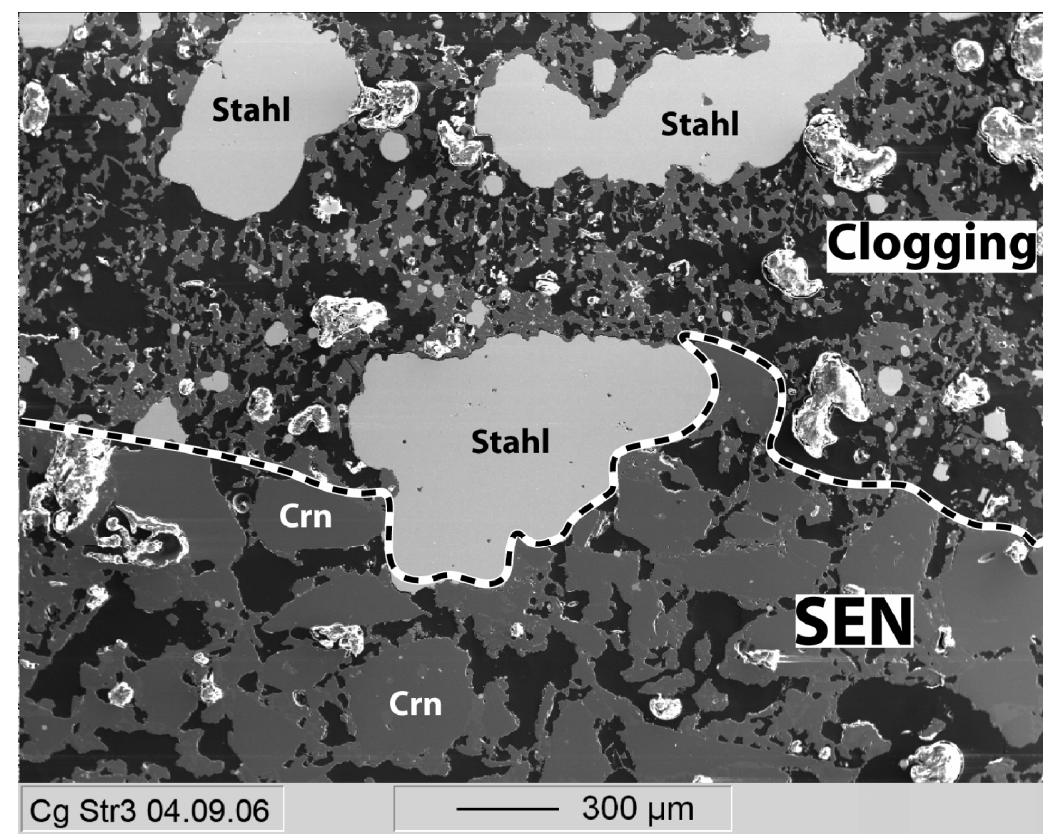

Abbildung 89: Übersicht über den Kontaktbereich von Clogging zum FF-Material des Tauchrohres. Die schwarzweiß gestrichelte Linie zeigt die Grenze an. Die Tonerdekörner des Tauchrohres unterscheiden sich vor allen Dingen in ihrer Größe von denen des Cloggings.

\subsubsection{Schiebersand}

Es wurden vier Schiebersande mikroskopisch und mit Hilfe von Rückstreuelektronenbildern untersucht. Nach einer Rasterbildanalyse besteht der Schiebersand des Herstellers B zu 35 Vol.-\% aus Quarz und 59 Vol.-\% aus Chromit (siehe Abbildung 90). Die Chromit- und Quarzkörner sind z. T. hyp- bis idiomorph ausgebildet.

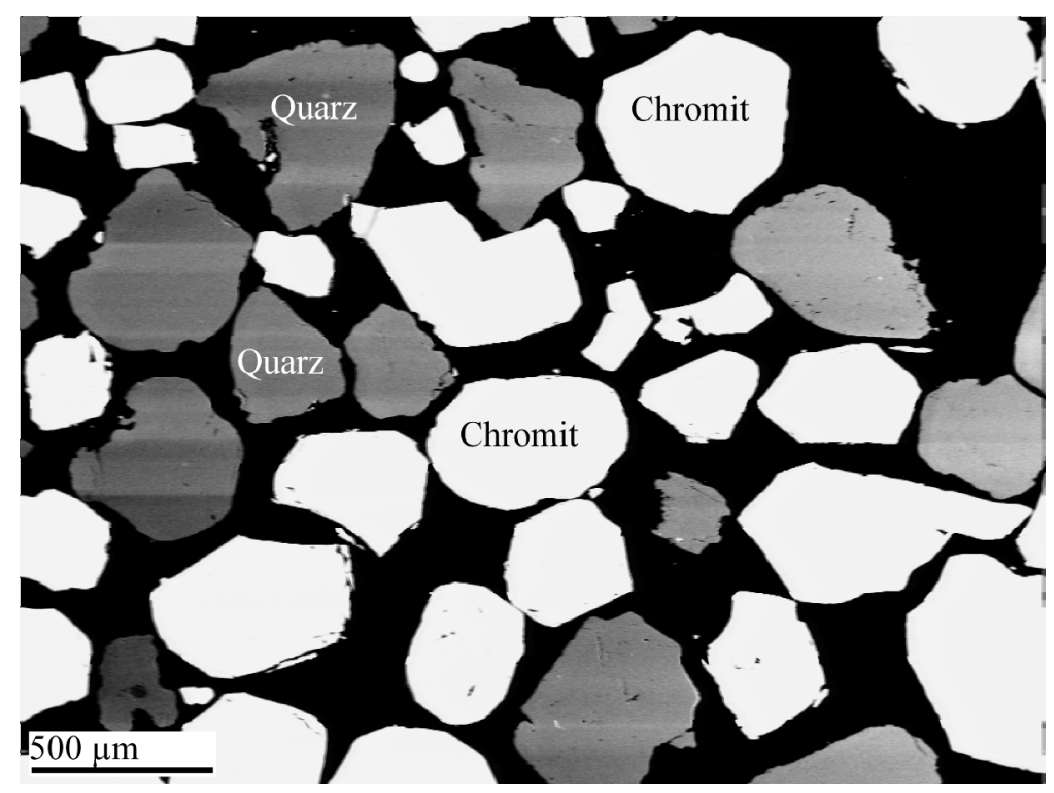

Abbildung 90: Rückstreuelektronenbild des Schiebersandes von Hersteller B.

Ebenfalls kommen im Schiebersand des Herstellers A03 Quarz und feinkörniger, schlecht kristallisierter Mikroklin vor (siehe Abbildung 91 und Abbildung 92). Beide Phasen sind eng 
miteinander verwachsen. Bei den Untersuchungen wurde jeweils nur ein Korn von Klinopyroxen und Mikroklin gefunden.

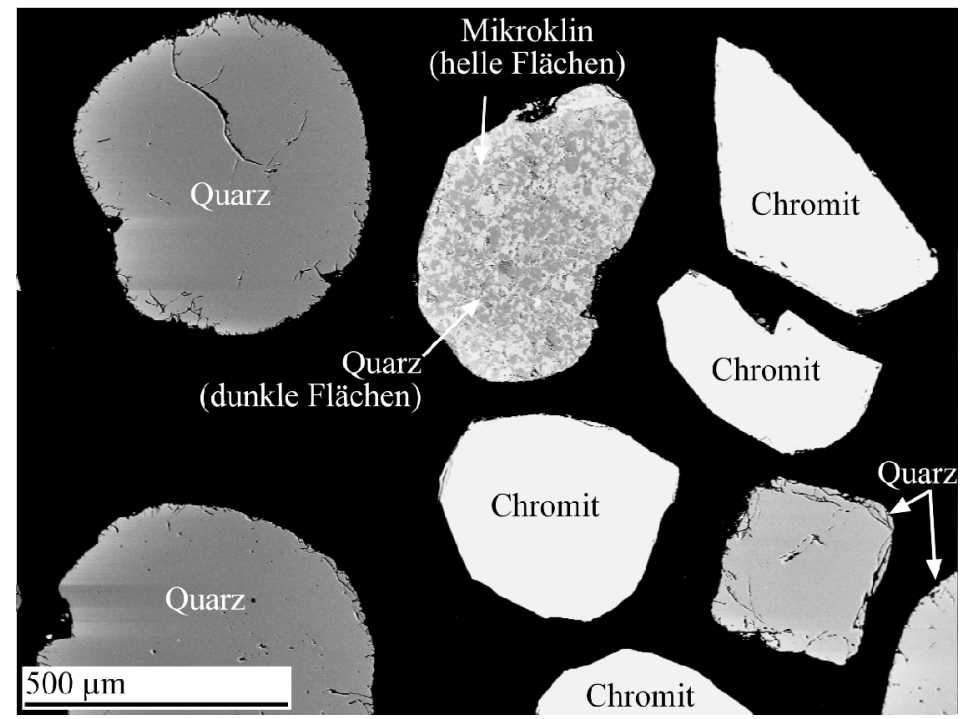

Abbildung 91: Rückstreuelektronenbild gerundeter Quarzkörner und kubischer Chromitkörner (Hersteller A03).

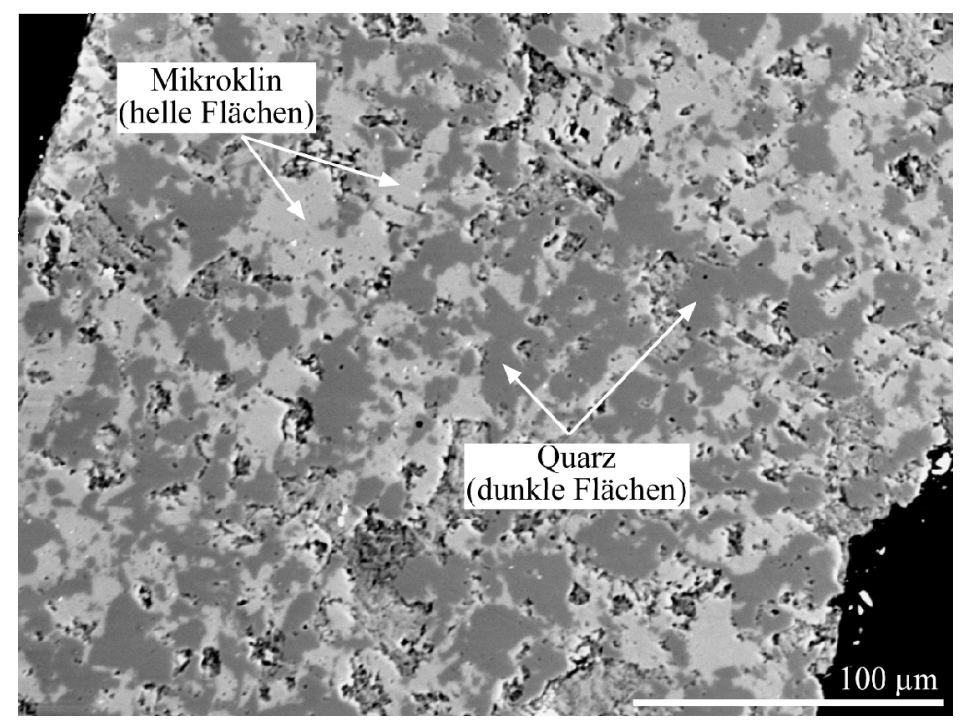

Abbildung 92: Rückstreuelektronenbild des mikrokristallinen triklinen Mikroklins (Kalifeldspat) in Verbindung mit feinkörnigem Quarz (Hersteller A03).

\subsubsection{Pfannensteine}

Das Gefüge der Pfannensteine besteht aus Periklas in mikrokristalliner Kohlenstoffmatrix. Einige Körner erscheinen im Durchlicht weiß und bilden große Einkristalle. Der kubische Periklas zeigt in $\{100\}$ eine Spaltbarkeit (siehe Abbildung 93). Diese großen Minerale steigern mit ihrer hohen Kornrohdichte die Qualität der Schmelzmagnesia, sie werden in der besonders korrosiven Schlackenzone eingesetzt. Andere Körner sind rundlich, kleiner und von rotbräunlicher Farbe. Sie sind korrodiert; an den Korngrenzen sind ausgeprägte Reaktionssäume aus- 
gebildet. Diese Minerale sind zu größeren Periklasfragmenten aggregiert, ähnlich einem sphärolithischen Gefüge (siehe Abbildung 93 und Abbildung 94).

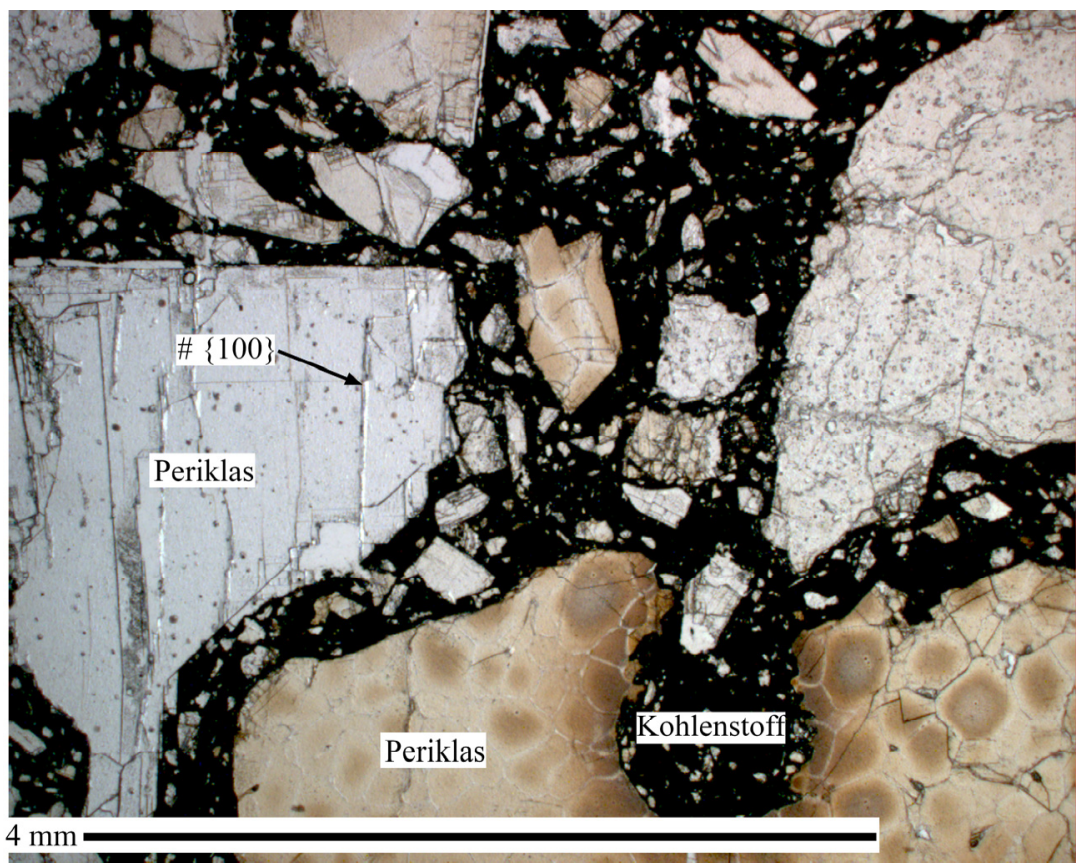

Abbildung 93: Durchlichtmikrophotographie des Pfannensteins D mit rotbräunlichem, korrodiertem Periklas und weißem, höherwertigen Periklas in feinkörniger Kohlenstoffmatrix.

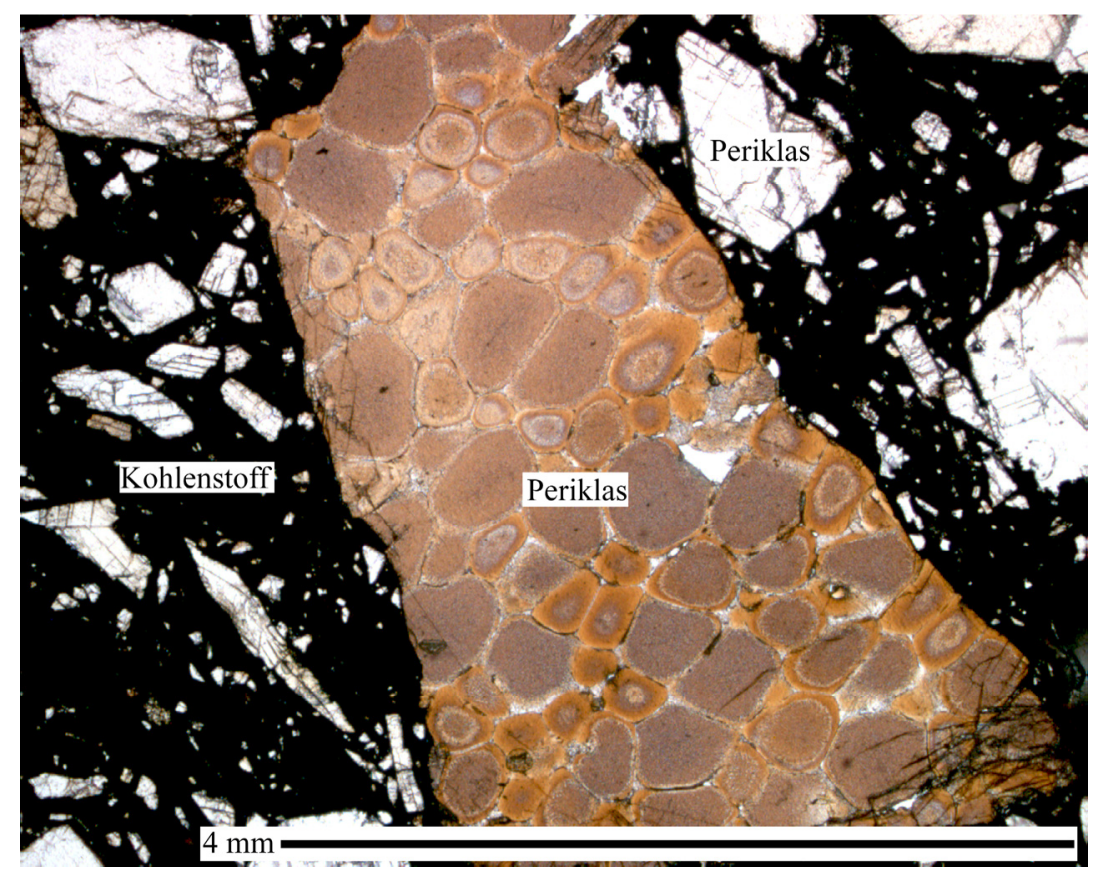

Abbildung 94: Durchlichtmikrophotographie eines getemperten, pechgebundenen MagnesiaKohlenstoffsteins (Pfannenstein B) mit einem großen, rotbräunlichen Periklasfragment neben weißen Periklaskristallen in feinkörniger Kohlenstoffmatrix. 


\subsection{Diskussion}

Die untersuchten oxidischen Ausscheidungen bestehen vorwiegend aus Tonerde und Hibonit. Ein Charakteristikum von Clogging im Vergleich zu SEN-Unterseiten-Präzipitaten ist dessen im Mittel rd. dreimal höherer Spinellgehalt.

Clogging und SEN-Unterseiten-Präzipitate besitzen mit Tonerde und Hibonit zwei domi-

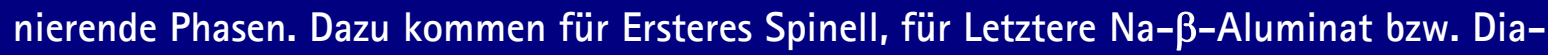
oyudaoit.

Bei den beobachteten Spinellen handelte es sich um eisenreichen Spinell und Hercynit (siehe Abbildung 87), die wahrscheinlich sekundär gebildet wurden. Beim Herausziehen des Tauchrohres aus der Kokille am Ende einer Gießsequenz werden möglicherweise $\mathrm{Al}_{2} \mathrm{O}_{3}$ (z. B. Desoxidationsprodukte), MgO (z. B. von Mitlaufschlacke oder FF-Material) und Fe bei Luftkontakt oxidiert nach:

$$
\left\langle\mathrm{Al}_{2} \mathrm{O}_{3}\right\rangle+(\mathrm{MgO})+2[\mathrm{Fe}]+\frac{1}{2}\left\{\mathrm{O}_{2}\right\} \rightarrow\left\langle\mathrm{Fe}, \mathrm{MgAl}_{2} \mathrm{O}_{4}\right\rangle+(\mathrm{FeO})
$$

Spinell wird durch eine kubisch-flächenzentrierte Zelle von $\mathrm{O}^{2-}$-Ionen mit dem Gitterparameter $\mathrm{a}_{0}=0,808 \mathrm{~nm}$ aufgebaut (SHOU-YONG et al. 2000). In stöchiometrischem Spinell besetzen die $\mathrm{Mg}^{2+}$-Ionen acht Tetraederlücken in der Elementarzelle, in den Oktaederlücken sitzen 16 $\mathrm{Al}^{3+}$-Ionen. Wird $\mathrm{Mg}^{2+}$ durch $\mathrm{Al}^{3+}$ auf der Tetraederposition substituiert, werden überstöchiometrische Spinelle des Typs $\mathrm{MgO} \times n\left(\mathrm{Al}_{2} \mathrm{O}_{3}\right)$ gebildet. Der diadoche Einbau von $\mathrm{Al}^{3+}$ reicht dabei von $n=1$ bis $n=7,3$ (SHOU-YONG et al. 2000 und Referenzen darin). Dies führt zu einer Verkleinerung des Gitterparameter $\mathrm{a}_{0}$, da der Ionenradius von $\mathrm{Al}^{3+}$ (53 pm) kleiner ist als der des $\mathrm{Mg}^{2+}$ (72 pm). Die Rietveldanalyse ergab für die Spinelle der untersuchten oxidischen Ablagerungen einen Gitterparameter von im Mittel $\mathrm{a}_{0}=8,0695 \AA$. Nach HILL et al. (1979) und FISCHER (1967) hat Spinell ein $\mathrm{a}_{0}$ von 8,0832 Å (siehe Abbildung 69). Damit handelt es sich bei diesen Phasen um überstöchiometrische Spinelle, bei denen $\mathrm{Mg}^{2+}$ durch $\mathrm{Al}^{3+}$ substituiert ist.

SEN-Unterseiten-Präzipitate unterscheiden sich vom Clogging insbesondere durch einen im Mittel rd. elfmal höheren Na- $\beta$-Aluminat- bzw. Diaoyudaoitgehalt. Die Ursache für die erhöhten $\mathrm{Na}_{2} \mathrm{O}$ - und $\mathrm{K}_{2} \mathrm{O}$-Gehalte in SEN-Unterseiten-Ablagerungen ist noch nicht geklärt. Gießpulver ist eine Quelle für Alkalien, besitzt aber ein deutlich höheres Na/K-Verhältnis. Reisschalenasche weist im Vergleich zu den oxidischen Ablagerungen ein stark erhöhtes K/Na-Verhältnis auf (Abbildung 95). 


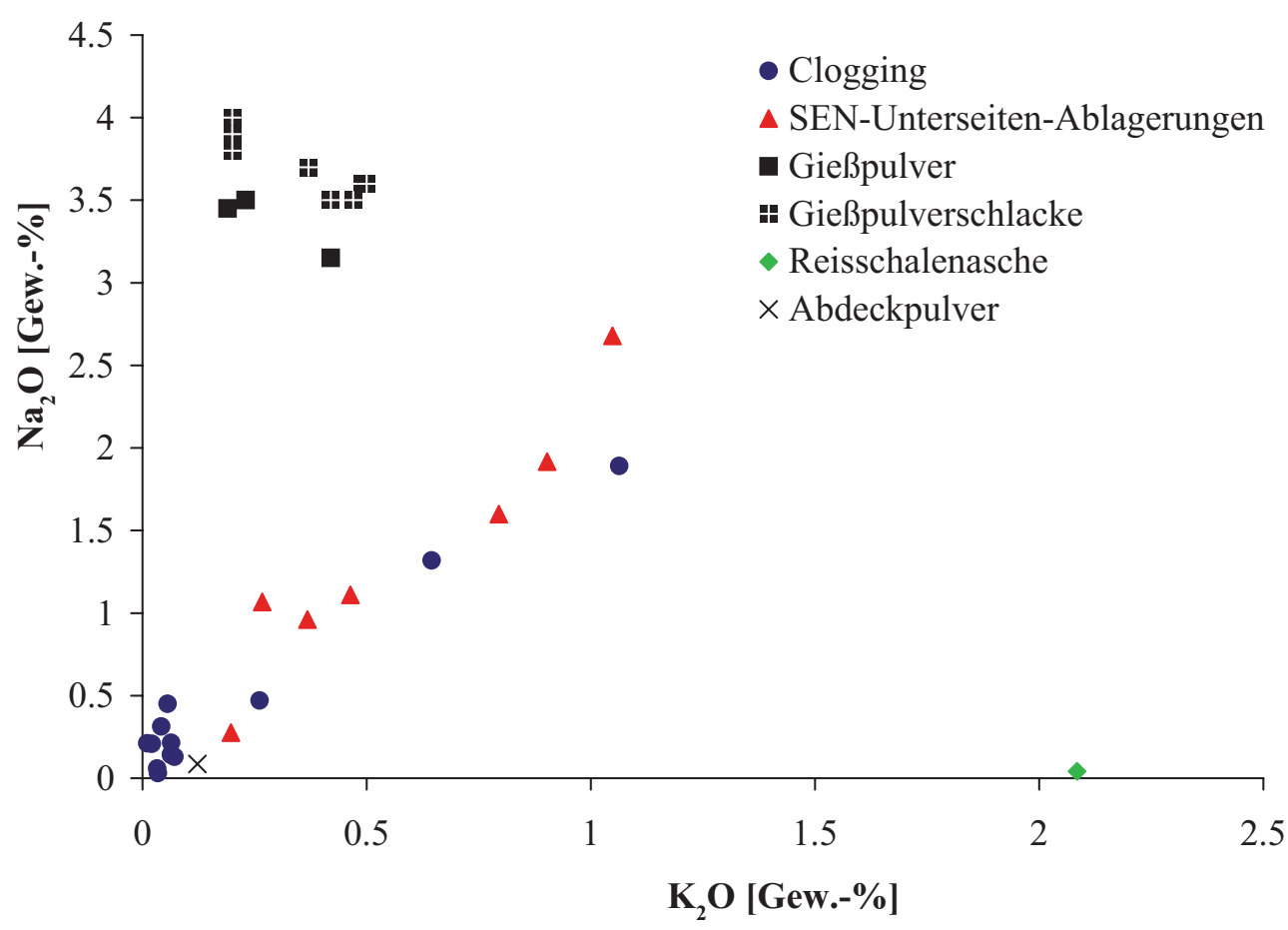

Abbildung 95: $\mathrm{Na}_{2} \mathrm{O}$ gegen $\mathrm{K}_{2} \mathrm{O}$ von oxidischen Ablagerungen und möglichen Alkalienquellen.

Gingen die Gehalte der Alkalien allein auf Gießpulver und Reisschalenasche zurück, müssten die Proben mit den höchsten Na- und K-Gehalten zu etwa 50 Gew.-\% aus Reisschalenasche bestehen. Das würde zu $\mathrm{SiO}_{2}$-Gehalten von über $50 \mathrm{Gew.- \%}$ führen, die bei oxidischen Ausscheidungen im Tauchrohr und an dessen Unterseite nie beobachtet wurden. Dass die Gießpulverschlacke mehr $\mathrm{Na}_{2} \mathrm{O}$ enthält als das Gießpulver selbst, deutete entweder darauf hin, dass es noch eine weitere Alkalienquelle gibt oder dass andere Elemente/Verbindungen aus dem Gießpulver entfernt werden (Abbildung 95). Mikrochemische Untersuchungen ergaben, dass Clogging am Kontakt zum Stahl meist aus reinen Korundkörnern besteht (Abbildung 84); in Richtung der Tauchrohrwandung wurden zunehmend Na- $\beta$-Aluminatphasen beobachtet. Dies kann darauf hindeuten, dass Alkalien während des Gießens aus dem SEN herausgebrannt werden. Die Reduktion von $\mathrm{Na}_{2} \mathrm{O}$ und $\mathrm{K}_{2} \mathrm{O}$ aus dem FF-Material des Tauchrohres könnte zur Bildung von Na- $\beta$-Aluminat beitragen. Dabei würde der Bindungskohlenstoff des FF-Materials mit den Oxiden des Tauchrohres über Redoxreaktionen zu gasförmigem Natrium führen nach:

$$
\left\langle\mathrm{Na}_{2} \mathrm{O}\right\rangle+\langle\mathrm{C}\rangle \rightarrow 2\{\mathrm{Na}\}+\{\mathrm{CO}\}
$$

Die mobilen Gasphasen würden durch den Unterdruck im Tauchrohr Richtung Stahl transportiert und beim Kontakt mit der Schmelze nach folgender Gleichung wieder oxidiert werden:

$$
34\{\mathrm{CO}\}+2\{\mathrm{Na}\}+22[\mathrm{Al}] \rightarrow\left\langle\mathrm{Na}_{2} \mathrm{Al}_{22} \mathrm{O}_{34}\right\rangle+34[\mathrm{C}]
$$


Die Korrelation zwischen Spinell- und MgO-Gehalt (siehe Abbildung 68) zeigt einen stark positiven Zusammenhang. Der Fehler der Rietveldanalyse liegt hierfür bei rd. $\pm 3 \%$. Damit zeigt die Rietveldanalyse, dass Magnesium im Clogging fast ausschließlich im Spinell vorkommt. Die ebenfalls gute Korrelation zwischen Hibonit- und CaO-Gehalt zeigt Entsprechendes für das Calcium (siehe Abbildung 70).

Der Tonerdegehalt von Clogging korreliert in einem fast perfekt positiven Zusammenhang mit dem Netto- $\mathrm{Al}_{2} \mathrm{O}_{3}$-Gehalt von Clogging (Abbildung 71). Bei null Prozent Tonerde liegt der $\mathrm{Al}_{2} \mathrm{O}_{3}$-Gehalt bei 13 Gew.-\%, er müsste jedoch bei null liegen. Dieser Versatz im $\mathrm{Al}_{2} \mathrm{O}_{3}$ Gehalt deutet entweder auf eine fehlende Phase oder auf einen fehlenden Glasanteil hin. In den Diffraktogrammen wurden alle Hauptphasen identifiziert, allerdings wurde bei der Rietveldrechnung nicht der Glasanteil quantifiziert. Tonerdearme Proben weisen die höchsten Hibonit- und Glasgehalte auf. Das Calcium stammt entweder aus dem CaO-reichen Abdeckpulver der Tundishschlacke oder aus der Pfannenschlacke und wird wahrscheinlich als flüssige Mitlaufschlacke in das Clogging eingetragen. Legt man in Abbildung 71 durch die Cloggingdatenpunkte eine Regressionsgerade, die durch den Ursprung geht, öffnet sich mit abnehmenden Tonerde- und $\mathrm{Al}_{2} \mathrm{O}_{3}$-Gehalten eine „Schere“. Dieser Bereich kann einem fehlenden Glasanteil zugeordnet werden und den Versatz im $\mathrm{Al}_{2} \mathrm{O}_{3}$-Gehalt erklären.

Abbildung 73 zeigt eine gute Korrelation zwischen $\delta^{18} \mathrm{O}$ und dem Spinellgehalt von Clogging. Nach der Regressionsgeraden müsste Clogging, das zu 100 Gew.-\% aus Spinell besteht, einen $\delta^{18} \mathrm{O}$ von $\sim+22 \%$ aufweisen. Die MgO-Quellen im Prozess haben $\delta^{18} \mathrm{O}-$ Werte von +7 bis $+13 \%$ (VTR-Masse und Pfannensteine). MgO-Quellen mit solch hohen $\delta^{18} \mathrm{O}-$ Werten wurden nicht gemessen. In diesem Fall können auch über die Kopplung von Isotopie mit quantitativer Mineralogie die Anteile von VTR-Masse und/oder Pfannensteinen am Clogging nicht bestimmt werden.

In Kapitel 3.4.1.1 wurde gezeigt, dass Cloggingproben mit einem $\mathrm{Al}_{2} \mathrm{O}_{3}$-Gehalt $>94 \%$ im Mittel einen $\delta^{18} \mathrm{O}$ von ungefähr $+5 \%$ haben. Entsprechendes zeigt sich bei der Darstellung von $\delta^{18} \mathrm{O}$ gegen den Tonerdegehalt von Clogging (Abbildung 72). Der $\delta^{18} \mathrm{O}-$ Wert nimmt mit dem Tonerdegehalt ab. Bei über 80 Gew.-\% Tonerde liegt der Mittelwert für Clogging bei rd. $+5 \%$. Damit kommen Chemie und Mineralogie zum gleichen Ergebnis.

Die Beugungsdiagramme der Tundishschlacken zeigen bei drei Proben erhöhte Gehalte von Chromit (siehe Kapitel 4.2.2). Dabei handelt es sich um Chromit aus dem Schiebersand, der von der Schlacke absorbiert wurde. Die niedrige Isotopie des Chromits hat einen Einfluss auf den $\delta^{18} \mathrm{O}$ der Tundishschlacke. Bei dem in Kapitel 3.4.4 aufgestellten Vierkomponentenmo- 
dell wird allein von assimilierten Tonerdeeinschlüssen in der Tundishschlacke ausgegangen. Die neueren Untersuchungen zeigen, dass aufsteigender Chromit hierbei auch zu berücksichtigen ist. Die anderen Tundishschlackenproben enthielten überwiegend Glas, was die Identifizierung kristalliner Phasen verhinderte. Der beobachtete Gehlenit in der Tundishschlacke geht auf die Bildung niedrigschmelzender Eutektika von $\mathrm{CaO}$ und $\mathrm{Al}_{2} \mathrm{O}_{3}$ aus dem Abdeckpulver mit $\mathrm{SiO}_{2}$ aus der Reisschalenasche zurück. Der kristalline Anteil dieser Proben besteht aus rd. 80 Gew.-\% Abdeckpulver und rd. 20 Gew.-\% Reisschalenasche. 


\section{Diskussion}

\section{Wozu können Sauerstoffisotope im Zusammenhang mit Clogging nützlich sein?}

In dieser Studie wurden Sauerstoffisotope als Marker genutzt, um die Herkunft nichtmetallischer Ablagerungen in Tauchrohren und an deren Unterseiten beim Stranggießen von Stahl zu klären.

\section{Welche Zusammensetzung hat Clogging?}

Nichtmetallische Ablagerungen gehen hauptsächlich auf die Oxidation des im Stahl gelösten Aluminiums zurück (siehe Kapitel 2.1 und 12.1) (BANNENBERG 1995, PlesCHIUTSCHNIGG 1989, ZIMMERMANN 1974). Die analysierten Ausscheidungen variieren im $\delta^{18} \mathrm{O}$ von +2 bis $+13 \%$ (siehe Kapitel 2.4.1.4 und 3.4.1.1). Die Sauerstoffisotopie von Clogging im Tauchrohr ist vergleichbar mit der von SEN-Unterseiten-Präzipitaten (siehe Abbildung 36). Die chemische Zusammensetzung nichtmetallischer Ausscheidungen wird mit 89 bis 98 Gew.-\% durch $\mathrm{Al}_{2} \mathrm{O}_{3}$ dominiert, was für aluminiumberuhigte Stähle typisch ist (PACK et al. 2005). Die hohen $\mathrm{Al}_{2} \mathrm{O}_{3}$-Gehalte spiegeln sich in hohen Gehalten von Tonerde (10 bis 95 Gew.-\%) und Hibonit (5 bis 90 Gew.-\%) wider (siehe Kapitel 4.2.1). Aus der isotopischen, chemischen und mineralogischen Zusammensetzung wird geschlussfolgert, dass es sich bei Clogging und SENUnterseiten-Präzipitaten um genetisch gleiche Materialien handelt.

\section{Gibt es Isotopenfraktionierung bei der Oxidation von Aluminium aus Stahlschmelzen?}

Eine Grundlage, um Sauerstoffisotope zu nutzen, ist, dass bei der Oxidation gelösten Aluminiums keine Fraktionierung zwischen O-Quelle und gebildeter Tonerde auftritt. In Teil I und II sowie in einer Voruntersuchung wurden Hochtemperaturexperimente durchgeführt, mit denen überprüft werden sollte, ob Isotopenfraktionierung bei der Oxidation von Al aus flüssigen Stahlschmelzen auftritt. Es wurden Fe,Al-Legierungen mit 0,28, 0,68, und 0,87 Gew.-\% sowie reines Al bei einer Sauerstofffugazität von $\log f \mathrm{O}_{2}=-14,8$ bis $-10,5$ in einem $\mathrm{H}_{2} / \mathrm{CO}_{2}$ Gasstrom bei $1600^{\circ} \mathrm{C}$ eine Stunde lang kontrolliert partiell oxidiert (siehe Kapitel 2.3 und 3.3). Eine Stahlschmelze mit 0,03 Gew.-\% Al puffert einen $\log f \mathrm{O}_{2}$ von $-14,9\left(1550^{\circ} \mathrm{C}\right)$ und gleicht im $f \mathrm{O}_{2}$ den reduziertesten Experimenten. Durch die reduzierenden Bedingungen bei den Experimenten dieser Studie wurde lediglich Tonerde, aber nicht wie in den Vorversuchen $\left(\log f \mathrm{O}_{2}=-4,0\right)$ Hercynit oder Wüstit gebildet. Der $\delta^{18} \mathrm{O}$ der synthetisierten Tonerde und der der Ofenatmosphäre wurden gasmassenspektrometrisch bestimmt. Die Fraktionierung zwischen Ofenatmosphäre und $\mathrm{Al}_{2} \mathrm{O}_{3}$ variierte zwischen $\Delta^{18} \mathrm{O}_{\text {Ofenatmosphäre - gebildeter Tonerde }}=$ 
$+13,8 \%\left(\log f \mathrm{O}_{2}=-14,8\right)$ und $\Delta^{18} \mathrm{O}_{\text {Ofenatmosphäre - gebildeter Tonerde }}=+10,6 \%$ o $\left(\log f \mathrm{O}_{2}=-10,5\right)$.

Bei den Vorversuchen wurde bei $\log f \mathrm{O}_{2}=-4,0$ eine Fraktionierung von $\Delta^{18} \mathrm{O}_{\text {Ofenatmosphäre - }}$ gebildeter Tonerde $=+3,4 \%$ festgestellt. Je höher $\mathrm{fO}_{2}$ war, desto niedriger war die Fraktionierung zwischen der gebildeten Tonerde und der Ofenatmosphäre (siehe Abbildung 33). Der AlOxidationsgrad der Legierungen liegt zwischen 35 und 99\%. Es ließ sich kein Zusammenhang zwischen dem Oxidationsgrad der Legierung und dem $\delta^{18} \mathrm{O}$ der gebildeten Tonerde feststellen (siehe Abbildung 23). Auch zeigte sich keine Korrelation zwischen dem Al-Ausgangsgehalt der Legierungen und dem $\delta^{18} \mathrm{O}$ der gebildeten Tonerde (siehe Abbildung 23).

Bei Temperaturen von $1600^{\circ} \mathrm{C}$ liegen in einem $\mathrm{H}_{2} / \mathrm{CO}_{2}$-Gasstrom neben den Einsatzgasen vorwiegend $\mathrm{CO}$ und $\mathrm{H}_{2} \mathrm{O}$ als oxidierende Spezies vor. Nach Berechnungen von RICHET et al. (1977) beträgt die Gleichgewichtsfraktionierung bei $1300^{\circ} \mathrm{C}$ zwischen $\mathrm{O}_{2}$ und $\mathrm{CO} 3,7 \%$ und zwischen $\mathrm{O}_{2}$ und $\mathrm{H}_{2} \mathrm{O}$ 2,4\%o. Es wird angenommen, dass die Gleichgewichtsfraktionierung zwischen $\mathrm{CO}$ bzw. $\mathrm{H}_{2} \mathrm{O}$ und Tonerde so ähnlich ist wie die zwischen $\mathrm{O}_{2}$ und Tonerde $\left(\Delta^{18} \mathrm{O}_{\mathrm{O}_{2} \text {-Tonerde }}=+0,4 \%\right.$, PACK et al. 2005) (siehe Kapitel 2.3.4). Obwohl dieser Wert mit einiger Unsicherheit behaftet ist, zeigt die Größenordnung, dass die beobachtete Fraktionierung mit der Gleichgewichtsfraktionierung allein nicht erklärt werden kann. Bedingungen für einen Rayleighprozess waren nicht gegeben, da das Sauerstoffangebot im Gasstrom bei den Experimenten immer gleich war.

Kinetische Fraktionierung wäre eine mögliche Ursache für die beobachtete große Fraktionierung. In Gasen ist die mittlere kinetische Energie $E_{\text {kin }}$ aller Teilchen gleich groß mit:

$$
E_{\text {kin }}=\frac{1}{2} m v^{2}
$$

für alle Massen $m$ und Geschwindigkeiten $v$. CO ist ein Sauerstoffträger in der Ofenatmosphäre. Das Verhältnis der Geschwindigkeiten $v$ von ${ }^{12} \mathrm{C}^{16} \mathrm{O}$ zu ${ }^{12} \mathrm{C}^{18} \mathrm{O}$ ist:

$$
\frac{v\left({ }^{12} \mathrm{C}^{16} \mathrm{O}\right)}{v\left({ }^{12} \mathrm{C}^{18} \mathrm{O}\right)}=\sqrt{\frac{30}{28}}=1,035=\alpha
$$

Die Geschwindigkeit von ${ }^{12} \mathrm{C}^{16} \mathrm{O}$ ist um 3,5\% höher als die von ${ }^{12} \mathrm{C}^{18} \mathrm{O}$. Für $\mathrm{CO}_{2}$ ist $\alpha=$ 1,022, für $\mathrm{H}_{2} \mathrm{O}$ ist $\alpha=1,054$ (siehe Tabelle 6). Leichte Isotopologe bewegen sich schneller als schwere und kollidieren häufiger mit der Legierung (siehe Abbildung 25). Nach einer Modellvorstellung wird die Bildung von $\mathrm{Al}_{2} \mathrm{O}_{3}$ in eine Hin- und eine Rückreaktion eingeteilt. Bei der Hinreaktion wird durch kinetische Fraktionierung isotopisch leichte Tonerde gebildet. Für 


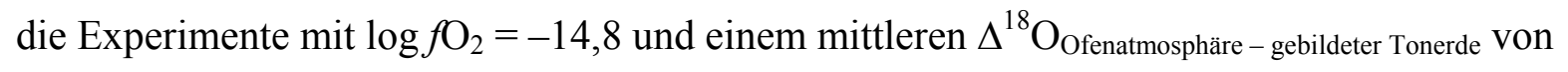
$+12,9 \%$ kann die durch CO gebildete Tonerde maximal 34,5\%o leichter sein als das CO. Diese Oxidation des Al durch CO wird hier als Hinreaktion bezeichnet. Durch die Rückreaktion wird eine Equilibrierung der gebildeten Tonerde mit dem Ofengas angestrebt. Für CO als oxidierende Spezies findet diese zu rd. 50\% statt (siehe auch Abbildung 26).

Die Ergebnisse der Experimente zeigen, dass es bei der Oxidation von Al aus flüssigen Stahlschmelzen bei Temperaturen von $1600^{\circ} \mathrm{C}$ zu einer erheblichen O-Isotopenfraktionierung kommen kann. Die beobachteten Zusammenhänge sind völlig neu, bisher wurde angenommen, dass bei solch hohen Temperaturen, wie sie bei der Stahlherstellung herrschen, keine Fraktionierung bei der Oxidation legierten Aluminiums auftritt (PACK 2000, PACK et al. 2005).

Bei der Stahlherstellung enthält die Schmelze nach dem Frischen in der Regel zwischen 600 und 1000 ppm O, der beim Desoxidieren durch die Zugabe von Al abgesättigt wird (siehe TOULOUSE et al. in press und Referenzen dort, sowie Kapitel 1.4 und 2.3.4). Der O-Gehalt der Schmelze sinkt dabei auf 37 ppm (DEKKERS 2003, VERMEULEN et al. 2000) bzw. bei ULCGüten auf 27 ppm (DEKKERS et al. 2004a, DEKKERS et al. 2004b) ab. Damit werden beim Desoxidieren zwischen 94 und 97\% des gelösten Sauerstoffs umgesetzt. Daher sind die Experimente nicht auf das Desoxidieren anzuwenden, da kinetische Fraktionierung bei einem quantitativen Umsatz keine Rolle spielt.

\section{Welche Isotopie hat Luft-O $\mathrm{O}_{2}$ im Vergleich zu Clogging? Spielt kinetische Fraktionierung}

\section{hier eine Rolle?}

Die untersuchten oxidischen Präzipitate in und unter Tauchrohren weichen im $\delta^{18} \mathrm{O}$ stark von dem des Luft- $\mathrm{O}_{2} \mathrm{ab}$, daher erscheint ihre Bildung durch einfache Reoxidation des Stahls an Luft unwahrscheinlich (ToulousE et al. in press). Ein potentieller Prozess, der zur Reoxidation des Stahls führen könnte, wäre die Diffusion von Luft- $\mathrm{O}_{2}$ durch das Tauchrohr. Nach TUTTLE et al. (2004) wird die Bildung von Clogging durch eine etwaige Permeabilität von Tauchrohren nicht beeinflusst, was darauf hindeutet, dass Clogging nicht auf Partikel zurückgeht, die erst im Tauchrohr gebildet wurden. Ein solcher Diffusionsprozess entspricht durch den kontinuierlichen Gasfluss eher den experimentellen Bedingungen als dem Desoxidieren in der Pfanne und würde also niedrige $\delta^{18} \mathrm{O}$-Werte oxidischer Ablagerungen ergeben. Bei der Diffusion von Luft durch das Tauchrohr würde bevorzugt isotopisch leichter Sauerstoff das Aluminium im Stahl oxidieren. Auf diese Weise könnten kinetisch fraktionierte Tonerdepartikel einen niedrigeren $\delta^{18} \mathrm{O}$ als Luft- $\mathrm{O}_{2}$ aufweisen. Bei diesem Prozess wäre $f \mathrm{O}_{2}$ wahrschein- 
lich deutlich unter dem der Luft mit $\log f \mathrm{O}_{2}=0,21$. Bei den Vorversuchen wurde bei $\log f \mathrm{O}_{2}$ $=-4,0$ eine Fraktionierung von rd. 3\%o festgestellt. Daraus wird abgeleitet, dass Tonerdeeinschlüsse, die jedoch durch direkten Kontakt des Stahls mit Luft z. B. beim Angießen im Tundish gebildet wurden, um weniger als 3\%o von der Isotopie des $\mathrm{Luft}_{2} \mathrm{O}_{2}$ abweichen müssten. Dies setzt voraus, dass $\mathrm{O}_{2}$ gleichermaßen reaktiv ist wie die oxidierenden Spezies bei den Experimenten, da sonst die Equilibrierung durch das reaktionsträge $\mathrm{O}_{2}$ herabgesetzt wird und trotz hohem $f \mathrm{O}_{2}$ eine große Fraktionierung zwischen gebildeter Tonerde und $\mathrm{O}_{2}$ auftreten kann. Nichtmetallische Ablagerungen mit $\delta^{18} \mathrm{O}$-Werten ähnlich dem von Luft-O $\mathrm{O}_{2}$ wurden weder in dieser Studie noch in denen von PACK (2000) und PACK et al. (2005) beobachtet (siehe Kapitel 3.4.1).

Somit konnte aus sauerstoffisotopischer Sicht kinetische Fraktionierung von Luft- $\mathrm{O}_{2}$ als Quelle für die Bildung von Clogging und SEN-Unterseiten-Präzipitaten nicht völlig ausgeschlossen werden.

\section{Welche Isotopie hat Prozess- $\mathrm{O}_{2}$ im Vergleich zu Clogging?}

Basierend auf den beobachteten $\delta^{18} \mathrm{O}-$ Werten der abgelagerten Oxide wird angenommen, dass es sich dabei nicht um In-Situ-Bildungen im Tauchrohr handelt, sondern dass die Präzipitate aus Partikeln aufgebaut sind, die bei der Desoxidation in der Pfanne und bei der Reoxidation des Stahls im Schattenrohr und/oder dem Tundish entstehen. Auch THOMAS and BAI (2001) gehen davon aus, dass Tauchrohrclogging auf die Ablagerung von Oxiden zurückgeht, die bereits vor dem Erreichen des Tauchrohres im Stahl vorlagen.

Um die Isotopie von Desoxidationsprodukten zu analysieren, wurde der $\delta^{18} \mathrm{O}$ von Rückstandsisolaten untersucht. Diese wurden aus Lollyproben gewonnen, die am Ende des Frischens vor Zugabe des Desoxidationsmittels aus dem Konverter gezogen wurden. Die $\delta^{18} \mathrm{O}-$ Werte der Rückstandsisolate liegen zwischen +22 und $+24 \pm 1 \%$ (siehe Kapitel 3.4.3 und 3.5). Obwohl der Fehler bei der Messung von Rückstandsisolaten aufgrund ihrer extrem feinkörnigen Struktur größer ist als bei der Messung von makroskopischen Mineralkörnern, ist der $\delta^{18} \mathrm{O}$ der Rückstandsisolate mit dem des korrespondierenden Prozess- $\mathrm{O}_{2}$ vergleichbar (siehe Abbildung 52). Somit tritt keine oder nur eine kleine Fraktionierung von 0 bis $2 \%$ sowohl bei der Lösung von O im Stahl als auch bei der Bildung von Desoxidationsprodukten in der Pfanne auf. Daraus wird abgeleitet, dass oxidische Präzipitate, die ausschließlich aus Desoxidationsprodukten zusammengesetzt sind, einen $\delta^{18} \mathrm{O}$ von +22 bis $+24 \%$ aufweisen sollten (Toulouse et al. in press). 
Es wurde gezeigt, dass der $\delta^{18} \mathrm{O}$ von Frisch- $\mathrm{O}_{2}$ aus dem Stahlwerk in Duisburg zwischen $+23,5$ und $+25,4 \%$ variiert und im Mittel mit $+24,3 \%$ den gleichen $\delta^{18} \mathrm{O}$ wie Frisch- $\mathrm{O}_{2}$ aus Dortmund hat (PACK 2000). Bei PACK et al. (2005) findet sich ein weiterer $\delta^{18} \mathrm{O}$ von Frisch$\mathrm{O}_{2}$ aus einem anderen Stahlwerk mit $+25,5 \%$. Frisch- $\mathrm{O}_{2}$ wird durch Luftverflüssigung und Trennung von $\mathrm{N}_{2}$ und $\mathrm{O}_{2}$ gewonnen (siehe Kapitel 3.4.2.2). Bei dem Prozess tritt lediglich eine kleine Fraktionierung auf, obwohl flüssiger Sauerstoff im Gleichgewicht mit gasförmigem $\mathrm{O}_{2}$ bei $-180^{\circ} \mathrm{C}$ um 5,5\%o relativ zur Gasphase in ${ }^{18} \mathrm{O}$ angereichert ist (siehe Kapitel 3.4.2.3 und Abbildung 50). Der hohe Durchsatz von $\mathrm{O}_{2}$ bei der Luftverflüssigung und -zerlegung bedingt einen $\delta^{18} \mathrm{O}$ nahe dem von atmosphärischem $\mathrm{O}_{2}$ (TOULOUSE et al. in press). An zwei Tagen im Dezember 2005 und Januar 2006 lag der $\delta^{18} \mathrm{O}$ von Frisch- $\mathrm{O}_{2}$ bei bis zu $+0,7 \%$. Es ist bisher nicht geklärt worden, wie solch ungewöhnlich niedrige $\delta^{18} \mathrm{O}$-Werte des Prozess- $\mathrm{O}_{2}$ entstehen konnten. Eine Möglichkeit sind Wartungsarbeiten an einem Luftzerleger Ende 2005, bei denen die $\mathrm{O}_{2}$-Produktion unterbrochen wurde. Da während dieser Zeit mit niedrigen $\delta^{18} \mathrm{O}$-Werten des Frisch- $\mathrm{O}_{2}$ weder Rückstandsisolate beprobt noch Cloggingproben genommen wurden, wird die Bildung von oxidischen Ablagerungen in Bezug auf Prozess- $\mathrm{O}_{2}$ mit einem $\delta^{18} \mathrm{O}$ von im Mittel $+24,3 \%$ diskutiert.

Aus dem deutlich niedrigeren $\delta^{18} \mathrm{O}$ oxidischer Präzipitate wird geschlussfolgert, dass Desoxidationsprodukte nicht die alleinige Ursache für Clogging und SEN-Unterseiten-Ablagerungen sein können; ein Reservoir mit niedrigem $\delta^{18} \mathrm{O}$ wird hierfür benötigt.

\section{Welche Isotopie haben Schlacken und FF-Materialien im Vergleich zu Clogging?}

Von PACK (2000) und PACK et al. (2005) wurde die Reduktion der Fayalitkomponente des Olivins aus der feuerfesten Auskleidung des Tundishs als O-Donator mit einem $\delta^{18} \mathrm{O}$ von rd. $+5 \%$ vorgeschlagen. Die Reduktion von Olivin wurde beobachtet und ist thermodynamisch möglich (PACK et al. 2003). In dieser Studie wurde kein Olivin in der feuerfesten Auskleidung von Pfanne und Tundish verwendet, sondern ausschließlich kohlenstoffgebundener Periklas (siehe Kapitel 4.2.4). Daher kann Olivin als O-Quelle ausgeschlossen werden.

Andere FF-Materialien und Schlacken können potentielle O-Quellen mit niedrigem $\delta^{18} \mathrm{O}$ sein. In dieser Studie wurden eine Reihe von FF-Materialien und Schlacken auf ihren $\delta^{18} \mathrm{O}$ hin untersucht (siehe Kapitel 3.4.4). Die $\delta^{18} \mathrm{O}$-Werte des Periklas aus Pfanne und Tundish liegen zwischen +7 und $+13 \%$. PACK et al. (2005) berichten von $\delta^{18} \mathrm{O}-$ Werten der Spritzmasse des Verteilers zwischen $+13,3$ und $+14,2 \%$, die dort untersuchten $\mathrm{MgO}^{*} \mathrm{CaO}-\mathrm{C}$-Steine der Pfannen weisen einen $\delta^{18} \mathrm{O}$ von $+12,6$ bis $+17,3 \%$ auf. Periklas ist nur schwer zu reduzieren und 
ist daher als Sauerstoffquelle zu vernachlässigen. Andere Materialien wie Konverter-, Pfannen- und Tundishschlacke weisen höhere $\delta^{18} \mathrm{O}$-Werte als Clogging und SEN-UnterseitenAblagerungen auf (siehe Abbildung 57). Der CaO-Gehalt oxidischer Ablagerungen variiert zwischen 0,1 und 7,7 Gew.-\%, der MgO-Gehalt von 0,1 bis 6,8 Gew.-\% (siehe Tabelle 18). Bei PACK (2000) finden sich maximale Gehalte für $\mathrm{CaO}$ und $\mathrm{MgO}$ von bis zu 14 bzw. 13 Gew.-\%. Damit ist der Anteil von Mitlaufschlacken bzw. FF-Materialien aus Pfanne und Tundish an den oxidischen Präzipitaten von untergeordneter Bedeutung für die Diskussion der Isotopendaten, weil ihr Anteil in jedem Fall <16 Gew.\% beträgt (TOULOUSE et al. in press). Dieser Prozentsatz ergibt sich aus den $\mathrm{CaO}$ - und $\mathrm{MgO}$-Anteilen der oxidischen Ablagerungen.

\section{Welche Isotopie hat Chromit im Vergleich zu Clogging?}

Das einzige FF-Material unter den analysierten Prozessmaterialien mit einem niedrigen $\delta^{18} \mathrm{O}$ ist der Chromit aus dem Schiebersand mit einem durchschnittlichen $\delta^{18} \mathrm{O}$ von $-3,5 \%$ (siehe Abbildung 59). Vier verschiedene Chromitprodukte dreier unterschiedlicher Schiebersandhersteller weisen den gleichen niedrigen $\delta^{18} \mathrm{O}$ auf. Allein bei TAYLOR (1968) wurde ein veröffentlichter $\delta^{18} \mathrm{O}$-Wert von Chromit gefunden. Danach hat Chromit aus dem ultramafischen Union Bay-Komplex (südöstliches Alaska) einen $\delta^{18} \mathrm{O}$ von $+4,5 \%$. Die ähnlichen $\delta^{18} \mathrm{O}$-Werte der untersuchten Chromite lassen annehmen, dass alle Chromite von derselben Lagerstätte stammen. Diese Schlussfolgerung wird durch mikrochemische Untersuchungen mittels EMS unterstützt. Quarz im Schiebersand ist isotopisch variabler, da er als Quarzsand aus unterschiedlichen Reservoiren stammt, die bei fluviatilem Transport gemischt wurden (TOULOUSE et al. in press). Nach dem Öffnen der Schieberplatten gelangt der Schiebersand in den Tundish. Ein Teil des Schiebersandes steigt in der Schmelze im Tundish auf und wird von der Schlacke absorbiert. Ein anderer Teil wird durch das legierte Al im Stahl reduziert. Die NettoChromitreduktion erfolgt nach (siehe ToulousE et al. in press):

$$
\begin{aligned}
& \overbrace{\left\langle\mathrm{Fe}_{0.52}^{2+} \mathrm{Mg}_{0.48}^{2+}\left[\mathrm{Fe}_{0.22}^{3+} \mathrm{Cr}_{1.21}^{3+} \mathrm{Al}_{0.57}^{3+}\right] \mathrm{O}_{4}\right\rangle}^{\text {Chromit aus dem Schiebersand }}+\overbrace{0,39[\mathrm{Al}]}^{\text {legiertes Al }} \rightleftharpoons \\
& 0,74[\mathrm{Fe}]+1,21[\mathrm{Cr}]+0,48\left\langle\mathrm{MgAl}_{2} \mathrm{O}_{4}\right\rangle+\underbrace{2,08[\mathrm{O}]}_{\text {reagiert mit Al zu Al} 2 \mathrm{O}_{3}}
\end{aligned}
$$

Die Reaktion zeigt, dass bei der Reduktion von $1 \mathrm{~kg}$ Chromit 0,17 kg O frei werden, die für die Bildung von freiem $\mathrm{Al}_{2} \mathrm{O}_{3}$ mit dem gelösten $\mathrm{Al}$ im Stahl zur Verfügung stehen. Bei der nachfolgend beschriebenen Modellierung wird vorausgesetzt, dass diese Tonerde den gleichen $\delta^{18} \mathrm{O}$ wie Chromit besitzt. Daher ist Chromit aus dem Schiebersand die einzige beobach- 
tete O-Quelle im Prozess, die direkt zu einem niedrigen $\delta^{18} \mathrm{O}$-Wert oxidischer Ablagerungen beitragen kann.

Da der $\delta^{18} \mathrm{O}$ von Clogging und SEN-Unterseiten-Präzipitaten deutlich höher ist als der $\delta^{18} \mathrm{O}$ von Chromit, wird angenommen, dass Chromit nicht die einzige O-Quelle für diese Ablagerungen sein kann, sondern dass Clogging und SEN-Unterseiten-Präzipitate aus einer Mischung von Tonerde unterschiedlicher Reservoire aufgebaut sind.

\section{Welchen Anteil haben Chromit, Prozess- $\mathrm{O}_{2}$ und Luft-O $\mathrm{O}_{2}$ am Clogging?}

Das Mischen von Tonerde verschiedener Reservoire soll zunächst als Zweikomponentenmodell vgl. Kapitel 3.5 diskutiert werden. Ein Endglied soll dabei Tonerde sein, die auf die Reduktion des Chromits aus dem Schiebersand zurückgeht. Pro Pfanne wird rd. 80 kg Schiebersand eingesetzt, der rd. 56 kg Chromit enthält. Die mittlere molare Masse des untersuchten Chromits ist $195 \mathrm{~g} / \mathrm{mol}$. Bei der Reduktion von einem Mol Chromit werden 2,08 Mol O frei, die für die Bildung freier Tonerde zur Verfügung stehen (siehe oben). Dies führt pro Pfanne zu maximal $20 \mathrm{~kg}$ Tonerde, die durch Oxidation von $\mathrm{Al}$ entstehen und auf die Reduktion des Chromits aus dem Schiebersand zurückgehen.

Das andere Endglied soll Tonerde sein, bei der es sich um Desoxidationsprodukte aus der Pfanne handelt. Nach DEKKERS (2002) liegt der Gesamtsauerstoffgehalt im Stahl nach dem Desoxidieren bei 30 ppm, wobei 25 ppm als Oxid und 5 ppm gelöst im Stahl sind. Dies entspricht einer Löslichkeit von 5 ppm O in der Stahlschmelze bei 0,03 Gew.\% Al (TURKDOGAN 1996). Bei DEKKERS (2003) findet sich ein Gesamtsauerstoffgehalt im Stahl nach dem Desoxidieren von 28 bis 46 ppm. Abzüglich des gelösten Sauerstoffanteils im Stahl von 5 ppm O (dieser steht nicht für die Bildung von Tonerde zur Verfügung) ergibt sich ein O-Gehalt von 23 bis 41 ppm in Form von Tonerdeeinschlüssen, die von der Pfanne in den Tundish transferiert werden (TOULOUSE et al. in press). In dem Stahlwerk, aus dem die beprobten nichtmetallischen Ablagerungen stammen, beträgt die Pfannenkapazität $265 \mathrm{t}$, was zu maximal 13 bis 23 $\mathrm{kg}$ Tonerde pro Pfanne führt. Es wurde gezeigt, dass Desoxidationsprodukte aus der Pfanne einen $\delta^{18} \mathrm{O}$ von rd. $+24 \%$ aufweisen.

Werden Tonerdepartikel in den angegebenen Proportionen aus der Chromitreduktion mit Desoxidationsprodukten aus der Pfanne gemischt, ergeben sich $\delta^{18} \mathrm{O}-$ Werte für oxidische Ablagerungen von $+7,3$ bis $+11,3 \%$ mit einem Mittelwert von $+9,5 \%$. Nach dem Zweikomponentenmodell führt dies zu einem Anteil von 39 bis 53 Gew.- $\% \mathrm{Al}_{2} \mathrm{O}_{3}$ aus Desoxidationsprodukten und 47 bis 61 Gew. $\% \mathrm{Al}_{2} \mathrm{O}_{3}$ aus der Chromitreduktion in nichtmetallischen Ablagerungen. 
Der mittlere $\delta^{18} \mathrm{O}$ von Clogging aus Duisburg liegt bei $+6,8 \%$, d. h. die Isotopie ist um rd. 3\%o niedriger als der nach dem Zweikomponentenmodell berechnete mittlere $\delta^{18} \mathrm{O}$.

Atmosphärischer Sauerstoff ist eine omnipräsente O-Quelle für oxidische Ablagerungen. Die minimale Beteiligung von Luft- $\mathrm{O}_{2}$ kann anhand des Stickstoffgehaltes des Stahls bestimmt werden. Hierbei ist zu beachten, dass sich Sauerstoff schneller im Stahl löst als Stickstoff (DEKKERS 2003). Die Differenz zwischen dem Stickstoffgehalt des Stahls im Tundish und dem in der Pfanne ist ein Maß für die Reoxidation der Schmelze durch $\mathrm{Luft}_{2} \mathrm{O}_{2}$ bei dieser Transferoperation (ZHANG and THOMAS 2003b). Die Aufstickung des Stahls zwischen Pfanne und Tundish liegt in Duisburg bei 1 bis $2 \mathrm{ppm}$. Das $\mathrm{N}_{2} / \mathrm{O}_{2}-$ Massenverhältnis troposphärischer Luft ist 78/21 =3,7. Bei einem Stickstoffanstieg von 1 bis 2 ppm errechnet sich damit eine Beteiligung von mindestens 0,3 bis 0,5 ppm atmosphärischen Sauerstoffs, die zur Oxidation von legiertem Al bereitstehen (TOULOUSE et al. in press). Basierend auf einer Pfannenkapazität von 265 t ergibt sich durch Reoxidation des $\mathrm{Luft}_{2} \mathrm{O}_{2}$ ein Minimum an gebildeter Tonerde pro Pfanne von 0,2 bis $0,3 \mathrm{~kg}$. Letztere hat einen $\delta^{18} \mathrm{O}$ von $+23,5 \%$ (DoLE et al. 1954) und ist damit isotopisch nicht von Prozess- $\mathrm{O}_{2}$ als Verursacher nichtmetallischer Präzipitate zu unterscheiden.

Wird Tonerde, die durch Oxidation mittels Luft- $\mathrm{O}_{2}$ entstanden ist, mit Tonerde aus Prozess$\mathrm{O}_{2}$ und Tonerde aus der Chromitreduktion in den beschriebenen Proportionen gemischt, ergibt sich ein $\delta^{18} \mathrm{O}$ für oxidische Ablagerungen von $+7,4$ bis $11,4 \%$ und eine Beteiligung von 46 bis 61 Gew.-\% Schiebersand, 39 bis 53 Gew.-\% Desoxidationsprodukten und 0,3 bis 0,9 Gew.-\% Luft-O ${ }_{2}$ (siehe Abbildung 6 in Kapitel 12.5). Die Sauerstoffisotopenzusammensetzung oxidischer Ablagerungen ist im Mittel um 0,6\% niedriger als das Minimum des nach dem Dreikomponentenmodell berechneten $\delta^{18} \mathrm{O}$-Bereichs.

Das Modell hat jedoch eine Schwachstelle, die in weiteren Arbeiten zu untersuchen ist. Die Reduktion von Chromit führt nicht nur zur Bildung von Tonerde, sondern auch zu der von Spinell (siehe oben). Das aus der Reduktionsgleichung abgeleitete Spinell/TonerdeMassenverhältnis ist $0,97, \mathrm{~d}$. h. die Reduktion von Chromit produziert Tonerde und Spinell in annähernd gleichen Proportionen. Bei der Reduktion von Chromit aus $80 \mathrm{~kg}$ Schiebersand werden neben maximal $20 \mathrm{~kg}$ Tonerde maximal $20 \mathrm{~kg}$ Spinell gebildet. Werden $20 \mathrm{~kg}$ Tonerde und $20 \mathrm{~kg}$ Spinell aus der Chromitreduktion mit 13 bis $23 \mathrm{~kg}$ Tonerde aus Desoxidationsprodukten und 0,2 bis 0,3 kg Tonerde aus der Reoxidation durch $\mathrm{Luft}_{-} \mathrm{O}_{2}$ gemischt, ergibt sich ein $\delta^{18} \mathrm{O}$-Bereich von $+3,4$ bis $+6,8 \%$ mit einem Mittelwert von $+5,2 \%$ oür oxidische Ablagerungen. Dieser ist rd. 2\%o niedriger als der Mittelwert von Clogging aus Duisburg. Der 
Einfluss von Spinell aus der Chromitreduktion ist im Hinblick auf die chemische Zusammensetzung oxidischer Ablagerungen jedoch problematisch.

Bei angenommenen 46 bis 61 Gew.-\% Chromit als O-Quelle führt der gebildete Spinell zu $\mathrm{MgO}-$ Gehalten in den Oxiden von 6 bis 8 Gew.-\%. Die niedrigsten MgO-Gehalte oxidischer Ablagerungen liegen bei 0,1 Gew.-\% (siehe Kapitel 3.4.1.1). Nach dem Modell würde die Beteiligung von Spinell und Tonerde aus der Chromitreduktion in diesem Fall bei maximal rd. 1 Gew.-\% liegen. Derart geringe Mengen Sauerstoff aus der Chromitreduktion würden $\delta^{18} \mathrm{O}$-Werte von Clogging und SEN-Unterseiten-Ablagerungen von bis $\mathrm{zu}+24 \%$ ergeben, die weder in dieser Studie noch in denen von PACK (2000) und PACK et al. (2005) beobachtet wurden. Daraus wird geschlussfolgert, dass frei werdendes $\mathrm{MgAl}_{2} \mathrm{O}_{4}$ nicht zur Ansatzbildung in und unter Tauchrohren beiträgt.

Um die gemessenen $\delta^{18} \mathrm{O}-$ Werte und die niedrigen $\mathrm{MgO}-$ Gehalte von Clogging und SENUnterseiten-Präzipitaten erklären zu können, ist eine mechanische Separation der freien Tonerde vom Spinell erforderlich. Ein denkbarer Prozess wäre, dass bei der Chromitreduktion im Tundish frei werdender Sauerstoff vorübergehend im Stahl gelöst würde und erst nach einem gewissen Schmelztransport bei möglicherweise niedrigeren Temperaturen in Richtung Tauchrohr mit dem gelösten $\mathrm{Al} z u \mathrm{Al}_{2} \mathrm{O}_{3}$ reagieren würde. Ein Teil dieser Partikel würde zu Tauchrohrclogging beitragen. Der residuale Spinell von der Chromitreduktion würde in die Tundishschlacke abgeschieden werden (siehe Abbildung 7 in Kapitel 12.5).

Höhere Gehalte von $\mathrm{FeO}$ und $\mathrm{Cr}_{2} \mathrm{O}_{3}$ sowie die röntgenographische Identifizierung von Chromit in einigen Tundishschlackenproben zeigen, dass ein Teil des Chromits bereits vor einer möglichen Reduktion absorbiert wird (siehe Kapitel 4.2.2). Neben reduzierbarem Chromit besteht Schiebersand vorwiegend aus Quarzsand. $\mathrm{SiO}_{2}$ ist nach dem Richardson-JeffesDiagramm stabiler als Fe- und Cr-Oxide (TURKDOGAN 1996) und wird daher als weniger bedeutend für die Oxidation des legierten $\mathrm{Al}$ angenommen. Überdies besitzt Quarz eine geringere Dichte als Chromit, wodurch er schneller in die Tundishschlacke abgeschieden wird (siehe Kapitel 3.5). Deswegen wurde Quarz bei den Massenbilanzierungen nicht berücksichtigt. 


\section{Zusammenfassung}

In den letzten Dekaden hat die Sekundärmetallurgie aufgrund wachsenden Druckes auf die Stahlhersteller nach wirtschaftlicher Erzeugung immer mehr belastbarer Stähle für neue Anwendungszwecke stetig an Bedeutung zugenommen. Die Ansprüche an den Reinheitsgrad der Stähle steigen dabei von Jahr zu Jahr, dieser wird maßgeblich durch nichtmetallische Einschlüsse bestimmt. Bei Ablagerung dieser meist oxidischen Ausscheidungen z. B. in Tauchrohren verstopfen die Ansätze die Fließkanäle, es entsteht Clogging. Letzteres kann sich neben schädlichen Einflüssen auf das Produkt auch prozessschädigend auswirken. Mit Hilfe von Sauerstoffisotopen kann ein Beitrag zur Ursachenklärung von Clogging geleistet werden.

Sauerstoffisotope wurden als Marker benutzt, um die O-Quelle für die Bildung nichtmetallischer Einschlüsse/Ablagerungen beim Stranggießen von Stahl zu erforschen und damit Verursacher des Problems festzustellen. Auf der Basis von Massenbilanzierungen wurde ein quantitatives Modell zur Herkunft oxidischer Ablagerungen entwickelt. Die Studie kommt zu dem Ergebnis, dass die Entstehung nichtmetallischer Ablagerungen in Tauchrohren und an deren Unterseiten mit der Reduktion von Chromit aus dem Schiebersand zusammenhängt. Sein Anteil wird auf rd. 50 bis 60 Gew.-\% quantifiziert. Als weitere O-Quellen für die Oxidation von Al wurden Prozess- und Luft- $\mathrm{O}_{2}$ festgestellt. Letzterer macht mindestens einen Anteil von 1\% aus.

Um die ermittelten O-Quellen zu minimieren, müssen der Prozess selbst und die verwendeten Prozessmaterialien wie die feuerfesten Auskleidungen der metallurgischen Gefäße, die Schlackenbildner und insbesondere der Schiebersand möglichst chemisch inert gehalten werden. Dafür sind wegen reduzierender Prozessbedingungen bereits stark reduzierte Phasen Voraussetzung. Im Schiebersand ist nach Möglichkeiten der Substitution des Chromits durch weniger leicht reduzierbare Minerale mit gleichen physikalischen Eigenschaften zu suchen. Der Anteil von Prozess- $\mathrm{O}_{2}$ kann möglicherweise durch weiter verbesserte Abscheidebedingungen von Desoxidationsprodukten in Pfanne und Tundish verringert werden. Die Reoxidation des Stahls durch Luft-O $\mathrm{O}_{2}$ tritt vor allen Dingen beim Angießen auf, wenn die Schmelze noch nicht durch eine schützende Schlackenschicht bedeckt ist. Durch Flutung des Tundishs mit Argon vor dem Angießen könnte die ohnehin schon sehr geringe Reoxidation potentiell weiter erniedrigt werden, wobei jedoch die Kosten für eine etwaige Argonflutung den Nutzen übersteigen dürften. 


\section{Ausblick}

\section{1 ${ }^{17}$ O-Messung als Marker für Clogging}

Das in dem vorherigen Kapitel beschriebene Dreikomponentenmodell wurde auf Basis von konventioneller Sauerstoffisotopenanalytik und Massenbilanzierungen aufgestellt. Durch Messung des dritten stabilen Sauerstoffisotops ${ }^{17} \mathrm{O}$ kann die Modellvorstellung überprüft werden. ${ }^{17} \mathrm{O}-$ Messungen an refraktären Phasen wie Tonerde sind sowohl die Präparation der Proben als auch die Messung im Massenspektrometer betreffend aufwendig und schwierig, aber in Göttingen möglich. Das Hauptproblem sind Stickstoff-Fluor-Verbindungen, die auf dem Verhältnis von Masse/Ladung des Isotopologs ${ }^{17} \mathrm{O}^{16} \mathrm{O}$ interferieren. Dies stellt hohe Reinheitsansprüche an das eingesetzte Fluorgas und erfordert eine weitestgehende Reinigung des Sauerstoffs. Darüber hinaus sind Korrekturrechnungen der interferierenden Schmutzgase notwendig. PACK et al. (2007) haben ein Verfahren entwickelt, mit dem fehlerhafte ${ }^{17} \mathrm{O}$ Messungen auch ohne Abtrennung überlagernder Schmutzgase korrigiert werden können (siehe Kapitel 13).

Die Fraktionierung des ${ }^{17} \mathrm{O} /{ }^{16} \mathrm{O}$-Verhältnisses ist etwa halb so groß wie die des ${ }^{18} \mathrm{O} /{ }^{16} \mathrm{O}$ Verhältnisses (PACK et al. 2007, SHARP 2007). Dieser Zusammenhang wird als massenabhängige Fraktionierung bezeichnet und lässt alle irdischen Gesteine im Dreiisotopendiagramm des Sauerstoffs ( $\delta^{17} \mathrm{O}$ gegen $\delta^{18} \mathrm{O}$ ) entlang einer Geraden mit der Steigung $\beta \approx 0,52$ liegen. In Abbildung 96 ist $\delta^{17} \mathrm{O}$ gegen $\delta^{18} \mathrm{O}$ von terrestrischen Gesteinen und relativ dazu die Lage von Mondgesteinen und Meteoriten dargestellt. Der Mond ist genetisch betrachtet ein Teil der Erde und hat deswegen die gleiche Lage wie die Gesteine. $\delta$ '-Werte sind linearisierte Formen der $\delta$-Notation (siehe hierzu MILLER 2002) und berechnen sich nach:

$$
1000 \ln \left(\frac{\delta^{x} \mathrm{O}_{\text {SMOW }}}{1000}+1\right)=\delta^{\prime x} \mathrm{O}_{\text {SMOW }} \text { mit } x=17,18
$$

Mit dieser Form wird ein vollständig linearer Zusammenhang zwischen $\delta^{17} \mathrm{O}$ und $\delta^{18} \mathrm{O}$ hergestellt, wodurch experimentelle Daten linear gefittet werden können. Die rote Gerade in Abbildung 96 wird als terrestrische Fraktionierungslinie (TFL) bezeichnet und illustriert Variationen von ${ }^{17} \mathrm{O} /{ }^{16} \mathrm{O}$ als Funktion von ${ }^{18} \mathrm{O} /{ }^{16} \mathrm{O}$. Dieser Zusammenhang ist die Konsequenz massenabhängiger Fraktionierung zwischen Mineralen, Wasser und Magmen während chemischer Reaktionen (RUMBLE et al. 2006). Die Steigung der TFL hängt mit dem relativen Verhältnis der Differenzen der Isotopenmassen zusammen: 


$$
\frac{\left(\mathrm{m}_{{ }^{17} \mathrm{O}}-\mathrm{m}_{{ }_{16} \mathrm{O}}\right)}{\left(\mathrm{m}_{{ }^{18} \mathrm{O}}-\mathrm{m}_{16 \mathrm{O}}\right)}=0,50 \text { mit } \mathrm{m}_{\mathrm{i}} \text { für die Masse der jeweiligen Isotope }
$$

\section{Alle feuerfesten Erzeugnisse wie z. B. der Schiebersand liegen auf der TFL, da sie alle „von}

der Erde abstammen".

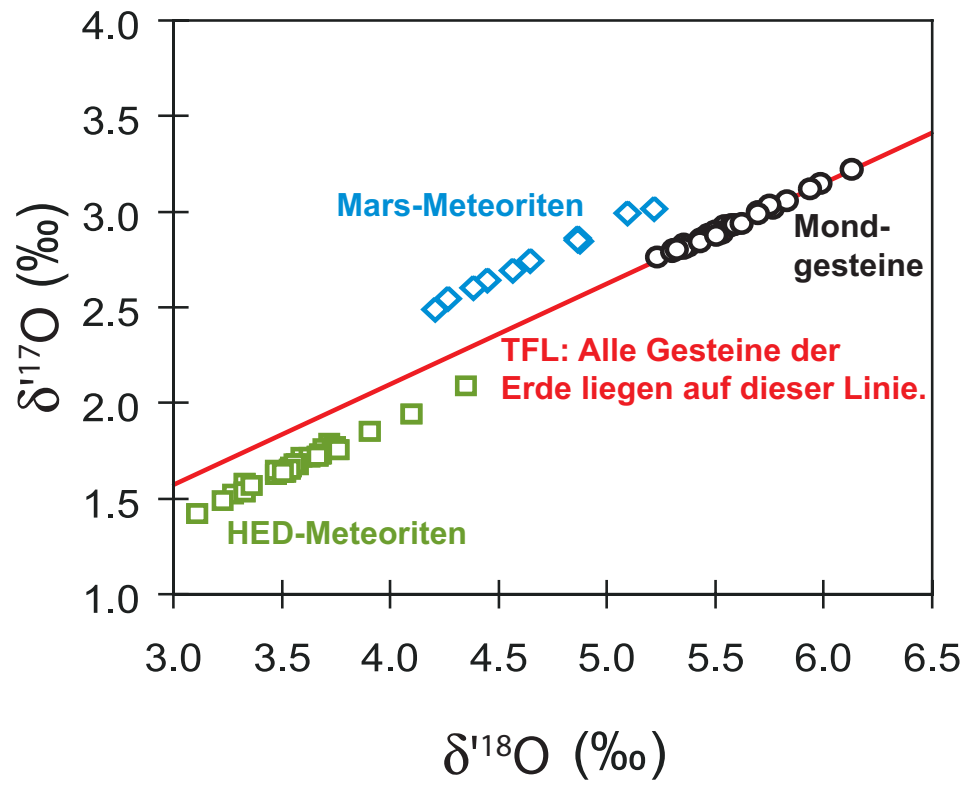

Abbildung 96: Dreiisotopendiagramm von irdischen Gesteinen (rote Linie) und relativ dazu die Lage von Mondgesteinen und Meteoriten (verändert nach WIECHERT et al. 2004).

Die Steigung der TFL varriert zwischen 0.5240 und 0.5266 (RUMBLE et al. 2007). Nach MiLLER (2002) beträgt sie 0,5247, PACK et al. (2007) geben eine von 0,5237 an. Die Steigung stellt eine Mischung aus kinetischer und Gleichgewichtsfraktionierung dar, wobei letztere im Falle von Gesteinen und Mineralen überwiegt. Alle Materialien, die von dieser Geraden abweichen, werden als isotopisch anomal bezeichnet. Eine O-Isotopenanomalie kann durch sogenannte massenunabhängige Fraktionierung (CLAYTON et al. 1973, THIEMENS and HEIDENREICH 1983) oder durch massenabhängige, kinetische Fraktionierung entstehen, deren Fraktionierungslinie eine etwas flachere Steigung als die TFL hat (Young et al. 2002). Die Größe der O-Isotopenanomalie wird als $\Delta^{\prime 17} \mathrm{O}_{\mathrm{TFL}}$ angegeben mit:

$$
\Delta^{\prime 17} \mathrm{O}_{\mathrm{TFL}}=\delta^{\prime 17} \mathrm{O}_{\mathrm{SMOW}}-\beta_{\mathrm{TFL}} * \delta^{\prime 18} \mathrm{O}_{\mathrm{SMOW}}
$$

Luftsauerstoff hat einen weltweit konstanten $\delta^{18} \mathrm{O}$ von $+23,5 \%$ (DOLE et al. 1954). YounG et al. (2002) schreiben, dass Meerwasser im Gleichgewicht mit Luft- $\mathrm{O}_{2}$ einen $\delta^{18} \mathrm{O}_{\text {SMOW }}$ von annähernd null haben müsste. Nach UREY (1947) liegt der $\delta^{18} \mathrm{O}$ von Meerwasser bei $25^{\circ} \mathrm{C}$ bei $+6 \%$. Die Erhöhung des $\delta^{18} \mathrm{O}$ von troposphärischem $\mathrm{O}_{2}$ gegenüber Meerwasser $\left(\delta^{18} \mathrm{O}_{\mathrm{SMOW}}=\right.$ 0\%o) liegt an einem stationären Zustand zwischen der Produktion von $\mathrm{O}_{2}$ durch Photosynthese 
von Land- und Meerpflanzen und der Respiration, d. h. $\mathrm{O}_{2}$-Aufnahme. Bei der Photosynthese wird $\mathrm{O}_{2}$ mit einem $\delta^{18} \mathrm{O}$-Wert von $0 \%$ bis $+4 \%$ produziert. Der Gasaustausch findet bei Pflanzen über deren Stomata statt. Das sind kleine Spaltöffnungen, die von zwei bohnenförmigen Schließzellen gebildet werden. Bei der Respiration wird der Sauerstoff kinetisch fraktioniert und es werden bevorzugt ${ }^{16} \mathrm{O}$-haltige Moleküle von den Pflanzen aufgenommen, was $\mathrm{zu}$ einer Verschiebung im $\delta^{18} \mathrm{O}$ von rd. 20\%o gegenüber dem des Luft- $\mathrm{O}_{2}$ führt. Der residuale Sauerstoff wird dabei an ${ }^{18} \mathrm{O}$ angereichert. Dieser Prozess wird als Dole-Effekt bezeichnet (DOLE 1935).

Es ist nun beobachtet worden, dass $\mathrm{Luft}-\mathrm{O}_{2}$ eine Isotopenanomalie von $\Delta^{\prime 17} \mathrm{O}=-0,344 \%$ hat (PACK et al. 2007). Young et al. (2002) führen diese Isotopenanomalie allein auf kinetische Fraktionierung während der Respiration zurück. LUZ et al. (1999) dagegen schreiben, dass isotopisch anomaler Sauerstoff aus der Stratosphäre in die Troposphäre gelangt, wo er sich mit isotopisch normalem $\mathrm{O}_{2}$ mischt. Auch ANGERT et al. (2003) sehen den Grund für den negativen $\Delta^{\prime 17} \mathrm{O}_{\mathrm{TFL}}$ von Luft- $\mathrm{O}_{2}$ in der Zumischung von stratosphärischem $\mathrm{O}_{2}$ (siehe Abbildung 97).

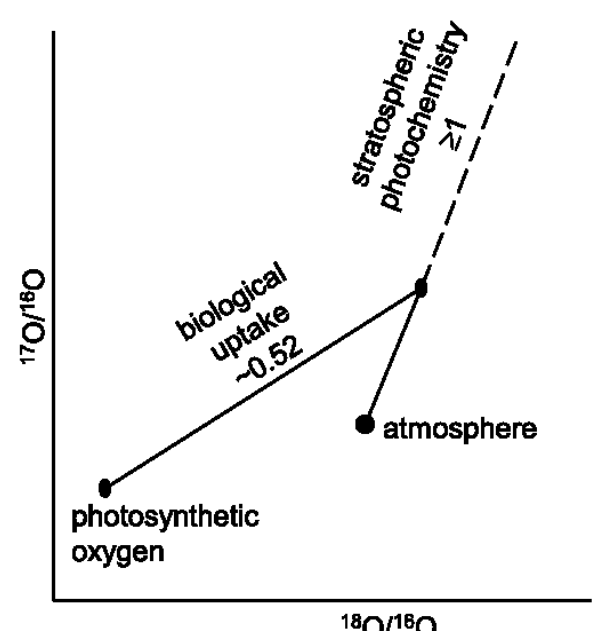

Abbildung 97: Schematisches Dreiisotopendiagramm zur Illustration der Anomalie von Luft- $\mathrm{O}_{2}$. Troposphärisches $\mathrm{O}_{2}$ entsteht bei der Photosynthese und wird massenabhängig bei der Respiration und massenunabhängig durch photochemische Reaktionen in der Stratosphäre fraktioniert. Die Balance zwischen beiden Prozessen bestimmt die Dreiisotopenzusammensetzung (ANGERT et al. 2003).

MiLler (2002) und PACK et al. (2007) sagen, dass der negative $\Delta^{\prime 17} \mathrm{O}$ von Luft- $\mathrm{O}_{2}$ eine Mischung aus kinetisch fraktioniertem Sauerstoff (durch die Respiration) und dem Eintrag von isotopisch anomalem stratosphärischem $\mathrm{O}_{2}$ ist (siehe Abbildung 98). 


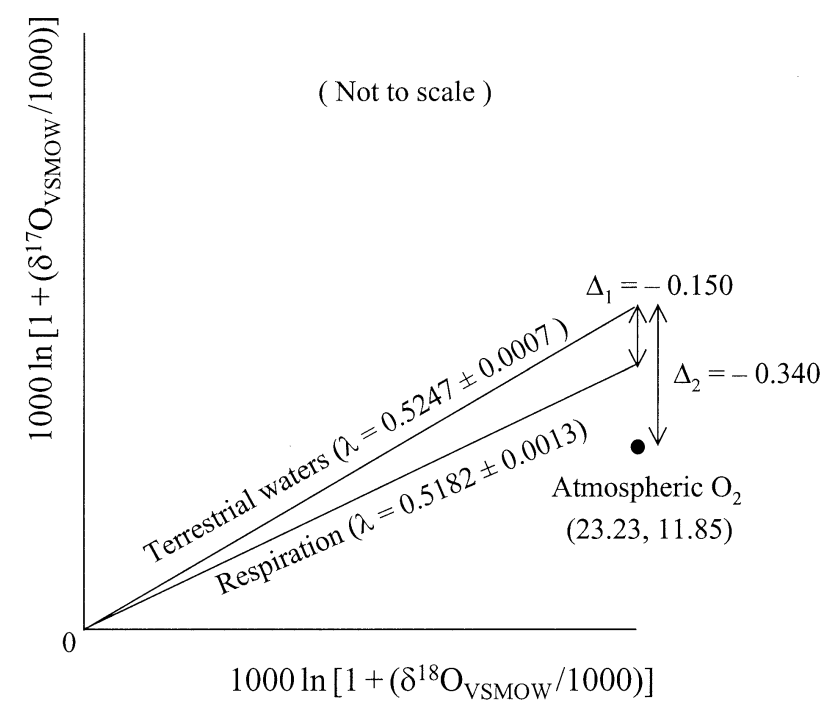

Abbildung 98: $\delta^{17} \mathrm{O}$ gegen $\delta^{18} \mathrm{O}$ von Luft- $\mathrm{O}_{2}$ als Produkt von kinetischer Fraktionierung während der Respiration und Eintrag von anomalem stratosphärischem $\mathrm{O}_{2}$ (MILLER 2002).

Angewandt auf die Stahlherstellung würde das bedeuten, dass nichtmetallische Präzipitate im Tauchrohr, die zu 100\% durch Oxidation mittels Luft- $\mathrm{O}_{2}$ gebildet wurden und bei deren Bildung kinetische Fraktionierung keine Rolle spielt, den gleichen $\Delta^{17} \mathrm{O}_{\mathrm{TFL}}$ wie troposphärisches $\mathrm{O}_{2}$ besitzen. Damit müsste das $\Delta^{\prime 17} \mathrm{O}_{\mathrm{TFL}}$ ein effizientes Werkzeug zur Identifizierung und Quantifizierung atmosphärischer O-Quellen sein.

An dieser Stelle soll die Bildung von Clogging allein durch kinetisch fraktionierten Luft- oder Prozess- $\mathrm{O}_{2}$ diskutiert werden. Die Betrachtung bezieht sich hierbei auf die kinetische Fraktionierung des Verhältnisses von ${ }^{17} \mathrm{O}$ zu ${ }^{16} \mathrm{O}$ zum Verhältnis von ${ }^{18} \mathrm{O}$ zu ${ }^{16} \mathrm{O}$. Jene zwischen ${ }^{18} \mathrm{O}$ und ${ }^{16} \mathrm{O}$ wurde in Kapitel 2.3.4 besprochen. Nach Young et al. (2002) und SHAHEEN (2005) ist bei massenabhängiger Fraktionierung der Fraktionierungsfaktor $\alpha$ für zwei Isotopenverhältnisse:

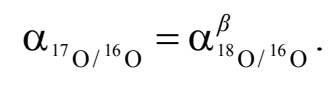

Für kinetische Fraktionierung ist

$$
\beta=\ln \left(\mathrm{M}_{1} / \mathrm{M}_{2}\right) / \ln \left(\mathrm{M}_{1} / \mathrm{M}_{3}\right)
$$

mit $\mathrm{M}_{i}$ für atomare oder molekulare Spezies. Danach errechnet sich im Dreiisotopendiagramm für atomaren Sauerstoff eine Gerade mit der Steigung $\beta=0,5147$ und für molekularen Sauerstoff eine Gerade mit $\beta=0,5076$ (siehe Abbildung 99). Die Geraden sind so dargestellt, dass sie durch den $\delta^{17} \mathrm{O}$ und $\delta^{\prime 18} \mathrm{O}$ von Luft-O ${ }_{2}$ verlaufen. 


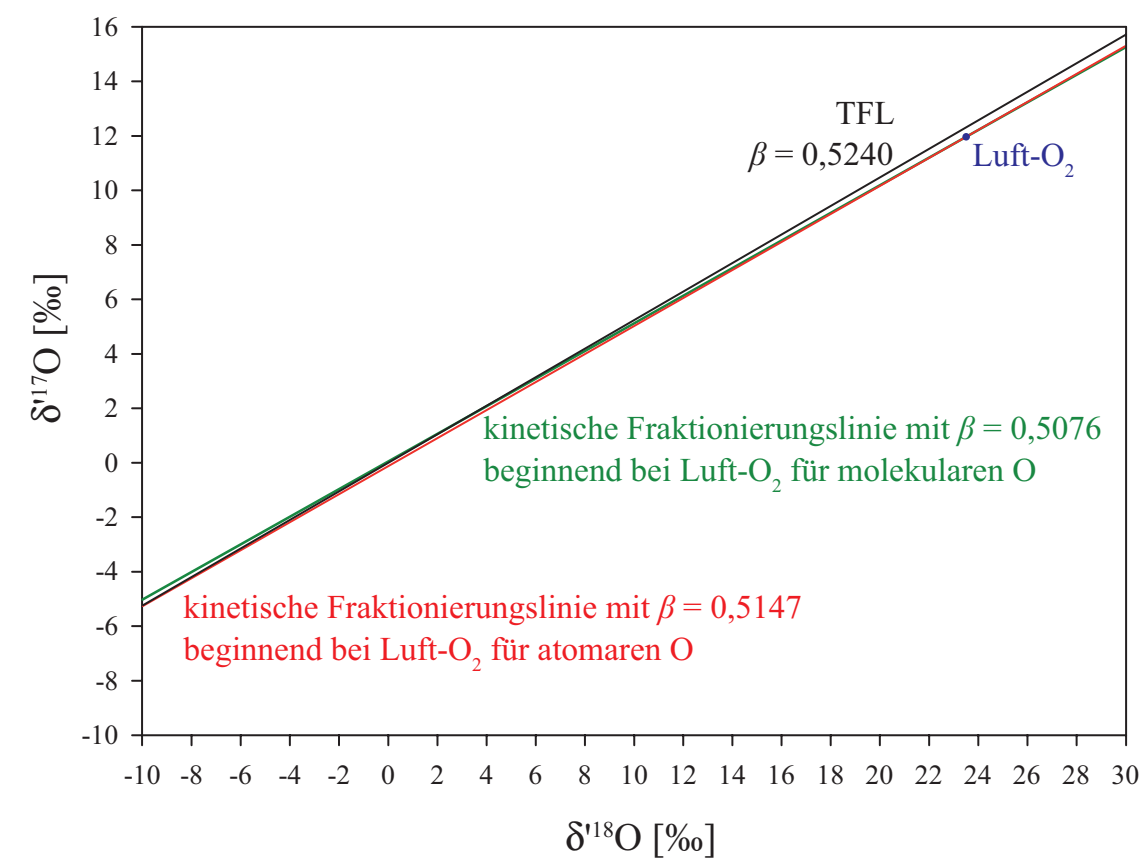

Abbildung 99: $\delta^{\prime 17} \mathrm{O}$ gegen $\delta^{18} \mathrm{O}$ von irdischen Gesteinen mit $\beta=0,5240$ (schwarze Gerade $=\mathrm{TFL}$ ). Die rote Gerade ist die theoretisch berechnete kinetische Fraktionierungslinie für atomaren Sauerstoff $(\beta=0,5147)$, die grüne Gerade ist jene für molekularen Sauerstoff $(\beta=0,5076)$.

Trägt man $\Delta^{\prime 17} \mathrm{O}$ gegen $\delta^{\prime 18} \mathrm{O}$ auf, wird der Unterschied zwischen der TFL und den kinetischen Fraktionierungslinien deutlicher (siehe Abbildung 100).

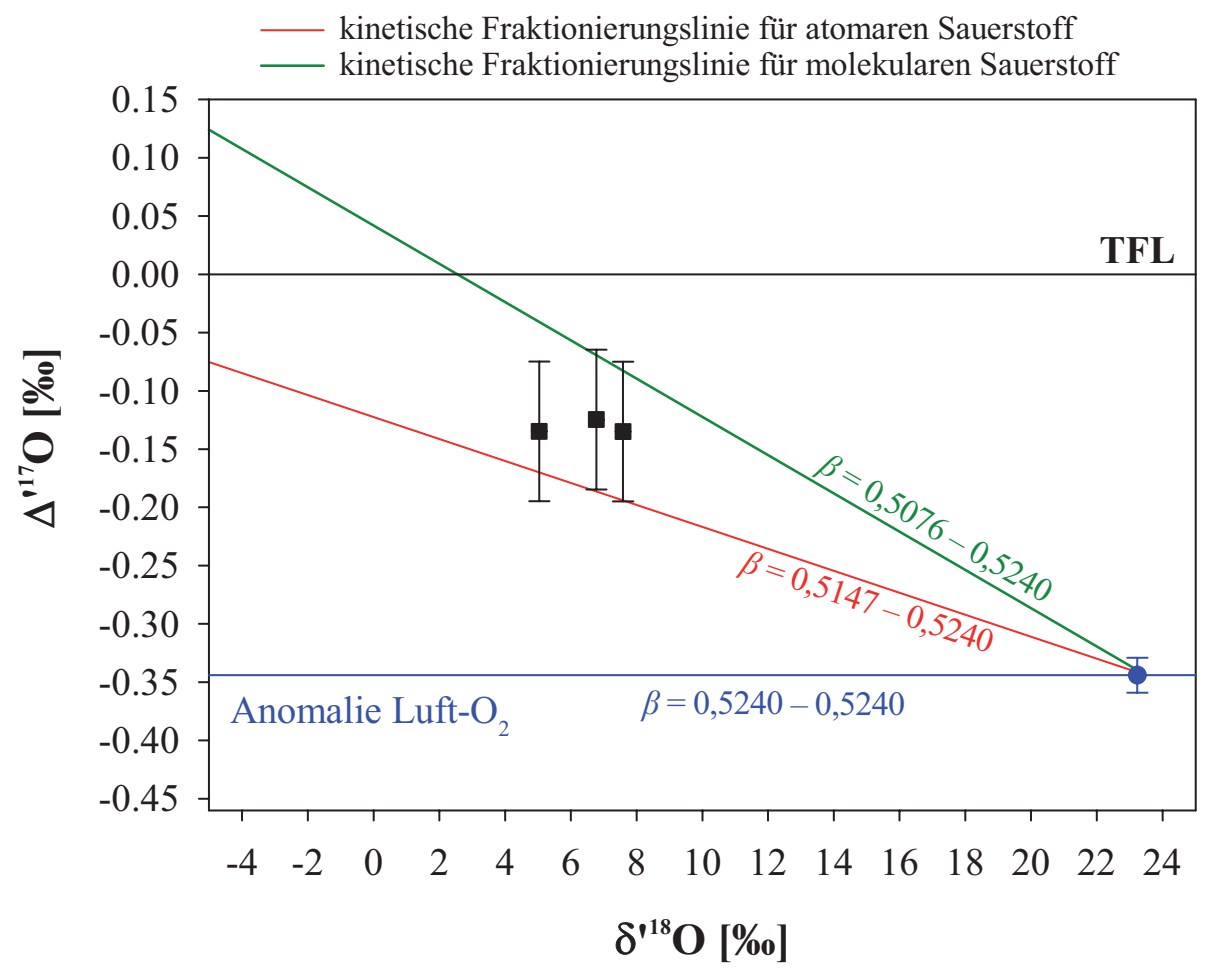

Abbildung 100: $\Delta^{17} \mathrm{O}$ gegen $\delta^{18} \mathrm{O}$ von Luft- $\mathrm{O}_{2}$ (blauer Punkt) und davon ausgehend zwei theoretisch berechnete kinetische Fraktionierungslinien für atomaren und molekularen Sauerstoff. Die schwarzen Quadrate zeigen die Lage dreier Cloggingproben an. 
Die Analyse dreier Cloggingproben ergab im Mittel ein $\Delta^{\prime 17} \mathrm{O}$ von $-0,13 \%$. Einzelanalysen haben einen Fehler im $\Delta^{\prime 17} \mathrm{O}$ von $\pm 0.06 \%$. Damit liegt Clogging geringfügig oberhalb der theoretisch berechneten kinetischen Fraktionierungslinie für atomaren und etwas unterhalb der für molekularen Sauerstoff (siehe Abbildung 100). Die Proben liegen im Fehler der Messung auf beiden Fraktionierungslinien. Bezogen auf Prozess- $\mathrm{O}_{2}$ erscheint die Fraktionierungslinie für atomaren Sauerstoff von größerer Relevanz, da der Prozess- $\mathrm{O}_{2}$ in atomarer Form $\left(\mathrm{O}^{0}\right)$ im Stahl gelöst ist (TURKDOGAN 1996). Für Luft-O ${ }_{2}$ muss möglicherweise auch die Fraktionierungslinie für molekularen Sauerstoff betrachtet werden, wenn Luft- $\mathrm{O}_{2}$ den Stahl direkt oxidiert, ohne vorher gelöst zu werden. Es müssen mehr Messungen durchgeführt werden, um den Messfehler zu verkleinern und die Lage von Clogging im Dreiisotopendiagramm eindeutiger darstellen zu können. Dadurch könnte die Bildung von Clogging durch kinetische Fraktionierung von Prozess- oder Luft- $\mathrm{O}_{2}$ überprüft werden.

In Abbildung 101 ist $\delta^{\prime 17} \mathrm{O}$ gegen $\delta^{\prime 18} \mathrm{O}$ terrestrischer Gesteine dargestellt und relativ dazu die Lage des Prozess- $\mathrm{O}_{2}$. Zwölf Prozess- $\mathrm{O}_{2}$-Proben $(\mathrm{N}=19)$ ergaben im Mittel eine Anomalie im

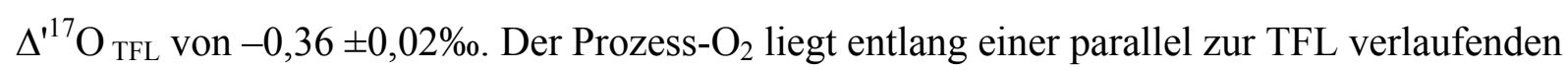
Linie. Dies zeigt an, dass beim Lindeverfahren auch bei einer sehr großen Fraktionierung zwischen ${ }^{18} \mathrm{O}$ und ${ }^{16} \mathrm{O}$ von $>20 \%$ keine Fraktionierung im $\Delta^{17} \mathrm{O}_{\mathrm{TFL}}$ auftritt, und ermöglicht damit erst die Verwendung von $\Delta^{\prime 17} \mathrm{O}_{\mathrm{TFL}}$ als Marker.

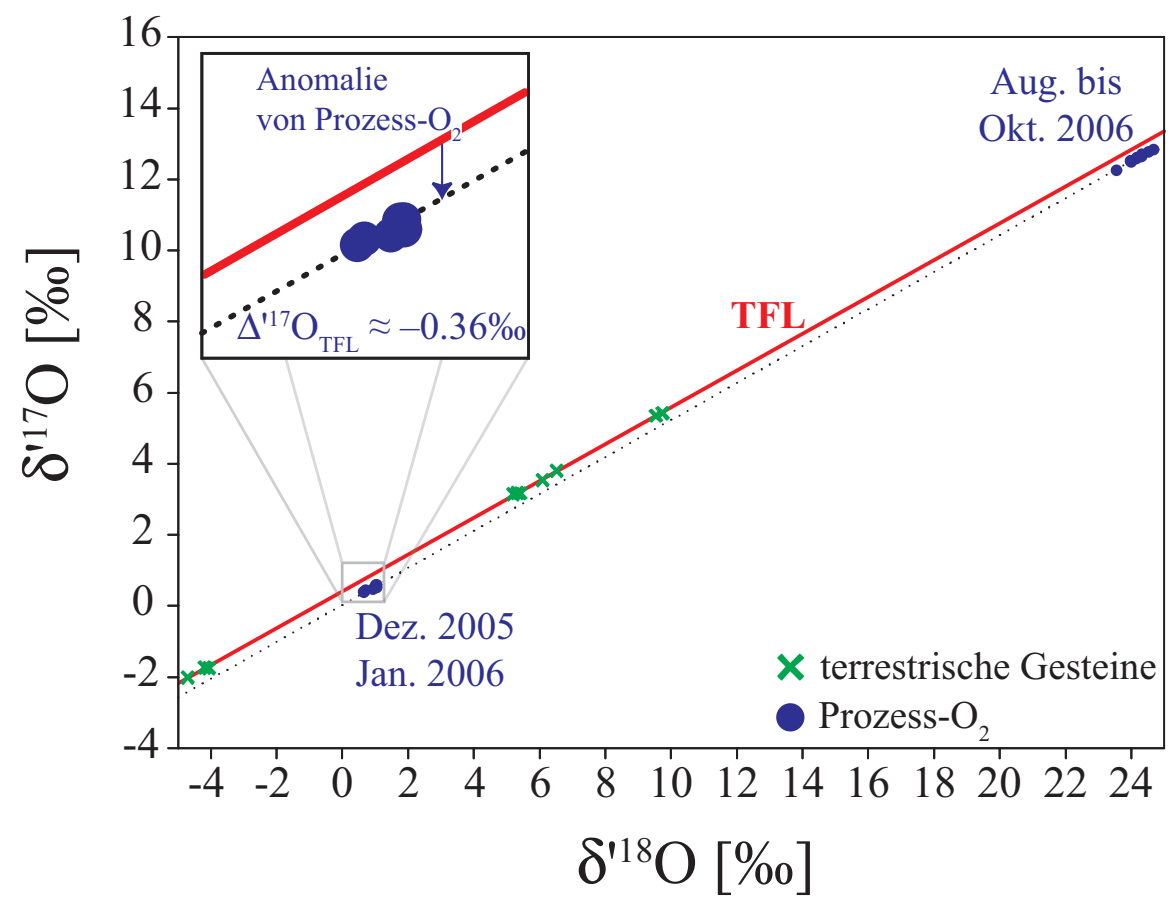

Abbildung 101: Dreiisotopendiagramm von irdischen Gesteinen (rote Linie) und relativ dazu die Lage des Prozess- $\mathrm{O}_{2}$ (gestrichelte Linie). 
Wie in Kapitel 3.4.3 dargestellt, haben Rückstandsisolate aus dem Konverter die Isotopie von Prozess- $\mathrm{O}_{2}$. Daher müssten Rückstandsisolate aus dem Konverter, vorausgesetzt, dass keine Fraktionierung auftritt, die gleiche Anomalie wie Prozess- $\mathrm{O}_{2}$ besitzen. Bisher wurden an zwei Rückstandsisolaten ${ }^{17} \mathrm{O}$-Messungen durchgeführt, die mit $\Delta^{\prime 17} \mathrm{O}_{\mathrm{TFL}}=-0,21 \%$ o und $-0,43 \%$, im Mittel mit $-0,32 \%$ die gleiche Anomalie wie Luft- $\mathrm{O}_{2}$ haben. Das heißt, dass beim Frischen im Konverter und Desoxidieren in der Pfanne keine Fraktionierung im $\Delta^{\prime 17} \mathrm{O}_{\mathrm{TFL}}$ auftritt.

In Abbildung 102 ist der $\Delta^{17} \mathrm{O}_{\mathrm{TFL}}$ von drei Chromiten aus dem Schiebersand, drei Cloggingproben und der von Prozess- $\mathrm{O}_{2}$ dargestellt. Der Chromit liegt bei $\Delta^{\prime 17} \mathrm{O}_{\mathrm{TFL}}=0 \%$ auf der Geraden, auf der alle terrestrischen Gesteine liegen (horizontale schwarze Linie). Der Prozess- $\mathrm{O}_{2}$ liegt auf einer horizontalen Linie bei $\Delta^{17} \mathrm{O}_{\mathrm{TFL}} \approx-0,36 \%$. Clogging liegt zwischen diesen beiden horizontalen Linien bei $\Delta^{17} \mathrm{O}_{\mathrm{TFL}} \approx-0,13 \%$. Danach hat Clogging einen Anteil von rd. 36\% Prozess- $\mathrm{O}_{2}$ und rd. 64\% Chromit. Der 64\%ige terrestrische Anteil lässt sich auch entlang der schräg verlaufenden Mischungslinie (gestrichelte Linie) ablesen (siehe Abbildung 102).

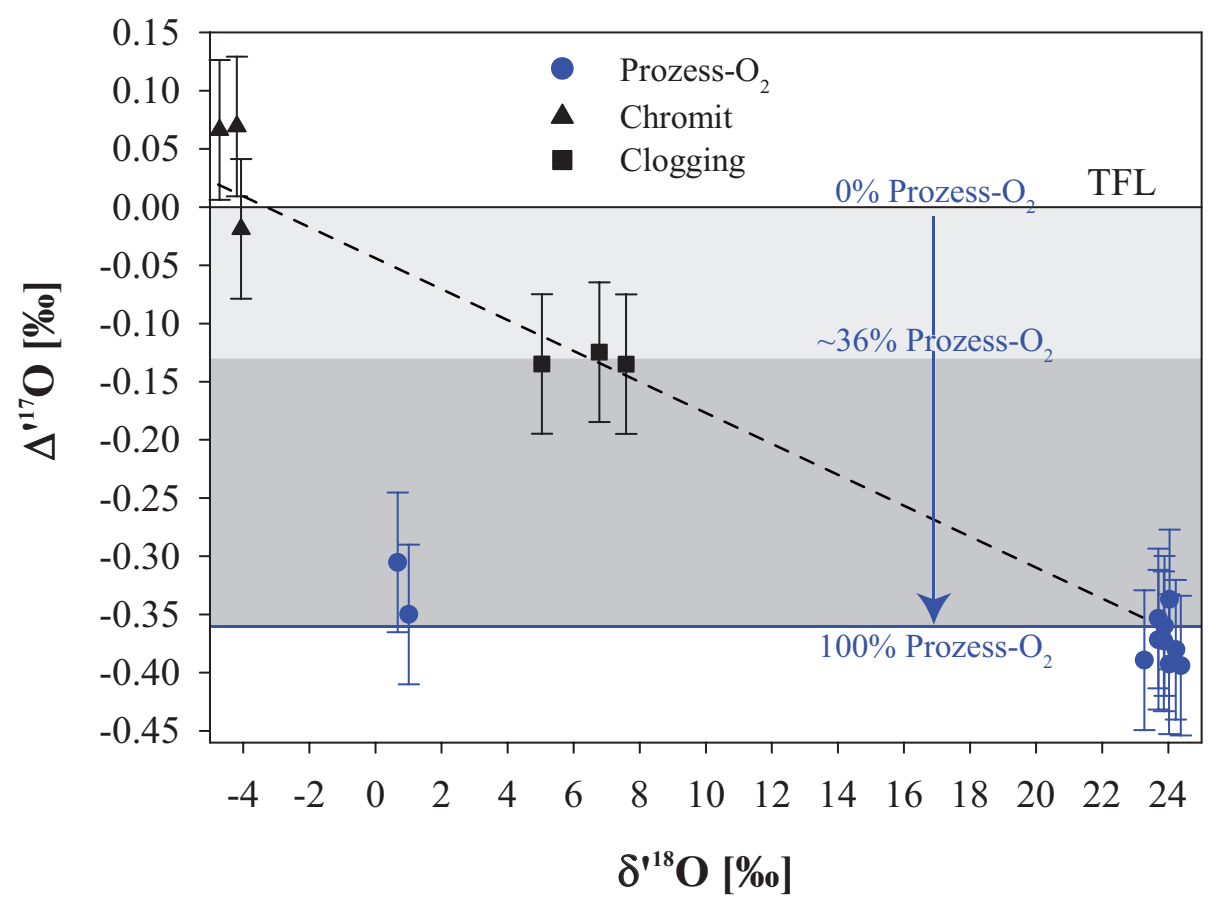

Abbildung 102: $\Delta^{, 17} \mathrm{O}_{\mathrm{TFL}}$ gegen $\delta^{18} \mathrm{O}$ von Chromit, Clogging und Prozess- $\mathrm{O}_{2}$. Die gestrichelte Linie ist eine Mischungslinie.

Das zeigt, dass durch ${ }^{17} \mathrm{O}-$ Messungen direkt Aussagen über die Anteile von verschiedenen OQuellen gemacht werden können. Damit sind diese Messungen konventioneller O-Isotopenanalytik überlegen, bei der lediglich einzelne Isotopien miteinander verglichen werden 
können. Bei der Anwendung von $\Delta^{17} \mathrm{O}_{\mathrm{TFL}}$ als Marker muss ein potentieller Anteil kinetischer Fraktionierung bei der Bildung von Clogging stets berücksichtigt werden.

Mit ${ }^{17}$ O-Messungen kann der Werkzeugkasten zur Klärung der Herkunft nichtmetallischer Ausscheidungen beim Stranggießen von Stahl um ein bedeutendes Tool erweitert werden. Das $\Delta^{17} \mathrm{O}_{\text {TFL }}$ ermöglicht direkt qualitative und quantitative Aussagen über den Anteil atmosphärischer wie terrestrischer 0-Quellen.

Um die aufgestellte Modellvorstellung zu überprüfen, müssen Rückstandsisolate aus dem

Tundish genommen werden. Ihr $\delta^{18} \mathrm{O}$ und $\Delta^{{ }^{17}} \mathrm{O}_{\mathrm{TFL}}$ können Aufschluss darüber geben, ob der Restsauerstoff im Stahl nach dem Desoxidieren kinetisch fraktioniert wird und dadurch niedrige $\delta^{18} \mathrm{O}$-Werte von Clogging erzeugt werden können. Falls dies nicht der Fall sein sollte, könnte kinetische Fraktionierung möglicherweise ausgeschlossen und das Modell bestätigt werden.

\subsection{Weitere Möglichkeiten der Untersuchung von Clogging}

In-Situ-O-Isotopenmessungen von Phasen des Cloggings mittels Ionensonde können benutzt werden, um zu klären, welche Phase was für eine Isotopie mitbringt, und schließlich können über die quantitative Mineralogie die Anteile der einzelnen Cloggingverursacher benannt werden.

Die Messung von Stickstoffisotopen $\left(\delta^{15} \mathrm{~N}\right)$ im Stahl kann Aufschluss über mögliche Fraktionierungen zwischen Luft- und/oder Prozess- $\mathrm{N}_{2}$ und im Stahl beobachtete TiN-Einschlüsse geben (siehe Abbildung 103).

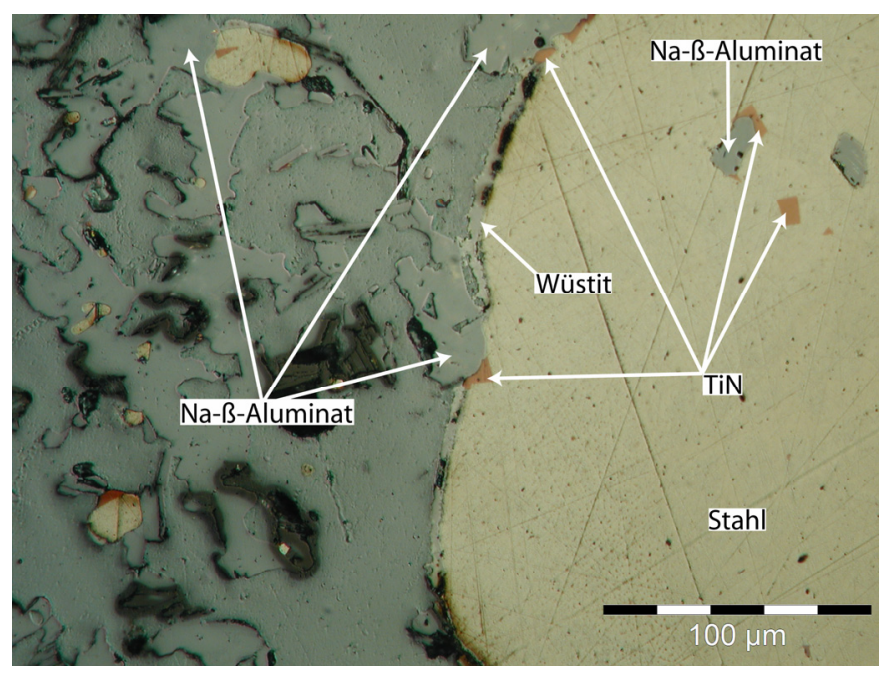

Abbildung 103: Auflichtmikrophotographie der Cloggingprobe vom 04.09.06, Strang 3 mit TiN- und Na- $\beta$-Aluminateinschlüssen im Stahl. Das große Stahlkorn ist von einem Wüstitsaum umgeben. Bei den sich gegenüber dem Einbettungsmedium erhebenden Phasen handelt es sich ebenfalls um Na- $\beta$ Aluminate. 
Dies könnte einen Hinweis auf die Fraktionierung von O-Isotopen geben, da Stickstoff damit auch an Reaktionen beteiligt ist. Eine etwaige Fraktionierung von Stickstoffisotopen im Prozess würde auf eine mögliche Fraktionierung von Sauerstoffisotopen hinweisen.

Spurenelementanalytik inklusive der Messung von Seltenerden mittels Laser Ablation Inductively Coupled Plasma Mass Spectrometry kann genutzt werden, um potentielle Verursacher von Clogging z. B. anhand ihrer spezifischen Seltenerdmuster zu identifizieren und anschlieBend mittels Massenbilanzierungen entsprechend Kapitel 3.4.4 zu quantifizieren. 


\section{Literatur}

Ahlborg, K., Fruehan, R. J., Potter, M. S., Badger, S. R., and Casuccio, G. S. (2003): Inclusions in Aluminum-Killed Steel with Varying Calcium Additions. Conference Proceedings: ISSTech, 177-194.

Allmann, R. (2003): Röntgenpulverdiffraktometrie. Rechnergestützte Auswertung, Phasenanalyse und Strukturbestimmung. Spinger-Verlag, Berlin, 275.

Angert, A., Rachmilevitch, S., Barkan, E., and Luz, B. (2003): Global biogeochemical cycles 17 (1), 1030.

Asprey, L. B. (1976): Journal of Fluorine Chemistry 7, 359-361.

Bannenberg, N. (1995): Demands on refractory material for clean steel production. In: Global Development of Refactories. Technical Association of Refractories, 36-59.

Basu, S., Choudhary, S. K., and Girase, N. U. (2004): ISIJ International 44 (10), 1653-1660.

Berns, H. (1999): Was ist Stahl? Eine Stahlkunde für jedermann. Spinger-Verlag, Berlin, 122.

Binns, R., Lyons, A. R. A., Peyton, A. J., and Pritchard, W. D. N. (2001): Meas. Sci. Technol. 12, 132-1138.

Chialvoa, A. A. and Horita, J. (2006): The Journal of Chemical Physics 125, 034510.

Clayton, R. N., Grossman, L., and Mayeda, T. K. (1973): Science 182 (4111), 485-488.

Coplen, T.B., Hopple, J.A., Böhlke, J.K., Peiser, H.S., Rieder, S.E., Krouse, H.R., Rosman, K.J.R., Ding, T., Vocke, R.D., Jr., K.M. Révész, Lamberty, A., Taylor, P., and Bièvre, P. De (2002): Compilation of Minimum and Maximum Isotope Ratios of Selected Elements in Naturally Occurring Terrestrial Materials and Reagents. United States Geological Survey, 98.

Dawson, S. (1990): Iron \& Steelmaker 4, 33-42.

Deines, P., Nafziger, R. H., Ulmer, G. C., and Woermann, E. (1976): Metallurgical Transactions B-Process Metallurgy 7 (1), 143.

Dekkers, R. (2002): Non-metallic inclusions in liquid steel. Dissertation, Katholieke Universiteit Leuven, 195.

Dekkers, R. (2003): Aardkundige Mededelingen 13, 109-120.

Dekkers, R., Blanpain, B., Plessers, J., and Wollants, P. (2004a): Does hydrogen affect the oxide cleanliness of liquid steel? Conference Proceedings: Non-metallic Inclusions Control and Continuous Improvement of Processes based on Objective Measurement, 24.

Dekkers, R., Blanpain, B., Plessers, J., and Wollants, P. (2004b): Steel cleanliness and hydrogen in liquid steel. Conference Proceedings: 7th Int. Conf. on Molten Slags, Fluxes and Salts, 753-762. 
Dekkers, R., Blanpain, B., and Wollants, P. (2002): Reoxidation: The Cause of Nozzle Clogging. Conference Proceedings: Electric Furnace Conference Proceedings, 287-294.

Dekkers, R., Blanpain, B., and Wollants, P. (2004c): AIST Transactions, Iron and Steel Technology, 93-98.

Dekkers, R., Blanpain, B., Wollants, P., Haers, F., Gommers, B., and Vercruyssen, C. (2003): Steel Research International 74 (6), 351-355.

Dekkers, R., Blanpain, B., Wollants, P., Haers, F., Vercruyssen, C., and Peeters, L. (1999): Evolution of non-metallic inclusions in liquid low alloyed aluminium killed steels. Conference Proceedings: Proceedings of the Extraction and Processing Division of the Minerals, Metals and Materials Society, Pittsburgh, 269-277

Dekkers, R., Wollants, P., Blanpain, B., Haers, F., Vercruyssen, C., and Gommers, B . (2001): Deoxidation Mechanism in Low Carbon Steel Killed with Aluminium. Conference Proceedings: 2nd Int. Congress on the Science \& Technology of Steelmaking, 655665.

DIN, Hrsg. (2000): DIN EN 10020: Begriffbestimmungen für die Einteilung der Stähle. Beuth Verlag GmbH, Berlin

DIN, Hrsg. (2005): DIN Taschenbuch 402: Stahl und Eisen - Gütenormen 2. Betonstahl, Stähle für den Stahlbau, Flacherzeugnisse für Kaltumformung ohne Überzüge, Flacherzeugnisse mit Überzügen, Kaltprofile, Verpackungsblech und -band. Normen und Werkstoffblätter. Beuth Verlag GmbH, Berlin, 776.

Dole, M. (1935): Journal of the American Chemical Society 57 (12), 2731.

Dole, M., Lane, G. A., Rudd, D. P., and Zaukelies, D. A. (1954): Geochimica et Cosmochimica Acta 6, 65-78.

Elfsberg, J. (2003): Oscillation Mark Formation in Continuous Casting Processes. Dissertation, Royal Institute of Technology, Stockholm, 56.

Fischer, P. (1967): Zeitschrift fuer Kristallographie, Kristallgeometrie, Kristallphysik, Kristallchemie 124, 275-302.

Fix, I. (2004): Anwendungen der quantitativen Röntgendiffraktometrie (QXRD) in der pharmazeutischen Analytik. Dissertation, Rheinische Friedrich-Wilhelms-Universität Bonn, 214.

Fuhr, F. and Cicutti, C. (2003): Relationship Between Nozzle Deposits and Inclusion Composition in the Continuous Casting of Steel Conference Proceedings: ISSTech, 165-175.

Fujita, K. and Abe, O. (2006): Geophysical Research Letters 33 (18), L18503.

Gudenau, H. W. (1989a): Eisenhüttenmännische Verfahrenstechnik. Hochofen. Materialsammlung zur Vorlesung. Institut für Eisenhüttenkunde, RWTH Aachen, 274. 
Gudenau, H. W. (1989b): Eisenhüttenmännische Verfahrenstechnik. Vom Erz zum Stahl. Materialsammlung zum Praktikum "Eisenhüttenmännische Verfahrenstechnik". Institut für Eisenhüttenkunde, RWTH Aachen, 497.

Gudenau, H. W. (2002): Metallurgie. Materialsammlung zum Praktikum Metallurgie. Institut für Eisenhüttenkunde, RWTH Aachen, 561.

Hagen, B. (2002): Rekonstruktion von Metamorphosepfaden mit stabilen Isotopen: Möglichkeiten der Laserfluorinierung am Beispiel des Sächsischen Granulitgebirges. Dissertation, Rheinische Friedrich-Wilhelms-Universität Bonn, 179.

Halwax, E. (1998): Materials Science Forum 278-281, 93-98.

Hill, R. J., Craig, J. R., and Gibbs, G. V. (1979): Phys. Chem. Minerals 4, 317-339.

Hilty, D. C. and Kay, D. A. R. (1985): Conference Proceedings: Electric Furnace Steelmaking, 237.

Hiraga, Y., Yashima, Y., and Fujii, K. (1995): Journal of the Technical Association of Refractories 15 (1), 22-27.

Hoefs, J. (2004): Stable isotope geochemistry. Springer Verlag, Berlin, 244.

Höller, H. (1999): Veitsch-Radex Rundschau, 30-39.

International Atomic Energy Agency, Hrsg. (2007): http://wwwpub.iaea.org/MTCD/publications/PDF/Newsletters/WE-NL-20.pdf, 08.10.2007, 13:42 Uhr.

International Iron and Steel Institute, Hrsg. (2007): http://steeluniversity.org, 04.10.2007, 21:45 Uhr.

Jönsson, P., Andersson, M., Nakajima, K., Appelberg, A., Tilliander, A., Shibata, H., and Kitamura, S. (2005): Current Advances in Materials and Processes (CAMP-ISIJ) 18 (4), 849-852.

Juvonen, P. (2004): Effects of Non-Metallic Inclusions on Fatigue Properties of Calcium Treated Steels. Dissertation, University of Technology, Helsinki, 103.

Kemey, F. L. (1998): Tundish Nozzle Clogging - Measurments and Prevention. Conference Proceedings: McLean Symposium Proceedings (ISS, Warrendale, PA), 103-110.

Kiessling, R. (1997): Non-metallic Inclusions in Steel. Part I-IV, The Institute of Materials, London, 400.

Kleber, I., Bautsch, H.-J., and Bohm, J. (1990): Einführung in die Kristallographie. Verlag Technik GmbH, Berlin, 416.

Klug, H. P. and Alexander, L. E. (1974): X-Ray Diffraction Procedures for polycrystalline and amorphous materials. John Wiley \& Sons New York, 966. 
Knüppel, H. (1970): Desoxidation und Vakuumbehandlung von Stahlschmelzen. Band I: Thermodynamische und kinetische Grundlagen. Verlag Stahl und Eisen M.B.H., 6970.

Krischner, H. (1990): Einführung in die Röntgenfeinstrukturanalyse. Vieweg Verlag, Braunschweig, 193.

Lange, K. W. (1988): Int. Mater. Rev. 33 (2), 53.

Leibfried, G. and Dietrich, H. (1951): Zeitschrift für Physik A Hadrons and Nuclei 131 (1), 113-129.

Linde (keine Angabe): Prinzip der Luftzerlegung. 13.

Luo, C. (2001): Modeling the Behavior of Inclusions in Plastic Deformation of Steels. Dissertation, KTH, Stockholm, 64.

Luz, B., Barkan, E., Bender, M. L., Thiemens, M. H., and Boering, K. A. (1999): Nature 400, $547-550$.

McKinney, C. R., McCrea, J. M., Epstein, S., Allen, H. A., and Urey, H. C. (1950): Review of Scientific Instruments 21, 724-730.

Miller, M. F. (2002): Geochimica et Cosmochimica Acta 66 (11), 1881-1889.

Morrison, W. B. (1989): Ironmaking Steelmakaing 16 (2), 123.

Mukai, K. and Zeze, M. (2003): Steel Research International 74 (3), 131-138.

Nicholson, A. and Gladman, T. (1986): Ironmaking Steelmaking 13, 53-69.

Oeters, F. (1989): Metallurgie der Stahlherstellung. Verlag Stahleisen mbH, Düsseldorf, 503.

Ogibayashi, S. (1994): Taikabutsu (Refractories) 46 (4), 166-178.

Ovtchinnikov, S. (2001): Kontrollierte Erstarrung und Einschlußbildung bei der Desoxidation von hochreinen Stahlschmelzen. Dissertation, Technische Universität Bergakademie Freiberg, 193.

Ovtchinnikov, S., Kazakov, S., and Janke, D. (2003): Ironmaking \& Steelmaking 30 (6), 487496.

Pack, A. (2000): Tracing the origin of oxide inclusions in continously cast steel - An interdisciplinary approach. Dissertation, Rheinische Friedrich-Wilhelms-Universität Bonn,164.

Pack, A. (2006): Über die Analyse stabiler Sauerstoffisotope und ihre Verwendung zur Lösung von Problemen aus den Bereichen Geo- und Kosmochemie sowie der angewandten Mineralogie. Habilitationsschrift, Leibniz Universität Hannover

Pack, A., Hoernes, S., Göbbels, M., Bross, R., and Buhr, A. (2005): European Journal of Mineralogy 17 (3), 483-493. 
Pack, A., Hoernes, S., Walther, T., and Bross, R. (2003): European Journal of Mineralogy 15 (1), 193-205.

Pack, A., Toulouse, C., and Przybilla, R. (2007): Rapid Communications in Mass Spectrometry 21, 1-8.

Pleschiutschnigg, F.-P. (1989): Das Verhalten oxidischer Einschlüsse sowie ihre Kontrolle in Al-beruhigtem Stahl während der Stahlerzeugung und des Stranggießens. Habilitation, RWTH Aachen, 182.

Plöckinger, E. (1963): Journal of The Iron and Steel Institute 201 (7), 576.

Poirier, J. and Thillou, B. (1994): Stahl und Eisen (Special issue, XXXVII), International Colloquium on Refractories, Aachen, 114-118.

Rackers, K. G. and Thomas, B. G. (1995): Clogging in Continuous Casting Nozzles. Conference Proceedings: Steelmaking Conference Proceedings, 723-734.

Richard, K. (1969): Chemie Ingenieur Technik. Verfahrenstechnik, Technische Chemie, Apparatewesen 41 (8), 485-490.

Richet, P., Bottinga, Y., and Javoy, M. (1977): Annual Review of Earth and Planetary Sciences 5 65-110.

Rietveld, H. M. (1967): Acta Crystallographica 22, 151-152.

Robinson, S. W., Matrin, I. W., and Pickering, F. B. (1979): Met. Technol., 157-169.

Rumble, D., Miller, M. F., Franchi, I. A., and Greenwood, R. C. (2006): Lunar and Planetary Science XXXVII, 1.

Rumble, D., Miller, M. F., Franchi, I. A., and Greenwood, R. C. (2007): Geochimica et Cosmochimica Acta 71 (14), 3592-3600.

Sasai, K. and Mizukami, Y. (2002): Journal of Technical Association of Refratories 22 (3), 179-186.

Sasai, K. and Mizukami, Y. (2003): Journal of Technical Association of Refratories 23 (3), 156-163.

Savarino, J., Kaiser, J., Morin, S., Sigman, D. M., and Thiemens, M. H. (2007): Atmospheric Chemistry and Physics 7, 1925-1945.

Shaheen, R. (2005): Investigation of the Oxygen Isotope Exchange Between Carbon Dioxide and Ozone via O(1D). Dissertation, Universtität Heidelberg, 128.

Shannon, G. N. and Sridhar, S. (2004): Separation of A12O3 Inclusions Across Interfaces Between Molten Steel Ladle-, Tundish- and Mold-Slags. Conference Proceedings: Metal Separation Technologies III, 259-268.

Sharp, Z. D. (1990): Geochimica et Cosmochimica Acta 54 (5), 1353-1357.

Sharp, Z. D. (2007): Principles of Stable Isotope Geochemistry. 344. 
Shou-Yong, J., Li-Bin, L., Ning-Kang, H., Jin, Z., and Yong, L. (2000): Journal of Materials Science Letters 19, 225-227.

Taylor, H. P. Jr. (1968): Contributions to Mineralogy and Petrology 19, 1-71.

Thiemens, M. H. and Heidenreich, J. E. (1983): Science 219 (4588), 1073-1075.

Thomas, B. G. and Bai, H. (2001): Tundish Nozzle Clogging - Application of Computational Models. Conference Proceedings: 18rd Process Technology Division Conference Proceedings, 895-912.

Toulouse, C., Pack, A., Ender, A., and Petry, S. (in press): Steel Research International, 18.

Turkdogan, E. T. (1996): Fundamentals of Steelmaking. The Institute of Materials, London, 331.

Tuttle, R.B., Peaslee, K.D., and Smith, J.D. (2004): Effects of Nozzle Permeability on Clogging during Continuous Casting. Conference Proceedings: AISTech 2004 Proceedings, 669-680.

Urey, H. C. (1947): Journal of the Chemical Society, 562-581.

US Department of Energy, Hrsg. (2007): Cold Work Embrittlement of Interstitial Free Steel. www.p2pays.org/ref\%5C08/07460.pdf, 09.10.2007, 10:11 Uhr.

van der Eijk, C., Grong, Ø., and Walmsley, J. (2000): Effects of Deoxidation Practice on the Inclusion Formation in Low Alloy Structural Steels. Conference Proceedings: Sixth International Conference on Molten Slags, Fluxes and Salts, 23.

Verein Deutscher Eisenhüttenleute, Hrsg. (1989): Stahlfibel. Verlag Stahleisen mbH, Düsseldorf, 135 .

Verein Deutscher Eisenhüttenleute, Hrsg. (1990): Stahl-Eisen-Liste. Liste der in der Bundesrepublik Deutschland hergestellten Stähle. Verlag Stahleisen mbH, Düsseldorf, 250.

Vermeulen, Y., Coletti, B., Wollants, P., Blanpain, B., and Haers, F. (2000): Steel Research International 71 (10), 391-395.

Weddige, H.-J. (2001): Stahl im Wettbewerb der Werkstoffe. Dissertation, Technische Universität Bergakademie Freiberg, 188.

Wiechert, U. H., Halliday, A. N., Palme, H., and Rumble, D. (2004): Earth and Planetary Science Letters 121, 373-382.

Wijk, O. (1995): Inclusion engineering. Conference Proceedings: Scaninject VII, Part I, MEFOS, 7th International Conference on Refining processes, Lulea, Schweden, 35-67.

Willi, A. V. (1983): Isotopeneffekte bei chemischen Reaktionen. Georg Thieme Verlag, 180.

Young, A. (1993): The Rietveld method. In: International Union of Crystallography monographs on crystallography. 298.

Young, E. D., Gally, A., and Nagahara, H. (2002): Geochimica et Cosmochimica Acta 66 (6), 1095-1104. 
Zhang, L. and Thomas, B. G. (2002): Evaluation and Control of Steel Cleanliness - Review. Conference Proceedings: 85th Steelmaking Conference Proceedings, ISS-AIME, Warrendale, $P A, 431-542$.

Zhang, L. and Thomas, B. G. (2003a): XXIV National Steelmaking Symposium 1, 38-183.

Zhang, L. and Thomas, B. G. (2003b): ISIJ International 43 (3), 271-291.

Zheng, Y. F. (1991): Geochimica et Cosmochimica Acta 55 (8), 2299-2307.

Zimmermann, A. (1974): Die Entstehung oxidische Einschlüsse im Stahl unter besonderer Berücksichtigung des Einflusses feuerfester Materialien des Systems SiO2-A12O3. Technische Universität Clausthal, 50. 


\section{Abbildungen}

Abbildung 1: Kubisch-innenzentriertes Gitter des Ferrits (KLEBER et al. 1990) 5

Abbildung 2: Photo eines Karosseriebauteils für PKWs und dessen typische chemische Zusammensetzung (ULC-IF-Stahl) (INTERNATIONAL IRON AND STEEL INSTITUTE 2007).

Abbildung 3: Der für die Stahlherstellung relevante Teil des Fe-C-Diagramms. Die gestrichelten Linien begrenzen Stabilitätsfelder des metastabilen Systems $\left(\mathrm{Fe}_{-} \mathrm{Fe}_{3} \mathrm{C}\right)$, die durchgezogenen Linien die des stabilen Systems (Fe-C). Letzteres ist in der Praxis der Stahlherstellung weniger von Bedeutung (verändert nach TURKDOGAN 1996).

Abbildung 4: Kubisch-flächenzentrierte Elementarzelle mit $h k l$-Achsenindizierung. Beim Austenit ist Kohlenstoff im Zentrum der Kristallstruktur gelöst (LEIBFRIED and DIETRICH 1951).

Abbildung 5: Variation des Gitterparameters a von reinem Eisen als Funktion der Temperatur (modifiziert nach TURKDOGAN 1996).

Abbildung 6: Dargestellt sind die beiden wichtigsten Verfahrensrouten: Hochofen - Konverter -

Pfannenbehandlung - Gießen - Walzen und Lichtbogenofen - Konverter - Pfannenbehandlung - Gießen -

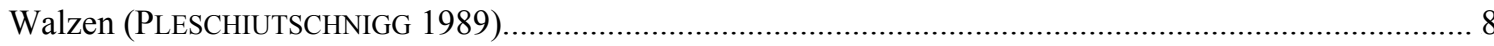

Abbildung 7: Einsatzstoffe und Produkte des Hochofens (GUDENAU 1989a). ............................................. 9

Abbildung 8: Temperatur- und Druckabhängigkeit der Aktivität von Kohlenstoff in $\mathrm{CO} / \mathrm{CO}_{2}$-Gasgemischen

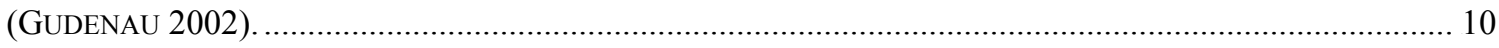

Abbildung 9: Skizze des LD-Konverters während der Hauptphase der Entkohlung (GUDENAU 2002). ............. 12

Abbildung 10: Abbrandverlauf der Elemente beim Konverterprozess (GUDENAU 2002). .............................. 13

Abbildung 11: O-Gehalt in Fe-Schmelzen in Abhängigkeit vom Al-Gehalt (KNÜPPEL 1970).......................... 15

Abbildung 12: RH-Vakuumanlage mit feuerfesten Einlaufstutzen zur Überführung der Schmelze aus der Pfanne in den Unterteil des Vakuumbehandlungsgefäßes (verändert nach INTERNATIONAL IRON AND STEEL

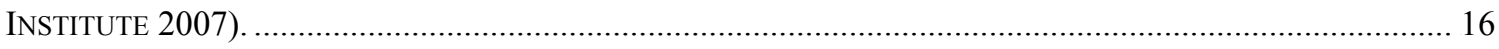

Abbildung 13: Skizze eines Querschnitts einer Stranggussanlage (verändert nach BINNS et al. 2001).............. 17

Abbildung 14: Form von Einschlüssen im Stahl vor (links) und nach dem Walzen (rechts). (a) duktile

Einschlüsse, (b) spröde Einschlüsse und (c) nicht verformbare, sphärische Einschlüsse mit einem duktilen Rand (DEKKERS 2003) 20

Abbildung 15: Asymmetrische Einschlusskontamination eines Strangs, verursacht durch asymme-trischen Gießstrahl aufgrund von Clogging im Tauchrohr (ZHANG and THOMAS 2003b).

Abbildung 16: Versuchsaufbau zur Oxidation der Fe,Al-Legierungen in einem vertikalen Gasmischungsofen.

Die Proben wurden in einem Korundtiegel unter reduzierender Atmosphäre partiell oxidiert (rechts Photo des Probenhalters im Rohrofen).

Abbildung 17: Vertikaler Gasmischungsofen (links), Kieselglasbehälter zum Quenchen des Tonerdetiegels nach

Beendigung des Experimentes (rechts).

Abbildung 18: IR-Laserfluorinierungsanlage am Mineralogisch-Petrologischen Institut der Rheinischen

Friedrich-Wilhelms-Universität Bonn (verändert nach PACK 2000).

Abbildung 19: IR-Laserfluorinierungsanlage mit $\mathrm{CO}_{2}$-Laser, Hochvakuumline (Weg des Gases durch rote Linie vereinfacht angedeutet) und Gasmassenspektrometer am CNR in Pisa (Italien)..... 
Abbildung 20: Rasteraufnahme der synthetisierten Tonerde (REM, EDS).

Abbildung 21: Fe,Al-Legierungen mit 0,28, 0,68 und 0,87 Al [Gew.\%] Ausgangszusammensetzung (blaue Säulen) und Al-Gehalte nach den Experimenten (grüne Säulen).

Abbildung 22: O-Isotopenanalyse der synthetisierten Tonerde unter $\mathrm{H}_{2} / \mathrm{CO}_{2}$-Gasstrom und die der Ofenatmosphäre $\left(\mathrm{H}_{2} / \mathrm{CO}_{2}\right.$-Gasstrom).

Abbildung 23: Abgetragen ist $\delta^{18} \mathrm{O}$ von Ofenatmosphäre und Tonerde gegen die anteilig gebildete Tonerde $f$. . 33

Abbildung 24: $\delta^{18} \mathrm{O}$ von Sauerstoff und Tonerde als Funktion des noch nicht reagierten Sauerstoffanteils $(f)$ und des Fraktionierungsfaktors $(\alpha)$ zwischen Sauerstoff und Tonerde. (A) $\delta^{18} \mathrm{O}$ von $\mathrm{O}_{2}$ und Tonerde für unterschiedliche Fraktionierungsfaktoren $\left(\alpha_{\mathrm{O}_{2} \text {-Tonerde }}>1\right)$. Die aktuell berechnete Fraktionierung zwischen $\mathrm{O}_{2}$ und Tonerde ist $0,4 \%$. (B) $\delta^{18} \mathrm{O}$ von $\mathrm{O}_{2}$ und Tonerde für unterschiedliche Fraktionierungsfaktoren ( $\alpha_{\mathrm{O}_{2}-\text { Tonerde }}<1$ ) (aus PACK et al. 2005). 36

Abbildung 25: Modell der experimentellen Versuchsbedingungen mit leichten Molekülen mit hohen Geschwindigkeiten (lange Pfeile) und schweren Molekülen mit niedrigen Geschwindigkeiten (kurze Pfeile).

Abbildung 26: Fraktionierung zwischen Ofenatmosphäre und gebildeter Tonerde für unterschiedliches $\mathrm{fO}_{2}$ (semiquantitative Darstellung).

Abbildung 27: Sekundärelektronenbild der Cloggingprobe vom 05.09.05, Strang 2, Stahlwerk A.

Abbildung 28: Diffraktogramm und Rietveldanalyse von Tauchrohrclogging; blaue Linie = gemessenes

Diffraktogramm, rote Linie $=$ angepasstes Diffraktogramm (Rietveldanalyse), graue Line $=$

Differenzkurve, senkrechte Striche = Peaklagen der Phasen.

Abbildung 29: Photo von Tauchrohr 2 mit Clogging. Der rote Kreis zeigt den Bereich der Probennahme

(05.09.05, Strang 2, Stahlwerk A). Das Tauchrohr hat eine Länge von rd. einem Meter und einen Innendurchmesser von rd. $9 \mathrm{~cm}$ im Bereich D.

Abbildung 30: Zusammenfassendes Histogramm der O-Isotopie von Tauchrohrclogging und einige mögliche O-

Quellen. Dargestellt sind alle bisher gemessenen Tauchrohrcloggingproben.

Abbildung 31: Zeichnung des Versuchsaufbaus zur Extraktion von $\mathrm{O}_{2}$ aus den Inertgasen $\mathrm{Ar}$ und $\mathrm{N}_{2}$.

Abbildung 32: Photo der $\mathrm{CO}_{2}$-Linie mit Probengasflasche (links), Ofen zur $\mathrm{O}_{2}$-Konvertierung (Mitte rechts) und

Drehschieberpumpe (hinten rechts) zur Evakuierung der Anlage.

Abbildung 33: Aufgetragen ist der $\delta^{18} \mathrm{O}$-Wert der gebildeten Tonerde gegen $\log f \mathrm{O}_{2}$. Der $\delta^{18} \mathrm{O}$-Wert der

Ofenatmosphäre ist als horizontale Linie dargestellt.

Abbildung 34: Zusammensetzung der Fe,Al-Legierungen vor (blaue Säulen) und nach (grüne Säulen) den

Experimenten (die Legierungen wurden nach den Experimenten mikrochemisch analysiert).

Abbildung 35: Zusammenfassendes Histogramm aller bisher gemessenen $\delta^{18} \mathrm{O}-\mathrm{Werte}$ von nichtmetallischen

Ablagerungen im Tauchrohr und an dessen Unterseite.

Abbildung 36: $\delta^{18} \mathrm{O}-$ Werte von Clogging und SEN-Unterseiten-Ablagerungen aus Teil II an acht Tagen. 54

Abbildung 37: Aufgetragen ist der $\delta^{18} \mathrm{O}-$ Wert von SEN-Unterseiten-Ablagerungen gegen den Abstand vom FF-

Material der SEN-Unterseite (Teil II) und Daten aus PACK et al. (2005).

Abbildung 38: Dargestellt ist die SEN-Unterseiten-Ablagerung vom 24.10.06, Strang 4, Stahlwerk B.

Abbildung 39: Zusammenhang zwischen der Isotopie von oxidischen Ablagerungen beim Stranggießen von

Strang 3 und 4 . 
Abbildung 40: Zusammenhang zwischen der Chemie oxidischer Ablagerungen von Strang 3 und 4. 56

Abbildung 41: Korrelation zwischen $\delta^{18} \mathrm{O}$ und $\mathrm{Al}_{2} \mathrm{O}_{3}$-Gehalt von Clogging.

Abbildung 42: Photos des Tauchrohres nach dem Versuch mit offenem Stopfen. Links sind etwa $1 \mathrm{~cm}$ mächtige Anfrierungen von Stahl unmittelbar nach dem Gießen zu sehen. Rechts ist Clogging aus dem oberen SENBereich (Richtung Stopfen) abgebildet. Die Anfrierung besteht aus Stahl mit einem Netzwerk aus Clogging. 57

Abbildung 43: Isotopie von Clogging und von Luft- $\mathrm{O}_{2}$ beim Versuch mit offenem Stopfen. 58

Abbildung 44: Zeichnung des Tauchrohres, das beim Versuch mit offenem Stopfen zum Einsatz kam. In rot sind die $\delta^{18} \mathrm{O}$-Werte von Clogging in Promille angegeben. 58

Abbildung 45: Organigramm über die Blassauerstofflieferung von Linde und Air Liquide an die ThyssenKrupp Steel AG. Zwei Drittel des $\mathrm{O}_{2}$ kommen von Linde, ein Drittel stammt von Air Liquide.

Abbildung 46: Luftzerleger (links) mit den Produkten GOX und LOX am Boden und $\mathrm{N}_{2}$ am Kopf der Säule und ein Ausschnitt (rechts), der den prinzipiellen Aufbau einer Rektifikationssäule zeigt (verändert nach LINDE).

Abbildung 47: Fraktionierung zwischen Dampf und Wasser bei Kondensation aus einer Wolke nach dem Rayleighschen Destillationsgesetz (siehe auch Kapitel 2.3.4) (HoEFs 2004).

Abbildung 48: Versuchsaufbau zur Bestimmung der Fraktionierung zwischen GOX und LOX.

Abbildung 49: Aufgetragen ist der $\Delta^{18} \mathrm{O}_{\text {LOX-GOX }}$ gegen unterschiedliche Equilibrierungszeiten $\left(\Delta^{18} \mathrm{O}_{\text {LOX-GOX }}=\right.$ $\left.\delta^{18} \mathrm{O}_{\mathrm{LOX}}-\delta^{18} \mathrm{O}_{\mathrm{GOX}}\right)$

Abbildung 50: Aufgetragen ist der $\Delta^{18} \mathrm{O}_{\text {LOX-GOX }}$ gegen die Temperatur (verändert nach CHIALVOA and HORITA 2006).

Abbildung 51: $\delta^{18} \mathrm{O}$ des Prozess-O ${ }_{2}$ von Teil I und II inkl. der Proben von GOX und LOX (alle Proben außer GOX und LOX wurden im Stahlwerk B genommen).

Abbildung 52: Isotopie von Frisch- $\mathrm{O}_{2}$ und Rückstandsisolaten aus dem Konverter an drei Tagen.

Abbildung 53: Dargestellt sind Tundishschlackenanalyen als Ergebnis der chemischen Analyse im System CaO$\mathrm{Al}_{2} \mathrm{O}_{3}-\mathrm{SiO}_{2}$. Bis auf zwei Proben liegen alle im Liquidusfeld $\left(1600^{\circ} \mathrm{C}\right)$.

Abbildung 54: Dreikomponentenmodell von Tundishschlacke bestehend aus assimilierter Tonerde aus dem

Stahl, Reisschalenasche und Abdeckpulver.

Abbildung 55: Lineares Gleichungssystem mit vier Gleichungen und vier Unbekannten zur Berechnung der Anteile von RA, AP, To und VTR an der Tundishschlacke.

Abbildung 56: Berechnete Zusammensetzung der Tundishschlacke und ihre $\delta^{18} \mathrm{O}-\mathrm{Werte}$ (gemessen und berechnet).

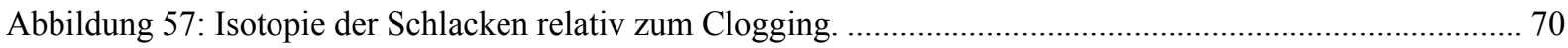

Abbildung 58: Isotopie von FF-Materialien und relativ dazu die Lage von Blassauerstoff aus Teil II. .............. 71

Abbildung 59: Isotopie der verschiedenen Phasen von Schiebersanden unterschiedlicher Hersteller. 72

Abbildung 60: Rückstreuelektronenbild eines Enstatitkorns mit Verwachsungen von Chromit und Klinopyroxen (Schiebersand Hersteller A03). 72

Abbildung 61: Illustration des Zweikomponentenmodells für die Herkunft von Clogging. 76

Abbildung 62: Prinzip der Braggschen Röntgenbeugung an einer Netzebenenschar. d ist der Netzebenenabstand, $\theta$ ist der Glanz- oder Braggwinkel... 
Abbildung 63: Strahlengang eines Pulverdiffraktometers mit Bragg-Brentano-Geometrie mit Blendensystem und sekundärem Monochromator (ALLMANN 2003)

Abbildung 64: Dargestellt sind die Gehalte in Gew.-\% der eingewogenen Korund-Spinell-Mischungen gegen die berechneten Gehalte. Das Diagramm zeigt nur geringe Abweichungen (bis rd. 6\%) zwischen eingewogenen und berechneten Gehalten bei der quantitativen Analyse der untersuchten Korund-SpinellMischungen mittels Win XPOW.

Abbildung 65: Vergleichendes Beispiel einer Rietveldverfeinerung der SEN-Unterseiten-Ablagerung vom 05.09.06, Strang 4. Der obere Fit zeigt eine deutlich bessere Anpassung an das beobachtete Diffraktogramm. Beim unteren Fit werden deutlich mehr Peaks nicht abgedeckt, die Restintensität schlägt sich in einer nach oben abweichenden Differenzkurve nieder.

Abbildung 66: Beugungsdiagramm und Rietveldplot von Clogging vom 04.09.06, Strang 3. Die Probe hat einen Tonerdegehalt von $\geq 95$ Gew.-\%. Bei 45 Grad $2 \theta$ ist ein unidentifizierter Peak, der möglicherweise zu elementarem Eisen gehört.

Abbildung 67: Beugungsdiagramm und Rietveldplot der SEN-Unterseiten-Ablagerung vom 24.10.06, Strang 3.

Abbildung 68: Spinell- gegen MgO-Gehalt von Clogging und SEN-Unterseiten-Ablagerungen...... 88

Abbildung 69: Gitterparameter $\mathrm{a}_{0}$ von Spinellen $\left(\mathrm{MgAl}_{2} \mathrm{O}_{4}\right)$ der oxidischen Ablagerungen im Tauchrohr und an dessen Unterseite (schwarze Punkte). Relativ dazu ist die Lage von Hercynit $\left(\mathrm{FeAl}_{2} \mathrm{O}_{4}\right)$, Spinell $\left(\mathrm{MgAl}_{2} \mathrm{O}_{4}\right)$ und nichtstöchiometrischen Spinellen $\left[\mathrm{MgO} \times n\left(\mathrm{Al}_{2} \mathrm{O}_{3}\right)\right]$ aus Literaturdaten dargestellt. Für $n>1$ sitzt $\mathrm{Al}^{3+}$

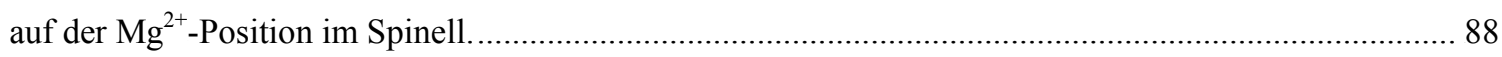

Abbildung 70: Hibonit- gegen $\mathrm{CaO}-G e h a l t$ von Clogging und SEN-Unterseiten-Ablagerungen....................... 89

Abbildung 71: Tonerdegehalt gegen Netto- $\mathrm{Al}_{2} \mathrm{O}_{3}$-Gehalt von Clogging. Die Lage von SEN-Unterseiten-

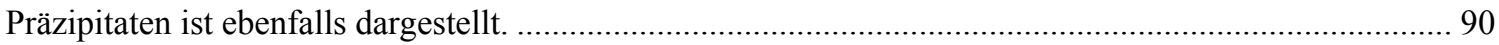

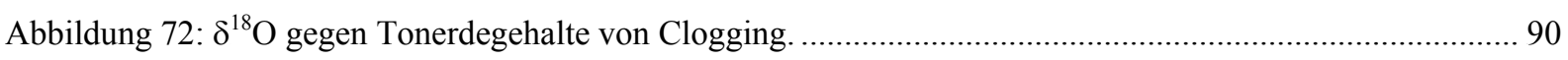

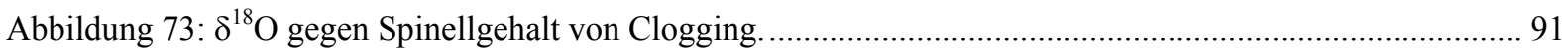

Abbildung 74: Tundishschlackenprobe vom 28.08.06, Strang 3. Das Diffraktogramm zeigt einen hohen Glasanteil im Pulver. Als einzige kristalline Phase wurde Cristobalit $\left(\mathrm{SiO}_{2}\right)$ identifiziert.

Abbildung 75: Tundishschlackenprobe vom 01.09.06, Strang 3. Als einzige Phase wurde Gehlenit $\left(\mathrm{Ca}_{2} \mathrm{Al}_{2} \mathrm{SiO}_{7}\right)$ identifiziert.

Abbildung 76: Tundishschlackenprobe vom 29.08.06, Strang 3. Auffallend ist der Chromit, der insgesamt nur in

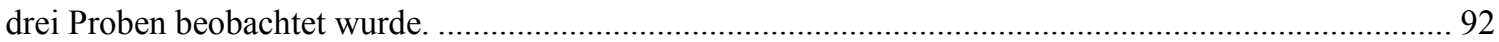

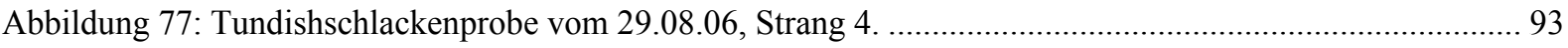

Abbildung 78: Tundishschlackenprobe vom 23.10.06, Strang 3. 93

Abbildung 79: Das Beugungsdiagramm des Schiebersandes von Hersteller B (Gesamtanalyse) hat den stärksten Reflex bei 27 Grad $2 \theta$ (Chromit). Der zweitstärkste Reflex liegt bei 36 Grad $2 \theta$ und gehört zum Quarz. . 94 Abbildung 80: Dargestellt ist das Beugungsdiagramm des separierten Enstatit. Der Untergrund ist durch amorphe Anteile im Pulver erhöht. Das Pulver wurde aufgrund geringer Probenmenge als Schwemmpräparat gemessen.

Abbildung 81: Das Beugungsdiagramm zeigt den hochsymmetrischen Periklas des Pfannensteins C, der nur vier Reflexe aufweist. Bei 26 Grad $2 \theta$ liegt der stärkste Peak des Graphits. Ein nennbarer Untergrund ist nicht zu erkennen, was auf einen sehr hohen kristallinen Anteil im Pulver schließen lässt. 
Abbildung 82: Diffraktogramm des FF-Materials eines Tauchrohres, das direkten Kontakt zur Schmelze hatte (nach dem Gießen)

Abbildung 83: Unterteilung von Clogging in drei Hauptlithologien (Aufsichtschnitt senkrecht zur Längsachse des Tauchrohres). Am Kontakt zur Stahlschmelze treten die Körner meist in einem losen Verband auf. In einem Zwischenbereich sind meist isometrische bis leistenförmige Einsprenglinge in einer glasigen Matrix zu beobachten. Am Kontakt zum FF-Material des SEN bildet das Clogging typischerweise ein korallenartiges Netzwerk aus.

Abbildung 84: Sekundärelektronenbild von porösem, korundreichem Clogging am Kontakt zur Stahlschmelze (Probe vom 04.09.06, Strang 3). Auffallend ist hier ein relativ großes Melilithkorn.

Abbildung 85: Sekundärelektronenbild von Clogging (28.08.06, Strang 3) mit idiomorphen Korund- und Hibonitkristallen in einer glasigen Al-, Si-, Ca-Matrix. Aufgrund seiner größeren mittleren Ordnungszahl erscheint der Hibonit relativ zum Korund heller im elektronenoptischen Bild.

Abbildung 86: Sekundärelektronenbild von Clogging (28.08.06, Strang 3). Die leistenartigen Hiboniteinsprenglinge bilden ein sperriges intersertales Gefüge in glasiger Grundmasse.

Abbildung 87: Sekundärelektronenbild von Clogging (31.08.06, Strang 4). Hypidiomorphe Hercynitkörner in einer dichten Anordnung mit rundlichen Wüstitkristallen.

Abbildung 88: Auflichtmikrophotographie von Clogging am Kontakt zum Tauchrohr (Probe vom 29.08.06, Strang 3).

Abbildung 89: Übersicht über den Kontaktbereich von Clogging zum FF-Material des Tauchrohres. Die schwarzweiß gestrichelte Linie zeigt die Grenze an. Die Tonerdekörner des Tauchrohres unterscheiden sich vor allen Dingen in ihrer Größe von denen des Cloggings.

Abbildung 90: Rückstreuelektronenbild des Schiebersandes von Hersteller B. 101

Abbildung 91: Rückstreuelektronenbild gerundeter Quarzkörner und kubischer Chromitkörner (Hersteller A03).

Abbildung 92: Rückstreuelektronenbild des mikrokristallinen triklinen Mikroklins (Kalifeldspat) in Verbindung mit feinkörnigem Quarz (Hersteller A03).

Abbildung 93: Durchlichtmikrophotographie des Pfannensteins D mit rotbräunlichem, korrodiertem Periklas und weißem, höherwertigen Periklas in feinkörniger Kohlenstoffmatrix

Abbildung 94: Durchlichtmikrophotographie eines getemperten, pechgebundenen Magnesia-Kohlenstoffsteins (Pfannenstein B) mit einem großen, rotbräunlichen Periklasfragment neben weißen Periklaskristallen in feinkörniger Kohlenstoffmatrix.

Abbildung 95: $\mathrm{Na}_{2} \mathrm{O}$ gegen $\mathrm{K}_{2} \mathrm{O}$ von oxidischen Ablagerungen und möglichen Alkalienquellen. 105

Abbildung 96: Dreiisotopendiagramm von irdischen Gesteinen (rote Linie) und relativ dazu die Lage von Mondgesteinen und Meteoriten (verändert nach WIECHERT et al. 2004).

Abbildung 97: Schematisches Dreiisotopendiagramm zur Illustration der Anomalie von $\mathrm{Luft}-\mathrm{O}_{2}$. Troposphärisches $\mathrm{O}_{2}$ entsteht bei der Photosynthese und wird massenabhängig bei der Respiration und massenunabhängig durch photochemische Reaktionen in der Stratosphäre fraktioniert. Die Balance zwischen beiden Prozessen bestimmt die Dreiisotopenzusammensetzung (ANGERT et al. 2003).

Abbildung 98: $\delta^{17} \mathrm{O}$ gegen $\delta^{18} \mathrm{O}$ von Luft- $\mathrm{O}_{2}$ als Produkt von kinetischer Fraktionierung während der Respiration und Eintrag von anomalem stratosphärischem $\mathrm{O}_{2}$ (MILLER 2002)...... 
Abbildung 99: $\delta^{17} \mathrm{O}$ gegen $\delta^{\prime 18} \mathrm{O}$ von irdischen Gesteinen mit $\beta=0,5240$ (schwarze Gerade $=\mathrm{TFL}$ ). Die rote Gerade ist die theoretisch berechnete kinetische Fraktionierungslinie für atomaren Sauerstoff $(\beta=0,5147)$, die grüne Gerade ist jene für molekularen Sauerstoff $(\beta=0,5076)$.

Abbildung 100: $\Delta^{\prime 17} \mathrm{O}$ gegen $\delta^{\prime 18} \mathrm{O}$ von Luft-O $\mathrm{O}_{2}$ (blauer Punkt) und davon ausgehend zwei theoretisch berechnete kinetische Fraktionierungslinien für atomaren und molekularen Sauerstoff. Die schwarzen Quadrate zeigen die Lage dreier Cloggingproben an.

Abbildung 101: Dreiisotopendiagramm von irdischen Gesteinen (rote Linie) und relativ dazu die Lage des Prozess- $\mathrm{O}_{2}$ (gestrichelte Linie).

Abbildung 102: $\Delta^{\prime 17} \mathrm{O}_{\mathrm{TFL}}$ gegen $\delta^{\prime 18} \mathrm{O}$ von Chromit, Clogging und Prozess- $\mathrm{O}_{2}$. Die gestrichelte Linie ist eine Mischungslinie.

Abbildung 103: Auflichtmikrophotographie der Cloggingprobe vom 04.09.06, Strang 3 mit TiN- und Na- $\beta$ Aluminateinschlüssen im Stahl. Das große Stahlkorn ist von einem Wüstitsaum umgeben. Bei den sich gegenüber dem Einbettungsmedium erhebenden Phasen handelt es sich ebenfalls um Na- $\beta$-Aluminate. 125 


\section{Tabellen}

Tabelle 1: Einteilung der Stähle nach chemischer Zusammensetzung und Hauptgüteklassen (DIN 2000)........... 3

Tabelle 2: Grenzwerte zwischen legierten und unlegierten Stählen nach (DIN 2000).................................... 4

Tabelle 3: Typische Zusammensetzung von in Deutschland erzeugtem Roheisen in Gew.-\% (GUDENAU 2002). 10

Tabelle 4: Hochofenschlackenzusammensetzung in Gew.-\% nach GUDENAU (1989a).................................. 11

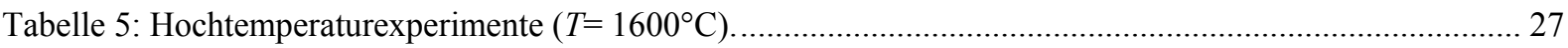

Tabelle 6: Geschwindigkeitsquotient $\left(v_{1} / v_{2}\right)$ und Fraktionierung $(\Delta)$ zwischen Gasspezies und der zuerst

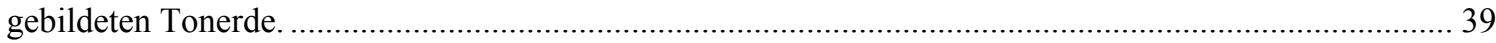

Tabelle 7: RFA von Tauchrohrclogging aus Stahlwerk A und B (Auszug der RFA) ................................... 41

Tabelle 8: Übersicht über die genommenen Proben nichtmetallischer Ablagerungen in Tauchrohren und an deren

Unterseiten im Stahlwerk B (US = Ablagerungen an der SEN-Unterseite). ........................................ 49

Tabelle 9: Übersicht über die genommen Schlackenproben im Stahlwerk B. ................................................. 50

Tabelle 10: Übersicht über die im Mai 2006 durchgeführten Hochtemperaturexperimente................................50

Tabelle 11: Einfluss von Luft- $\mathrm{O}_{2}$ am Gesamt-O bei nicht inertisiert gezogenen Lollyproben aus dem Konverter.

Tabelle 12: Menge an Tonerde, die pro Pfanne maximal aus Al gebildet werden könnte, wenn ausreichend Sauerstoff zur Verfügung stünde.

Tabelle 13: Menge an Tonerde, die pro Pfanne bei gegebenem Sauerstoffgehalt maximal gebildet werden kann.

Tabelle 14: Maximale Menge an Tonerde, die pro Pfanne aus Chromit gebildet werden kann. 75

Tabelle 15: Einwaagen der fünf Korund-Spinell-Mischungen und die jeweiligen prozentualen Gewichtsanteile

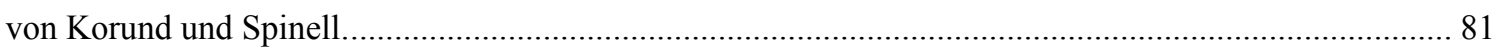

Tabelle 16: Ergebnisse der quantitativen Phasenbestimmung mit Win XPOW .............................................8 82

Tabelle 17: Übersicht über die röntgenographisch untersuchten feuerfesten Erzeugnisse der Pfanne.................96

Tabelle 18: RFA in Gew.-\% von Clogging (Cg) und Ablagerungen an der SEN-Unterseite (Cg US) aus Teil II.

Tabelle 19: RFA in Gew.-\% von Tundishschlackenproben (Tu) aus Teil II.

Tabelle 20: $\delta^{18} \mathrm{O}$-Werte oxidischer Ausscheidungen aus der Voruntersuchung, aus Teil I und aus Teil II. Alle Proben wurden im continuous-flow Modus gemessen.

Tabelle 21: $\delta^{18} \mathrm{O}-$ Werte von Prozessmaterialien. Alle Proben wurden im continuous-flow Modus gemessen... 144

Tabelle 22: $\delta^{\prime 18} \mathrm{O}-, \delta^{\prime 17} \mathrm{O}$ - und $\Delta^{\prime 17} \mathrm{O}-\mathrm{Werte}$ von Prozess- $\mathrm{O}_{2}$, Chromit aus dem Schiebersand und von drei Cloggingproben. Alle Daten wurden dual-inlet Modus gemessen.

Tabelle 23: Übersicht über die Ergebnisse der Rietveldanalyse von Clogging ( $\mathrm{Cg})$ und SEN-Unterseiten-

Präzipitaten (Cg US) aus Teil II. Crn = Korund, Spl = Spinell, Hem = Hämatit, NaMg-Al = Na-Mg-

Aluminat, $\beta-\mathrm{Al}=\mathrm{Na}-\beta-$ Alumninat, Dia $=$ Diaoyudaoit, Gra $=$ Graphit, $\mathrm{Ru}=$ Rutil. 146 


\section{Anhang}

\subsection{Chemische Analyse}

\subsubsection{Oxidische Ausscheidungen}

Tabelle 18: RFA in Gew.-\% von Clogging (Cg) und Ablagerungen an der SEN-Unterseite (Cg US) aus Teil II.

\begin{tabular}{|c|c|c|c|c|c|c|c|c|c|}
\hline Probe & MnO & $\mathrm{SiO}_{2}$ & $\mathrm{TiO}_{2}$ & $\mathrm{Al}_{2} \mathrm{O}_{3}$ & $\mathrm{CaO}$ & MgO & $\mathrm{ZrO}_{2}$ & $\mathrm{~K}_{2} \mathrm{O}$ & $\mathrm{Na}_{2} \mathrm{O}$ \\
\hline Cg Str3 060828 & 0.3 & 0.6 & 0.3 & 91.5 & 1.3 & $\overline{5.8}$ & 0.0 & 0.1 & 0.1 \\
\hline Cg Str3 060829 & 0.2 & 0.5 & 0.3 & 93.3 & 0.3 & 2.4 & 0.0 & 1.1 & 1.9 \\
\hline Cg Str3 060831 & 0.2 & 0.3 & 0.0 & 91.6 & 1.3 & 5.9 & 0.0 & 0.3 & 0.5 \\
\hline Cg Str3 060904 & 0.0 & 0.7 & 1.5 & 96.6 & 0.6 & 0.4 & 0.0 & 0.0 & 0.1 \\
\hline Cg Str3 060905 & 0.2 & 1.2 & 1.9 & 94.7 & 1.0 & 0.4 & 0.0 & 0.1 & 0.5 \\
\hline Cg Str3 061024 Kontakt m. SEN & 0.2 & 0.1 & 1.2 & 90.7 & 5.6 & 0.9 & 1.1 & 0.0 & 0.2 \\
\hline Cg Str3 061024 Kontakt m. Schmelze & 0.3 & 0.1 & 1.1 & 88.5 & 7.7 & 0.9 & 1.2 & 0.0 & 0.2 \\
\hline Cg Str4 060828 & 0.3 & 0.9 & 0.2 & 90.6 & 1.1 & 6.8 & 0.0 & 0.0 & 0.0 \\
\hline Cg Str4 060831 & 0.3 & 0.4 & 0.0 & 93.0 & 0.7 & 3.6 & 0.1 & 0.6 & 1.3 \\
\hline Cg Str4 060904 & 0.1 & 0.9 & 2.5 & 95.0 & 1.0 & 0.1 & 0.2 & 0.1 & 0.1 \\
\hline Cg Str4 060905 & 0.0 & 0.2 & 1.7 & 97.6 & 0.1 & 0.1 & 0.0 & 0.1 & 0.1 \\
\hline Cg Str4 061023 & 0.1 & 0.6 & 1.9 & 95.2 & 0.7 & 0.4 & 1.0 & & \\
\hline Cg Str4 061024 Kontakt m. SEN & 0.1 & 0.1 & 0.8 & 91.9 & 4.8 & 1.2 & 0.9 & 0.1 & 0.2 \\
\hline Cg Str4 061024 Kontakt m. Schmelze & 0.2 & 0.1 & 1.3 & 90.3 & 5.8 & 0.9 & 1.1 & 0.0 & 0.3 \\
\hline Cg Str4 061026 & 0.1 & 0.1 & 1.7 & 95.8 & 0.3 & 1.4 & 0.5 & 0.0 & 0.2 \\
\hline Min & 0.0 & 0.1 & 0.0 & 88.5 & 0.1 & 0.1 & 0.0 & 0.0 & 0.0 \\
\hline Max & 0.3 & 1.2 & 2.5 & 97.6 & 7.7 & 6.8 & 1.2 & 1.1 & 1.9 \\
\hline Mittelwert & 0.2 & 0.4 & 1.1 & 93.1 & 2.2 & 2.1 & 0.4 & 0.2 & 0.4 \\
\hline Cg US Str3 060904 & 0.1 & 2.0 & 2.4 & 90.1 & 2.5 & 0.8 & 0.6 & 0.5 & 1.1 \\
\hline Cg US Str3 060905 & 0.1 & 0.9 & 2.2 & 92.6 & 2.1 & 0.6 & 0.2 & 0.4 & 1.0 \\
\hline Cg US Str3 061024 & 0.5 & 0.1 & 1.1 & 89.1 & 4.5 & 1.0 & 1.0 & 0.9 & 1.9 \\
\hline Cg US Str4 060904 & 0.1 & 1.1 & 2.5 & 89.3 & 2.4 & 0.7 & 1.5 & 0.8 & 1.6 \\
\hline Cg US Str4 060905 & 0.0 & 0.7 & 2.0 & 93.3 & 3.0 & 0.3 & 0.2 & 0.2 & 0.3 \\
\hline Cg US Str4 061023 & 0.2 & 0.2 & 2.0 & 91.6 & 1.2 & 0.6 & 0.6 & 1.0 & 2.7 \\
\hline Cg US Str4 061024 Kontakt m. Schmelze & 0.2 & 0.1 & 1.2 & 88.7 & 6.0 & 1.6 & 0.8 & 0.3 & 1.1 \\
\hline Cg US Str4 061026 & 0.1 & 0.6 & 4.8 & 91.4 & 1.5 & 0.8 & 0.8 & & \\
\hline Min & 0.0 & 0.1 & 1.1 & 88.7 & 1.2 & 0.3 & 0.2 & 0.2 & 0.3 \\
\hline Max & 0.5 & 2.0 & 4.8 & 93.3 & 6.0 & 1.6 & 1.5 & 1.0 & 2.7 \\
\hline Mittelwert & 0.2 & 0.7 & 2.3 & 90.7 & 2.9 & 0.8 & 0.7 & 0.6 & 1.4 \\
\hline Mittelwert Gesamt & 0.2 & 0.5 & 1.5 & 92.3 & 2.4 & 1.6 & 0.5 & 0.3 & 0.7 \\
\hline
\end{tabular}




\subsubsection{Tundishschlacken}

Tabelle 19: RFA in Gew.-\% von Tundishschlackenproben (Tu) aus Teil II.

\begin{tabular}{|c|c|c|c|c|c|c|c|c|c|c|}
\hline & $\mathrm{FeO}$ & MnO & $\mathrm{SiO}_{2}$ & $\mathrm{TiO}_{2}$ & $\mathbf{A l}_{2} \mathbf{O}_{3}$ & $\mathrm{CaO}$ & MgO & $\mathrm{ZrO}_{2}$ & $\mathbf{K}_{2} \mathbf{O}$ & $\mathrm{Na}_{2} \mathrm{O}$ \\
\hline Tu Str3 060828 & 3.28 & 7.88 & 45.70 & 2.11 & 19.70 & 14.10 & 5.34 & 0.03 & 0.99 & 0.09 \\
\hline Tu Str3 060829 & 12.86 & 4.64 & 34.80 & 1.02 & 24.20 & 1.77 & 6.23 & 0.03 & 0.47 & 0.04 \\
\hline Tu Str3 060831 & 2.62 & 3.13 & 39.80 & 0.74 & 29.50 & 17.60 & 4.82 & 0.03 & 0.60 & 0.09 \\
\hline Tu Str3 060901 & 0.80 & 0.62 & 17.70 & 2.55 & 37.70 & 36.90 & 2.91 & 0.05 & 0.32 & 0.09 \\
\hline Tu Str3 060905 & 3.85 & 1.30 & 32.40 & 5.21 & 28.50 & 20.40 & 4.24 & 0.04 & 0.60 & 0.26 \\
\hline Tu Str3 061023 & 9.58 & 1.81 & 44.70 & 4.74 & 18.40 & 8.68 & 4.82 & 0.06 & 1.04 & 0.10 \\
\hline Tu Str3 & 0.69 & 2.3 & 25.40 & 3.02 & 31.00 & 29.80 & 5.14 & 0.12 & 0.44 & 0.10 \\
\hline $\mathrm{Tu} \mathrm{St}$ & 1.54 & 81 & 21.60 & 7.02 & 33.60 & 30.00 & 2.52 & 0.10 & 0.42 & 0.10 \\
\hline $\mathrm{TuSt}$ & 2.34 & 74 & 35.90 & 2.27 & 24.70 & 20.80 & 5.39 & 0.07 & 0.74 & 0.12 \\
\hline $\mathrm{Tu} \mathrm{St}$ & 10.39 & 4.38 & 47.00 & 1.07 & 22.30 & 1.22 & 4.71 & 0.03 & 0.78 & 0.04 \\
\hline Tu St & 2.69 & 3.15 & 39.60 & 0.72 & 30.00 & 17.30 & 4.77 & 0.03 & 0.61 & 0.07 \\
\hline $\mathrm{Tu}$ Str & 2.87 & 0.70 & 23.00 & 2.44 & 35.60 & 32.10 & 2.77 & 0.06 & 0.47 & 0.07 \\
\hline Tu Str4 060905 & 3.74 & 1.44 & 29.70 & 5.77 & 31.60 & 21.60 & 3.62 & 0.04 & 0.63 & 0.18 \\
\hline Tu Str4 061023 & 6.25 & 2.01 & 33.40 & 5.14 & 25.60 & 19.80 & 6.04 & 0.08 & 0.86 & 0.10 \\
\hline Tu Str4 061024 & 3.14 & 6.33 & 30.30 & 3.68 & 27.00 & 21.60 & 5.90 & 0.12 & 0.58 & 0.10 \\
\hline Tu Str4 061026 & 2.57 & 1.70 & 21.60 & 6.72 & 31.60 & 28.60 & 2.78 & 0.10 & 0.52 & 0.10 \\
\hline Min & 0.7 & 0.6 & 17.7 & 0.7 & 18.4 & 1.2 & 2.5 & 0.0 & 0.3 & 0.0 \\
\hline Max & 12.9 & 7.9 & 47.0 & 7.0 & 37.7 & 36.9 & 6.2 & 0.1 & 1.0 & 0.3 \\
\hline Mittelwert & 4.3 & 3.1 & 32.7 & 3.4 & 28.2 & 20.1 & 4.5 & 0.1 & 0.6 & 0.1 \\
\hline
\end{tabular}




\subsection{Sauerstoffisotopenanalyse}

\subsubsection{Oxidische Ausscheidungen}

Tabelle 20: $\delta^{18} \mathrm{O}$-Werte oxidischer Ausscheidungen aus der Voruntersuchung, aus Teil I und aus Teil II. Alle Proben wurden im continuous flow-Modus gemessen.

\begin{tabular}{|l|c|c|}
\hline Voruntersuchung & $\delta^{18} \mathbf{O}$ & $\mathrm{N}$ \\
\hline \hline 706-710, VT1, Strang 1 & 2.13 & 3 \\
\hline 724-728, VT4, Strang 1 & 5.97 & 2 \\
\hline 445-450, Strang 1 & 2.50 & 2 \\
\hline Teil I & 9.25 & 1 \\
\hline 2 D innen Wandung & 10.50 & 1 \\
\hline 2 D innen Zone 2 & 13.42 & 1 \\
\hline 2 D innen Zone 1 & 7.95 & 1 \\
\hline 1 E innen & 10.97 & 1 \\
\hline 2 C innen & 7.08 & 1 \\
\hline Probe 7 innen & 5.65 & 1 \\
\hline Probe 7 außen & \multicolumn{2}{|l|}{} \\
\hline Teil II & 10.49 & 2 \\
\hline Cg Str3 060828 & 8.32 & 2 \\
\hline Cg Str3 060829 & 8.50 & 3 \\
\hline Cg Str3 060831 & 4.51 & 2 \\
\hline Cg Str3 060904 & 4.07 & 2 \\
\hline Cg Str3 060905 & 7.54 & 1 \\
\hline Cg Str3 061024 Kontakt m. SEN & 6.55 & 1 \\
\hline Cg Str3 061024 Kontakt m. Schmelze & 9.55 & 2 \\
\hline Cg Str4 060828 & 7.82 & 2 \\
\hline Cg Str4 060831 & 6.62 & 2 \\
\hline Cg Str4 060904 & 6.15 & 2 \\
\hline Cg Str4 060905 & 4.86 & 1 \\
\hline Cg Str4 061023 & 10.41 & 1 \\
\hline Cg Str4 061024 Kontakt m. SEN & 7.47 & 1 \\
\hline Cg Str4 061024 Kontakt m. Schmelze & 4.81 & 18 \\
\hline Cg Str4 061026 & 7.97 & 2 \\
\hline Cg US Str3 060904 & 3.45 & 2 \\
\hline Cg US Str3 060905 & 6.88 & 2 \\
\hline Cg US Str3 061024 & 7.83 & 2 \\
\hline Cg US Str4 060904 & 3.60 & 2 \\
\hline Cg US Str4 060905 & 5.21 & 1 \\
\hline Cg US Str4 061023 & 6.09 & 2 \\
\hline Cg US Str4 061024 Kontakt m. Schmelze & 5.85 & 1 \\
\hline Cg US Str4 061026 & \multicolumn{2}{|l}{} \\
\hline \multicolumn{3}{|l|}{} \\
\hline
\end{tabular}




\subsubsection{Schlacken und Prozessmaterialien}

Tabelle 21: $\delta^{18} \mathrm{O}-$ Werte von Prozessmaterialien. Alle Proben wurden im continuous flow-Modus gemessen.

\begin{tabular}{|l|c|c|}
\hline Probe & $\boldsymbol{\delta}^{\mathbf{1 8}} \mathbf{O}[\mathbf{6 0}]$ & $\mathbf{N}$ \\
\hline \hline Tu Str3 060828 & 15.89 & 1 \\
\hline Tu Str4 060828 & 13.18 & 1 \\
\hline Tu Str3 060829 & 9.10 & 1 \\
\hline Tu Str4 060829 & 7.91 & 1 \\
\hline Tu Str3 060831 & 14.06 & 1 \\
\hline Tu Str4 060831 & 15.17 & 1 \\
\hline Tu Str3 060901 & 14.11 & 1 \\
\hline Tu Str4 060901 & 15.14 & 1 \\
\hline Tu Str3 060905 & 12.38 & 1 \\
\hline Tu Str4 060905 & 14.06 & 1 \\
\hline Tu Str3 061023 & 14.64 & 1 \\
\hline Tu Str4 061023 & 15.33 & 1 \\
\hline Tu Str3 061024 & 14.94 & 1 \\
\hline Tu Str4 061024 & 14.76 & 1 \\
\hline Tu Str3 061026 & 14.30 & 1 \\
\hline Tu Str4 061026 & 14.65 & 1 \\
\hline & & \\
\hline Pfannenschlacke 060905 & 16.74 & 1 \\
\hline Pfannenschlacke 061023 & 18.16 & 1 \\
\hline & & \\
\hline Konverter Schlacke 061023 & 19.04 & 1 \\
\hline Konverter Schlacke 061024 & 19.46 & 2 \\
\hline & & \\
\hline Chromit Schiebersand Hersteller A02 & -4.12 & 1 \\
\hline Chromit Schiebersand Hersteller C & -3.51 & 1 \\
\hline Chromit Schiebersand Hersteller B & -2.96 & 1 \\
\hline Quarz Schiebersand Hersteller A03 & 11.58 & 1 \\
\hline Quarz Schiebersand Hersteller A02 & 10.74 & 1 \\
\hline Quarz Schiebersand Hersteller A02 & 10.90 & 1 \\
\hline Quarz Schiebersand Hersteller C & 10.41 & 1 \\
\hline Quarz Schiebersand Hersteller C & 10.25 & 1 \\
\hline Quarz Schiebersand Hersteller B & 11.31 & 1 \\
\hline Quarz Schiebersand Hersteller B & 13.95 & 1 \\
\hline Enstatit Schiebersand Hersteller A02 & 6.87 & 1 \\
\hline Enstatit Schiebersand Hersteller C & 6.29 & 1 \\
\hline Enstatit Schiebersand Hersteller B & 6.18 & 1 \\
\hline Enstatit Schiebersand Hersteller A03 & 5.78 & 1 \\
\hline & & \\
\hline VTR-Masse Ox2 060905 & 11.52 & 1 \\
\hline VTR-Masse Ox2 060905 & 13.25 & 1 \\
\hline & & \\
\hline Abdeckpulver & 17.03 & 1 \\
\hline Pfannenstein A & 9.40 & 1 \\
\hline Pfannenstein B & 6.99 & 1 \\
\hline Pfannenstein C & & 1 \\
\hline Pfannenstein D & \\
\hline
\end{tabular}




\subsection{3 ${ }^{17}$ O-Messung von Prozess- $\mathrm{O}_{2}$, Chromit und Clogging}

Tabelle 22: $\delta^{18} \mathrm{O}-, \delta^{17} \mathrm{O}$ - und $\Delta^{\prime 17} \mathrm{O}-$ Werte von Prozess- $\mathrm{O}_{2}$, Chromit aus dem Schiebersand und von drei Cloggingproben. Alle Daten wurden im dual inlet-Modus gemessen.

\begin{tabular}{|c|c|c|c|c|}
\hline Probe & $\delta^{18} \mathrm{O}$ & $\delta^{\prime 17} \mathrm{O}$ & $\Delta^{\prime 17} \mathbf{O}_{\mathrm{TFL}}$ & $\mathbf{N}$ \\
\hline Prozess- $\mathrm{O}_{2} 20.12 .2005$ & 1.01 & 0.54 & -0.35 & 4 \\
\hline Prozess- $\mathrm{O}_{2} 24.01 .2006$ & 0.67 & 0.40 & -0.31 & 3 \\
\hline Prozess- $\mathrm{O}_{2} 28.08 .2006$ & 24.02 & 12.57 & -0.39 & 2 \\
\hline Prozess- $\mathrm{O}_{2} 29.08 .2006$ & 24.03 & 12.63 & -0.34 & 1 \\
\hline Prozess- $\mathrm{O}_{2} 31.08 .2006$ & 25.06 & 13.23 & -0.28 & 1 \\
\hline Prozess- $\mathrm{O}_{2} 04.09 .2006$ & 23.88 & 12.53 & -0.36 & 1 \\
\hline Prozess- $\mathrm{O}_{2} 05.09 .2006$ & 24.22 & 12.69 & -0.38 & 1 \\
\hline Prozess- $\mathrm{O}_{2} 23.10 .2006$ & 23.86 & 12.50 & -0.37 & 1 \\
\hline Prozess- $\mathrm{O}_{2} 24.10 .2006$ & 23.69 & 12.43 & -0.35 & 1 \\
\hline Prozess- $\mathrm{O}_{2} 26.10 .2006$ & 23.27 & 12.18 & -0.39 & 1 \\
\hline $24.10 .06 \mathrm{GOX}$ & 23.70 & 12.42 & -0.37 & 2 \\
\hline $24.10 .06 \mathrm{LOX}$ & 24.37 & 12.75 & -0.39 & 1 \\
\hline Chromit Schiebersand Hersteller B & -4.19 & -2.13 & 0.07 & 1 \\
\hline Chromit Schiebersand Hersteller C & -4.07 & -2.15 & -0.02 & 1 \\
\hline Chromit Schiebersand Hersteller A02 & -4.72 & -2.41 & 0.07 & 1 \\
\hline Cg Str4 31.08.06 & 7.59 & 3.85 & -0.13 & 1 \\
\hline Cg oberer SEN-Bereich Str4 061026 & 5.04 & 2.51 & -0.13 & 1 \\
\hline Cg Str4 24.10.06 Kontakt m. Schmelze & 6.77 & 3.43 & -0.12 & 1 \\
\hline
\end{tabular}




\subsection{Rietveldanalyse}

Tabelle 23: Übersicht über die Ergebnisse der Rietveldanalyse von Clogging $(\mathrm{Cg})$ und SEN-

Unterseiten-Präzipitaten (Cg US) aus Teil II. Crn = Korund, $\mathrm{Spl}=$ Spinell, Hem = Hämatit, NaMg-Al

$=\mathrm{Na}-\mathrm{Mg}$-Aluminat, $\beta-\mathrm{Al}=\mathrm{Na}-\beta$-Aluminat, $\mathrm{Dia}=$ Diaoyudaoit, $\mathrm{Gra}=$ Graphit, $\mathrm{Ru}=\mathrm{Rutil}$.

\begin{tabular}{|l|c|c|c|c|c|c|c|c|c|}
\hline Probe & Crn & Spl & Hib & Hem & NaMg-Al & B-Al & Dia & Gra & Ru \\
\hline \hline Cg Str3 060828 & 58.38 & 26.88 & 13.80 & & 0.94 & & & & \\
\hline Cg Str3 060829 & 55.70 & 12.02 & 26.22 & 3.48 & 2.61 & & & & \\
\hline Cg Str3 060831 & 61.00 & 29.01 & 6.23 & & 2.99 & 0.78 & & & \\
\hline Cg Str3 060904 & 94.56 & & 5.44 & & & & & & \\
\hline Cg Str3 060905 & 80.57 & 5.35 & 9.13 & 4.96 & & & & & \\
\hline Cg Str3 061024 Kontakt m. SEN & 29.09 & & 70.91 & & & & & & \\
\hline Cg Str3 061024 Kontakt m. Schmelze & 9.93 & & 90.07 & & & & & & \\
\hline Cg Str4 060828 & 54.27 & 33.28 & 12.35 & & 0.10 & & & & \\
\hline Cg Str4 060831 & 60.85 & 15.96 & 19.47 & & 1.79 & 1.93 & & & \\
\hline Cg Str4 060904 & 80.00 & & 20.01 & & & & & & \\
\hline Cg Str4 060905 & 90.00 & 4.05 & & 5.92 & & & & 0.01 & \\
\hline Cg Str4 061023 & 79.40 & 5.46 & 15.14 & & & & & & \\
\hline Cg Str4 061024 Kontakt m. SEN & 32.93 & & 67.07 & & & & & & \\
\hline Cg Str4 061024 Kontakt m. Schmelze & 22.25 & & 77.75 & & & & & & \\
\hline Cg Str4 061026 & 88.59 & 7.72 & & 2.84 & & & & & \\
\hline Cg US Str3 060904 & 65.20 & & 26.00 & 4.30 & & 3.44 & & & 1.09 \\
\hline Cg US Str3 060905 & 80.69 & & 11.77 & 5.58 & & 1.95 & & & \\
\hline Cg US Str3 061024 & 13.84 & & 61.83 & 4.34 & & & 19.99 & & \\
\hline Cg US Str4 060904 & 47.90 & & 42.00 & 4.55 & & 4.32 & & & 1.27 \\
\hline Cg US Str4 060905 & 54.18 & & 39.67 & 4.54 & & & & 1.08 & \\
\hline Cg US Str4 061023 & 38.30 & 1.92 & 29.80 & & & & 29.99 & & \\
\hline Cg US Str4 061024 Kontakt m. Schmelze & 9.68 & & 77.73 & 2.33 & & & 10.26 & & \\
\hline Cg US Str4 061026 & 37.43 & 9.65 & 38.90 & 5.33 & & & 8.69 & & \\
\hline
\end{tabular}




\title{
12 Manuskript TOULOUSE et al. (in press)
}

\section{Stable Oxygen Isotopes for Tracing the Origin of Clogging in Continuous Casting Submerged Entry Nozzles}

\author{
Christoph Toulouse ${ }^{1,2)}$, Andreas Pack ${ }^{2)}$, Alfred Ender ${ }^{1)}$, Stefan Petry ${ }^{1)}$ \\ 1) ThyssenKrupp Steel AG, Metallurgy Division, Steel Production, QC \& Process Development, Kaiser-Wilhelm-Straße 100, \\ 47166 Duisburg, Germany ${ }^{2)}$ Georg-August-Universität Göttingen, Geowissenschaftliches Zentrum, Abteilung Isotopengeologie, \\ Goldschmidtstraße 1, 37077 Göttingen, Germany
}

An approach for tracing the origin of submerged entry nozzle (SEN) clogging that occurs during continuous casting of Al-killed steel is presented. This approach consists of using stable oxygen isotope ratios. IR laser fluorination in combination with gas mass spectroscopy is performed to determine the oxygen isotope composition of alumina-rich precipitates (clogging) and possible oxygen sources like refractory materials, slags, process and atmospheric oxygen. Three oxygen sources for clogging are identified. A quantitative model is presented.

Keywords: Clogging, alumina, refractory, submerged entry nozzle, oxygen isotopes

\subsection{Introduction}

Since 30 years clogging in continuous casting SENs has been a production problem [1, 2]. Clogging reduces the flow passage diameter, which results in a decrease in net casting throughput up to stop casting [2]. This reduces productivity and increases costs because less heats can be cast and SENs have to be changed more frequently [3]. Furthermore, nozzle restriction leads to undesired flow pattern in the mold [3, 4]. Erosion of clogging can cause surface defects $[5,6]$ and deteriorates steel cleanness.

Clogging of Al-killed steel is predominantly composed of alumina [7-9], which forms by reaction of $\mathrm{Al}$ with oxygen $\mathrm{Al}_{2} \mathrm{O}_{3}$ :

$$
2 \mathrm{Al}^{\text {liquid steel }}+3 \mathrm{O} \rightleftharpoons \mathrm{Al}_{2} \mathrm{O}_{3}^{\text {solid }}
$$

In this reaction oxygen can be dissolved in the steel or may directly oxidize alloyed Al from an extrinsic oxygen source (e.g. air $\mathrm{O}_{2}$ or refractory materials).

In the past, investigations concentrated on phenomenological analysis [2, 5, 10-13] like morphological studies [14]. In that study it was concluded from oxide particles shapes that reoxidation is the main cause of nozzle clogging, at least after ladle treatment and most probably in the SEN. The oxygen source for the formation of alumina-rich precipitates in SENs, however, remained unexplained [15]. 
Atmospheric $\mathrm{O}_{2}$ and process $\mathrm{O}_{2}$ are possible oxygen sources for clogging. All oxygen-bearing refractory materials and slags, which have melt contact, are potential oxygen sources for clogging. In addition, refractory materials and slags could be involved via erosion and carryover, respectively.

In our approach we use the oxygen isotope composition of clogging in comparison with potential oxygen sources. This is expressed as $\delta^{18} \mathrm{O}$ which is a measure for the ${ }^{18} \mathrm{O} /{ }^{16} \mathrm{O}$ ratio in clogging relative to the ${ }^{18} \mathrm{O} /{ }^{16} \mathrm{O}$ ratio in seawater (Equation 2).

$\delta^{18} \mathrm{O}=\left(\frac{\left(\frac{{ }^{18} \mathrm{O}}{{ }^{16} \mathrm{O}}\right)_{\text {Sample }}}{\left(\frac{{ }^{18} \mathrm{O}}{{ }^{16} \mathrm{O}}\right)_{\text {VSMOW }}}-1\right) \times 1000[\%]$

Seawater is the international reference standard and has per definition $\delta^{18} \mathrm{O}=0 \%$ (Vienna Standard Mean Ocean Water $=$ VSMOW). In this study isotope fractionation is used as term to express the variability of the ratio of ${ }^{18} \mathrm{O}$ to ${ }^{16} \mathrm{O}$.

Pack et al. [16] were the first who carried out oxygen isotope analysis of clogging and refractory materials. They concluded that olivine from the basic tundish lining may be oxygen donator for the formation of non-metallic inclusions. This conclusion is confirmed by petrographic and thermodynamic studies by Pack et al. [17].

\subsection{Sampling}

Samples were taken in a steel plant of the ThyssenKrupp Steel AG in Duisburg (Germany). In spring 2004, 3 clogging samples were taken on 3 different days. In 2005, 7 clogging samples were taken from 3 different SENs on 3 different days. On 8 days between August and October 2006, 23 oxide deposition samples were taken from 13 SENs. Oxide precipitates were sampled inside the SEN (clogging) and at its bottom side (Figure 1). 


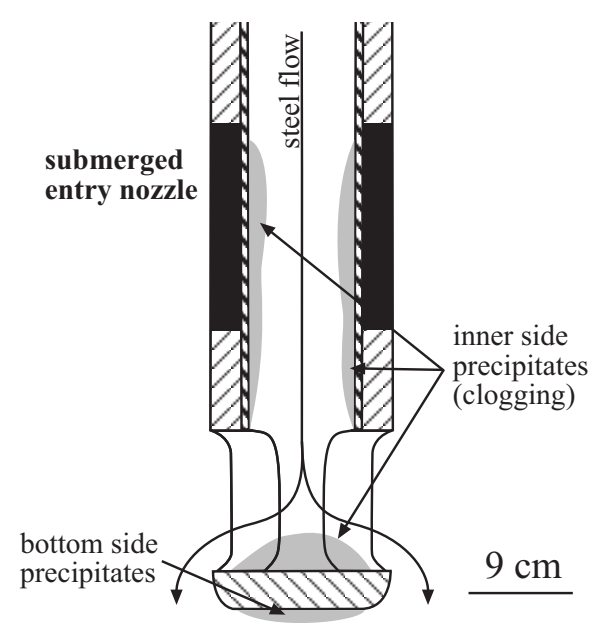

Figure 1. Sketch illustrating the occurrence of SEN oxide precipitates. Material was sampled from inside the SEN (clogging) and from its bottom side.

Two process $\mathrm{O}_{2}$ samples were taken in December 2005 and January 2006. One process $\mathrm{O}_{2}$ sample was taken on each of the 8 sampling days between August and October 2006.

Slag samples were taken from the converter $(\mathrm{N}=3)$, the ladle $(\mathrm{N}=3)$ and from the tundish $(\mathrm{N}=16)$.

Furthermore the following samples were taken from unused refractories:

1. Basic refractory bricks from the ladle $(\mathrm{N}=6), 3$ different manufactures

2. Slide gate powder $(\mathrm{N}=4), 3$ manufacturers

3. Basic tundish lining $(\mathrm{N}=2), 1$ manufacturers

During October 2006, 3 lolly specimens were sampled on 3 days from the converter; each of them at the end of the blowing process. Isolate residues were made from these samples.

\subsection{Analytical Methods}

Bulk chemical analysis of clogging and process materials were performed using X-ray fluorescence spectrometry. The concentrations of $\mathrm{Na}, \mathrm{Mg}, \mathrm{Al}, \mathrm{Si}, \mathrm{K}, \mathrm{Ca}, \mathrm{Ti}, \mathrm{Cr}, \mathrm{Mn}$ and $\mathrm{Zr}$ were analyzed. Samples were crushed and iron metal was removed using a magnet. Since clogging (oxide fraction) is nearly Fe-free, remaining Fe was subtracted from the analyses, which were then normalized to $100 \mathrm{wt} . \%$ of the oxides.

Micro chemical analyses were performed on polished sections using a CAMECA SX-100 electron probe micro analyzer (EPMA). Estimating $\mathrm{Fe}^{3+}$ concentrations from microprobe analyses using stoichiometric criteria were done after a method by Droop [18]. 
The mineralogical composition was determined by using a PHILIPS PW 1800 X-ray powder diffractometer in Bragg-Brentano geometry. Evaluation of diffraction patterns was performed using the program Win XPOW from STOE \& Cie GmbH.

Lolly specimens were deoxidized with Al. To extract isolate residue of a steel sample, potentiostatic isolation was applied. With this technique, the steel is dissolved and fine-grained $\mathrm{Al}_{2} \mathrm{O}_{3}$ can be recovered for analysis.

Oxygen isotope ratios were measured by means of mass spectrometry with $\mathrm{O}_{2}$ as analyte. $\delta^{18} \mathrm{O}$ is calculated from the ratio of the ${ }^{18} \mathrm{O}^{16} \mathrm{O}$ to ${ }^{16} \mathrm{O}{ }^{16} \mathrm{O}$ molecules in comparison with reference gas with known $\delta^{18} \mathrm{O}$. Silicates and oxides were analyzed using IR laser fluorination. At this, a $\mathrm{CO}_{2}$ laser heats the sample in a $\mathrm{F}_{2}$ atmosphere to $\sim 2000^{\circ} \mathrm{C}$. Oxygen extraction for alumina can be expressed as:

$$
2 \mathrm{Al}_{2} \mathrm{O}_{3}+6 \mathrm{~F}_{2} \rightarrow 4 \mathrm{AlF}_{3}+3 \mathrm{O}_{2}
$$

Excess $\mathrm{F}_{2}$ reacts with $\mathrm{NaCl}$ to $\mathrm{NaF}$ and $\mathrm{Cl}_{2}$, which is separated from sample $\mathrm{O}_{2}$ by freezing out at $-196^{\circ} \mathrm{C}$ in an U-tube. Oxygen gas is trapped at $-196^{\circ} \mathrm{C}$ on a $5 \AA$ molecular sieve, from which it is injected into the source of SIRMS Finnigan Delta Plus gas mass spectrometer. One analysis takes about 20 minutes, but requires only $1 \mathrm{mg}$ oxides or silicates.

Accuracy and precision of the solid sample measurements were determined by repeated measurement of garnet reference standard (University of Wisconsin Garnet-2, $\delta^{18} \mathrm{O}=+5.8 \%$ [19]) to $\pm 0.2 \%$ o $(1 \sigma)$. For powdered clogging samples we give an error in the range of $\pm 0.4 \%$ o $(1 \sigma)$. For extremly fine-grained isolate residue an error in the range of $\pm 1 \%(1 \sigma)$ is estimated. Process $\mathrm{O}_{2}$ samples are directly injected via a dual-inlet system into the gas mass spectrometer, so that a possible preparation-caused error cannot appear. For these analyses an error in the range of $\pm 0.1 \%$ o $(1 \sigma)$ is given. For a more detailed descriptions of the IR laser fluorination it is referred to [16, 20-22].

\subsection{Results}

\subsubsection{Clogging}

Clogging samples that were taken from the steel plant in Duisburg between August and October $2006(\mathrm{~N}=15)$, were chemically analyzed. Main chemical components in clogging are $\mathrm{Al}_{2} \mathrm{O}_{3}, \mathrm{CaO}, \mathrm{MgO}$ and $\mathrm{TiO}_{2}$. The most significant element oxides with values less than 1 wt.\% are $\mathrm{Na}_{2} \mathrm{O}, \mathrm{SiO}_{2}$ and $\mathrm{ZrO}_{2}$ (Table 1). The $\mathrm{Cr}_{2} \mathrm{O}_{3}$ content of clogging is $<0.1$ wt.\%. 
Table 1. Average chemical composition of clogging and bottom side precipitates from ThyssenKrupp Steel AG in Duisburg (XRF data given in wt.\%, $\mathrm{N}=23$ ).

\begin{tabular}{|c|c|c|c|}
\hline & clogging & $\begin{array}{c}\text { bottom side } \\
\text { precipitates }\end{array}$ & average \\
\hline $\mathrm{MnO}$ & 0.2 & 0.2 & 0.2 \\
\hline $\mathrm{SiO}_{2}$ & 0.4 & 0.7 & 0.5 \\
\hline $\mathrm{TiO}_{2}$ & 1.1 & 2.3 & 1.5 \\
\hline $\mathrm{Al}_{2} \mathrm{O}_{3}$ & 93.1 & 90.7 & 92.3 \\
\hline $\mathrm{CaO}$ & 2.2 & 2.9 & 2.4 \\
\hline $\mathrm{MgO}$ & 2.1 & 0.8 & 1.6 \\
\hline $\mathrm{ZrO}_{2}$ & 0.4 & 0.7 & 0.5 \\
\hline $\mathrm{K}_{2} \mathrm{O}$ & 0.2 & 0.6 & 0.3 \\
\hline $\mathrm{Na}_{2} \mathrm{O}$ & 0.4 & 1.4 & 0.7 \\
\hline $\mathrm{Total}$ & 100 & 100 & 100 \\
\hline
\end{tabular}

The $\mathrm{Al}_{2} \mathrm{O}_{3}$ contents of clogging vary between 89 and 98 wt.\%. CaO contents vary between 0.1 and 7.7 wt.\%, and that of $\mathrm{MgO}$ between 0.1 and $6.8 \mathrm{wt} . \%$. The bottom side precipitates $(\mathrm{N}=$ 8) have $\mathrm{Al}_{2} \mathrm{O}_{3}$ contents between 89 and 93 wt.\%. The $\mathrm{CaO}$ contents of bottom side precipitates range between 1.2 and $6.0 \mathrm{wt} . \% . \mathrm{MgO}$ in bottom side precipitates varies between 0.3 and 1.6 wt.\%. On an average, bottom side precipitates are richer in alkalis with values of 1.4 wt.\% $\mathrm{Na}_{2} \mathrm{O}$ and 0.6 wt. $\% \mathrm{~K}_{2} \mathrm{O}$ compared to clogging with 0.4 wt. $\% \mathrm{Na}_{2} \mathrm{O}$ and 0.2 wt. $\% \mathrm{~K}_{2} \mathrm{O}$ (Table 1).

In 15 clogging and 8 bottom side precipitate samples taken between August and October 2006 from the steel plant in Duisburg, ten different phases have been identificated: corundum $\left(\mathrm{Al}_{2} \mathrm{O}_{3}\right)$, hibonite $\left(\mathrm{CaAl}_{12} \mathrm{O}_{19}\right)$, spinel $\left(\mathrm{MgAl}_{2} \mathrm{O}_{4}\right)$, diaoyudaoite $\left(\mathrm{NaAl}_{11} \mathrm{O}_{17}\right)$, Na- $\beta$-aluminate $\left(\mathrm{Na}_{2} \mathrm{Al}_{22} \mathrm{O}_{34}\right)$, hematite $\left(\mathrm{Fe}_{2} \mathrm{O}_{3}\right), \mathrm{Na}-\mathrm{Mg}$-aluminate $\left(\mathrm{NaMg}_{2} \mathrm{Al}_{15} \mathrm{O}_{25}\right)$, graphite $(\mathrm{C})$ and rutile $\left(\mathrm{TiO}_{2}\right)$ and iron.

70 oxygen isotope analyses were obtained from 33 oxide precipitate samples from the steel plant in Duisburg. Figure 2 shows $\delta^{18} \mathrm{O}$ values of oxide precipitates inside the SEN and those from the bottom side in comparison with literature data from Pack [21]. 


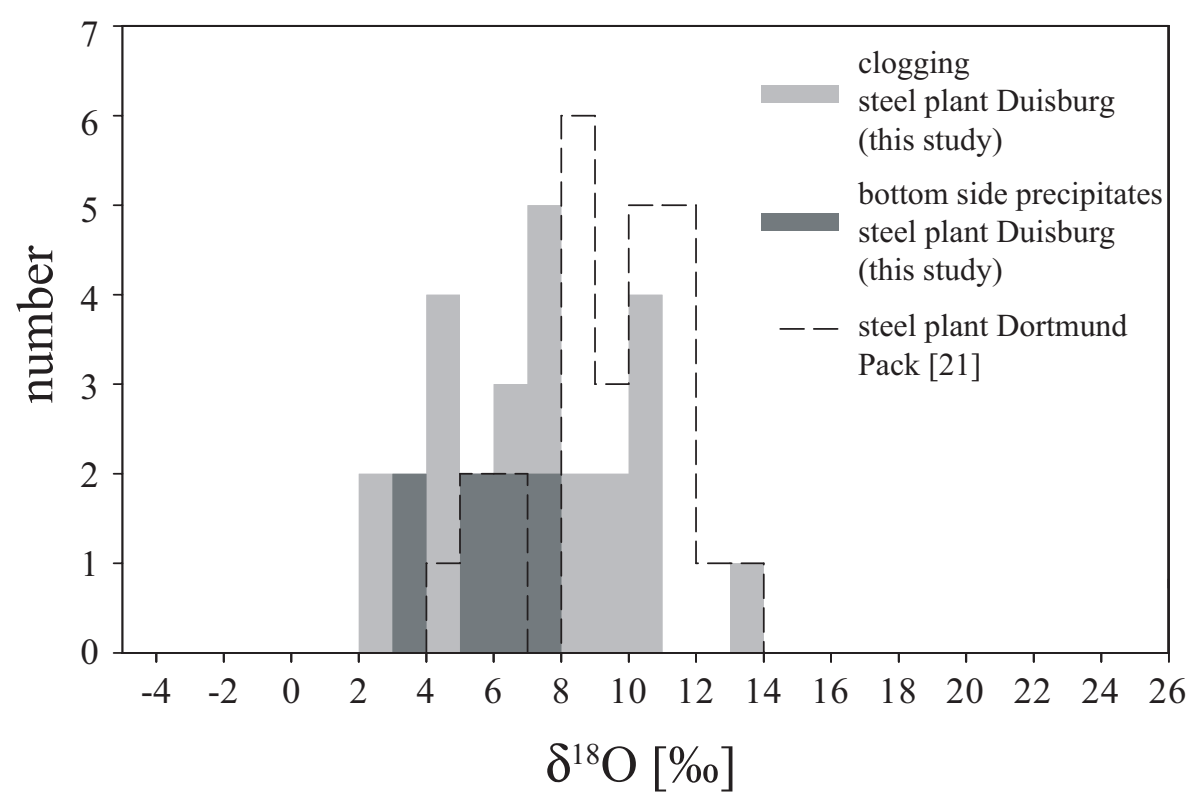

Figure 2. Recapitulatory histogram of $\delta^{18} \mathrm{O}$ values of oxide precipitates inside the SEN (clogging) and those at bottom side (gray shaded areas). Data from Pack [21] are shown for comparison (dashed line).

$\delta^{18} \mathrm{O}$ values of clogging from ThyssenKrupp Steel AG in Duisburg vary between +2.1 and $+13.4 \%$. The bottom side precipitates from ThyssenKrupp Steel AG in Duisburg show $\delta^{18} \mathrm{O}$ values from +3.5 to $+8.0 \%$.

\subsubsection{Process oxygen}

Two process oxygen samples that were taken in December 2005 and January 2006 have $\delta^{18} \mathrm{O}$ values of +0.7 and $+1.0 \%$, respectively. In August and October $2006(\mathrm{~N}=8)$ the $\delta^{18} \mathrm{O}$ of process oxygen in the same steel plant lay between +23.5 and $+25.4 \%$. Atmospheric $\mathrm{O}_{2}$ has a world-wide constant $\delta^{18} \mathrm{O}$ of $+23.5 \%$ [23].

\subsubsection{Isolate residues}

Isolate residues are composted of almost pure $\mathrm{Al}_{2} \mathrm{O}_{3}$ as corundum. The material is very finegrained. Figure 3 shows the results of oxygen isotope measurements of isolate residues and corresponding process $\mathrm{O}_{2}$ during three days. The $\delta^{18} \mathrm{O}$ values of isolate residues vary between +21.6 and $+24.2 \%$. Process $\mathrm{O}_{2}$ during these days has $\delta^{18} \mathrm{O}$ values from +23.5 to $+24.2 \%$. 


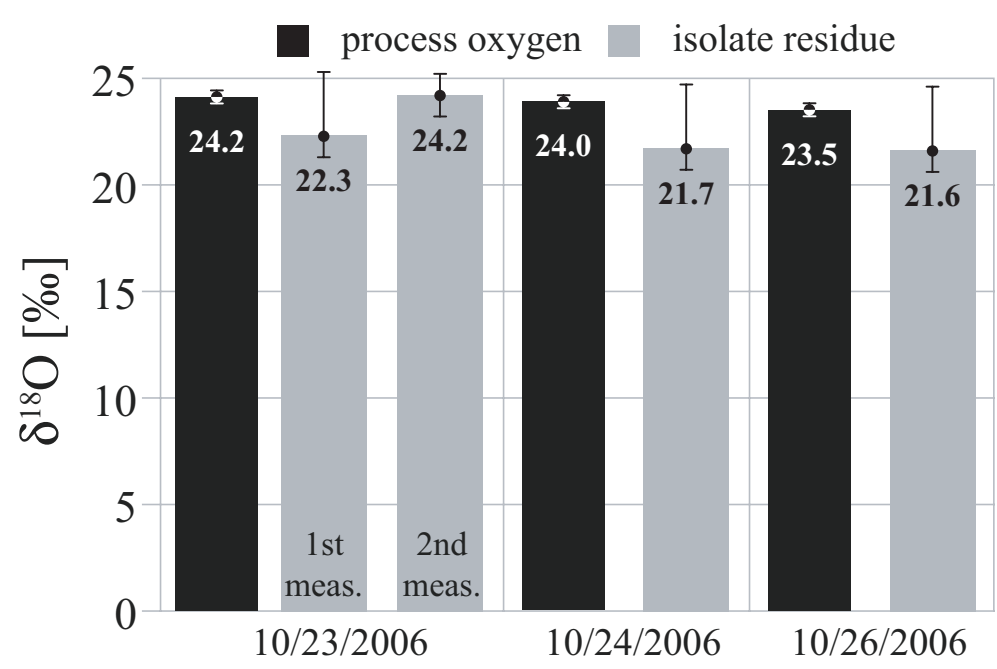

Figure 3. Oxygen isotope composition of process $\mathrm{O}_{2}$ and isolate residue from the converter.

\subsubsection{Refractory materials and slags}

The basic tundish lining consists of $89 \mathrm{wt} . \% \mathrm{MgO}$ as periclase, which has $\delta^{18} \mathrm{O}$ values of +11 to $+13 \%$ ( $(\mathrm{N}=2)$. The oxide fraction of basic refractory bricks from the ladle consists of $>96$ wt. $\% \mathrm{MgO}$ as periclase, which has $\delta^{18} \mathrm{O}$ values of +7 to $+9 \%(\mathrm{~N}=4)$. The tundish slag is variable in composition with $18-47$ wt.\% $\mathrm{SiO}_{2}, 18-38$ wt. $\% \mathrm{Al}_{2} \mathrm{O}_{3}, 1-37$ wt. $\% \mathrm{CaO}, 1-13$ wt. $\% \mathrm{FeO}$ and $3-6$ wt. $\% \mathrm{MgO}$. The measured $\mathrm{Cr}_{2} \mathrm{O}_{3}$ content varies between 0.3 and 8 wt.\% and is correlated with higher $\mathrm{FeO}$ concentrations. Three $\mathrm{FeO}$-rich samples were analyzed by $\mathrm{X}$-ray powder diffraction. They contain chromite as crystalline phase. $\delta^{18} \mathrm{O}$ values of the tundish slags are between +8 and $+16 \%(\mathrm{~N}=16)$. The ladle slag consists of $28-43 \mathrm{CaO}, 13-$ 29 wt. $\% \mathrm{Al}_{2} \mathrm{O}_{3}, 12-25$ wt. $\% \mathrm{FeO}, 8-13 \mathrm{wt} . \% \mathrm{MgO}$ and varies in $\delta^{18} \mathrm{O}$ around +17 to $+18 \%$ ( $(\mathrm{N}=3)$. Converter slag consists of 53 wt. $\% \mathrm{CaO}, 22$ wt. $\% \mathrm{FeO}, 14$ wt. $\% \mathrm{SiO}_{2}, 3$ wt.\% $\mathrm{MnO}, 2$ wt.\% $\mathrm{MgO}, \mathrm{TiO}_{2}$ and $\mathrm{P}_{2} \mathrm{O}_{5}$ and has a $\delta^{18} \mathrm{O}$ value of $+19 \% 0(\mathrm{~N}=3)$. The $\mathrm{Cr}_{2} \mathrm{O}_{3}$ content of ladle and converter slag is below 1 wt.\%. Figure 4 shows the average chemical composition of the major element oxides and the $\delta^{18} \mathrm{O}$ values of converter, ladle and tundish slag. 


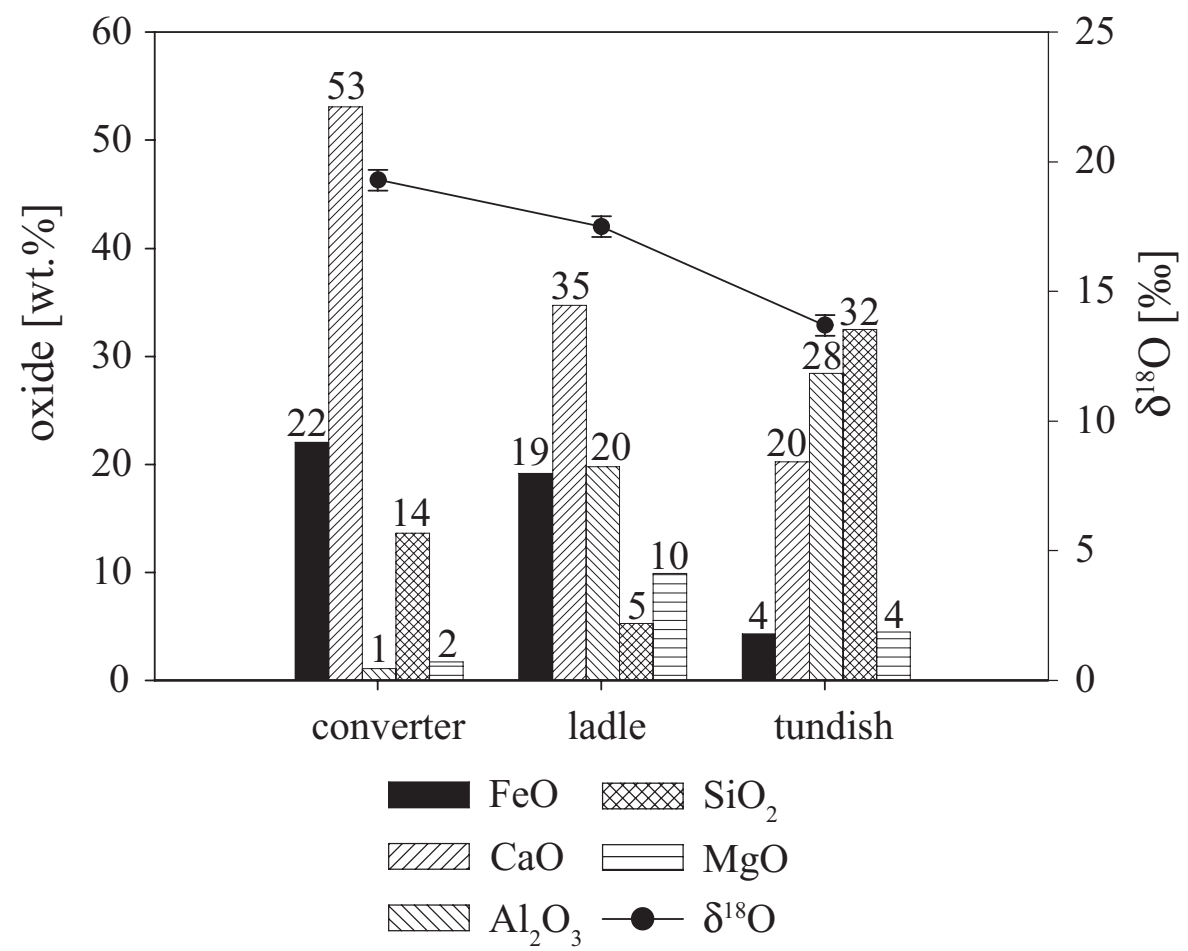

Figure 4. Average chemical and oxygen isotope composition of converter, ladle and tundish slag, respectively. The vertical columns show the contents of $\mathrm{FeO}, \mathrm{CaO}, \mathrm{Al}_{2} \mathrm{O}_{3}, \mathrm{SiO}_{2}$ and $\mathrm{MgO}$ in wt.\%. The black-filled circles show the $\delta^{18} \mathrm{O}$-values of the corresponding slags.

\subsubsection{Slide gate powder}

Slide gate powder consists of 33 wt. $\% \mathrm{Cr}_{2} \mathrm{O}_{3}, 28$ wt.\% $\mathrm{SiO}_{2}, 21$ wt. $\% \mathrm{Fe}_{2} \mathrm{O}_{3}, 11$ wt.\% $\mathrm{Al}_{2} \mathrm{O}_{3}$ and 7 wt. $\% \mathrm{MgO}$. Slide gate powder of all analyzed manufacturers contains of about $70 \mathrm{wt} . \%$ chromite, 30 wt. $\%$ quartz and $<3$ wt. $\%$ enstatite $\left(\mathrm{Mg}_{2} \mathrm{Si}_{2} \mathrm{O}_{6}\right)$. The micro chemical composition of chromite was determined by EPMA to fractions $\mathrm{Fe}_{0.52}^{2+} \mathrm{Mg}_{0.48}^{2+}\left[\mathrm{Fe}_{0.22}^{3+} \mathrm{Cr}_{1.21}^{3+} \mathrm{Al}_{0.57}^{3+}\right] \mathrm{O}_{4}$. The measured chromites have $\delta^{18} \mathrm{O}$ values of -4.1 to $-3.0 \%$, while quartz values vary between +10.3 and $+14.0 \%$. Enstatites lie between +6.2 and $+6.9 \%$ (Figure 5). 


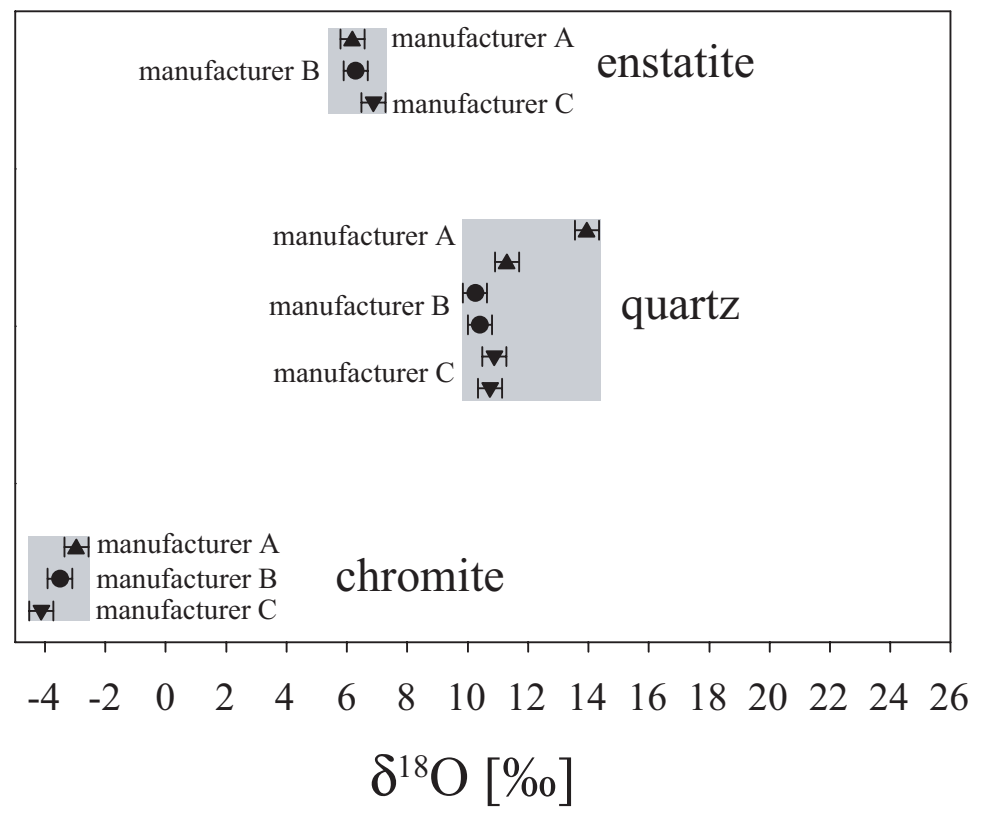

Figure 5. $\delta^{18} \mathrm{O}$ values of phases of slide gate powder.

\subsection{Discussion}

The $\delta^{18} \mathrm{O}$ values of oxide precipitations inside the SEN vary between +2 and $+13 \%$. This range is comparable to $\delta^{18} \mathrm{O}$ values measured on SEN clogging in Pack et al. [16], which varied between +5 and $+14 \%$ o (Figure 2). The isotope composition of bottom side precipitates is in the same range as $\delta^{18} \mathrm{O}$ of clogging from inside the SEN.

The chemical composition of clogging is dominated by alumina ( $89-98 \mathrm{wt} . \%)$. This is typical of clogging in low alloyed Al-killed steel [7-9]. We suggest that minor contribution of slag carry-over and/or erosion of basic refractories from ladle and/or tundish contribute to the observed $\mathrm{CaO}$ and $\mathrm{MgO}$ contents of $0.1-7.7$ wt.\% and $0.1-6.8$ wt.\%, respectively. The $\mathrm{CaO}$ and $\mathrm{MgO}$ concentrations reported by Pack [21] span a range up to 14 and $13 \mathrm{wt} \%$, respectively.

The influence of minor amounts of slag and/or refractories to the $\delta^{18} \mathrm{O}$ of clogging is, as first approximation, neglected in the following discussion since it is in any case $<16 \mathrm{wt} . \%$.

As in case of the study by Pack et al. [16], $\delta^{18} \mathrm{O}$ values of clogging are significantly different from the $\delta^{18} \mathrm{O}$ of air oxygen with $+23.5 \%$ [23]. It is therefore excluded that alumina-rich clogging forms by simple reoxidation of the steel through air contact. In such a scenario, $\delta^{18} \mathrm{O}$ of clogging is expected to be $\sim+23.5 \%$, if fractionation during oxidation of alloyed $\mathrm{Al}$ is neglected. 
One potential process of steel reoxidation is diffusion of $\mathrm{O}_{2}$ through the SEN walls. Faster diffusion of light ${ }^{16} \mathrm{O}^{16} \mathrm{O}$ relative to ${ }^{16} \mathrm{O}^{18} \mathrm{O}$ could potentially explain formation of SEN alumina precipitates that have $\delta^{18} \mathrm{O}$ lower than air $\mathrm{O}_{2}$. Diffusion, however, is a dynamic process in which also ${ }^{16} \mathrm{O}^{18} \mathrm{O}$ molecules will eventually reach the steel leading to formation of alumina with $\delta^{18} \mathrm{O}$ equal to air $\mathrm{O}_{2}$. Tuttle et al. [24] concluded that nozzle permeability has no effect on clogging. Based on the observed low $\delta^{18} \mathrm{O}$-values, we conclude that SEN clogging does not form in-situ, but represents a deposit of pre-existing particles from ladle and/or tundish. These particles may source from deoxidation in the ladle (i.e. deoxidation particles) and/or by reoxidation in the shroud tube and/or in the tundish. Thomas \& Bai [4] assume that the most important cause for clogs is the deposition of solid inclusions already present entering the tundish nozzle.

Carry-over of deoxidation particles may be a source of SEN clogging. In order to trace the isotope composition of deoxidation particles, we have analyzed isolate residues from lolly samples that were taken before steel is killed with Al. The samples were deoxidized by means of $\mathrm{Al}$. The formed $\mathrm{Al}_{2} \mathrm{O}_{3}$ was isolated from the lolly samples and analyzed for its $\delta^{18} \mathrm{O}$. Since, in the presence of excess $\mathrm{Al}$, nearly $100 \%$ of formerly dissolved $\mathrm{O}$ reacts to $\mathrm{Al}_{2} \mathrm{O}_{3}, \delta^{18} \mathrm{O}$ of $\mathrm{Al}_{2} \mathrm{O}_{3}$ is representative for $\delta^{18} \mathrm{O}$ of dissolved $\mathrm{O}$ in the steel in the ladle.

The $\delta^{18} \mathrm{O}$ values of isolate residues vary between +22 and $+24 \%$. For these samples a larger analytical uncertainty of $\pm 1 \%$ is given due to the very fine-grained consistency. Within error, $\delta^{18} \mathrm{O}$ of alumina isolate residues resemble $\delta^{18} \mathrm{O}$ of corresponding process $\mathrm{O}_{2}$ (Figure 3). It is therefore concluded that deoxidation particles, which have not directly been analyzed, largely mirror $\delta^{18} \mathrm{O}$ of dissolved $\mathrm{O}$ before killing, which in turn has $\delta^{18} \mathrm{O}$ of process $\mathrm{O}_{2}$. Although our analyses of isolate residues have a large analytical uncertainty, it is reasonable to draw the conclusion that no or only a little fractionation of $0-2 \%$ exists between $\delta^{18} \mathrm{O}$ of process $\mathrm{O}_{2}$ and deoxidation particles.

It has been demonstrated that process $\mathrm{O}_{2}$ typically has $\delta^{18} \mathrm{O}$ values that vary in a narrow range between +23.5 and $+25.4 \%$ o. Process $\mathrm{O}_{2}$ that was published by Pack et al. [16] from two different steel plants has $\delta^{18} \mathrm{O}=+24.3 \%$ and $+25.5 \%$, i.e. is within the range observed at ThyssenKrupp Steel AG in Duisburg. Process $\mathrm{O}_{2}$ is produced by air liquefaction. It is apparent that only little fractionation accompanies this process, although liquid $\mathrm{O}_{2}$ in equilibrium with gaseous $\mathrm{O}_{2}\left(\mathrm{t}=-180^{\circ} \mathrm{C}\right)$ is $5.5 \%$ enriched in ${ }^{18} \mathrm{O}$ relative to the gas phase [25]. The slight enrichment of process $\mathrm{O}_{2}$ relative to air $\mathrm{O}_{2}$ may be related to liquid/gas fractionation at low temperatures. High throughput of $\mathrm{O}_{2}$, however, maintains the $\delta^{18} \mathrm{O}$ close to the atmospheric value. 
On 2 different days in December 2005 and Janury 2006, $\delta^{18} \mathrm{O}$ of process $\mathrm{O}_{2}$ as low as $+0.7 \%$ o have been measured. It is not clear, which process is responsible for such extraordinary low $\delta^{18} \mathrm{O}$ of process $\mathrm{O}_{2}$. One possibility is maintenance work on one of the air liquefaction device during end of 2005. Since corresponding clogging or isolate residues samples have not been taken during the time of low $\delta^{18} \mathrm{O}$ process $\mathrm{O}_{2}$, we will discuss the formation of clogging with respect to process $\mathrm{O}_{2}$ with an average $\delta^{18} \mathrm{O}$ of $+24.3 \%$.

The $\mathrm{O}$ content of the steel in the ladle after refining decreases during killing due to formation of alumina from $600-800 \mathrm{ppm}$ to $37 \mathrm{ppm}[6,13]$. This corresponds to reaction of $94-95 \%$ dissolved $\mathrm{O}$ to solid alumina. Since that reaction is nearly quantitative, our conclusion that $\delta^{18} \mathrm{O}$ of deoxidation particles reflects $\delta^{18} \mathrm{O}$ of dissolved $\mathrm{O}$ before killing seems reasonable. We conclude that clogging made of pure deoxidation particles should exhibit $\delta^{18} \mathrm{O}$ of +22 to $+24 \%$. Since clogging has $\delta^{18} \mathrm{O}$ values significantly lower, we exclude that clogging is only product of precipitation of ladle deoxidation particles; a source with low $\delta^{18} \mathrm{O}$ is required.

It was suggested by Pack et al. [16, 21] that reduction of the fayalite content of olivine in the tundish lining provides a source of $\mathrm{O}$ with $\delta^{18} \mathrm{O} \approx+5 \%$. Reduction of olivine has been observed and is thermodynamically reasonable [17]. During the sampling campaign, however, no olivine was used in the tundish lining, which excludes olivine as source of low $\delta^{18} \mathrm{O}$ of clogging.

Other refractories and slags could potentially supply $\mathrm{O}$ with low $\delta^{18} \mathrm{O}$. In course of this study, a number of refractories and slags from ladle and tundish have been analyzed for their $\delta^{18} \mathrm{O}$. It is observed that periclase from ladle and tundish have $\delta^{18} \mathrm{O}$ values that vary between +7 and $+13 \%$, respectively. Pack et al. [16] report $\delta^{18} \mathrm{O}$ of periclase of basic tundish lining of +13.3 $-+14.2 \%$ and of $\mathrm{MgO}^{*} \mathrm{CaO}-\mathrm{C}$ bricks of $+12.6-+17.3 \%$. Periclase, however, is not regarded as major source of $\mathrm{O}$ to the steel. Other materials like ladle and tundish slags have $\delta^{18} \mathrm{O}$ values exceeding those of clogging (Figure 4). We conclude that, apart of minor slag carryover and/or erosion of refractories, slags and refractories do not play a major role as oxygen donators for the formation of clog build-ups in SENs.

The only refractory analyzed that has low $\delta^{18} \mathrm{O}$ is chromite of the slide gate powder with an average $\delta^{18} \mathrm{O}$ of $-3.5 \%$. Chromite from 3 manufacturers has identical yet low $\delta^{18} \mathrm{O}$ values. Only one $\mathrm{O}$ isotope analysis of chromite from Union Bay ultramafic complex, Cleveland Peninsula, Southeastern Alaska is published with $\delta^{18} \mathrm{O}=+4.5 \%$ [26]. The unusual low $\delta^{18} \mathrm{O}$ of chromites leads us to the suggestion that all source from the same deposit. This conclusion is supported by an identical chemical composition as determined by EPMA. Slide gate powder 
quartz is isotopically more variable and, most probably, made of quartz sand as indicated by the rounded grains. Quartz grains in sands are isotopically heterogenic since they source from different reservoirs and were mixed as river load. After opening the slide gates, the slide gate powder is transferred into the tundish. Following the opening of the slide gates, some material will be absorbed by the tundish slag and some will be reduced by $\mathrm{Al}$ in the steel melt.

The net chromite reduction reaction is expressed as:

$$
\begin{aligned}
& \overbrace{\mathrm{Fe}_{0.52}^{2+} \mathrm{Mg}_{0.48}^{2+}\left[\mathrm{Fe}_{0.22}^{3+} \mathrm{Cr}_{1.21}^{3+} \mathrm{Al}_{0.57}^{3+}\right] \mathrm{O}_{4}}^{\text {slide gate powder chromite }}+\overbrace{0.39 \mathrm{Al}_{1}^{\text {stel }}}^{\text {alloyed Al }} \rightleftharpoons
\end{aligned}
$$

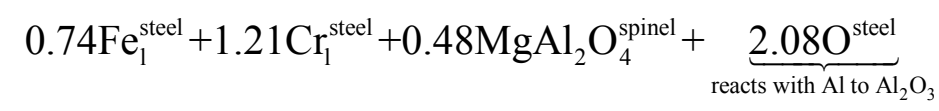

Equation 4 illustrates that reduction of $1 \mathrm{~kg}$ chromite in the liquid steel releases $0.17 \mathrm{~kg} \mathrm{O}$ that is available to react with $\mathrm{Al}$ to form free alumina. This alumina is expected of having $\delta^{18} \mathrm{O}$ equal to that of chromite, i.e. $-3.5 \%$. Alumina from chromite reduction is hence a suitable source for alumina with low $\delta^{18} \mathrm{O}$.

Since $\delta^{18} \mathrm{O}$ of clogging is considerably higher than $\delta^{18} \mathrm{O}$ of chromite, it is concluded that chromite can not be the only source of SEN clogging, but that clogging is a mixture of alumina from different reservoirs. Mixing of alumina from different sources shall first be discussed in terms of a 2-component model.

The one endmember shall be alumina that forms by reduction of chromite from slide gate powder. Per ladle $\sim 80 \mathrm{~kg}$ slide gate powder is used, which translates to $\sim 56 \mathrm{~kg}$ chromite. Chromite, as used in the slide gate powder has a molar mass of $195 \mathrm{~g} / \mathrm{mol}$. Reduction of one mol chromite liberates $2.08 \mathrm{~mol} \mathrm{O}$, which is available for the formation of free alumina (Equation 4). This is transformed to $20 \mathrm{~kg}$ alumina that can be formed by oxidation of $\mathrm{Al}$ through slide gate powder chromite per ladle. It is assumed that this alumina has $\delta^{18} \mathrm{O}=-$ $3.5 \%$.

The second component is alumina from deoxidation. According to Dekkers [27], who gives a content of $30 \mathrm{ppm} \mathrm{O}$ after deoxidation, $5 \mathrm{ppm}$ of that $\mathrm{O}$ occur dissolved in the melt and 25 ppm as solid alumina. This is in accordance to a solubility of $5 \mathrm{ppm} \mathrm{O}$ in a steel melt with 0.03 wt.\% Al [28]. Dekkers [6] gives a total oxygen content of the steel at the end of ladle metallurgy of $28-46 \mathrm{ppm}$. Subtraction the amount of dissolved O (not available for Al oxidation) gives $23-41 \mathrm{ppm} \mathrm{O}$ which is transferred in form of alumina particles from ladle to the tundish. The ladle capacity is $265 \mathrm{t}$ at ThyssenKrupp Steel AG in Duisburg, which results in $\sim 13$ to $23 \mathrm{~kg}$ alumina. We have shown that deoxidation particles have $\delta^{18} \mathrm{O} \approx+24 \%$. 
Mixing of alumina from chromite reduction and deoxidation particles in the given proportions results in $\delta^{18} \mathrm{O}$ of clogging between +7.3 and $+11.3 \%$ with an average value of $+9.5 \%$. According to the model, $39-53$ wt. $\%$ of $\mathrm{Al}_{2} \mathrm{O}_{3}$ in clogging source from deoxidation and $47-61$ wt. $\% \mathrm{Al}_{2} \mathrm{O}_{3}$ in clogging derived from chromite reduction. The average $\delta^{18} \mathrm{O}$ of clogging in the steel plant in Duisburg is $+6.8 \%$, i.e. it is by $0.5 \%$ lower than the range discussed in terms of the 2-component mixing model.

Atmospheric oxygen is an omnipresent potential source of clogging. The minimum contribution of air $\mathrm{O}_{2}$ can be estimated on base of the $\mathrm{N}$-content of the steel $\left(\mathrm{O}_{2}\right.$ is dissolved more rapidly than $\mathrm{N}_{2}$ [6]). The difference in nitrogen content of the steel between ladle and tundish indicates air entrapment during transfer operation [29]. The nitrogen pickup from ladle to tundish in the steel plant in Duisburg is in the range of $1-2 \mathrm{ppm}$. The $\mathrm{N}_{2} / \mathrm{O}_{2}$ mass-ratio of air is $78 / 21=3.7$. Given nitrogen pickup in the steel suggests a minimum of $0.3-0.5$ ppm oxygen from air that is available for the oxidation of alloyed Al. On base of a ladle capacity of $265 \mathrm{t}$, this corresponds to a minimum of $0.2-0.3 \mathrm{~kg}$ alumina per ladle that can form by reoxidation through air. Air $\mathrm{O}_{2}$ has $\delta^{18} \mathrm{O}=+23.5$, i.e. is, with respect to the origin of clogging, isotopically indistinguishable from process $\mathrm{O}_{2}$.

Mixing of air $\mathrm{O}_{2}$ derived alumina with alumina from chromite reduction and alumina from deoxidation at given proportions and $\delta^{18} \mathrm{O}$ values, gives a $\delta^{18} \mathrm{O}$ of clogging of +7.4 to $+11.4 \%$ o and a contribution of $46-61$ wt.\% slide gate powder, $39-53$ wt.\% deoxidation particles and $0.3-0.9$ wt.\% air $\mathrm{O}_{2}$ (Figure 6).

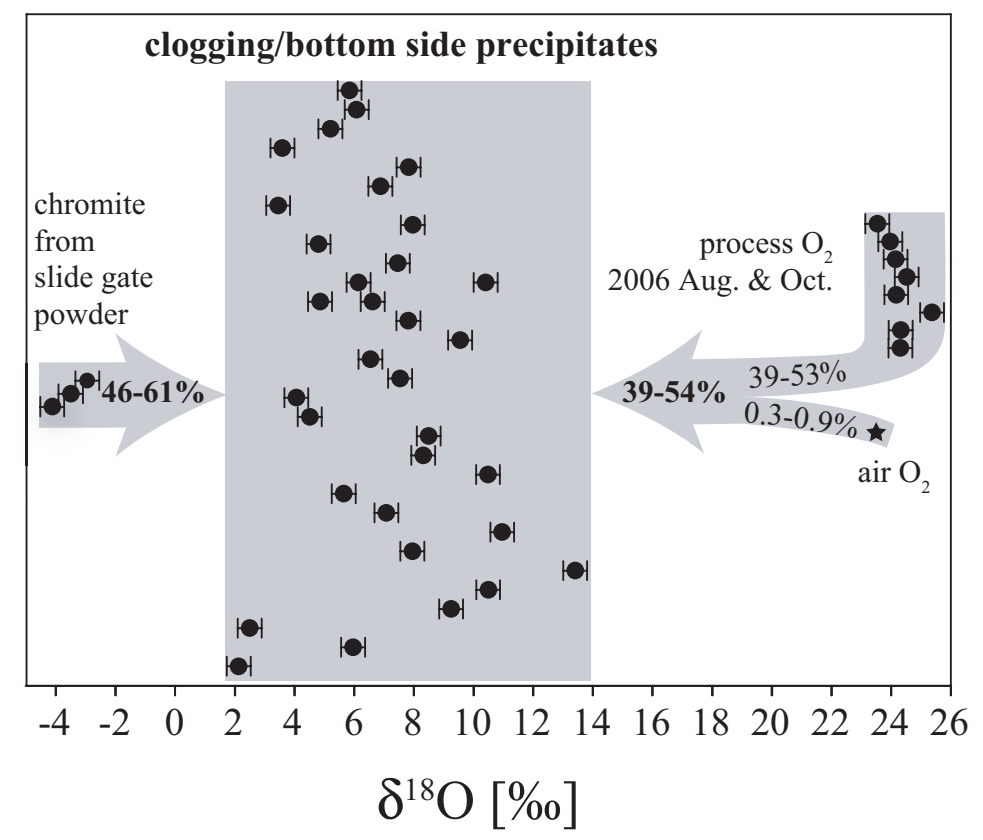

Figure 6. Sketch showing the 3-component model for the origin of clogging with its quantitative oxygen sources. 
The isotope composition of clogging is, on average, by $0.6 \%$ lower than suggested by the 3component model. The model, as demonstrated, has one major weakness, which has to be explored in further investigations. Reduction of chromite not only leads to formation of alumina, but also to formation of spinel (Equation 4). The spinel/alumina mass-ratio as derived from equation 4 is 0.97 , i.e. reduction of chromite produces alumina and spinel in approximately equal proportions. Reduction of chromite of $80 \mathrm{~kg}$ slide gate powder produces $20 \mathrm{~kg}$ spinel in addition to $20 \mathrm{~kg}$ alumina. Mixing of $20 \mathrm{~kg}$ alumina and $20 \mathrm{~kg}$ spinel from chromite reduction with $13-23 \mathrm{~kg}$ alumina from deoxidation and $0.2-0.3 \mathrm{~kg}$ alumina from air $\mathrm{O}_{2}$ gives clogging with $\delta^{18} \mathrm{O}$ between +3.4 to $6.8 \%$ and an average $\delta^{18} \mathrm{O}$ value of $+5.2 \%$. This $\delta^{18} \mathrm{O}$ is by $\sim 2 \%$ o lower than the observed average $\delta^{18} \mathrm{O}$ of clogging. It is, however, problematic with respect to the chemical composition of clogging.

Assuming between $46-61 \mathrm{wt} . \%$ chromite as oxygen source, the contribution of spinel from chromite reduction would lead to $\mathrm{MgO}$ concentrations of $6-8 \mathrm{wt}$ \% in clogging. The lowest content of $\mathrm{MgO}$ in clogging is $0.1 \mathrm{wt} . \%$. In terms of the model, this sets an upper limit of the contribution of free alumina and spinel from chromite reduction to $\sim 1 \mathrm{wt} . \%$. Such low amounts of $\mathrm{O}$ from chromite reduction in clogging would give $\delta^{18} \mathrm{O}$ of clogging up to $+24 \%$, which has not been observed in clogging samples in this study and in the study of Pack et al. [16]. Therefore we conclude that liberated $\mathrm{MgAl}_{2} \mathrm{O}_{4}$ from chomite reduction (Equation 4) does not contribute to SEN clogging.

Mechanical separation of spinel from free alumina is required to explain observed $\delta^{18} \mathrm{O}$ and low $\mathrm{MgO}$ contents of clogging. A feasible process involves temporal dissolution of $\mathrm{O}$ and transport away from the site of chromite reduction. Residual spinel from chromite reduction (Equation 4) is absorbed by the tundish slag, whereas free alumina is formed with the steel melt by reaction with dissolved excess O (Figure 7). A fraction of these alumina particles contribute to SEN clogging.

Higher $\mathrm{FeO}$ and $\mathrm{Cr}_{2} \mathrm{O}_{3}$ concentrations in some tundish slag samples, along with the identification of chromite by X-ray powder diffraction, show that some chromite is absorbed by the slag before reduction. This chromite will not provide $\mathrm{O}$ for the formation of alumina precipitates. Low $\mathrm{FeO}$ and $\mathrm{Cr}_{2} \mathrm{O}_{3}$ concentrations in most tundish slag samples, however, indicate effective chromite reduction. 


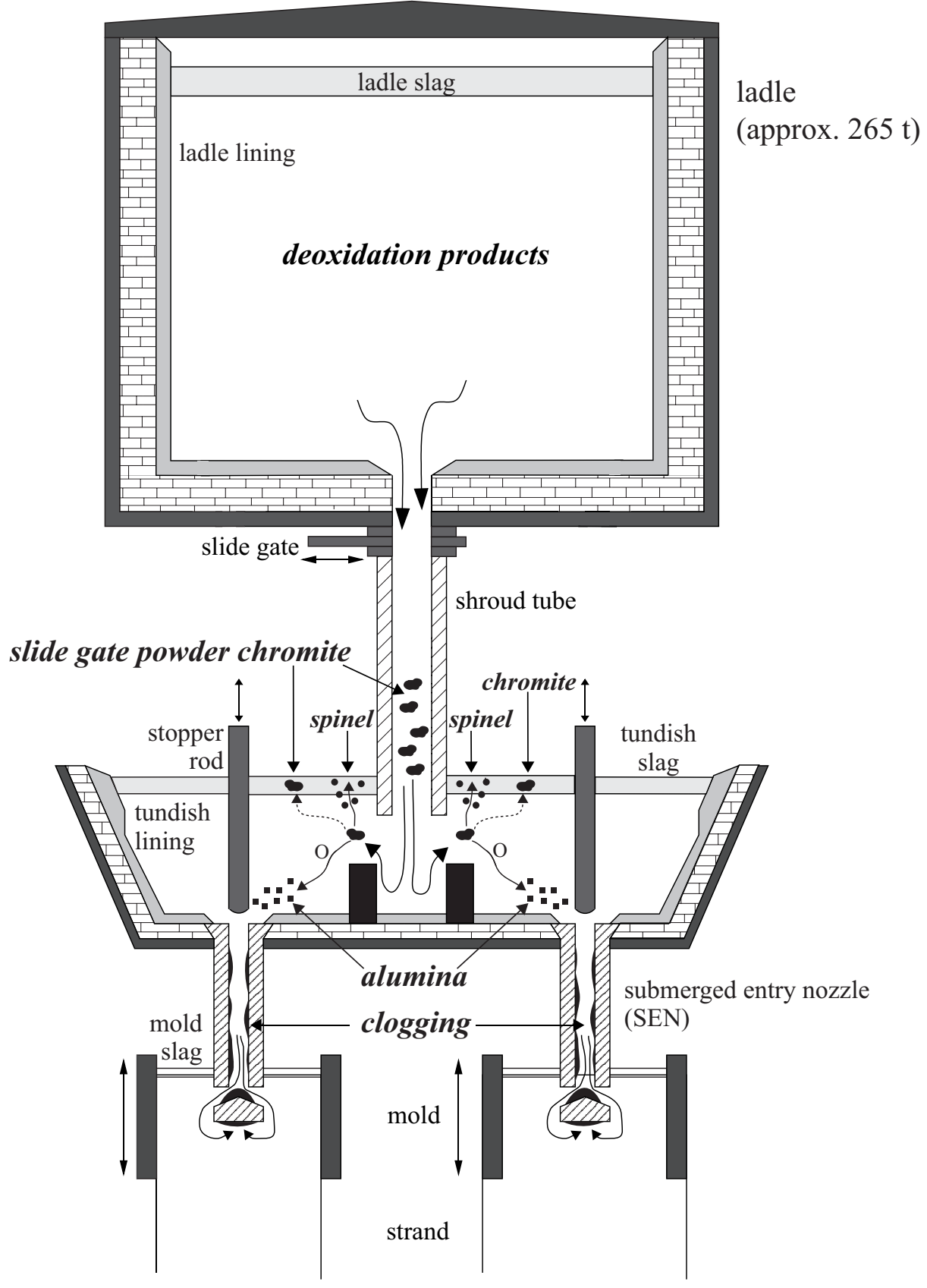

Figure 7. Sketch illustrating the reduction of slide gate powder chromite in the tundish of a continuous caster (modified after Pack [21]). Liberated $\mathrm{O}$ from the reaction will oxidize alloyed $\mathrm{Al}$ when $\mathrm{O}$ is spatially separated from chromite. A fraction of formed alumina (black-filled squares) is assumed to contribute to SEN clogging. The spinel (black-filled circles) from chromite reduction will also be absorbed by the tundish slag as minor amounts of chromite (indicated by the dashed line).

Slide gate powder is not only composed of reducible chromite, but also of quartz sand. Silica is harder to reduce compared to $\mathrm{Fe}$ - and $\mathrm{Cr}$-oxide and thus expected to contribute less to SEN clogging through $\mathrm{Al}$ oxidation. Furthermore, silica is less dense than chromite, which leads to faster floating of quartz grains and absorption into the tundish slag. We have therefore not considered quartz in the mass balance calculations. 


\subsection{Conclusions}

This paper demonstrates that oxygen isotopes analyses can be used as a tool to determine the origin of oxygen in clogging. Clogging as well as various potential sources of oxygen have been isotopically analyzed. Based on a mass balance calculation, we have developed a 3component model for the source of SEN clogging with contribution through slide gate powder, deoxidation particles and air $\mathrm{O}_{2}$.

According to this model, $46-61 \mathrm{wt} . \%$ of clogging originates from oxygen liberated during the reduction of chromite from the slide gate powder. $39-53 \mathrm{wt} . \%$ of clogging comes from carry-over of deoxidation particles from the ladle. The third component is air $\mathrm{O}_{2}$, for which a minimum contribution to SEN clogging of $0.3-0.9 \mathrm{wt} . \%$ is estimated.

\subsection{Acknowledgement}

We thank the ThyssenKrupp Steel AG for funding this research project. Also we would like to thank all collaborating people whose support made this work possible.

Contact: Christoph Toulouse

Georg-August-Universität

Geowissenschaftliches Zentrum

Abteilung Isotopengeologie

Goldschmidtstraße 1

37077 Göttingen, Germany

ThyssenKrupp Steel AG

Metallurgy Division, Steel Production, $Q C$ \& Process Development

Kaiser-Wilhelm-Straße 100

47166 Duisburg, Germany

\subsection{References}

[1] Rajtora, O. J., Smith, J. D. and Van Aken, D. C.: Effect of Nozzle Permeability on Alumina Accretion During Continuous Steel Casting, (2006), American Iron and Steel Institute, Technology Roadmap Program Office, Pittsburgh, PA 15220, USA

[2] Jönsson, P., Andersson, M., Nakajima, K., Appelberg, A., Tilliander, A., Shibata, H. and Kitamura, S.: Current Advances in Materials and Processes (CAMP-ISIJ), 18 (2005), 4, 849-852

[3] Rackers, K. G. and Thomas, B. G.: Clogging in Continuous Casting Nozzles, Steelmaking Conference Proceedings, (1995), Nashville, TN, USA, 78, 723-734

[4] Thomas, B. G. and Bai, H.: Tundish Nozzle Clogging - Application of Computational Models, 18rd Process Technology Division Conference Proceedings, (2001), Baltimore, MD, 18, 895-912 
[5] Dekkers, R., Blanpain, B. and Wollants, P.: Reoxidation: The Cause of Nozzle Clogging, Electric Furnace Conference Proceedings, (2002), 287-294

[6] Dekkers, R.: Aardkundige Mededelingen, 13 (2003), 109-120

[7] Bannenberg, N.: Demands on refractory material for clean steel production, (1995), In: Global Development of Refactories, Technical Association of Refractories, 36-59

[8] Pleschiutschnigg, F.-P.: Dr.-Thesis "Das Verhalten oxidischer Einschlüsse sowie ihre Kontrolle in Al-beruhigtem Stahl während der Stahlerzeugung und des Stranggießens", Aachen, (1989), p. 182

[9] Zimmermann, A.: Dr.-Thesis "Die Entstehung oxidische Einschlüsse im Stahl unter besonderer Berücksichtigung des Einflusses feuerfester Materialien des Systems SiO2A12O3", Clausthal, (1974), p. 50

[10] Basu, S., Choudhary, S. K. and Girase, N. U.: ISIJ International, 44 (2004), 10, $1653-$ 1660

[11] Dawson, S.: Iron \& Steelmaker, (1990), 4, 33-42

[12] Dekkers R., Blanpain, B. and Wollants, P.: AIST Transactions, Iron and Steel Technology, (2004), 93-98

[13] Vermeulen, Y., Coletti, B., Wollants, P., Blanpain, B. and Haers, F.: Steel Research International, 71 (2000), 10, 391-395

[14] Dekkers, R., Blanpain, B., Wollants, P., Haers, F., Gommers, B. and Vercruyssen, C.: Steel Research International, 74 (2003), 6, 351-355

[15] Hiraga, Y., Yashima, Y. and Fujii, K.: Journal of the Technical Association of Refractories, 15 (1995), 1, 22-27

[16] Pack, A., Hoernes, S., Göbbels, M., Bross, R. and Buhr, A.: European Journal of Mineralogy, 17 (2005), 3, 483-493

[17] Pack, A., Hoernes, S., Walther, T. and Bross, R.: European Journal of Mineralogy, 15 (2003), 1, 193-205

[18] Droop, G. T. R.: Mineralogical Magazine, 51 (1987), 431-435

[19] Valley, J. W., Kitchen, N., Kohn, M. J., Niendorf, C. R. and Spicizza, M. J.: Geochimica et Cosmochimica Acta, 59 (1995), 24, 5523-5531

[20] Sharp, Z. D.: Geochimica et Cosmochimica Acta, 54 (1990), 5, 1353-1357

[21] Pack, A.: Dr.-Thesis "Tracing the origin of oxide inclusions in continously cast steelAn interdisciplinary approach", (2000), p. 164

[22] Hagen, B.: Dr.-Thesis "Rekonstruktion von Metamorphosepfaden mit stabilen Isotopen: Möglichkeiten der Laserfluorinierung am Beispiel des Sächsischen Granulitgebirges", (2002), p. 179 
[23] Dole, M., Lane, G. A., Rudd, D. P. and Zaukelies, D. A.: Geochimica et Cosmochimica Acta, 6 (1954), 65-78

[24] Tuttle, R., Peaslee, K. D. and Smith, J. D.: Effect of Nozzle Permeability on Clogging, (2006), American Iron and Steel Institute, Technology Roadmap Program Office, Pittsburgh, PA 15220, USA.

[25] Chialvoa, A. A. and Horita, J.: The Journal of Chemical Physics, 125 (2006), 034510

[26] Taylor, H. P. J.: Contributions to Mineralogy and Petrology, 19 (1968), 1-71

[27] Dekkers, R.: Dr.-Thesis "Non-metallic inclusions in liquid steel", Leuven, (2002), p. 195

[28] Turkdogan, E. T.: Fundamentals of Steelmaking, (1996), p. 331

[29] Zhang, L. and Thomas, B. G.: ISIJ International, 43 (2003), 3, 271-291 


\title{
13 Manuskript PACK et al. (2007)
}

\section{Determination of oxygen triple isotope ratios of silicates without cryogenic separation of $\mathrm{NF}_{3}$ - technique with application to ana- lyses of technical $\mathrm{O}_{2}$ gas and meteorite classification}

\author{
A. Pack ${ }^{*}, 1$, C. Toulouse ${ }^{1,2}$, R. Przybilla ${ }^{1}$ \\ ${ }^{1}$ Universität Göttingen, Geowissenschaftliches Zentrum, Goldschmidtstraße 1, D-37077 Göttingen, Germany \\ ${ }^{2}$ ThyssenKrupp Steel AG, Metallurgy Division, Steel Production, QC \& Process Development, Kaiser-Wilhelm- \\ Straße 100, D-47166 Duisburg, Germany
}

*Correspondence author is Andreas Pack: apack@uni-goettingen.de

\begin{abstract}
A novel technique is described to determine $\Delta^{, 17} \mathrm{O}_{\text {TFL }}$ with high accuracy and precision by using IR laser fluorination with $F_{2}$ as reaction gas. The technique includes precise monitoring of the intensity ratio of the ${ }^{14} \mathrm{NF}_{2}(\mathrm{~m} / \mathrm{z}=52)$ to $\mathrm{O}_{2}$ signal. The correlation between intensity of ${ }^{14} \mathrm{NF}_{2}$ and positive error in $\delta{ }^{, 17} \mathbf{O}_{\text {vSMow }}$ allows correction of measured data to obtain reliable $\Delta^{, 17} \mathbf{O}_{\text {TFL }}$ values. The resultant error in $\Delta{ }^{, 17} \mathrm{O}_{\mathrm{TFL}}$ of a single measurement after correction is in the range of $\pm 0.06 \%$, i.e. sufficiently small to permit the technique to be useful for meteorite classification. Our data of technical $\mathrm{O}_{2}$, in combination with literature data, suggest a negative anomaly of tropospheric air $\mathrm{O}_{2}$ with $\Delta{ }^{, 17} O_{\text {TFL }}=-0.344 \pm 0.015 \%$ o $(1 \sigma)$.
\end{abstract}

\subsection{Introduction}

\subsubsection{Stable oxygen isotopes}

Oxygen is the third most abundant element in the solar system ${ }^{1}$. Terrestrial oxygen (in natural minerals, liquids and gases) is a mixture of three stable isotopes comprising of $\sim 99.8 \%{ }^{16} \mathrm{O}$, $\sim 0.04 \%{ }^{17} \mathrm{O}$ and $\sim 0.2 \%{ }^{18} \mathrm{O}$. In rocks, natural fluids and atmospheric gases, the ${ }^{18} \mathrm{O} /{ }^{16} \mathrm{O}$-ratio varies in a range from $-8 \%{ }^{2}$ to $+10 \%{ }^{3}$ compared to ratio of seawater ${ }^{4}$. Differences in oxygen isotope ratios are usually very small and therefore expressed in form of the $\delta$-notation

$$
\delta^{x} \mathrm{O}_{\text {vSMOW }}=\left(\frac{\left(\frac{{ }^{x} \mathrm{O}}{{ }^{16} \mathrm{O}}\right)_{\text {sample }}}{\left(\frac{{ }^{x} \mathrm{O}}{{ }^{16} \mathrm{O}}\right)_{\text {vsMow }}}-1\right) \times 1000
$$


with $x=17,18$. VSMOW denotes the oxygen isotope ratio of the international seawater standard, which has a ${ }^{17} \mathrm{O} /{ }^{16} \mathrm{O}$-ratio of $3.7990 \times 10^{-4}$ and ${ }^{18} \mathrm{O} /{ }^{16} \mathrm{O}$-ratio of $2.00517 \times 10^{-3}\left({ }^{5,6}\right)$.

The first scientific application of stable oxygen isotope variations $\left({ }^{18} \mathrm{O} /{ }^{16} \mathrm{O}\right)$ was in geology, namely the determination of palaeotemperatures by means of the fractionation between marine carbonates and water ${ }^{7,8}$. Today, stable oxygen isotope analyses are widely used in fields such as geology, cosmochemistry or life sciences.

\subsubsection{Mechanisms of isotope fractionation}

Mass-fractionation is used as general term to describe shifts in isotope ratios. In literature, mass-dependent fractionation is distinguished from so-called mass-independent isotope fractionation ${ }^{9}$.

Strictly speaking, all mechanisms related to fractionation among isotopes and isotopologues are mass-dependent.

Variations in isotope ratios may result from differences in isotopologue formation-rates during nucleosynthesis ${ }^{10}$, photochemical processes ${ }^{11}$, or differences bond strength of coexisting phases ${ }^{8,12,13}$. In kinetically-controlled reactions, isotopic fractionation results from the respective isotopologues reacting at slightly different rates. Kinetic fractionation is also important in processes where transport plays a role (e.g. diffusion) ${ }^{13}$. With few exceptions ${ }^{9,14}$ isotopic fractionation by physical or chemical processes results in changes of the ${ }^{17} \mathrm{O} /{ }^{16} \mathrm{O}$-ratio being approximately half the magnitude of the corresponding change to the ${ }^{18} \mathrm{O} /{ }^{16} \mathrm{O}$-ratio. This socalled "mass-dependent" fractionation results in terrestrial rocks fitting a line with slope $\sim 0.52$ in the $\delta^{17} \mathrm{O}_{\text {VSMOW }}$ vs $\delta^{18} \mathrm{O}_{\text {VSMow }}$ diagram. This line is often referred as the terrestrial fractionation line (TFL), with material that falls off the line as isotopically anomalous. An isotopic anomaly may be due to "mass-independent" fractionation processes ${ }^{15,16}$, but also (to a limited extent) may derive from mass-dependent fractionation processes that are characterized by a slope, which is slightly different from that of the reference line ${ }^{13}$.

The magnitude of an isotopic anomaly is described by the $\Delta^{, 17} \mathrm{O}_{\mathrm{TFL}}$, which is the vertical distance of the respective sample from the TFL in $\delta{ }^{, 17} \mathrm{O}_{\text {VSMOW Vs }} \delta{ }^{, 18} \mathrm{O}_{\text {VSMOW }}$ space, with

$$
\delta^{\prime x} \mathrm{O}_{\mathrm{VSMOW}}=10^{3} \ln \left(\frac{\delta^{x} \mathrm{O}_{\mathrm{VSMOW}}}{10^{3}}+1\right)
$$

and $x=17,18 . \delta^{\text {,x }} \mathrm{O}_{\text {VSMOw }}$ denotes the linearized form of the $\delta$-notation (see ${ }^{17}$ and ${ }^{18}$ for details). The isotope anomaly is defined as 


$$
\Delta^{\prime 17} \mathrm{O}_{\mathrm{TFL}}=\delta^{\prime 17} \mathrm{O}_{\mathrm{vSMOW}}-\beta \times \delta^{\prime 18} \mathrm{O}_{\mathrm{vSMOW}}
$$

$\beta$ is the slope of the TFL in the $\delta{ }^{17} \mathrm{O}_{\text {VSMOw vs }}{ }^{18} \mathrm{O}_{\text {VSMOw }}$ space. The value of $\beta$ for the TFL shows small variations $(0.5240-0.5266)$ that are, however, not related to differences in laboratory instrumentation ${ }^{19}$. Miller ${ }^{18}$ reported a $\beta$-value of 0.5247 . Since terrestrial rocks ${ }^{18,19}$ and waters ${ }^{20}$ fit on fractionation lines of similar (but not identical) slopes, little attention has been drawn to oxygen triple isotope analyses among geologists.

R. Clayton and his co-workers were the first to report chondritic components that fall far outside the $\mathrm{TFL}^{15}\left(\Delta^{, 17} \mathrm{O}_{\mathrm{TFL}} \geq-25 \%\right)$. They concluded that chondrites sampled material from different nucleosynthetic reservoirs, i.e. different stellar sources. More recently, isotope selfshielding is favored to have caused variations in the $\delta{ }^{17} \mathrm{O}_{\mathrm{vSMOW}} / \delta{ }^{18} \mathrm{O}_{\mathrm{VSMOW}}$-ratio in planets, asteroids and meteorite components ${ }^{11,21,22}$. Isotope analyses of solar wind that has been implanted in lunar metals show that asteroids and terrestrial planets differ in $\Delta^{, 17} \mathrm{O}_{\mathrm{TFL}}$ from the bulk solar system (represented by the Sun) ${ }^{23}$. This implies that asteroids and terrestrial planets have acquired their non-solar oxygen isotopic compositions during condensation and isotopic exchange in a gas irradiated by UV light, which places strong constraints on a few regions of the solar accretion disk where this is possible.

Since the observation of isotopically anomalous solar system and atmospheric components, a wealth of new information has been obtained through high precision determination of $\delta^{, 17} \mathrm{O}$ along with $\delta{ }^{, 18} \mathrm{O}$ (see review article by M. H. Thiemens ${ }^{9}$ ).

\subsection{Techniques and problems related to analysis of $\delta^{, 17} \mathrm{O}$ and $\delta^{, 18} \mathrm{O}$}

Precise analyses of $\delta{ }^{, 17} \mathrm{O}_{\mathrm{VSMOW}}$ and $\delta{ }^{18} \mathrm{O}_{\mathrm{VSMOW}}$ of rocks and minerals are obtained by mass spectrometry on $\mathrm{O}_{2}$ that is liberated whilst heating the sample by means of a wire-bound tube furnace ${ }^{24}$ or by means of an infra red laser ${ }^{18,19,25-31}$. In short, samples are reacted with $\mathrm{BrF}_{5}$ or $\mathrm{F}_{2}$ gas to form fluorides and $\mathrm{O}_{2}$, which is analyzed in a multi-collector gas mass spectrometer for $\delta{ }^{, 17} \mathrm{O}_{\mathrm{VSMOW}}$ and $\delta{ }^{18} \mathrm{O}_{\mathrm{VSMOW}}$. It has been shown that IR laser fluorination is generally superior to conventional fluorination in Ni-autoclaves, namely for refractory materials ${ }^{25,30}$. Small amounts of material required $(\sim 1 \mathrm{mg})$ are a further advantage of laser fluorination.

A common problem that is related to $\delta{ }^{17} \mathrm{O}_{\mathrm{VSMOW}}$ determination by means of fluorination is an isobaric interference on $\mathrm{m} / \mathrm{z}=33$ that gives $\delta{ }^{, 17} \mathrm{O}_{\mathrm{VSMOW}}$-values that range from correct (no interference) to erroneously high (up to $>100 \%$ ). 
The interfering species was suggested to be ${ }^{14} \mathrm{NF}$, a fragment of the $\mathrm{NF}_{3}$ molecule ${ }^{29,31-33}$. The origin of nitrogen can be intrinsic (e.g. N-rich enstatite chondrites), due to incomplete $\mathrm{F}_{2}$ cleaning or leaks.

Unless in case of isotope ratio monitoring gas mass spectrometry, analyses with a dual inlet system do not allow gas chromatographic separation of $\mathrm{NF}_{3}$ from $\mathrm{O}_{2}$.

Clayton and Mayeda ${ }^{32}$ purified samples of $\mathrm{O}_{2}$ by trapping at $-196^{\circ} \mathrm{C}$ on $13 \mathrm{X}$ molecular sieve, from which it was released using ethanol slush at $-115^{\circ} \mathrm{C}$. At this temperature $\mathrm{NF}_{3}$ is presumably kept on the molecular sieve and only $\mathrm{O}_{2}$ is released. They report precision of $\Delta^{, 17} \mathrm{O}_{\mathrm{TFL}}$ of $\pm 0.17 \%$ on the basis of a set of terrestrial samples. Other workers ${ }^{31}$ monitored the $\mathrm{m} / \mathrm{z}=52$ signal after each measurement. In the event of a signal at $\mathrm{m} / \mathrm{z}=52$ being detected, those authors adsorbed the $\mathrm{O}_{2}$ back from the sample bellow of the mass spectrometer, onto $13 \mathrm{X}$ molecular sieve cooled to $-196^{\circ} \mathrm{C}$. The temperature of the molecular sieve was then raised to $-115^{\circ} \mathrm{C}$, at which temperature $\mathrm{O}_{2}$ was released whilst $\mathrm{NF}_{3}$ was retained. The purified $\mathrm{O}_{2}$ was re-analyzed for isotopic composition. The precision of $\Delta^{, 17} \mathrm{O}_{\mathrm{TFL}}$ was reported to $\pm 0.024 \%{ }^{31}$. Rumble III et al. ${ }^{33}$ report that $\mathrm{NF}_{3}$ can be trapped at $80^{\circ} \mathrm{C}$ on $5 \AA$ molecular sieve; a temperature at which $\mathrm{O}_{2}$ is not trapped. Wiechert et al. ${ }^{29}$ report that $\mathrm{m} / \mathrm{z}=52$ was routinely monitored, but that no signal was observed. They report a precision of $\Delta{ }^{, 17} \mathrm{O}_{\mathrm{TFL}}$ of $\pm 0.05 \%$ for a single analysis.

\subsection{Objective of this study}

The fact that published high-precision $\Delta^{, 17} \mathrm{O}_{\mathrm{TFL}}$ data (obtained by conventional dual inlet gas mass spectrometry) were exclusively reported from laboratories that use $\mathrm{BrF}_{5}$ in combination with cryogenic trapping of $\mathrm{NF}_{3}$ may suggest that $\mathrm{NF}_{3}$ is less a problem with $\mathrm{BrF}_{5}$ than with $\mathrm{F}_{2} . \mathrm{BrF}_{5}$, however, is practically unavailable on the market any more.

We are aware of three laboratories (University of Bonn, University of Göttingen, CNS Pisa) that used or use $\mathrm{F}_{2}$ as reaction agent, in which large apparent excess $\Delta^{, 17} \mathrm{O}_{\mathrm{TFL}}$ (up to $>100 \%$ ) is measured, presumably due to massive isobaric interference through ${ }^{14} \mathrm{NF}$. Due to the problem of availability of $\mathrm{BrF}_{5}, \mathrm{~F}_{2}$ is likely to be the preferred fluorination agent for many existing and certainly for new laboratories. These laboratories may all face the same problem with too high $\Delta{ }^{17} \mathrm{O}_{\mathrm{TFL}}$ values.

Our own laboratory experiments showed that purification with different types of $13 \mathrm{X}$ molecular sieve failed to remove the large quantities of $\mathrm{NF}_{3}$ contamination in the laser line with $\mathrm{F}_{2}$ as 
reaction agent. Trapping of $\mathrm{NF}_{3}$ on $5 \AA$ molecular sieve has not been tested during this study, but has been shown by previous work ${ }^{33}$ to be very slow.

Therefore a method has been developed to overcome the analytical problems related to isobaric interference on $\mathrm{m} / \mathrm{z}=33$ without the necessity of differential cryogenic trapping of $\mathrm{NF}_{3}$. The goal is to obtain reliable and precise $\delta{ }^{, 17} \mathrm{O}_{\text {VSMOw }}$ and $\delta{ }^{, 18} \mathrm{O}_{\text {VSMOw }}$ measurements of silicate rocks and silicate and oxide minerals with an IR laser fluorination line and $\mathrm{F}_{2}$ as reaction gas.

High-precision analyses of the oxygen triple isotope composition of rocks and minerals are essential for the oxygen isotope characterization of meteorites, to assist their classification. A side issue was to show if technical $\mathrm{O}_{2}$ could be used to trace the magnitude of the isotope anomaly of tropospheric $\mathrm{O}_{2}$. We have directly compared analyses of technical $\mathrm{O}_{2}$ with analyses of terrestrial rocks and minerals, for which $\Delta^{, 17} \mathrm{O}_{\mathrm{TFL}}=0 \%$.

\subsection{Experimental}

\subsubsection{Sampling}

For the purpose of testing the technique, a set of 52 anhydrous minerals and rocks (San Carlos olivine, hematite, chromite, low-Ca pyroxene, corundum, chert, quartz, garnet, mid ocean ridge basalt) from igneous and low-grade metamorphic rocks has been analyzed in the time between January and June 2007. These minerals were assumed to fall on the TFL. We have not included any materials from ultra high-pressure rocks that were observed to fall on a fractionation line with a slightly different slope ${ }^{19}$. The minerals were handpicked single grains that were cleaned in ethanol and distilled water. Terrestrial samples were used as $\Delta^{, 17} \mathrm{O}_{\mathrm{TFL}}$ reference for $\mathrm{O}_{2}$ gas analyses and a meteorite specimen.

Technical $\mathrm{O}_{2}(>99.99 \%)$ was sampled from our reference gas bottle $\left(\delta^{18} \mathrm{O}_{\mathrm{VSMOW}}=12.5 \%\right)$ and at the ThyssenKrupp Steel plant in Duisburg (Germany), respectively. A total of 5 gas samples were measured with 4 samples collected in the steel plant in a time interval of several months. The gas was used for decarburization of the carbon-rich raw iron.

\subsubsection{Instrumentation}

The fluorination line used is similar to that described by Z. D. Sharp ${ }^{26}$. Major differences, however, are that $\mathrm{F}_{2}$ is used instead of $\mathrm{BrF}_{5}$ and that $\mathrm{O}_{2}$ is not reacted to $\mathrm{CO}_{2}$. 
1 - 2 mg silicate samples are loaded into a Ni sample holder and evacuated over night. Samples are fluorinated using a $50 \mathrm{~W} \mathrm{CO}_{2}$ laser $(\lambda=10.6 \mu \mathrm{m})$ and purified ${ }^{34} \mathrm{~F}_{2}$ gas $(\sim 20 \mathrm{mbar})$. Excess $\mathrm{F}_{2}$ is reacted with heated $\left(\sim 150^{\circ} \mathrm{C}\right) \mathrm{NaCl}$ to $\mathrm{NaF}$. $\mathrm{Cl}_{2}$ is collected in a cold trap at $196^{\circ} \mathrm{C}$. Sample $\mathrm{O}_{2}$ is then trapped at $-196^{\circ} \mathrm{C}$ on $5 \AA$ molecular sieve located close to the dual inlet system of a FINNIGAN DELTA+ mass spectrometer. The mass spectrometer is equipped with three Faraday cups (\#1 - \#3) with $3 \times 10^{8}, 3 \times 10^{11}$ and $1 \times 10^{11} \Omega$ feedback resistors for simultaneous measurement of $\mathrm{m} / \mathrm{z} 32,33$ and 34. After $6-10$ min trapping, $\mathrm{O}_{2}$ is expanded into the sample bellows of the mass spectrometer by means of heating the molecular sieve to $\sim 80^{\circ} \mathrm{C}$. The pressure in the bellows (sample and reference) was adjusted to obtain a signal between $\sim 2.5$ and $\sim 5 \mathrm{~V}$, respectively, on cup \#1. This range is well within the linear range of the FINNIGAN DELTA+ mass spectrometer.

After equilibration, the sample is analyzed using the conventional dual inlet system and compared with bottled reference $\mathrm{O}_{2}$ of known $\delta^{18} \mathrm{O}_{\text {VSMOW }}(+12.5 \%)$. The $\delta^{17} \mathrm{O}_{\text {VSMOw value of re- }}$ ference $\mathrm{O}_{2}$ was chosen as $+6.55 \%$. It will be demonstrated, however, that this value is incorrect. Therefore, data reported on base of the approximate assumption that bottle $\mathrm{O}_{2}$ has $\delta^{17} \mathrm{O}_{\text {VSMOW }}=+6.55 \%$, are expressed as $\delta{ }^{, 17} \mathrm{O}_{\text {VSMOW }}$ instead of $\delta{ }^{, 17} \mathrm{O}_{\text {VSMOW. }} \Delta^{, 17} \mathrm{O}_{\text {TFL values }}$ that are calculated on base of $\delta^{17} \mathrm{O}_{\mathrm{VSMOW}} *$ and $\delta^{18} \mathrm{O}_{\mathrm{VSMOW}}$ of reference $\mathrm{O}_{2}$ of +6.55 and $12.5 \%$, respectively, are specified as $\Delta^{, 17} \mathrm{O}_{\mathrm{TFL}}$.

Scans of the $m / z=52$ signal were performed by using the most sensitive detector (cup \#2).

$\mathrm{O}_{2}$ gas was samples in pre-evacuated $700 \mathrm{ml}$ steel flasks. The gas was expanded to the inlet system of the gas mass spectrometer by connecting the flasks using high-vacuum SWAGELOCK tube fittings and stainless steel bellow valves. No cryogenic trapping step was required.

\subsection{Results}

The uncorrected results of triple oxygen isotope analyses of terrestrial samples are displayed in the $\delta^{, 17} \mathrm{O}_{\text {VSMOW* Vs }} \delta{ }^{18} \mathrm{O}_{\text {VSMOW }}$ space in Figure $1(\mathrm{~N}=52)$. The materials span a range in $\delta^{18} \mathrm{O}_{\text {VSMOw }}$ from -4.7 to $+23.6 \%$. The data points displayed in Figure 1 source from two sets of experiments.

Those samples that are termed $\mathrm{NF}_{2}$-free in Figure $1(\mathrm{~N}=20$, open triangles) were selected from analytical sessions in the time between January and March 2007. The sample were chosen on base of their low $\Delta{ }^{, 17} \mathrm{O}_{\mathrm{TFL}} *$ values and/or on base of absence of a signal at $\mathrm{m} / \mathrm{z}=52$, 
which has been proven by magnetic field scans of the sample gas after analysis. Regression analysis gives a line with

$$
\delta{ }^{17} \mathrm{O}_{\text {VSMOW }}=0.524 \pm 0.006 \times \delta{ }^{18} \mathrm{O}_{\mathrm{VSMOW}}+0.40 \pm 0.06 \% \text {. }
$$

The regression parameters were calculated using the ISOPLOT macro for EXCEL ${ }^{35}$ $\left(\mathrm{R}^{2}>0.999\right)$. The intercept at $\delta^{, 17} \mathrm{O}_{\mathrm{VSMOW}}>0$ indicates that the bottle oxygen in use is isotopically anomalous, i.e. has an actual $\delta^{17} \mathrm{O}_{\mathrm{VSMOW}}<6.55 \%$.

The majority of natural silicate samples that were run in the sessions between January and March 2007, however, show apparent excess in ${ }^{17} \mathrm{O}$ through erroneously high $\delta{ }^{, 17} \mathrm{O}_{\text {Vsmow* }}$ values. Because scans of the $\mathrm{m} / \mathrm{z}=52$ signal were not carried out under the same conditions, we cannot include these samples to our correction procedure.

A second set of samples was analyzed between April and June 2007 (Figure 1, N = 32, open circles). Samples from this set were systematically scanned for a signal at $\mathrm{m} / \mathrm{z}=52$. Samples fall on or above a slope $\sim 0.5$ line in the $\delta^{17} \mathrm{O}_{\text {VsMow }}$ vs $\delta{ }^{18} \mathrm{O}_{\text {VSMOw }}$ space with apparent ${ }^{17} \mathrm{O}$ excess ranging from $\sim 0$ up to $\sim 40 \%$. The presence of erroneously high $\delta{ }^{, 17} \mathrm{O}_{\mathrm{VSMOW}} *$ values is not related to a specific sample material. A weak relation between the number of samples analyzed from a single holder and the apparent excess in ${ }^{17} \mathrm{O}$ is observed. The first samples usually showed the highest apparent excess in ${ }^{17} \mathrm{O}$, whereas those run at the end of a batch of 19 samples often showed little or no apparent ${ }^{17} \mathrm{O}$ excess.

In order to trace the origin of erroneously high $\delta^{, 17} \mathrm{O}_{\text {VSMOW* values, we have conducted mag- }}$ netic field scans across the ${ }^{14} \mathrm{NF}_{2}{ }^{+}$peak $(\mathrm{m} / \mathrm{z}=52)$ after each run. $\mathrm{NF}_{2}$ and the presumably interfering $\mathrm{NF}$ are fragments of the $\mathrm{NF}_{3}$ molecule. Scanning data for gas that was extracted from a garnet are shown in Figure 2 (open circles). Reference $\mathrm{O}_{2}$ was scanned for background subtraction (Figure 2, solid circles). Four parameters $\left(\mathrm{I}_{\mathrm{a}}, \mathrm{I}_{\mathrm{b}}, \mathrm{I}_{\mathrm{b}}^{\mathrm{O}_{2}}, \mathrm{I}_{\mathrm{b}}^{\mathrm{NF}_{2}}\right)$ are indicated in Figure 2. $\mathrm{I}_{\mathrm{a}}$ is the intensity of the $\mathrm{O}_{2}$ background before the $\mathrm{m} / \mathrm{z}=52$ peak. In order to correct erro-

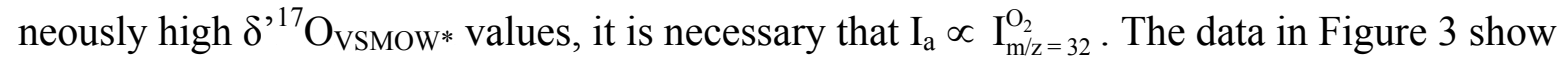
that this condition is fulfilled and that $\mathrm{I}_{\mathrm{a}}$ can be used as measure for $\mathrm{I}_{\mathrm{m} / \mathrm{z}=32}^{\mathrm{O}_{2}}$, i.e. for the $\mathrm{O}_{2}$ partial pressure in the source. Parameter $\mathrm{I}_{\mathrm{b}}^{\mathrm{O}_{2}}$ is the contribution of the $\mathrm{O}_{2}$ background to the intensity of the $\mathrm{m} / \mathrm{z}=52$ peak. $\mathrm{I}_{\mathrm{b}}^{\mathrm{O}_{2}}$ has to be subtracted from the total signal $\mathrm{I}_{\mathrm{b}}$ in order to obtain the net intensity $\mathrm{I}_{\mathrm{b}}^{\mathrm{NF}_{2}}$ of the ${ }^{14} \mathrm{NF}_{2}$ fragment. The net intensity $\mathrm{I}_{\mathrm{b}}^{\mathrm{NF}_{2}}$, in relation to the $\mathrm{O}_{2}$ partial pressure is a measure for the amount of ${ }^{14} \mathrm{NF}$ that interferes with ${ }^{33} \mathrm{O}_{2}$; it is assumed that $\mathrm{I}_{\mathrm{b}}^{\mathrm{NF}_{2}} \propto \mathrm{I}_{\mathrm{m} / \mathrm{z}=33}^{\mathrm{NF}}$. The value of $\mathrm{I}_{\mathrm{b}}^{\mathrm{O}_{2}}$, however, is inaccessible for samples that contain $\mathrm{NF}_{2}$. 
Therefore, we have determined the relation between $\mathrm{I}_{b} \mathrm{O}_{2}$ and $\mathrm{I}_{\mathrm{a}}$ from scans of reference $\mathrm{O}_{2}$, which is assumed to be devoid of $\mathrm{NF}_{2}$ and NF. A linear relation is observed for all samples, which allows determination of $\mathrm{I}_{b}^{\mathrm{O}_{2}}$ as function of $\mathrm{I}_{\mathrm{a}}$ for samples with $\mathrm{I}_{\mathrm{b}}^{\mathrm{NF}_{2}}>0 \mathrm{mV}$ (Figure 4).

Knowing the relation between $\mathrm{I}_{\mathrm{a}}$ and $\mathrm{I}_{\mathrm{b}}^{\mathrm{NF}_{2}}$ and knowing the value of $\mathrm{I}_{\mathrm{b}}$ allows calculation of the $\mathrm{I}_{\mathrm{b}}^{\mathrm{NF}_{2}}$ net intensity by means of the relation $\mathrm{I}_{\mathrm{b}}^{\mathrm{NF}_{2}}=\mathrm{I}_{\mathrm{b}}-\mathrm{I}_{\mathrm{b}}^{\mathrm{O}_{2}}$. The contribution of ${ }^{14} \mathrm{NF}_{3}$ to the ${ }^{17} \mathrm{O}^{18} \mathrm{O}$ signal is assumed to be proportional to the $\mathrm{I}_{\mathrm{b}}^{\mathrm{NF}_{2}} / \mathrm{I}_{\mathrm{a}}$-ratio with $\mathrm{I}_{\mathrm{a}} \propto \mathrm{I}_{\mathrm{m} / \mathrm{z}=32}^{\mathrm{O}_{2}}$ (Figure 3).

The relation between apparent ${ }^{17} \mathrm{O}$ excess and the $\mathrm{I}_{\mathrm{b}}^{\mathrm{NF}_{2}} / \mathrm{I}_{\mathrm{a}}$-ratio is shown in Figure 5 . The ${ }^{17} \mathrm{O}$ excess is expressed in terms of $\Delta{ }^{, 17} \mathrm{O}_{\mathrm{TFL}}{ }^{*}=\delta{ }^{, 17} \mathrm{O}_{\mathrm{VSMOW}}$, uncorrected $-0.5237 \times \delta{ }^{18} \mathrm{O}_{\mathrm{VSMOW}}$. Data for individual days fall on single lines that show small variations in slope $(\mathrm{m})$ and intercept $(\mathrm{k})$. The correct $\delta{ }^{17} \mathrm{O}_{\mathrm{vSmow}}$ values are calculated using the relation:

$$
\delta^{\prime 17} \mathrm{O}_{\text {VSMOW }}=\delta^{\prime 17} \mathrm{O}_{\text {VSMOW*, uncorrected }}-\mathrm{m} \times \frac{\mathrm{I}_{\mathrm{b}}^{\mathrm{NF}_{2}}}{\mathrm{I}_{\mathrm{a}}}-\mathrm{k}
$$

with $\mathrm{m}$ and $\mathrm{k}$ being the regression parameters of the relation of apparent $\Delta^{, 17} \mathrm{O}_{\mathrm{TFL}} \mathrm{VS} \mathrm{I}_{\mathrm{b}}^{\mathrm{NF}_{2}} / \mathrm{I}_{\mathrm{a}}$. The values for $\Delta^{, 17} \mathrm{O}_{\mathrm{TFL}}$ are generally a function of $\beta$, which was taken from the dataset of apparently $\mathrm{NF}_{3}$-free samples. The validity of this choice is illustrated in Figure 6, which shows $\delta^{, 17} \mathrm{O}_{\text {VSMOW Vs }} \delta{ }^{18} \mathrm{O}_{\text {VSMOW }}$ (top) and $\Delta^{, 17} \mathrm{O}_{\mathrm{TFL}}$ as function of $\delta{ }^{, 18} \mathrm{O}_{\text {VSMOW }}$ (bottom). No systematic variation between $\Delta{ }^{17} \mathrm{O}_{\mathrm{TFL}}$ and $\delta{ }^{, 18} \mathrm{O}_{\mathrm{VSMOW}}$ is observed, which supports that $\beta$ is 0.5237 . In case of improper choice of $\beta$, a systematic increase or decrease of $\Delta^{, 17} \mathrm{O}_{\mathrm{TFL}}$ with $\delta{ }^{18} \mathrm{O}_{\mathrm{VSMOW}}$ would be observed for terrestrial samples. The overall error in $\Delta^{, 17} \mathrm{O}_{\mathrm{TFL}}$ of a single measurement is in the range of $\pm 0.06 \%$ (Figure 6 , bottom).

The weighted average slope is $\mathrm{m}_{\text {average }}=5.25 \pm 0.05(\mathrm{~N}=7,1 \sigma)$. The intercepts k range between +0.32 and $+0.52 \%$, respectively. The weighted average intercept is $0.348 \pm 0.014 \%$ ( $\mathrm{N}$ $=7,1 \sigma)$. Including data of $\mathrm{NF}_{3}$ free experiments $(\mathrm{k}=-0.40 \pm 0.06 \%$ ), an average intercept calculated to $\mathrm{k}=0.36 \pm 0.05 \%$. This intercept is related to the anomaly of bottle $\mathrm{O}_{2}$ with $\Delta{ }^{, 17} \mathrm{O}_{\mathrm{TFL}}=-0.36 \pm 0.05 \%$ o $(1 \sigma)$.

We have analyzed 5 samples of high-purity (>99.99\%) technical $\mathrm{O}_{2}$ with $\delta^{, 18} \mathrm{O}_{\text {VSMOw values }}$ of $+0.9,+12.5$ and $\sim+24 \%$ o $(\mathrm{N}=3)$, respectively. The corresponding $\Delta{ }^{, 17} \mathrm{O}_{\mathrm{TFL}} *$ values are $0.33,-0.33$ and -0.34 to $-0.37 \%$, respectively (Figure 7 ). We used a $\beta$ value of 5.237 for calculation of $\Delta^{, 17} \mathrm{O}_{\mathrm{TFL}}$. The error of $\Delta^{, 17} \mathrm{O}_{\mathrm{TFL}}$ for $\mathrm{O}_{2}$ samples is typically in the range of $\pm 0.05 \%$ $(1 \sigma)$ 
In order to test the correction procedure, we have analyzed meteorite material that was provided by A. Bischoff (Münster, Germany). The first analysis gave $\delta^{18} \mathrm{O}_{\mathrm{VSMOW}}=+3.6 \%$, $\delta{ }^{17} \mathrm{O}_{\mathrm{VSMOW}}=+1.7 \%$ and $\Delta,{ }^{, 17} \mathrm{O}_{\mathrm{TFL}}=-0.19 \%$. No signal that was related to the presence of

${ }^{14} \mathrm{NF}_{2}$ in the sample gas was observed for this sample (i.e. $\mathrm{I}_{\mathrm{b}}^{\mathrm{NF}_{2}}=0$ ). The second analysis gave $\delta{ }^{18} \mathrm{O}_{\text {VSMOW }}=+3.5, \delta{ }^{, 17} \mathrm{O}_{\text {VSMOW }}=+1.6 \%, \Delta{ }^{, 17} \mathrm{O}_{\mathrm{TFL}}=-0.22 \%$. The correct $\delta{ }^{17} \mathrm{O}_{\text {VSMOW value }}$ has been calculated on base of $\mathrm{I}_{\mathrm{b}}^{\mathrm{NF}_{2}}=50.1 \mathrm{mV}$, which corresponded to an apparent excess in ${ }^{17} \mathrm{O}$ of $+5.1 \%$. The meteorite was the basaltic eucrite Juvinas. Our results are shown along with published HED achondrite data from Wiechert et al. ${ }^{27}$ in Figure 8.

\subsection{Discussion}

Our raw data, i.e. uncorrected for ${ }^{14} \mathrm{NF}$ show a similar scatter $\delta{ }^{, 17} \mathrm{O}_{\text {VSMOw }}$ vs $\delta{ }^{, 18} \mathrm{O}_{\text {VSMOW }}$ space as data published in Rumble III et al. ${ }^{33}$ with the only difference that we have analyzed materials with a wider range in $\delta^{18} \mathrm{O}_{\mathrm{VSMOW}}$. Data either fall on or above the TFL (Figure 1). Absence of correlation between $\Delta^{, 17} \mathrm{O}_{\mathrm{TFL}}$ and $\delta^{, 18} \mathrm{O}_{\mathrm{SMOW}}$ indicates that photochemical reactions, such as observed by Thiemens and Heidenreich ${ }^{16}$ by interaction of $\mathrm{O}_{2}$ with UV light, are

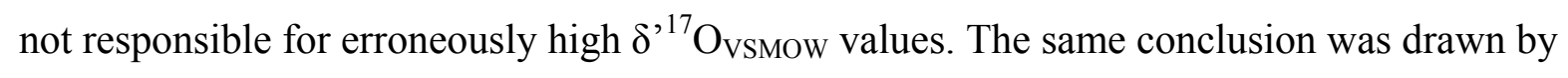
Rumble III et al. ${ }^{36}$ for their UV laser fluorination line.

The excellent correlation between the intensity $\mathrm{I}_{\mathrm{b}}^{\mathrm{NF}_{2}}$ of ${ }^{14} \mathrm{NF}_{2}{ }^{+}$signal on $\mathrm{m} / \mathrm{z}=52$ and the apparent deviation of terrestrial samples from the TFL is strong evidence that the ${ }^{14} \mathrm{NF}$ fragment is the only cause for erroneously high $\delta{ }^{, 17} \mathrm{O}_{\text {VSMOw }}$ values that are usually obtained in our laboratory. Although less well developed, a similar correlation between the $\mathrm{m} / \mathrm{z}=52$ peak and the $\Delta{ }^{, 17} \mathrm{O}_{\mathrm{TFL}}$ has been observed by Rumble III et al. ${ }^{33}$, who used an UV laser instead of a $\mathrm{CO}_{2}$ laser. The quality of the data in their Figure 6 indicates that these data were not suitable for a correction. We have not analyzed material that contained carbon. Therefore, we can only conclude that components such as $\mathrm{CF}_{4}$, as discussed by Robert et al. ${ }^{20}$ and Wiechert et al. ${ }^{27,29}$, do probably not play a role with respect to measurement of $\delta{ }^{, 17} \mathrm{O}_{\mathrm{VSMOW}}$ of carbon free silicates and oxides.

Our data show that $\Delta^{17} \mathrm{O}_{\mathrm{TFL}}$ analyses with accuracy and precision in the range of $\pm 0.06 \%$ are possible on $\mathrm{NF}_{3}$ contaminated samples, as is typical for IR laser fluorination with $\mathrm{F}_{2}$ as reaction agent. Cryogenic separation of $\mathrm{NF}_{3}$ either on $13 \mathrm{X}$ or $5 \AA$ molecular sieve is not required. The NF-correction can be applied for samples that show apparent ${ }^{17} \mathrm{O}$ excess as high as $+40 \%$. In order to avoid additional errors that are related to extrapolation, samples with un- 
known $\Delta{ }^{, 17} \mathrm{O}_{\mathrm{TFL}} *$ should show $\mathrm{I}_{\mathrm{b}}^{\mathrm{NF}_{2}} / \mathrm{I}_{\mathrm{a}}$-ratios that are comparable to the ratios of the terrestrial samples that are used for the correction.

We have obtained a $\beta$ value of 0.5237 for terrestrial rocks and minerals. This is identical to the $\beta$ value of $0.5240 \pm 0.0015$ that has recently been reported by Rumble et al. ${ }^{19}$ for terrestrial rocks (excluding ultra high-pressure garnets).

Our data indicate that the bottle $\mathrm{O}_{2}$ that is used as reference gas $\left(\delta^{18} \mathrm{O}_{\mathrm{VSMOW}}=+12.5 \%\right)$ is isotopically anomalous with $\Delta{ }^{17} \mathrm{O}_{\mathrm{TFL}}=-0.36 \pm 0.05 \%$. Bottle $\mathrm{O}_{2}$ is produced by liquefaction from air and is thus likely to inherit the anomaly of tropospheric $\mathrm{O}_{2}$. Four samples of technical $\mathrm{O}_{2}$ from a steel plant show, within error, identical $\Delta^{, 17} \mathrm{O}_{\mathrm{TFL}}$-values, although they show wide variation in $\delta^{18} \mathrm{O}_{\text {VSMOW. }}$. Robert et al. ${ }^{20}$ erroneously assumed that their industrial reference $\mathrm{O}_{2}$ falls on the TFL. Recasting their data into the $\delta{ }^{, 17} \mathrm{O}$ and $\delta{ }^{18} \mathrm{O}$ notation gives $\Delta^{, 17} \mathrm{O}_{\mathrm{TFL}}=-$ $0.342 \%$ o $(\beta=0.5279)$ for their reference $\mathrm{O}_{2}$. Miller et al. ${ }^{18}$ calculated a $\Delta^{, 17} \mathrm{O}_{\mathrm{TFL}}$ value of tropospheric $\mathrm{O}_{2}$ to $-0.340 \%$. He used published isotope data ${ }^{37,38}$. In a recent abstract, Young and Zigeler ${ }^{39}$ reported $\Delta^{, 17} \mathrm{O}_{\mathrm{TFL}}=-0.347 \%$ for tropospheric $\mathrm{O}_{2}$ relative to rocks and minerals. Within error, our analyses of technical $\mathrm{O}_{2}$, along with literature data ${ }^{20}$, show the same $\Delta{ }^{, 17} \mathrm{O}_{\mathrm{TFL}}$ than tropospheric $\mathrm{O}_{2}{ }^{18,39}$ (Figure 7). The fact that technical $\mathrm{O}_{2}$ shows a wide variation in $\delta^{18} \mathrm{O}_{\mathrm{VSMOW}}$, but no correlated variation in $\Delta^{, 17} \mathrm{O}_{\mathrm{TFL}}$ (Figure 7) indicates that fractionation during air liquefaction and rectification does fall on a line with $\beta$ equal to that defined by rocks and minerals.

We conclude that technical $\mathrm{O}_{2}$ inherits the isotope anomaly of air $\mathrm{O}_{2}$, from which it is produced. The average $\Delta^{, 17} \mathrm{O}_{\mathrm{TFL}}$ of tropospheric $\mathrm{O}_{2}$ is $-0.344 \pm 0.015 \%$. This value was calculated from our data of technical $\mathrm{O}_{2}(\mathrm{~N}=5)$ and from literature data on technical and tropospheric $\mathrm{O}_{2}{ }^{18,20,39}(\mathrm{~N}=8)$. If the anomaly results solely from kinetic fractionation during respiration, $\beta_{\text {respiration would be }-0.344 \% 0 / 23.5 \%}=-0.015$ lower than the $\beta_{\mathrm{TFL}}$. Assuming $\beta_{\mathrm{TFL}}=0.524^{19 \text {,this }}$

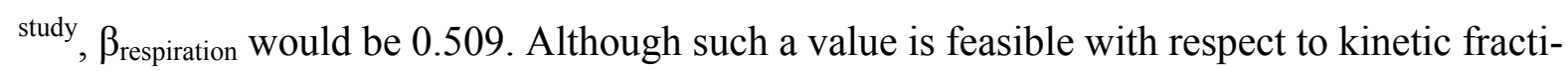
onation $^{13}$, it was shown by experimental work by Luz et al. ${ }^{40 \text { (and references therein) }}$ that respiration fractionates oxygen along a line with $\beta_{\text {respiration }}=0.518$. It was suggested by Miller et al. ${ }^{18}$ that this indicates that the $\Delta,{ }^{17} \mathrm{O}_{\mathrm{TFL}}$ of tropospheric $\mathrm{O}_{2}$ is caused by respiratory fractionation and influx of anomalous stratospheric $\mathrm{O}_{2}$. In view of our data, respiration accounts for an anomaly of $(0.518-0.524) \times 23.5 \%=-0.141 \%$, which is $41 \%$ of the total anomaly of tropospheric $\mathrm{O}_{2}\left(\Delta^{, 17} \mathrm{O}_{\mathrm{TFL}}=-0.344 \%\right)$. The remaining $59 \%$ of the anomaly should be caused by influx of isotopically anomalous $\mathrm{O}_{2}$ from the stratosphere. Photosynthetic $\mathrm{O}_{2}$ is regarded to be isotopically normal $^{41}$. 
Wiechert et al. ${ }^{27}$ reported a mean $\Delta{ }^{, 17} \mathrm{O}_{\mathrm{TFL}}$ for eucrites, howardites and diogenites (excluding Ibitira) of $-0.219 \pm 0.004 \%$ (see their data in Figure 8). Our mean datum of $\Delta^{, 17} \mathrm{O}_{\mathrm{TFL}}=-$ $0.21 \pm 0.03 \%$ for Juvinas is, within error, identical to the latter value showing that our technique is applicable to cosmochemical problems. Clayton et al. ${ }^{42}$ reported $\delta^{18} \mathrm{O}_{\mathrm{VSMOW}}=+3.48 \%$ and $\Delta^{17} \mathrm{O}_{\mathrm{TFL}}=-0.28 \%$ for Juvinas. They gave an error for $\Delta^{17} \mathrm{O}_{\mathrm{TFL}}$ of $\pm 0.1 \%$. Our data of $\delta{ }^{18} \mathrm{O}_{\mathrm{VSMOW}}=+3.6$ and $\Delta,{ }^{17} \mathrm{O}_{\mathrm{TFL}}=-0.21 \%$ are identical to data from the more recent determination by Wiechert et al. ${ }^{27}$.

\subsection{Conclusions}

Interference of the ${ }^{14} \mathrm{NF}$ fragment on the ${ }^{17} \mathrm{O}^{16} \mathrm{O}$ isotopologue is detrimental to precise $\delta^{17} \mathrm{O}_{\text {VSMOw }}$ analyses of silicates and oxides by means of laser fluorination with $\mathrm{F}_{2}$ as reagent. It is demonstrated that the contribution of ${ }^{14} \mathrm{NF}^{+}$on $\mathrm{m} / \mathrm{z}=33$ is proportional to the amount of the ${ }^{14} \mathrm{NF}_{2}$ fragment $(\mathrm{m} / \mathrm{z}=52)$ in the sample gas. Analyses of a small set of samples with known $\delta{ }^{17} \mathrm{O}_{\mathrm{VSMOW}} / \delta{ }^{18} \mathrm{O}_{\mathrm{VSMOW}}$-ratio (i.e. terrestrial rocks and minerals with $\Delta^{, 17} \mathrm{O}_{\mathrm{TFL}}=0 \%$ ) allow correction of the measured $\delta{ }^{17} \mathrm{O}$ values in order to obtain correct $\Delta{ }^{, 17} \mathrm{O}_{\mathrm{TFL}}$ data. The correction can be applied to analyses of samples with unknown $\Delta^{, 17} \mathrm{O}_{\mathrm{TFL}}$, like carbon free meteorites. The technique, however, has not yet been tested for carbon containing materials like carbonaceous chondrites or ureilites. For carbon free silicates and oxides, the overall uncertainty in $\Delta^{, 17} \mathrm{O}_{\mathrm{TFL}}$ is in the range of $\pm 0.06 \%$, i.e. comparable to results from laboratories that either have no $\mathrm{NF}_{3}$ contamination of that can separate $\mathrm{NF}_{3}$ from $\mathrm{O}_{2}$ in the sample gas. $\Delta{ }^{, 17} \mathrm{O}_{\mathrm{TFL}}$ data of technical $\mathrm{O}_{2}$ with wide variety in $\delta^{18} \mathrm{O}$ are, within error, identical to analyses of tropospheric $\mathrm{O}_{2}$. We suggest that tropospheric has a $\Delta^{, 17} \mathrm{O}_{\mathrm{TFL}}=-0.344 \pm 0.015 \%$ with $41 \%$ of this anomaly caused by respiratory fractionation and 59\% caused by influx of stratospheric $\mathrm{O}_{2}$ to the troposphere.

A shortcoming of the method that is described here is that a number of terrestrial rocks has to be measured before analyzing a sample with unknown $\Delta{ }^{17} \mathrm{O}_{\mathrm{TFL}}$. This is time-consuming and leads to a considerable reduction of the sample throughput.

\subsection{Acknowledgements}

A. Bischoff (Münster) is thanked for providing meteorite material. Funding through the Deutsche Forschungsgemeinschaft (grant PA909/2-1, Emmy-Noether Program), ThyssenKrupp Steel AG (Duisburg, Germany) and the University of Göttingen (Germany) is acknowledged. Helpful and detailed comments by M. Miller and an anonymous reviewer helped improving the manuscript. 


\subsection{References}

1. Lodders K. Astrophys. J. 2003; 591: 1220.

2. $\quad$ Fujita K, Abe O. Geophys. Res. Lttrs. 2006; 33: L18503.

3. Savarino J, Kaiser J, Morin S, Sigman DM, Thiemens MH. Atmos. Chem. Phys. 2007; 7: 1925 .

4. Coplen TB, Hopple JA, Böhlke JK, Peiser HS, Rieder SE, Krouse HR, Rosman KJR, Ding T, Vocke RDJ, Révész KM, Lamberty A, Taylor P, DeBièvre P. Compilation of Minimum and Maxium Isotope Ratios of Selected Elements in Naturally Occurring Terrestrial Materials and Reagents. United States Geological Survey: Reston, 2002; 98.

5. $\quad$ Baertschi P. Earth Planet. Sci. Lttrs. 1976; 31: 341.

6. $\quad$ Li W, Ni B, Jin D, Chang TL. Kexue Tongbao 1988; 33: 1610.

7. $\quad$ Epstein S, Buchsbaum R, Lowenstam H, Urey HC. J. Geol. 1951; 62: 417.

8. Urey HC, Epstein S, McKinney CR. Geol. Soc. Am. Bull. 1951; 62: 399.

9. Thiemens MH. Ann. Rev. Earth Planet. Sci. 2006; 34: 217.

10. Nagashima K, Krot AN, Yurimoto H. Nature 2004; 428: 921.

11. Clayton RN. Nature 2002; 415: 860.

12. Urey HC. J. Chem. Soc. 1947; 40: 562.

13. Young ED, Galy A, Nagahara H. Geochim. Cosmochim. Acta 2002; 66: 1095.

14. Bao HM, Thiemens MH, Farquhar J, Campbell DA, Lee CCW, Heine K, Loope DB. Nature 2000; 406: 176.

15. Clayton RN, Grossman L, Mayeda TK. Science 1973; 182: 485.

16. Thiemens MH, Heidenreich JEI. Science 1983; 219: 1073.

17. Hulston JR, Thode HG. J. Geophys. Res. 1965; 70: 3475.

18. Miller MF. Geochim. Cosmochim. Acta 2002; 66: 1881.

19. Rumble D, Miller MF, Franchi IA, Greenwood JP. Geochim. Cosmochim. Acta 2007; 71: 3592 .

20. Robert F, Rejou MA, Javoy M. Earth Planet. Sci. Lttrs. 1992; 108: 1.

21. Navon O, Wasserburg GJ. Earth Planet. Sci. Lttrs. 1985; 73: 1.

22. Lyons JR, Young ED. Nature 2005; 435: 317.

23. Hashizume K, Chaussidon M. Nature 2005; 434: 619.

24. Taylor BE. in PA De Groot (ed.) Handbook of Isotope Analytical Techniques. Elsevier: 2004; 400. 
25. Eiler JM. in JW Valley, DR Cole (eds.) Stable Isotope Geochemistry. Mineralogical Society of America: Washington, D.C., 2001; 319.

26. Sharp ZD. Geochim. Cosmochim. Acta 1990; 54: 1353.

27. Wiechert UH, Halliday AN, Palme H, Rumble D. Earth Planet. Sci. Lttrs. 2004; 221: 373.

28. Wiechert U, Halliday AN, Palme H, Rumble D. Geochim. Cosmochim. Acta 2002; 66: A834.

29. Wiechert U, Halliday AN, Lee DC, Snyder GA, Taylor LA, Rumble D. Science 2001; 294: 345 .

30. Pack A, Hoernes S, Göbbels M, Broß R, Buhr A. Eur. J. Min. 2005; 17: 483.

31. Miller MF, Franchi IA, Sexton AS, Pillinger C, T. Rap. Comm. Mass. Spectrom. 1999; 13: 1211 .

32. Clayton RN, Mayeda TK. Earth Planet. Sci. Lttrs. 1983; 62: 1.

33. Rumble III D, Farquhar J, Young ED, Christensen CP. Geochim. Cosmochim. Acta 1997; 61: 4229.

34. Asprey LB. J. Fluor. Chem. 1976; 7: 359.

35. Ludwig KR. Berkeley Geochronology Center Special Publ. 2003; 4: 71 pp.

36. Rumble DI, Farquhar J, Young ED, Christensen CP. Geochim. Cosmochim. Acta 1997; 61: 4229.

37. Luz B, Barkan E, Bender ML, Thiemens MH, Boering KA. Nature 1999; 400: 547.

38. Kroopnick P, Craig H. Science 1972; 175: 54.

39. Young ED, Ziegler K. Eos Transactions, AGU Joint Assembly 2006; 87: V43C.

40. Luz B, Barkan E. Geochim. Cosmochim. Acta 2005; 69: 1099.

41. Helman Y, Barkan E, Eisenstadt D, Luz B, Kaplan A. Plant Physiol. 2005; 138: 2292.

42. Clayton RN, Onuma N, Mayeda TK. Earth Planet. Sci. Lttrs. 1976; 30: 10. 


\subsection{Figures}

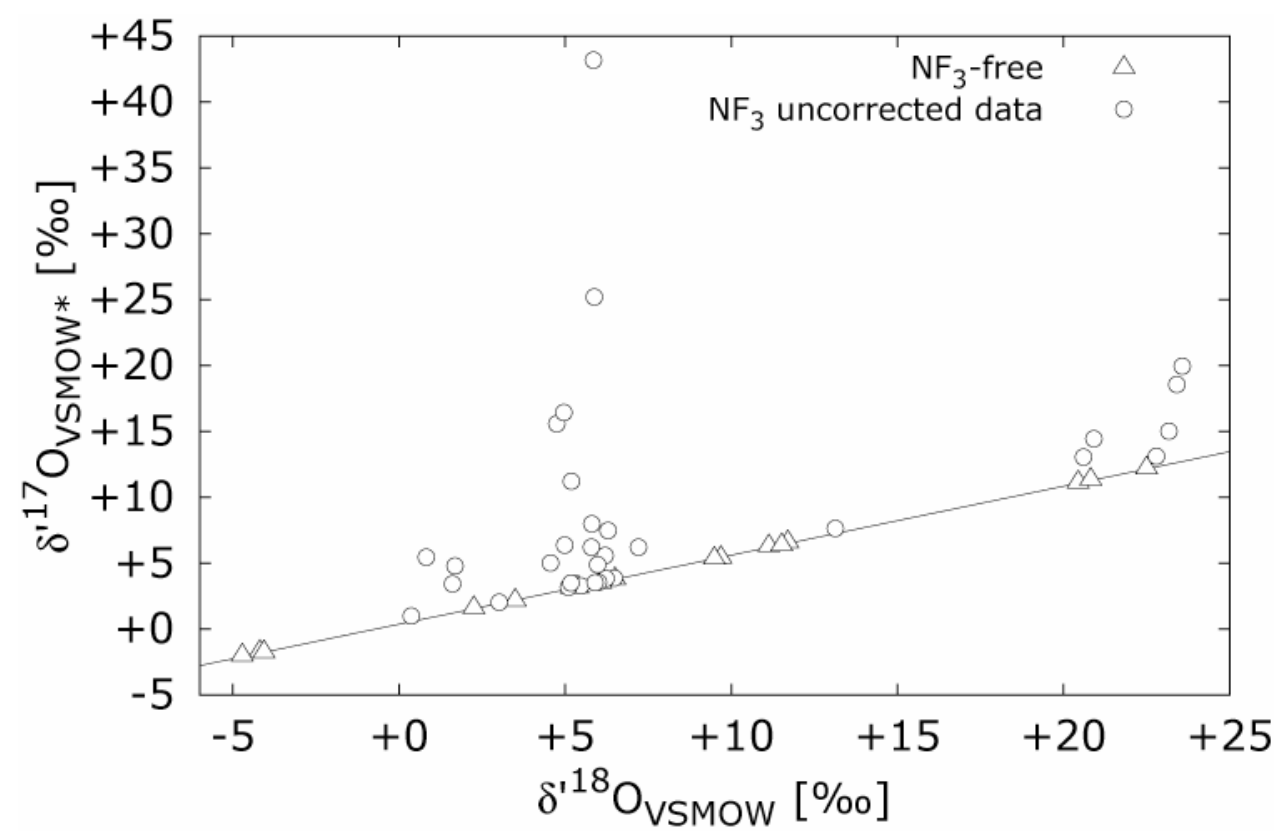

Figure 1. (top) Plot of $\delta^{, 17} \mathrm{O}_{\text {VSMOw }}$ vs $\delta{ }^{18} \mathrm{O}_{\text {VSMOw }}$ of anhydrous minerals from terrestrial igneous rocks. The analyses displayed as solid circles were used for the regression analysis which gives $\delta^{, 17} \mathrm{O}_{\text {VSMOW }}=0.524 \times \delta{ }^{18} \mathrm{O}_{\text {VSMOW }}+0.40 \%$.

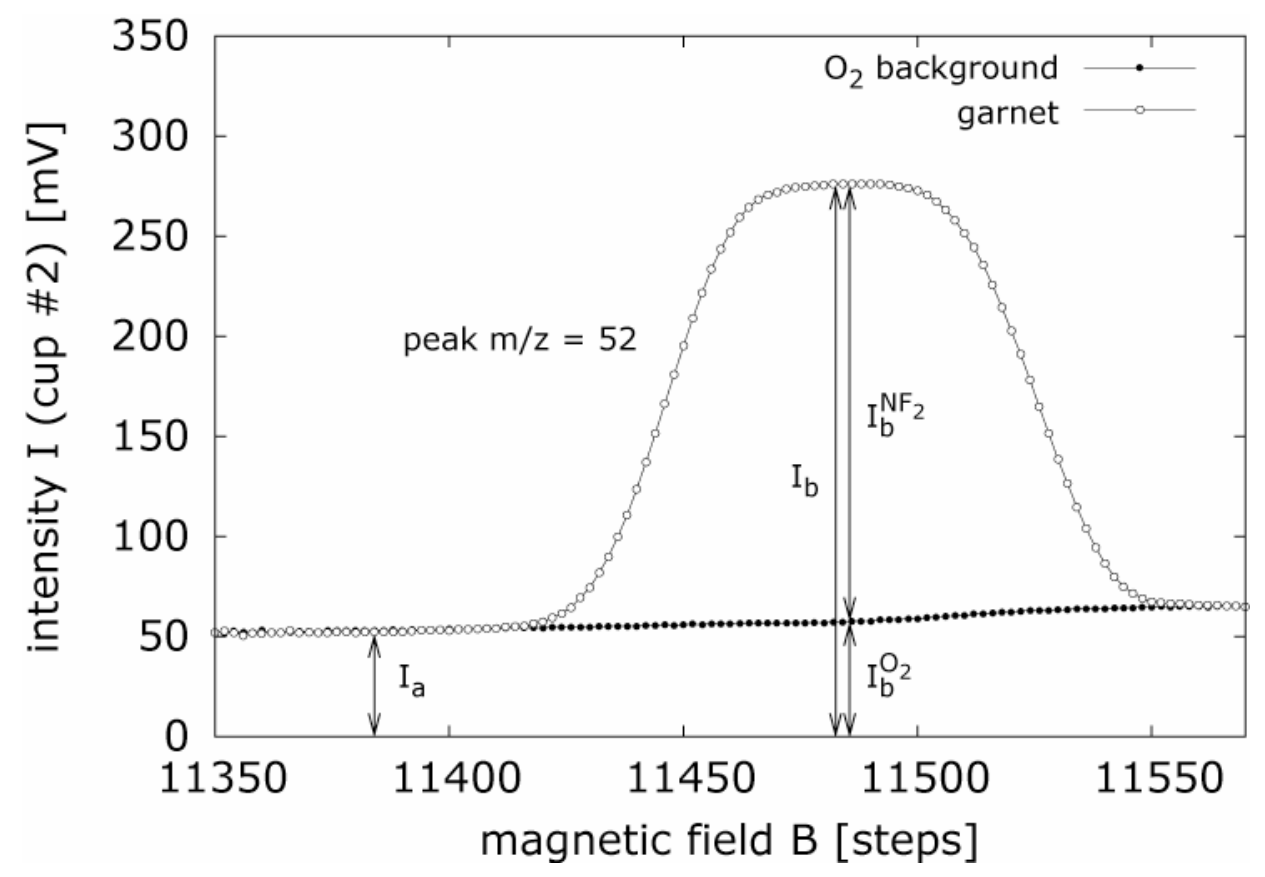

Figure 2. Plot of the intensity on detector cup \#2 vs the magnetic field strength across the $\mathrm{m} / \mathrm{z}=52$ peak. Shown are data for bottle $\mathrm{O}_{2}$ and $\mathrm{O}_{2}$ that was extracted from a terrestrial garnet. 


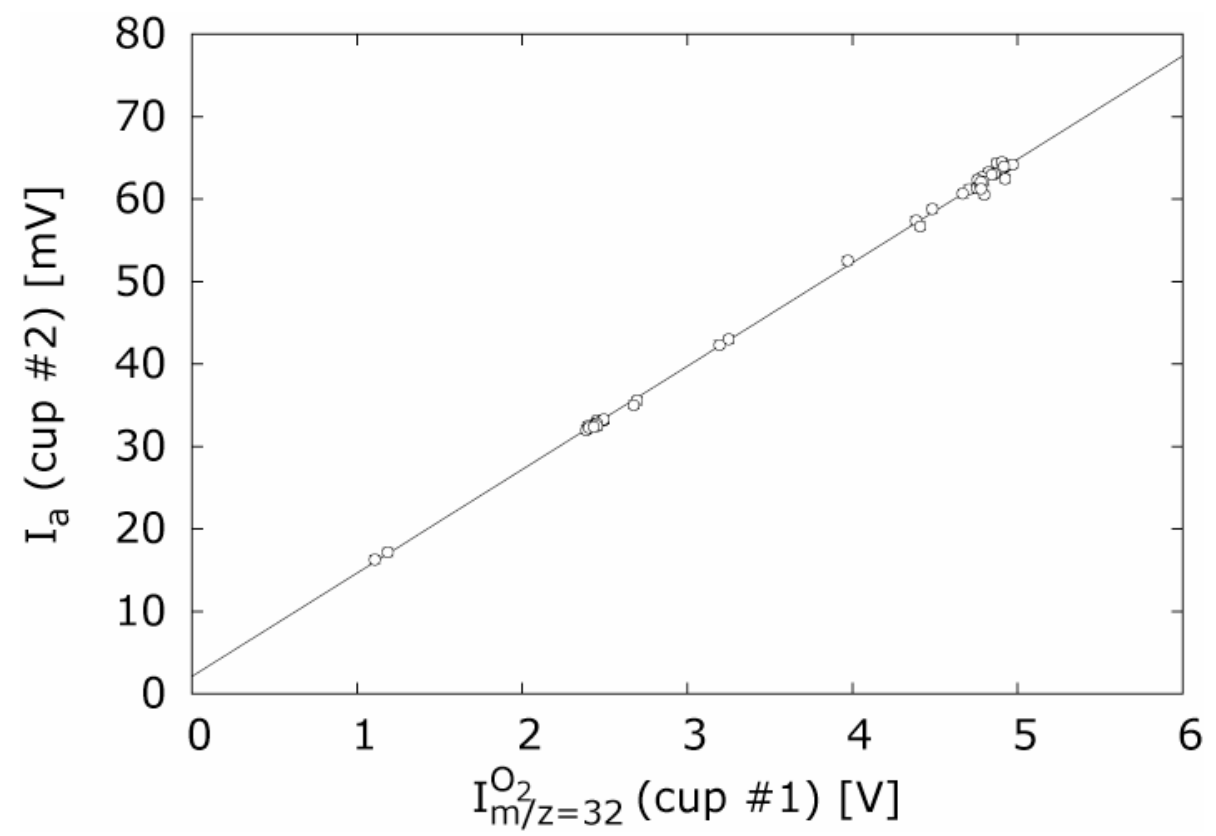

Figure 3: Plot of $\mathrm{I}_{\mathrm{a}}$ vs $\mathrm{I}_{\mathrm{m} / \mathrm{z}=32}^{\mathrm{O}_{2}}$ of reference $\mathrm{O}_{2}$ and minerals and rocks.

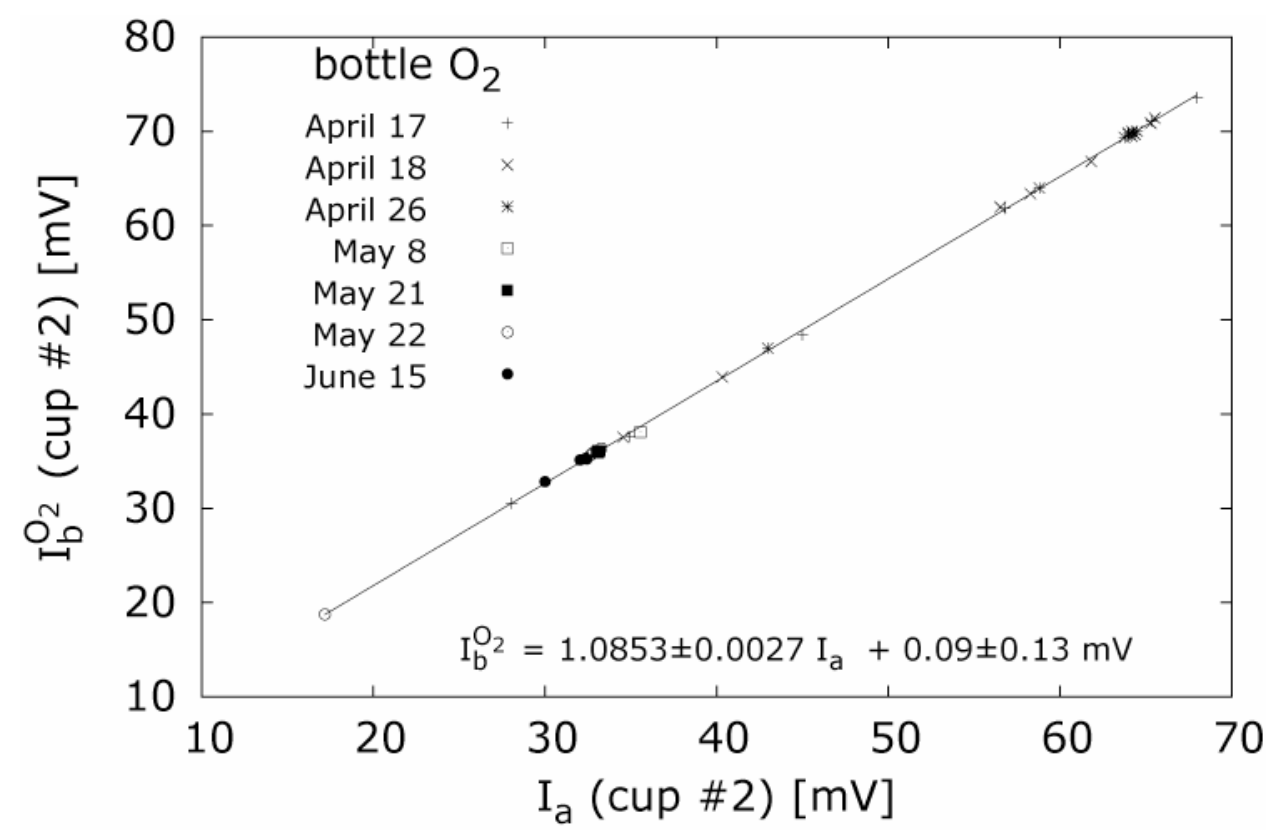

Figure 4: Plot of $\mathrm{I}_{\mathrm{b}}^{\mathrm{O}_{2}}$ vs $\mathrm{I}_{\mathrm{a}}$ for reference $\mathrm{O}_{2}$ samples with the result of the linear regression calculation. 


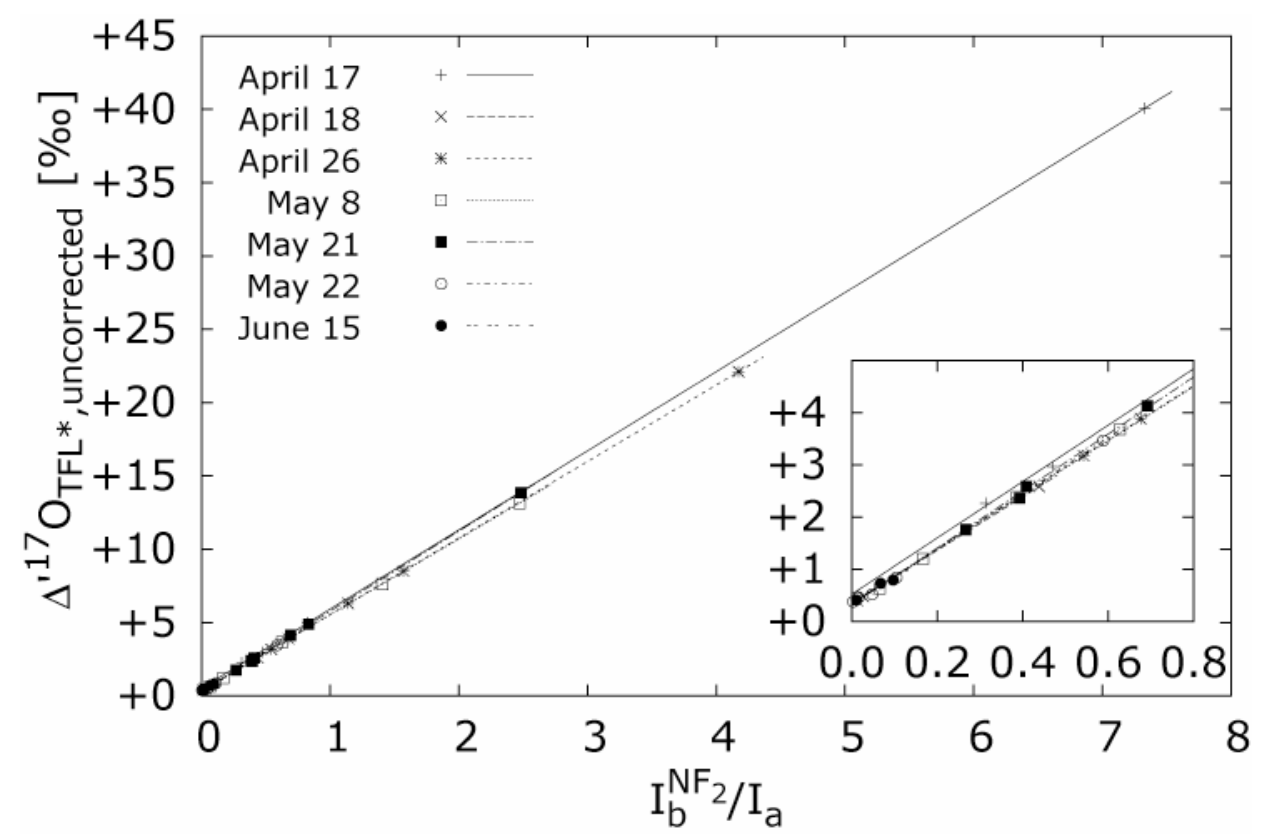

Figure 5. Plot of the uncorrected $\Delta^{, 17} \mathrm{O}_{\mathrm{TFL}} *$ values vs the $\mathrm{I}_{\mathrm{b}}^{\mathrm{NF}_{2}} / \mathrm{I}_{\mathrm{a}}$ intensity ratios. The linear regression lines are shown for the different days.

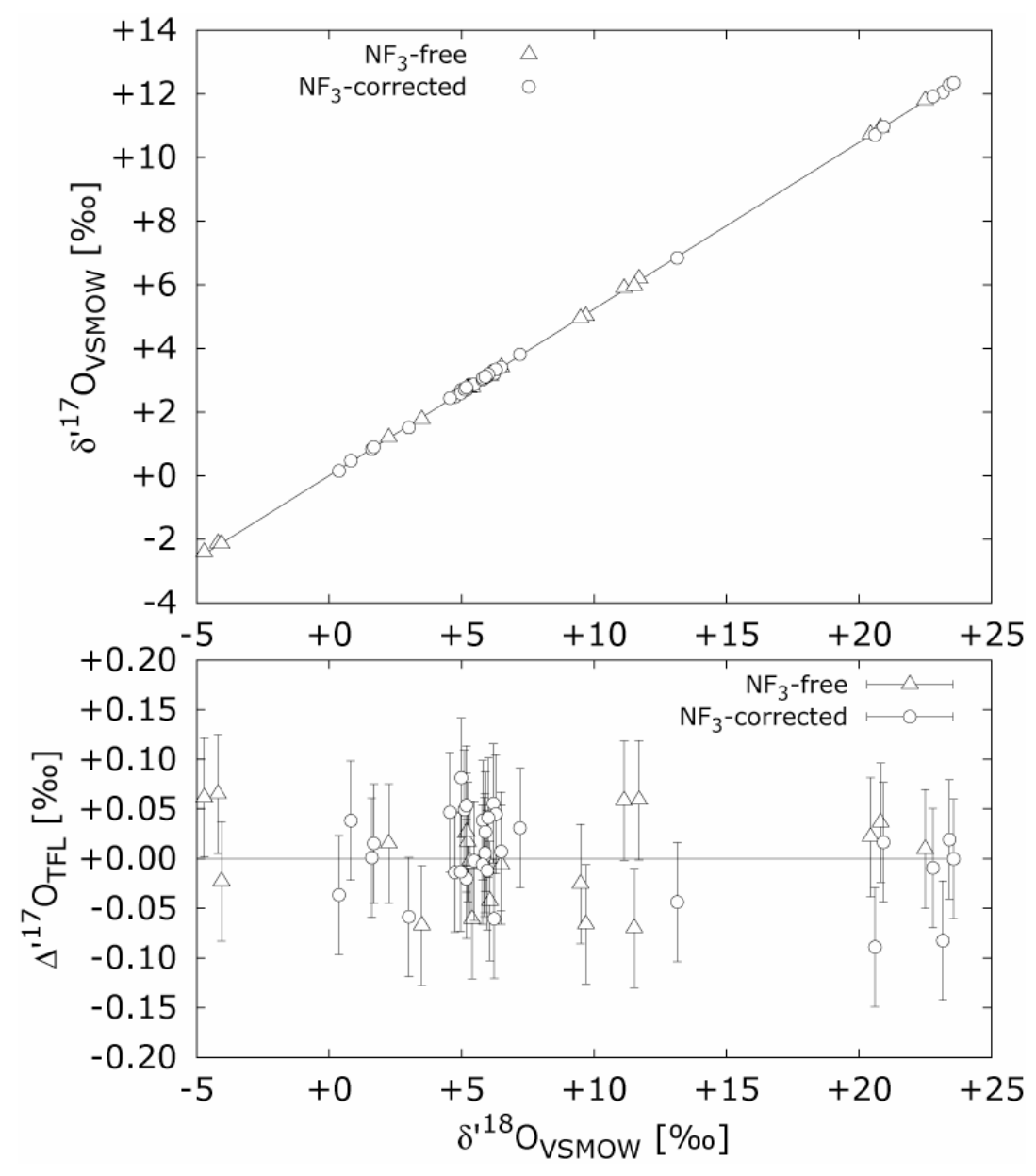

Figure 6. (top) Plot showing the corrected $\delta^{, 17} \mathrm{O}_{\text {VSMOw }}$ Vs $\delta^{, 18} \mathrm{O}_{\text {VSMOw }}$ of terrestrial mineral and rock samples $(\mathrm{N}=52)$. The data fall on a single line with slope $\beta=0.5237$. (bottom) Plot of $\Delta^{17} \mathrm{O}_{\text {TFL VS }}$ $\delta^{, 18} \mathrm{O}_{\text {VSMOw }}$ of the same samples. The vertical error bars correspond to an uncertainty of $\pm 0.06 \%$ in $\Delta{ }^{, 17} \mathrm{O}_{\mathrm{TFL}}$. 


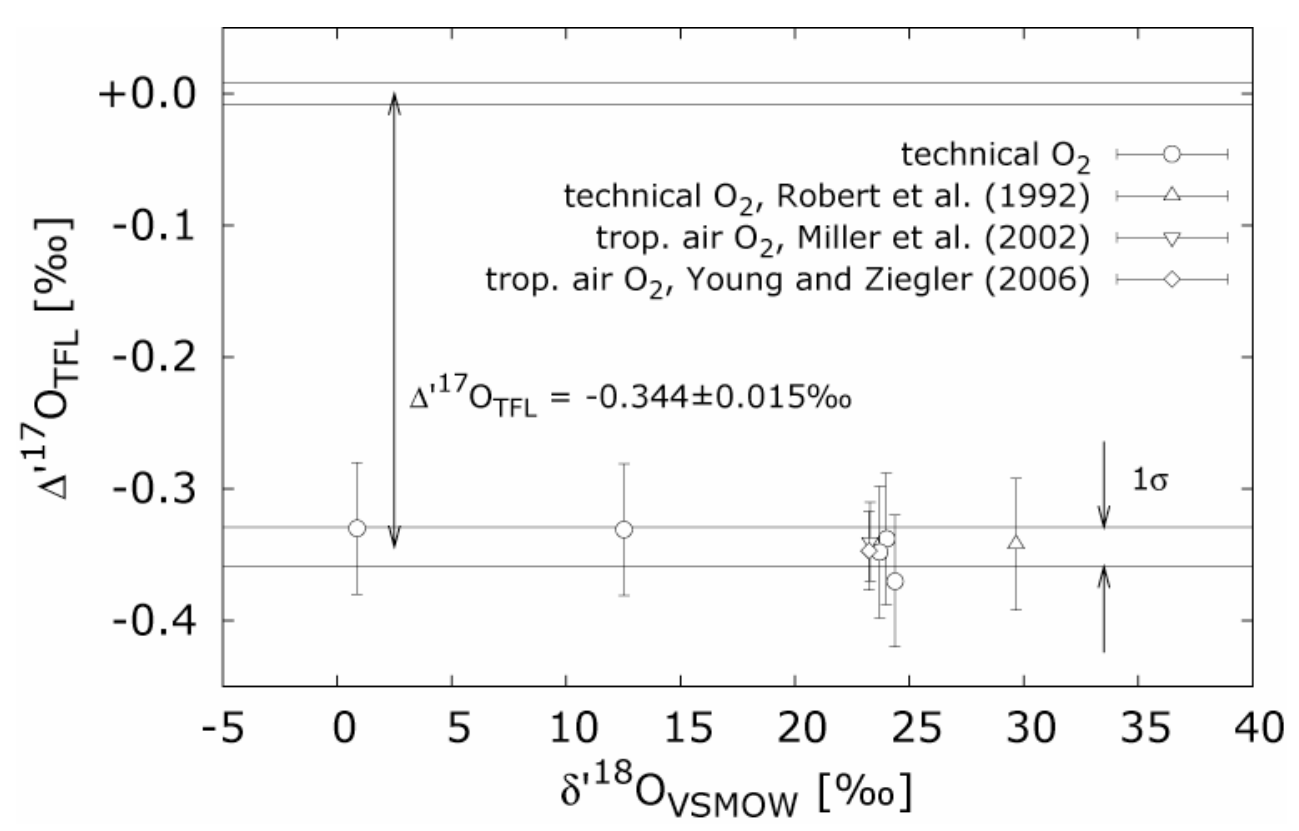

Figure 7. Plot of $\Delta{ }^{, 17} \mathrm{O}_{\mathrm{TFL}}$ VS $\delta^{, 18} \mathrm{O}_{\mathrm{VSMOW}}$ of technical $\mathrm{O}_{2}$ (this study; Robert et al. ${ }^{20}$ ) and of tropospheric $\mathrm{O}_{2}$ from Miller et al. ${ }^{18}$ and Young and Ziegler ${ }^{39}$. The error for the mean value of rocks and minerals on the TFL was calculated using data from this study $(\mathrm{N}=52)$ and data from Robert et al. ${ }^{20}$ (excluding water analyses).

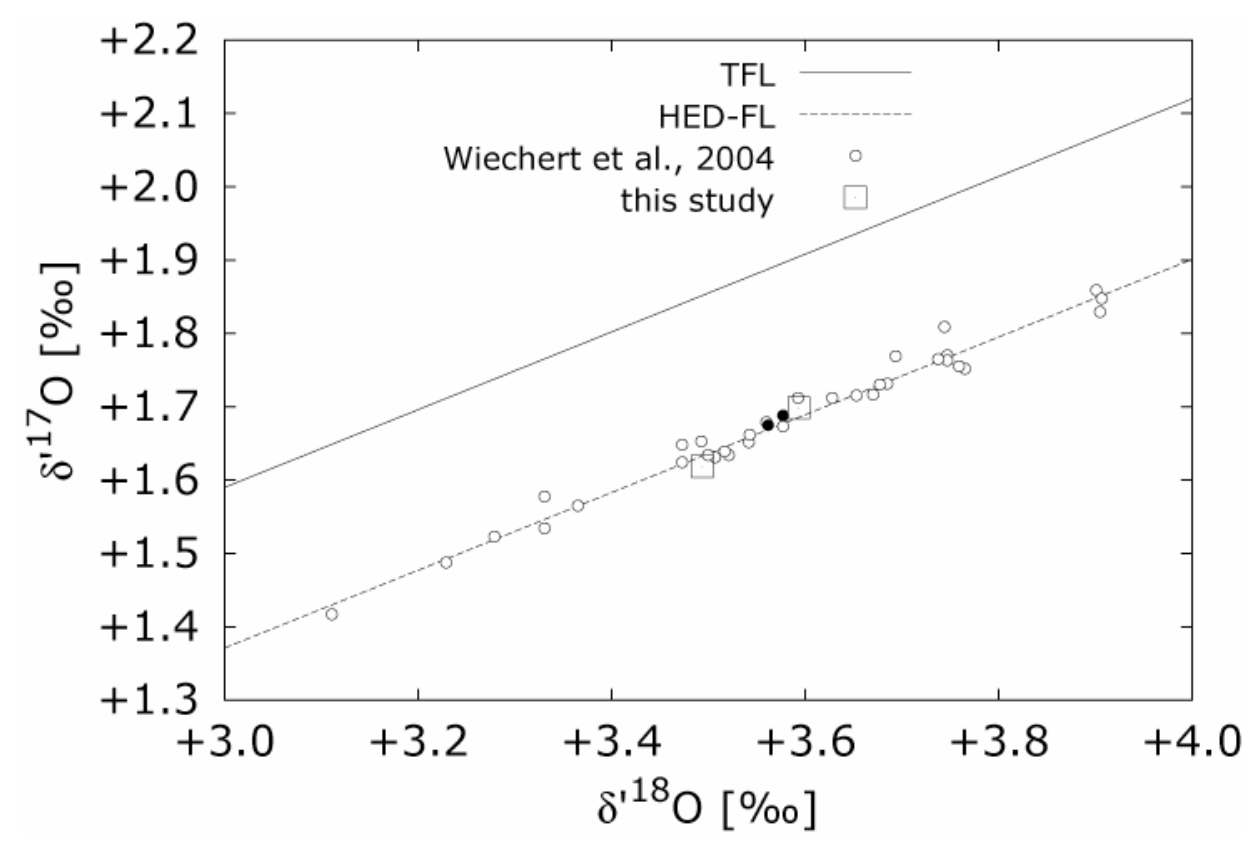

Figure 8. Oxygen three isotope diagram showing data from HED meteorites from Wiechert et al. ${ }^{27}$ (open circles), including Juvinas (solid circles). Juvinas data from this study are shown as open squares. HED-FL denotes the HED parent body mass fractionation line. 


\title{
14 Curriculum Vitae
}

\author{
Persönliche Daten Christoph Toulouse \\ Geburtsdatum/-ort: 21.11.1975 in Aachen \\ Familienstand: ledig \\ Nationalität: deutsch/französisch
}

Sprachen

Muttersprachen Deutsch und Französisch

Schulbildung

06.1997

Abitur am Maria-Wächtler-Gymnasium in Essen

\section{Zivildienst}

$08.1997-08.1998$

Schwerbehindertenfahrdienst beim Deutschen Roten Kreuz in Essen

Hochschulbildung

$10.1998-12.2004$

Studium der Geographie, Geologie und Kartographie/Fernerkundung an der RuhrUniversität Bochum

Diplom-Gesamtnote: ,sehr gut“

$11.2005-12.2007$

Promotion im Hauptfach Mineralogie mit den verwandten Fachgebieten Isotopengeologie und Kristallographie an der Leibniz Universität Hannover und der GeorgAugust-Universität Göttingen

Note Dissertation: ,sehr gut“"

Note Disputation: ,,sehr gut"“

\section{Anstellungen}

$09.2004-09.2005$

Zunächst Anstellung als studentische Hilfskraft, später als Dipl.-Geogr. in der Arbeitsgruppe Geotechnik der Emscher und Lippe Gesellschaft für Wassertechnik mbH in Essen

$11.2005-03.2007$

Anstellung als wissenschaftlicher Mitarbeiter am Institut für Mineralogie an der Leibniz Universität Hannover

$04.2007-14.09 .2007$

Anstellung als wissenschaftlicher Mitarbeiter am Geowissenschaftlichen Zentrum, Abteilung Isotopengeologie der Georg-August-Universität Göttingen

seit 15.09.2007

Anstellung als Fachkoordinator Metallurgie im Direktionsbereich Rohstahl der ThyssenKrupp Steel AG in Duisburg 